University of Tennessee Health Science Center UTHSC Digital Commons

\title{
$5-2015$
}

\section{Functional Activity and Switching of Novel Cannabinergic Ligands}

Bret Alan Koertge

University of Tennessee Health Science Center

Follow this and additional works at: https://dc.uthsc.edu/dissertations

Part of the Medicinal and Pharmaceutical Chemistry Commons

\section{Recommended Citation}

Koertge, Bret Alan , "Functional Activity and Switching of Novel Cannabinergic Ligands" (2015). Theses and Dissertations (ETD). Paper 364. http://dx.doi.org/10.21007/etd.cghs.2015.0164.

This Dissertation is brought to you for free and open access by the College of Graduate Health Sciences at UTHSC Digital Commons. It has been accepted for inclusion in Theses and Dissertations (ETD) by an authorized administrator of UTHSC Digital Commons. For more information, please contact jwelch30@uthsc.edu. 


\title{
Functional Activity and Switching of Novel Cannabinergic Ligands
}

\author{
Abstract \\ Pursuant to the discovery of the cannabinoid receptors, research in this field has grown exponentially over \\ the last 2 decades. With their utility in various disease states such as heart disease, cancer, stroke, \\ neurodegenerative and inflammation, cannabinoids stand poised to become a great therapeutic agent. \\ This research seeks to better understand the functional mechanism of cannabinoids, in the hopes of \\ ascertaining which molecular attributes confer desirable selectivity and functional activity. \\ Looking first at classical benzchromene core analogues, we have shown that presence of an aromatic \\ substitution at $\mathrm{C}-1$ ' imparts a $\mathrm{CB} 1$ agonist, $\mathrm{CB} 2$ antagonist. This is a unique mechanism and one of the \\ first examples of an agent with dual affinity and opposing activity. \\ In the triaryl series of compounds, more insight is gained for the functional pharmacophore of \\ cannabinoids. With this series of compounds we are able to learn that minor changes are able to confer \\ profound differences, such as C-1'-gem-dimethyl derivatives are agonists while C-1' ketones are inverse \\ agonists. \\ Within these triaryl series, newly synthesized derivatives help to round out the functional pharmacology \\ for these compounds - allowing a more direct comparison to the previously synthesized classical core \\ compounds. In total, a more thorough understanding of the function, which follows form of several \\ cannabinergic compounds, is gained through this exercise and research. \\ Document Type \\ Dissertation \\ Degree Name \\ Doctor of Philosophy (PhD) \\ Program \\ Pharmaceutical Sciences \\ Research Advisor \\ Bob M. Moore, II, Ph.D. \\ Keywords \\ ACTOne, Cannabinoid, Functional Activity, Homology, Pharmacology, THC

\section{Subject Categories} \\ Medicinal and Pharmaceutical Chemistry | Medicine and Health Sciences | Pharmacy and Pharmaceutical \\ Sciences

\section{Comments} \\ One year embargo expired May 2016
}


FUNCTIONAL ACTIVITY AND SWITCHING OF NOVEL CANNABINERGIC LIGANDS

\author{
A Dissertation \\ Presented for \\ The Graduate Studies Council \\ The University of Tennessee \\ Health Science Center
}

\author{
In Partial Fulfillment \\ Of the Requirements for the Degree \\ Doctor of Philosophy \\ From The University of Tennessee
}

By

Bret Alan Koertge

May 2015 
Copyright (C) 2015 by Bret Alan Koertge. All rights reserved. 


\section{DEDICATION}

This dissertation is dedicated to my loving and supportive wife, Stephanie Nicole Koertge, who has endured, enabled and pushed me for all the years of research this document represents.

Also, to my children, may you always keep your curiosity and wit. 


\section{ACKNOWLEDGEMENTS}

I would like to take this opportunity to thank my major professor, Dr. Bob M. Moore, II for his guidance, support and motivation-by-any-means. Additionally, I wish to extend an enormous debt of gratitude and gratefulness to my remaining committee members, Dr. Isaac Donkor, Dr. Wei Li, Dr. Trevor Sweatman and Dr. George Wood, whose guidance during the research, testing and writing processes has been invaluable and instrumental in the success of this document's culmination.

I would also like to thank my colleagues, Dr. Chaela S. Presley, Dr. Steven N. Gurley, Dr. Suni M. Mustafa and Ammaar H. Abidi for their support and the countless useful discussions provided by each throughout the years. I also extend a debt of gratitude and thanks to past members of our lab group for their work in synthesis, which afforded me the compounds with which to perform the functional tests encompassed herein: Dr. Himanshu Bhattacharjee, Dr. Mathingi Krishmanurthy and Dr. Asha Nadipuram. Also, I extend a special thank you to Peihong Guan for teaching me proper cell culture techniques, which served me well during this work.

Knowing that I would not be who or where I am today without the love and support of my family, I would be remiss if a heartfelt thanks was not given to them. I wish to extend the deepest gratitude to my mother, Karen Pinkstaff, father, Larry Koertge, stepmother, Sherri Koertge, sister, Tara Cardinal and brother, Jordan Kohlhouse for all their moral support and guidance over the years of academic pursuit that has led to penning this document into existence. Last, but certainly not least, to my wife and children who have endured evenings and nights without me as I completed research or pounded away on a keyboard, and to them I give my ineffable thanks as we look forward to our next great adventure.

Finally, I wish to thank the College of Pharmacy at the University of Tennessee, Health Science Center for affording me the opportunity to conduct my research and expand my academic horizons. 


\begin{abstract}
Pursuant to the discovery of the cannabinoid receptors, research in this field has grown exponentially over the last 2 decades. With their utility in various disease states such as heart disease, cancer, stroke, neurodegenerative and inflammation, cannabinoids stand poised to become a great therapeutic agent. This research seeks to better understand the functional mechanism of cannabinoids, in the hopes of ascertaining which molecular attributes confer desirable selectivity and functional activity.

Looking first at classical benzchromene core analogues, we have shown that presence of an aromatic substitution at C-1' imparts a CB1 agonist, CB2 antagonist. This is a unique mechanism and one of the first examples of an agent with dual affinity and opposing activity.

In the triaryl series of compounds, more insight is gained for the functional pharmacophore of cannabinoids. With this series of compounds we are able to learn that minor changes are able to confer profound differences, such as C-1'-gem-dimethyl derivatives are agonists while $\mathrm{C}-1$ ' ketones are inverse agonists.

Within these triaryl series, newly synthesized derivatives help to round out the functional pharmacology for these compounds - allowing a more direct comparison to the previously synthesized classical core compounds. In total, a more thorough understanding of the function, which follows form of several cannabinergic compounds, is gained through this exercise and research.
\end{abstract}




\section{TABLE OF CONTENTS}

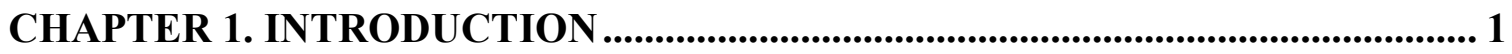

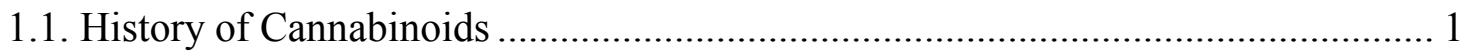

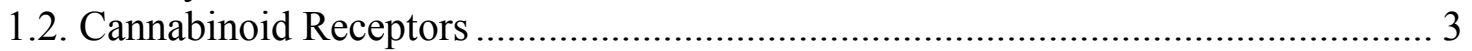

1.2.1. History and background of cannabinoid receptors........................................ 3

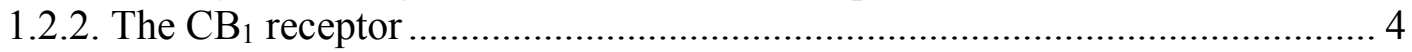

1.2.3. The $\mathrm{CB}_{2}$ receptor .............................................................................. 5

1.2.4. The GPR55 receptor - A putative cannabinoid receptor ……………….......... 5

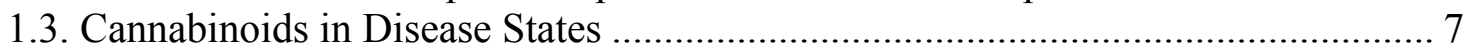

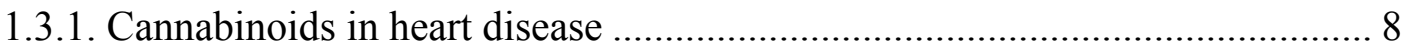

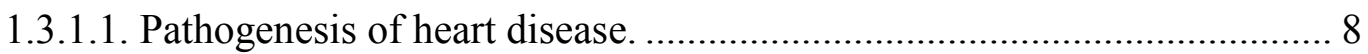

1.3.1.2. Cannabinoids as anti-hypertensive agents............................................. 8

1.3.1.3. Cannabinoids as anti-ischemic agents post-MI..................................... 9

1.3.1.4. Cannabinoids as anti-inflammatory agents post-MI. .............................. 9

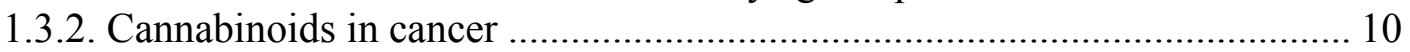

1.3.2.1. Cancer rates in The United States of America. ...................................... 10

1.3.2.2. Cannabinoids in lung and bronchial cancers......................................... 10

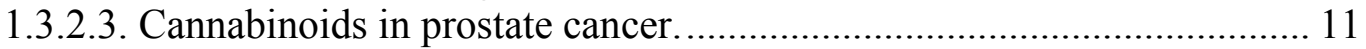

1.3.2.4. Cannabinoids in breast cancer.......................................................... 11

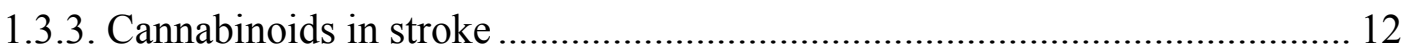

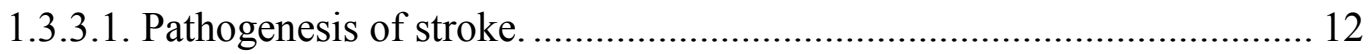

1.3.3.2. Cannabinoids as anti-inflammatory agents post-stroke........................... 12

1.3.3.3. Cannabinoids as anti-ischemic agents post-stroke. .............................. 13

1.3.4. Cannabinoids in Alzheimer's Disease......................................................... 14

1.3.4.1. Background of Alzheimer's Disease.................................................. 14

1.3.4.2. Cannabinoids in Alzheimer's Disease................................................... 14

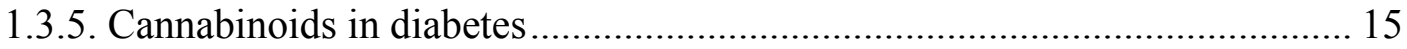

1.3.5.1. Endocannabinoids' control of metabolism.......................................... 15

1.3.5.2. Endocannabinoids in type-2 diabetes and metabolic disorders.............. 16

1.3.5.3. Endocannabinoids in abdominal obesity and hyperglycemia. ................ 17

1.3.5.4. Use of $\mathrm{CB}_{1}$ antagonists and inverse agonists in type-2 diabetes............ 18

1.4. Goals of the Current Project .......................................................................... 19

\section{CHAPTER 2. ASSAY DEVELOPMENT FOR THE FUNCTIONAL ACTIVITY ANALYSIS OF NOVEL CANNABINERGIC COMPOUNDS.................................... 21}

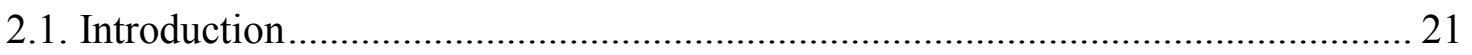

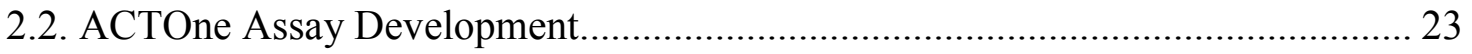

2.2.1. Manufacturer recommended procedure ....................................................... 23

2.2.1.1. Commonalities of ACTOne assay protocols......................................... 23

2.2.1.2. $\mathrm{CB}_{1}$-specific parameters of the ACTOne assay. ................................... 23

2.2.1.3. $\mathrm{CB}_{2}$-specific parameters of the ACTOne assay. ..................................... 23

2.2.1.4. Parental, HEK-CNG-specific parameters of the ACTOne assay........... 24

2.2.2. Assay stimulus standardization ............................................................. 24

2.2.2.1. Manufacturer recommended cell stimulus. ........................................... 24

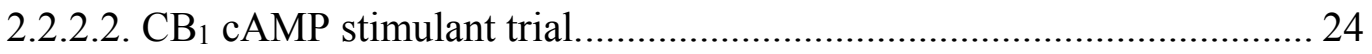




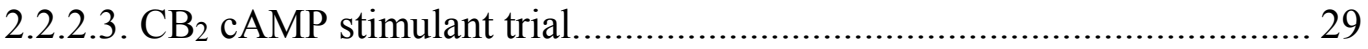

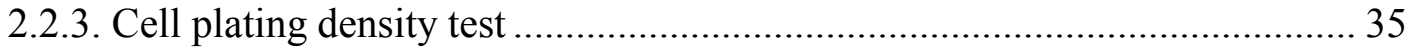

2.2.4. Assay plate selection ......................................................................... 40

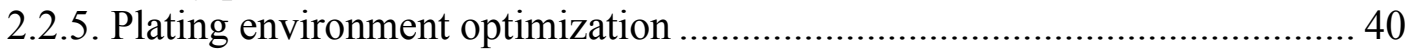

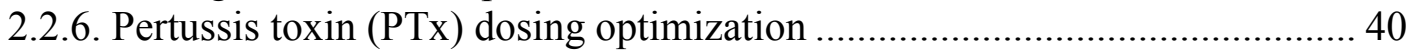

2.2.6.1. Duration of PTx pre-treatment. ......................................................... 41

2.2.6.2. Addition order of PTx treatment. ........................................................ 41

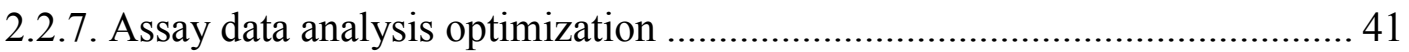

2.2.7.1. GraphPad Prism template building.................................................... 46

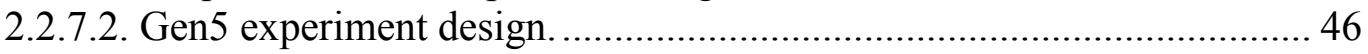

2.2.7.3. Gen5 "Power Export" template building................................................ 47

2.2.7.4. Master extraction template. ................................................................ 50

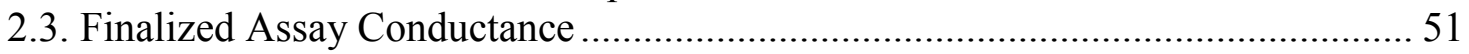

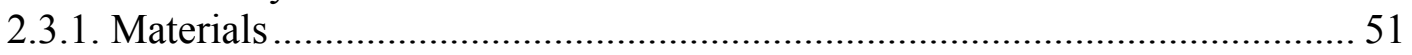

2.3.2. Cell culture and frozen cell preparation ...................................................... 51

2.3.3. CNG channel-coupled cAMP assay …………......................................... 51

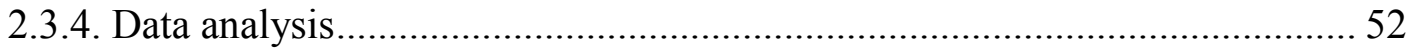

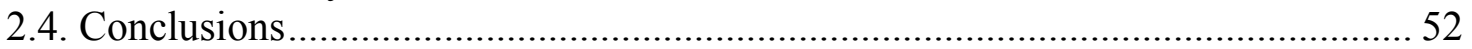

\section{CHAPTER 3. FUNCTIONAL EVALUATION OF A NOVEL CLASS OF} PHENYL-SUBSTITUTED SIDE CHAIN ANALOGUES OF $\Delta^{8}$-THC .....................53

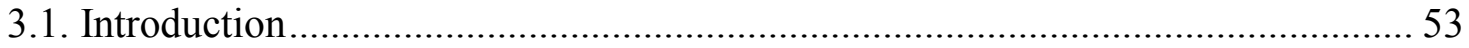

3.2. Classical Cannabinoids - Modifying C-3 Substituents to Increase Binding ......... 56

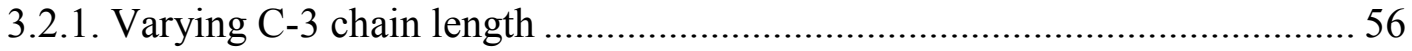

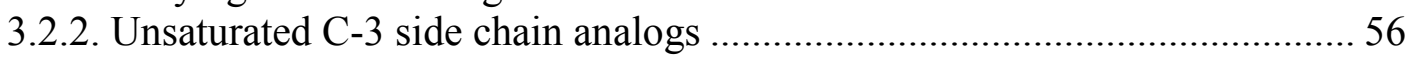

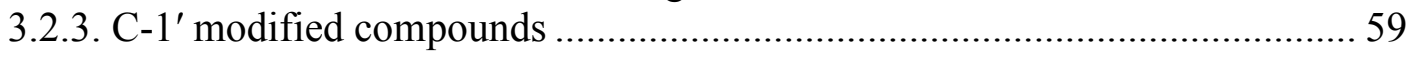

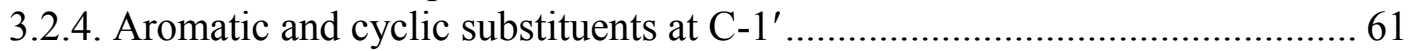

3.3. Novel Series of C-1'-Dimethyl-Aryl- $\Delta^{8}$-THC Analogs (KM Series) ..................... 64

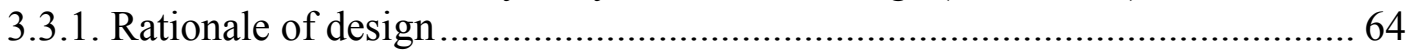

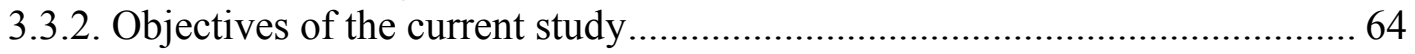

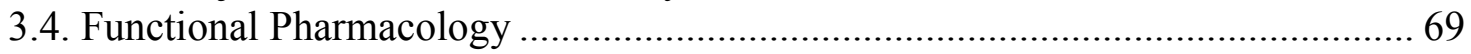

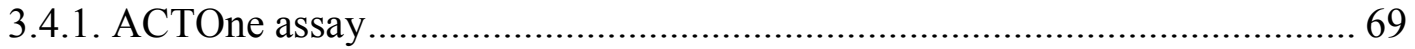

3.4.2. $\mathrm{CB}_{1}$ functional activity for the $\mathrm{KM}$ series ........................................................ 69

3.4.3. $\mathrm{CB}_{2}$ functional activity for the $\mathrm{KM}$ series .................................................. 74

3.4.4. CNG functional activity for the $\mathrm{KM}$ series ................................................ 74

3.4.5. $\mathrm{CB}_{1} \mathrm{PTx}$-sensitive $\mathrm{G}_{\mathrm{i} / \mathrm{o}}$-inactivation for the $\mathrm{KM}$ series ................................. 74

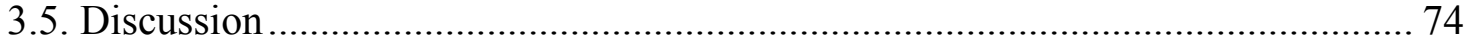

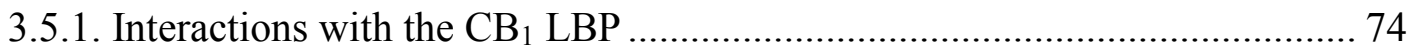

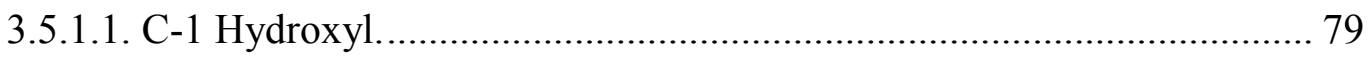

3.5.1.2. C-3 lipophilic side chain................................................................... 79

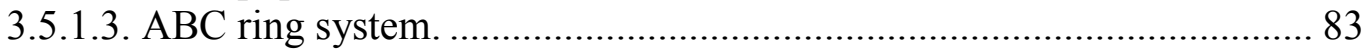

3.5.1.4. (Optional) C-11 ("Northern") hydroxyl. ............................................... 83

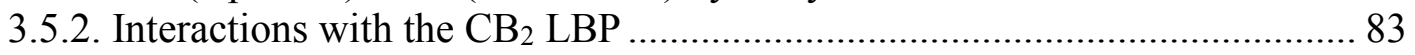

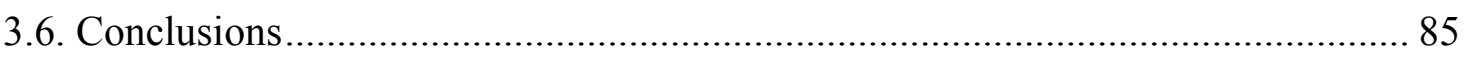

CHAPTER 4. FUNCTIONAL EVALUATION OF A NOVEL CLASS OF HETEROCYCLIC CORE ANALOGUES OF HEXAHYDRO $\Delta^{8}$-THC .................... 88 


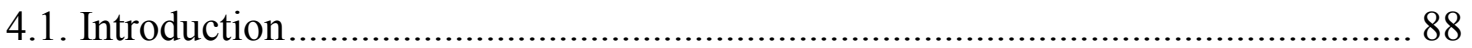

4.2. Classical Cannabinoids - Modifying the $\mathrm{A} / \mathrm{B} / \mathrm{C}$ Ring ........................................... 89

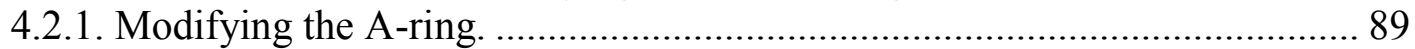

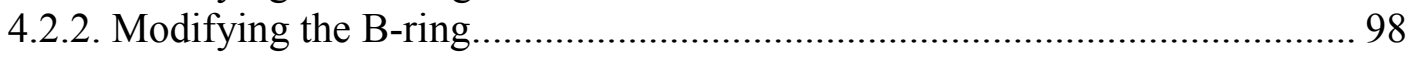

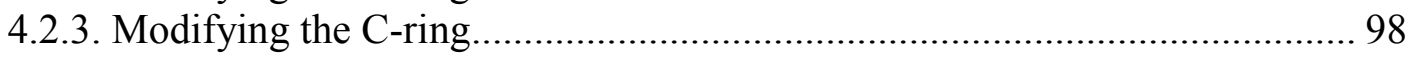

4.3. Non-Classical Cannabinoids - Heterocyclic Ring-Containing Compounds ....... 100

4.3.1. Aminoalkylindoles (AAIs) ............................................................... 100

4.3.1.1. N-1 substitutions of aminoalkylindoles............................................. 104

4.3.1.2. C-2 substituents of aminoalkylindoles................................................ 104

4.3.1.3. C-3 substituents of aminoalkylindoles. ............................................... 106

4.3.1.4. Indole Ring Substituents and Modifications........................................ 109

4.3.2. Diarylpyrazoles ...................................................................................... 109

4.3.2.1. N-1 substituents of diarylpyrazoles...................................................... 111

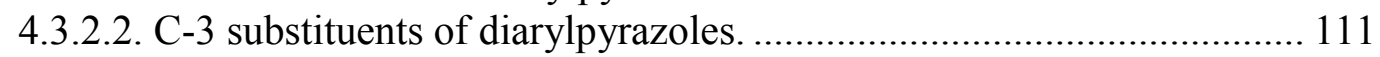

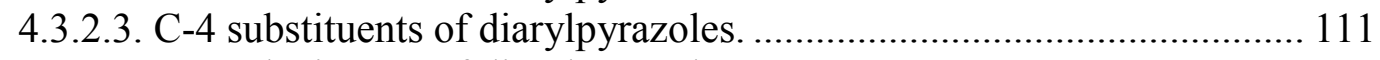

4.3.2.4. C-5 substituents of diarylpyrazoles. .................................................... 115

4.4. Novel Series of C-1'-Dimethyl-Hexahydro-Pyridine/Pyrimidine-THC Analogs

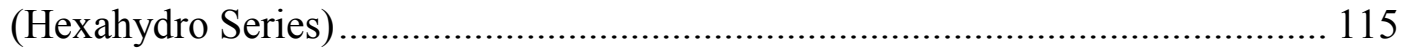

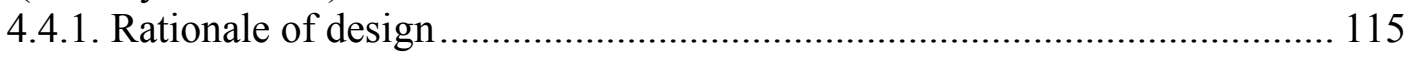

4.4.2. Objectives of the current study ………………….................................. 120

4.5. Functional Pharmacology ............................................................................... 120

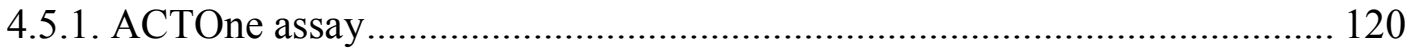

4.5.2. $\mathrm{CB}_{1}$ functional activity for the Hexahydro series ....................................... 122

4.5.3. $\mathrm{CB}_{2}$ functional activity for the Hexahydro series........................................ 122

4.5.4. CNG parental functional activity for the Hexahydro series ......................... 122

4.5.5. $\mathrm{CB}_{1} \mathrm{PTx}$-sensitive $\mathrm{G}_{\mathrm{i} / \mathrm{o}}$-inactivation for the Hexahydro series ..................... 122

4.5.6. $\mathrm{CB}_{2} \mathrm{PTx}$-sensitive $\mathrm{G}_{\mathrm{i} / \mathrm{o}}$-inactivation for the Hexahydro series .................... 130

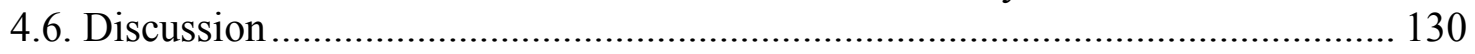

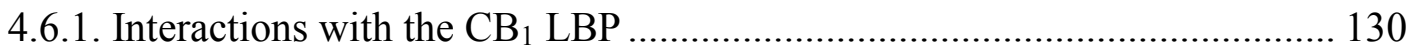

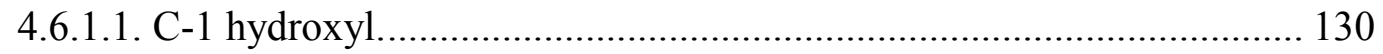

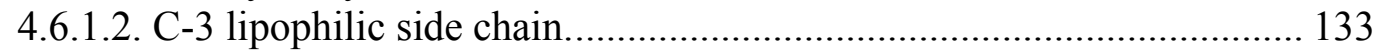

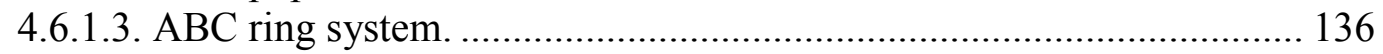

4.6.1.4. (Optional) C-11 ("Northern") hydroxyl. .............................................. 136

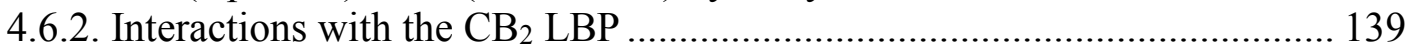

4.6.2.1. C-1 Hydroxyl, benzchromene oxygen and (optional) C-6 hydroxyl.... 139

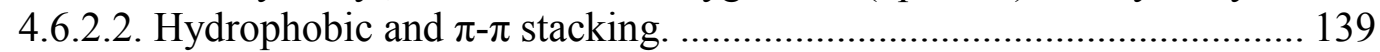

4.6.2.3. Proposed interactions of the hexahydro series with the $\mathrm{CB}_{2} \mathrm{LBP}$....... 139

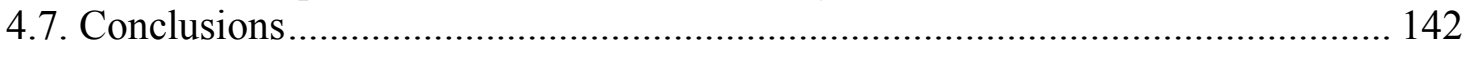

\section{CHAPTER 5. FUNCTIONAL EVALUATION OF A NOVEL CLASS OF TRIARYL CANNABINERGIC LIGANDS}

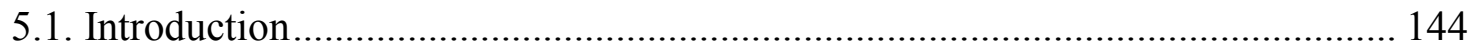

5.2. Non-Classical Cannabinoids .......................................................................... 146

5.2.1. AC-bicyclic and ACD-tricyclic compounds ............................................. 146

5.2.2. Hybrid cross-over compounds................................................................ 146

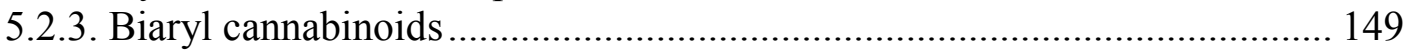

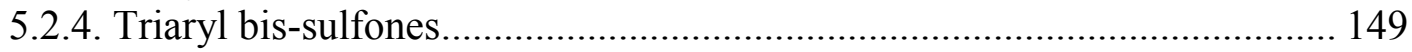


5.3. Novel Series of Triaryl Cannabaergic Ligands ................................................. 152

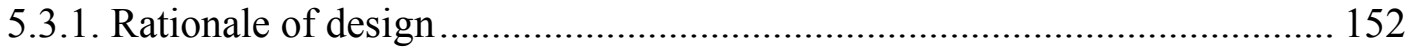

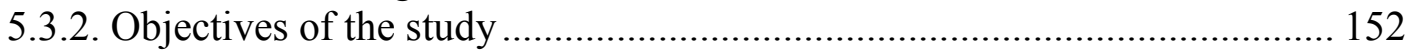

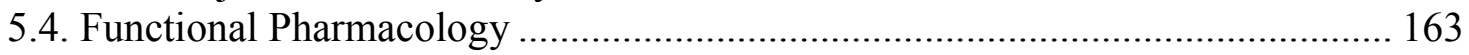

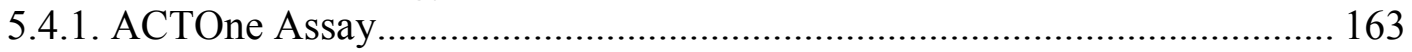

5.4.2. Functional activity for HB triaryl series .................................................... 163

5.4.2.1. $\mathrm{CB}_{1}$ functional activity for the $\mathrm{HB}$ series............................................ 163

5.4.2.2. $\mathrm{CB}_{2}$ functional activity for the HB Series. ........................................ 167

5.4.2.3. CNG parental functional activity for the HB series. ........................... 167

5.4.2.4. $\mathrm{CB}_{1} \mathrm{PTx}$-sensitive $\mathrm{G}_{\mathrm{i} / \mathrm{o}}$-inactivation for the $\mathrm{HB}$ series. ......................... 167

5.4.2.5. $\mathrm{CB}_{2} \mathrm{PTx}$-sensitive $\mathrm{G}_{\mathrm{i} / \mathrm{o}}$-inactivation for the HB series......................... 167

5.4.3. Functional activity for SMM Triaryl series................................................. 167

5.4.3.1. $\mathrm{CB}_{1}$ functional activity for the SMM series. ...................................... 167

5.4.3.2. $\mathrm{CB}_{2}$ functional activity for the SMM series...................................... 175

5.4.3.3. CNG parental functional activity for the SMM series. ........................ 175

5.4.3.4. $\mathrm{CB}_{1}$ PTx-sensitive $\mathrm{G}_{\mathrm{i} / \mathrm{o}}$-inactivation for the SMM series. ..................... 175

5.4.3.5. $\mathrm{CB}_{2} \mathrm{PTx}$-sensitive $\mathrm{G}_{\mathrm{i} / \mathrm{o}}$-inactivation for the SMM series. …………...... 175

5.4.4. Functional activity for pyridine triaryl series ............................................ 175

5.4.4.1. $\mathrm{CB}_{1}$ functional activity for the Pyridine Triaryl series.......................... 175

5.4.4.2. $\mathrm{CB}_{2}$ functional activity for the pyridine triaryl series.......................... 184

5.4.4.3. CNG parental functional activity for the pyridine triaryl series........... 184

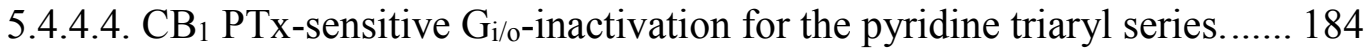

5.4.4.5. $\mathrm{CB}_{2} \mathrm{PTx}$-sensitive $\mathrm{G}_{\mathrm{i} / \mathrm{o}}$-inactivation for the pyridine triaryl series....... 184

5.4.5. Functional activity for pyrimidine triaryl series .......................................... 192

5.4.5.1. CB1 functional activity in the pyrimidine triaryl series....................... 192

5.4.5.2. $\mathrm{CB}_{2}$ functional activity in the pyrimidine triaryl series........................ 192

5.4.5.3. CNG parental functional activity in the pyrimidine triaryl series........ 192

5.4.5.4. $\mathrm{CB}_{1} \mathrm{PTx}$-sensitive $\mathrm{G}_{\mathrm{i} / \mathrm{o}}$-inactivation in the pyrimidine triaryl series.... 192

5.4.5.5. $\mathrm{CB}_{2}$ PTx-sensitive $\mathrm{G}_{\mathrm{i} / \mathrm{o}}$-inactivation in the pyrimidine triaryl series.... 192

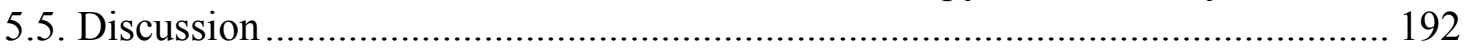

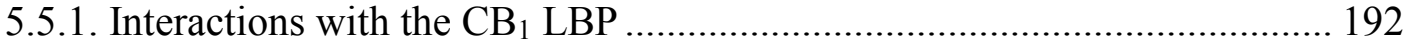

5.5.1.1. $\mathrm{CB}_{1}$ LBP interactions with non-classical ketone moiety....................... 192

5.5.1.2. $\mathrm{CB}_{1} \mathrm{LBP} \pi-\pi$ stacking interactions.................................................... 200

5.5.1.3. $\mathrm{CB}_{1}$ LBP additional known interactions of non-classical agents. ........ 202

5.5.1.4. $\mathrm{CB}_{1}$ LBP interactions of the hydrophobic pocket. .............................. 202

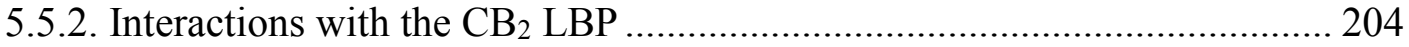

5.5.2.1. C-1 Hydroxyl, Benzchromene Oxygen and (optional) C-6 Hydroxyl. 204

5.5.2.2. Hydrophobic and $\pi-\pi$ stacking. ......................................................... 205

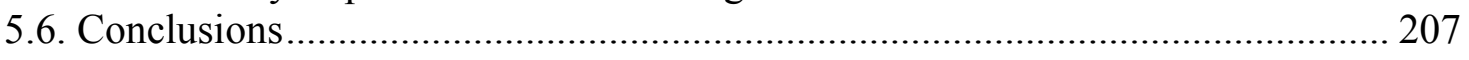

CHAPTER 6. FUNCTIONAL PHARMACOPHORE ............................................... 210

6.1. $\mathrm{CB}_{1}$-Specific Proposed Functional Switches ……………………………….... 210

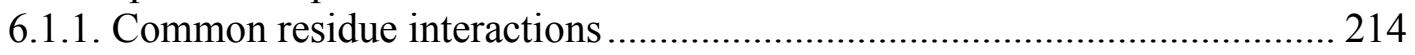

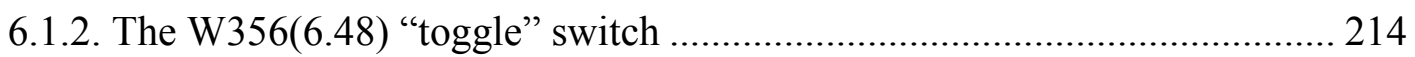

6.1.3. The lack of hydrophilic interaction within the LBP .................................... 218

6.1.4. C-1' importance beyond binding affinity ………..................................... 218

6.2. CB2-Specific Proposed Functional Switches ....................................................... 222 
6.2.1. Common residue interactions

6.2.2. The Di-hydroxy A-ring

6.2.3. The C-1' substituent effect

225

6.2.4. The D-ring substituent effect......................................................... 225

6.3. Summary

225

LIST OF REFERENCES ......................................................................................... 229

VITA 


\section{LIST OF TABLES}

Table 3.1. Binding affinities for the KM series of compounds..................................68

Table 3.2. $\quad \mathrm{EC}_{50}$ values for $\mathrm{KM}$ series functional assays ............................................. 71

Table 4.1. $\quad \mathrm{CB}_{1} / \mathrm{CB}_{2} \mathrm{~K}_{\mathrm{i}}$ ratios for selected compounds........................................95

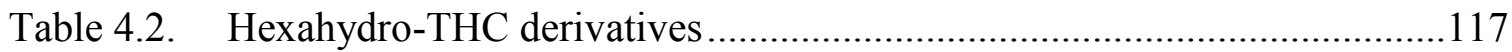

Table 4.3. Binding affinities for hexahydro series .............................................. 118

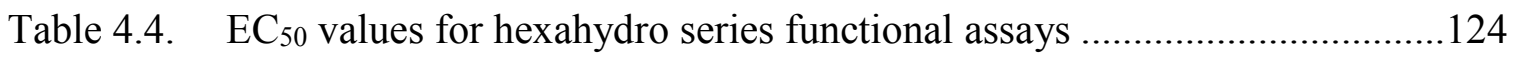

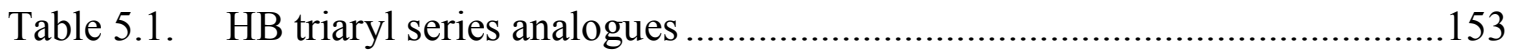

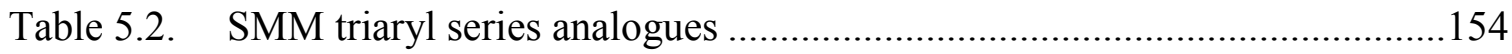

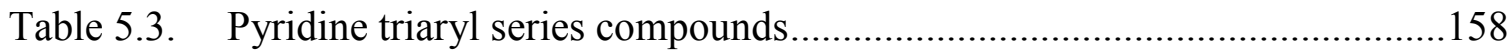

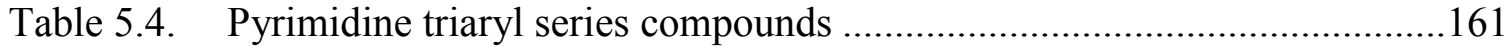

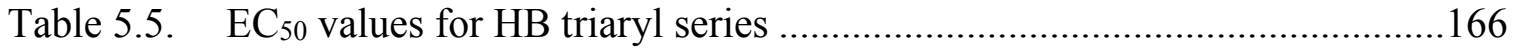

Table 5.6. $\quad \mathrm{EC}_{50}$ values for SMM triaryl series...................................................... 174

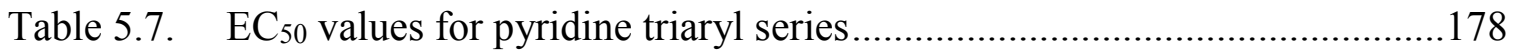

Table 5.8. $\quad$ EC $_{50}$ values for pyrimidine triaryl series .............................................196 


\section{LIST OF FIGURES}

Figure 1.1. Structures of compounds isolated from Cannabis sativa ...............................2

Figure 1.2. Synthetic and endogenous cannabinoid compounds.....................................6

Figure 2.1. Isoproterenol $\mathrm{EC}_{50}$ curve at 50 minutes post-addition................................25

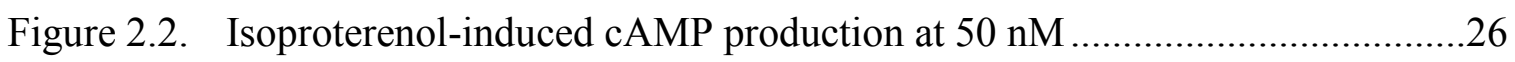

Figure 2.3. NECA $\mathrm{EC}_{50}$ curve at 50 minutes post-addition .........................................27

Figure 2.4. NECA-induced cAMP production in $\mathrm{CB}_{1}$ and $\mathrm{CB}_{2}$ cells ............................28

Figure 2.5. $\quad \mathrm{CB}_{1}$ adrenergic drug test for stimulation of cAMP production .....................30

Figure 2.6. $\mathrm{CB}_{2}$ adrenergic drug test for stimulation of cAMP production .....................31

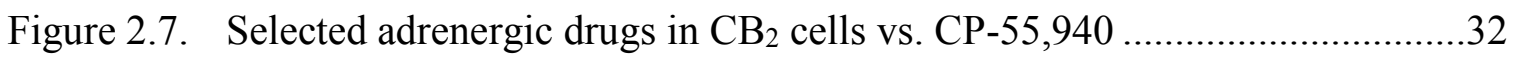

Figure 2.8. Manufacturer-recommended cAMP stimulants .............................................33

Figure 2.9. Forskolin as a cAMP stimulant in $\mathrm{CB}_{1}$ and $\mathrm{CB}_{2}$ cells .................................34

Figure 2.10. Forskolin response in $\mathrm{CB}_{1}$ and $\mathrm{CB}_{2}$ vs. time .........................................36

Figure 2.11. All viable cAMP stimuli at $500 \mathrm{nM}$ in $\mathrm{CB}_{1}$ and $\mathrm{CB}_{2}$ cells ..........................37

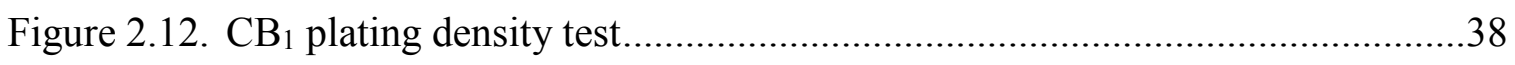

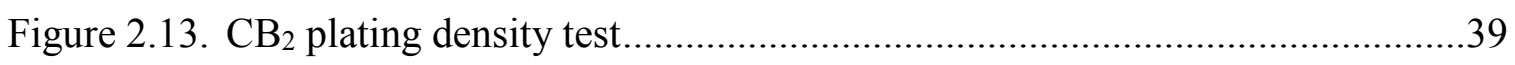

Figure 2.14. $\mathrm{CB}_{1}$ cells with 1 hour pretreatment of PTx vs. CP-55,940 ..........................42

Figure 2.15. $\mathrm{CB}_{1}$ cells with overnight PTx pretreatment vs. CP-55,940 .........................43

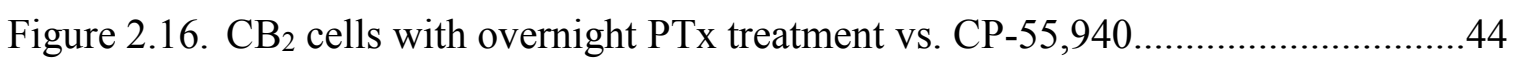

Figure 2.17. $\mathrm{CB}_{2}$ cells with PTx addition order test..................................................45

Figure 3.1. Classical cannabinoid with pharmacophore elements ...................................54

Figure 3.2. Design progression of the KM series of compounds .....................................55

Figure 3.3. $\mathrm{C}-3$ chain length analogues and the effect on $\mathrm{CB}_{1}$ binding affinity .............57

Figure 3.4. Unsaturated C-3 side chain analogs …………………….........................58 
Figure 3.5. Acidic and basic modifications to the terminus of $\Delta^{8}$-THC C-3 substituents

Figure 3.6. Cyclic modifications at $\mathrm{C}-1^{\prime}$ which affect $\mathrm{CB}_{1}$ and $\mathrm{CB}_{2}$ binding affinity ....62

Figure 3.7. Aromatic modifications of $\Delta^{8}-\mathrm{THC}$ at $\mathrm{C}-3$ which affect $\mathrm{CB}_{1}$ and $\mathrm{CB}_{2}$ binding affinity.....

Figure 3.8. Bicyclic and tricyclic $\mathrm{C}-3$ substituents of $\triangle 8-\mathrm{THC}$ that alter $\mathrm{CB}_{1}$ and $\mathrm{CB}_{2}$ binding affinity

Figure 3.9. Comparison of the conformational space for adamantyl and bornyl side chains of various $\triangle 8$-THC analogues

Figure 3.10. KM series analogues

Figure 3.11. Drug plate layout and concentrations

Figure 3.12. $\mathrm{CB}_{1}$ functional activity for meta-substituted $\mathrm{KM}$ series compounds .72

Figure 3.13. $\mathrm{CB}_{1}$ functional activity of para-substituted derivatives of KM series .73

Figure 3.14. $\mathrm{CB}_{1}$ functional activity of misc. KM series derivatives .75

Figure 3.15. $\mathrm{CB}_{2}$ functional activity of $\mathrm{KM}$ series compounds, 50 minutes after drug addition

Figure 3.16. CNG functional activity of KM series compounds, 50 minutes after compound addition.

Figure 3.17. $\mathrm{CB}_{1}$ PTx-sensitive $\mathrm{G}_{\mathrm{i} / \mathrm{o}}$-inactivation functional activity, 50 minutes after compound addition.

Figure 3.18. $\mathrm{CB}_{1} \mathrm{LBP}$ interactions with $\mathrm{C}-1$ hydroxyl and benzchromene oxygen .........80

Figure 3.19. $\mathrm{CB}_{1} \mathrm{LBP}$ proposed hydrophobic $\mathrm{C}-3$ pocket

Figure 3.20. $\mathrm{CB}_{1} \mathrm{LBP}$ proposed additional hydrophobic interactions of 67 with the $\mathrm{C}$ 3 pocket

Figure 3.21. $\mathrm{CB}_{1} \mathrm{LBP} \pi-\pi$ stacking interactions and key residue interactions

Figure 3.22. $\mathrm{CB}_{2}$ LBP steric hinderance of $c$ is and trans isomers of WIN-55,212-2 ......86

Figure 3.23. Comparison of analogous functional groups of WIN-55,212-2, KM 233 and WIN-55,212-3

Figure 4.1. Classical cannabinoid with pharmacophoric elements .90

Figure 4.2. Design progression for the hexahydro series of compounds .91 


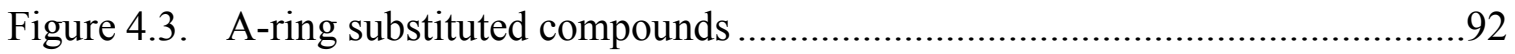

Figure 4.4. Amino and aminomethyl derivatives ...................................................94

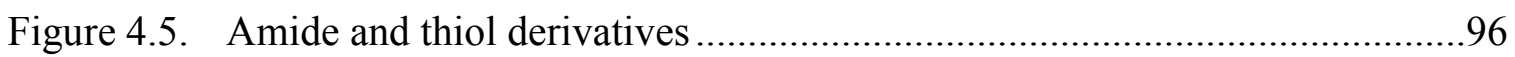

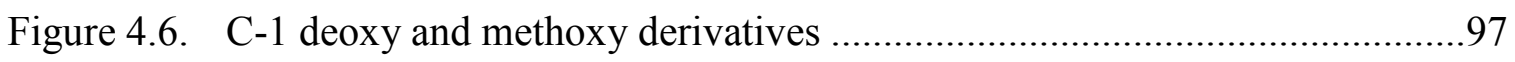

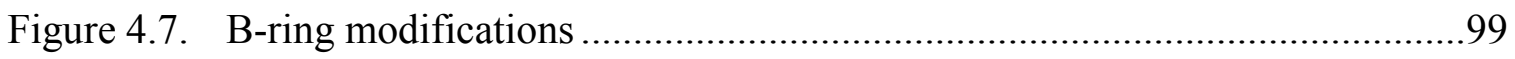

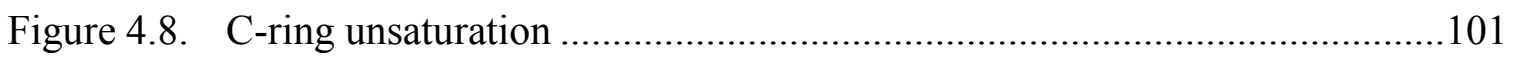

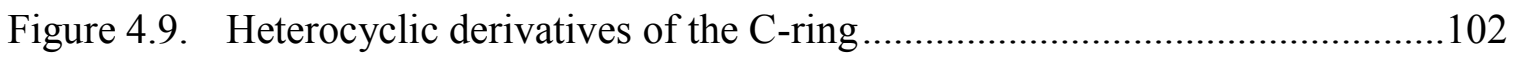

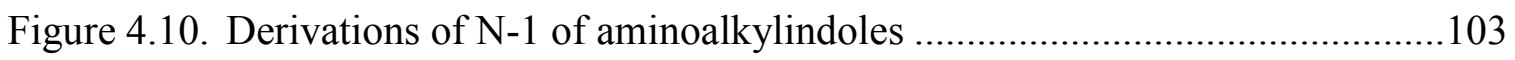

Figure 4.11. Modifications to C-2 of aminoalkylindoles ......................................... 105

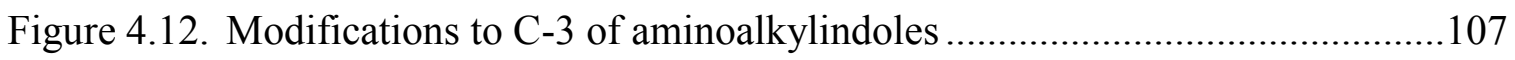

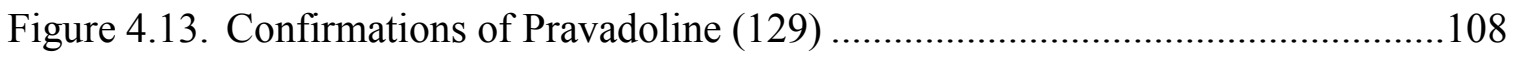

Figure 4.14. Known standards of diarylpyrazole class of cannabinoid ligands .............110

Figure 4.15. Alkyl N-1 modifications of diarylpyrazoles .......................................112

Figure 4.16. Aromatic and cycloalkyl modifications to N-1 of diarylpyrazoles.............113

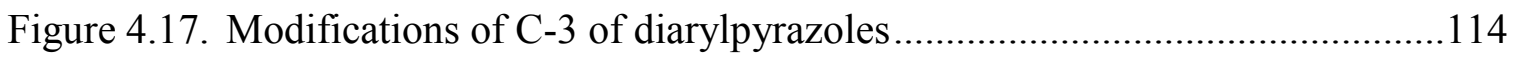

Figure 4.18. Conformationally constrained and non-traditional diarylpyrazoles ..........116

Figure 4.19. NMR structure confirmation of the pyridine hexahydro compounds .........119

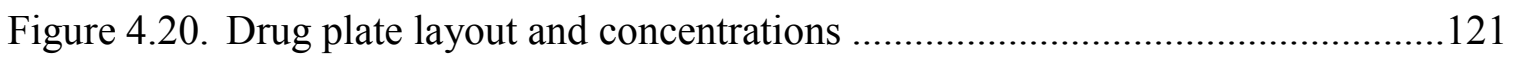

Figure 4.21. $\mathrm{CB}_{1}$ functional activity of hexahydro series compounds, 50 minutes after compound addition.

Figure 4.22. $\mathrm{CB}_{1}$ functional activity for select hexahydro series compounds, 50 minutes after compound addition.

Figure 4.23. $\mathrm{CB}_{2}$ functional activity for hexahydro series, 50 minutes after compound addition

Figure 4.24. $\mathrm{CB}_{2}$ functional activity for select hexahydro series compounds, 50 minutes after compound addition.

Figure 4.25. HEK-CNG parental cell functional activity for hexahydro series, 50 minutes after compound addition. 
Figure 4.26. $\mathrm{CB}_{1}$ PTx-sensitive $\mathrm{G}_{\mathrm{i} / \mathrm{o}}$-inactivation of functional activity for hexahydro series, 50 minutes after compound addition.

Figure 4.27. $\mathrm{CB}_{2} \mathrm{PTx}$-sensitive $\mathrm{G}_{\mathrm{i} / \mathrm{o}}$-inactivation of functional activity for hexahydro series, 50 minutes after compound.

Figure 4.28. $\mathrm{CB}_{1} \mathrm{LBP}$ - amide and benzchromene oxygen interactions of 170, as compared to HU-210

Figure 4.29. $\mathrm{CB}_{1}$ hydrophobic $\mathrm{C}-3$ substituent binding pocket

Figure 4.30. $\mathrm{CB}_{1} \mathrm{LBP}$ - comparison of the aliphatic and aromatic hexahydro interactions

Figure 4.31. Stabilization of the $\mathrm{CB}_{1} \mathrm{LBP}$ in the inactive state

Figure 4.32. $\mathrm{CB}_{1} \mathrm{LBP}$ proposed $\pi-\pi$ stacking and key residue interactions

Figure 4.33. $\mathrm{CB}_{2}$ LBP steric hinderance of $c$ is and trans isomers of WIN-55,212-2 .....140

Figure 4.34. Comparison of analogous functional groups of WIN-55,212-2, 172 and WIN-55,212-3

Figure 4.35. Proposed $\mathrm{CB}_{2} \mathrm{LBP}$ residues for compound 170

Figure 5.1. Triaryl series design flow

Figure 5.2. Non-classical cannabinoids.

Figure 5.3. Hybrid cannabinoid ligands

Figure 5.4. Biaryl cannabinoid ligands

Figure 5.5. Triaryl bis-sulfone derivatives

Figure 5.6. Generic triaryl structure nomenclature

Figure 5.7. Drug plate layout and concentrations

Figure 5.8. $\mathrm{CB}_{1}$ functional activity of $\mathrm{HB}$ triaryl series, 50 minutes after compound addition

Figure 5.9. $\mathrm{CB}_{2}$ functional activity of $\mathrm{HB}$ triaryl series, 50 minutes after compound addition

Figure 5.10. CNG functional activity of HB triaryl series, 50 minutes after compound addition

Figure 5.11. $\mathrm{CB}_{1} \mathrm{PTx}$-sensitive $\mathrm{G}_{\mathrm{i} / \mathrm{o}}$-inactivation of $\mathrm{HB}$ triaryl series, 50 minutes after compound addition. 
Figure 5.12. $\mathrm{CB}_{2} \mathrm{PTx}$-sensitive $\mathrm{G}_{\mathrm{i} / \mathrm{o}}$-inactivation of $\mathrm{HB}$ triaryl series, 50 minutes after compound addition

Figure 5.13. $\mathrm{CB}_{1}$ functional activity of SMM triaryl series, 50 minutes after compound addition.

Figure 5.14. $\mathrm{CB}_{1}$ functional activity of select SMM triaryl series compounds, 50 minutes after compound addition.

Figure 5.15. $\mathrm{CB}_{2}$ functional activity of SMM triaryl series 176

Figure 5.16. $\mathrm{CB}_{2}$ functional activity of select $\mathrm{SMM}$ triaryl series compounds.

Figure 5.17. CNG functional activity for SMM triaryl series 179

Figure 5.18. $\mathrm{CB}_{1}$ PTx-sensitive $\mathrm{G}_{\mathrm{i} / \mathrm{o}}$-inactivation for selected SMM triaryl series compounds

Figure 5.19. $\mathrm{CB}_{2} \mathrm{PTx}$-sensitive $\mathrm{G}_{\mathrm{i} / \mathrm{o}}$-inactivation for select $\mathrm{SMM}$ triaryl series compounds

Figure 5.20. $\mathrm{CB}_{1}$ functional data for pyridine triaryl compounds

Figure 5.21. $\mathrm{CB}_{1}$ functional activity of known binders within pyridine triaryl series ....183

Figure 5.22. $\mathrm{CB}_{1}$ active compounds with unknown $\mathrm{K}_{\mathrm{i}}$ values.

Figure 5.23. $\mathrm{CB}_{2}$ functinal data for pyridine triaryls 186

Figure 5.24. CB2 functional agonists of the pyridine triaryl series

Figure 5.25. $\mathrm{CB}_{2}$ functional activity of non-agonists with known binding within the pyridine triaryl series.

Figure 5.26. HEK-CNG functional assay with pyridine triaryl series

Figure 5.27. $\mathrm{CB}_{1} \mathrm{PTx}$-sensitive $\mathrm{G}_{\mathrm{i} / \mathrm{o}}$-inactivation with pyridine triaryl series. 190

Figure 5.28. $\mathrm{CB}_{2} \mathrm{PTx}$-sensitive $\mathrm{G}_{\mathrm{i} / \mathrm{o}}$-inactivation with pyridine triaryl series.....

Figure 5.29. $\mathrm{CB}_{1}$ functional activity for pyrimidine triaryl series.

Figure 5.30. $\mathrm{CB}_{2}$ functional activity of pyrimidine triaryl series

Figure 5.31. $\mathrm{CB}_{2}$ functional activity for select agonists in pyrimidine triaryl series

Figure 5.32. HEK-CNG functional activity of the pyrimidine triaryl series

Figure 5.33. $\mathrm{CB}_{1} \mathrm{PTx}$-sensitive $\mathrm{G}_{\mathrm{i} / \mathrm{o}}$-inactivation for select pyrimidine triaryl compounds 
Figure 5.34. $\mathrm{CB}_{2}$ PTx-sensitive $\mathrm{G}_{\mathrm{i} / \mathrm{o}}$-inactivation for select pyrimidine triaryl compounds

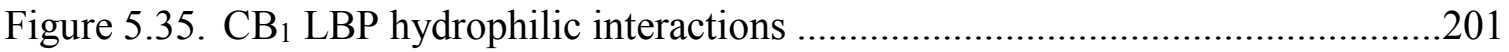

Figure 5.36. $\mathrm{CB}_{1}$ hydrophobic $\mathrm{C}-3$ substituent binding pocket ................................203

Figure 5.37. $\mathrm{CB}_{2}$ required D-ring and A-ring elements for compound $195 \ldots \ldots \ldots \ldots \ldots \ldots . . . .206$

Figure 5.38. $\mathrm{CB}_{2}$ required $\mathrm{C}-1^{\prime}$ elements for pyridine triaryls ....................................208

Figure 5.39. $\mathrm{CB}_{2}$ required $\mathrm{C}-6$ and $\mathrm{C}-\mathrm{1}^{\prime}$ elements for pyrimidine triaryls ....................209

Figure 6.1. Representative examples from each of the novel cannabinergic agents.....211

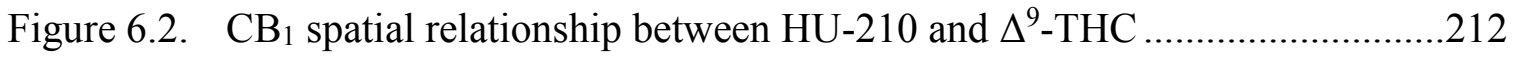

Figure 6.3. $\mathrm{CB}_{1}$ spatial relationship between Compound 67 and $\Delta^{9}-\mathrm{THC} \ldots \ldots \ldots \ldots \ldots . . .213$

Figure 6.4. $\mathrm{CB}_{1} \mathrm{LBP}$ common residues for classical core compound 67 ..................215

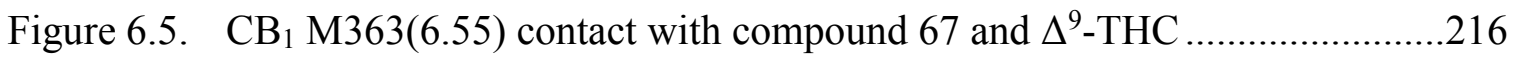

Figure 6.6. $\quad \mathrm{CB}_{1} \mathrm{LBP}$ W356(6.48) "toggle" switch presumed to be activated by compound 67

Figure 6.7. CB $_{1}$ LBP W356(6.48) "toggle" switch presumed to be deactivated by compound 172 .

Figure 6.8. $\mathrm{CB}_{1}$ interaction of 214 and 192 with the W356 "toggle" switch ...............220

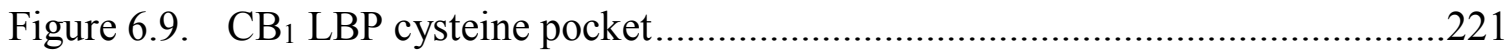

Figure 6.10. $\mathrm{CB}_{2}$ compounds demonstrating the requirement for di-hydroxy A-ring ....223

Figure 6.11. $\mathrm{CB}_{2} \mathrm{C}-1^{\prime}$ substituents and functional changes ........................................224

Figure 6.12. $\mathrm{CB}_{2} \mathrm{C}-1^{\prime}$ substituent effects - switch from agonist to inverse agonist........226

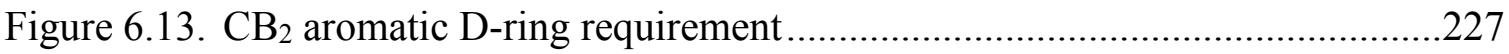




\section{CHAPTER 1. INTRODUCTION}

\subsection{History of Cannabinoids}

Cannabinoids have enjoyed a very long and somewhat sordid history. Originally grown for its hemp fiber for use in the fashioning of clothes, ropes and paper, it has a history of therapeutic use in India and China for nearly 5000 years. ${ }^{1}$ The use of Cannabis sativa preparations for treatment of a variety of pathological conditions in ancient Indian, Chinese, and Egyptian civilizations is well-documented. ${ }^{2}$ This documentation and literature is rife with therapeutic indications for Cannabis with usage as an analgesic, ${ }^{3,4}$ an anesthetic, ${ }^{5}$ an appetite stimulant, ${ }^{6}$ an anti-inflammatory agent, ${ }^{7}$ and several other ailments and afflictions. ${ }^{2}$ Although it took time to spread throughout the Western world for recreational and medicinal use, the true evolution of (-)-trans- $\Delta^{9}$-tetrahydrocannabinol $\left(\Delta^{9}\right.$-THC, the active psychotropic agent of the Cannabis sativa plant) (Figure 1.1) into a therapeutic agent has a much more recent history.

Early in the nineteenth century, natural product interest and research began to gain a foothold, and several alkaloids such as morphine, cocaine and strychnine were isolated from plant materials. The search for the psychoactive constituent of marijuana began during this period of rapid growth in the field of pharmacognosy. It was the common belief for many years that the principal active constituent of Cannabis sativa was in fact an alkaloid. This would hold true until Marshall and coworkers isolated a mixture of terpenes from an ether extract of Indian hemp in $1896,{ }^{8}$ which produced the same pharmacological effects observed with Cannabis sativa use. This provided the first observational evidence that the principal active compound was not an alkaloid but a terpene. In the early 1930s Cahn and coworkers isolated cannabinol (Figure 1.1) and determined a partial structure after conducting extensive chemical degradation studies. ${ }^{9}$ This discovery was followed in rapid succession by the synthesis of several derivatives of cannabinol and $\Delta^{6 \mathrm{a}, 10 \mathrm{a}}$-tetrahydrocannabinol $\left(\Delta^{6 \mathrm{a}, 10 \mathrm{a}}-\mathrm{THC}\right.$ ) (Figure 1.1) by the Adams group $^{10,11}$ and the Todd group. ${ }^{12}$ The pharmacological activities of cannabinol and $\Delta^{6 \mathrm{a}, 10 \mathrm{a}}-\mathrm{THC}$, as tested by the Gayer areflexia test for suppression of corneal reflexes in the rabbit, were observed to be similar to those of the Cannabis extract, but were significantly less potent.

The isolation and structural characterization of $\Delta^{9}-$ THC, was reported in 1964 by Gaoni and Mechoulam. ${ }^{13}$ The activity of this cannabinoid was subsequently studied in a number of animal models. These studies ranged from evaluation of the cataleptic reaction in mice $^{14}$ to the effects on motor activity and gross behavior in mice and rats. ${ }^{15}$ There was also extensive experimentation in rhesus monkeys ${ }^{16}$ which ultimately led to the pharmacological characterization of cannabinoids. Following these reports, several other constituents of marijuana such as cannabidiol, cannabigerol, cannabichromene, cannabicyclol (Figure 1.1) and related cannabinoid acids were isolated and characterized. The structural characterization of $\Delta^{9}$-THC provided a springboard to stimulate interest in research on cannabinoids. This resulted in the design and synthesis of several structurally diverse classes of cannabinoids. Studies on the effects of various analogues in animal models showed a positive correlation between structure and biological activity indicating 


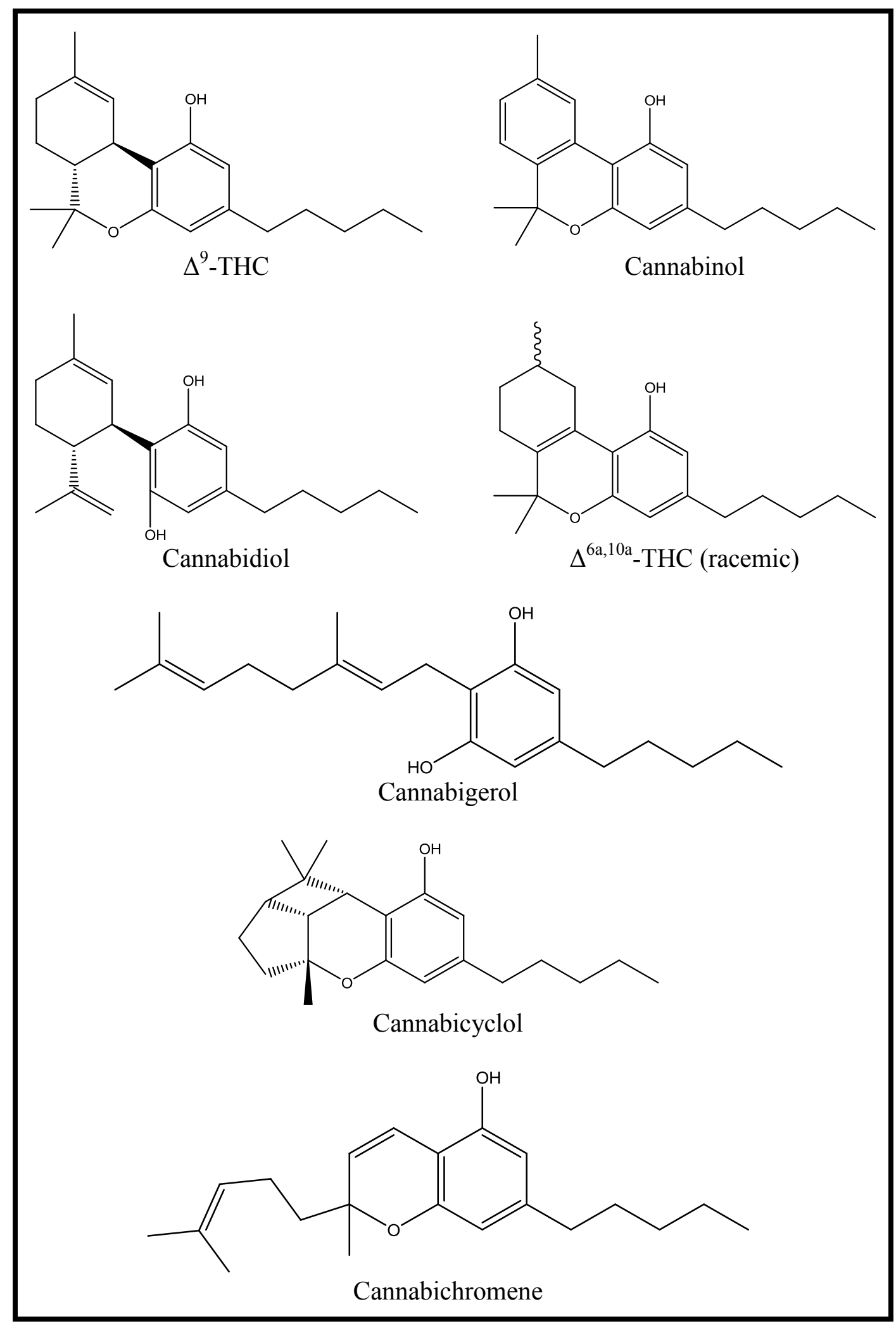

Figure 1.1. Structures of compounds isolated from Cannabis sativa 
the possible involvement of a defined receptor for the cannabinoids. This variety of compounds then aided in the discovery and cloning of the cannabinoid receptors and the subsequent identification of their endogenous ligands.

\subsection{Cannabinoid Receptors}

\subsubsection{History and background of cannabinoid receptors}

The beginning of the exponential growth seen in the field of cannabinoid research in the last two decades can be traced to the identification, isolation and cloning of the cannabinoid receptors. After the discovery of $\Delta^{9}$-THC, but before the discovery of the cannabinoid receptors, research on cannabinoids in the late 1970s and in the early 1980s was based on the assumption that the pharmacological effects attributed to cannabinoids were mediated not via cannabinoid receptors but by dissolution into and perturbation of cellular membranes. Due to their high lipophilicity, it was thought that cannabinoids behaved like general anesthetics in terms of their mechanism of action. ${ }^{17}$ However, the highly stereospecific activity associated with several cannabinoids ${ }^{18}$ suggested receptor mediated action. Then, in 1988, Devane and coworkers ${ }^{19}$ utilized a tritium labeled synthetic cannabinoid to tag specific sites in the brain and on neuronal cells. This then provided strong evidence for the presence of a specific cannabinoid receptor in the rat brain. This discovery directly preceded the isolation of the cDNA of this receptor, its expression in Chinese hamster ovary $(\mathrm{CHO})$ cells and subsequent naming as the cannabinoid receptor type $1\left(\mathrm{CB}_{1}\right) \cdot{ }^{20}$ In 1993 , a second cannabinoid receptor subtype, cannabinoid receptor type $2\left(\mathrm{CB}_{2}\right)$, was isolated and cloned from the human promyelocytic cell line HL60 by Munro and coworkers. ${ }^{21}$ An amino terminal variant of the human $\mathrm{CB}_{1}$ receptor has also been reported, ${ }^{22}$ in which a 167 base portion of the $\mathrm{CB}_{1}$ coding sequence is spliced out of the mRNA - termed $\mathrm{CB}_{1 \mathrm{~A}}$.

The cannabinoid receptors belong to the superfamily of G-protein coupled receptors ${ }^{14}$ (GPCRs), are single polypeptides with seven transmembrane $\alpha$-helices, an extracellular, glycosylated N-terminus and intracellular $\mathrm{C}$-terminus. The $\mathrm{CB}_{1}$ and $\mathrm{CB}_{2}$ receptor subtypes each possess $68 \%$ amino acid identity within their transmembrane regions but only $48 \%$ homology throughout the entire peptide ${ }^{14}$ - a trait that is unique among the $\mathrm{CB}_{1}$ and $\mathrm{CB}_{2}$ receptors. Many other GPCR families share approximately $75 \%$ homology among the subtypes. Also, it is noteworthy that the $\mathrm{CB}_{1}$ receptor is highly conserved across mouse, rat and human; however, there is only an $81 \%$ amino acid identity of the $\mathrm{CB}_{2}$ receptor across rat and human variants. ${ }^{23}$

Whether there are additional cannabinoid receptors is still a question that plagues many researchers in this field. This is, in part, due to the observation that the endogenous cannabinoid ligand, anandamide, induces spinal anti-nociception via a different mechanism than $\Delta^{9}$-THC. ${ }^{24}$ In 2000, Di Marzo and coworkers carried out studies ${ }^{25}$ using transgenic mice ${ }^{26}$ that lack the $\mathrm{CB}_{1}$ receptor to evaluate the $\mathrm{CB}_{1}$ mediated effects of anandamide and $\Delta^{9}$-THC. They found that in these knockout mice anandamide still produced $\mathrm{CB}_{1}$-like effects such as catalepsy, analgesia, and loss of spontaneous activity whereas $\Delta^{9}$-THC did not. This suggested the presence of a new cannabinoid receptor in 
the brain. Recent studies have also shown that a number of the cannabinoid ligands bind to the orphan receptor GPR55. ${ }^{27-29}$ This receptor is reported to be expressed in several tissues and might function in regulation of vascular tone and immune-cell migration. ${ }^{29}$ Anandamide is also known to act on other classes of receptors such as the vanilloid receptor, $\mathrm{TRPV}_{1}$. Anandamide activates $\mathrm{TRPV}_{1}$ and produces membrane currents or increases in intracellular calcium levels. ${ }^{30-32}$ The presence of these multiple sites for cannabinoid action (along with the aforementioned possibility for yet undiscovered sites) coupled with several signaling pathways and associated second messengers is compelling evidence for the complex pharmacology associated with cannabinoids.

\subsubsection{The $\mathrm{CB}_{1}$ receptor}

The $\mathrm{CB}_{1}$ receptor is a $52.8 \mathrm{kDa}, 472$ amino acid polypeptide, found in both the $\mathrm{CNS}$ and periphery in both neural and non-neural tissues. $\mathrm{CB}_{1}$ is coupled to inhibitory $\mathrm{G}_{\mathrm{i} / \mathrm{o}}$ proteins ${ }^{33}$ which bind to its third intracellular loop and its C-terminus. ${ }^{34} \mathrm{CB}_{1}$ receptors may also exert an action on $\mathrm{G}_{\mathrm{s}}$ proteins to stimulate cyclic AMP (cAMP). ${ }^{35}$ The amino acid residues in transmembrane regions 3,5 and 6 of the $\mathrm{CB}_{1}$ receptor are believed to be involved in binding with cannabinergic ligands. ${ }^{36}$ Activation of $\mathrm{CB}_{1}$ causes inhibition of adenylyl cyclase and a reduction in cAMP levels. Reduction of cAMP affects phosphorylation by protein kinase A which causes modulation of ion channels and other secondary messengers. Activation of voltage-sensitive outwardly rectifying $\mathrm{K}+$ channels, ${ }^{37}$ inwardly rectifying $\mathrm{K}+$ channels $^{38}$ and inhibition of voltage gated $\mathrm{N}, \mathrm{L}, \mathrm{P}$ and Q-type $\mathrm{Ca}^{+2}$ channels ${ }^{39}$ are observed in response to $\mathrm{CB}_{1}$ receptor activation.

The $\mathrm{CB}_{1}$ receptor regulates different members of the mitogen activated protein kinase (MAP kinase) family of enzymes such as:

1. extracellular signal-regulated kinase $(\mathrm{ERK})^{40-42}$

2. c-Jun N-terminal kinase ${ }^{43,44}$

3. $\mathrm{p} 38.44,45$

Activation of MAP kinase is associated with:

1. activation of immediate early genes like c-Fos and Egr1 (Krox-24) ${ }^{41}$

2. activation of focal adhesion kinase (important for integrating cytoskeletal changes associated with signal transduction events)

3. activation of phospholipases (PL), PLA, PLC, and PLD ${ }^{46}$

4. activation of the $\mathrm{Na}^{+} / \mathrm{H}^{+}$exchanger ${ }^{47}$

5. activation of phospholipase A2, which in turn causes release of arachidonic acid leading to the synthesis of prostaglandins. ${ }^{48}$

There is also evidence of a second pathway initiated by $\mathrm{CB}_{1}$ receptor stimulation coupled with sphingomyelinase activation and subsequent release of ceramide. ${ }^{42}$ Ceramide then causes activation of the Raf-1/MAP kinase cascade ${ }^{49}$ and also carnitine palmitoyltransferase I in mitochondrial membranes to stimulate ketogenesis and fatty acid oxidation. ${ }^{50}$ The elevation of intracellular ceramide have also been suggested to be 
one of the possible factors responsible for the apoptotic effects of cannabinoids seen on ceramide-related cancers such as glioblastoma. ${ }^{51}$

\subsubsection{The $\mathrm{CB}_{2}$ receptor}

The $\mathrm{CB}_{2}$ cannabinoid receptor, also referred to as the peripheral cannabinoid receptor, is a $41 \mathrm{kDa}, 360$ amino acid polypeptide predominantly expressed in the immune system. ${ }^{52,53}$ Its amino acid sequence is quite different from that of $\mathrm{CB}_{1}$, especially in the amino terminal domain, which is significantly shorter and not as well conserved in the $\mathrm{CB}_{2}$ receptor when compared to the $\mathrm{CB}_{1}$ receptor. The amino acid residues involved in ligand recognition are also found, as it was for the $\mathrm{CB}_{1}$ receptor, in transmembrane domains 3,5 and 6 for the $\mathrm{CB}_{2}$ receptor. ${ }^{54}$

Like the $\mathrm{CB}_{1}$ receptor, $\mathrm{CB}_{2}$ couples to the inhibitory $\mathrm{G}_{\mathrm{i} / \mathrm{o}}$ proteins that bind to its third intracellular loop and C-terminus. Activation of the $\mathrm{CB}_{2}$ receptor also causes inhibition of adenylyl cyclase and therefore a reduction in cAMP levels, as it was with $\mathrm{CB}_{1}$. Most of the known signaling pathways of $\mathrm{CB}_{2}$ are identical to those observed with $\mathrm{CB}_{1}$. However, unlike the $\mathrm{CB}_{1}$ receptor, the $\mathrm{CB}_{2}$ receptor has no effect on ion channels. ${ }^{38}$

After the discovery of the $\mathrm{CB}_{2}$ receptor it was believed that it was found only in the immune system and was completely absent from the CNS. ${ }^{55}$ More recently, however, $\mathrm{CB}_{2}$ expression has been confirmed in the brain on microglia, ${ }^{56}$ blood vessels, ${ }^{56}$ and on some neurons. ${ }^{57,58} \mathrm{CB}_{2}$ has a much higher prevalence than $\mathrm{CB}_{1}$ within the immune system. The expression level of the $\mathrm{CB}_{2}$ gene in immune tissues is 10 to 100 times the level of the $\mathrm{CB}_{1}$ gene. ${ }^{59}$ In fact, the $\mathrm{CB}_{2}$ mRNA content of the spleen and tonsils is almost equivalent to the $\mathrm{CB}_{1}$ mRNA content in the CNS. ${ }^{59}$ This first led researchers to suggest that $\mathrm{CB}_{2}$ receptor might play an important role in immune function - more specifically the down-regulation thereof. It is no surprise then that many reports have been made over the years regarding the deleterious effects of marijuana smoking on host defense mechanisms where marijuana smokers were found to be more susceptible to bacterial and viral infections. ${ }^{60} \mathrm{CB}_{2}$-specific ligands and specific targeting of the immune system is possible by virtue of the fact that between transmembrane domains 1 and 7 , including the loop regions, the $\mathrm{CB}_{2}$ receptor is only $44 \%$ identical to the $\mathrm{CB}_{1}$ receptor.

\subsubsection{The GPR55 receptor - A putative cannabinoid receptor}

The GPR55 receptor, first identified and cloned by O'Dowd in 1999, ${ }^{61,62}$ found favor as a putative cannabinoid receptor by an in silico screen due to an amino acid sequence in the binding region that is similar. Research groups at the pharmaceutical companies Glaxo-Smith-Kline and AstraZeneca then extensively characterized the receptor in the hope that it would be responsible for the blood pressure lowering effects of cannabinoids. GPR55 demonstrates activation by endogenous, phyto-, and synthetic cannabinoids but GPR55 knockout mice generated at Glaxo-Smith-Kline showed no alteration in the cannabinoid induced blood pressure regulation after administration of the GPR55 agonist abnormal-cannabidiol (Figure 1.2). 


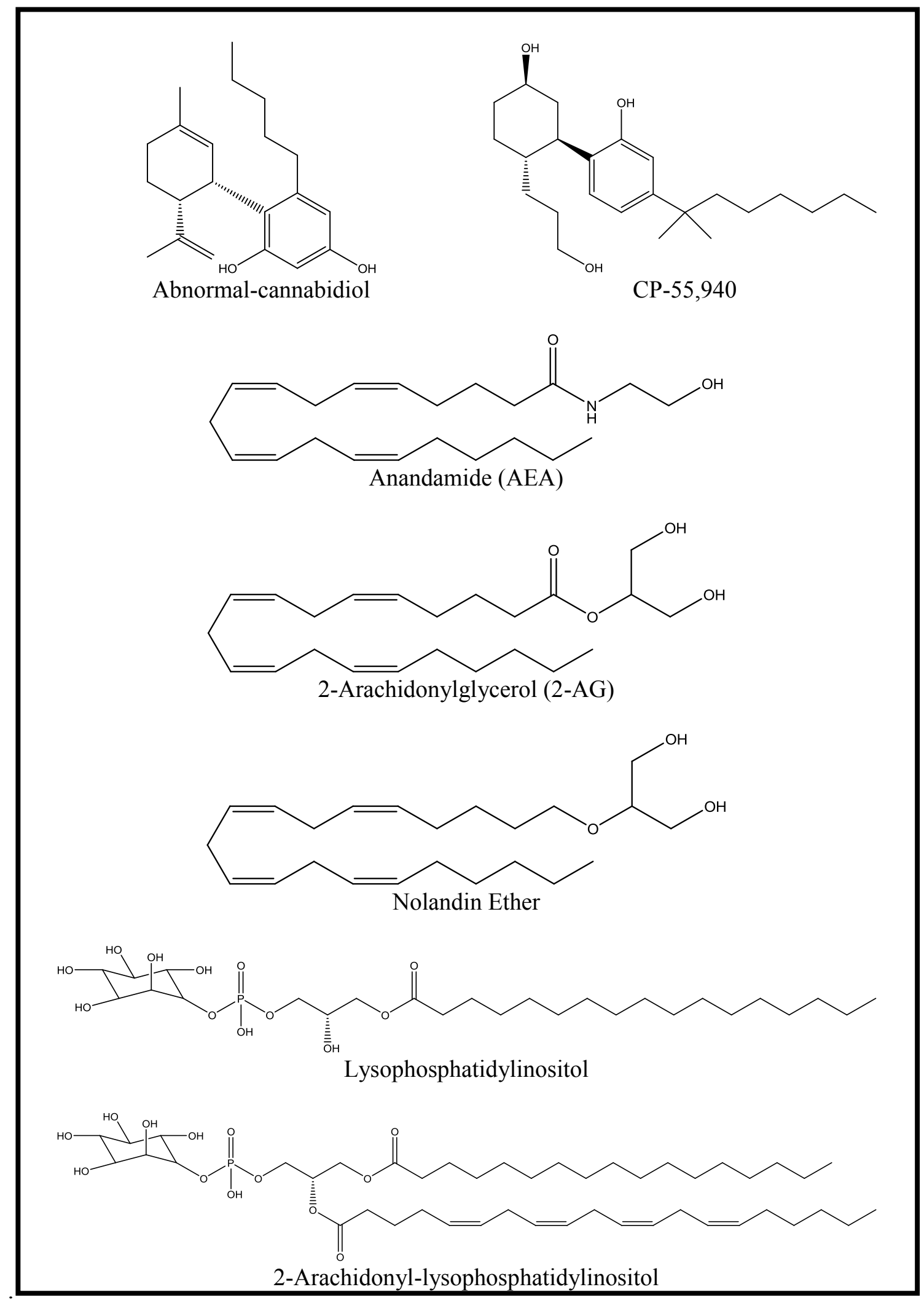

Figure 1.2. Synthetic and endogenous cannabinoid compounds 
Some of the known $\mathrm{CB}_{1}$ and $\mathrm{CB}_{2}$ ligands also bind to GPR55. The receptor is activated by $\Delta^{9}$-THC and cannabidiol, ${ }^{29}$ synthetic cannabinoids such as CP-55,940 and the endocannabinoids anandamide (AEA), 2-arachidonoylglycerol (2-AG), and nolandin ether (Figure 1.2) ${ }^{29}$ Recent research suggests that lysophosphatidylinositol and its 2arachidonoyl derivative (Figure 1.2) may be the endogenous ligands for GPR $55^{63,64}$ thus giving rise to it being a potential LPI receptor. ${ }^{65}$ GPR 55 appears likely to be a possible target for treatment of inflammation and pain as with the other cannabinoid receptors. ${ }^{66-69}$

While the $\mathrm{CB}_{1}$ and $\mathrm{CB}_{2}$ receptors couple to $\mathrm{G} \alpha_{\mathrm{i} / \mathrm{o}}$ proteins, GPR55 is coupled to $\mathrm{G} \alpha_{13}$ and activation of the receptor leads to stimulation of a number of GTPases such as Ras homolog gene family, member A (RhoA), cell division control protein 42 homolog (cdc42) and Ras-related C3 botulinum toxin substrate 1 (rac1). ${ }^{27}$ Members of this GTPase superfamily appear to regulate a diverse array of cellular events, including the control of cell growth, cytoskeletal reorganization, and the activation of protein kinases. ${ }^{70}$ Stimulation of GPR55 induces F-actin formation under the control of Ga13, RhoA and Rho-associated, coiled-coil containing protein kinase (ROCK). ${ }^{63}$ GPR55 activation also induces intracellular calcium release and activation of the transcription factors nuclear factor kappa-light-chain-enhancer of activated B cells (NF- $\kappa \mathrm{B})$, nuclear factor of activated T-cells (NFAT) and cAMP response element-binding (CREB). ${ }^{63}$ The physiological role of GPR55 remains unclear. Mice with a target deletion of the GPR55 gene show no specific phenotype. ${ }^{71}$ GPR55 is has been found to be widely expressed in the brain, especially in the cerebellum. It is found in the gastrointestinal tract in the jejunum and ileum and also in the adrenal glands but is apparently not highly expressed elsewhere in the periphery. ${ }^{29}$ Osteoblasts and osteoclasts have also been shown to express GPR55 and this has been shown to regulate bone cell function. ${ }^{72}$

\subsection{Cannabinoids in Disease States}

In the United States of America, heart disease (Section 1.3.1), cancer (encompassing all variants under the auspice thereof) (Section 1.3.2), stroke (Section 1.3.3), Alzheimer's Disease (Section 1.3.4) and diabetes (Section 1.3.5) are five of the top seven causes of deaths. Many of these disease states have complex pathogenesis and are amalgamations of several years of poor habits and lifestyles of individuals; however, once diagnosed, treatment of many of these disease states is tenuous, at best. There are data that strongly point to the usefulness of cannabinoids in all of these disease states. It is for the betterment of treatment not only for these diseases, but for cannabinoid use therein that this project finds its utility and mission - to better design a cannabinergic agents we must first understand how they function. While it is well and good to understand the need for a $\mathrm{CB}_{1}$ antagonist and a $\mathrm{CB}_{2}$ receptor agonist to positively impact cerebral ischemia in stroke, ${ }^{73}$ understanding the functional activity of drugs prior to their use in vivo is essential for targeted drug design and resource optimization. Furthermore, use of this information to develop a functional pharmacophore of cannabinoids helps to expedite and further hone the synthetic design of cannabinergic ligands. However, as with any project, especially one with this potential benefit, one must understand the target disease state to maximize the management thereof. Briefly, I will examine five of the top seven disease states and the positive role cannabinoids may play in each. 


\subsubsection{Cannabinoids in heart disease}

1.3.1.1. Pathogenesis of heart disease. While the true derivation and progression of this disease is innately complex and multifactorial, there are certain key elements that lend themselves to the progression of the disease. One of these main components of heart disease is atherosclerosis, which is an inflammatory disease, at its root. ${ }^{74,75}$ This inflammation is a response to damage done to the blood vessels as a result of plaque deposit or rupture or small intima tears caused by high blood pressure. Cannabinoids find usefulness in treating this pathogenesis from acting as: antihypertensive agents (Section 1.3.1.2), anti-ischemic agents post-MI (Section 1.3.1.3) or anti-inflammatory agents (Section 1.3.1.4).

1.3.1.2. Cannabinoids as anti-hypertensive agents. While the cardiovascular effects of cannabinoids has been known since the 1960s, harnessing their power to positively impact the cardiovascular system has languished. ${ }^{76}$ The first possible impact cannabinoids may have on this process is in the realm of blood pressure control. The peripheral effects of systemically administered cannabinoids in vivo seem to predominate in the overall activity of these compounds. ${ }^{77} \mathrm{IV}$ administration of anandamide causes a triphasic response in the blood pressure of anaesthetized rats which transitions from a transient drop, to a short-lived spike and ending in a prolonged third phase of lower blood pressure $^{78}-$ similar to the onset of higher doses of clonidine. ${ }^{79}$ The initial phase of lowered blood pressure appears to be due to vagal output, as atropine and cervical vagotomy eradicate this initial drop. ${ }^{80}$ The secondary, more sustained, drop in blood pressure shows signs of being $\mathrm{CB}_{1}$-mediated through presynaptic inhibition of sympathetic output in the periphery. ${ }^{78,80}$ In addition to the sympathetic outflow inhibition, data exists which suggest that there is a direct vasodilatory effect of cannabinoids, as HU-210 has been shown to lower blood pressure in spite of sympathetic blockade. ${ }^{80,81}$

Overall, anandamide is hypothesized to work by the aforementioned reduction of peripheral vascular resistance as well as a decrease in cardiac contractility. ${ }^{82,83}$ This dual mechanism for blood pressure control is consistent with many other drugs currently on the market for the treatment and management of hypertension. Additionally, mitigation of these effects occurs by administration of the $\mathrm{CB}_{1}$ inverse agonist SR-141716A. ${ }^{83}$

In humans, there is a noted tachycardia and minor pressor effect upon acute administration of cannabinoids; however, this gives way to bradycardia and hypotension with repeated dosing. ${ }^{84,85}$ It is worth noting, that there are differences in levels of wakefulness between the laboratory animals and the human test subjects. ${ }^{86}$ A comparison of this effect was noted, where monkeys in a sterile, predictable laboratory environment demonstrated the same bradycardia effect noted in previous animal models after initial dosing. ${ }^{87}$ This effect, while notable and profound for exogenously administered cannabinoids flies somewhat in the face of convention when one considers the normotensive state of $\mathrm{CB}_{1}$ knockout mice ${ }^{88}$ and FAAH-deficient mice had a normal hemodynamic profile. ${ }^{89}$ Taken as a whole, these results show how endogenous cannabinoids do not exert tonic control of the cardiovascular system, under normal conditions; however, under abnormal conditions (i.e. hypertension), endocannabinoid tone may blunt the elevation of blood pressure and cardiac contractility. ${ }^{90}$ 
Many in vitro studies have corroborated some of the current in vivo mechanisms, as well as generate hypotheses in their own right. First, in vitro experiments conducted in isolated, Langendorff-apparatus-perfused, rat hearts ${ }^{91}$ and in isolated, electrically stimulated human atrial muscle, ${ }^{92}$ have demonstrated HU-210's and anandamide's ability to decrease cardiac output, as was the case in vivo. ${ }^{93}$ A second set of experiments sought to elucidate the mechanism of HU-210's vasodilator effects in spite of sympathetic blockade; however, those studies have done little to tease apart a true mechanism. ${ }^{77,94,95}$ This variability of mechanism also varies from species to species, where a rat aorta may experience a $20 \%$ reduction, ${ }^{96}$ while rabbit aorta experiences an $80 \%$ relaxation. ${ }^{97}$ It is certain that cannabinoid-induced arachidonic acid production ${ }^{98}$ and/or nitric oxide (NO) production $^{99}$ are not mechanistic routes for the vasodilatory actions thereof in most species or vascular beds, suggesting that cannabinoid vasodilation is site-specific. A final mechanism put to in vitro testing is that of alternate receptor activation, more namely transient receptor potential vanilloid, type $1\left(\mathrm{TRPV}_{1}\right)$ and the stimulation thereof to release calcitonin gene-related peptide (CGRP) from sensory nerves. This has shown itself to be of minor consequence, as $\mathrm{CB}_{1}$ knockout $^{88}$ and TRPV knockout $^{82}$ mice both present with normal hemodynamic profiles. The potential for involvement of TRPV 1 and GPCR do provide some insight into the sex-linked differences of vasodilation of cannabinoids - with females experiencing a higher degree of vasodilation, possibly due to higher levels of estrogens, a well-documented vasodilator. ${ }^{100}$

\subsubsection{Cannabinoids as anti-ischemic agents post-MI. Following a}

myocardial infarction (MI), the ischemic damage that occurs to the cardiac tissue is of top concern when evaluating a patient for long-term prognosis. Mitigation of the infarct is of the utmost importance, to ensure a full recovery with little to no loss of cardiac function. Cannabinoids, especially the endocannabinoids anandamide ${ }^{101}$ and palmitoyethanolamide ${ }^{102}$ have shown promise in minimizing the infarct size of experimental models of myocardial ischemia; however, there is evidence the mechanisms thereof are due to an alternate receptor pathway that has yet to be fully characterized. ${ }^{101,103}$ In contradiction to the novel pathway theory, there is data that suggest $\mathrm{CB}_{1}$ and $\mathrm{CB}_{2}$ are involved as a cohort, with or without a novel receptor site. $\mathrm{CB}_{1-}$ and $\mathrm{CB}_{2}$-selective agents have been shown ineffective in minimizing the infarct size. Selective antagonism of $\mathrm{CB}_{1}$ or $\mathrm{CB}_{2}$ by SR-141716A or SR-144528, respectively abolishes anandamide's ability to influence infarct size. ${ }^{101}$

1.3.1.4. Cannabinoids as anti-inflammatory agents post-MI. As previously mentioned, atherosclerosis is one of the major causes of heart disease, and is itself a state of chronic inflammation. ${ }^{104}$ Even at low doses, THC demonstrates a blockade to the advancement of atherosclerotic lesions in a murine model thereof. ${ }^{105}$ Validating the mechanism of this being a $\mathrm{CB}_{2}$-mediated effect on the atherosclerotic lesion, ${ }^{106}$ pretreatment with SR-144528 serves to block the beneficial effects of THC in this model. ${ }^{105}$ The same study that helped validate a $\mathrm{CB}_{2}$-mediated regulation of atherosclerotic plaque formation also provided evidence of reduced $\mathrm{T}_{1}$-helper response and blockade of macrophage migration to the site, both of which contribute to early development processes in atherosclerosis. Further evidence to implicate $\mathrm{CB}_{2}$ as the prime target arose from an in vivo murine model of established atherosclerosis and in vitro macrophage migration. These data, which show that peritoneal macrophages isolated 
from CB2 knockout mice as well as pretreatment of wild-type macrophages with SR144528, did not respond to THC-induced migration. ${ }^{105}$ Finally, there was evidence demonstrating the presence of $\mathrm{CB}_{2}$ receptor expression on the atherosclerotic vessels, at the site of plaque formation, but vessels devoid of disease showed no such expression of $\mathrm{CB}_{2}$ receptors. ${ }^{105}$ Taken in totality, this suggests $\mathrm{CB}_{2}$-selective agonists may be a desirable therapeutic agent in the treatment and/or prevention of atherosclerosis; however, there is a need for more in vivo work of this hypothesis before any definitive answer regarding the vitality of this as a treatment option.

\subsubsection{Cannabinoids in cancer}

1.3.2.1. Cancer rates in The United States of America. Cancer is an all-encompassing disease state, and it is as this cohort that it becomes the second leading killer of persons in The United States of America. Under this auspice, there are many subtypes of cancer to consider, and treatment modalities differ among them. The most recent compiled data from The American Cancer Society (2009) shows that among men and women, lung $\&$ bronchus cancer (Section 1.3.2.2) is the leading type of cancer for both groups - approximately 61 deaths per 100,000 males and approximately 40 deaths per 100,000 females. Looking specifically at the gender-related cancers, prostate cancer (Section 1.3.2.3) is the second highest killer of men at approximately 22 deaths per 100,000 males, and breast cancer (Section 1.3.2.4) ranks second amongst women at approximately 22 deaths per 100,000 females. The pathogenesis of each cancer is far more complex and involved of a process to cover in detail within this document. However, the crux of any cancer is that cells lose their normal apoptotic signaling and persist at an abnormal rate of cell division, eventually entering into lymphatic circulation and metastasizing to distant sites and causing similar hyperproliferative growths.

1.3.2.2. Cannabinoids in lung and bronchial cancers. Being the leading type of cancer in The U.S.A. for both men and women, it is logical that we begin the examination of cannabinoids in cancer with lung and bronchial cancers. Within lung and bronchial cancers, there are several subtypes still, with non-small cell lung cancer (NSCLC) being the most prevalent subtype.107 In NSCLC, CB1 and CB2 receptors are overexpressed $24 \%$ and $55 \%$, respectively, of the cases studied.108 There exists several reports that point to EGFR hyperexpression and hyperactivation, which promotes resistance of lung cancer to current mainstays of chemotherapy. Given, too, that there is known cross-talk of GPCRs and EGFR, where GPCRs transactivate EGFRs, 109 use of CB1 and/or CB2 agents to inhibit the untoward effects of EGFR may present therapeutically viable option. This EGFR effect has seen positive results in vitro with WIN-55,212-2, a non-selective cannabinoid receptor agonist, significantly impeding the growth of A549 cancer cells by $60-70 \% .108$ The CB2-selective agonist JWH-015 also reduced cell viability by 40 $50 \% .108$

On the converse of this data lies a much more dubious section of data. In some studies, THC at low doses $(100-300 \mathrm{nM})$ demonstrated a pro-proliferative action in NCI-H292 cells via cross-talk between cannabinoid receptors and EGFR; however, this same study showed that $\mu \mathrm{M}$ concentrations of THC demonstrated anti-proliferative 
action. ${ }^{110}$ Another similar study demonstrated the upregulation of Ki67, a proproliferative marker, in the lungs of chronic smokers of marijuana. ${ }^{111}$ It is important to note that in both of these cases, the pro-proliferative effects occur with either smoked marijuana or at levels achievable by smoking marijuana. That is to say, when smoked, Cannabis sativa delivers a very small amount of THC with each dose, and that this method of drug delivery is not the most viable option. To discount entirely the use of THC and/or its derivatives from this data is not prudent - it merely highlights and emphasizes the need for more selective, potent agents with an optimized dosage route for lung and bronchial cancers.

1.3.2.3. Cannabinoids in prostate cancer. The second leading cause of cancerrelated death among men is prostate cancer. With a 1 in 6 chance of developing this cancer over the course of a male's life, ${ }^{107}$ it becomes of great importance to identify not only preventative measures but treatment options to positively impact persons afflicted with this disease state. In a pair of studies conducted by Sánchez, et. al. $50 \mathrm{nM}$ doses of THC caused a small, but statistically significant increase in the proliferation of LNCaP and PC3 cells - an action mimicked by 2-arachidonoylglycerol (2-AG) but opposed by anandamide. ${ }^{112,113}$ Further just-cause for the use of cannabinoids in prostate cancer is that other groups have demonstrated that doses of anandamide above $2 \mu \mathrm{M}$ have shown an anti-proliferative effect in PC3, LNCaP and DU145 cells, while 2-AG was proproliferative at the same concentrations. ${ }^{114,115}$ Similar to the process noted with THC in lung and bronchial cancer, it could be due to a dose and/or receptor affinity issue, as anandamide is a full agonist at both $\mathrm{CB}_{1}$ and $\mathrm{CB}_{2}$, whereas $\mathrm{THC}$ is only a partial agonist at both and 2-AG is a full agonist with a higher $\mathrm{CB}_{1}$ selectivity. Thus, my hypothesis would be that an effective cannabinoid in prostate cancer would be a potent, full agonist of $\mathrm{CB}_{1}$ and $\mathrm{CB}_{2}$, with little or no specificity. However, due to the untoward effects of central $\mathrm{CB}_{1}$ agonist, we would desire a peripherally administered and restricted compound. Overall, there is viability for cannabinoids in prostate cancer, and an area that will hopefully see a burgeoning of data in the near future.

1.3.2.4. Cannabinoids in breast cancer. As prostate was the second leading killer of men, breast cancer is the second leading killer of women, with a similar rate of occurrence at 1 in 8 women. ${ }^{107}$ Equilibrium predominates in breast cancer, with conflicting reports of THC being pro- or anti-proliferative. Some studies in MCF-7 cells have demonstrated $\Delta^{9}$-THC as a pro-proliferative agent, up to $5 \mu \mathrm{M} ;{ }^{116,117}$ however, this was contradicted by another group with the same cell line - showing that up to $1 \mathrm{mM}$, THC did not promote proliferation but did block $17 \beta$-estradiol-induced proliferation. ${ }^{118}$ Studies in MDA-MB-231 cells have also yielded a dichotomy of data, with some reports of THC being pro-proliferative ${ }^{116}$ and others showing inhibition of growth in this cells line. ${ }^{119}$ Data also exists from McAllister, et. al. of anti-proliferative effects of THC in MDA-MB-468 and EVSA-T cells. ${ }^{119}$

In vivo models of breast cancer show an equally confusing gamut of data. A xenotransplant of 4T1 paw cells into BALB/c mice saw an increase in tumor burden with $25 \mathrm{mg} / \mathrm{kg}$ of THC injected IP for 21 days. ${ }^{116}$ A separate model of MMTV-neu mice, which spontaneously develop mammary tumors, saw a reduction of not only tumor burden but also a decrease in the rate and extent of metastasis to the lungs. ${ }^{120}$ Overall, as 
is the case with cancer, data is conflicting and varies widely in the literature. This could be simply due to differences in in vitro or in vivo model systems, the drugs utilized, the dosage forms or even the cancers themselves. Moreover, there is positive data regarding cannabinoids, which would suggest pursuit of cannabinoids in breast cancer to be a fruitful endeavor, and one that commands a spot on the research docket.

\subsubsection{Cannabinoids in stroke}

1.3.3.1. Pathogenesis of stroke. The two major mechanisms causing brain damage in stroke are ischemia and hemorrhage. Ischemic stroke, which represents about $80 \%$ of all strokes, results from decreased or absent circulating blood which, in-turn deprives neurons of life-sustaining substrates. The effects of ischemia are fairly rapid because the brain does not store glucose, the chief energy substrate, and is incapable of anaerobic metabolism. ${ }^{121}$ Non-traumatic intracerebral hemorrhage represents approximately $10 \%$ to $15 \%$ of all strokes. Intracerebral hemorrhage originates from deep penetrating vessels and causes injury to brain tissue by disrupting connecting pathways and causing localized pressure injury. In either case, destructive biochemical substances released from a variety of sources play an important role in tissue destruction.

Formation of microscopic thrombi responsible for impairment of microcirculation in the cerebral arterioles and capillaries is a complex phenomenon. Formation of a micro thrombus results from ischemia-induced activation of destructive vasoactive enzymes released by endothelium, leucocytes, platelets and other neuronal cells. Mechanical "plugging" by leucocytes, erythrocytes, platelets and fibrin ensues. ${ }^{122}$

Inflammatory response to tissue injury initiated by the rapid production of many different inflammatory mediators, tumor necrosis factor (TNF) being one of the key agents, activation of microglia and adhesion and migration of peripheral leukocytes. ${ }^{123}$ Leukocyte recruitment to the ischemic areas occurs as early as thirty minutes after ischemia and reperfusion. In addition to contributing to mechanical obstruction of microcirculation, the leucocytes also activate vasoactive substances such as oxygen free radicals, arachidonic acid metabolites (cytokines), and nitric acid. The cellular effects of these mediators include vasodilatation, vasoconstriction, increased permeability, increased platelets aggregation, increased leukocyte adherence to the endothelial wall, and immunoregulation. It is through modulation of this inflammatory process that the utility of cannabinoids in stroke find their foothold as a therapeutic option.

1.3.3.2. Cannabinoids as anti-inflammatory agents post-stroke. There are several reports of the endocannabinoids, anandamide and 2-AG, released locally following neuronal damage to influence the inflammatory and cell differentiation processes. ${ }^{7,124}$ Of particular interest, in conjunction with this release is evidence of $\mathrm{CB}_{1}$ expression on T-lymphocytes, which may result from endocannabinoid stimulation. ${ }^{125-129}$ This helps to bolster the proposed mechanism of anandamide, which is in abundance near the infarcted brain area, locally inhibiting T-lymphocyte proliferation. ${ }^{130}$ While this function of $\mathrm{CB}_{1}$ is undoubtedly important, the lion's share of cannabinoid work as antiinflammatory agents post-stroke is through $\mathrm{CB}_{2}$. 
$\mathrm{CB}_{2}$ has long been known to be expressed on immune organs such as the thymus and spleen, ${ }^{53}$ as well as circulating inflammatory cells such as T-lymphocytes, B-lymphocytes, NK cells, monocytes and neutrophils; ${ }^{59,131,132}$ however, the more recent confirmation of $\mathrm{CB}_{2}$ on astrocytes, ${ }^{133}$ microglia, ${ }^{134}$ neural subpopulations and oligodendroglial progenitors ${ }^{135}$ points to a more direct regulation of $\mathrm{CB}_{2}$ in these brain inflammatory cells. ${ }^{136}$ In microglial cells, the level of $\mathrm{CB}_{2}$ expression is highly dependent upon the activation state of the cell as it is responding to inflammation, infection or stress. ${ }^{7,137}$ It is during the early inflammatory activation phase where $\mathrm{CB}_{1}$ and $\mathrm{CB}_{2}$ show their highest levels of upregulation, ${ }^{7,138-140}$ thus early treatment, within 90 minutes of symptom onset, of the post-ischemic attack is preferred for optimal outcome. In fact, use of JWH-133, a $\mathrm{CB}_{2}$-selective agonist, demonstrated not only a reduction of microglial activation and inflammatory gene expression in a mouse middle cerebral artery occlusion and focal cerebral ischemia model but an improvement of brain infarction size and clinical outcomes. ${ }^{141}$ Confirmation of this mechanism bears itself out in $\mathrm{CB}_{2}$ knockout mice, where infarct size is considerably larger in the knockout mice as compared to the wild type. ${ }^{142}$

A final component of cannabinoids in the inflammatory process of ischemia could be via impact of the CD4+ and CD25+ T cells, as they data suggests they to play a role in neuroprotection of mice after focal cerebral ischemia. ${ }^{143,144}$ The cannabinoid mechanism in these cells may be due to agonist's ability to affect proliferation, polarization ${ }^{7,145}$ and/or cytolytic capacity ${ }^{146}$ of the T cells. Studies have helped to confirm this action, with WIN-55,212-2 showing favor toward lymphocyte migration within the spleen ${ }^{147}$ and 2AG greatly minimizing mitogen-induced proliferation of mouse splenocytes. ${ }^{148}$ While this data provides positive news for the use of cannabinoids as anti-inflammatory agents following a stroke, contrasting data from in vitro and in vivo models persist and pose a problem for future therapeutic use of cannabinoid agents.

1.3.3.3. Cannabinoids as anti-ischemic agents post-stroke. The ischemic process of neuronal cell death is due to the cascade of events during the first few hours after the onset of ischemia. In these early hours, reactive oxygen species (ROS), calcium $\left(\mathrm{Ca}^{+2}\right)$ and sodium $\left(\mathrm{Na}^{+}\right)$increase in the cytosol and are directly proportional to necrosis and apoptosis of neurons. ${ }^{149}$ The increase in $\mathrm{Ca}^{+2}$ occurs via NMDA-medicated pathways and is due to a lack of oxygen and glucose during the ischemic attack. ${ }^{150}$ Given that blockade of the $\mathrm{Ca}^{+2}$ channels is associated with neural protection, $\mathrm{Ca}^{+2}$ represents a pivotal target in modifying the progression of neuronal cell death during ischemia. ${ }^{151}$

As is the case in several facets of cannabinoid research, we do not yet fully know the exact mechanisms for neuroprotection of the endocannabinoids and synthetic cannabinoids, and data conflict in some aspects. For example, some groups report that $\mathrm{CB}_{1}$ agonist activity is the means to neural protection, ${ }^{152}$ while others have used SR-141716A, a known $\mathrm{CB}_{1}$ inverse agonist, to show the same neuroprotective action via a TPVR 1 -mediated pathway. ${ }^{153}$ Some things are quite clear, though, with regard to cannabinoid actions on ischemic neurons.

One such known is that activation of $\mathrm{CB}_{1}$ causes propagation of several pro-survival pathways involving mitogen-activated protein kinase (MAPK) kinase 1/2 
(MEK1/2), extracellular signal-regulated kinase (ERK1/2) and nuclear factor-kappa B $(\mathrm{NF} \kappa \mathrm{B}) .{ }^{154,155}$ In addition to potential for initiating pro-survival pathways, amelioration of both global and regional blood flow was observed with low and high doses of THC in humans; ${ }^{156}$ however, more recent studies have shown a potential link between chronic cannabis use in a youth and recurrent ischemic stroke. ${ }^{157}$ An even more recent, prospective study of 48 young adults with prior ischemic stroke demonstrated multifocal intracranial artery stenosis in those with cannabis consumption. ${ }^{158}$

While this may seem daunting news for use of cannabinoids in the ischemic stroke setting, it is not a mutually exclusive issue. Keeping this in perspective, the human studies are utilizing dried and smoked cannabis, of which THC is the main cannabinoid present, and as previously addressed, THC is a poor agent in many of the disease states examined. In addition, smoking cannabis could by its own volition, put the person in a hypoxic state - albeit for a brief period, but this is not a clinically insignificant variable to take into account when dealing with ischemic stroke. Finally, these studies need more refinement in terms of selective $\mathrm{CB}_{1}$ and $\mathrm{CB}_{2}$ agents, testing the gamut of activity from agonist to antagonist to inverse agonist before making any definitive conclusions about cannabinoid use in this disease state.

\subsubsection{Cannabinoids in Alzheimer's Disease}

1.3.4.1. Background of Alzheimer's Disease. Alzheimer's Disease (AD) is becoming more and more prevalent a disease as we have an increasingly aged population and this is an age-related disease. $\mathrm{AD}$ is hallmarked with a progressive loss of cognition and memory, and is the most common form of dementia among the elderly. ${ }^{159}$ Pathological characterization of AD shows neurofibrillary tangles, amyloid-beta $(\mathrm{A} \beta)$ plaques and degradation of the synapses, which causes a loss of neurons specifically in the hippocampus and neighboring areas of the cerebral cortex. Evidence also exists to implicate a sustained activation of microglia in the plaque-bearing areas, causing an area of inflammation ${ }^{160,161}$ and oxidative stress. ${ }^{162,163}$

1.3.4.2. Cannabinoids in Alzheimer's Disease. The previously known high level of $\mathrm{CB}_{1}$ in the hippocampal and cerebral cortex regions of the brain ${ }^{164}$ gave cursory probability of endocannabinoid involvement in the disease progression and pathogenesis. The microglial cells in the plaque-bearing areas have an increase in $\mathrm{CB}_{1}$ and $\mathrm{CB}_{2}$ expression, ${ }^{165}$ while hippocampal and basal ganglia $\mathrm{CB}_{1}$ expression overall is reduced in AD. ${ }^{165,166}$ Despite their high expression in the plaque regions of the brain, cannabinoid receptors are nitrosylated, ${ }^{165}$ which functionally impairs the GPCRs by altering cannabinoid coupling to G-proteins. One final aspect of cannabinoid involvement is the increase of FAAH within astrocytes associated with the plaques. ${ }^{167}$ As FAAH is responsible for metabolizing anandamide and $2-\mathrm{AG}$, this suggests that endocannabinoids are involved in the plaque-formation process.

There has been a good amount of pre-clinical data, which suggests that cannabinoid use is associated with positive outcomes in some of the neuropathological hallmarks of AD. ${ }^{165}$ One such study demonstrated an increase in $2-\mathrm{AG}$ pursuant to $\mathrm{A} \beta$ induced hippocampal damage, which points to this endocannabinoid being 
neuroprotective. ${ }^{168}$ Another process important to the neurodegenerative process of $\mathrm{AD}$, as it was in stroke, is the increase in $\mathrm{Ca}^{+2}$ influx which leads to neuronal damage. Currently, the FDA has approved memantadine for use in $\mathrm{AD}$, and it works as an antagonist of the NMDA receptor to limit $\mathrm{Ca}^{+2}$ influx into the neuron to slow disease progression. Similar to memantadine, HU-211 - a synthetic, "unnatural" enantiomer of HU-210 - works as a selective NMDA receptor inhibitor and consequently helps to protect neurons from degradation. ${ }^{169,170} \mathrm{As} \mathrm{AD}$ is a very complex process, this potential crossover is but one of many ways in which cannabinoids can find usefulness.

Another possible mechanism of neural protection for cannabinoids is demonstrated when $\mathrm{CB}_{2}$ is selectively stimulated, which leads to suppression of microglia activity and thus a decrease in the production of TNF- $\alpha$ and nitric oxide. ${ }^{171}$ Another study of WIN-55,212-2 and HU-210 demonstrated a similar decrease in microglia activation and TNF- $\alpha$ release. ${ }^{165}$ Conclusions from these studies strongly point to the hypothesis that $\mathrm{CB}_{2}$ overexpression in this instance is a marker and mechanism for neural protection. Another potential agent for use is cannabidiol, which has shown efficacy in blocking glutamate-induced excitotocicity, ${ }^{172}$ reduction of proinflammatory mediators, ${ }^{173}$ scavenging of reactive oxygen species to reduce lipid peroxidation ${ }^{174}$ and inhibition of tau protein hyperphosphorylation - an additional AD neuropathological feature. ${ }^{175}$

While there is currently nothing available for patients suffering from AD to completely halt or reverse the progression of the disease, cannabinoids provide a potent agent that can augment and mirror current therapies on the market. Developing cannabinoids to halt the process of neurodegeneration, or delay it much more than current therapies available, remains an ever-present goal of research in this field. Cannabinoids have already found a place in AD treatment by combatting the severe aggression that accompanies the latter stages of the disease. ${ }^{176}$ Overall, AD is another exciting and useful arena for cannabinoid use and research.

\subsubsection{Cannabinoids in diabetes}

1.3.5.1. Endocannabinoids' control of metabolism. There exists data which show a correlation betwixt endocannabinoids and leptin, an adipocyte-derived signaling hormone which are proportional to the fed/satiated state of the animal (i.e. the more wellfed and satiated the animal, the higher the leptin levels). Leptin's relationship to endocannabinoids seems to be inversely related. Studies demonstrate an increase in endocannabinoids in the hypothalamus of food-deprived rats ${ }^{177}$ - specifically 2-AG levels after a 24 hour fast. ${ }^{178}$ The latter of these studies, conducted by Hanus et al., extend the animals out for 12 days of semi starvation and show a direct, negative correlation to 2AG concentrations and the duration of starvation. ${ }^{178}$ Taken together, one may conclude that in the acute phase 2-AG levels rise; however, they eventually decline in a prolonged fasting state due to lack of biosynthesis, thus perpetuating the cycle of maintaining a fasted state - as in anorexia. Another study shows the more direct effect of leptin on reducing anandamide and 2-AG in the hypothalamus. ${ }^{179}$ As a whole, endocannabinoids show themselves as potential co-regulators for metabolism and the drive for satiety.

To that point, a variety of tissues regulates endocannabinoids, especially in the 
visceral periphery. The duodenum, in periods of food deprivation, increases the local levels of anandamide and 2-AG - hypothetically, to act upon sensory and vagal nerves of the brain stem and regulate satiety. ${ }^{180,181}$ As with any hormone system, strict regulation of the endocannabinoid system in adipose tissue must occur. PPAR- $\gamma$ stimulation can inhibit 2-AG in mature $3 \mathrm{t} 3 \mathrm{~F} 442 \mathrm{~A}$ mouse adipocytes, ${ }^{182}$ and decreases $\mathrm{CB}_{1}$ expression and increases FAAH expression in human adipocytes. ${ }^{183}$ This data would suggest $\mathrm{CB}_{1}$ receptors are involved in the early differentiation of adipocytes, possibly upstream of PPAR- $\gamma$, and is subsequently down regulated once PPAR- $\gamma$ is at its peak and differentiation is complete. Concomitantly, PPAR $-\delta$ - itself activated via physical exertion - inhibits $\mathrm{CB}_{1}$ receptor expression. ${ }^{184}$ Lastly, there exists an inverse relationship among both insulin and leptin and endocannabinoid levels, ${ }^{182,185}$ which may augment FAAH expression. ${ }^{186}$

The sustained increase in either endocannabinoids or $\mathrm{CB}_{1}$, or both, in several peripheral organs and tissues of obese or hyperglycemic animals has gained widespread acceptance. Further, data indicate an increase of endocannabinoids in adipocytes and $\beta$ cells. ${ }^{182}$ The effect upon 2-AG, appears to be a PPAR- $\gamma$-mediated effect on the biosynthesis of 2-AG from mature hypertrophic adipocytes. ${ }^{182}$ In both diet-induced obese (DIO) mice and Zucker rats, compared to lean, age-matched animals, endocannabinoids are starkly decreased in subcutaneous fat, ${ }^{180,187}$ this data may then indicate that in obese and/or hyperglycemic rodents there is clearly a hypoactive endocannabinoid system in the adipose store, whereas in the mesenteric fat of DIO mice endocannabinoid levels are unaltered. ${ }^{187}$

\subsubsection{Endocannabinoids in type-2 diabetes and metabolic disorders. As}

previously mentioned, the endocannabinoid system plays an integral role in regulating the metabolic processes of the body, and its reach extends to many different organ systems. One such example demonstrates how $\mathrm{CB}_{1}$ receptors of hypertrophic adipocytes, when subjected to constant agonist activity, suppress adiponectin expression ${ }^{182,188}$ - helping to explain why a $\mathrm{CB}_{1}$ antagonist raises adiponectin in adipocytes in obese mice more readily than lean mice; ${ }^{189}$ and how $\mathrm{CB}_{1}$ antagonists restore the lean phenotype to the expression of adiponectin-depending genes in the adipose tissue of DIO mice. ${ }^{190}$ This information, coupled with the known protective role of adiponectin against insulin resistance and atherogenic inflammation, may lead one to believe that blockade of the endocannabinoid system is the key to mitigating obesity and thusly insulin resistance and atherogenic inflammation. However, as with many hormone systems - endocannabinoids, included ubiquitous and totalitarian control may result in untoward effects. Though, recent data do show $\mathrm{CB}_{1}$ playing a key role in the loss of visceral fat and the enhancement of thermogenesis in subcutaneous fat. ${ }^{191}$

Mitigation of fat accumulation and type is but one of several factors that play into metabolic disorders and into type- 2 diabetes. Another hallmark of type- 2 diabetes is the hyperinsulinemia resulting from insulin resistance. $\mathrm{CB}_{1}$ stimulation has been shown to augment basal insulin release by $\beta$-cells, ${ }^{182,187,192}$ which may result in $\beta$-cell hypertrophy and subsequent dysfunction, perpetuating the development of type-2 diabetes. A more recent study demonstrated that rimonabant (SR-141716A) decreases basal insulin hypersecretion in Zucker diabetic rat isolated pancreatic islets, without affecting the 
glucose-stimulated insulin secretion. ${ }^{193}$ Coupled with this $\mathrm{CB}_{1}$ overstimulation-induced $\beta$-cell damage, hyperactive $\mathrm{CB}_{1}$ receptors in the liver of the mouse may be a root cause of insulin resistance and hepatosteatosis. ${ }^{194}$ Lastly, the paramount characteristic of type-2 diabetes is the resistance of peripheral tissues, especially skeletal muscle, to insulin. Blockade of $\mathrm{CB}_{1}$ ameliorates this trait and increases glucose uptake and AMP kinase expression of skeletal muscle. ${ }^{195,196}$

As one can plainly see, dysregulation of the endocannabinoid system, more specifically the over activity thereof, shows strong ties to the development of metabolic syndrome and subsequently or concomitantly type- 2 diabetes. A trio of studies involving rimonabant in Zucker rats further demonstrated this correlation. The first gave indication that with oral treatment of rimonabant at $30 \mathrm{mg} / \mathrm{kg}$ the presence of hepatic steatosis and other related metabolic syndrome features were eradicated. ${ }^{197}$ Further, this study showed an improvement of hepatomegaly and markers associated with hepatic damage. ${ }^{197}$ Finally, this study there was a noted improvement in blood lipids toward a more normal and healthy state. ${ }^{197}$ Most notable from this study is that this was shown via pair-fed controls that the effect was not merely a result of diet, alone. The second study demonstrated the utility of rimonabant in attenuating the morality and chronic renal failure seen in obesity and long-term diabetes, again as compared to pair-fed but untreated rats. ${ }^{198}$ The study further demonstrated a sustained lowering of body weight, transient hypophagia, increased adiponectin and a confirmation of the first study's rectification of dyslipidemia. ${ }^{198}$ The third study sought to examine the inflammation component, and in so doing demonstrated that rimonabant could successfully lower the neutrophil and macrophage levels in obese Zucker rats as a model for both glucose intolerance and metabolic syndrome. ${ }^{198}$ As in the previous 2 studies, rimonabant successfully attenuated eating, rectified dyslipidemia and decreased atherosclerotic lesions. ${ }^{198}$ This study did take the data a step further to show a decrease in proinflammatory cytokines MCP-1 and interleukin-12 (IL-12). ${ }^{198}$ As a constellation, these studies and data point to the potential of the peripheral, rather than the central, endocannabinoid system as being a viable target for the treatment of obesity-related disease states.

1.3.5.3. Endocannabinoids in abdominal obesity and hyperglycemia. There is no denying that there is a positive correlation between elevated intra-abdominal obesity and type-2 diabetes - given, too, the further direct correlation to an increase in atherogenic inflammation and an increase in cardiovascular events. ${ }^{199}$ As previously discussed, the endocannabinoid system lends itself to regulation and differentiation of adipose tissue when left unchecked. It has been demonstrated that 2-AG, but not anandamide, is increased in visceral, but not subcutaneous, fat; ${ }^{182}$ an increase in $\mathrm{CB}_{1}$ expression and endocannabinoid turnover (i.e. increased biosynthetic and degradation enzyme expression) in obese individuals; ${ }^{183}$ and a decrease in endocannabinoid synthesis in subcutaneous fat. ${ }^{187}$ More recently, an observation of the subcutaneous fat of obese, type-2 diabetes patients demonstrates a ubiquitous lowering of endocannabinoid tone. ${ }^{200}$ These data point to the very real and very serious potential for a dysregulated endocannabinoid system to cause an increase in abdominal fat deposit in lieu of subcutaneous fat - promoting an increase in the cardiometabolic risks for patients. 
Further adding to the positive association of the endocannabinoid system, intraabdominal obesity and type- 2 diabetes, a direct relationship has been shown between intra-abdominal obesity and circulating 2-AG. ${ }^{201,202}$ It was through these studies that a more direct correlation between intra-abdominal fat and circulating 2-AG manifest as cardiometabolic risk factors - such as low HDL, high triglycerides, low insulin sensitivity, glucose intolerance ${ }^{201}$ and decreased plasma adiponectin. ${ }^{202}$ Further causal link between hyperglycemia and increased 2-AG was observed even in non-obese individuals $^{182}$ - giving more credential to a dysregulated endocannabinoid system as an independent risk factor in type- 2 diabetes. This was echoed by a prospective, though small, study of viscerally obese men who underwent 12 months of therapeutic lifestyle changes to dietary habits and physical activity. The results showed an across-the-board reduction in not only body mass and fat composition, but a stark decrease in 2-AG levels. ${ }^{203}$ Further, the data showed an inverse correlation between 2-AG and HDL levels along with a direct correlation between $2-\mathrm{AG}$ and insulin resistance. ${ }^{203}$ On its surface, this data suggests 2-AG as the culpable target; however, one must be mindful of the fact that endocannabinoids are not generally hormone-like in traditional function, so further study is needed to determine if $2-\mathrm{AG}$ is secondary to increased peripheral production especially in obesity.

1.3.5.4. Use of $C B_{1}$ antagonists and inverse agonists in type-2 diabetes. The central tenant in the management of type- 2 diabetes is and will forever remain therapeutic lifestyle changes such as improved dietary habits and increased physical activity; however, there is always the desire to augment these changes to improve outcomes for patients. For a time, rimonabant showed great promise as this agent - successfully completing four (4) phase III and two (2) phase IIIB clinical trials ${ }^{204-207}$ and approved for use in the EU on June 21, 2006. Its success was short-lived, though, as it the European Medicines agency pulled the drug from the market on October 23, 2008 over concerns of serious psychiatric problems and even suicide. It was not until January 16, 2009 that the European Medicines Agency formally and completely withdrew its approval. Other potential agents, such as Merck's taranabant ${ }^{208-210}$ and Pfizer's otenabant ${ }^{211,212}$ have ceased to continue with clinical trial because of similar problems arising during clinical trial.

These drugs are worth mentioning, because during their initial investigation they showed great promise themselves and provide valuable insight into the potential of cannabinergic drugs' utility in this arena. The rimonabant in obesity (RIO) trials demonstrated rimonabant's utility in reducing not only food intake but also the signs, symptoms and complications of metabolic syndrome. ${ }^{213}$ Expansion of these trials into the RIO-diabetes trial showed the beneficial effects of current standard treatment with rimonabant in not only improving body composition but regulating and stabilizing glucose control. ${ }^{205}$ Further, the ARPEGGIO trial began to expose the weaknesses of rimonabant, highlighting the increase in anxiety and depression (both with 14\% occurrence) among the treatment groups. The SERENADE study showed an increase in both anxiety and depression, though with less frequency $(5.8 \%)$ than ARPEGGIO. The untoward psychotropic effects, which have long plagued cannabinoid therapies, would lead to the downfall of rimonabant, and unfortunately the entire class, both in development as well as deployment. 
As is the case with many new drug classes, there are zeniths and nadirs to contend with, and with the current explosion and interest in cannabinoids, we are only now beginning to understand this intricately woven and tightly controlled system. As one can see from this brief presentation, cannabinoids present a very viable target for use in controlling metabolic syndrome and type-2 diabetes; however, there needs to be more study on separation of the beneficial and untoward effects. There are efforts underway to peripherally restrict cannabinoids, ${ }^{214}$ but the possibility exists that compounds may be synthesized which could specifically exert beneficial effects without the need to be restricted to one compartment or the other. Given all of the aforementioned potential for cannabinoids and the relatively limited amount of understanding we have for this system, I think we have only just begun to see the utility of these compounds and how we can develop and use them to benefit and impact a variety of disease states, type- 2 diabetes being one of many.

\subsection{Goals of the Current Project}

Knowing now how positively one may influence some of the top disease states of people via cannabinoids, this project takes on a great deal of merit. This project seeks to develop a more guided approach to the design of cannabinoid agents. This begins with the validation and refinement of an assay to ascertain functional activity (Chapter 2). Then, detailed look at current knowledge of structural pharmacophore data at the C-3 position of classical cannabinoids, and an exploration of a novel series of C-3-modified classical cannabinoids (Chapter 3). Building from the success of the C-3-modified classical cannabinoids, I examine the effects on functional activity resulting from the modification of the classical benzchromene core (Chapter 4). Considering all previous data, I will examine the hybridization of non-classical and classical features to result in another novel triaryl class of cannabinoids (Chapter 5). Finally, taking into account the current literature and body of evidence for modelling $\mathrm{CB}_{1}$ and $\mathrm{CB}_{2}$, coupling that with my own rudimentary modelling and functional data, a rough functional pharmacophore was developed to guide future design (Chapter 6).

As one can see, cannabinoids have a wide-ranging and potentially blockbuster impact to healthcare and treatment of disease states. This project seeks to gain more knowledge and insight into the function of novel cannabinergic agents synthesized in our lab. Through this work, knowledge is generated to elucidate which, if any, components of classical and triaryl-core cannabinoids can result in agonist, antagonist, inverse agonist or a mixture thereof, as defined by the cubic ternary complex model. Within this model for GPCR functional activity, there is an augmentation of the extended ternary complex model. Inherent in both of these models is the specificity of certain drugs for a particular state. The first assumption made is that the receptor is in equilibrium with an active and inactive state (i.e. ready to signal downstream or not). Agonists of this model target the active state, and thus shift the equilibrium toward the active state of the receptor. Inverse agonists target the inactive conformation of the receptor, and so the equilibrium shifts toward the inactive state. Antagonists do not discriminate nor target a particular conformation of the receptor, thus the equilibrium is not changed and the total available receptor decreases. The added component to the cubic ternary complex model is that the 
receptor, in the active or inactive state - ligand bound or unbound - can bind and sequester G-proteins into a non-signaling state. This added allocation of thermodynamic activity allows for the added activity of an inverse agonist. With this model, inverse agonists could bind the inactive state and subsequently sequester G-proteins away from signaling pathways. This is actually a noted occurrence with cannabinoids, in particular. ${ }^{40}$

Through this project's documentation, I will demonstrate the unique aspects of classical and non-classical cannabinoids which impart equally unique functional activity. Seemingly, simple interchanges at the $\mathrm{C}-1^{\prime}$ position cause a non-classical agent to change from an agonist at $\mathrm{CB}_{2}$ to an inverse agonist. Additionally, bioisosteric replacement of a carbocyclic ring to a heterocyclic ring in classical cannabinoids cause a similar shift from agonist to inverse agonist, and within the heterocyclics, transition from a single to a dual heteroatom heterocyclic ring yields inverse agonist at $\mathrm{CB}_{1}$ and $\mathrm{CB}_{2}$, respectively. Taking these unique functional activities, along with the theories presented in the cubic ternary complex model, I will attempt to reconcile and possibly implicate key residue interactions within the $\mathrm{CB}_{1}$ and $\mathrm{CB}_{2}$ ligand-binding pocket, which may result in these unique activities. 


\section{CHAPTER 2. ASSAY DEVELOPMENT FOR THE FUNCTIONAL ACTIVITY ANALYSIS OF NOVEL CANNABINERGIC COMPOUNDS}

\subsection{Introduction}

As previously discussed, there exists a desire for selectively targeting $\mathrm{CB}_{1}$ and $\mathrm{CB}_{2}$ receptors to help maximize therapeutic benefit and minimize negative side effects namely the psychotropic effects of $\mathrm{CB}_{1}$ agonists. Along with that, there is a need and desire to know the functional activity of drugs (agonist, antagonist, inverse agonist) to help elucidate the downstream effect(s) of a drug. To help ascertain the functional activity of cannabinoid drugs, two assays hold the lion's share of utilization $\left(\left[{ }^{35} \mathrm{~S}\right]-\mathrm{GTP} \gamma \mathrm{S}\right.$ and $\beta$-arrestin); however, I will show the development and utility of a third assay that is currently available.

Far and away the most dominant force in functional activity analysis has been the $\left[{ }^{35} \mathrm{~S}\right]-\mathrm{GTP} \gamma \mathrm{S}$ assay. It is useful in that it allows one to obtain the potency, efficacy and antagonist affinity without the need to have downstream amplification for agonist compounds. ${ }^{215}$ While this is unquestionably useful, I believe, there is a deficiency inherent within that assay. The deficiency lies in the fact that it is an essentially irreversible reaction (in the presence of $\mathrm{mM} \mathrm{Mg}^{+2}$ ) 216,217 , such that once an agonist binds to the receptor being assayed, $\left[{ }^{35} \mathrm{~S}\right]-\mathrm{GTP} \gamma \mathrm{S}$ binds to the $\mathrm{G} \alpha$-subunit, forming a hydrolysis-resistant $\mathrm{G} \alpha-\left[{ }^{35} \mathrm{~S}\right]-\mathrm{GTP} \gamma \mathrm{S}$ complex, preventing hydrolysis by the GTPase of $\mathrm{G} \alpha$ and mitigating rejoining with the $\mathrm{G} \beta \gamma$-complex subunit. Measurement of activation is accomplished by filtering the membrane preparation and counting the radioactivity left on the filter(s). This does not mimic more physiologic conditions, whereupon agonist binding to the receptor GDP dissociates from and GTP binds to the G $\alpha$-subunit, eventually being hydrolyzed back to GDP by the GTPase domain of the G $\alpha$-subunit and allowing the $\mathrm{G} \alpha$-subunit to rejoin the G $\beta \gamma$-complex subunit. While the $\left[{ }^{35} \mathrm{~S}\right]-\mathrm{GTP} \gamma \mathrm{S}$ assay is a very sensitive assay, able to detect very minute $\mathrm{EC}_{50}$ values of compounds, its irreversible nature may present issues when moving drugs from the bench top into a living and dynamic system. Additionally, it has been shown that while the reaction is nearly irreversible, cannabinoids show evidence of promoting the dissociation of $\left[{ }^{35} \mathrm{~S}\right]-\mathrm{GTP} \gamma \mathrm{S}$ from $\mathrm{G} \alpha,{ }^{218}$ thus leaving us with false data for the compounds to be tested.

Another assay utilized is the PathHunter ${ }^{\circledR} \beta$-arrestin assay from DiscoveRx. This assay utilizes a fragmented $\beta$-galactosidase enzyme to detect GPCR activation. In this assay system, a small fragment of the $\beta$-galactosidase enzyme is fused to the GPCR of interest; the remaining larger fragment of $\beta$-galactosidase is co-expressed as an $\mathrm{N}$ terminal deletion mutant. Upon activation of the GPCR, binding of the small and large fragments forms the activated $\beta$-galactosidase enzyme. Activation of this enzyme leads to stimulation of chemiluminescent dye and subsequent detection on a plate reader. While there have been reports of this assay's use in detecting and qualifying inverse agonists; ${ }^{219}$ however, when examining the data presented, it is evident that this assay is not as sensitive at detecting inverse agonists relative to agonists. Additionally, nowhere within the manufacturer literature nor in various other group's use of this assay has this been shown. ${ }^{220,221}$ While it does accomplish the aforementioned goal of more closely 
mimicking physiological conditions by not relying upon an essentially irreversible activity, lack of or reduced ability to detect inverse agonist activity makes this a lessviable candidate. Additionally, while manufacturer data suggests that engineering of the GPCR of interest does not alter the binding affinity for substrate, it does impart an added variable, which should not be fully discounted in biological assays and renders this assay even less desirable.

A bridging of the gap between a need to mimic physiological conditions and to detect inverse agonist activity lies with the ACTOne assay available from Codex Biosolutions. This assay utilizes, HEK-293 cells transduced with the GPCR of interest along with a proprietary cyclic-nucleotide gated ion (CNG) channel, which opens in response to increased intracellular cAMP and allows for the influx of $\mathrm{K}^{+}, \mathrm{Na}^{+}$and $\mathrm{Ca}^{++}$. A phosphodiesterase inhibitor (Ro 20-1724) is added to the assay well in excess $(25 \mu \mathrm{M})$ to prevent the degredation of cAMP and allow for its real-time detection via the CNG channel activation. Detection of this ion flow results from the presence of an extracellular cationic-dependent dye, whose fluorescence shows on a plate reader at $540 \mathrm{~nm}$ excitation and $590 \mathrm{~nm}$ emission. As the extracellular concentration of cations decreases, a result of increased intracellular cAMP and subsequent influx of cations via the CNG channel, emission detected by the plate reader begins to increase, accordingly. Thus, in the case of $\mathrm{CB}_{1}$ and $\mathrm{CB}_{2}$, which are coupled to the inhibitory $\mathrm{G}_{\mathrm{i} / \mathrm{o}}$, an agonist of either of these receptors will cause a decrease in intracellular cAMP and an increase in extracellular cations - this increase of cations complexes the cation-sensitive dye so that it does not emit upon excitation. Therefore, the emission detection by the plate reader directly correlates to the amount of cAMP produced in each of the wells of a plate - allowing for a real-time examination of cAMP levels, without any engineering, alteration or complexation of the GPCR of interest. It is for its simplicity, ability to detect agonist, antagonists and inverse agonists as well as a reversible, non-engineered GPCR presence that this assay found utilization. However, initial conductance of the assay was not without its problems, and refinement and optimization was paramount in assuring accuracy, consistency and comparability of the data across all drugs and receptor types of interest.

Having selected the ACTOne assay based on the aforementioned parameters, conductance of the assay proved to be problematic, with responses and $\mathrm{EC}_{50}$ values for well-established cannabinoid compounds waxing and waning from day to day and even from plate to plate within the same day's runs. Given that there are several variables at play in such a dynamic assay, I took the time and effort to tease apart the individual variables to regulate, stabilize and optimize each step of the assay - ensuring accuracy and consistency in the data gathered. I conducted experiments to modify the manufacturer recommended procedure (Section 2.2.1) to optimize: cAMP stimulants (Section 2.2.2), plating density (Section 2.2.3), plate selection (Section 2.2.4), plating environment (Section 2.2.5), pertussis toxin dosing (Section 0) and order of addition (Section 2.2.6.2), data analysis (Section 2.2.7) and finally arrived at a streamlined procedure (Section 2.3). The data presented herein shows the process of honing the assay into a tool useful in the procurement of functional activity of novel series of cannabinoids generated by my predecessors in the group. 


\subsection{ACTOne Assay Development}

\subsubsection{Manufacturer recommended procedure}

2.2.1.1. Commonalities of ACTOne assay protocols. Overall, the treatment of the cells among the 3 assay protocols $\left(\mathrm{CB}_{1}, \mathrm{CB}_{2}\right.$ and $\left.\mathrm{HEK}-\mathrm{CNG}\right)$ is the same, with growth recommended in DMEM with 10\% FBS, $250 \mu \mathrm{g} / \mathrm{mL}$ G418 and $1 \mu \mathrm{g} / \mathrm{mL}$ puromycin. Each of the cell types are to be kept at $37^{\circ} \mathrm{C}$ with $5 \% \mathrm{CO}_{2}$, and not allowed to grow beyond $90 \%$ confluence - overgrowth may significantly reduce the cell's response to a ligand and take several passages to regenerate.

The initial recommendation for cell plating is that one should use a Poly-D-Lysine coated plate to facilitate cell adherence, and plate cells the preceding day so that they may grow overnight. For the 96-well plate, which is what I used, the manufacturer recommends plating 70,000 cells/100 $\mu \mathrm{L} /$ well; however, there is the ever-present caveat that one must optimize the cell number for each assay.

Following the overnight growth at $37^{\circ} \mathrm{C}$, add ACTOne Membrane Potential Dye to each of the wells in the plate at a volume equal to the volume of cell suspension added the previous day $-100 \mu \mathrm{L}$, in my case. The dye is to dwell for two hours at room temperature, in a dark place. In the event a serum-free environment is required, remove the media and replace with DPBS containing $0.2 \%$ to $0.5 \%$ BSA prior to adding the dye.

The differences among the three protocols prompted an initial hypothesis that the assay needed examination with greater scrutiny. The purpose of this examination is to maintain consistency across the three protocols so that data obtained from conductance thereof is able to be cross-compared and correlated.

2.2.1.2. CB1-specific parameters of the ACTOne assay. Differences among the three assay protocols begin with the agent used to stimulate adenylyl cyclase. In the case of the $\mathrm{CB}_{1}$ protocol, the agent of choice is isoproterenol - exerting its effects via the

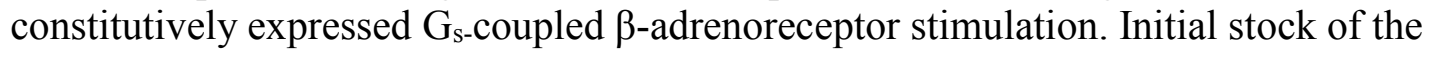
compound to be tested, adenylyl cyclase stimulant and PDE-inhibitor (Ro 20-1724) are prepared at five times the final testing concentrations due to the fact that they will be diluted 1:5 when added to the assay plate ( $50 \mu \mathrm{L}$ compound stock added to $200 \mu \mathrm{L}$ of cell suspension and membrane potential dye). Consistent in all wells of the assay is Ro 20-1724 at $25 \mu \mathrm{M}$ and isoproterenol at $300 \mathrm{nM}$, final concentrations. The compound of interest has its concentration varied from column to column in a given row to obtain a dose response curve on a logarithmic scale.

2.2.1.3. $\mathrm{CB}_{2}$-specific parameters of the ACTOne assay. Similarly to the $\mathrm{CB}_{1}$ protocol, the final concentration of Ro 20-1724 is $25 \mu \mathrm{M}$ in each well; however, the adenylyl cyclase stimulant is different and at a different concentrations. For the $\mathrm{CB}_{2}$ protocol, NECA becomes the agent of choice to stimulate adenylyl cyclase via $\mathrm{G}_{\mathrm{s}^{-}}$ coupled adenosine $A_{2 b}$ receptors constitutively expressed on HEK293 cells. Final concentration of NECA is $250 \mathrm{nM}$ in each of the assay wells. Additionally, in the documentation from the manufacturer, CP-55,940, which is the standard I use for 
non-selective $\mathrm{CB}$-agonists, was at ten times higher concentrations in the $\mathrm{CB}_{1}$ protocol tests $v$. the $\mathrm{CB}_{2}$ protocol tests - though the $\mathrm{EC}_{50}$ for $\mathrm{CP}-55,940$ is $0.2 \mathrm{nM}$ and $0.3 \mathrm{nM}$ for $\mathrm{CB}_{1}$ and $\mathrm{CB}_{2}$, respectively, in the literature. ${ }^{222}$

2.2.1.4. Parental, HEK-CNG-specific parameters of the ACTOne assay. For the parental cells, since there is no over-expression of our GPCR of interest, this protocol is useful in determining the $\mathrm{EC}_{50}$ of the adenylyl cyclase stimulant, as well as confirming the $\mathrm{G}_{\mathrm{i} / \mathrm{o}}$ activity observed is not due to another, constitutively expressed, GPCR of HEK293 cells. It is of an interesting note that the manufacturer reports the $\mathrm{EC}_{50}$ of NECA in HEK293 cells to be $611 \mathrm{nM}$; however, as previously mentioned, the $\mathrm{CB}_{2}$ protocol only calls for a $250 \mathrm{nM}$ NECA concentration in each of the assay wells.

\subsubsection{Assay stimulus standardization}

Since this assay relies on cAMP as the substrate for $\mathrm{CNG}$ channel activation and subsequent impact upon the cation-sensitive dye, a reproducible and consistent source of adenylyl cyclase induction is essential for continuity of data and analysis. Recommended within the manufacturer's directions is the use of isoproterenol for $\mathrm{CB}_{1}$ cells and NECA for both $\mathrm{CB}_{2}$ and parental $\mathrm{CNG}$ cells to stimulate cAMP production and subsequent $\mathrm{CNG}$ channel activation of the cells. However, to maintain more consistency within the assay, comparing the three cells lines across multiple plates on multiple days, it was my hypothesis that the stimulus should be consistent across all runs in order for the results to be deemed comparable and consistent.

2.2.2.1. Manufacturer recommended cell stimulus. Initially, isoproterenol was employed at $50 \mathrm{nM}$, being $>10 \mathrm{X}$ the $\mathrm{EC}_{50}$ of isoproterenol at $\mathrm{CB}_{1}\left(\mathrm{CB}_{1}=2.56 \mathrm{nM} ; \mathrm{CB}_{2}=\right.$ $12.3 \mathrm{nM}$ at 50 minutes after addition) (Figure 2.1). As one can see immediately, the response gained from isoproterenol in the $\mathrm{CB}_{2}$ cells was virtually nil at 50 minutes. While generally good in the $\mathrm{CB}_{1}$ assay, there was still some inconsistency therein, coupled with a waning of the response in the $\mathrm{CB}_{2}$ assay after 10 minutes of the kinetic run time (Figure 2.2) isoproterenol lost favor as a truly viable candidate. Considering the next manufacturer recommended stimulus, NECA, was tried at $100 \mathrm{nM}$, being $>2 \mathrm{X}$ the $\mathrm{EC}_{50}$ of NECA $\left(\mathrm{CB}_{1}=40.0 \mathrm{nM} ; \mathrm{CB}_{2}=316 \mathrm{nM}, 50\right.$ minutes after addition) (Figure 2.3). There was a glimmer of hope for NECA, inasmuch as providing a nearly equal response, albeit at an $\mathrm{EC}_{50} \sim 8 \mathrm{X}$ that of $\mathrm{CB}_{1}$; however, these hopes were dashed even at nearly $2 \mathrm{X}$ the $\mathrm{EC}_{50}$ of NECA at $\mathrm{CB}_{2}$, making this agent not readily cross-comparable (Figure 2.4).

2.2.2.2. CB1 cAMP stimulant trial. In an effort to maintain a consistent stimulation of cAMP production, utilizing the knowledge that manufacturer recommended agents were adrenergic agonists, eight adrenergic agonists were screened as potentials for the $\mathrm{CB}_{1}$ assay. The agents are: medetomiodine $(\alpha-2$ agonist), norepinephrine (non-selective $\alpha$ - and $\beta$-agonist), clonidine ( $\alpha-2$ agonist), epinephrine (non-selective $\alpha$ - and $\beta$-agonist), oxymetazoline (non-selective $\alpha$-agonist), yohimbine $(\alpha-2$ antagonist), pseudoephedrine (non-selective $\alpha$-agonist), isoproterenol (non-selective $\beta$ agonist) and NECA (adenosine receptor agonist). Evaluation of the data is relative, such 


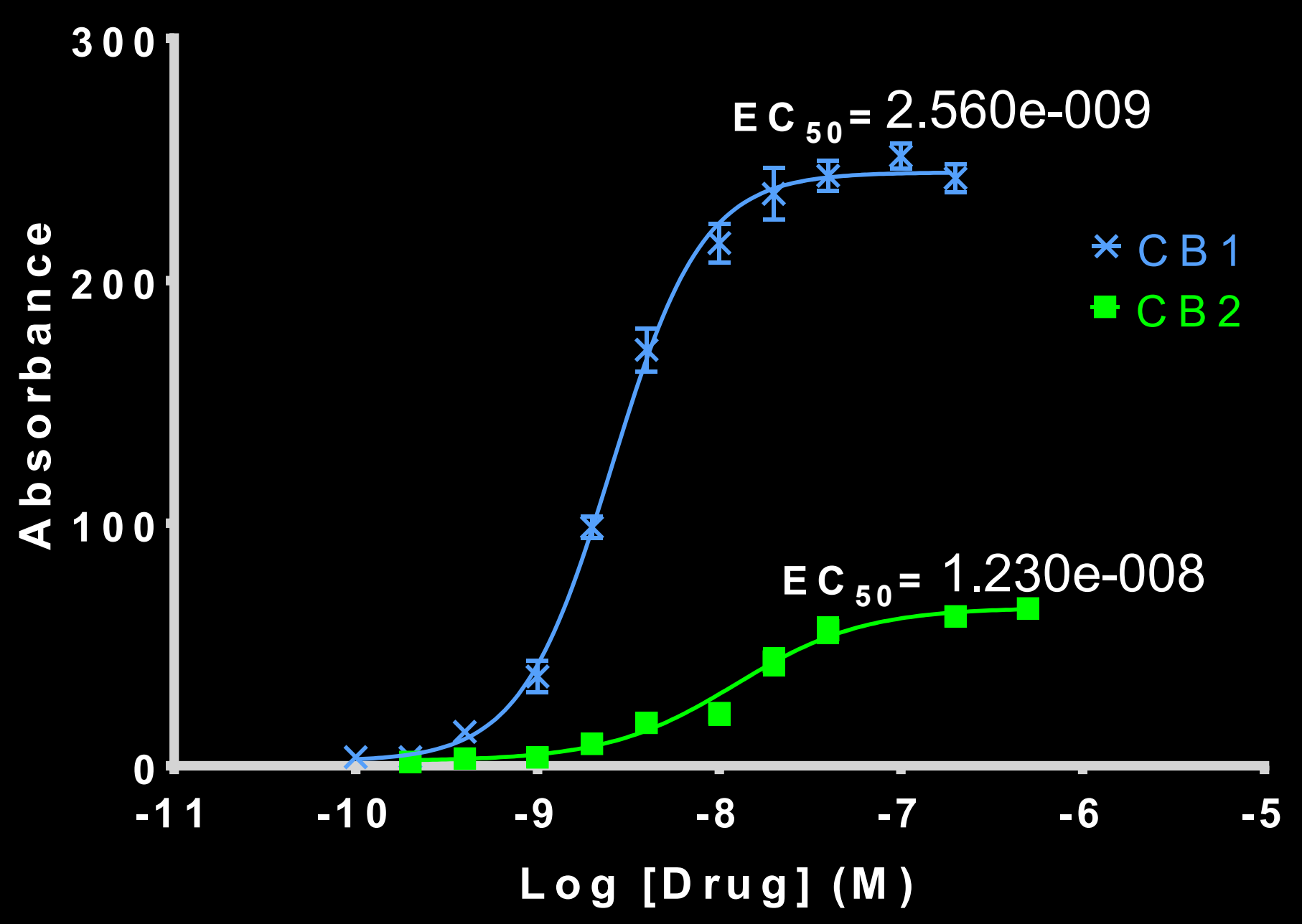

Figure 2.1. Isoproterenol EC $_{50}$ curve at 50 minutes post-addition 


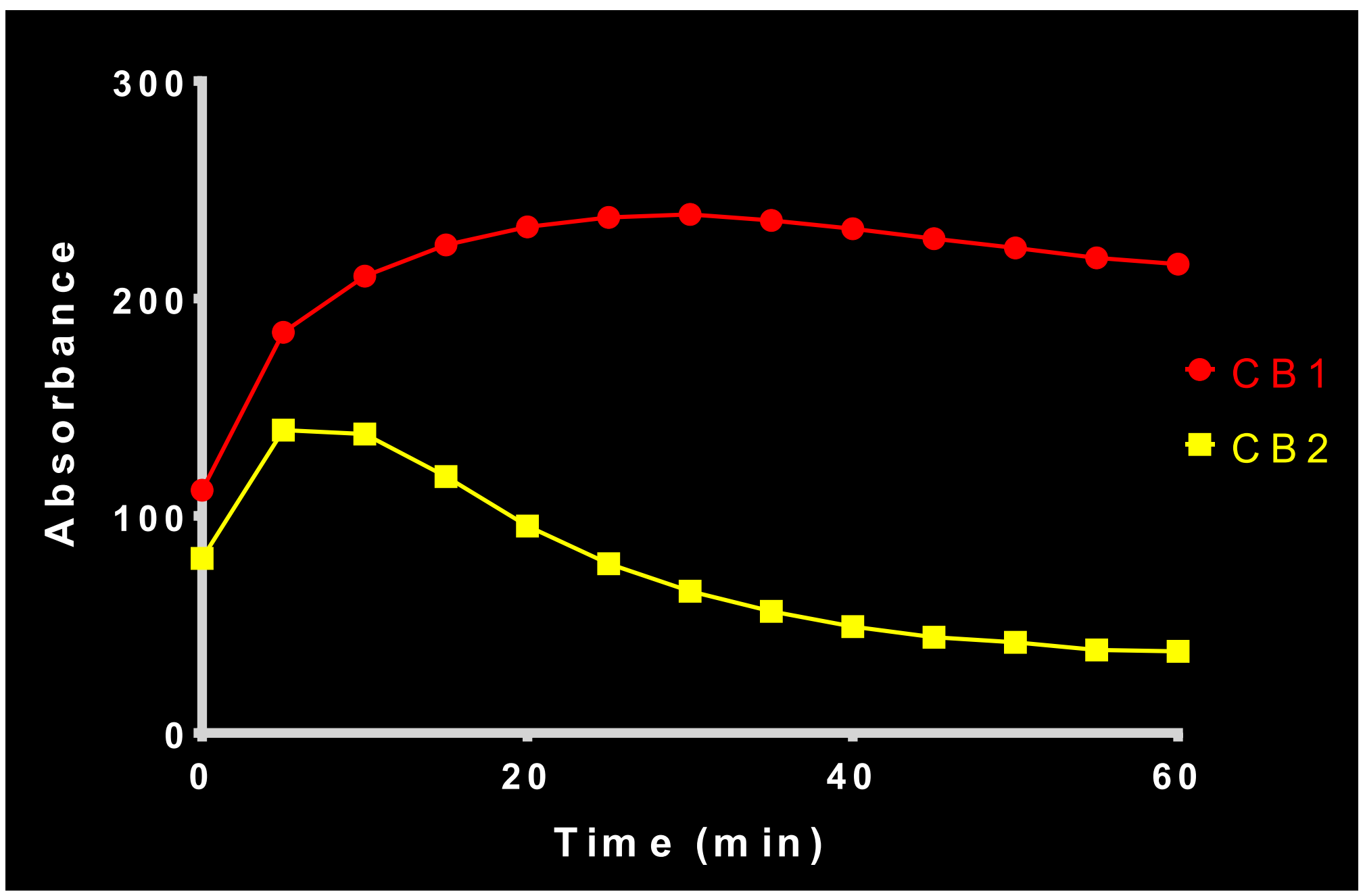

Figure 2.2. Isoproterenol-induced cAMP production at $50 \mathrm{nM}$ 


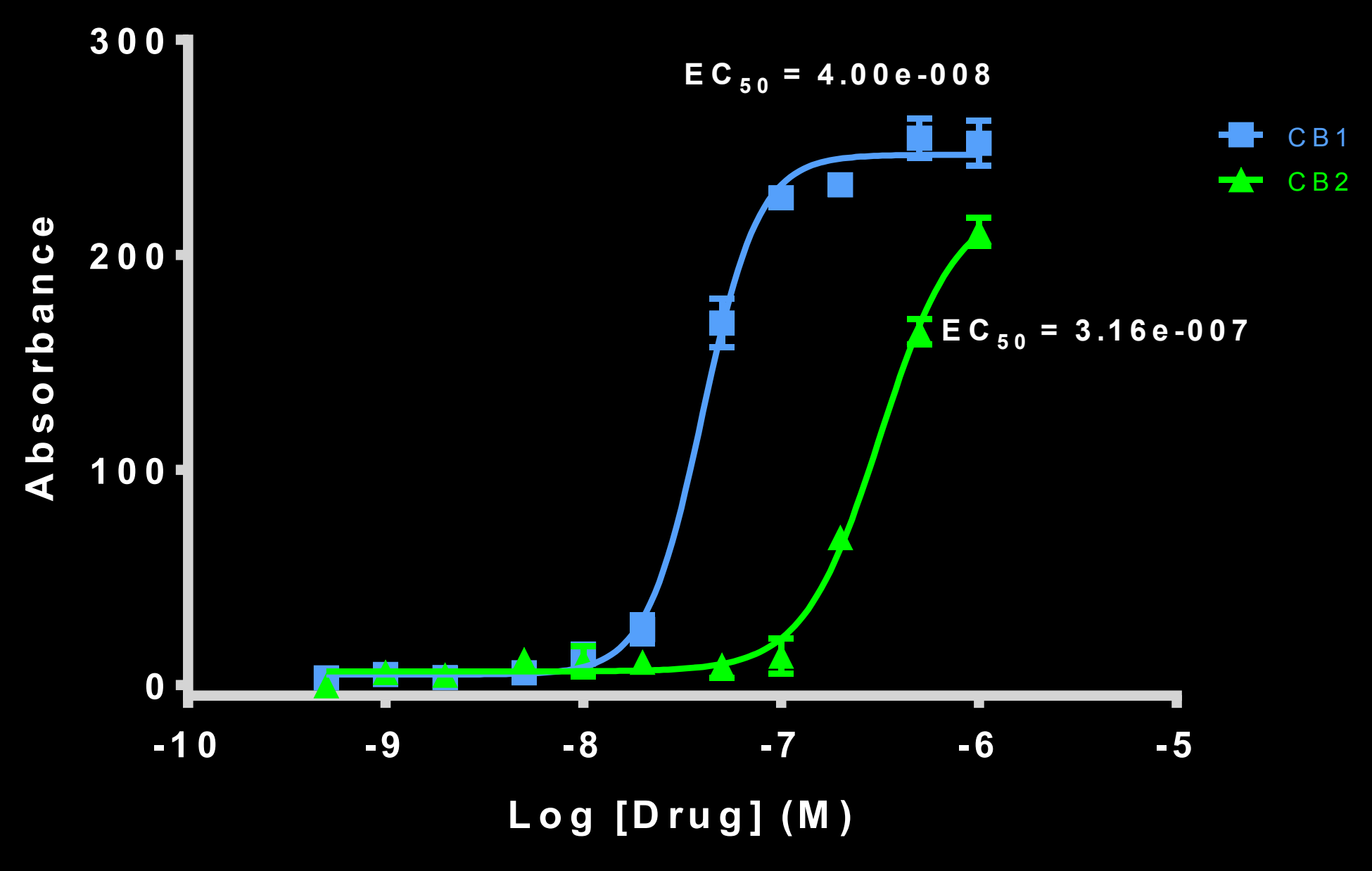

Figure 2.3. NECA EC50 curve at 50 minutes post-addition 


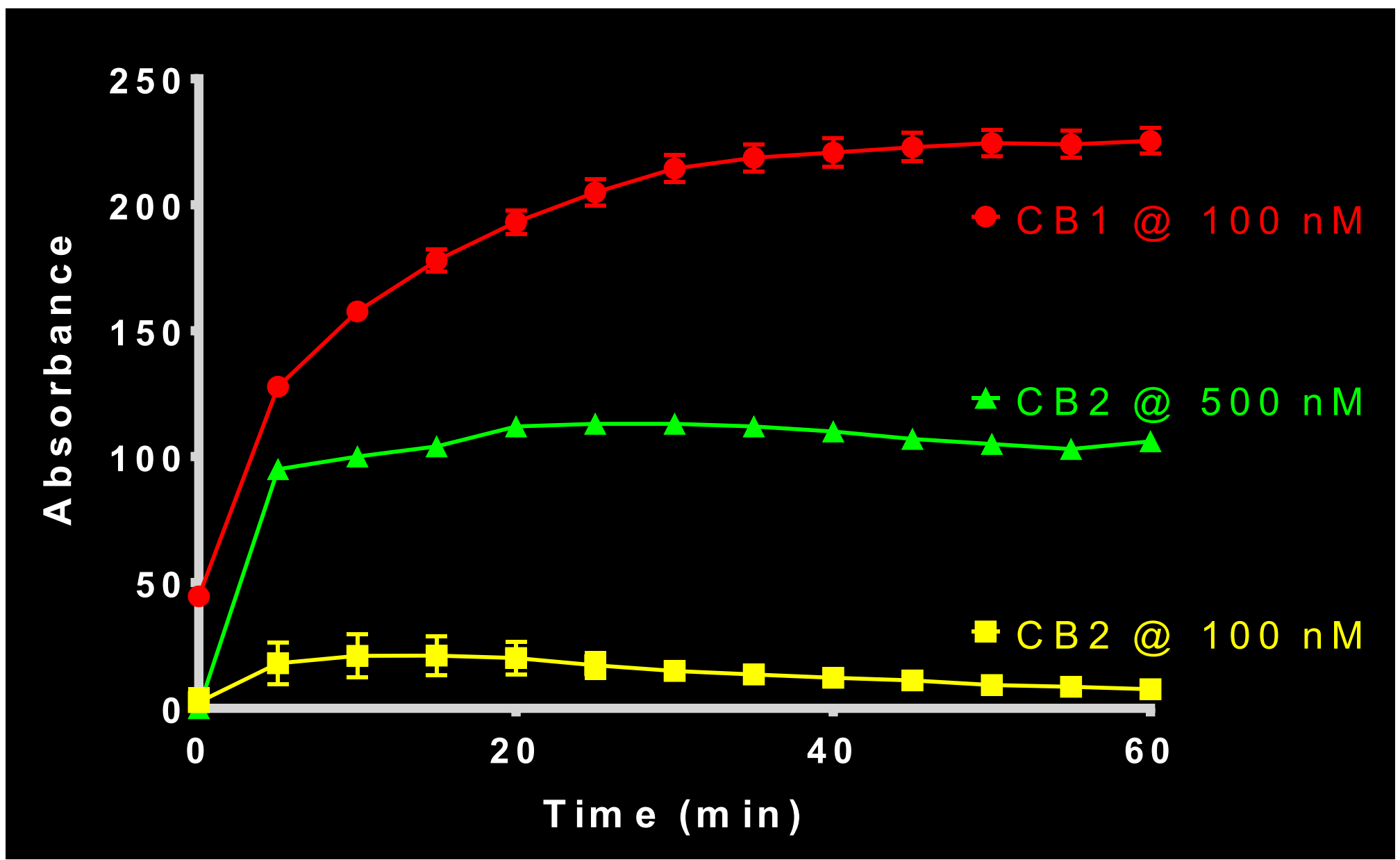

Figure 2.4. NECA-induced cAMP production in $\mathrm{CB}_{1}$ and $\mathrm{CB}_{2}$ cells 
that the highest value on the assay plate corresponds to the highest possible value for all of the drugs. This analysis allows for a more direct comparison of the data, to show that isoproterenol $\left(\mathrm{EC}_{50}=14 \mathrm{nM}\right.$; Lit: $\left.123 \mathrm{nM}^{223}\right)$, NECA $\left(\mathrm{EC}_{50}=147.5 \mathrm{nM}\right.$; Lit: $\mathrm{A}_{1}=26$ $\left.\mathrm{nM},{ }^{224} \mathrm{~A}_{2 \mathrm{~A}}=26.1 \mathrm{nM},{ }^{225} \mathrm{~A}_{2 \mathrm{~B}}=1400 \mathrm{nM},{ }^{226} \mathrm{~A}_{3}=129 \mathrm{nM}^{227}\right)$ and epinephrine $\left(\mathrm{EC}_{50}=\right.$ $110.5 \mathrm{nM}$; Lit: $8.2 \mathrm{nM}^{228}$ ) will each stimulate cAMP production in the $\mathrm{CB}_{1}$ cells to a similar level (Figure 2.5).

2.2.2.3. $\mathrm{CB}_{2} \mathbf{C A M P}$ stimulant trial. Similar to the aforementioned trial of agonists in the $C B_{1}$ cells, evaluation of various adrenergic agonists for the $\mathrm{CB}_{2}$ assay was conducted with: norepinephrine (non-selective $\alpha$ - and $\beta$-agonist), epinephrine (nonselective $\alpha$-and $\beta$-agonist), oxymetazoline (non-selective $\alpha$-agonist), pseudoephedrine (non-selective $\alpha$-agonist), formoterol ( $\beta_{2}$-selective agonist), isoproterenol (non-selective $\beta$-agonist), and NECA (adenosine receptor agonist) (Figure 2.6). Data was calculated, as it was for the $\mathrm{CB}_{1}$ assay, as a relative effectiveness for each of the drugs. As a result of this analysis epinephrine, formoterol, isoproterenol and NECA appear to be potential agents for further testing and elucidation of properties; however, as previously discussed isoproterenol and NECA have viability and consistency issues with the $\mathrm{CB}_{2}$ assay.

To fully test the $\mathrm{CB}_{2}$ assay stimulants, these potential stimulants, isoproterenol, formoterol, epinephrine and NECA, were tested at their respective literature cited $\mathrm{EC}_{50}$ $\left(150 \mathrm{nM},{ }^{223} 350 \mathrm{nM}, 6 \mathrm{nM}^{228}\right.$ and $125 \mathrm{nM},{ }^{227}$ respectively) and $\mathrm{EC}_{90}(2500 \mathrm{nM}, 1000 \mathrm{nM}$, $1000 \mathrm{nM}$ and $1000 \mathrm{nM}$, respectively) $v$. CP-55,940. Data from this study demonstrate that NECA, at its $\mathrm{EC}_{90}$, is the only one of the 4 capable of stimulating cAMP production 50 minutes after addition to the assay plate (Figure 2.7); however, it still shows much evidence of variability, and this is not at all desired.

When examining the two cell lines, it is apparent that the data may not be accurately compared using equivalent doses of isoproterenol or NECA (Figure 2.8), nor is it possible with any of the additional adrenergic agents tested. For the $\mathrm{CB}_{1}$ cells, a final concentration of $50 \mathrm{nM}$ of isoproterenol in each well was enough to elucidate a response from the cells; however, this same dose in $\mathrm{CB}_{2}$ cells began to decline only 10 minutes after addition to the cells (Figure 2.8). NECA was considered next, and at a final concentration of $100 \mathrm{nM}$ in each well for $\mathrm{CB}_{1}$, we again see a nice rise in cAMP production that is consistent over the entirety of the 60 minute kinetic run; however, in $\mathrm{CB}_{2}$, the same dose of NECA would not elucidate a strong enough response to be detectable (Figure 2.8). Given the fact that isoproterenol is inconsistent with respect to time between the two assays and NECA is inconsistent with respect to dose, a more consistent and directly comparable stimulant of cAMP production was needed to help stabilize the cAMP levels in the assay and allow for more accurate cross-comparison.

Forskolin is a well-known, direct stimulant of adenylyl cyclase in assays measuring cAMP production, with a $12 \mu \mathrm{M} \mathrm{EC}_{50 .}{ }^{229,230}$ To apply this to my assay, a forskolin dose response curve was conducted to ascertain the dose necessary for use in both the $\mathrm{CB}_{1}$ and $\mathrm{CB}_{2}$ assays. In both the $\mathrm{CB}_{1}$ and $\mathrm{CB}_{2}$ assays, the dose response was quite stark as the dose was increased, with an $\mathrm{EC}_{50}$ of $112 \mathrm{nM}$ and $164 \mathrm{nM}$ for $\mathrm{CB}_{1}$ and $\mathrm{CB}_{2}$, respectively and an $\mathrm{EC}_{90}$ of $292 \mathrm{nM}$ and $296 \mathrm{nM}$ for $\mathrm{CB}_{1}$ and $\mathrm{CB}_{2}$, respectively (Figure 2.9). The $\mathrm{EC}_{50}$ and $\mathrm{EC}_{90}$ values in both $\mathrm{CB}_{1}$ and $\mathrm{CB}_{2}$ are quite close and both 


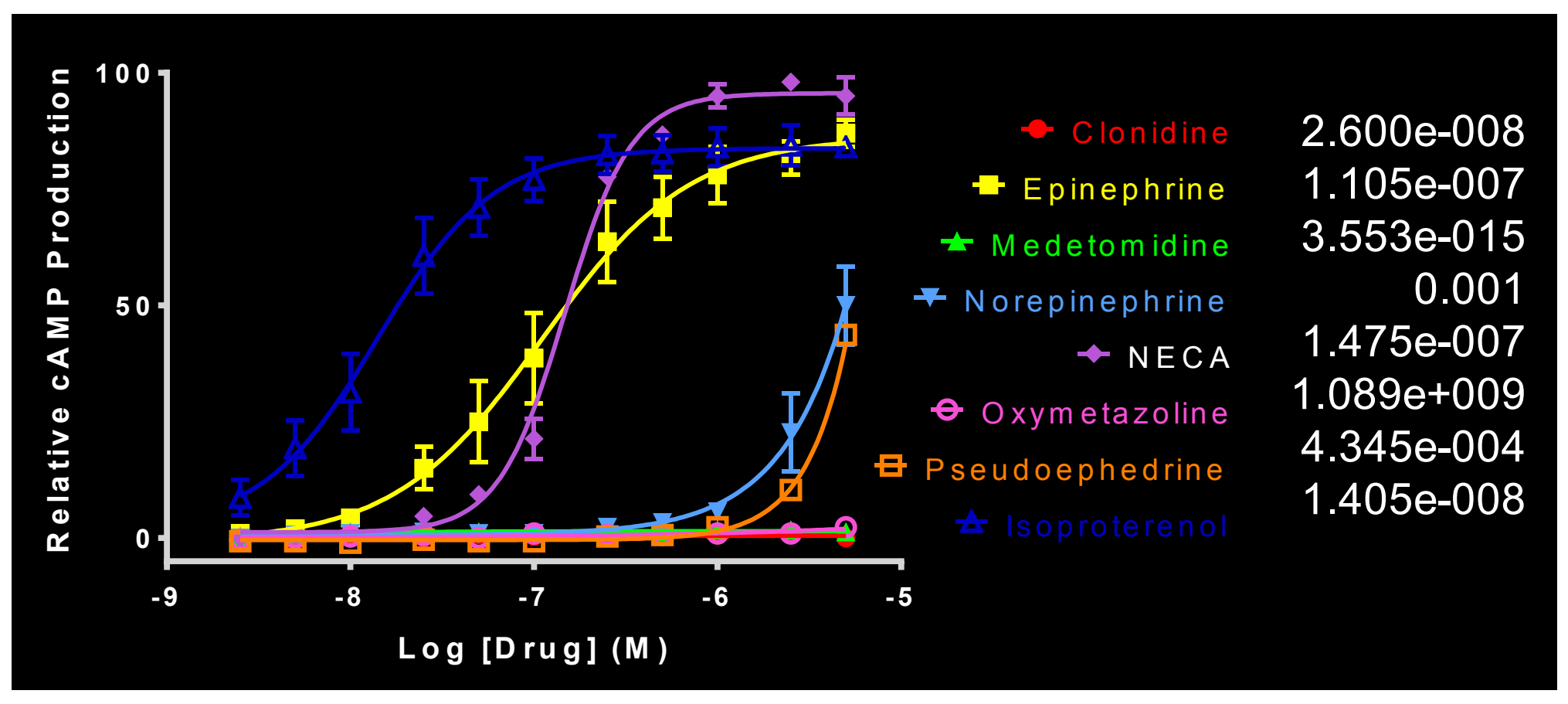

Figure 2.5. $\quad \mathrm{CB}_{1}$ adrenergic drug test for stimulation of cAMP production 


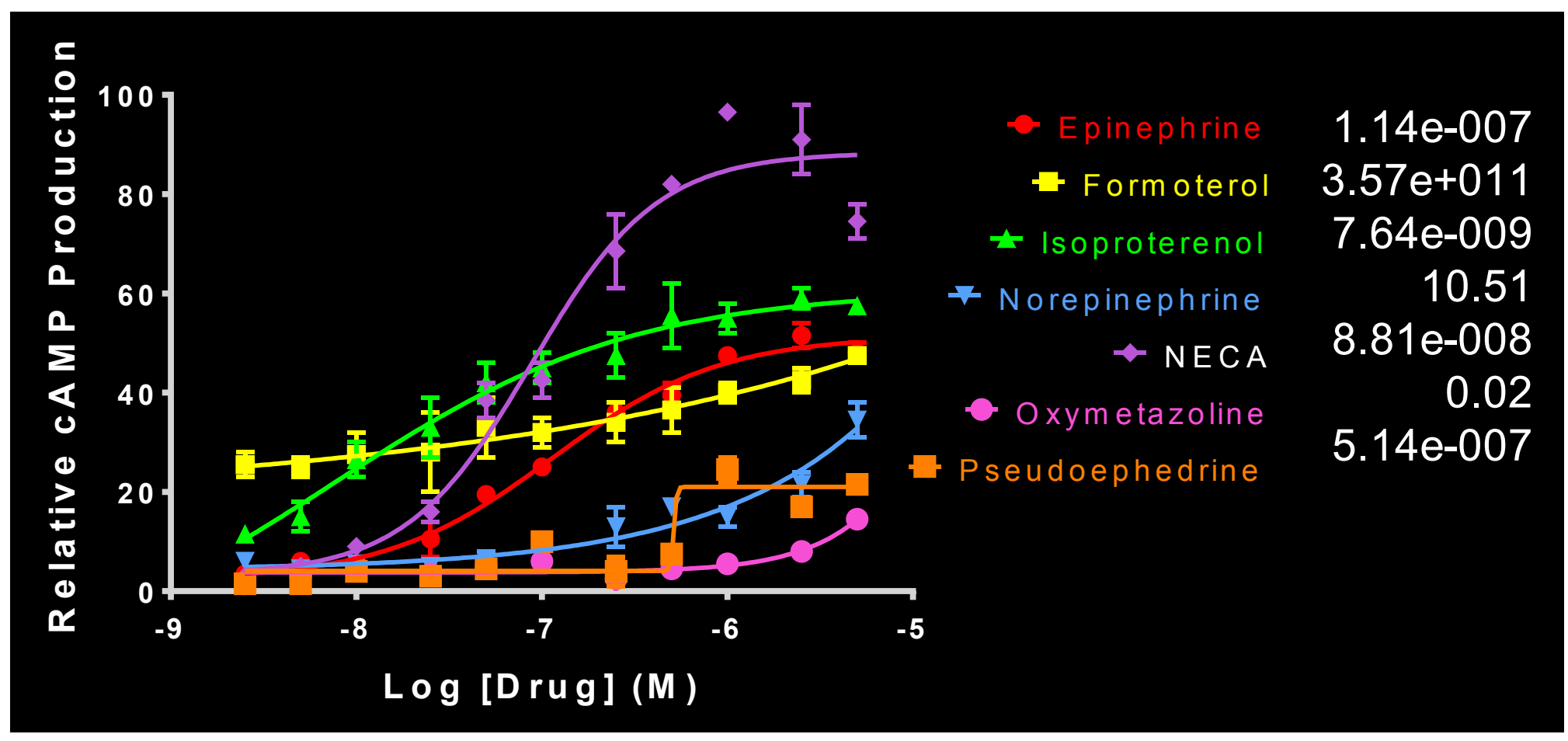

Figure 2.6. $\quad \mathrm{CB}_{2}$ adrenergic drug test for stimulation of $\mathrm{CAMP}$ production 


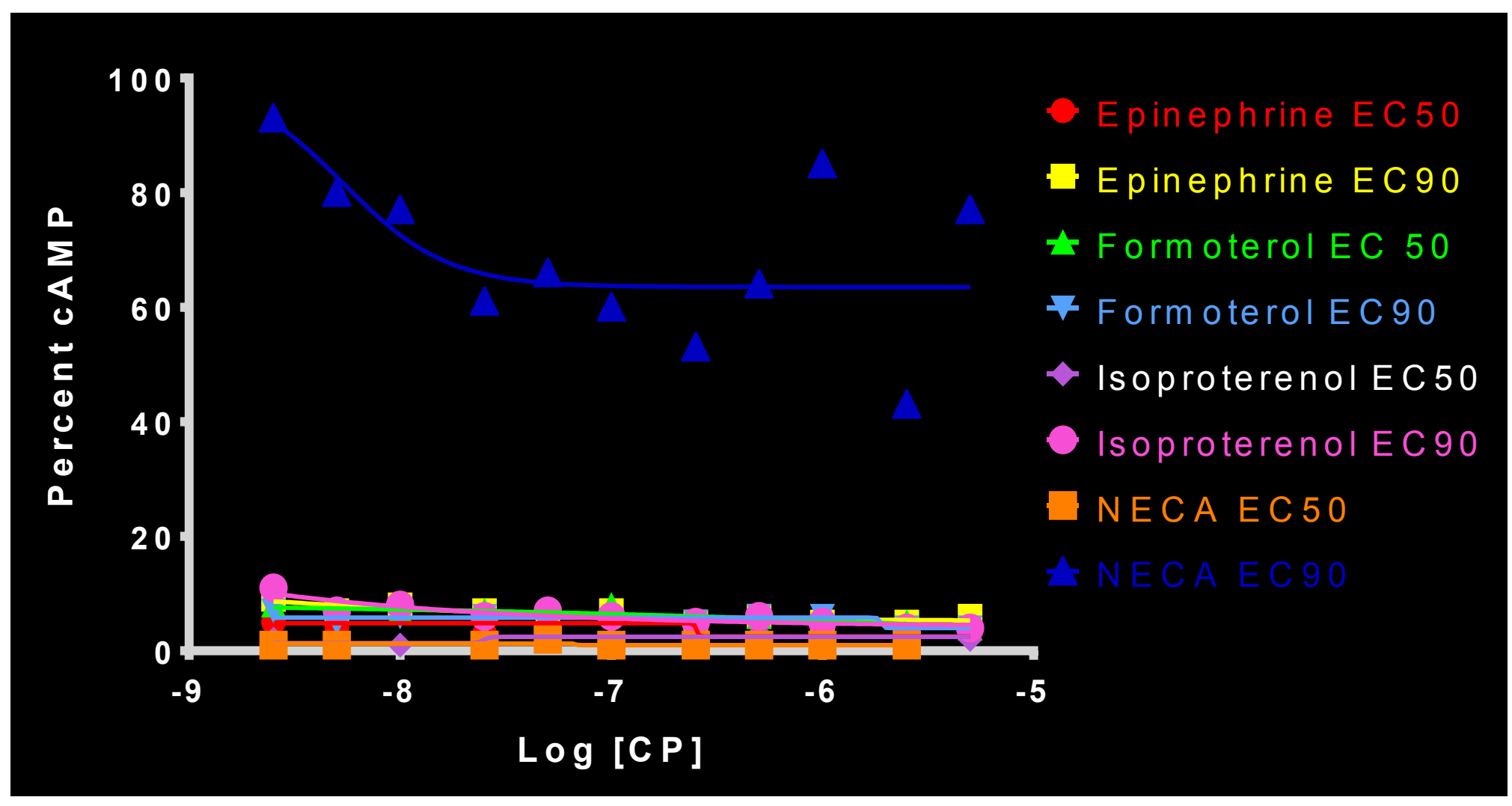

Figure 2.7. Selected adrenergic drugs in $\mathrm{CB}_{2}$ cells vs. $\mathrm{CP}-\mathbf{5 5 , 9 4 0}$ 


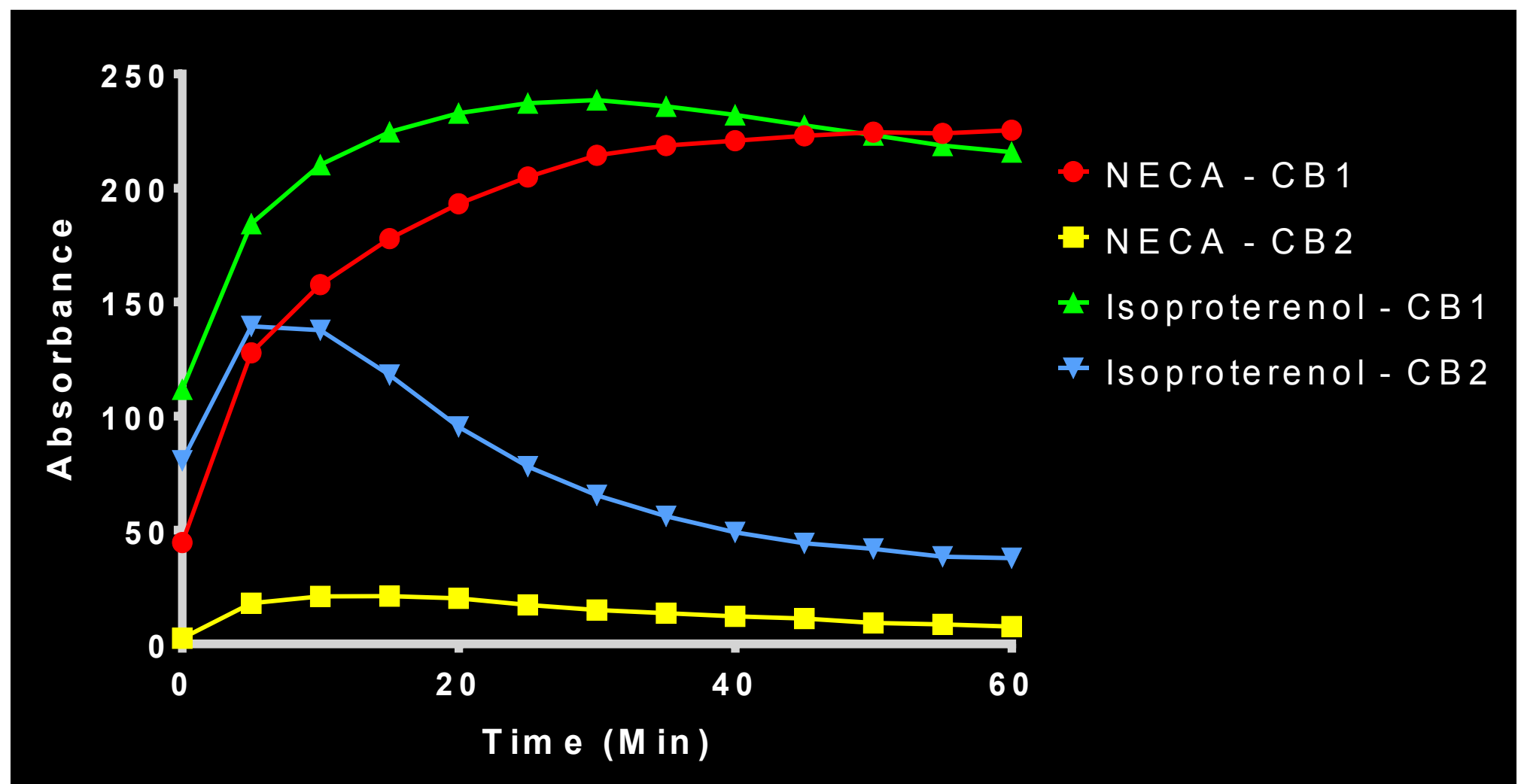

Figure 2.8. Manufacturer-recommended cAMP stimulants

Notes: Isoproterenol is $50 \mathrm{nM}$ and NECA is $100 \mathrm{nM}$ for both $\mathrm{CB}_{1}$ and $\mathrm{CB}_{2}$. 


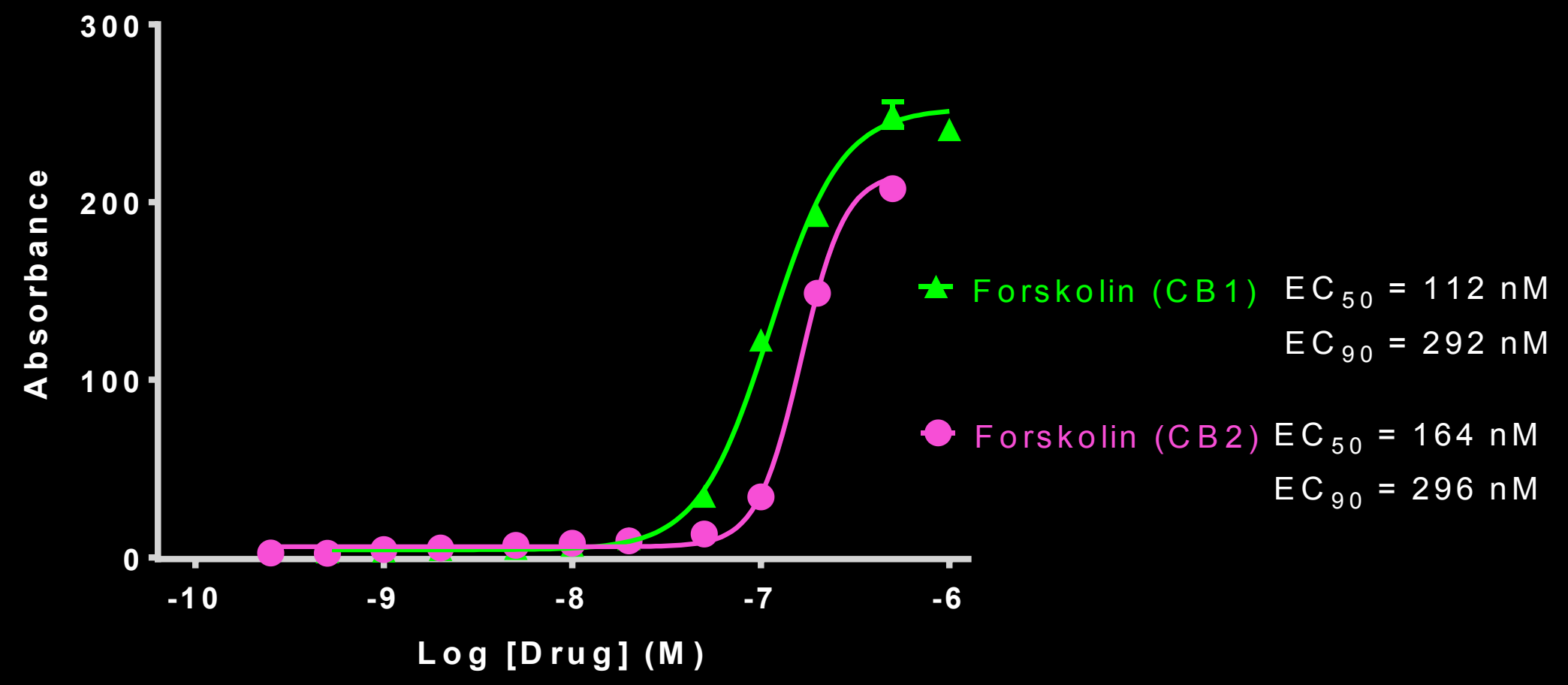

Figure 2.9. Forskolin as a cAMP stimulant in $\mathrm{CB}_{1}$ and $\mathrm{CB}_{2}$ cells 
achieved and held at or near maximum response within 20 minutes in both $\mathrm{CB}_{1}$ and $\mathrm{CB}_{2}$ (Figure 2.10). Given that there was not an appreciable difference in response of $\mathrm{CB}_{1}$ or $\mathrm{CB}_{2}$ cells between $1 \mu \mathrm{M}$ and the approximate $\mathrm{EC}_{90}$ for both $(300 \mathrm{nM}), 800 \mathrm{nM}$ was chosen as the final concentration of forskolin to use in each well of the assay across all three cell lines. Comparing equivalent $(500 \mathrm{nM})$ concentrations of isoproterenol, NECA and forskolin between the $\mathrm{CB}_{1}$ and $\mathrm{CB}_{2}$ cells (Figure 2.11), we see clear evidence that any of the three $\mathrm{CAMP}$ stimulants work well in $\mathrm{CB}_{1}$. However, at $\mathrm{CB}_{2}$, isoproterenol starts to wane after only 10 minutes, NECA, while comparable to forskolin, does not have the sustained plateau and consistency needed to assure reliability within this assay.

\subsubsection{Cell plating density test}

Having now ascertained which stimulus to utilize in order to induce cAMP production, the next pertinent parameter to address is that of plating density. To test for activity with each of the varying cell densities, with constant levels of forskolin to serve as the stimulus for cAMP production, I selected two non-selective cannabinoid agonists (CP-55,940 and WIN-55,212-2), a CB 2 -selective agonist (HU-308), a CB 1 -selective inverse agonist (SR-141716A) and a $\mathrm{CB}_{2}$-selective inverse agonist (SR-144528). In addition, as a point of reference, the manufacturer recommends plating of the cells at $70 \mathrm{~K}$ cells/well, and in a 96-well plate (as I use) there would be $100 \mu \mathrm{L}$ of cell suspension present.

For the $\mathrm{CB}_{1}$ cells, six plates with densities of $80 \mathrm{~K}$ cells/well, $70 \mathrm{~K}$ cells/well, $60 \mathrm{~K}$ cells/well, $50 \mathrm{~K}$ cells/well, $40 \mathrm{~K}$ cells/well and $30 \mathrm{~K}$ cells/well would serve to answer this question. Looking at the three agents used in this test (CP-55,940, SR-141716A and WIN-55,212-2), it is initially evident that there is not a great deal of difference among the varying densities (Figure 2.12). Looking at the $\mathrm{EC}_{50}$ and $\mathrm{R}^{2}$ values for each of the three compounds leaves me with $40 \mathrm{~K}$ cells/well as the most viable option. This density strikes the best balance for all three of the known compounds for $\mathrm{EC}_{50}$ and $\mathrm{R}^{2}$ value, and this is the density utilized for $\mathrm{CB}_{1}$ functional assays for the remainder of the testing.

For the $\mathrm{CB}_{2}$ cells, six places with densities of $70 \mathrm{~K}$ cells/well, $60 \mathrm{~K}$ cells/well, $50 \mathrm{~K}$ cells/well, $40 \mathrm{~K}$ cells/well, $30 \mathrm{~K}$ cells/well and $20 \mathrm{~K}$ cells/well would serve to optimize the plating density for $\mathrm{CB}_{2}$ cells. Considering, again, the $\mathrm{EC}_{50}$ and $\mathrm{R}^{2}$ values for the three agents used (CP-55,940, SR-144528 and HU-308), the most viable option becomes $60 \mathrm{~K}$ cells/well (Figure 2.13). Again, this gives us the strongest balance between a good $\mathrm{EC}_{50}$ of all three compounds with an acceptable, though not perfect, $\mathrm{R}^{2}$ value. Henceforth, $60 \mathrm{~K}$ cells/well will be the density utilized in plating $\mathrm{CB}_{2}$ for functional assay.

Though there is no optimization for the parental HEK-CNG cells, because it was impossible to actually generate a change in cAMP due to the lack of cannabinoid receptors, I did institute parameters to provide valid comparisons. Given that the constitutively expressed receptors are identical, I deemed it acceptable to take the average of $\mathrm{CB}_{1}$ and $\mathrm{CB}_{2}$ plating densities as the plating density for the HEK-CNG cells. Thus, $50 \mathrm{~K}$ cells/well was the plating density for the HEK-CNG cell assays conducted as the control for all of the compounds tested herein. 


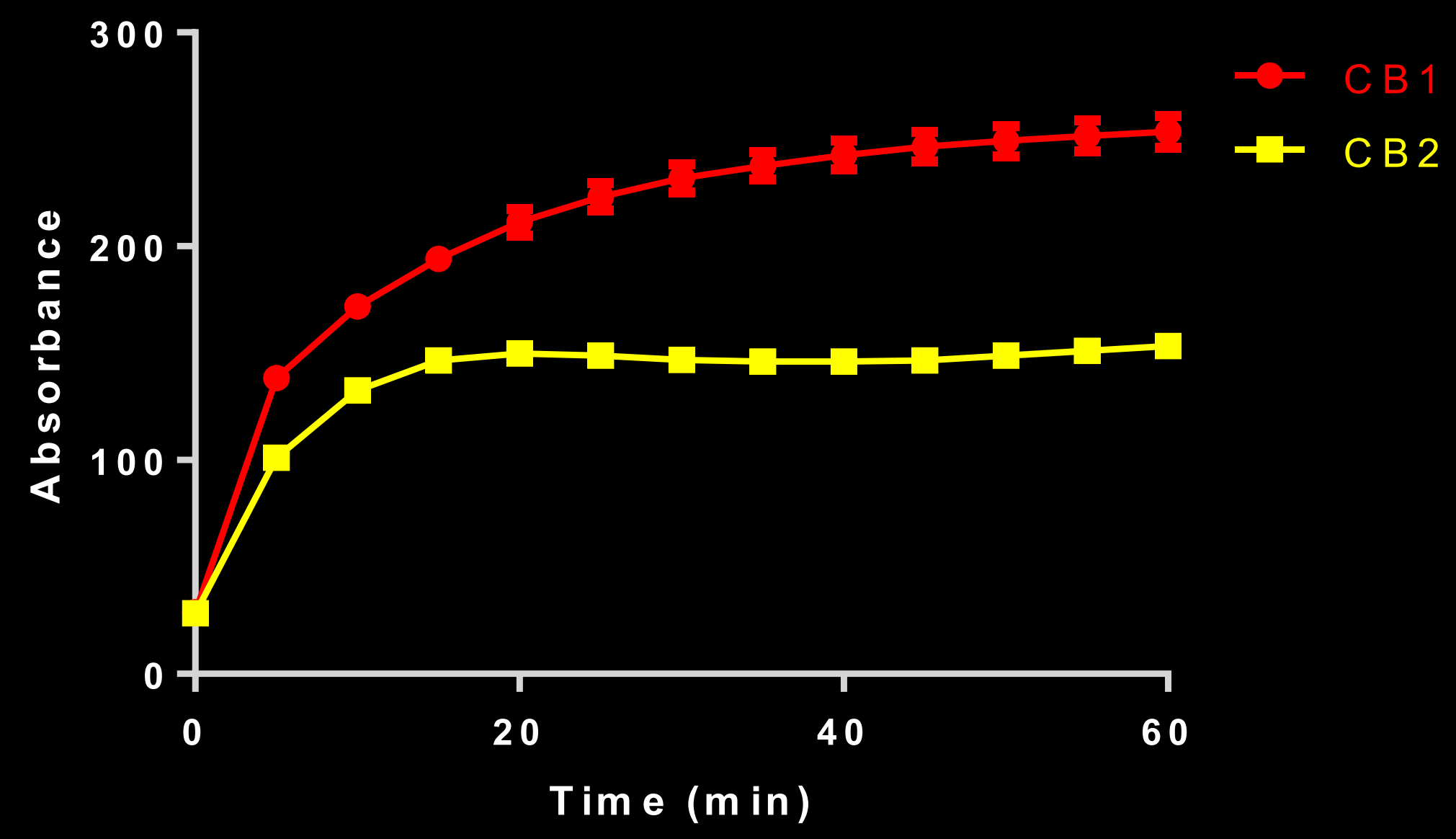

Figure 2.10. Forskolin response in $\mathrm{CB}_{1}$ and $\mathrm{CB}_{2}$ vs. time 


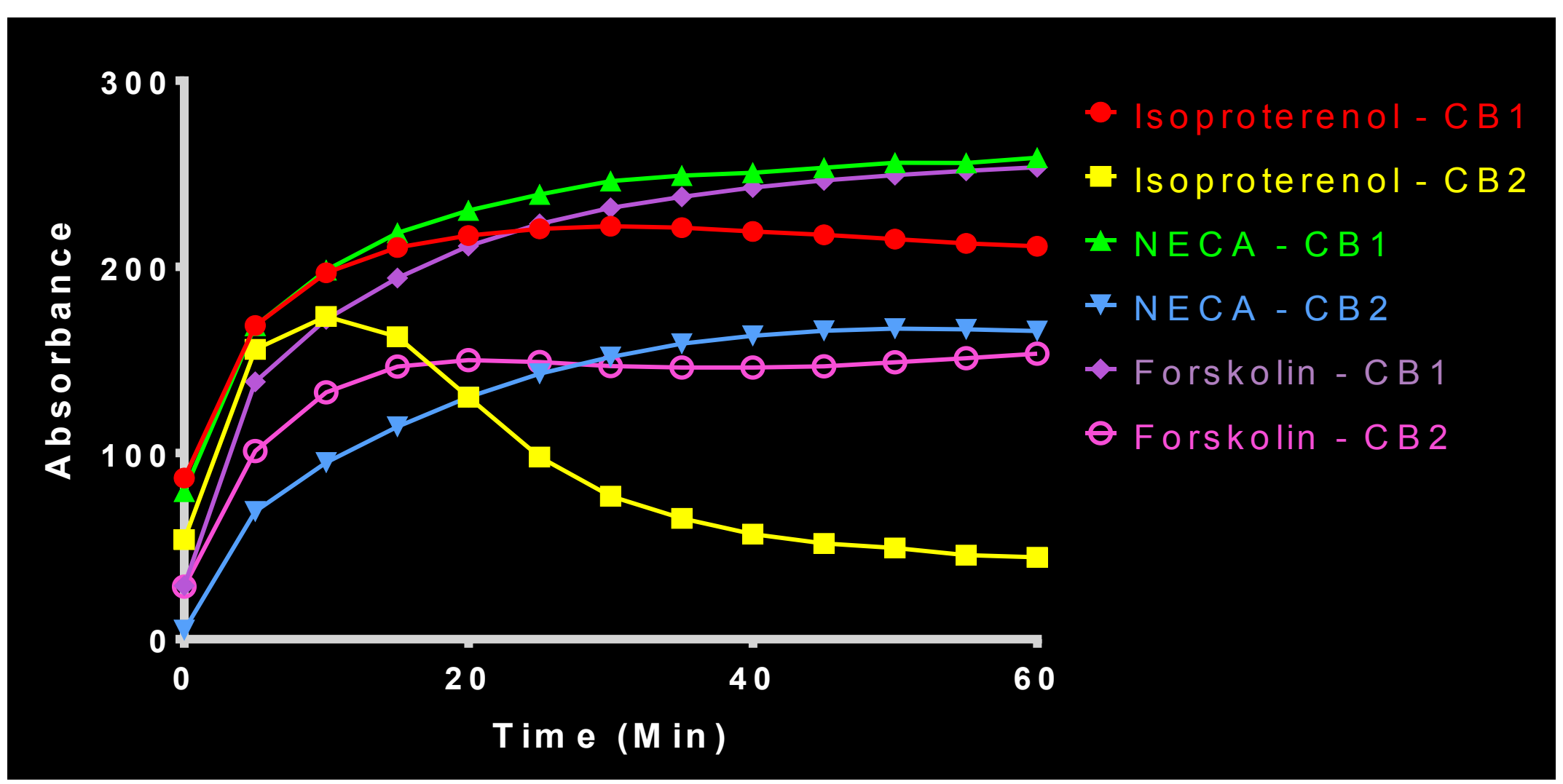

Figure 2.11. All viable cAMP stimuli at $500 \mathrm{nM}$ in $\mathrm{CB}_{1}$ and $\mathrm{CB}_{2}$ cells 
C P $-55,940$

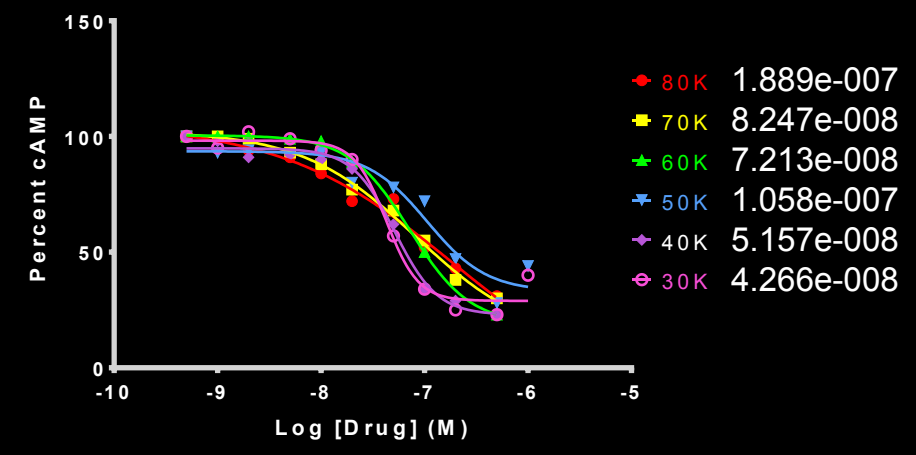

\begin{tabular}{|l|c|c|c|c|c|c|}
\hline & $80 K$ & $70 K$ & $60 K$ & $50 K$ & $40 K$ & $30 K$ \\
$\mathbf{R}^{2}$ & 0.9813 & 0.9947 & 0.9994 & 0.9363 & 0.9903 & 0.9797 \\
\hline
\end{tabular}

S R - 141716A

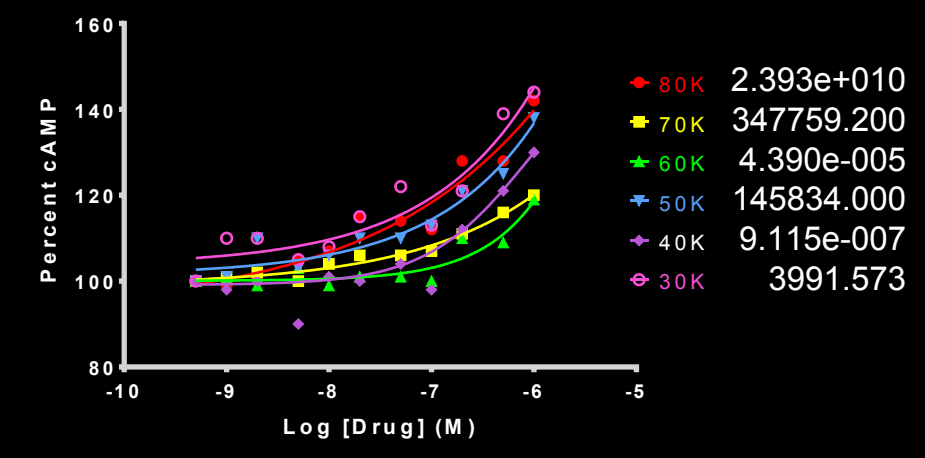

\begin{tabular}{|l|c|c|c|c|c|}
\hline & 70K & 60K & 50K & 40K & 30K \\
$\mathbf{R}^{2}$ & 0.9736 & 0.8795 & 0.9434 & 0.9952 & 0.8951 \\
\hline
\end{tabular}

W IN 55,212-2

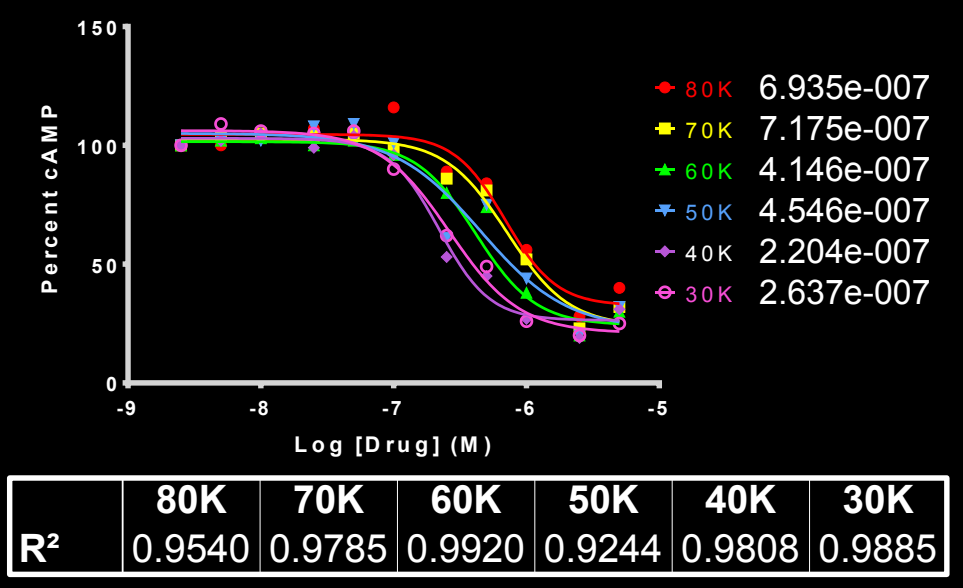

Figure 2.12. $\mathrm{CB}_{1}$ plating density test 


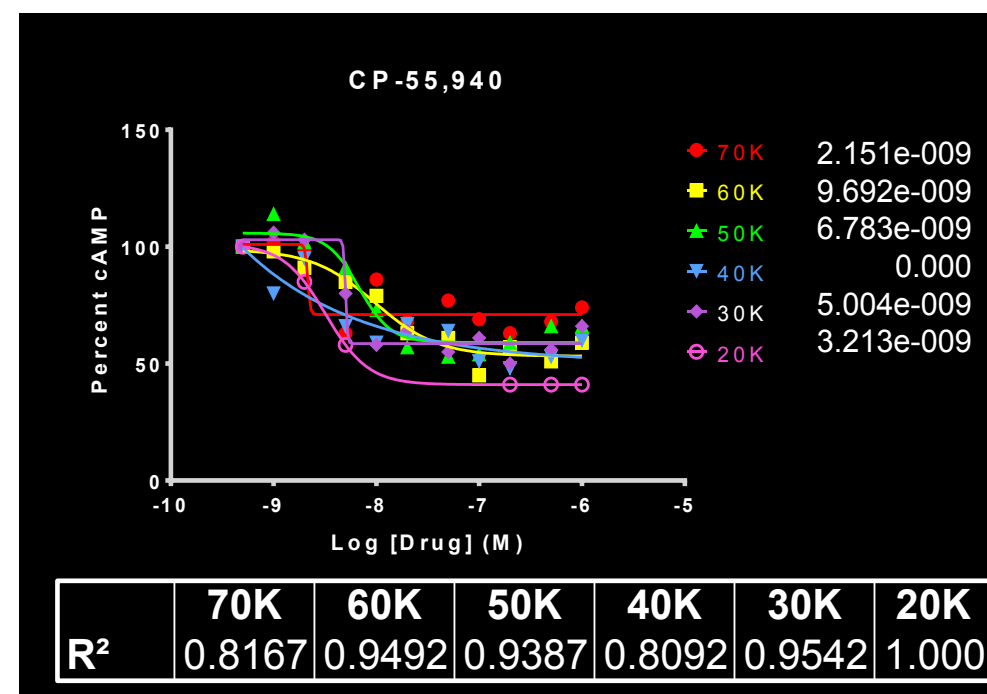

SR - 144528

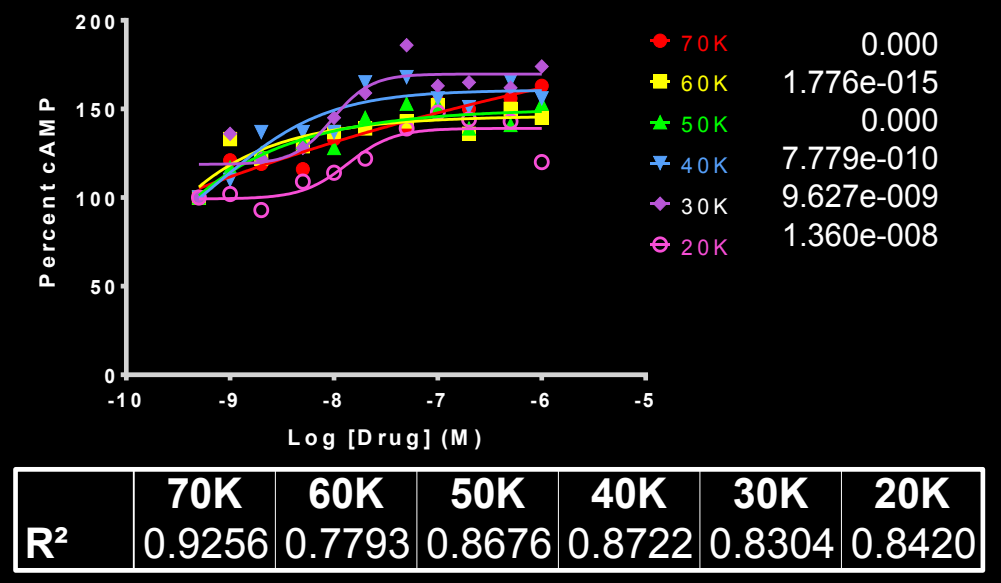

H U -30 8

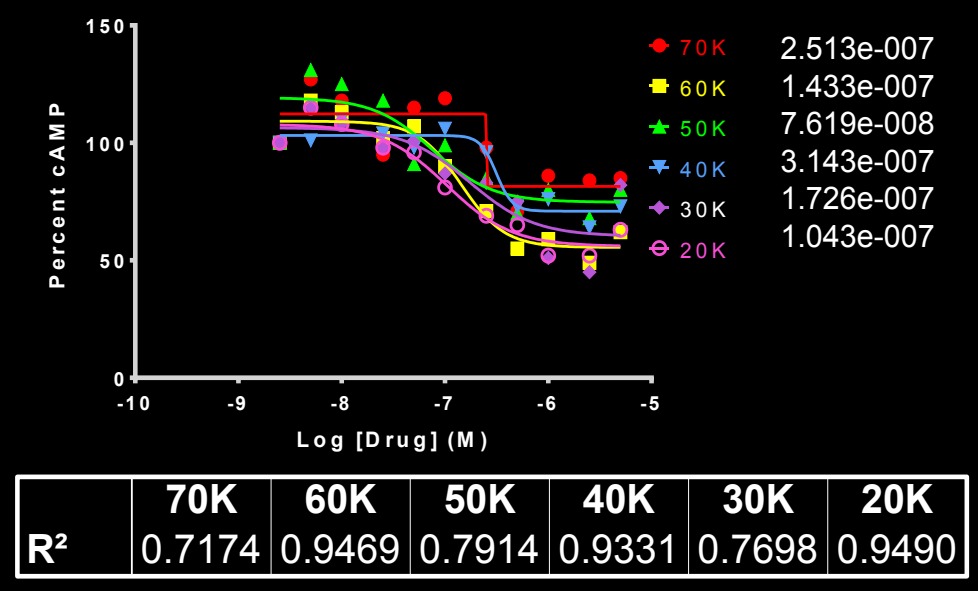

Figure 2.13. $\quad \mathrm{CB}_{2}$ plating density test 


\subsubsection{Assay plate selection}

As stated in the manufacturer's recommendations, cells should be plated overnight on Poly-D-Lysine plates; however, cost of Poly-D-Lysine relative to more widely available polystyrene plates warranted a test. Plating at identical densities from the same batch of cells, I conducted tests of dye mixing times in polystyrene plates to compare to the Poly-D-Lysine results previously run at room temperature. Four (4) assay plates subjected to 1 hour, 45 minutes, 30 minutes or 15 minutes of dye mixing time in polystyrene plates served as the base for this test. Overall, the 1-hour dye mixing time was superior, as it was in the Poly-D-Lysine plates; however, there was a notable difference in the confluence of the cells in each well with the polystyrene plates. There seemed to be a difference in rate of growth, as plating 50,000 cells/well did not achieve the same $80-90 \%$ confluence that was noted with the Poly-D-Lysine plates. Due to this inconsistency of cell growth, the results were not directly comparable to the results of the Poly-D-Lysine plates. The decision was to forego the polystyrene plates and utilize the Poly-D-Lysine plates to help ensure consistency of results and minimize the number of cells needed to achieve confluence.

\subsubsection{Plating environment optimization}

Documentation from Codex Biosolutions states that once the cells are plated there is no need to feed them; however, there is a balance that must be stuck between keeping the cells viable for assay and not complexing the drugs being assayed, as it is well-known that cannabinoids are highly protein bound. ${ }^{231}$ Normal growth media for the cells is DMEM with $10 \% \mathrm{FBS} ; 1 \% \mathrm{P} / \mathrm{S} ; 250 \mu \mathrm{g} / \mathrm{mL} \mathrm{G} 418$ and $1 \mu \mathrm{g} / \mathrm{mL}$ puromycin, and is what initially was utilized for plating, minus the selection antibiotics of G418 and puromycin. The initial test was to see what, if any, difference exists between FBS and BSA and to compare different concentrations of the two against one another. Initial concentrations of FBS tested were $10 \%, 5 \%$ and $1 \%$. Initial concentrations of BSA tested were $1 \%, 0.5 \%$ and $0.25 \%$. In all three of the tested concentrations of BSA there was no discernible curve with any of the cAMP production stimulants (epinephrine, formoterol, isoproterenol and NECA) tested. Within the FBS concentrations tested, all three were comparable; however, 1\% FBS did show slightly better across all tested cAMP stimulants, so it warranted further testing.

Using isoproterenol as the cAMP stimulant, FBS at 4 different concentrations $(1 \%, 0.75 \%, 0.5 \%$ and $0.25 \%)$ were examined. The $1 \%$ FBS plate demonstrated inconsistency from the previous run, and subsequently lower concentrations of FBS proved even more erratic. So, ultimately, the decision was made to continue plating and running the assays in DMEM with $10 \% \mathrm{FBS}$ and $1 \% \mathrm{P} / \mathrm{S}$.

\subsubsection{Pertussis toxin (PTx) dosing optimization}

In examining $\mathrm{CB}_{1}$ and $\mathrm{CB}_{2}$, which are $\mathrm{PTx}$-sensitive $\mathrm{G}_{\mathrm{i} / \mathrm{o}}$-coupled receptors, one of the assays necessary to run is a PTx-sensitive $\mathrm{G}_{\mathrm{i} / \mathrm{o}}$-inactivation of the coupling of $\mathrm{CB}_{1}$ or $\mathrm{CB}_{2}$ receptors to $\mathrm{G}_{\mathrm{i} / \mathrm{o}}$ prior to drug treatment and conductance of the assay. While it is 
true that the HEK-CNG cells help to rule out the drug effects on any other constitutively expressed receptor, it does not validate that the actions are due to $\mathrm{CB}_{1}$ and/or $\mathrm{CB}_{2} \mathrm{G}_{\mathrm{i} / \mathrm{o}}$ GPCR activity.

2.2.6.1. Duration of PTx pre-treatment. The first question to answer with regard to PTx treatment of the cells is how long it is necessary to pretreat the cells. I examined two methods when testing this question, both easily fitting into the normal procedure for the assay and thus their selection and utility. The first was to add PTx directly before the membrane potential dye, which would allow 1 hour of dwell time for the PTx prior to drug addition. At all 8 concentrations tested $(400 \mathrm{ng} / \mathrm{mL}, 200 \mathrm{ng} / \mathrm{mL}, 80$ $\mathrm{ng} / \mathrm{mL}, 40 \mathrm{ng} / \mathrm{mL}, 20 \mathrm{ng} / \mathrm{mL}, 8 \mathrm{ng} / \mathrm{mL}, 4 \mathrm{ng} / \mathrm{mL}$ and $0 \mathrm{ng} / \mathrm{mL})$, CP-55,940 was still able to exert its full effect of inhibiting forskolin-induced cAMP production (Figure 2.14).

The next method employed was to add the PTx into the cell suspension reservoir at the time of plating and allow the PTx to dwell in the cell suspension overnight, at least 12 hours prior to drug addition. At all 7 concentrations tested $(400 \mathrm{ng} / \mathrm{mL}, 200 \mathrm{ng} / \mathrm{mL}, 80$ $\mathrm{ng} / \mathrm{mL}, 40 \mathrm{ng} / \mathrm{mL}, 20 \mathrm{ng} / \mathrm{mL}, 8 \mathrm{ng} / \mathrm{mL}$ and $4 \mathrm{ng} / \mathrm{mL})$, CP-55,940 was unable to exert its full effect of inhibiting forskolin-induced cAMP production (Figure 2.15). Since literature precedence had set PTx-sensitive $\mathrm{G}_{\mathrm{i} / \mathrm{o}}$-inactivation dosing in a range from 8 $\mathrm{ng} / \mathrm{mL}$ to $100 \mathrm{ng} / \mathrm{mL}$, I selected $4 \mathrm{ng} / \mathrm{mL}$ to conserve the most resources and still maintain a inactivation of $\mathrm{G}_{\mathrm{i} / \mathrm{o}}$ activity in the assay. Based on these data, I concluded that the optimal procedure for PTx-sensitive $\mathrm{G}_{\mathrm{i} / \mathrm{o}}$-inactivation is $4 \mathrm{ng} / \mathrm{mL}$ with an overnight pretreatment.

To confirm these findings in $\mathrm{CB}_{2}$, the same seven concentrations $(400 \mathrm{ng} / \mathrm{mL}, 200$ $\mathrm{ng} / \mathrm{mL}, 80 \mathrm{ng} / \mathrm{mL}, 40 \mathrm{ng} / \mathrm{mL}, 20 \mathrm{ng} / \mathrm{mL}, 8 \mathrm{ng} / \mathrm{mL}$ and $4 \mathrm{ng} / \mathrm{mL}$ ) of PTx were added to the $\mathrm{CB}_{2}$ cells and allowed to sit overnight before screening against CP-55,940. As was the case in $\mathrm{CB}_{1}, \mathrm{CP}-55,940$ was unable to inhibit forskolin-induced cAMP production across the entire spectrum of concentrations tested (Figure 2.16). As a result of this study, and to maintain continuity of assay conditions across the cell types, $4 \mathrm{ng} / \mathrm{mL}$ was selected as the target concentration for future PTx-sensitive $\mathrm{G}_{\mathrm{i} / \mathrm{o}}$-inactivation studies of the $\mathrm{CB}_{2}$ cells.

2.2.6.2. Addition order of PTx treatment. Knowing that $4 \mathrm{ng} / \mathrm{mL}$ with an overnight dwell time is necessary before inhibition of the $\mathrm{G}_{\mathrm{i} / \mathrm{o}}$ is achieved, the question remained of how to best administer the PTx. This question became relevant due to the perception of a lack of cell adhesion to the plates when mixed into the cell suspension. To test this, 4 plates: no PTx, PTx in cell suspension, PTx in PBS added at plating and PTx in PBS added 2 hours after plating, were tested. The result was that there is no difference in how the PTx is added, just that it dwells overnight (Figure 2.17).

\subsubsection{Assay data analysis optimization}

As previously mentioned, readings of the assay plates utilizes a BioTek Synergy 2 running Gen5 software. GraphPad Prism generates the graphs and non-linear curve fitting with $\mathrm{EC}_{50}$ calculations. While the Gen5 software is capable of generating graphs and $\mathrm{EC}_{50}$ curves, the manipulation of the data was not as automated and high-throughput as 
was desired. In addition, extraction of data from Gen5 software and importation into
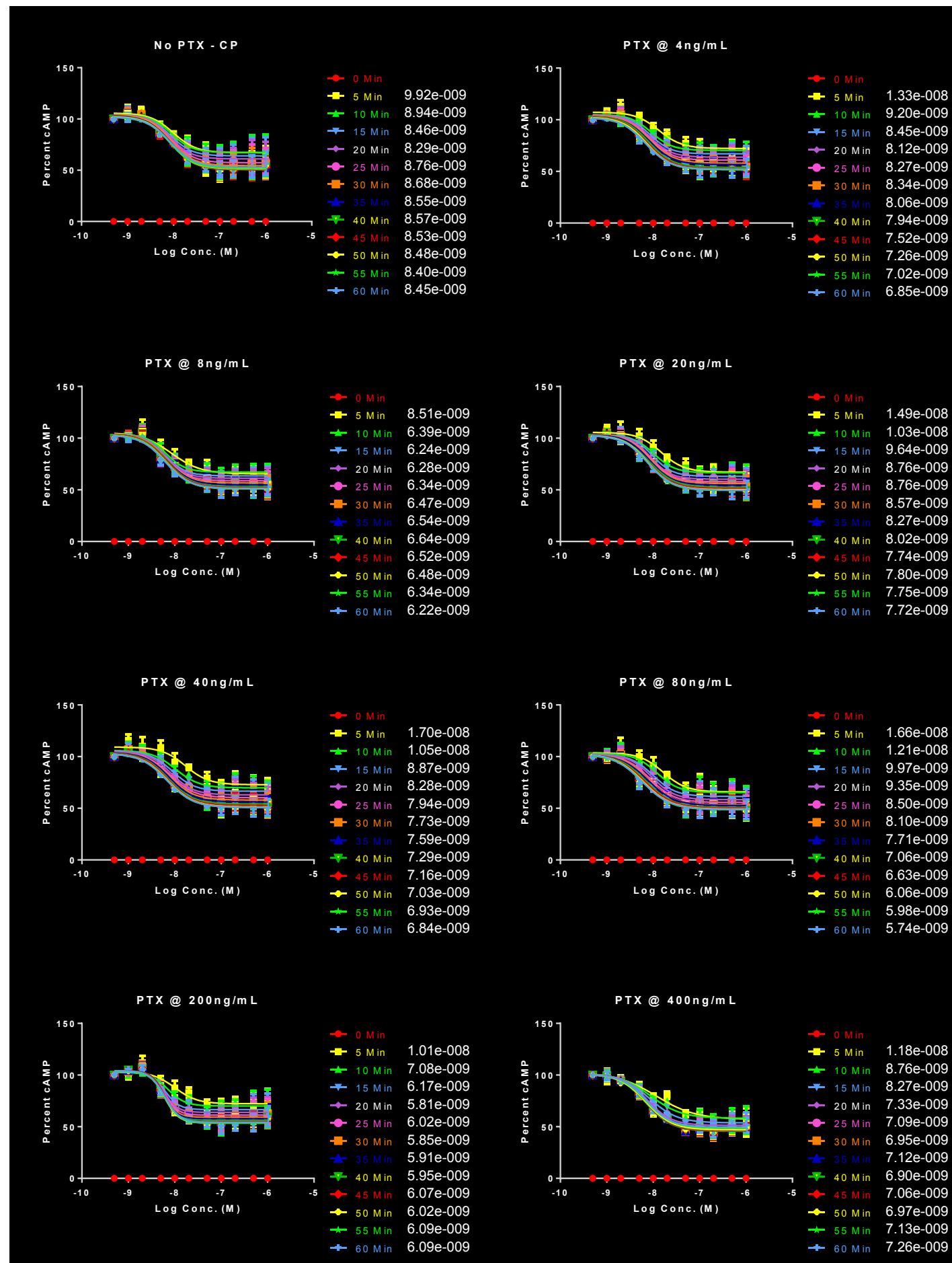

Figure 2.14. CB1 cells with 1 hour pretreatment of PTx vs. CP-55,940 


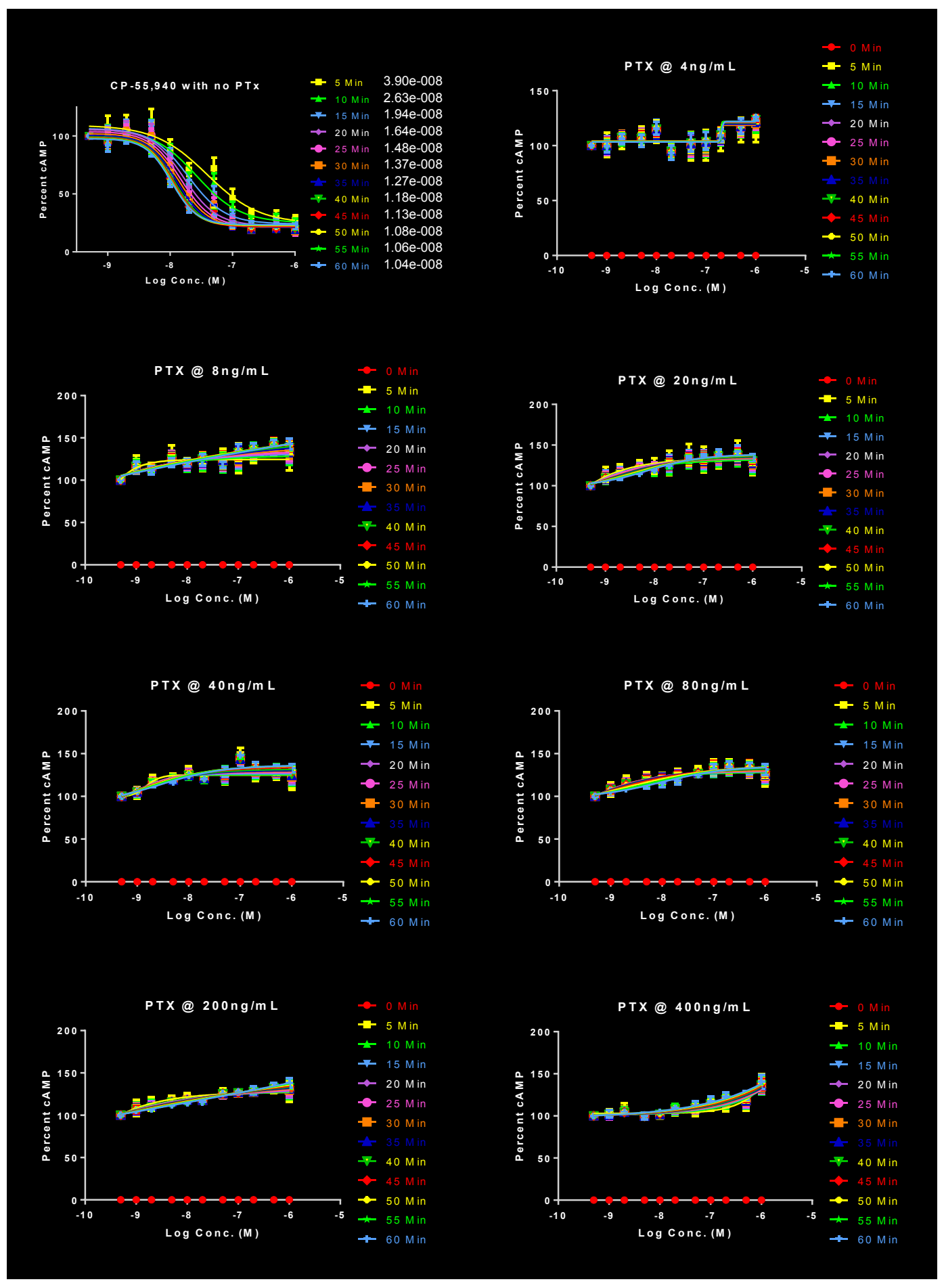

Figure 2.15. $\mathrm{CB}_{1}$ cells with overnight PTx pretreatment vs. CP-55,940 


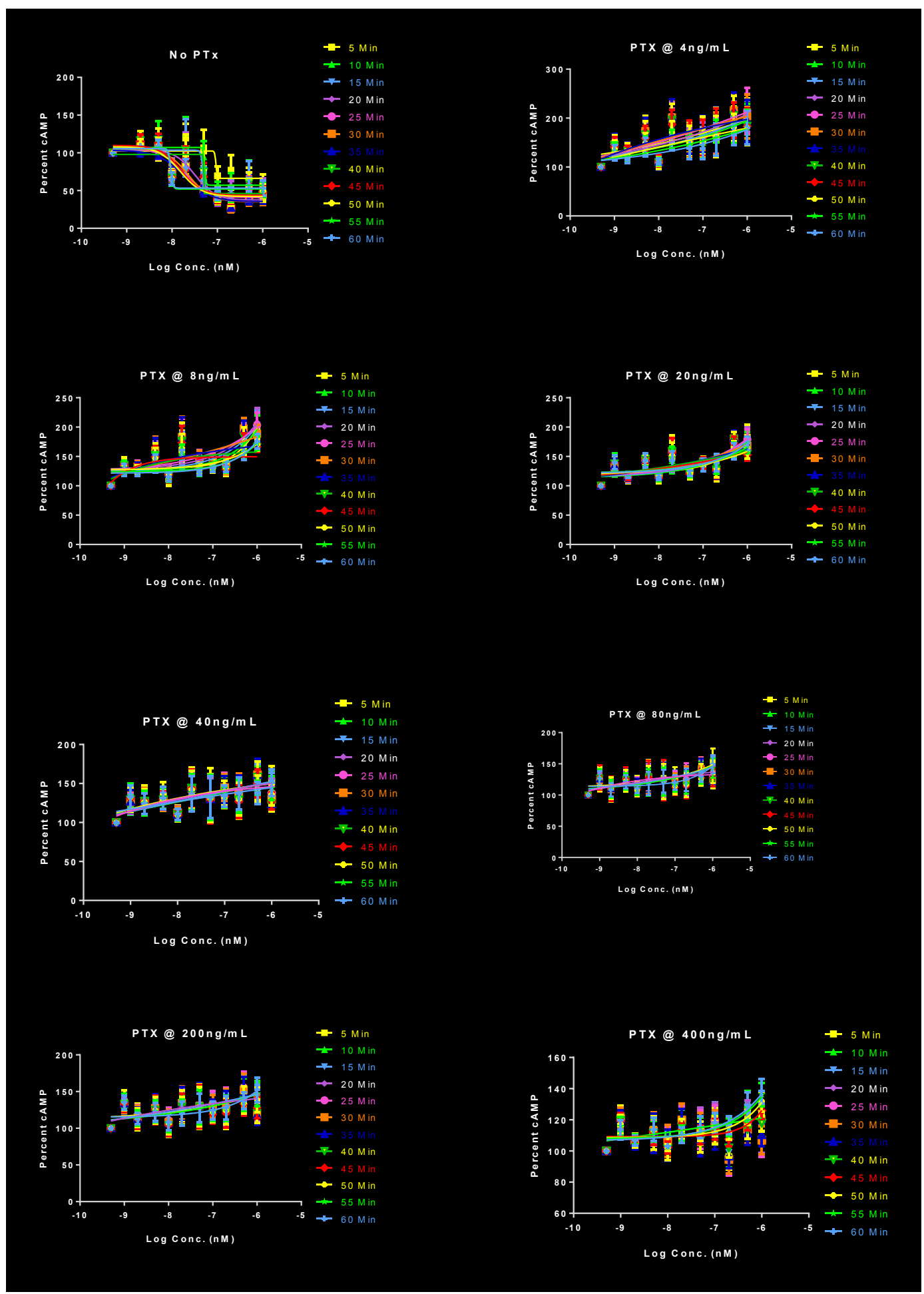

Figure 2.16. $\quad \mathrm{CB}_{2}$ cells with overnight PTx treatment vs. CP-55,940 


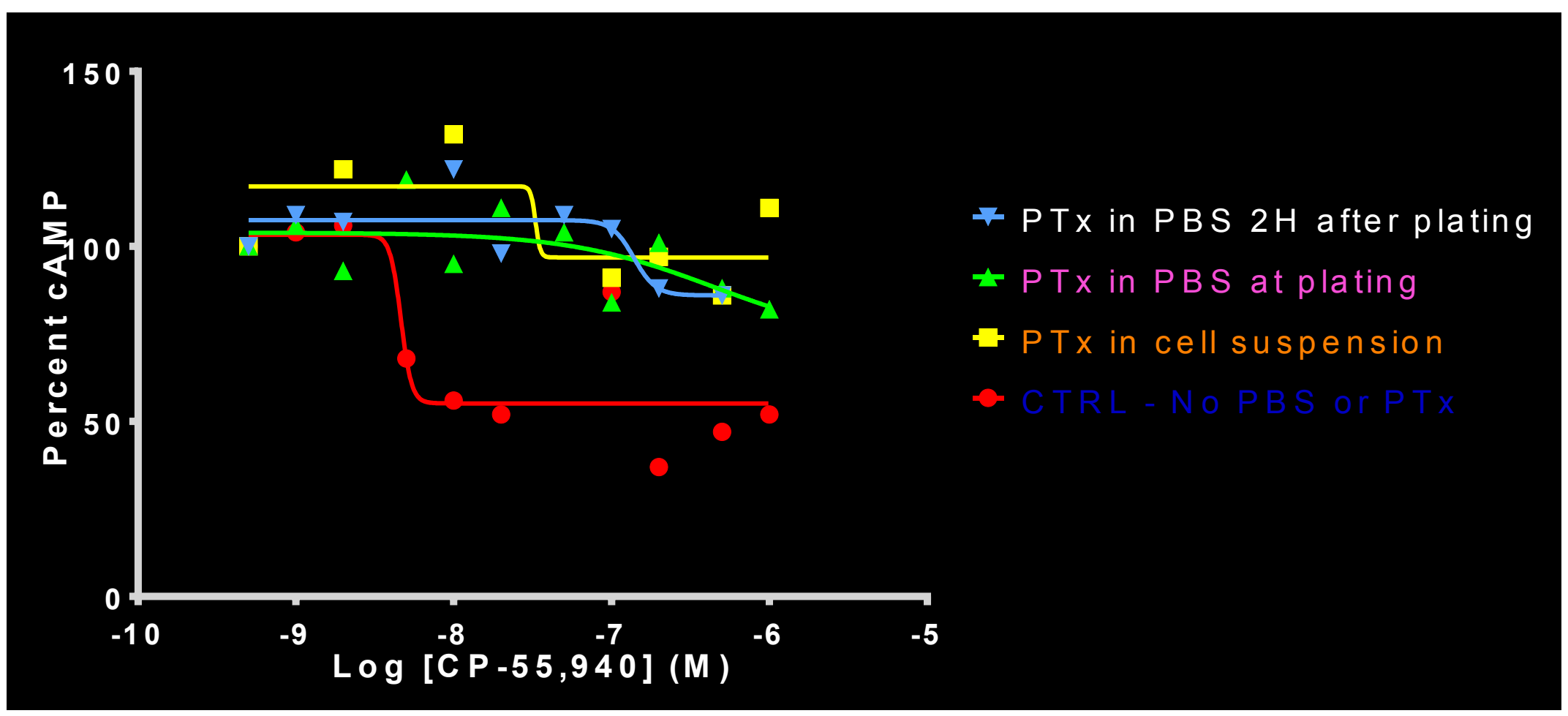

Figure 2.17. $\mathrm{CB}_{2}$ cells with $\mathrm{PTx}$ addition order test 
GraphPad Prism was a cumbersome and tedious process. Utilizing the "Power Export" function of the Gen5 software allowed me to develop custom VBA macros - visual basic applications, which allows for user-defined functions and automates processes and accessing Windows APIs - for use in Microsoft Excel, which would automate the process of data normalization and subsequent importation into and analysis by GraphPad Prism.

2.2.7.1. GraphPad Prism template building. The initial step in this process was to import manually data from one plate of an assay to construct a template in GraphPad Prism. The template contains eight XY scatter with single $\mathrm{Y}$ value data sheets and graphs. Analysis of all eight sheets was accomplished through non-linear regression with the built-in "log(agonist) vs. response - Variable slope" equation with automatic outlier elimination and an output of a summary $\mathrm{EC}_{50}$ table. After all eight data sheets, results sheets and graphs existed, the data tables had their contents removed and the resulting shell project saved as a template, with the name "IndividualPlates." This template resided in a subfolder in the directory utilized by Gen 5 for data storage and exportation - in this case: "C: $\backslash$ Data $\backslash$ Templates $\backslash . "$

Since our typical run will be for $\mathrm{n}=6$ for each drug, there needs to be data combined from across six separate plates into one master file for each compound. As such, a combined data template was necessary to create. To accomplish this task, a project was created such that contained therein was nine XY scatter with 6 replicate $Y$ value data sheets and graphs. The data sheets were populated with data and analysis of all 9 sheets was accomplished through non-linear regression with the built-in " $\log$ (agonist) vs. response - Variable slope" equation with automatic outlier elimination and an output of a summary $\mathrm{EC}_{50}$ table. Following the analysis and subsequent creation of the results sheets and population of the graphs, data tables had their contents removed, and the resultant shell project saved as a template, with the name "MultiplePlates." This template resides in a subfolder in the directory to be utilized by Gen5 for data storage and exportation - in this case: "C: $\mid$ DatalTemplates $\backslash . "$

2.2.7.2. Gen5 experiment design. The first task in the design of this experiment was to ensure that all data remains in a readily accessible manner for quick retrieval and obvious content. Utilizing the parameters of the Gen5 protocol options, I created a nomenclature system that automatically generates after the user inputs key, required, data at the start of an experiment. A central repository of data, simply "C: $\mid$ Data, ," came to be for this task. Within that folder, a subfolder would be created for the protocol being used (CB1 Functional, CB2 Functional or HEK-CNG Functional), i.e. "C: $\mid$ DatalCB1 Functional.$"$ Within the subdivisions of data, further compartmentalization of the experiments based on the $<$ Barcode $><$ PlateID $>$ fields input by the user at the initial read of each plate is implemented. The $<$ Barcode $>$ is the notebook number of the individual conducting the assay in the format of "3-letter initials"-"notebook number"-"page in notebook", e.g. BAK-1B-57. The $<$ PlateID $>$ field is the project title for that series of runs to make it more readily identifiable as to the contents thereof, e.g. "cAMP Stimulant Dose Curves." With this nomenclature, files would be stored based on the assay run and then further segregated by the notebook number, allowing for quick callback to the experimental notes, e.g. " $\mathrm{C}: \mathrm{Data} \backslash \mathrm{CB} 1$ Functional $\backslash \mathrm{BAK}-1 \mathrm{~B}-57$ _cAMP Stimulant Dose Curves\." 
The next task was to ensure that file names followed a similarly intuitive nomenclature for ease of reference to the experimental notes in the notebook and the obvious contents of each file, were they separated from their parent folder(s). The naming scheme is set in the protocol options of each of the 3 protocols being utilized, and follows a system similar to that of the folder nomenclature: " $<$ Protocol $><$ PlateID $>$ (autoenumeration)_< Barcode $><$ Date $>$ (YYMMDD)." The $<$ Protocol $>$, as previously mentioned is CB1 Functional, $\mathrm{CB} 2$ Functional or HEK-CNG Functional. <PlateID $>$ and $<$ Barcode $>$ are the same as previously mentioned. The (autoenumeration) field is a 4 digit, sequential series of numbers that increases with each run, so that 6 plates with identical $<$ Protocol $>$, $<$ PlateID $>,<$ Barcode $>$ and $<$ Date $>$ will all have unique (autoenumeration) values to identify which of the 6 runs it is. Lastly, $<$ Date $>$ is the current experiment day's date; however, in Gen5 you cannot select the format of the date, so it always shows as YYMMDD. An example of a filename with full path for the first run in a series of 6 would be: "C: $\mid$ Data $\backslash \mathrm{CB} 1$ Functional $\backslash \mathrm{BAK}-1 \mathrm{~B}-57$ cAMP Stimulant Dose Curves $\backslash$ CB1 Functional_cAMP Stimulant Dose Curves (0001)_BAK-1B57_130516 (YYMMDD).xpt.” This nomenclature system allows for quick reference to the notebook, as well as the person responsible for the run and the content of each of the files - all aiding in expediting data acquisition and analysis.

To accomplish the task of making the data acquisition as automated as possible, the Gen5 software was customized with a unique experiment design that was copied across all three types of cell assays. The first customization was in the sequence of events when reading a plate. The sequence for each plate run in these experiments is:

1. Set Temperature: Set point $25^{\circ} \mathrm{C}$

2. Read: (F) 540/25,590/20

3. Pause

a. Plate In/Out - Plate Out

b. Popup: "Add DRUG to wells, place plate on reader and then click OK"

c. Plate In/Out - Plate In

4. Start Kinetic [Run 1:00:00, Interval 0:01:00]

a. Read: (F) 540/25,590/20

5. End Kinetic

The automation of steps helped to ensure that there was consistency among the days and persons running the assays to aid in lack of omission of steps.

At the completion of the kinetic run, the "Power Export" function was set to commence, automatically. This function a Microsoft Excel template that the user may customize to output certain parameters of each experimental run. The "Power Export" file was named exactly as the experiment from which it was generated, e.g. " $\mathrm{C}: \mid \mathrm{Data} \backslash \mathrm{CB} 1$ Functional $\backslash B A K-1 B-57$ cAMP Stimulant Dose Curves $\backslash$ CB1 Functional_cAMP Stimulant Dose Curves (0001) BAK-1B-57_130516 (YYMMDD).xlsm.”

2.2.7.3. Gen5 "Power Export" template building. One of the key components in facilitating fast and accurate data analysis was the utilization of the "Power Export" 
function of Gen5. Properly configured, this generated a Microsoft Excel spreadsheet that could perform all necessary data calculations and then automatically import them into GraphPad Prism utilizing templates previously mentioned for these experiments. The first step in building this template is to ensure that all pertinent data transfers on the spreadsheet that exported from Gen5 into Microsoft Excel. To accomplish this, the "Read 2" matrix migrated, beginning at cell A1. Beginning at cell A65, I placed the "Field Group," which contained all of the experiment's nomenclature information, i.e. protocol name, plate ID, barcode and reading date/time. Beginning at cell A70, I placed the "Well IDs" group, which listed the names of the drugs contained in each well. With these fields in place, every time the experiment performs a "Power Export" all of the data from Gen5 consistently migrates into a formatted Microsoft Excel spreadsheet.

With the data now in a more malleable form in Microsoft Excel, it is time to build the customized VBA macros that appended to the "Power Export" template inside each of the three protocols. Numerous cycles of trial and error, as well as questions asked with regard to how best to view the data led to a rough draft of the macro design. Taking this, along with how best to normalize/standardize across different plates on different days with different receptor types led to the finalized product.

Primarily, creation of a "Declarations" module is paramount, because this facilitates the passage of variables from module to module without having to worry about improper nomenclatures or pass through commands. See Supplementary Information $\mathbf{1 . 1}$ for full template code of the Declarations module.

Next, I created a "switchboard" module that would be responsible for calling all of the subsequent modules for data analysis. Through this module all data is packaged, normalized to internal standards and importated into GraphPad Prism. See

Supplementary Information 1.2 for full template code of "Extract" module.

With this "switchboard" in place, it is time to start examining all of its moving parts, beginning with the first call in the process, which is to select the evaluation parameters - accomplished via the "CalcType" form. This form contains radio buttons, which allow the user to select various analytical parameters for the data, such as the time frame, X-axis component, normalization method and calculation method. After the user selects one of each of these parameters, the "Run Extraction" button is clicked, causing the global options to be updated with the new inputs and control the remainder of the data extraction and manipulation process. See Supplementary Information 1.3 for full template code of the "CalcType" form module.

The normalization method chosen, dictates the next VBA function initialized. The "NormData" module contains all four of the data normalization methods that I had employed at one time or another; however, the one used in the analysis of all the forthcoming data was "Norm2." Wherein the wells with the lowest concentration of drug (column 11 on the assay plate) served as the experimental max for that row at any given point in time and the "Blank" cells (column 12, rows A and B of the assay plate) serve as the experimental minimum. The use of the lowest drug concentration as the experimental maximum helped to address the drifting of data, which was noted during the early runs of 
the assay. The data were then normalized values via Equation 2.1:

$$
\frac{A_{n}+(x-A)\left(B_{n}-A_{n}\right)}{B-A}
$$

Equation 2.1

Wherein $\mathrm{A}=$ Experimental Basal Response, $\mathrm{B}=$ Experimental Maximum Response, $A_{n}=$ Normalized Minimum Value (0), $B_{n}=$ Normalized Maximum Value (100) and $\mathrm{x}=$ raw data value to be normalized. See Supplementary Information 1.4 for full template code of the "NormData" module.

The next parameter manipulated has to do with the time interval of interest. All data presented in subsequent chapters utilized the 5 minutes time interval, i.e. values from each of the concentrations of drug were examined at every 5 minutes up to 60 minutes. However, in some cases it is useful to examine based on the maximum and minimum values for the control wells (column 12, rows E and F of the assay plate) to determine the optimal time interval. See Supplementary Information 1.5 for full template code of "FiveMin" and "ZenNadir" modules.

Within both the "FiveMin" and "ZenNadir" modules are references to necessary information for their completion. The first of which is the concentrations for each of the wells in the assay plate. If the file does not exist for this, the VBA code will call a form for the user to input the beginning concentration of each row (column 1) for the assay plate in nM. After inputting the concentrations in the form and clicking "OK," VBA code runs to translate the $\mathrm{nM}$ values into $\log _{10}$ values for use in GraphPad Prism and creates a "Concentrations.txt" file so that the values do not have to be re-input by the user for subsequent runs of the same plate layout in a day. See Supplementary Information 1.6 for full template code of the "Concentrations" module.

As part of the Gen5 experiment setup, the user should input the row names into the software; however, in the event that this step goes overlooked, a form will pop up during analysis of the data asking the user to input the row names. Upon inputting the names and clicking OK, the code runs to create a name file and apply those names to the current spreadsheet. See Supplementary Information 1.7 for full template code of the "Names" module.

Following the time, concentration and name executions, it is now time to run the appropriate calculation module to refine the data, if need be. All functional data presented henceforth will have no additional calculations applied to it beyond the aforementioned normalization method. The 6 possible calculation methods are: take only the normalized values (the method employed in all forthcoming analysis); to subtract the Ro-containing control wells (column 12, rows $\mathrm{C}$ and $\mathrm{D}$ of the assay plate); to subtract the forskolincontaining control wells (column 12, rows E and F); to divide by the lowest concentration of drug (column 11, same row as data being evaluated and a the same point in time); to divide the drug at a given time to that well's value at time $=0 \mathrm{~min}$; or to divide each drug by the forskolin-containing control (column 12, rows E and F). See Supplementary Information 1.8 for full template code of the different calculation modules. 
After any of the calculations are completed, you will notice that there is a call to organize the raw data. This is a simple transposition of the data to ready it for importation into GraphPad Prism. See Supplementary Information 1.9 for full template code of the "Organize" module. With the data now normalized, calculated, organized and ready for importation into GraphPad Prism, it is necessary to create individual data sheets that GraphPad will use to import into the shelled data sheets that exist in the template. See Supplementary Information 1.10 for full template code of the "DataSheets" module. After this, the code loops continuously to await completion of the Prism importation.

After creating the data sheets for each of the 8 drugs on a plate, the VBA macro will generate a script to execute when it calls the GraphPad Prism program. See Supplementary Information $\mathbf{1 . 1 1}$ for full template code of the "PrismScript" module. After the GraphPad Prism script completes its data importation, it creates a "Done.txt" file, which is the signal for the all-the-while-looping Microsoft Excel spreadsheet to copy itself into the master book, clean up the temporary files and close. I wanted to copy the extracted, normalized and calculated data into a master file, because I wanted a mechanism to collect all 6 runs in one file to replicate this process for the $n=6$ analysis of each of the drugs tested. See Supplementary Information $\mathbf{1 . 1 2}$ for full template code for the "Copy" module.

With all of these VBA macros and forms in place and functioning, it is necessary to insert an easy mechanism to set into motion the "switchboard" and the subsequent cascade of events I've gone through. To accomplish this task, I inserted an object onto the main sheet of the Gen5 "Power Export" template - for this, I used an unfilled, unoutlined rectangle that I sized to the A2 cell of the sheet. After inserting this, right clicking the object and clicking "Assign Macro" allowed me to select the "Extract" macro as its designated target. Once saved, this template will be the default "Power Export" for this protocol, and the protocol need only be replicated in triplicate and assigned the 3 aforementioned names. Once the "Power Export" executes at the completion of a run, I need only click on the object previously inserted that is assigned the "Extract" macro to automate data manipulation and importation into GraphPad Prism.

2.2.7.4. Master extraction template. As each of the runs for a set of drugs completes, and as previously mentioned, the data sheet from each mirrors into a master Excel workbook located in the same folder as all of the other current data for the in-progress 8 compounds. After the completion of the sixth run, it is necessary to perform a similar, albeit more simple, action on the master workbook to extract the data from each individual run, group them together and export the data from Excel into GraphPad Prism.

As was the case with the individual run template, the first and foremost module to create is the "Declarations" module to allow for passage of variables among the different functions contained in the project. See Supplementary Information 1.13 for details of this VBA code.

Since this workbook is merely copying already transformed data from within itself into one, centralized, sheet, the amount of VBA code necessary is quite limited relative to the previous template. Still contained within it is the scripting necessary to 
generate data sheets for importation into GraphPad Prism. Therefore, with the click of a "button," set up just as we did for the individual runs - an object assigned to the "Extract" macro - we can automate the bundling of all six runs for the eight ligands contained in each day's runs and compile a master sheet of data. See Supplementary Information 1.14 for details of the VBA code.

Execution of this series of macros allows for the easy bundling of 6 plates worth of data for 8 different ligands at 11 concentrations each over 60 minutes. Combination of the individual plate macros with the master workbook macros reduces data analysis time by hours, which allows for a higher, more accurate throughput of compounds.

\subsection{Finalized Assay Conductance}

\subsubsection{Materials}

Forskolin, purchased from Tocris Bioscience/R\&D Systems (Minneapolis, MN). Ro 20-1724 (PDE inhibitor), G418, Puromycin, Pen/Strep (100U/100 $\mu$ g per mL), DPBS, Hank's 1x Buffer, DMEM, Direct Blue 71, HEPES and pluronic acid were purchased from ThermoFisher Scientific (Waltham, MA). Fetal Bovine Serum (FBS) purchased from Atlanta Biologicals (Lawrenceville, GA). The transfected HEK-293 cells coexpressed with modified CNG channels along with Cannabinoid Receptor Type 1 (CBR-1) or Cannabinoid Receptor Type 2 (CBR-2) and their parental cells (without Cannabinoid Receptor Type 1 or 2; HEK-CNG) were purchased from Codex BioSolutions - so named ACT:One Cells (Gaithersburg, MD). Membrane potential dye was made by combining 1 L Hank's 1x Buffer with 5.2 g HEPES (20 mM final concentration) and adjusting $\mathrm{pH}$ to 7.4 . To the buffered solution we add $2.9 \mathrm{~mL}$ of DiSBAC stock - freshly prepared by adding $11.91 \mathrm{mg}$ DiSBAC to $3 \mathrm{~mL}$ DMSO -700 $\mu \mathrm{L} 10 \%$ pluronic acid and $1.874 \mathrm{~g}$ Direct Blue 71 . Cell culture media for the CBR1 and CBR2 containing cells was made by combining $890 \mathrm{~mL}$ DMEM, $100 \mathrm{~mL}$ FBS, $10 \mathrm{~mL} \mathrm{P/S}$, $1250 \mu \mathrm{L}$ G418 and $100 \mu \mathrm{L}$ Puromycin.

\subsubsection{Cell culture and frozen cell preparation}

HEK-CNG, $\mathrm{CB}_{1}$ and $\mathrm{CB}_{2}$ transfected cells were maintained in DMEM medium containing $10 \% \mathrm{FBS}, 1 \% \mathrm{Pen} / \mathrm{Strep}, 250 \mathrm{mcg} / \mathrm{mL} \mathrm{G} 418$ at $37{ }^{\circ} \mathrm{C}$ in $5 \% \mathrm{CO}_{2}$; however, the CBR-1 and CBR-2 transfected cells were cultured in medium containing an additional $1 \mu \mathrm{g} / \mathrm{mL}$ Puromycin. The cells, seeded at a density of $2.8 \times 10^{6}$ cells, in a T225 flask (Corning; Corning, NY) containing $40 \mathrm{~mL}$ of the appropriate culture media and were allowed to grow until reaching $80 \%$ to $90 \%$ confluence.

\subsubsection{CNG channel-coupled cAMP assay}

Cells from 1 appropriately $85-90 \%$ confluent flask were harvested. The cells were resuspended in $10 \%$ FBS DMEM with $1 \%$ pen/strep medium at 500,000 cells $/ \mathrm{mL}$ for 
CBR-1 and HEK-CNG and 300,000 cells/mL for CBR-2. $100 \mu \mathrm{L}$ of resuspended cells was dispensed into each well of 6 clear, 96-well, Poly-D-Lysine plates (BD via Fisher Scientific) using a 12-channel multi-channel pipet (ThermoFisher Scientific; Waltham, MA). It is important to note that the plating method needs to be such that the cell suspension must be plated by row, rather than by column, to alleviate the false-positive change in cAMP noticed on some of my data. Thus, plate column 12, then row 1 (columns 1 through 11), row 2 (columns 1 through 11), etc. for each plate. After overnight culture at $37{ }^{\circ} \mathrm{C}$ and $5 \% \mathrm{CO}_{2}$ the cells were $75 \%$ to $80 \%$ confluent.

After the overnight incubation period, I added $100 \mu \mathrm{L}$ of membrane potential dye to each well of the culture plate and allowed to sit in a dark area at room temperature for 60 minutes. After the 60 minute incubation period a baseline read in a BioTek Synergy 2 plate reader with an excitation of 540/25 nm and an emission of 590/20 nm. Following the baseline read, $50 \mu \mathrm{L}$ of ligand stock solution containing: appropriate drug concentration (beginning at either $25 \mu \mathrm{M}$ or $5 \mu \mathrm{M}[5 \mu \mathrm{M}$ or $1 \mu \mathrm{M}$, final], Table 1 ), $4 \mu \mathrm{M}$ Forskolin (800 nM, final) and $125 \mu \mathrm{M}$ Ro 20-1724 (25 $\mu \mathrm{M}$, final) in DPBS with $2.5 \%$ DMSO was added to the cell culture plate. Columns 1 through 11 contain drug, and Column 12 contains control wells. Control wells are: "Blank" (cells, media and dye), "Ro" ("Blank" + 25 M Ro 20-1724 [Final]), "Ro/For" ("Ro" + 800 nM Forskolin [Final]) and "CP CTRL" ("Ro/For" + $5 \mu \mathrm{M}$ CP 55,940 [Final]). The plate was then placed on the plate reader with 1 read per well per minute with an excitation of 540/25 $\mathrm{nm}$ and an emission of 590/20 $\mathrm{nm}$ for 60 minutes.

\subsubsection{Data analysis}

I analyzed primary data with customized VBA macros, developed internally, in Microsoft Excel (Redmond, WA). Column 11 (lowest [drug]), determined the experimental maximum response (100\% activity) at each individual time point, and the experimental basal response was determined by the average response given by the 2 "Blank" wells. All data were normalized with these minimum and maximum values via Equation 2.1. The $\mathrm{EC}_{50}$ values were calculated from concentration-response curves by non-linear regression analysis utilizing GraphPad Prism Software (San Diego, CA).

\subsection{Conclusions}

In this chapter, I have demonstrated optimization of the ACTOne GPCR assay from Codex Biosolutions to ensure reproducible, cost-effective and reliable data in a high-throughput manner. Additionally, I have provided an example of one method to enhance the Gen5 plate reading software with Microsoft Excel's powerful VBA macro capabilities to yield a fast and effective means of analyzing large quantities of data generated from conductance of functional assays.

Future directions for this project could be a further honing of the assay to gain even more cost-savings, speed and accuracy of data acquisition. Namely, a more serious trial and investigation into the use of the more cost-effective polystyrene plates over the Poly-D-Lysine coated plates. 


\section{CHAPTER 3. FUNCTIONAL EVALUATION OF A NOVEL CLASS OF PHENYL-SUBSTITUTED SIDE CHAIN ANALOGUES OF $\Delta^{8}$-THC}

\subsection{Introduction}

Classical cannabinoids are tricyclic compounds possessing a benzopyran core these are of either natural origin or synthetic analogues thereof. The naturally occurring, prototypes for these compounds are $\Delta^{9}$-THC and $\Delta^{8}$-THC, which both bind to $\mathrm{CB}_{1}$ and $\mathrm{CB}_{2}$ receptors and exert a partial agonist activity. ${ }^{14}$ Within the classical compounds impacting the $\mathrm{CB}_{1}$ receptor there exists novel means of dealing with anti-nociception, mitigation of chemotherapeutic-induced nausea and vomiting, decreasing intra-ocular pressure in glaucoma, increasing appetite in patients with wasting syndromes, reduction of muscle spasticity caused by diseases such as multiple sclerosis and treating diarrhea by slowing intestinal motility are present. ${ }^{232,233} \mathrm{With} \mathrm{CB}_{2}$ receptors serving the body as modulators of cytokine release, agents targeting these compounds show great promise in inflammation, especially in instances of inflammation-induced organ damage. ${ }^{136,165}$ The drawback exists in separating $\mathrm{CB}_{1}$ and $\mathrm{CB}_{2}$ activity to retain or augment the desired therapeutic outcome while simultaneously mitigating the negative psychotropic effects, ascribed to $\mathrm{CB}_{1}$, of such compounds. This desire for such potentially impactful compounds for various disease states which have favorable therapeutic use profiles has led to a bevy of research into the classical cannabinoids with regard to their pharmacology and structural activity relationships/requirements (SAR).

Current knowledge of cannabinoid SAR has centered heavily around the classical, benzopyran core, and from these studies one may gather three pharmacophoric elements exist for classical cannabinoids to exert cannabimimetic activity (Figure 3.1):

1. C-1 phenolic hydroxyl group

2. Trans ring junction at the $\mathrm{B}$ and $\mathrm{C}$ rings

3. Lipophilic C-3 alkyl side chain

Literature precedent shows that lengthening of the C-3 alkyl chain (Section 3.2.1) causes an increase in the binding affinity of the compounds, with an optimal length of 7 to 8 carbons. Additionally, unsaturation (Section 3.2.2), especially about the C-1',2' bond affords an even greater enhancement of binding affinity and selectivity, with the linear alkyne and cis $1^{\prime}, 2^{\prime}$ alkene showing optimal activity. Another area of exploration was the substitution of C-1' and C-2' (Section 3.2.3), which demonstrated the importance of this position, with 1',1'-dimethylation being optimal. Compilation of this knowledge (Figure 3.2) led to the synthesis of cycloalkyl ${ }^{234}$ and aromatic $^{235}$ substituents (Section 3.2.4) introduced at $\mathrm{C}-1$ '.

This study seeks to utilize the ACTOne Assay (Chapter 2) to explore the functional ramifications of an aromatic substitution at C-1' (Section 3.3). Taking this functional activity data, I will then reconcile these outcomes against homology models and current literature standards' binding to propose hypothetical interactions with $\mathrm{CB}_{1}$ and $\mathrm{CB}_{2}$, to further develop the SAR and unique mechanism of these compounds. 


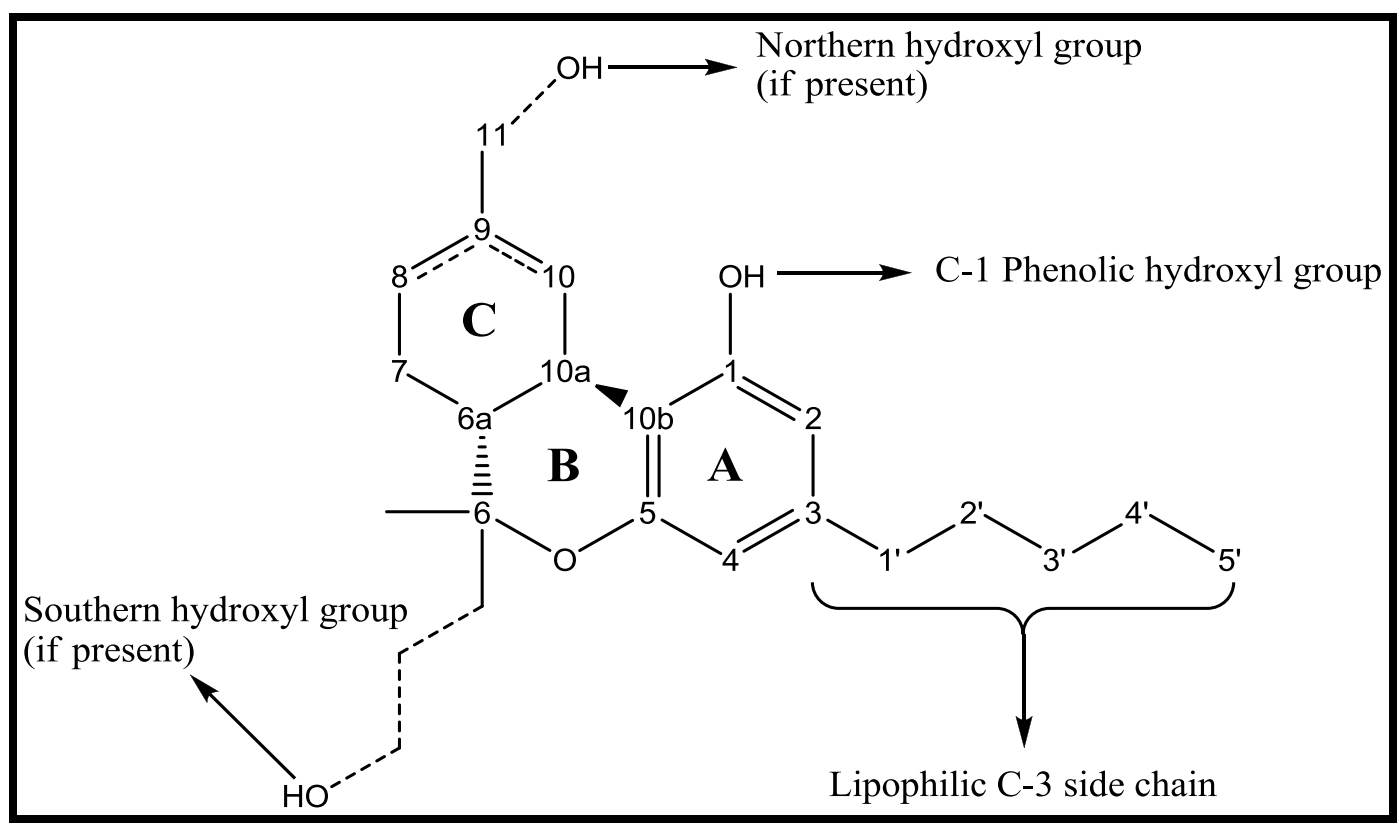

Figure 3.1. Classical cannabinoid with pharmacophore elements 


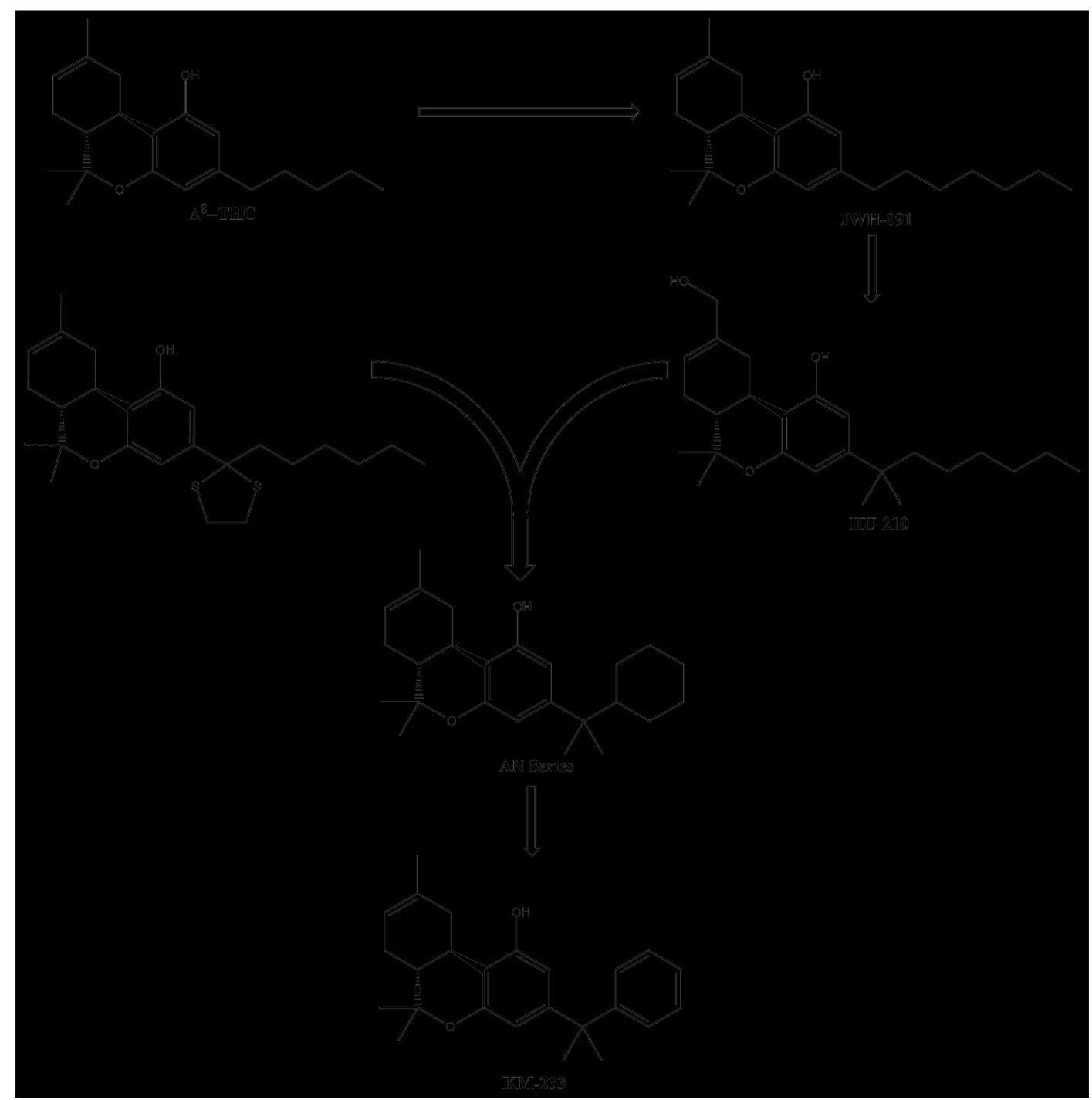

Figure 3.2. Design progression of the KM series of compounds 


\subsection{Classical Cannabinoids - Modifying C-3 Substituents to Increase Binding}

For the purposes of examining this novel series of compounds, I will leave C-1 phenolic hydroxyl and the trans ring junction pharmacophoric elements intact, and I will not bring into account the optional elements of the northern and southern hydroxyl groups.

\subsubsection{Varying $C-3$ chain length}

The most logical starting point for modification of the $\mathrm{C}-3$ postion was to substitute the $n$-pentyl of $\Delta^{8}$-THC (1) $\left(\mathrm{CB}_{1} \mathrm{Ki}=47.6 \mathrm{nM} ; \mathrm{CB}_{2} \mathrm{~K}_{\mathrm{i}}=39.3 \mathrm{nM}\right)$ with varying lengths of C-3 substituents (Figure 3.3). This ranged from $n$-butyl (2) $\left(\mathrm{CB}_{1} \mathrm{~K}_{\mathrm{i}}=\right.$ $65 \mathrm{nM})$ to $n$-hexyl (3) $\left(\mathrm{CB}_{1} \mathrm{~K}_{\mathrm{i}}=41 \mathrm{nM}\right)$ to $n$-heptyl (4) $\left(\mathrm{CB}_{1} \mathrm{~K}_{\mathrm{i}}=22 \mathrm{nM}\right)$ and to $n$-octyl (5) $\left(\mathrm{CB}_{1} \mathrm{~K}_{\mathrm{i}}=8.5 \mathrm{nM}\right) .{ }^{236}$ Data from this study show that $n$-octyl (5) has the highest affinity, with $n$-heptyl (4) a near second.

\subsubsection{Unsaturated $\mathrm{C}-3$ side chain analogs}

A next logical step is to undertake the introduction of unsaturation into the C-3 side chain (Figure 3.4) to probe the binding pockets. Conformationally restricted aliphatic chains to helped to elucidate the spatial configuration of the LBP of $\mathrm{CB}_{1}$ and $\mathrm{CB}_{2}$ receptors about the hydrophobic $\mathrm{C}-3$ binding pocket. Beginning the process of unsaturation one may turn attention first to the $\mathrm{C}-1$ ' heptyne derivative $(6)\left(\mathrm{CB}_{1} \mathrm{~K}_{\mathrm{i}}=0.65\right.$ $\left.\mathrm{nM} ; \mathrm{CB}_{2} \mathrm{~K}_{\mathrm{i}}=3.1 \mathrm{nM}\right)^{237}$ and note that it has, at best, a modest proclivity for $\mathrm{CB}_{1}$ over $\mathrm{CB}_{2}$ but good affinity for both overall. Stepping down the unsaturation at $\mathrm{C}-1^{\prime}$, one may examine the $C$-1'-heptene derivatives as the Z-isomer (7) $\left(\mathrm{CB}_{1} \mathrm{~K}_{\mathrm{i}}=0.8 \mathrm{nM} ; \mathrm{CB}_{2} \mathrm{~K}_{\mathrm{i}}=9.5\right.$ $\mathrm{nM})^{238}$ and E-isomer $(8)\left(\mathrm{CB}_{1} \mathrm{~K}_{\mathrm{i}}=1.2 \mathrm{nM} ; \mathrm{CB}_{2} \mathrm{~K}_{\mathrm{i}}=5.3 \mathrm{nM}\right){ }^{238}$ It is noteworthy that these are $\mathrm{C}$-11-hydroxy compounds, but they do not differ, in the case of the Z-isomer at $\mathrm{CB}_{1}$, from the C-9-methyl compounds $\left(\mathrm{CB}_{1} \mathrm{~K}_{\mathrm{i}}=0.86 \mathrm{nM}\right) .{ }^{236}$ Taking these three compounds as a group, it is evident that $\mathrm{CB}_{1}$ affinity is unaffected among the three; however, the $\mathrm{CB}_{2} / \mathrm{CB}_{1}$ ratio changes. Most notably, this change in the ratio occurs with compound 7 , suggesting that $\mathrm{CB}_{2}$ has less tolerance for acute angle orientation and unsaturation of $\mathrm{C} 1$ '- $\mathrm{C} 2$ '.

Moving more distal along the $\mathrm{C}-3$ substituent chain one may examine a large number of $\mathrm{C}-2^{\prime}$ unsaturated compounds, beginning first with the $2^{\prime}$-hexynyl derivative (9) $\left(\mathrm{CB}_{1} \mathrm{~K}_{\mathrm{i}}=11 \mathrm{nM}\right){ }^{236}{ }^{2}$-octynyl $(\mathbf{1 0})\left(\mathrm{CB}_{1} \mathrm{~K}_{\mathrm{i}}=4.9 \mathrm{nM}\right)$ does well so long as there is a free phenolic hydroxyl; however, when $\mathrm{C}-1$ is methoxy the affinity drops considerably $\left(\mathrm{CB}_{1} \mathrm{~K}_{\mathrm{i}}=189 \mathrm{nM}\right) .{ }^{236} 2^{\prime}$-nonynyl $(\mathbf{1 1})\left(\mathrm{CB}_{1} \mathrm{~K}_{\mathrm{i}}=3.7 \mathrm{nM}\right)^{236}$ continues to improve upon the binding affinities of both 2 '-hexynyl and 2 '-octynyl, suggesting that with an optimized angle through unsaturation the binding pocket extends further. In terms of the $2^{\prime}$-alkenes, the Z-isomers of both $2^{\prime}$-heptene $(\mathbf{1 2})\left(\mathrm{CB}_{1} \mathrm{~K}_{\mathrm{i}}=1.55 \mathrm{nM}\right)^{239}$ and $2^{\prime}$-octene $(\mathbf{1 3})\left(\mathrm{CB}_{1} \mathrm{~K}_{\mathrm{i}}=\right.$ $3.19 \mathrm{nM})^{236}$ showed the most promise, with $2^{\prime}$-pentene-5'-hydroxy (14) $\left(\mathrm{CB}_{1} \mathrm{~K}_{\mathrm{i}}=125\right.$ $\mathrm{nM})^{239}$ and 2'-heptene- 6 '-acetamide $(15)\left(\mathrm{CB}_{1} \mathrm{~K}_{\mathrm{i}}=43.4 \mathrm{nM}\right)^{239}$ paling in comparison. The lack of affinity in the hydrophilic derivatives points strongly to the conclusion that this is a relatively exclusively lipophilic pocket of the receptor. 


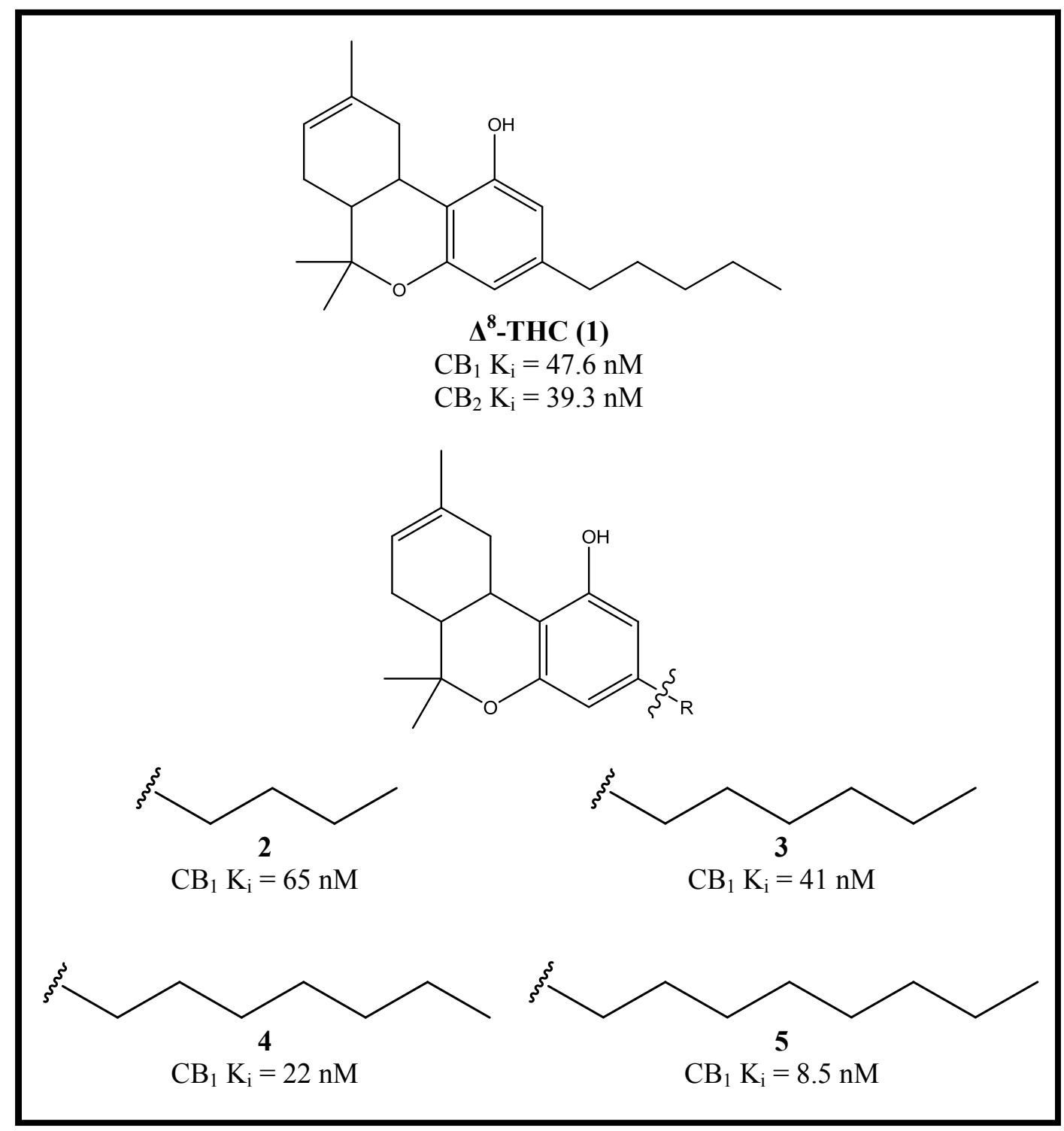

Figure 3.3. $\quad \mathrm{C}-3$ chain length analogues and the effect on $\mathrm{CB}_{1}$ binding affinity 


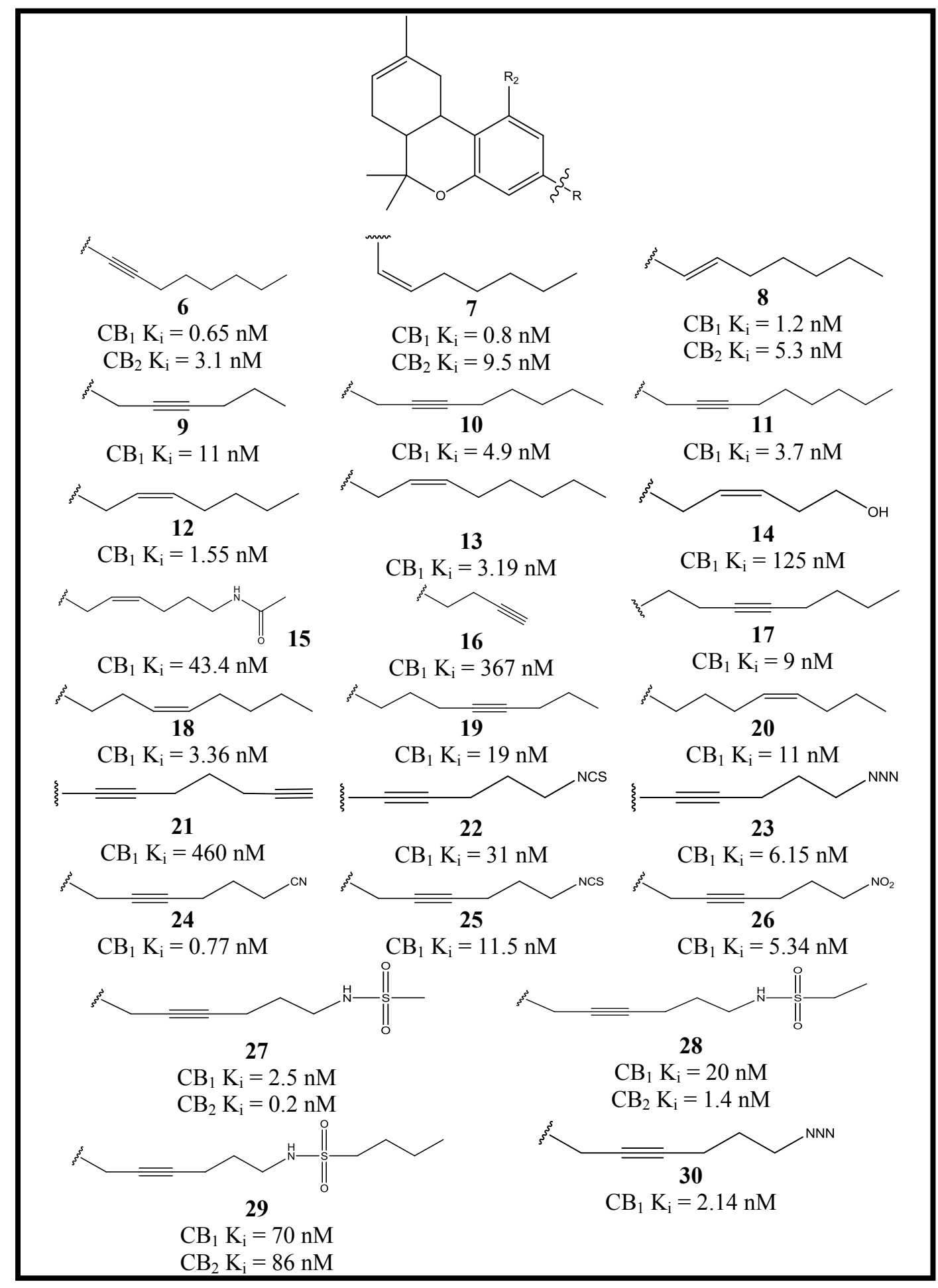

Figure 3.4. Unsaturated $\mathrm{C}-3$ side chain analogs

Notes: $\mathrm{R}_{2}$ is $\mathrm{OH}$ in all compounds except compound $\mathbf{1 1}$ where $\mathrm{R}_{2}$ is methoxy. 
Continuing the trek of unsaturation, one may move one carbon more distal on the C-3 chain, where relatively few modifications exist to date. 3'-butynyl (16) $\left(\mathrm{CB}_{1} \mathrm{~K}_{\mathrm{i}}=367\right.$ $\mathrm{nM})^{236}$ fared much worse than $3^{\prime}$-octynyl $(17)\left(\mathrm{CB}_{1} \mathrm{~K}_{\mathrm{i}}=9 \mathrm{nM}\right)^{236}$, but neither did as well as the Z-isomer of $3^{\prime}$-octenyl $(\mathbf{1 8})\left(\mathrm{CB}_{1} \mathrm{~K}_{\mathrm{i}}=3.36 \mathrm{nM}\right)^{236}$ (Figure 3.4). This data suggests that the hydrophobic pocket of $\mathrm{CB}_{1}$ turns, and the more rigid, straight alkynes likely have some steric issues hindering their binding affinity.

Moving next to the $4^{\prime}$ position, there are 2 derivatives of note, $4^{\prime}$-octynyl (19) $\left(\mathrm{CB}_{1} \mathrm{~K}_{\mathrm{i}}=19 \mathrm{nM}\right)$ and the Z-isomer of 4'-octenyl (20) $\left(\mathrm{CB}_{1} \mathrm{~K}_{\mathrm{i}}=11 \mathrm{nM}\right)^{236}$ (Figure 3.4). These two suggest, again, that the binding pocket of $\mathrm{CB}_{1}$ has a curve to it and that the more distal on the $\mathrm{C}-3$ side chain the unsaturation is, especially to the alkyne (linear) state, the less likely one is to fit that curve and thus one loses binding affinity.

Next, sites of multiple unsaturation are examined, beginning with $1^{\prime}, 6^{\prime}$-heptadiyne (21) $\left(\mathrm{CB}_{1} \mathrm{~K}_{\mathrm{i}}=460 \mathrm{nM}\right) .{ }^{236} 2^{\prime}$-pentyne derivatives with a 5' substitution (Figure 3.4) seem to fair best as far as the multiple unsaturation derivatives go, which may seem counterintuitive given 21's lack of affinity. However, the presence of 5'-CN (22) $\left(\mathrm{CB}_{1} \mathrm{~K}_{\mathrm{i}}\right.$ $=31 \mathrm{nM})^{236}$ or $5^{\prime}-\mathrm{NNN}(\mathbf{2 3})\left(\mathrm{CB}_{1} \mathrm{~K}_{\mathrm{i}}=6.15 \mathrm{nM}\right)^{236}$ demonstrates that in the case of multiple unsaturation, the LBP requires a $\mathrm{H}$-bond accepting terminus to bind with higher affinity. This trend continues when one looks at the 2'-hexyne derivatives containing 6'$\mathrm{CN}(\mathbf{2 4})\left(\mathrm{CB}_{1} \mathrm{~K}_{\mathrm{i}}=0.77 \mathrm{nM}\right), 6^{\prime}-\mathrm{NCS}(\mathbf{2 5})\left(\mathrm{CB}_{1} \mathrm{~K}_{\mathrm{i}}=11.5 \mathrm{nM}\right)$ or $6^{\prime}-\mathrm{NO}_{2}(\mathbf{2 6})\left(\mathrm{CB}_{1} \mathrm{~K}_{\mathrm{i}}=\right.$ $5.34 \mathrm{nM}){ }^{236}$ This becomes most evident and useful comparing the 2'-heptyne-6'sulfonamide derivatives containing an $n$-methyl (27) $\left(\mathrm{CB}_{1} \mathrm{~K}_{\mathrm{i}}=2.5 \mathrm{nM} ; \mathrm{CB}_{2} \mathrm{~K}_{\mathrm{i}}=0.2\right.$ $\mathrm{nM}), n$-ethyl (28) $\left(\mathrm{CB}_{1} \mathrm{~K}_{\mathrm{i}}=20 \mathrm{nM} ; \mathrm{CB}_{2} \mathrm{~K}_{\mathrm{i}}=1.4 \mathrm{nM}\right)$ and $n$-butyl (29) $\left(\mathrm{CB}_{1} \mathrm{~K}_{\mathrm{i}}=70 \mathrm{nM}\right.$; $\mathrm{CB}_{2} \mathrm{~K}_{\mathrm{i}}=86 \mathrm{nM}$ ) substituted sulfonamide. ${ }^{240}$ This data suggests that the $\mathrm{CB}_{2} \mathrm{LBP}$ has a larger hydrophobic pocket that could be targeted over the $\mathrm{CB}_{1}$ LBP by extending the $\mathrm{C}-3$ substituent length just enough without causing an eventual hindrance to both - as is the case with comound 29. Also of interest is the affinity of $\mathbf{2 4}$ and the 5'-azidopent-2'-yne (30) $\left(\mathrm{CB}_{1} \mathrm{~K}_{\mathrm{i}}=2.14 \mathrm{nM}\right){ }^{241}$ The interest lies dually in the 3-fold difference of binding affinities and in that both are antagonists with 5 '-azidopent- 2 '-yne being a partial agonist while hept-2'-ynenitrile is neutral. ${ }^{241}$

\subsubsection{C-1' modified compounds}

As previously discussed in Section 3.2.1, optimal activity was found with $n$-heptyl (4) or $n$-octyl (5), but further derivation found those compounds with a $1^{\prime}, 1^{\prime}$ - or 1',2'-dimethyl substitution were more potent still. ${ }^{11,242,243} 1^{\prime}, 1^{\prime}$-dimethylheptyl (31) (CB $\left.\mathrm{K}_{\mathrm{i}}=0.89 \mathrm{nM} ; \mathrm{CB}_{2} \mathrm{~K}_{\mathrm{i}}=1.41 \mathrm{nM}\right)$ and $1^{\prime}, 2^{\prime}$-dimethylheptyl (32) $\left(\mathrm{CB}_{1} \mathrm{~K}_{\mathrm{i}}=0.46-0.84\right.$ $\mathrm{nM}$ ) provided the initial foundation of optimized chain length and substitution pattern for derivation into $1^{\prime}, 1^{\prime}$-dimethyl pentyl and hexyl with 5' or 6' substituted compounds (Figure 3.5). As the size of the substitution increases, the affinity for $\mathrm{CB}_{1}$ starts to numerically wane, as is exemplified by 1',1'-dimethyl-6'-hexanenitrile $(\mathbf{3 3})\left(\mathrm{CB}_{1} \mathrm{~K}_{\mathrm{i}}=\right.$ $0.36 \mathrm{nM}), 1^{\prime}, 1^{\prime}$-dimethyl-7'-heptanenitrile $(34)\left(\mathrm{CB}_{1} \mathrm{~K}_{\mathrm{i}}=0.6 \mathrm{nM}\right)$ and 1',1'-dimethyl-6'phenyl-7'-heptanenitrile (36) $\left(\mathrm{CB}_{1} \mathrm{~K}_{\mathrm{i}}=0.92 \mathrm{nM}\right) .{ }^{244}$ However, these values are not a therapeutically viable or targetable difference. A similar trend is noticed when comparing the 1',1'-dimethyl-6'-N,N dimethyl hexane $(37)\left(\mathrm{CB}_{1} \mathrm{~K}_{\mathrm{i}}=0.86 \mathrm{nM}\right)$ to 


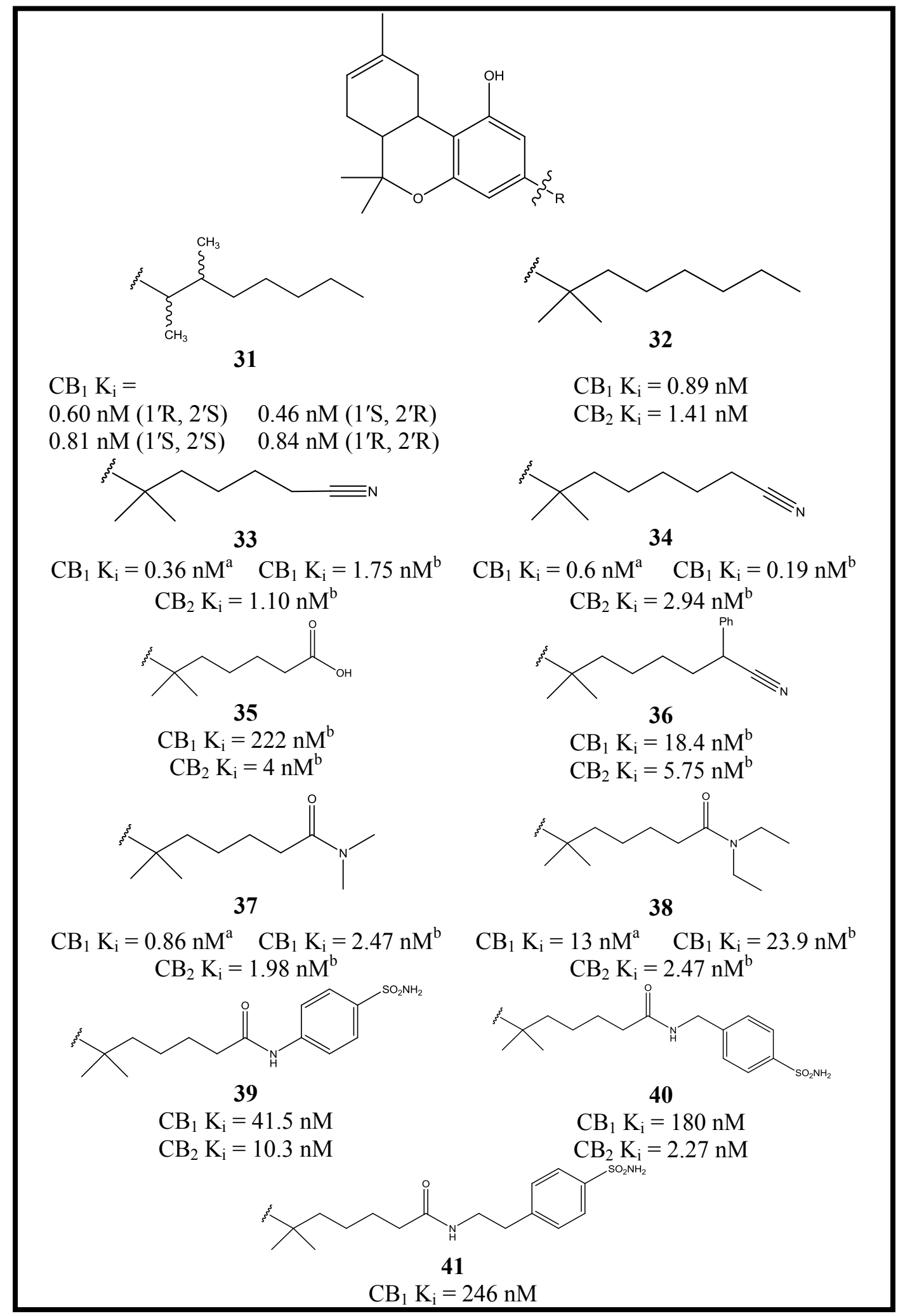

Figure 3.5. Acidic and basic modifications to the terminus of $\Delta^{8}$-THC C-3 substituents 
$1^{\prime}, 1^{\prime}$-dimehtyl-6'-N,N-diethylhexane (38) $\left(\mathrm{CB}_{1} \mathrm{~K}_{\mathrm{i}}=13 \mathrm{nM}\right) \cdot{ }^{244} p$-phenylsulfonamide derivatives $(39,40,41)$ overall fared worse than smaller substituents, but demonstrated the size constraints of the $\mathrm{CB}_{1}$ LBP. ${ }^{244}$ Interestingly, this increase in size was not as impactful on the $\mathrm{CB}_{2} \mathrm{~K}_{\mathrm{i}}$. This tolerance is somewhat evident in the case of $1^{\prime}, 1^{\prime}$-dimethyl6'-hexanenitrile (33) $\left(\mathrm{CB}_{1} \mathrm{~K}_{\mathrm{i}}=1.75 \mathrm{nM} ; \mathrm{CB}_{2} \mathrm{~K}_{\mathrm{i}}=1.1 \mathrm{nM}\right)$ versus 1', 1'-dimethyl-6'phenyl-7'-heptanenitrile (36) $\left(\mathrm{CB}_{1} \mathrm{~K}_{\mathrm{i}}=18.4 \mathrm{nM} ; \mathrm{CB}_{2} \mathrm{~K}_{\mathrm{i}}=5.75 \mathrm{nM}\right)$ and 1', $1^{\prime}$-dimethylN,N-diethylheptanamide (38) $\left(\mathrm{CB}_{1} \mathrm{~K}_{\mathrm{i}}=23.9 \mathrm{nM} ; \mathrm{CB}_{2} \mathrm{~K}_{\mathrm{i}}=2.47 \mathrm{nM}\right) .{ }^{239}$

In an attempt to exploit the apparent hydrophobic binding pocket that exists along with the $\mathrm{C}-3$ substituent binding pocket a series of $\mathrm{C}-1^{\prime}$ cyclic compounds were synthesized (Figure 3.6) for testing. ${ }^{245}$ This hydrophobic pocket is evidenced by the fact that $1^{\prime}, 1^{\prime}$-dimethyl compounds bind with far greater affinity than their methylene brethren, and further demonstrated by the lack of binding affinity for the 1'-hydroxyl (42) $\left(\mathrm{CB}_{1} \mathrm{~K}_{\mathrm{i}}=86.4 \mathrm{nM} ; \mathrm{CB}_{2} \mathrm{~K}_{\mathrm{i}}=65.6 \mathrm{nM}\right)$ and 1'-ketone (43) $\left(\mathrm{CB}_{1} \mathrm{~K}_{\mathrm{i}}=21.7 \mathrm{nM} ; \mathrm{CB}_{2} \mathrm{~K}_{\mathrm{i}}=\right.$ $83.7 \mathrm{nM})$. Comparing the C-3 heptyl (4) $\left(\mathrm{CB}_{1} \mathrm{~K}_{\mathrm{i}}=22 \mathrm{nM}\right)$ to $1^{\prime}, 1^{\prime}$-dimethylheptyl (32) $\left(\mathrm{CB}_{1} \mathrm{~K}_{\mathrm{i}}=0.83 \mathrm{nM} ; \mathrm{CB}_{2} \mathrm{~K}_{\mathrm{i}}=0.49 \mathrm{nM}\right)$ to 1'-cyclopentyl (44) $\left(\mathrm{CB}_{1} \mathrm{~K}_{\mathrm{i}}=0.45 \mathrm{nM} ; \mathrm{CB}_{2} \mathrm{~K}_{\mathrm{i}}\right.$ $=1.92 \mathrm{nM}), 1^{\prime}$-dithiolaneheptyl $(45)\left(\mathrm{CB}_{1} \mathrm{~K}_{\mathrm{i}}=0.32 \mathrm{nM} ; \mathrm{CB}_{2} \mathrm{~K}_{\mathrm{i}}=0.52 \mathrm{nM}\right)$ and $1^{\prime}$-dioxolane (46) $\left(\mathrm{CB}_{1} \mathrm{~K}_{\mathrm{i}}=0.52 \mathrm{nM} ; \mathrm{CB}_{2} \mathrm{~K}_{\mathrm{i}}=0.22 \mathrm{nM}\right)$ one may see that there is evidence of a dual-lobe hydrophobic binding pocket available about $\mathrm{C}-3$ substituent pocket. Interestingly, this too brings up an issue of size within both the $\mathrm{CB}_{1}$ and $\mathrm{CB}_{2}$ receptor LBP, as evidenced in the 1'-dithianeheptyl $(47)\left(\mathrm{CB}_{1} \mathrm{~K}_{\mathrm{i}}=1.8 \mathrm{nM} ; \mathrm{CB}_{2} \mathrm{~K}_{\mathrm{i}}=3.6\right.$ $\mathrm{nM})$ and 1'-benzodithioleheptyl $(48)\left(\mathrm{CB}_{1} \mathrm{~K}_{\mathrm{i}}=56.9 \mathrm{nM} ; \mathrm{CB}_{2} \mathrm{~K}_{\mathrm{i}}=257 \mathrm{nM}\right){ }^{245}$ This is an example, though, of a means to gain a 5 -fold selection for $\mathrm{CB}_{1}$ over $\mathrm{CB}_{2}$ receptor subtypes. This auxiliary hydrophobic pocket is not a primary binding pocket, as evidenced in the $\mathrm{C}$ - $1^{\prime}$-dithiolane derivative that is without an additional $\mathrm{C}-1$ ' substituent (49) $\left(\mathrm{CB}_{1} \mathrm{~K}_{\mathrm{i}}=168 \mathrm{nM} ; \mathrm{CB}_{2} \mathrm{~K}_{\mathrm{i}}=103 \mathrm{nM}\right){ }^{245}$ There is some loss of affinity when the C$1^{\prime}$ contains both dithiolane and cylcohexyl substituents $(\mathbf{5 0})\left(\mathrm{CB}_{1} \mathrm{~K}_{\mathrm{i}}=1.86 \mathrm{nM} ; \mathrm{CB}_{2} \mathrm{~K}_{\mathrm{i}}=\right.$ $1.05 \mathrm{nM}$ ), suggesting that there exists a balance to be struck between hydrophobic binding and steric bulk too near the benzchromene core (Figure 3.6). However, this same $\mathrm{C}-1$ '-cyclohexyl in conjunction with the $1^{\prime}, 1^{\prime}$-dimethyl results in one of the strongest nonselective binding compounds to date (51) $\left(\mathrm{CB}_{1} \mathrm{~K}_{\mathrm{i}}=0.57 \mathrm{nM} ; \mathrm{CB}_{2} \mathrm{~K}_{\mathrm{i}}=0.65 \mathrm{nM}\right){ }^{234}$

\subsubsection{Aromatic and cyclic substituents at $\mathrm{C}-\mathbf{1}^{\prime}$}

As one may consider the tolerance for bulky substituents at C-1' and couple that with our previously mentioned knowledge of unsaturation, a next logical step is to introduce aromatic and bi/tricyclic substituents in at C-1'. Interestingly, much like the C$1^{\prime}$ cyclohexyl derivatives of the previous section, the best binding is achieved when the molecule contains moieties capable of binding to both the upper and lower hydrophobic pockets of the LBP. This is evidenced by comparing $p$-substituted aryl derivatives when the aryl substituents were joined to the benzchromene ring core via direct $\mathrm{C}-3$ linkage ${ }^{246}$ or $1^{\prime}, 1^{\prime}$-dimethyl linkage ${ }^{235}$ (Figure 3.7). Examination of these comparator compounds one may see that the $1^{\prime}, 1^{\prime}$-dimethyl compounds far exceed the binding affinity for the direct $\mathrm{C}-3$ conjugation; however, the directly conjugated compounds do offer some selectivity as the $p$-substituent increases in size, but this is minimal in relation to the $\mathrm{CB}_{1} / \mathrm{CB}_{2}$ ratios achieved with the 1'-gem-dimethyl compounds. 


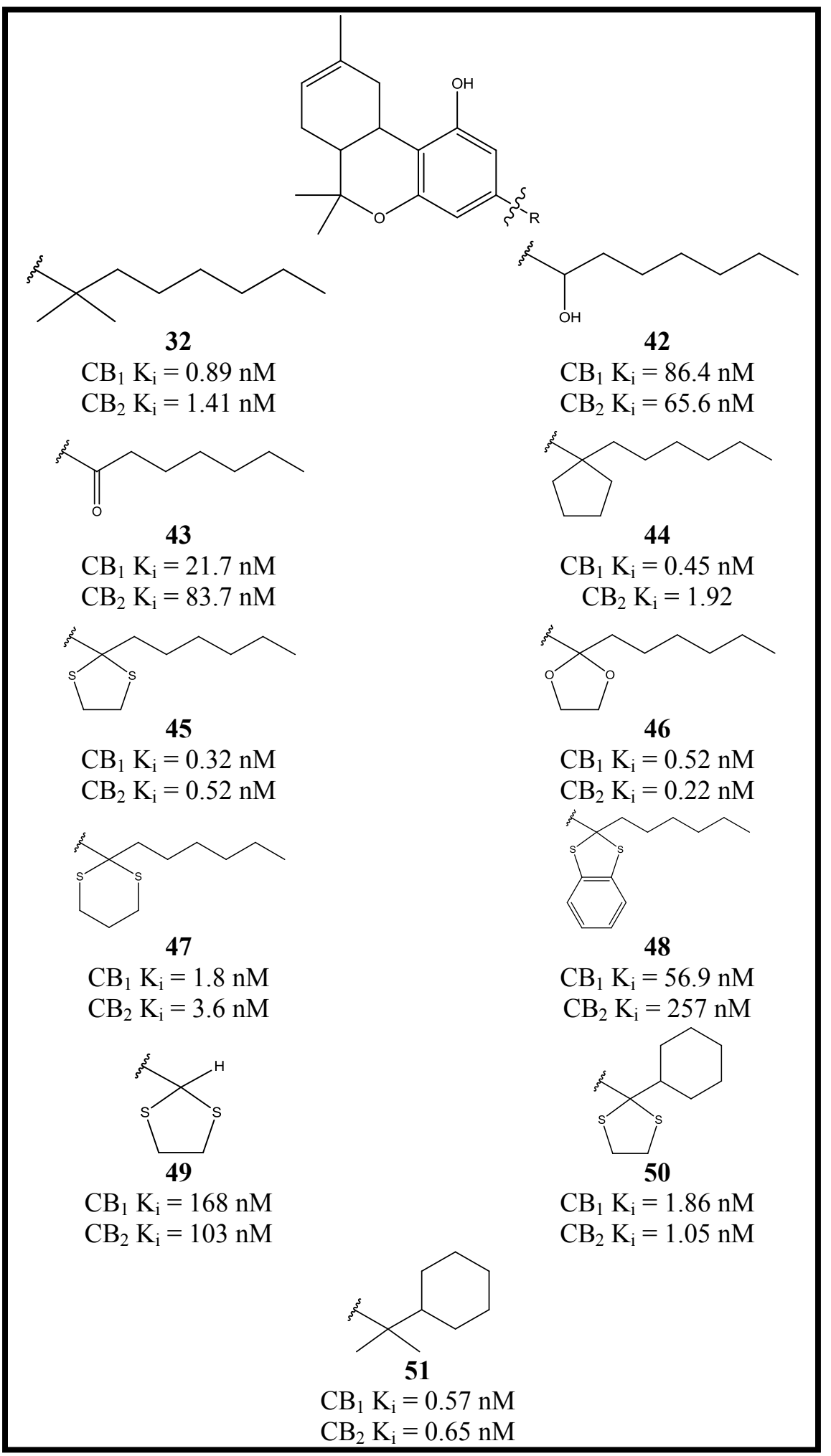

Figure 3.6. Cyclic modifications at $C-1^{\prime}$ which affect $\mathrm{CB}_{1}$ and $\mathrm{CB}_{2}$ binding affinity 


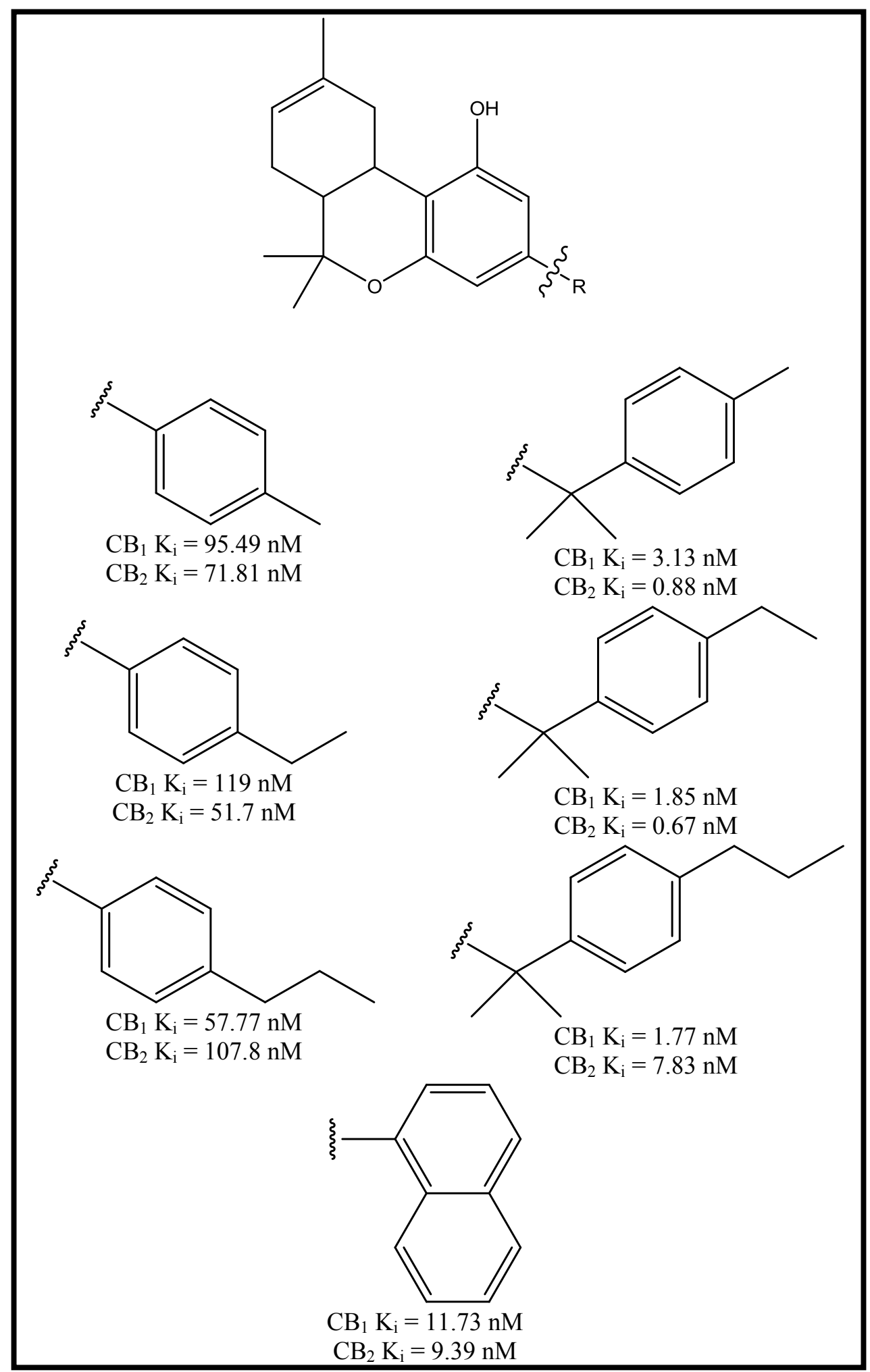

Figure 3.7. Aromatic modifications of $\Delta^{8}-\mathrm{THC}$ at $\mathrm{C}-3$ which affect $\mathrm{CB}_{1}$ and $\mathrm{CB}_{2}$ binding affinity 
The apparent difference in size is intriguing, as the compounds that seem to prefer $\mathrm{CB}_{2}$ over $\mathrm{CB}_{1}$ tend to prefer bulky $\mathrm{C}-3$ substituents, whereas the $\mathrm{CB}_{1}$-prefering ligands prefer a more compact $C-3$ substituent (Figure 3.8; Figure 3.9). There seems to be a breaking point in this conformational freedom where one loses both selectivity for $\mathrm{CB}_{2}$ and overall binding affinity. If one would compare $\mathrm{C}-3$ isobornyl (54) $\left(\mathrm{CB}_{1} \mathrm{~K}_{\mathrm{i}}=60.2\right.$ $\left.\mathrm{nM} ; \mathrm{CB}_{2} \mathrm{~K}_{\mathrm{i}}=6.1 \mathrm{nM}\right)$ to $\mathrm{C}-3$ 1-adamantyl (53) $\left(\mathrm{CB}_{1} \mathrm{~K}_{\mathrm{i}}=6.8 \mathrm{nM} ; \mathrm{CB}_{2} \mathrm{~K}_{\mathrm{i}}=52.0 \mathrm{nM}\right)$, one may see that there exists a difference between receptor preference (Figure 3.8). ${ }^{247}$ The adamantyl substituent has much less conformational space requirements compared to those of the isobornyl (Figure 3.9). It is noteworthy that the C-3 bornyl (52) $\left(\mathrm{CB}_{1} \mathrm{~K}_{\mathrm{i}}=\right.$ $8.9 \mathrm{nM} ; \mathrm{CB}_{2} \mathrm{~K}_{\mathrm{i}}=7.4 \mathrm{nM}$ ), which bears a similar $\mathrm{Y}$-axis conformational space to isobornyl, lacks the $\mathrm{Z}$-axis conformational space occupied by isobornyl, and thus it is not selective for $\mathrm{CB}_{1}$ nor $\mathrm{CB}_{2}$, albeit quite potent. There does still exist a limit within both the $\mathrm{CB}_{1}$ and $\mathrm{CB}_{2} \mathrm{LBP}$ as evidenced in the $\mathrm{C}-3$ bornylmethyl $(\mathbf{5 6})\left(\mathrm{CB}_{1} \mathrm{~K}_{\mathrm{i}}=32.7 \mathrm{nM} ; \mathrm{CB}_{2} \mathrm{~K}_{\mathrm{i}}\right.$ $=30.5 \mathrm{nM})$ and isobornylmethyl $(\mathbf{5 8})\left(\mathrm{CB}_{1} \mathrm{~K}_{\mathrm{i}}=49 \mathrm{nM} ; \mathrm{CB}_{2} \mathrm{~K}_{\mathrm{i}}=49.5 \mathrm{nM}\right)$ compounds. These compounds have a larger conformational space requirement, which appears to mitigate any preference for one receptor subtype over the other and lowers the binding affinity to near that of THC. To further support this hypothesis, consider the adamantyl derivatives of THC synthesized by Lu, et al (Figure 3.8), ${ }^{248}$ The C-3 1-adamantyl (53) has less conformational requirements (Figure 3.9). However, 2-adamantyl exhibits much more conformational freedom - a trait further extrapolated by the (2-adamantylidene)methyl (57) $\left(\mathrm{CB}_{1} \mathrm{~K}_{\mathrm{i}}=48.6 \mathrm{nM} ; \mathrm{CB}_{2} \mathrm{~K}_{\mathrm{i}}=8.9 \mathrm{nM}\right)$ derivative. The presence of the unsaturation within the (2-adamantylidene)methyl affords a rigid enough frame so as to maximize the $\mathrm{Y}$ - and $\mathrm{Z}$-axes freedom while still maintaining affinity and specificity for the $\mathrm{CB}_{2}$ receptor LBP. As one moves to a (1-adamantyl)methyl (59) ( $\mathrm{CB}_{1}$ $\left.\mathrm{K}_{\mathrm{i}}=29.3 \mathrm{nM} ; \mathrm{CB}_{2} \mathrm{~K}_{\mathrm{i}}=26.9 \mathrm{nM}\right)$ or (2-adamantyl)methyl $(\mathbf{6 0})\left(\mathrm{CB}_{1} \mathrm{~K}_{\mathrm{i}}=79.7 \mathrm{nM} ; \mathrm{CB}_{2}\right.$ $\mathrm{K}_{\mathrm{i}}=76 \mathrm{nM}$ ) the freedom afforded by the fully saturated methylene linkage causes too much freedom and a loss of both specificity and affinity for $\mathrm{CB}_{1}$ and $\mathrm{CB}_{2}$ occurs.

\subsection{Novel Series of C-1'-Dimethyl-Aryl- $\Delta^{8}$-THC Analogs (KM Series)}

\subsubsection{Rationale of design}

Amalgamation of all of these data gathered from previous series of cannabinoid compounds led to the work of former members of my lab group, which saw the synthesis of cycloalkyl ${ }^{234}$ and aromatic ${ }^{235}$ substituents introduced at C-1' (Figure 3.10). All of the compounds of this group saw marked improvement in affinity for $\mathrm{CB}_{1}$ and $\mathrm{CB}_{2}$ receptors over that of THC (Table 3.1). With such an increase in binding affinity, a need existed to establish a functional activity for these compounds.

\subsubsection{Objectives of the current study}

Utilizing this novel series of compounds, I explored the functional pharmacology of each in relation to $\mathrm{CB}_{1}, \mathrm{CB}_{2}$ and the parental $\mathrm{HEK}-293-\mathrm{CNG}$ cell lines to understand the mechanism of action (MoA) in a cell-based reporter system. Furthermore, to aid in

explanation of the observed functional data, I will reconcile known literature with regard 


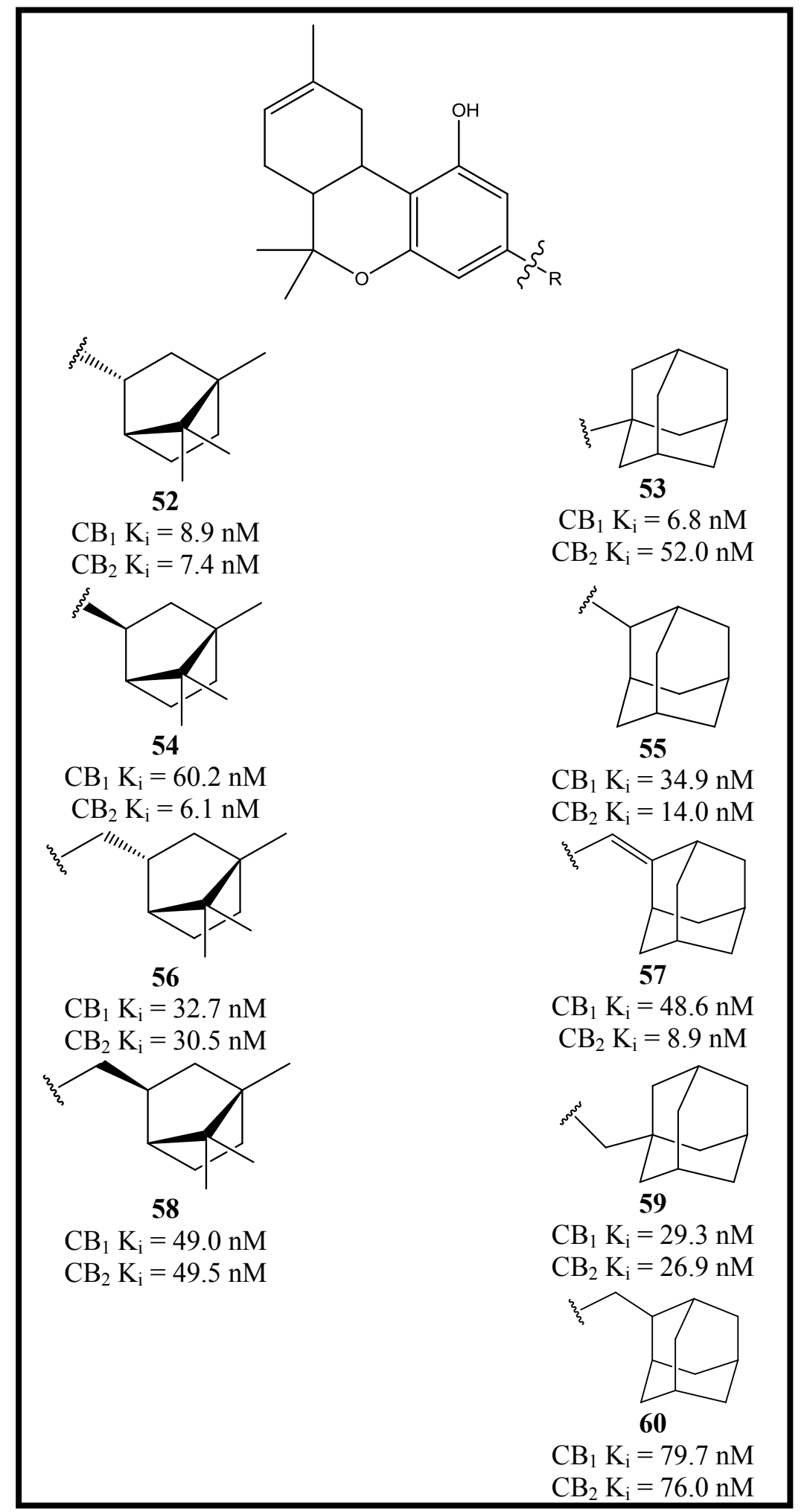

Figure 3.8. Bicyclic and tricyclic $\mathrm{C}-3$ substituents of $\triangle 8$-THC that alter $\mathrm{CB}_{1}$ and $\mathrm{CB}_{2}$ binding affinity 


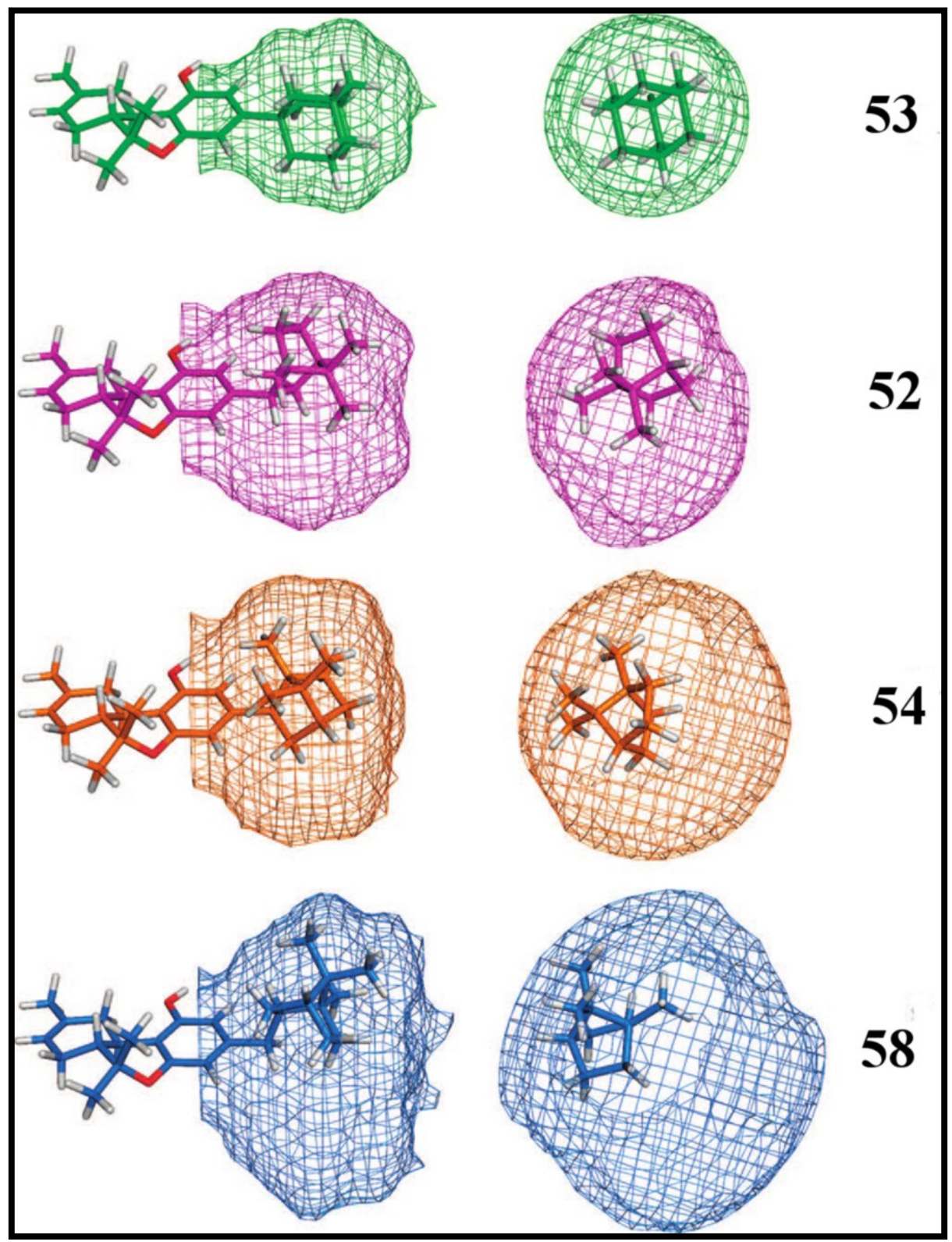

Figure 3.9. Comparison of the conformational space for adamantyl and bornyl side chains of various $\triangle 8$-THC analogues

Notes: Accessible conformers within $8 \mathrm{kcal}$ mol-1 of the global energy minimum for 3(adamant-1'-yl)- $\Delta 8$-THC (53, green), 3-bornyl- $\Delta 8$-THC (54, magenta), 3-isobornyl- $\Delta 8$ THC (52, orange), and 3-bornylmethyl- $\Delta 8$-THC (58, blue). The accessible conformational space for 3-isobornylmethyl- $\triangle 8$-THC (not shown) is similar to that of $\mathbf{5 8}$. The global minimum energy conformer for each ligand is shown in stick display. Adapted, with permission, from: Lu, D., Guo, J., Duclos, R. I., Bowman, A. L. \& Makriyannis, A. Bornyl- and Isobornyl- $\Delta 8$-tetrahydrocannabinols: A Novel Class of Cannabinergic Ligands. Journal of Medicinal Chemistry 51, 6393-6399, doi:10.1021/jm8005299 (2008). Copyright 2008 American Chemical Society. 


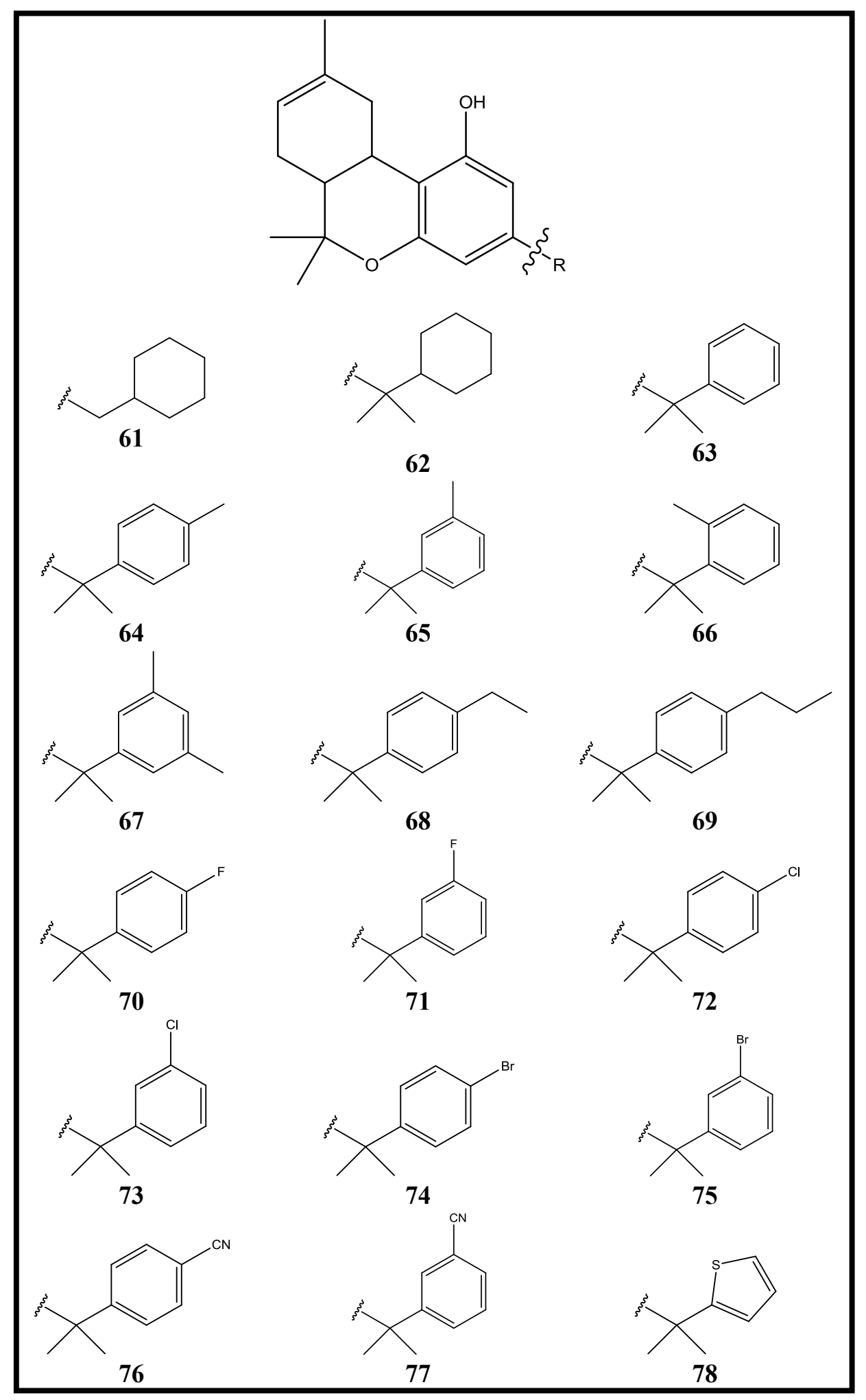

Figure 3.10. KM series analogues 
Table 3.1. Binding affinities for the KM series of compounds

\begin{tabular}{|c|c|c|}
\hline Compound & $\mathrm{CB}_{1} \mathrm{~K}_{\mathrm{i}}(\mathrm{nM})$ & $\mathrm{CB}_{2} \mathrm{~K}_{\mathrm{i}}(\mathrm{nM})$ \\
\hline 61 & - & - \\
\hline 62 & $0.57( \pm 0.05)$ & $0.65( \pm 0.04)$ \\
\hline 63 & $12.3( \pm 0.61)$ & $12.3( \pm 0.61)$ \\
\hline 64 & $3.13( \pm 0.37)$ & $0.88( \pm 0.05)$ \\
\hline 65 & $2.53( \pm 0.54)$ & $1.13( \pm 0.02)$ \\
\hline 66 & $34.4( \pm 2.84)$ & $10.65( \pm 1.27)$ \\
\hline 67 & $11.0( \pm 1.67)$ & $7.45( \pm 0.38)$ \\
\hline 68 & $1.85( \pm 0.16)$ & $0.67( \pm 0.05)$ \\
\hline 69 & $1.77( \pm 0.20)$ & $7.83( \pm 0.79)$ \\
\hline 70 & $76.1( \pm 1.55)$ & $12.4( \pm 0.24)$ \\
\hline 71 & $5.26( \pm 0.94)$ & $0.90( \pm 0.02)$ \\
\hline 72 & $18.8( \pm 1.39)$ & $1.68( \pm 0.20)$ \\
\hline 73 & $2.80( \pm 0.05)$ & $3.54( \pm 0.71)$ \\
\hline 74 & $5.03( \pm 0.39)$ & $1.54( \pm 0.16)$ \\
\hline 75 & $1.59( \pm 0.16)$ & $0.54( \pm 0.03)$ \\
\hline 76 & $9.25( \pm 0.23)$ & $2.53( \pm 0.23)$ \\
\hline 77 & $2.72( \pm 0.29)$ & $0.91( \pm 0.05)$ \\
\hline 78 & $1.08( \pm 0.04)$ & $0.27( \pm 0.01)$ \\
\hline
\end{tabular}


to binding and employ a $\mathrm{CB}_{1}$ homology model in an effort to provide some in silico hypothesis as to the potential residues involved in contributing to the functional activity.

\subsection{Functional Pharmacology}

\subsubsection{ACTOne assay}

Assay development and conductance details are found in Chapter 2. Briefly, stably transfected HEK-293 cells co-expressed with modified CNG channels along with $\mathrm{CB}_{1}$ or $\mathrm{CB}_{2}$ or their parental cells (without $\mathrm{CB}_{1}$ or $\mathrm{CB}_{2}$; HEK-CNG) were plated at an appropriate density in a clear, 96-well, Poly-D-Lysine plate and allowed to incubate at $37^{\circ} \mathrm{C}$ and $5 \%$ overnight. After the overnight incubation period, I added $100 \mu \mathrm{L}$ of ACTOne membrane potential dye to each well of the culture plate and allowed to sit in a dark area at room temperature for 60 additional minutes. After the 60 minute incubation period a baseline read in a BioTek Synergy 2 plate reader with an excitation of 540/25 $\mathrm{nm}$ and an emission of 590/20 nm. Following the baseline read, $50 \mu \mathrm{L}$ of drug stock solution containing (Figure 3.11): appropriate concentrations of ligand, Forskolin and Ro 20-1724 in DPBS with 2.5\% DMSO in the cell culture plate. The plate was then placed on the plate reader with 1 read per well per minute with an excitation of 540/25 nm and an emission of 590/20 nm for 60 minutes.

I analyzed primary data with customized VBA macros, developed internally, in Microsoft Excel (Redmond, WA). Column 11 (lowest [drug]) determined the experimental maximum response (100\% activity) at each individual time point, and the experimental basal response was determined by the average response given by the two "Blank" control wells. All data were normalized with these minimum and maximum values via Equation 2.1. The $\mathrm{EC}_{50}$ values were calculated from concentration-response curves by non-linear regression analysis utilizing GraphPad Prism Software (San Diego, CA).

\subsection{2. $\mathrm{CB}_{1}$ functional activity for the $K M$ series}

Plates were run as previously described (Section 3.4.1) in six replicates with CP-55,940 as a control. Compound 63 (KM-233), being the prototype, did not manifest as high of efficacy as several of the substituted aromatic ring analogs - especially those with $m e t a$-substitution(s). EC 50 values (Table 3.2) ranged from 34 to $516 \mathrm{nM}$ with the percent cAMP inhibition ranging from 10 to $70 \%, 50$ minutes after compound addition into the assay plate. Of particular note are the meta-substituted compounds $65,67,71,73$, 75 and 77 (Figure 3.12), which show an overall lowering of cAMP production near 70\%, which is equivalent to the standard of CP-55,940. EC 50 values were determined to be 41.8 $\mathrm{nM}, 41.2 \mathrm{nM}, 78.5 \mathrm{nM}, 99.5 \mathrm{nM}, 71.2 \mathrm{nM}$ and $127 \mathrm{nM}$ for compounds $\mathbf{6 5}, \mathbf{6 7}, \mathbf{7 1}, \mathbf{7 3}, \mathbf{7 5}$ and 77, respectively. From the para-substituted derivatives, only $68\left(\mathrm{EC}_{50}=34.0 \mathrm{nM}\right)$ was able to match CP-55,940 at $\sim 70 \%$ reduction of cAMP production, all others were less efficacious at $\sim 50 \%$ reduction (Figure 3.13) - highlighting the importance of $m e t a$-substitution in these derivatives. The remaining derivatives for this series 63,66 and 


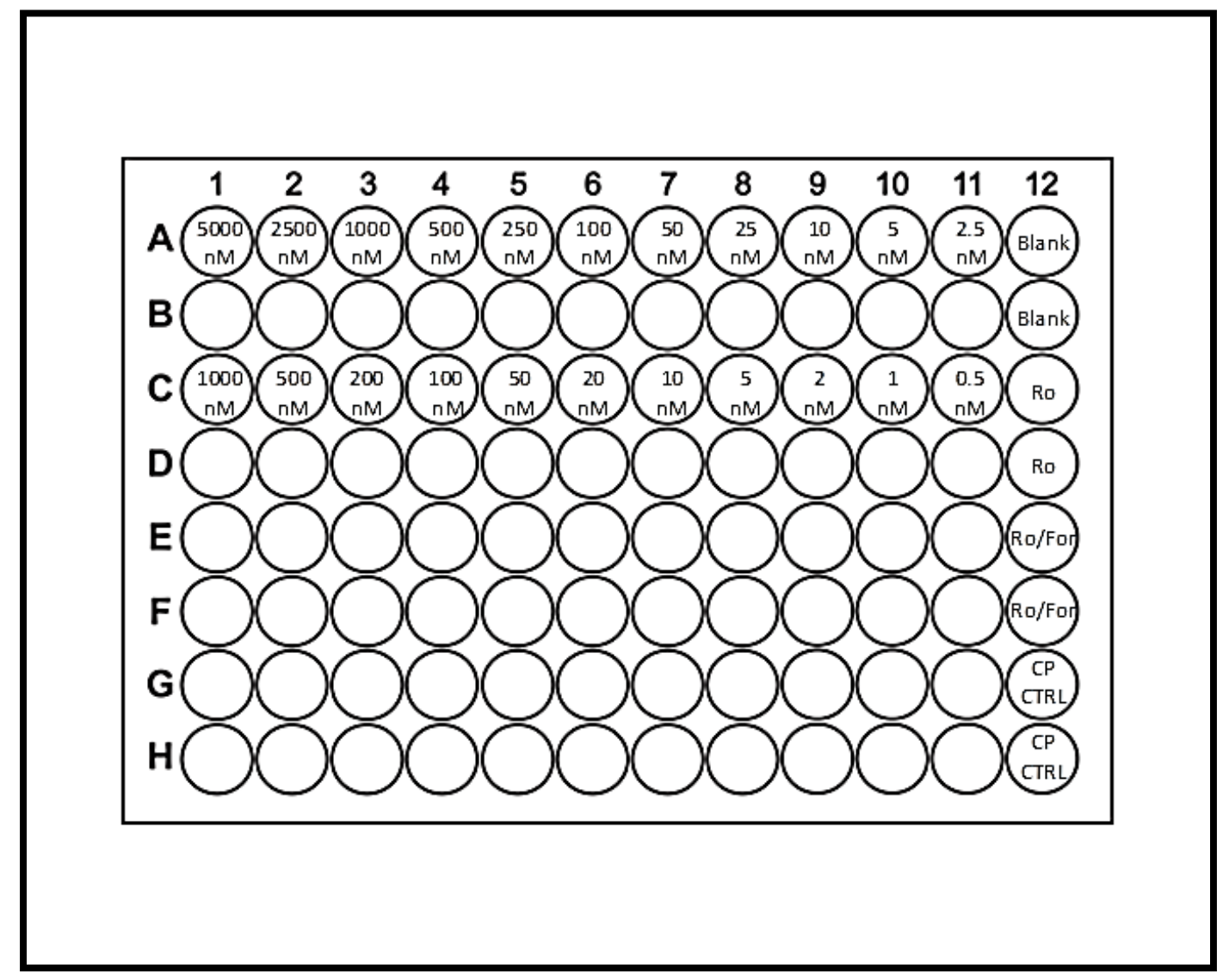

Figure 3.11. Drug plate layout and concentrations

Notes: Columns 1 through 11 contain test compound, and Column 12 contains control wells. All listed concentrations are the final concentrations once added to the testing plate. In the drug plate all compounds are at $5 \mathrm{X}$ that of the listed concentrations in Columns 1 through 11. Control wells (Column 12) are: "Blank" (cells, media and dye), "Ro" ("Blank" + $125 \mu \mathrm{M}$ Ro 20-1724 [25 $\mu \mathrm{M}$, Final]), "Ro/For" ("Ro" + $4 \mu \mathrm{M}$ Forskolin [800 nM, Final]) and "CP CTRL" ("Ro/For" + $25 \mu \mathrm{M}$ CP-55,940 [5 $\mu \mathrm{M}$, Final]). 
Table 3.2. $\quad \mathrm{EC}_{50}$ values for $\mathrm{KM}$ series functional assays

\begin{tabular}{|c|c|c|c|c|}
\hline Compound & $\begin{array}{c}\mathrm{CB}_{1} \\
\mathrm{EC}_{50}(\mathrm{nM})\end{array}$ & $\begin{array}{c}\mathrm{CB}_{2} \\
\mathrm{EC}_{50}(\mathrm{nM})\end{array}$ & $\begin{array}{c}\text { CNG } \\
\mathrm{EC}_{50}(\mathrm{nM})\end{array}$ & $\begin{array}{c}\mathrm{CB}_{1} \text { PTx } \\
\mathrm{EC}_{50}(\mathrm{nM})\end{array}$ \\
\hline 61 & $\mathrm{n} / \mathrm{a}$ & - & - & - \\
\hline 62 & $14.2^{\mathrm{a}}$ & - & - & - \\
\hline 63 & $204^{a}$ & $\mathrm{n} / \mathrm{a}$ & $\mathrm{n} / \mathrm{a}$ & $\mathrm{n} / \mathrm{a}$ \\
\hline 64 & $350^{\mathrm{a}}$ & $\mathrm{n} / \mathrm{a}$ & $\mathrm{n} / \mathrm{a}$ & $\mathrm{n} / \mathrm{a}$ \\
\hline 65 & $41.7^{\mathrm{a}}$ & $\mathrm{n} / \mathrm{a}$ & $\mathrm{n} / \mathrm{a}$ & $\mathrm{n} / \mathrm{a}$ \\
\hline 66 & $516^{\mathrm{a}}$ & $\mathrm{n} / \mathrm{a}$ & $\mathrm{n} / \mathrm{a}$ & $\mathrm{n} / \mathrm{a}$ \\
\hline 67 & $41.2^{\mathrm{a}}$ & $\mathrm{n} / \mathrm{a}$ & $\mathrm{n} / \mathrm{a}$ & $\mathrm{n} / \mathrm{a}$ \\
\hline 68 & $34.0^{\mathrm{a}}$ & $\mathrm{n} / \mathrm{a}$ & $\mathrm{n} / \mathrm{a}$ & $\mathrm{n} / \mathrm{a}$ \\
\hline 69 & $72.3^{\mathrm{a}}$ & $\mathrm{n} / \mathrm{a}$ & $\mathrm{n} / \mathrm{a}$ & $\mathrm{n} / \mathrm{a}$ \\
\hline 70 & $356^{\mathrm{a}}$ & $\mathrm{n} / \mathrm{a}$ & $\mathrm{n} / \mathrm{a}$ & $\mathrm{n} / \mathrm{a}$ \\
\hline 71 & $78.5^{\mathrm{a}}$ & $\mathrm{n} / \mathrm{a}$ & $\mathrm{n} / \mathrm{a}$ & $\mathrm{n} / \mathrm{a}$ \\
\hline 72 & $290^{\mathrm{a}}$ & $\mathrm{n} / \mathrm{a}$ & $\mathrm{n} / \mathrm{a}$ & $\mathrm{n} / \mathrm{a}$ \\
\hline 73 & $99.5^{\mathrm{a}}$ & $\mathrm{n} / \mathrm{a}$ & $\mathrm{n} / \mathrm{a}$ & $\mathrm{n} / \mathrm{a}$ \\
\hline 74 & $237^{\mathrm{a}}$ & $\mathrm{n} / \mathrm{a}$ & $\mathrm{n} / \mathrm{a}$ & $\mathrm{n} / \mathrm{a}$ \\
\hline 75 & $71.2^{\mathrm{a}}$ & $\mathrm{n} / \mathrm{a}$ & $\mathrm{n} / \mathrm{a}$ & $\mathrm{n} / \mathrm{a}$ \\
\hline 76 & $414^{\mathrm{a}}$ & $\mathrm{n} / \mathrm{a}$ & $\mathrm{n} / \mathrm{a}$ & $\mathrm{n} / \mathrm{a}$ \\
\hline 77 & $127^{\mathrm{a}}$ & $\mathrm{n} / \mathrm{a}$ & $\mathrm{n} / \mathrm{a}$ & $\mathrm{n} / \mathrm{a}$ \\
\hline 78 & $249^{a}$ & $\mathrm{n} / \mathrm{a}$ & $\mathrm{n} / \mathrm{a}$ & $\mathrm{n} / \mathrm{a}$ \\
\hline
\end{tabular}

Notes: $\mathrm{n} / \mathrm{a}$ : Not active, based on no statistically significant change in cAMP from baseline. "-" Indicates test not performed. a Indicates a decrease in cAMP compared to baseline. 


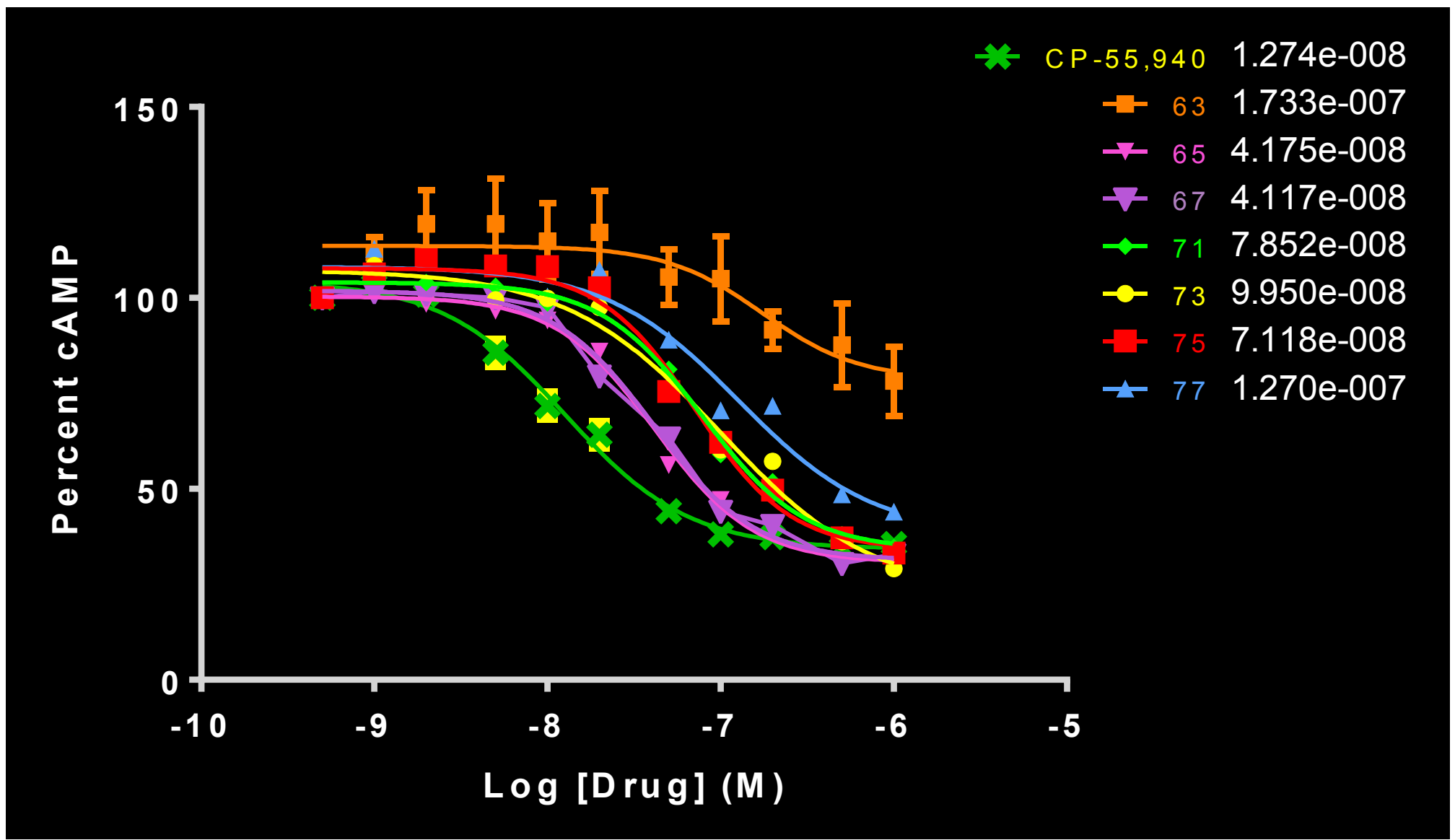

Figure 3.12. $\quad \mathrm{CB}_{1}$ functional activity for meta-substituted $\mathrm{KM}$ series compounds 


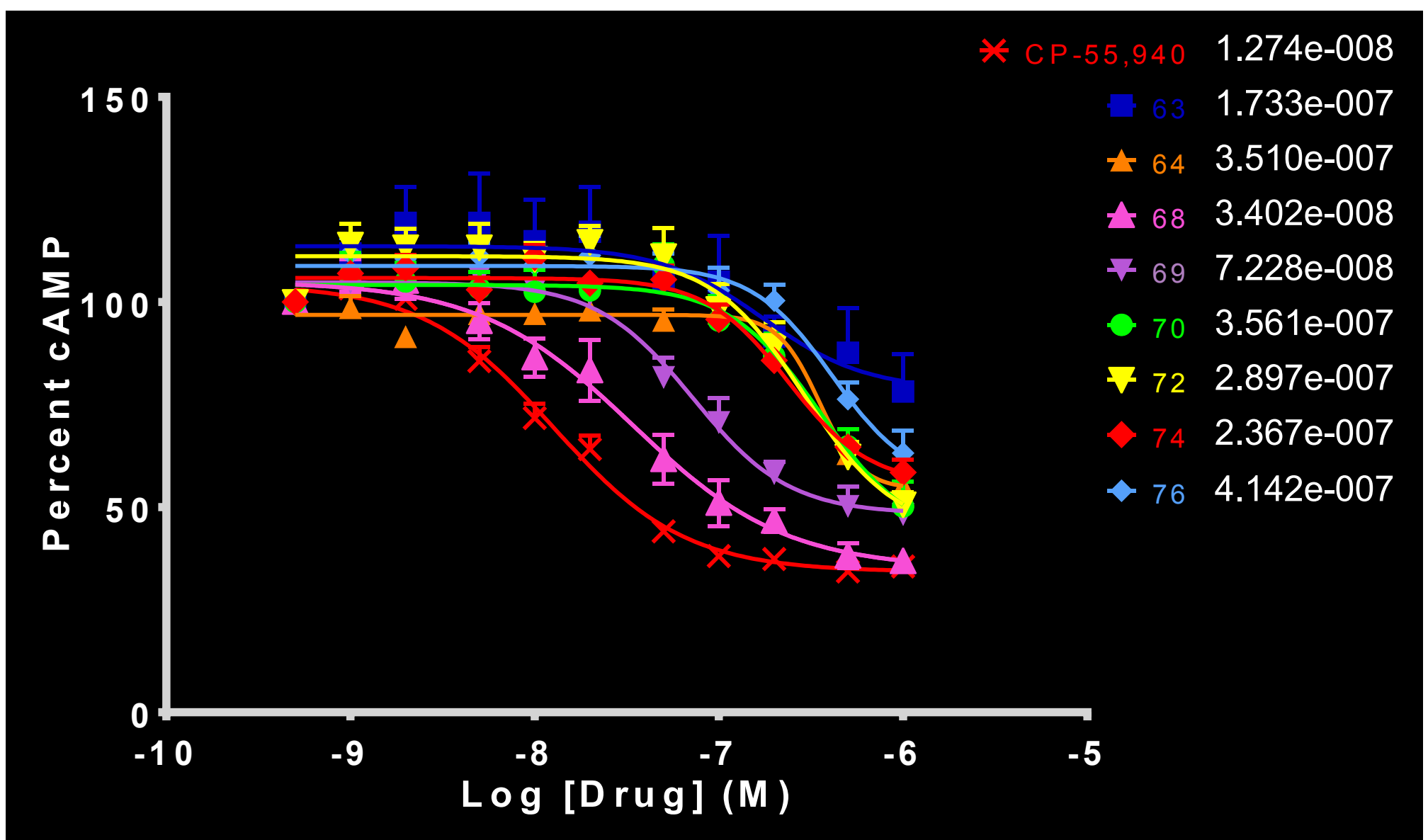

Figure 3.13. $\quad \mathrm{CB}_{1}$ functional activity of para-substituted derivatives of $\mathrm{KM}$ series 
78 only produced an approximately 30\% reduction in cAMP production (Figure 3.14), and while 63 is our prototype for this class, it is evident that substitution greatly improves its functional activity.

\subsection{3. $\mathrm{CB}_{2}$ functional activity for the $\mathrm{KM}$ series}

Plates were run as previously described (Section 3.4.1) in 6 replicates with CP-55,940 as a control for all 6 runs (Figure 3.15; Table 3.2). All compounds in the series demonstrated an inability to decrease cAMP production via $\mathrm{CB}_{2}$ activation.

\subsubsection{CNG functional activity for the KM series}

Plates were run as previously described in 3 replicates with CP-55,940 as a control for all 3 runs (Figure 3.16; Table 3.2). As fully expected, all of the compounds failed to elucidate any change in cAMP production, including our standard of CP-55,940. There is a small variance among the drugs, but this is not of true consequence as one is able to gather from the graph - especially relative to the full agonist graphs noted with these compounds in $\mathrm{CB}_{1}$ cells. This lack of activity with the parental HEK-293-CNG cells confirms that the $\mathrm{KM}$ series acts via $\mathrm{CB}_{1}$, and not via any other constitutively expressed receptors present in the HEK cells.

\subsection{5. $C_{1} P T x$-sensitive $G_{i / 0}$-inactivation for the $K M$ series}

Plates were run as previously described (Section 3.4.1) in 3 replicates with CP-55,940 as a control for all 3 runs (Figure 3.17; Table 3.2). All of the compounds in the series failed to change cAMP production, including our standard of CP-55,940, confirming a PTx-sensitive- $\mathrm{G}_{\mathrm{i} / \mathrm{o}}$-mediated $\mathrm{CB}_{1}$ effect. $\mathrm{CB}_{2} \mathrm{PTx}$-sensitive $\mathrm{G}_{\mathrm{i} / \mathrm{o}}$-inactivation assays were not completed due to a lack of agonist response in the previous assay.

\subsection{Discussion}

\subsubsection{Interactions with the $\mathrm{CB}_{1} \mathrm{LBP}$}

As previously discussed (Section 1.2.2), $\mathrm{CB}_{1}$ receptors are seven trans-membrane G-protein coupled receptors (GPCRs). ${ }^{14}$ There exist several homology models which have sought to elucidate the residues important for binding in domains 3, 6 and 7 as well as functional activity.

To generate hypotheses, gain some insight into the possible explanations and interplay between ligand and receptor, I performed docking of the compounds. Dr. Chang, University of California at Riverdale, provided 4 models: "ACEA" endocannabinoid model, "HU-210" - classical core model, "SR-141716A" - inverse agonist model and "WIN-55,212-2" - non-classical agonist model. ${ }^{249}$ The KM series of compounds, I believe, adopt a similar binding mode within the LBP to that of HU-210, thus this was the model used for docking studies. 


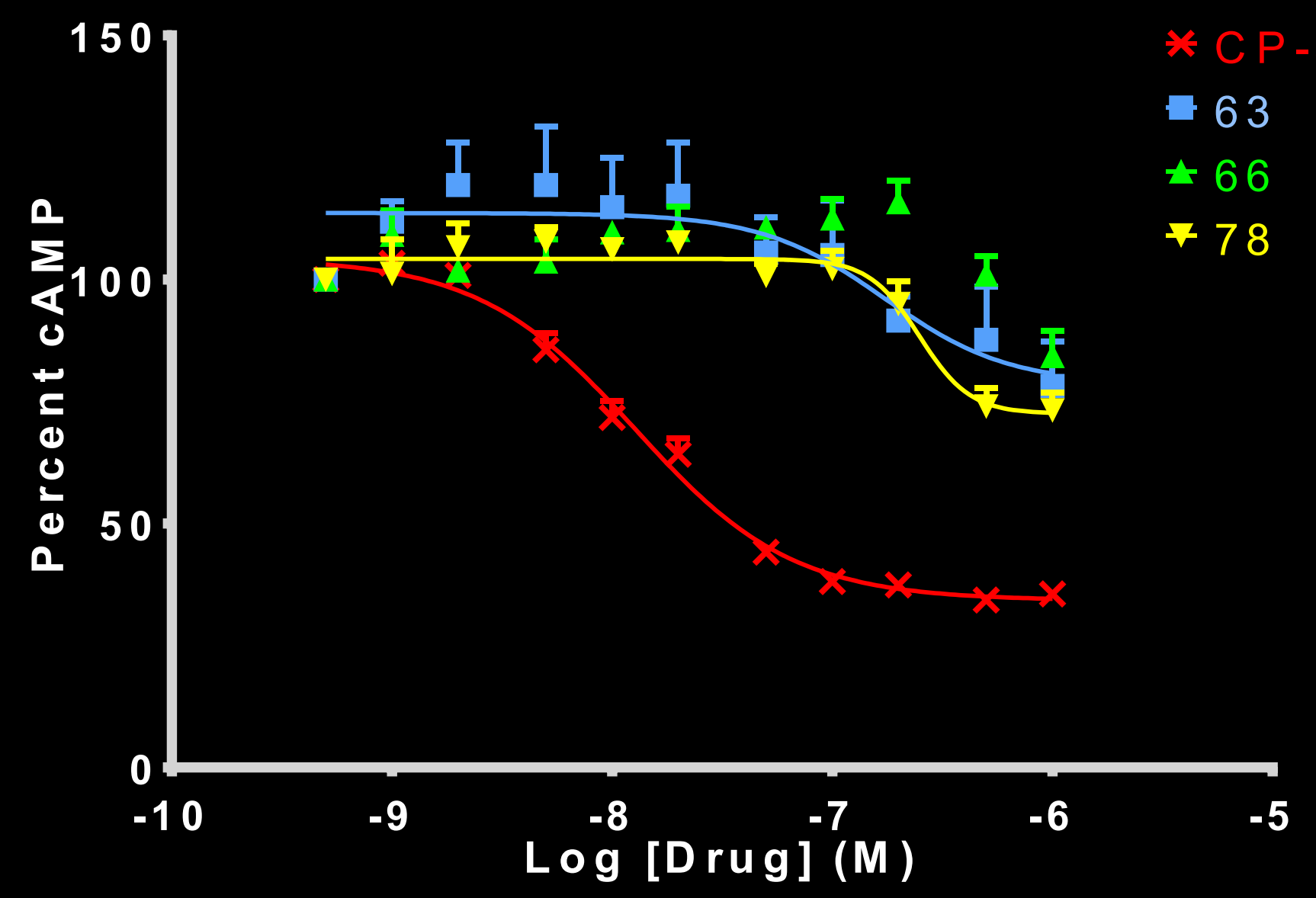

Figure 3.14. $\quad \mathrm{CB}_{1}$ functional activity of misc. KM series derivatives 


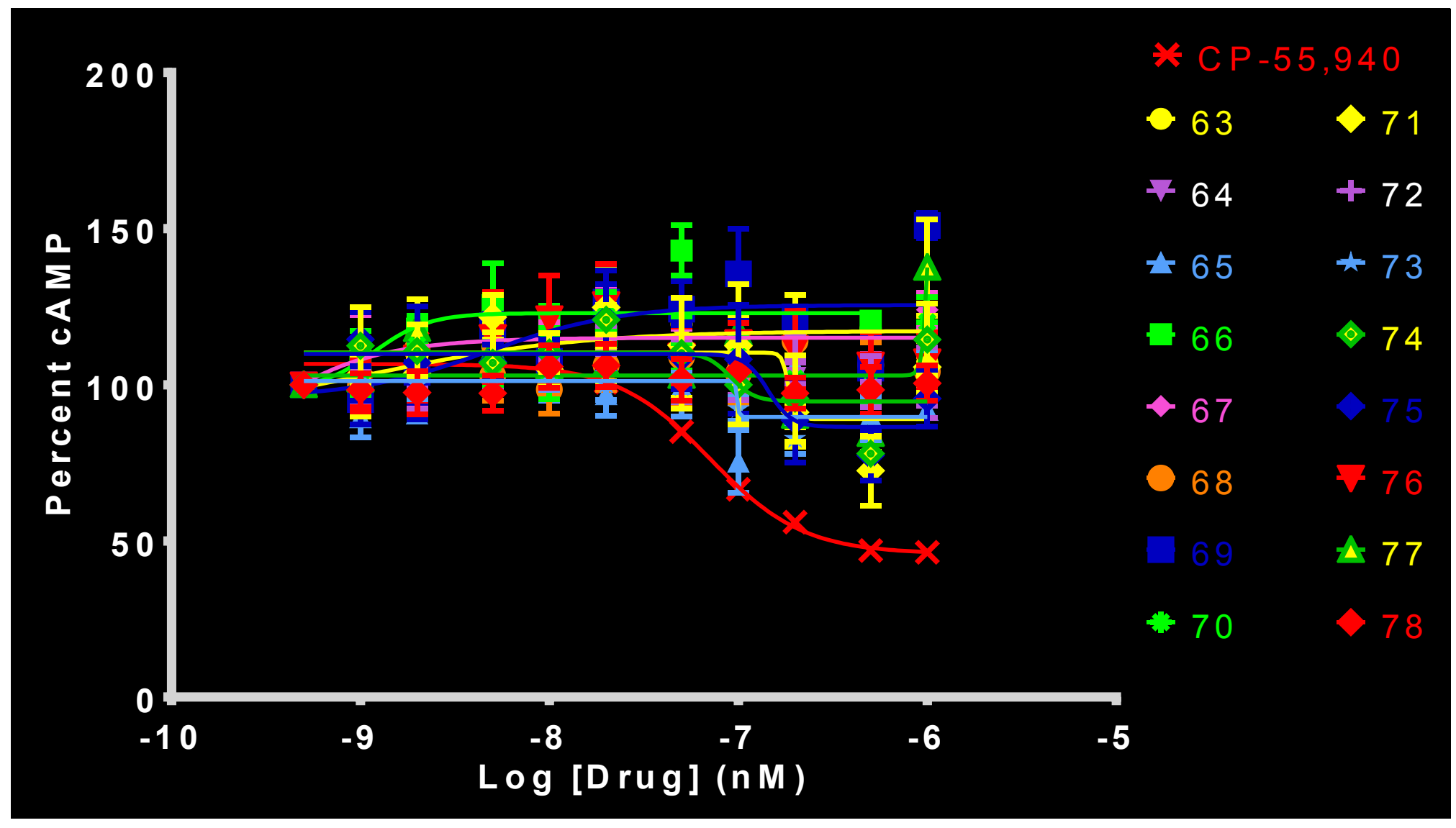

Figure 3.15. $\mathrm{CB}_{2}$ functional activity of $\mathrm{KM}$ series compounds, 50 minutes after drug addition 


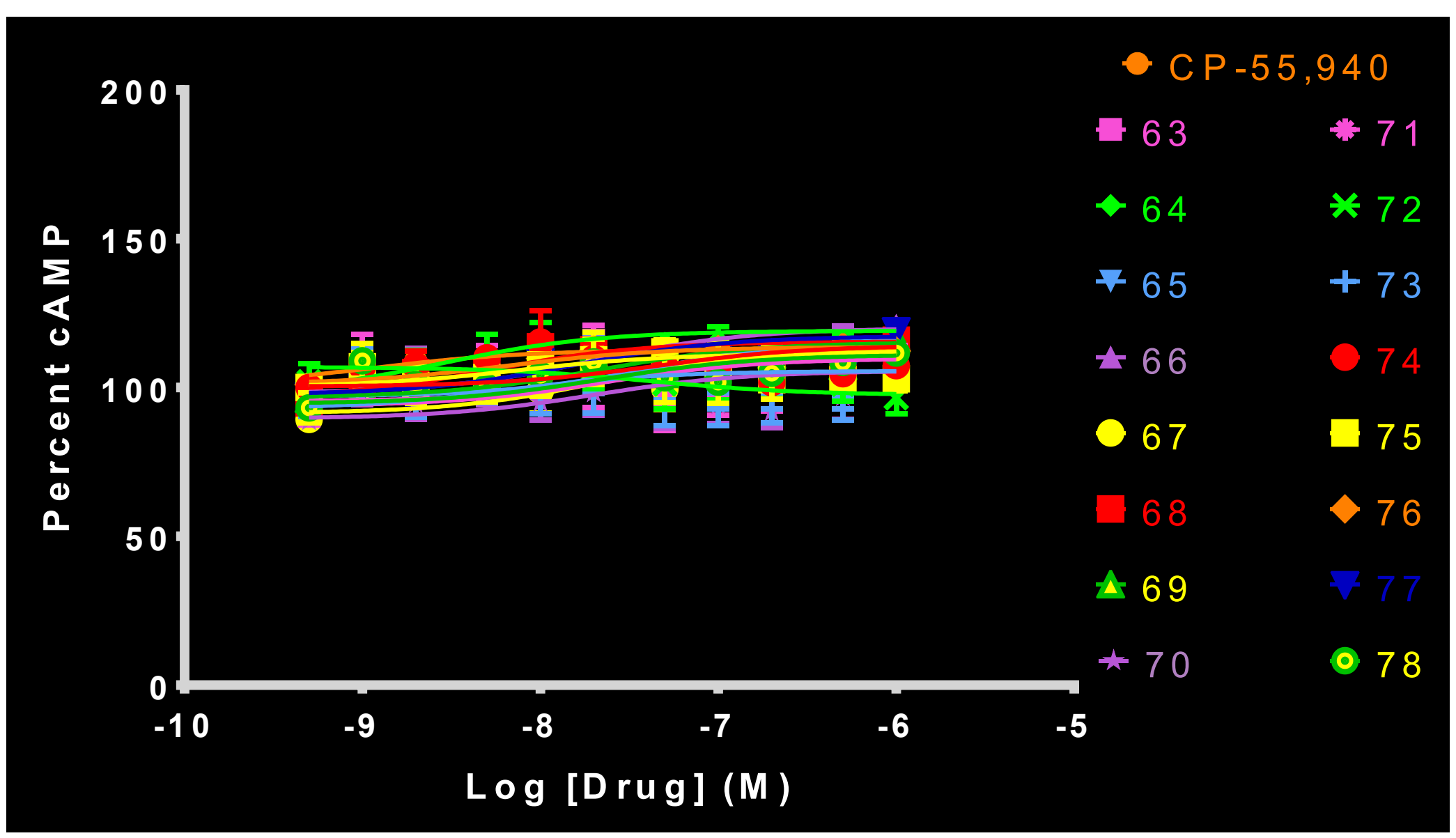

Figure 3.16. CNG functional activity of KM series compounds, 50 minutes after compound addition 


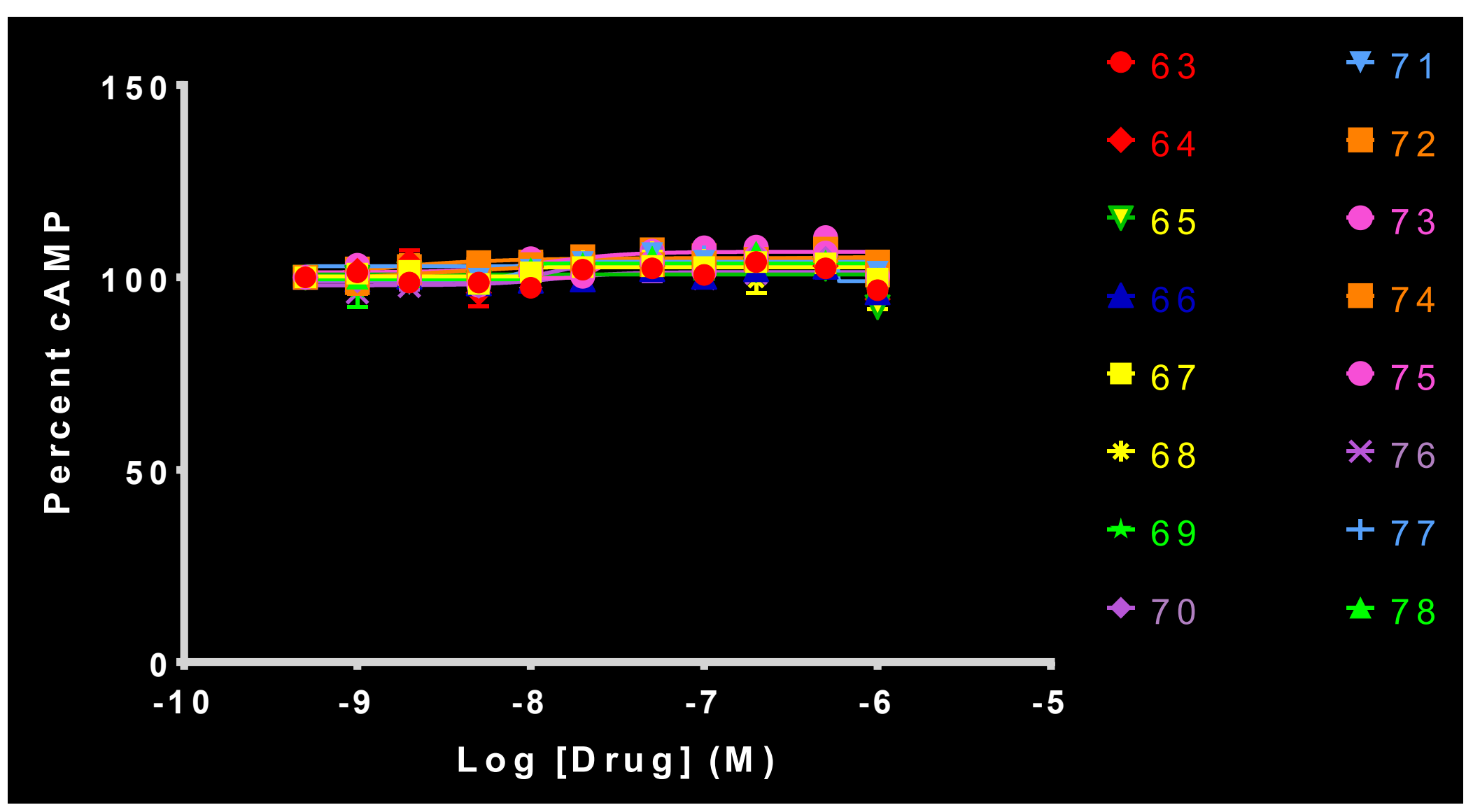

Figure 3.17. $\quad \mathrm{CB}_{1}$ PTx-sensitive $\mathrm{G}_{\mathrm{i} / \mathrm{o}}$-inactivation functional activity, 50 minutes after compound addition 
3.5.1.1. C-1 Hydroxyl. K192(3.28) plays a very important role in both receptor binding and function. Since it is a very flexible residue in the LBP, it can manifest conformations that allow it to hydrogen bond with either the C-1 hydroxyl, C-11 ("Northern") hydryoxyl, C-6 ("Southern") hydroxyl or even the benzochromene oxygen. ${ }^{250}$ However, newer data suggest that K192(3.28) plays a primary role as H-bond acceptor of the C-1 hydroxyl, ${ }^{251}$ presumably this occurs after being activated by donating a H-bond to F189(3.25) (Figure 3.18). ${ }^{249} \mathrm{~S} 383(7.35)$ interacts by donating a H-bond to either the benzchromene oxygen or the C-6 hydroxyl, if present (Figure 3.18A). ${ }^{251}$ Additionally, literature data suggest that the resting state of the $\mathrm{CB}_{1}$ receptor exists when the salt bridge between K192(3.28) and D366(6.58) is intact. ${ }^{252}$

Much the same as the established $\mathrm{CB}_{1}$ interactions of HU-210, I hypothesize that the KM series of compounds take advantage of the H-bonding with K192(3.28) with the C-1 hydroxyl and S383(7.35) with the benzchromene oxygen (Figure 3.18B) - as most classical cannabinoids do. Moreover than orienting the drug in the pocket, ligand interaction with K192(3.28) breaks the salt bridge between K192(3.38) and D366(6.58) a reported key stabilizing factor for the inactive state of the $\mathrm{CB}_{1}$ receptor. ${ }^{252}$

3.5.1.2. C-3 lipophilic side chain. A predominantly lipophilic pocket exists, seemingly as two lobes - as evidenced in the fact that $3-\left(1^{\prime}, 1^{\prime}\right.$-dimethylheptyl)- $\Delta^{8}$-THC binds with higher affinity than does 3-heptyl- $\Delta^{8}$-THC along with 3D-QSAR models which demonstrate this trait. ${ }^{253}$ In one homology model, residues V196(3.32), T197(3.33), F200(3.36), Y275(5.39), W279(5.43), L359(6.51) and M363(6.55) form a hydrophobic pocket which encompasses the A-ring and C-3 side chain of HU-210. ${ }^{254}$ More recent homology models form this pocket from I354(6.46), C355(6.47), W356(6.48), L359(6.51), L360(3.52) and M363(6.55) (Figure 3.19A). ${ }^{249}$ Interestingly, mutations studies conducted on this hydrophobic pocket show that M363A(6.55) mutation greatly impacts binding affinity for HU-210 but has virtually no impact on THC which would suggest that this is an important residue for $\mathrm{C}-1$ ' substituted derivatives. ${ }^{255}$

Due to the similar $\mathrm{CB}_{1}$ functional activity betwixt $\mathrm{HU}-210$ and the $\mathrm{KM}$ series, it is hypothesized that the interactions of the lipophilic $\mathrm{C}-3$ substituents are analogous (Figure 3.19B). The necessity of the M363(6.55) residue as demonstrated for HU-210 in the mutation binding studies bore itself out in the functional arena, as well. This further highlights the importance of the C-3 lipophilic side chain to both the binding affinity and the functional activity of these compounds. Residue W356(6.48) plays a key role in two ways. First, in the inactive state of the $\mathrm{CB}_{1}$ receptor, there is a proposed interaction of W356(6.48) and F200(3.36) - a "toggle switch." 256 Stabilization of this interaction may lead to the specialized antagonists known as inverse agonists, and this is precisely how SR-141716A binds in some homology models - a parallel stacking between these two residues creates an aromatic micro domain for SR-141716A. ${ }^{249}$ However, more recent homology models do not suggest this same binding mode for SR-141716A, and thus may indicate this is not as necessary an interaction. Second, interaction of W356(6.48) with the aryl ring of the KM Series draws the aryl ring more in contact with residues flanking W356(6.48), namely I354(6.46) and L359(6.51). It is this additional hydrophobic interaction (Figure 3.20) that I hypothesize gives compound 67 its superior functional effect, though its binding affinity is lower than related compounds. 


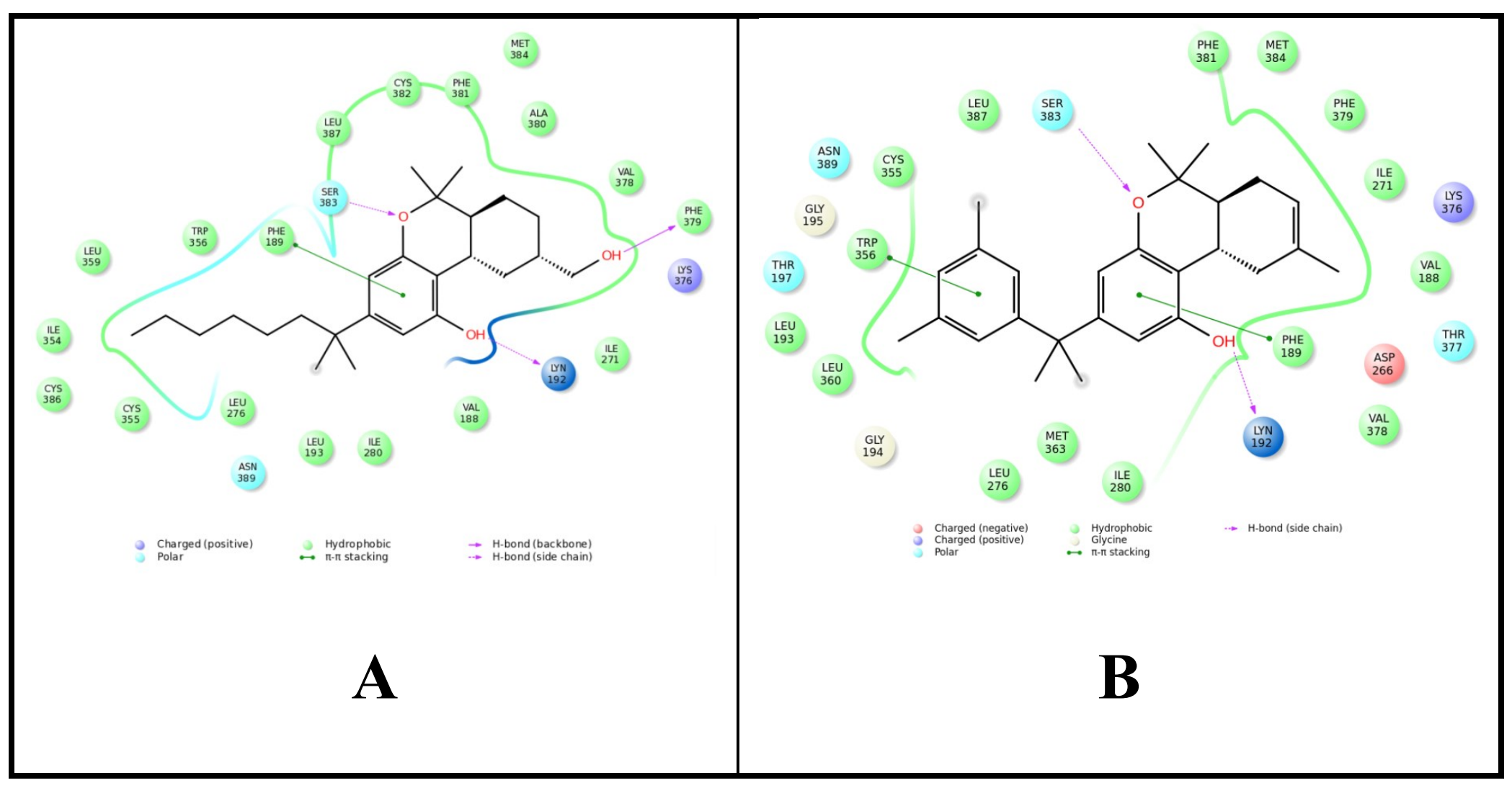

Figure 3.18. $\quad$ CB $_{1}$ LBP interactions with C-1 hydroxyl and benzchromene oxygen

Notes: Panel A depicts HU-210 H-bonding interactions as set forth in homology models. Panel B depicts the proposed interaction of Compound 67 with the LBP H-bonding groups. Homology models contributed by Dr. Chang, University of California, Riverdale. ${ }^{249}$ 


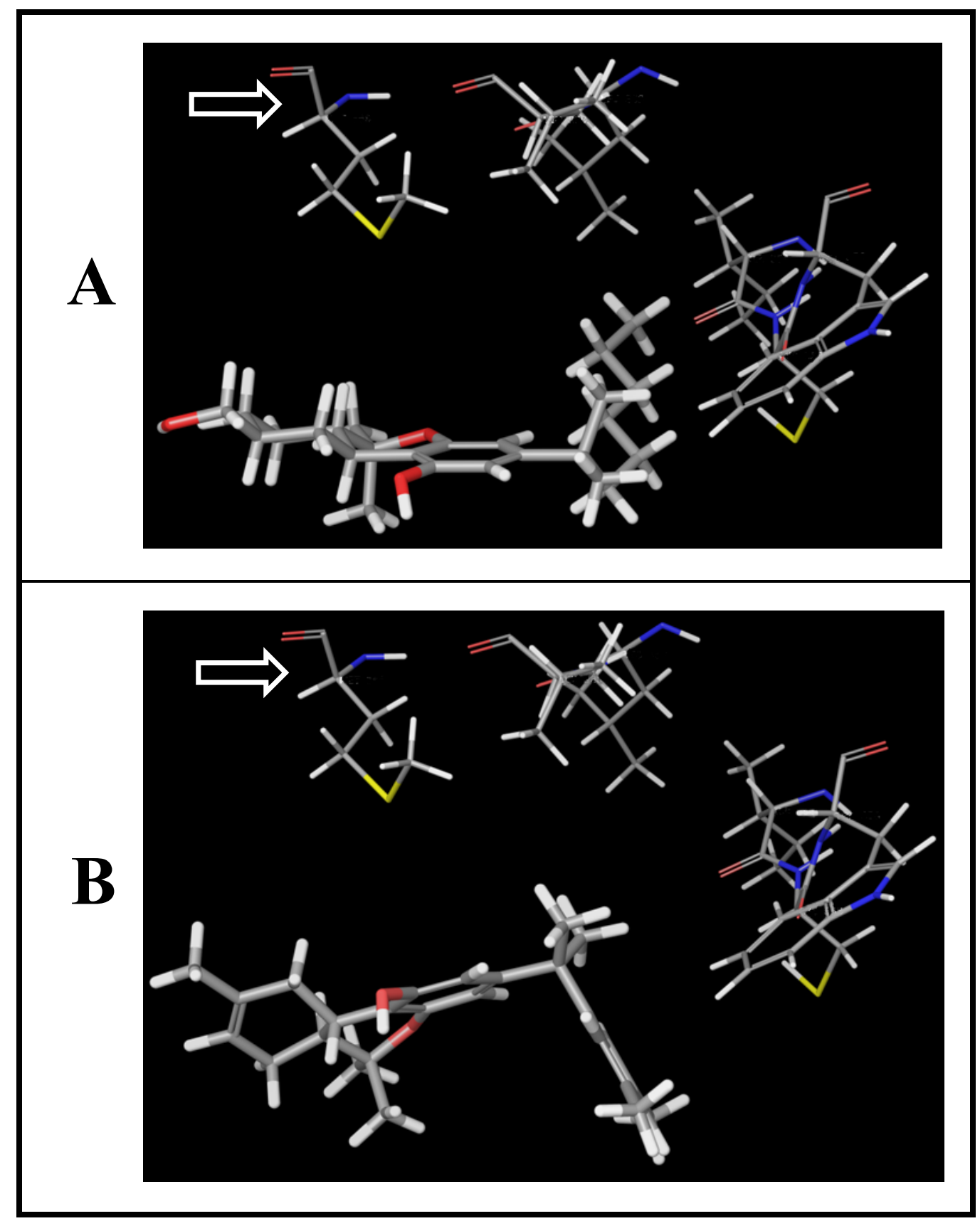

Figure 3.19. CB1 LBP proposed hydrophobic C-3 pocket

Notes: Panel A depicts the predicted HU-210 C-3 binding pocket of $\mathrm{CB}_{1}$. Panel $\mathrm{B}$ is the proposed Compound $67 \mathrm{C}-3$ binding pocket of $\mathrm{CB}_{1}$. The arrow highlights M363(6.55), demonstrated in mutation studies to interact with the C-1'-gem-dimethyls. Homology models were generously supplied by Dr. Chang, University of California, Riverdale. ${ }^{249}$ 


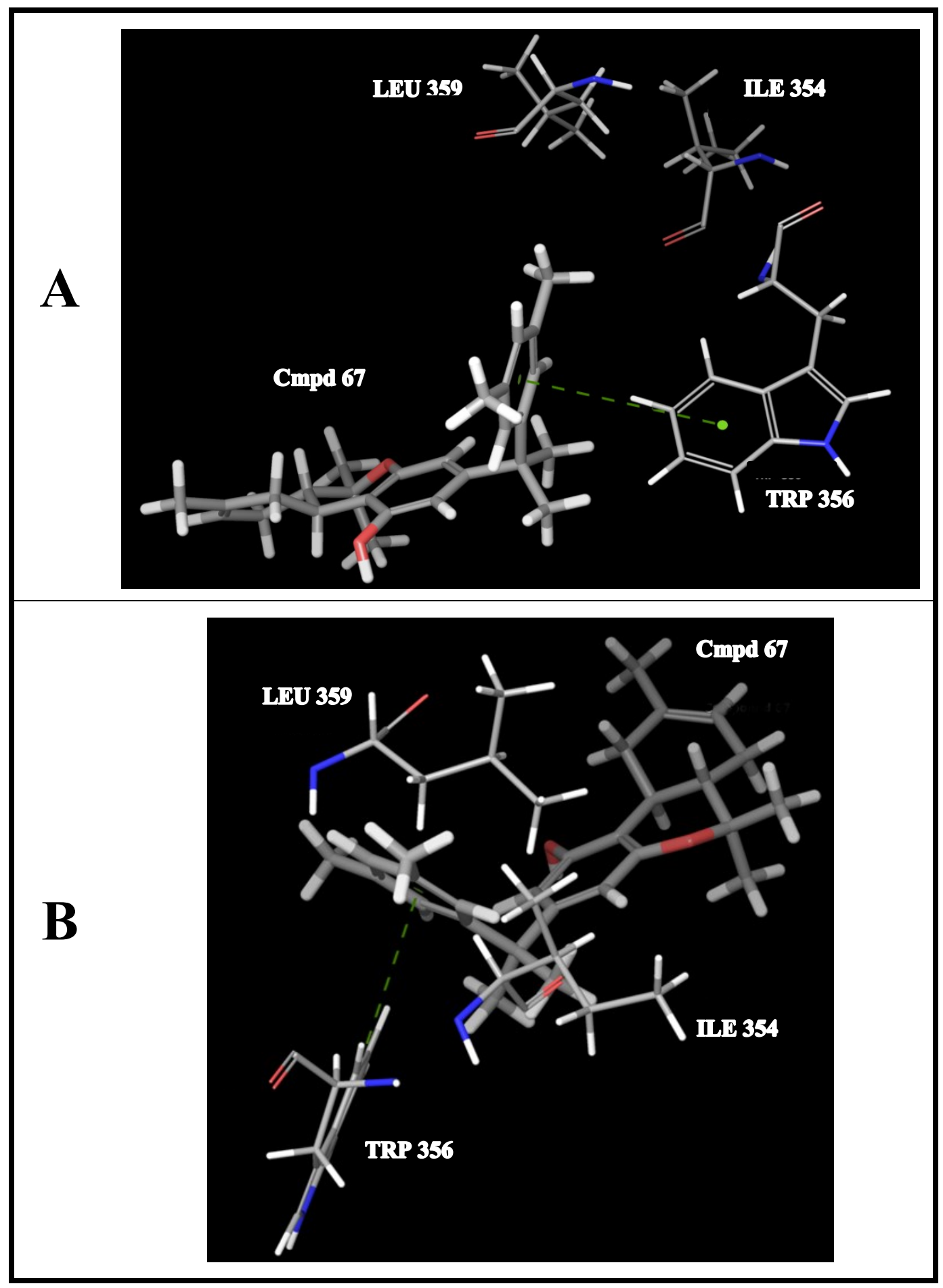

Figure 3.20. CB1 LBP proposed additional hydrophobic interactions of 67 with the C-3 pocket

Note: Panel A depicts the side view of $\mathbf{6 7}$ in the LBP. Panel B depicts the top view of $\mathbf{6 7}$ in the LPB. Dashed green lines indicate $\pi-\pi$ stacking interactions Homology models were supplied by Dr. Chang, University of California, Riverdale. ${ }^{249}$ 
3.5.1.3. ABC ring system. Residues $F 174(2.61)$ and $F 177(2.64)$ are suggested to not provide direct $\pi-\pi$ interactions with HU-210, but rather they help to stabilize the tricyclic (ABC ring) binding pocket domain of the LBP (Figure 3.21). ${ }^{255}$

Residues F200(3.36) and W279(5.43) appear to not play as important a role in terms of binding (in the case of AEA and CP-55,940) as they do in terms of receptor activation (Figure 3.21). ${ }^{257}$ That is to say, a ligand's hydrophobic substituents at the C3 position may bind well without interacting with these two residues, but agonists MUST have interaction with these residues to exert their effect. ${ }^{254} \mathrm{~F} 189(3.25)$ is key at providing a $\pi-\pi$ stacking (Figure 3.21) interaction with AEA, and is instrumental in binding of this to the pocket as its mutation to alanine causes a 7-fold drop in affinity for the receptor. ${ }^{258}$ F189(3.25) also seems to form the "ceiling of the anandamide binding pocket in $\mathrm{CB}_{1} . " 257$

As has been the case with many of the other interactions within the CB1 LBP, compound 67 shares many of the same interactions with HU-210; however, there is one key difference, which I believe helps compound 67 gain an edge. One of the key interactions within the CB1 LBP is the simultaneous interaction of the ligand with both F200(3.36) and W279(5.43). While HU-210 is obviously able to accomplish this by being an agonist at $\mathrm{CB} 1$, I believe that compound 67 is able to demonstrate a more favorable interaction with these residues via $\pi-\pi$ stacking interactions rather than strictly hydrophobic interactions present in HU-210.

3.5.1.4. (Optional) C-11 ("Northern") hydroxyl. With none of the compounds in the KM-series having this moiety present in the parent drug, I have not included it as part of this current study; however, it is included as part of this discussion, due to the fact that the primary metabolite of the KM series is a C-11 hydroxyl. ${ }^{259} \mathrm{~S} 383$ (7.35) shows itself to be equally important in binding of molecules along with stabilizing the LBP. S383(7.35) can hydrogen bond with the C-11 ("Northern") hydroxyl ${ }^{251}$ (if present) or the benzochromene oxygen ${ }^{249,254}$, but more importantly it helps to stabilize the LBP by maintaining the shape of the Trans-Membrane Helix 1-2-7 binding pocket. ${ }^{251}$ The "Northern" hydroxyl group may, theoretically, H-bond with K192(3.28).

\subsubsection{Interactions with the $\mathrm{CB}_{2} \mathrm{LBP}$}

As previously discussed (Section 1.2.3), $\mathrm{CB}_{2}$ receptors are also seven trans-membrane GPCRs; however, $\mathrm{CB}_{2}$ only shares $48 \%$ homology with $\mathrm{CB}_{1}$ - a trait unique to the $\mathrm{CB}_{1}$ and $\mathrm{CB}_{2}$ receptors among GPCRs, which generally share a much higher level of homology among receptors of the same family. ${ }^{14}$ The SAR of the $\mathrm{CB}_{2}$ LBP has yet to be fully developed, especially in terms of a reliable homology model or much less as a crystal structure. In the absence of a refined homology model, it is not possible to predict specific ligand amino acid interactions. However, very basic interpretations of the results are possible by comparing and contrasting to current literature standards. Specifically, the KM series possess high receptor affinity, and no functional activity, indicative of a neutral antagonist. Based on this, a plausible comparison between S-(-)-WIN-55,212-3 (a neutral antagonist) and R-(+)-WIN-55,212-2 (a weak agonist) and the activity of the KM series at $\mathrm{CB}_{2}$ may be presented. 


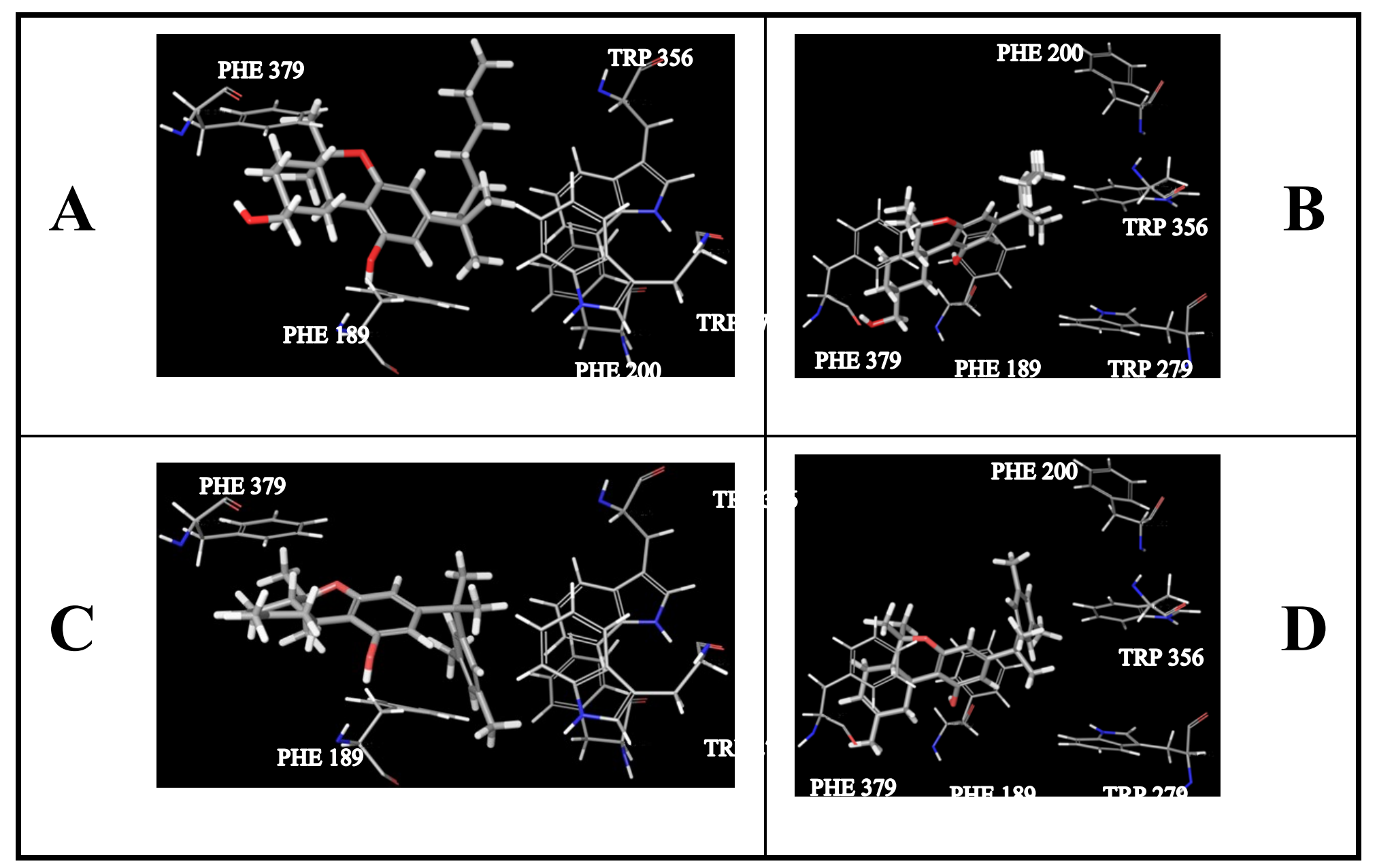

Figure 3.21. $\quad C_{1} \operatorname{LBP} \pi-\pi$ stacking interactions and key residue interactions

Notes: Panel A (top view) and Panel B (side view) depict the interactions of HU-210 within the CB 1 LBP. Panel C (top view) and Panel D (side view) depicts the proposed interactions of $\mathbf{6 7}$ in the $\mathrm{CB}_{1} \mathrm{LBP}$. Demonstrating the proximity of key receptor activators F200 and W279 as well as the $\pi-\pi$ stacking residue F189. Homology supplied by Dr. Chang. ${ }^{249}$ 
Attention now turns to the trans-B/C ring-junction. With data relatively limited for $\mathrm{CB}_{2}$ 's LBP, especially in the arena of classical core compounds, one must make some inferences based on available aminoalkylindole derivative homology model data. One such extrapolation lies in WIN-55,212-2's biologically assumed conformation to be s-trans-conformer. ${ }^{260}$ With the s-cis-conformer, there are suggested steric interactions with V113(3.32) (Figure 3.22). ${ }^{261}$ By extension, WIN-55,212-3, the S-(-) isomer of WIN-55,212-2 and antagonist, may also orient the morpholino side chain such to prevent critical amino acids interactions in the $\mathrm{CB}_{2} \mathrm{LBP}$ as discussed below.

Taking WIN-55,212-2 and ascribing its moieties to those of the representative example of KM-233 (63) (Figure 3.23), one can see that there are many similarities. While the similarities are numerous, the differences are profound as well, because the steric interactions of WIN-55,212-2 with V113(3.32) are non-existent with the KMseries. The steric issue comes in with what I hypothesize to be the "northern" aliphatic hydroxyl region, or C-11 substituted derivatives of $\Delta^{8}$-THC, thus the KM-series has little to contend with in this arena. It does raise an important question, though, for future design, synthesis and study of the classical core ligands - would incorporation of bulky C-11 substituents, especially conformationally (cis or trans) restricted derivatives, impact the $\mathrm{CB}_{2}$ selectivity and functional ability of compounds?

\subsection{Conclusions}

The KM Series of compounds represent a unique group of ligands for modulating the $\mathrm{CB}_{1}$ and $\mathrm{CB}_{2}$ receptor system. Increases in binding affinity come about from taking advantage of residues present in the binding pocket otherwise not adequately utilized, such as L359(6.51) and I354(6.46). Key interactions with the "toggle" switch at CB 1 (W356(6.48)) to hold it in the active state. Future study in this would test the hypothesis of the residue-ligand interactions via homology modeling studies. Additionally, testing the series as antagonists of $\mathrm{CB}_{2}$ against known agonists would functionally confirm this as a mechanism. 


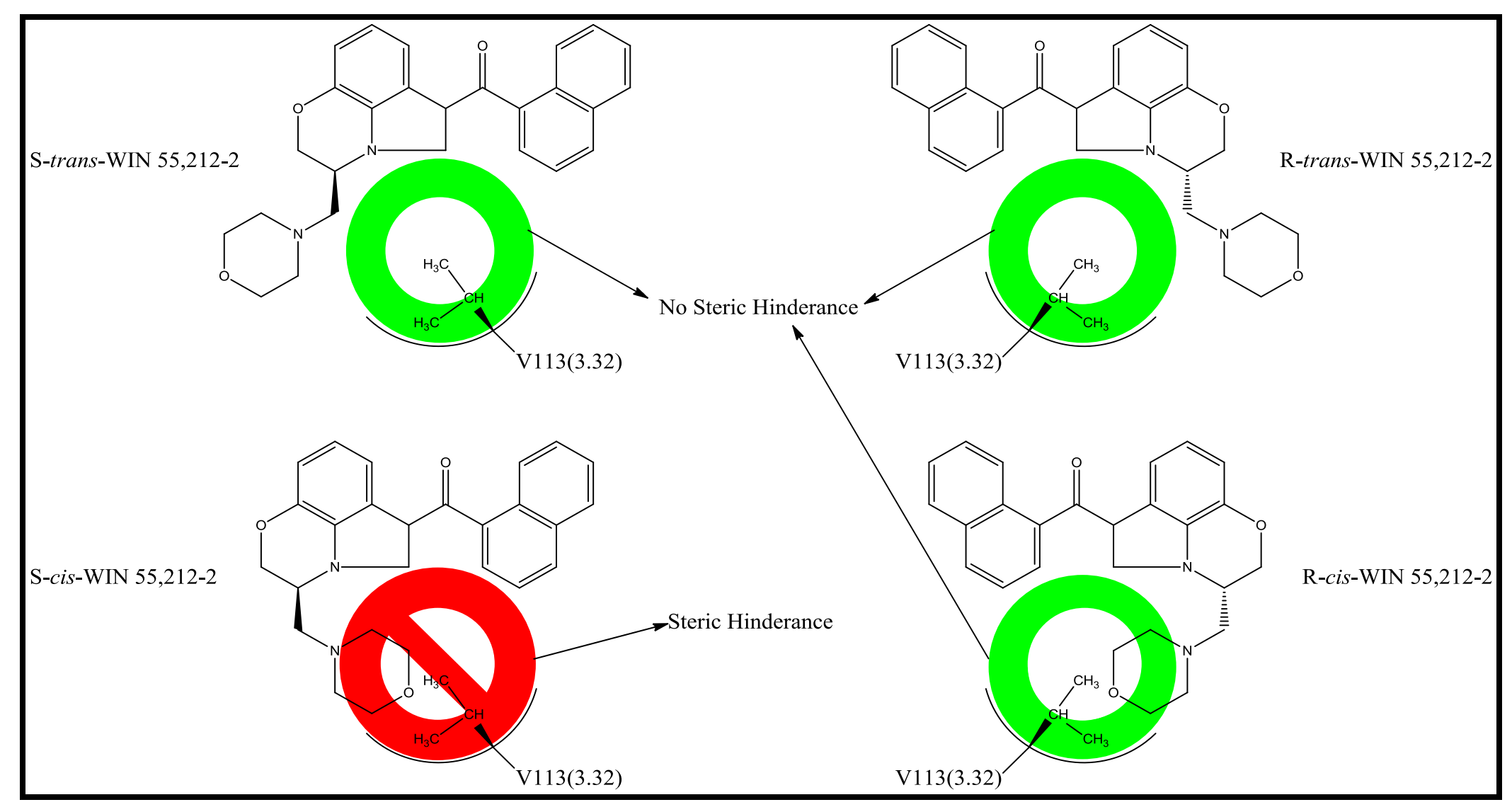

Figure 3.22. $\quad \mathrm{CB}_{2}$ LBP steric hinderance of cis and trans isomers of WIN-55,212-2 


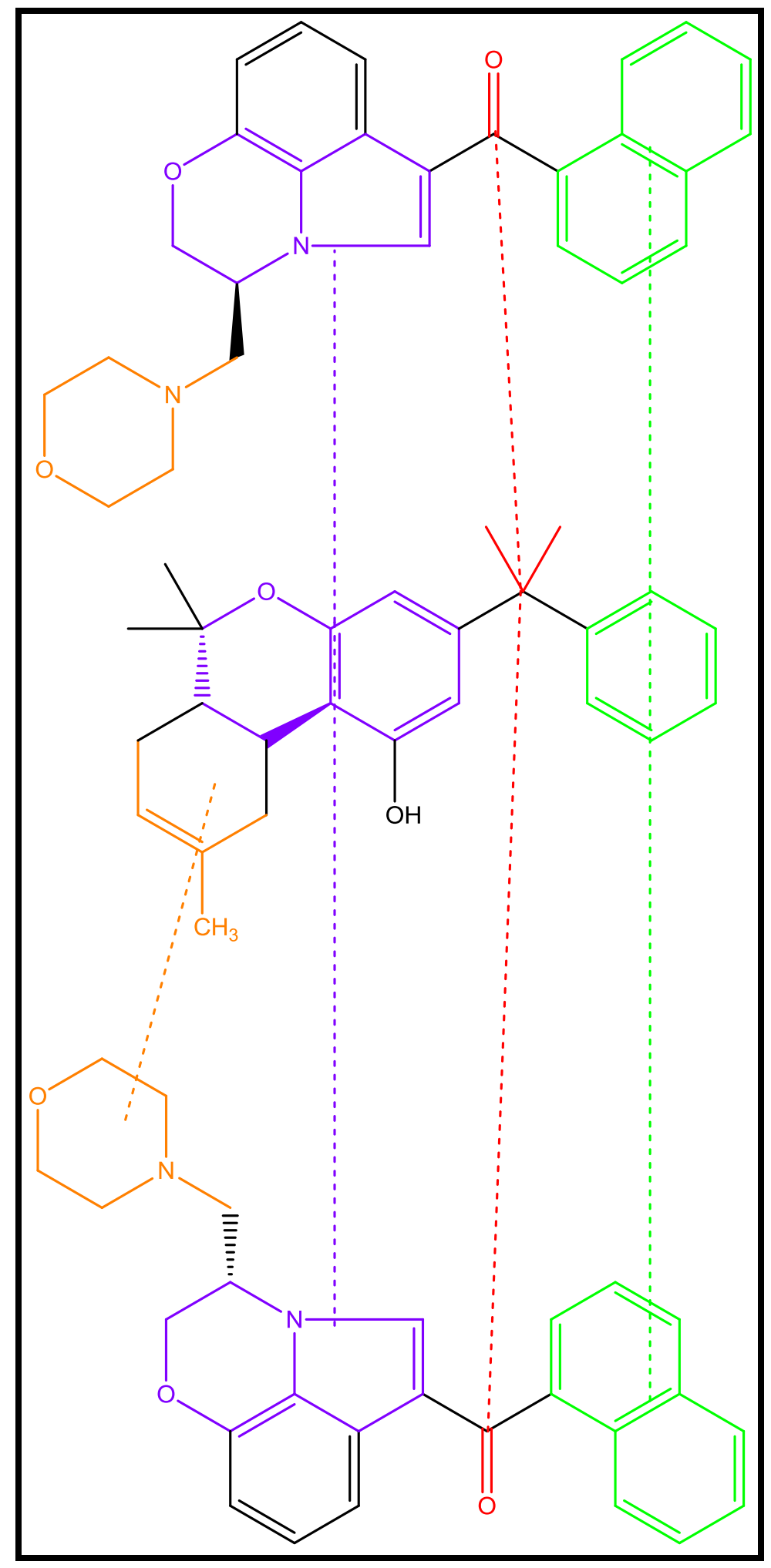

Figure 3.23. Comparison of analogous functional groups of WIN-55,212-2, KM 233 and WIN-55,212-3 


\section{CHAPTER 4. FUNCTIONAL EVALUATION OF A NOVEL CLASS OF HETEROCYCLIC CORE ANALOGUES OF HEXAHYDRO $\Delta^{8}$-THC}

\subsection{Introduction}

As previously discussed (Section 1.1), classical cannabinoids are tricyclic compounds possessing a benzopyran core - these are of either natural origin or synthetic analogues thereof. The naturally occurring, prototypes for these compounds are $\Delta^{9}$-THC and $\Delta^{8}-\mathrm{THC}$, which both bind to $\mathrm{CB}_{1}$ and $\mathrm{CB}_{2}$ receptors and exert a partial agonist activity. ${ }^{14}$ Within the classical compounds impacting the $\mathrm{CB}_{1}$ receptor novel means of dealing with anti-nociception, mitigation of chemotherapeutic-induced nausea and vomiting, decreasing intra-ocular pressure in glaucoma, increasing appetite in patients with wasting syndromes, reduction of muscle spasticity caused by diseases such as multiple sclerosis and treating diarrhea by slowing intestinal motility are present. ${ }^{14}$ It is well known in the literature that $\mathrm{CB}_{1}$ receptors are responsible for causing the untoward psychotropic effects of cannabinoid compounds. With $\mathrm{CB}_{2}$ receptors serving the body as modulators of cytokine release, agents targeting these compounds show great promise in inflammation, especially in instances of inflammation-induced organ damage. ${ }^{136,165}$ The drawback exists in separating $\mathrm{CB}_{1}$ and $\mathrm{CB}_{2}$ activity so as to retain or augment the desired therapeutic outcome while simultaneously mitigating the negative psychotropic effects of such compounds. This desire for such potentially impactful compounds for various disease states which have favorable therapeutic use profiles has led to a bevy of research into the classical cannabinoids with regard to their pharmacology and structural activity relationships/requirements (SAR).

For the classical cannabinoids it is established that substitution of the A-ring, aside from positions 1 and 3 , is generally detrimental to the $\mathrm{K}_{\mathrm{i}}$ of compounds, and substitution of the 1 position with anything other than hydroxyl is equally detrimental, though it can afford some selectivity for $\mathrm{CB}_{2}$ (Section 4.2.1). Manipulation of the B-ring of the classical core improves the potency of compounds over the parent THC compound (Section 4.2.2). Finally, desaturating the C-ring (as in ca nnabinol) serves to increase affinity and impart selectivity to compounds (Section 4.2.3). Considering these along with the previously mentioned C-3 modifications (Section 3.2), the classical core is rife with opportunities to exploit increases in affinity and specificity.

The non-classical cannabinoids provide a further insight on cannabinoid action as well. From the aminoalkylindoles (AAIs) it becomes apparent that size is of concern when substituting at the N-1 position, but this can be optimized to afford highly potent compounds (Section 4.3.1.1). From the AAIs one may also gather that $\mathrm{C}-2$ substitution does increase potency, but at the expense of $\mathrm{CB}_{2}$ specificity (Section 4.3.1.2). At C-3 one gathers that conformation and size play a crucial role in cannabinoid binding and activity (Section 4.3.1.3). Finally, the AAI compounds demonstrate how substitution of the indole ring system can result in highly potent and specific compounds (Section 4.3.1.4).

The other non-classical cannabinoid class, which provides valuable information in the development of this novel series of cannabinoids, is the well-known diarylpyrazole class. Much like the AAIs, size and electron-withdrawing substituents at N-1 aid in 
compound specificity (Section 4.3.2.1). C-3 derivatives of this class afford the knowledge that $\mathrm{CB}_{1}$ prefers a long and flexible moiety (Section 4.3.2.2). C-4 and C-5 derivatives for this class are relatively few; however, derivatives thereof provide knowledge that electron-withdrawing groups are preferred (Section 4.3.2.3 and Section 0). Summing all of this knowledge from classical (Figure 4.1) and non-classical cannabinoids gave rise to a true hybrid series of compounds, the pyridine- and pyrimidine-hexahydro-THC compounds (Figure 4.2).

The aim of this study is to utilize the ACTOne Assay (Chapter 2) to explore the functional ramifications of a heterocyclic A-ring, inconjunction with a hexahydro C-ring and C-1' substituents similar to the KM series (Chapter 3) (Section 4.4). Taking this functional data, I will then reconcile these outcomes against current literature standards' binding and homology models to propose hypothetical interactions with $\mathrm{CB}_{1}$ and $\mathrm{CB}_{2}-$ in an attempt to further understanding of cannabinoid SAR and the unique MoA for these compounds.

\subsection{Classical Cannabinoids - Modifying the A/B/C Ring}

Three pharmacophoric elements exist for classical cannabinoids to exert cannabimimetic activity (Figure 4.1):

1. C-1 phenolic hydroxyl group

2. Trans ring junction at the $\mathrm{B}$ and $\mathrm{C}$ rings $(6 \mathrm{aR}, 10 \mathrm{aR} \text { - required })^{18}$

3. Lipophilic C-3 alkyl side chain

For the purposes of examining this novel series of compounds, we will leave C-1 phenolic hydroxyl and the trans ring junction intact, and we will not bring into account the optional elements of the northern and southern hydroxyl groups. We will vary the A-ring with heterocyclic moieties and fully saturate the C-ring. Several sections of this chapter use a muricidal rat model as an in vivo characterization of cannabinoid activity. This model was utilized because several of the compounds were synthesized and tested prior to the isolation and characterization of the cannabinoid receptors, thus no $\mathrm{K}_{\mathrm{i}}$ values are reported, but the relative biological activity is. While this method does not provide a perfect 1:1 comparison, it does give a distinct picture the rank order of the compounds

\subsubsection{Modifying the A-ring.}

Variations about the A-ring are one of the most widely studied modifications within the tricyclic core. Several groups have manipulated this ring in an attempt to augment LBP interactions. Substitutions within the A-ring, with exception of the C-1 hydroxyl and $\mathrm{C}-3$ aliphatic, greatly reduce affinity of the compounds for $\mathrm{CB}_{1}$ receptors (Figure 4.3). ${ }^{262} \mathrm{C}$-4-bromo- $\Delta^{8}$-THC (79) $\left(\mathrm{CB}_{1} \mathrm{~K}_{\mathrm{i}}=5250 \mathrm{nM}\right)$ and C-4-nitro- $\Delta^{8}-\mathrm{THC}$ (80) $\left(\mathrm{CB}_{1} \mathrm{~K}_{\mathrm{i}}=1630 \mathrm{nM}\right)$ greatly reduce the affinity over $\Delta^{9}$-THC (1) $\left(\mathrm{CB}_{1} \mathrm{~K}_{\mathrm{i}}=44\right.$ $\mathrm{nM}) .{ }^{262}$ A C-2-nitro- $\Delta^{8}$-THC (82), C-2,4-diiodo- $\Delta^{8}$-THC (83) or C-2,4-dinitro- $\Delta^{8}$-THC (84) substitution abolishes all affinity for the compounds at $\mathrm{CB}_{1}\left(\mathrm{CB}_{1} \mathrm{~K}_{\mathrm{i}}=>10,000\right.$ $\mathrm{nM}) .{ }^{262}$ A C-2-Iodo- $\Delta^{8}-\mathrm{THC}(\mathbf{8 1})\left(\mathrm{CB}_{1} \mathrm{~K}_{\mathrm{i}}=89 \mathrm{nM}\right)$ substitution confers roughly a 


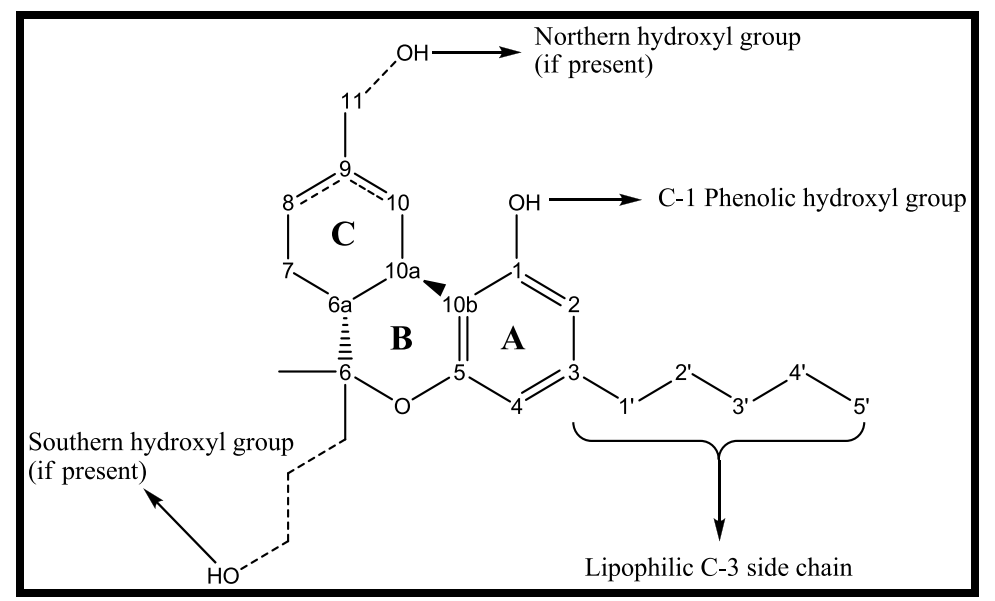

Figure 4.1. Classical cannabinoid with pharmacophoric elements 


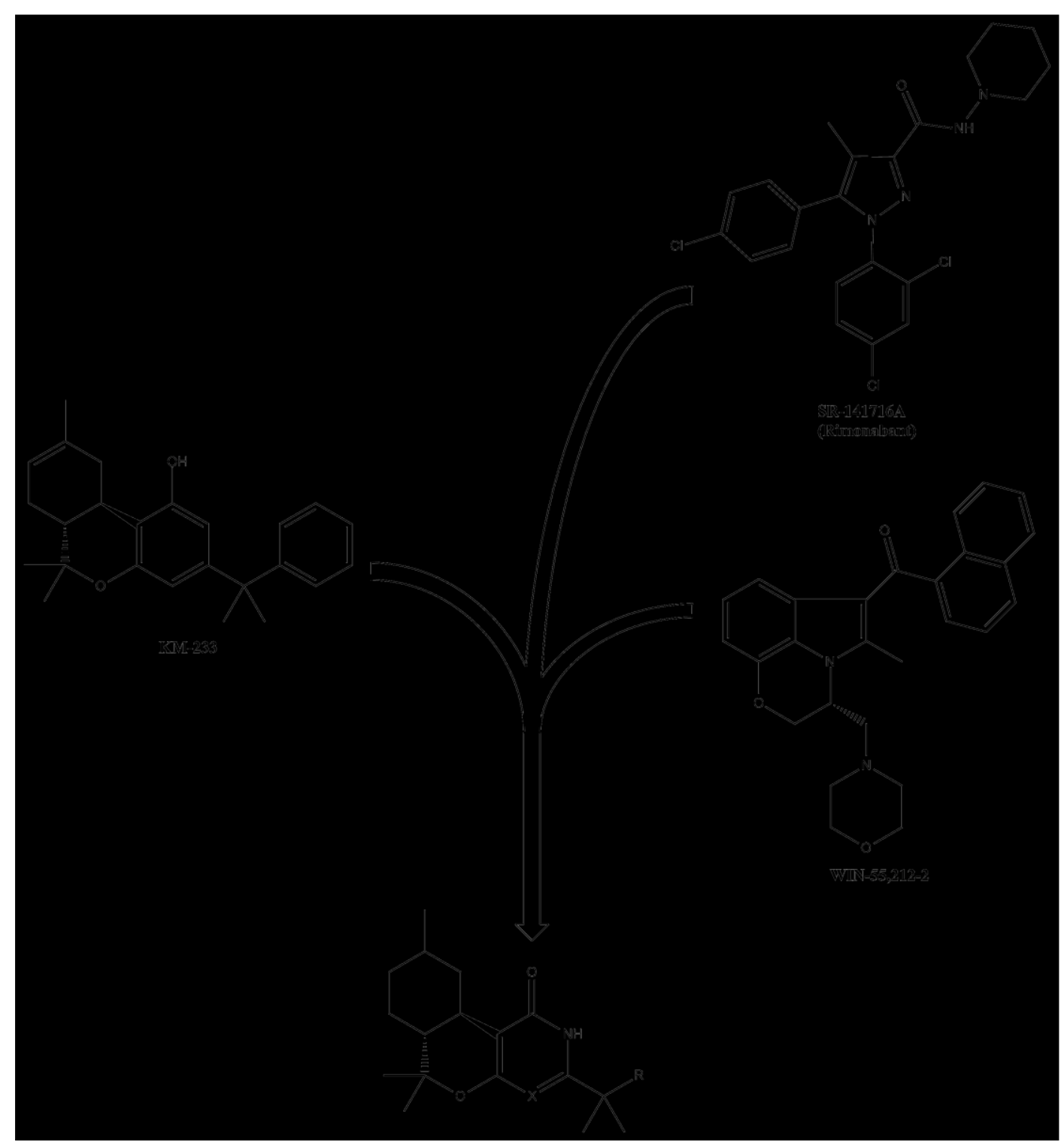

Figure 4.2. Design progression for the hexahydro series of compounds 


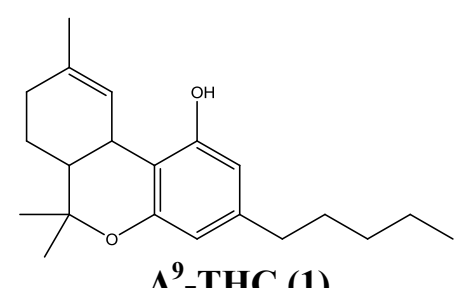

$\Delta^{9}$-THC (1)

$\mathrm{CB}_{1} \mathrm{~K}_{\mathrm{i}}=40 \mathrm{nM}^{\mathrm{a}}$

$\mathrm{CB}_{2} \mathrm{~K}_{\mathrm{i}}=36 \mathrm{nM}^{\mathrm{a}}$

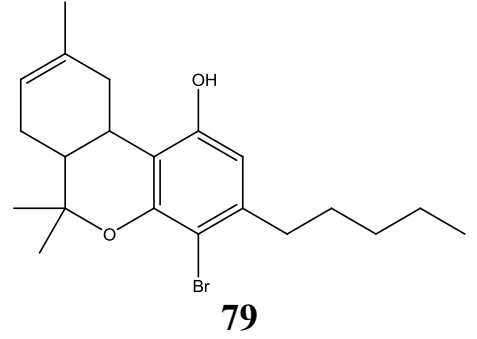

$\mathrm{CB}_{1} \mathrm{~K}_{\mathrm{i}}=5250 \mathrm{nM}$<smiles>CCCCCc1cc2c(c(O)c1I)C1CC(C)=CCC1C(C)(C)O2</smiles>

81

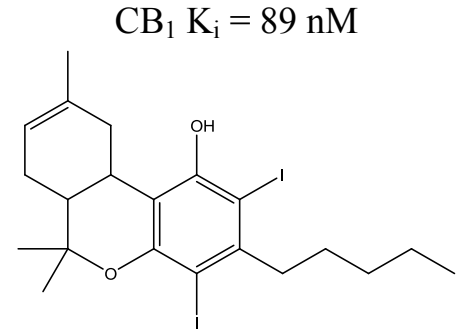

83

$\mathrm{CB}_{1} \mathrm{~K}_{\mathrm{i}}=10,600 \mathrm{nM}$

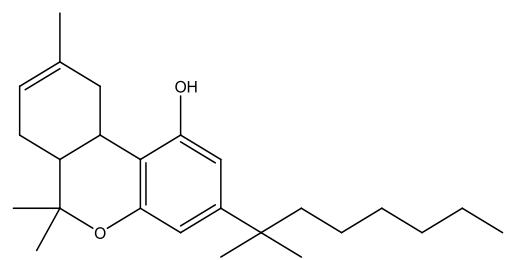

$\Delta^{8}$-THC-DMH (32)

$\mathrm{CB}_{1} \mathrm{~K}_{\mathrm{i}}=0.77 \mathrm{nM}^{\mathrm{b}}$

$\mathrm{CB}_{2} \mathrm{~K}_{\mathrm{i}}=0.27 \mathrm{nM}^{\mathrm{b}}$

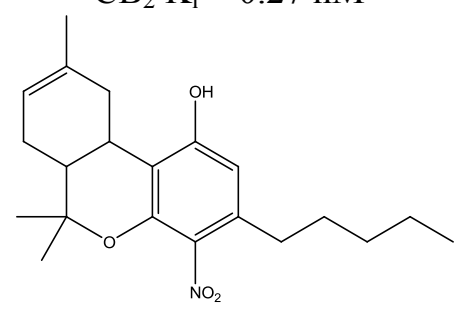

80

$\mathrm{CB}_{1} \mathrm{~K}_{\mathrm{i}}=1630 \mathrm{nM}$

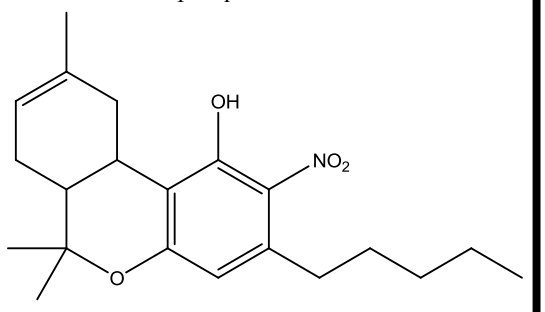

82

$\mathrm{CB}_{1} \mathrm{~K}_{\mathrm{i}}=>10,000 \mathrm{nM}$

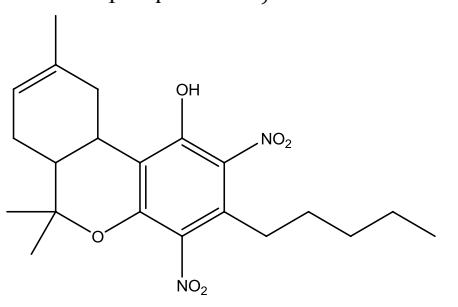

84

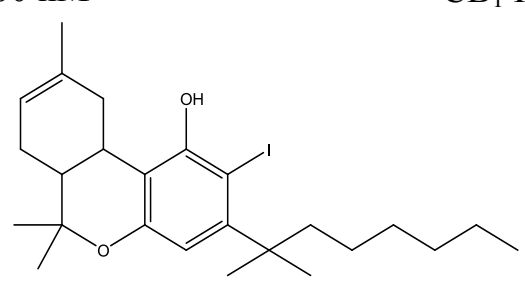

85

$\mathrm{CB}_{1} \mathrm{~K}_{\mathrm{i}}=61 \mathrm{nM}$

Figure 4.3. A-ring substituted compounds

${ }^{a}$ J.W. Huffman 265

b.W. Huffman ${ }^{242}$ 
2-fold decrease in binding affinity relative to $\Delta^{9}$-THC (1); however, a C-2-Iodo- $\Delta^{8}$-THC$\mathrm{DMH}(\mathbf{8 5})\left(\mathrm{CB}_{1} \mathrm{~K}_{\mathrm{i}}=61 \mathrm{nM}\right)$ gives almost an 80 -fold decrease in binding affinity at $\mathrm{CB}_{1}$ versus $\Delta^{8}$-THC-DMH (32) $\left(\mathrm{CB}_{1} \mathrm{~K}_{\mathrm{i}}=0.77 \mathrm{nM}\right){ }^{262}$ These data point to a need for the Aring to have a stable $\pi$-electron system for optimal $\pi-\pi$ stacking interactions in the LBP.

The most extensive modification to the $\mathrm{ABC}$ core ring system of the classical series of compounds takes place at C-1 of the A-ring, which naturally exists as a hydroxyl group. Data suggest that in the $\Delta^{6 \mathrm{a}, 10 \mathrm{a}}$ derivatives, it may be replaced by an amine and retain equivalent biological activity. In the muricidal rat model, used as a means of testing compounds due to the lack of $\mathrm{CB}$ receptor characterization at that time, to $\Delta^{9}$-THC only if the C-3 substituent contains C-1',2'-dimethylheptyl (86) $(5 \mathrm{mg} / \mathrm{kg})$ (Figure 4.4). ${ }^{263}$ Binding studies have confirmed in the $\Delta^{9}$-THC derivatives (Figure 4.4) that, while reduced in affinity relative to $\mathrm{C}$-1-hydroxyl, there is a manner of $\mathrm{CB}_{2}$ selectivity for C-1-amine (87) $\left(\mathrm{CB}_{1} \mathrm{~K}_{\mathrm{i}}=616 \mathrm{nM} ; \mathrm{CB}_{2} \mathrm{~K}_{\mathrm{i}}=215 \mathrm{nM}\right){ }^{264}$ Larger C-1amine substituents further reduce the affinity for both $\mathrm{CB}_{1}$ and $\mathrm{CB}_{2}$, but there are some instances where this garners some $\mathrm{CB}_{2}$ selectivity - e.g. $\mathrm{C}$-1-cyano $(\mathbf{8 8})\left(\mathrm{CB}_{1} \mathrm{~K}_{\mathrm{i}}=1720\right.$ $\left.\mathrm{nM}, \mathrm{CB}_{2} \mathrm{~K}_{\mathrm{i}}=387 \mathrm{nM}\right)$; methylcarbamate $(\mathbf{8 9})\left(\mathrm{CB}_{1} \mathrm{~K}_{\mathrm{i}}=>3000 \mathrm{nM}, \mathrm{CB}_{2} \mathrm{~K}_{\mathrm{i}}=1400 \mathrm{nM}\right)$; methylsulfite (90) $\left(\mathrm{CB}_{1} \mathrm{~K}_{\mathrm{i}}=>3000 \mathrm{nM}, \mathrm{CB}_{2} \mathrm{~K}_{\mathrm{i}}=1720 \mathrm{nM}\right)$; isonicotinamide (91) $\left(\mathrm{CB}_{1}\right.$ $\left.\mathrm{K}_{\mathrm{i}}=>3000 \mathrm{nM}, \mathrm{CB}_{2} \mathrm{~K}_{\mathrm{i}}=1770 \mathrm{nM}\right)$; methoxymethanamine (92) $\left(\mathrm{CB}_{1} \mathrm{~K}_{\mathrm{i}}=>3000 \mathrm{nM}\right.$, $\left.\mathrm{CB}_{2} \mathrm{~K}_{\mathrm{i}}=1400 \mathrm{nM}\right)$; methylurea (93) $\left(\mathrm{CB}_{1} \mathrm{~K}_{\mathrm{i}}=>3000 \mathrm{nM}, \mathrm{CB}_{2} \mathrm{~K}_{\mathrm{i}}=782 \mathrm{nM}\right)$; and methylacetamide (94) $\left(\mathrm{CB}_{1} \mathrm{~K}_{\mathrm{i}}=>3000 \mathrm{nM} ; \mathrm{CB}_{2} \mathrm{~K}_{\mathrm{i}}=1500 \mathrm{nM}\right)$ show this trend. ${ }^{264}$ This effect was even more profound for these derivatives when C-3-1',1'-dimethylheptyl was present in lieu of C-3- $n$-pentyl, especially in the case of C-3-CN, C-3-acetamide and C-3methylsulfite (Table 4.1) ${ }^{264}$ Acidic and amide derivations at $\mathrm{C}-1$ (Figure 4.5) were less active, with $\mathrm{N}$-methylformamide $(\mathbf{9 5})\left(\mathrm{CB}_{1} \mathrm{~K}_{\mathrm{i}}=>3000 \mathrm{nM} ; \mathrm{CB}_{2} \mathrm{~K}_{\mathrm{i}}=1260 \mathrm{nM}\right)$ and N,N-dimethylformamide (96) $\left(\mathrm{CB}_{1} \mathrm{~K}_{\mathrm{i}}=>3000 \mathrm{nM} ; \mathrm{CB}_{2} \mathrm{~K}_{\mathrm{i}}=1210 \mathrm{nM}\right)$ showing the most promise for $\mathrm{CB}_{2}$ selectivity and activity. ${ }^{264}$ The $\mathrm{C}-1$ substituent may not be a thiol group, this abolishes biological activity in the muricidal rat model, again this model is used due to the predating of $\mathrm{CB}$ receptor characterization, with C-3-n-pentyl (97) or C-3-1',2'-dimethylheptyl (98) (Figure 4.5). ${ }^{263}$ Interestingly, though, conversion of the C1 substituent to a methylthiol with C-3-n-pentyl (99) (Figure 4.5) yields a compound with lower binding affinity, but nearly a 2 -fold selectivity for $\mathrm{CB}_{2}$ over $\mathrm{CB}_{1}\left(\mathrm{CB}_{1} \mathrm{~K}_{\mathrm{i}}=\right.$ $\left.955 \mathrm{nM} ; \mathrm{CB}_{2} \mathrm{~K}_{\mathrm{i}}=465 \mathrm{nM}\right){ }^{264}$

Replacing C-1-hydroxyl with C-1-F causes a staunch drop in $\mathrm{CB}_{1}$ affinity and a moderate drop in $\mathrm{CB}_{2}$ affinity, which would allow for selective ligands at $\mathrm{CB}_{2}$ (Figure 4.6). As an example, when 3-heptyl- $\Delta^{8}$-THC (100) $\left(\mathrm{CB}_{1} \mathrm{~K}_{\mathrm{i}}=22 \mathrm{nM}\right)$ is compared to 1-fluoro-1-deoxy-3-heptyl- $\Delta^{8}$-THC (101) $\left(\mathrm{CB}_{1} \mathrm{~K}_{\mathrm{i}}=1560 \mathrm{nM} ; \mathrm{CB}_{2} \mathrm{~K}_{\mathrm{i}}=45 \mathrm{nM}\right)$ one sees the precipitous drop in $\mathrm{CB}_{1}$ affinity and immense $\mathrm{CB}_{2}$ selectivity present therein. ${ }^{266}$ It is noteworthy that there is no binding data available in current literature for 3-heptyl- $\Delta^{8}$-THC (100), so a true comparison of any $\mathrm{CB}_{2}$ activity gained or lost is not possible. I noticed a similar trend when comparing C-1-hydroxy and C-1-methyoxy derivatives of compounds (Figure 4.6). Examining 3-(6'-azido-2'-hexynyl)- $\Delta^{8}-\mathrm{THC}$ (102) $\left(\mathrm{CB}_{1} \mathrm{~K}_{\mathrm{i}}=2.14 \mathrm{nM} ; \mathrm{CB}_{2} \mathrm{~K}_{\mathrm{i}}=1.12 \mathrm{nM}\right)$ to 1-methoxy-3-(6'-azido-2'-hexynyl)$\Delta^{8}$-THC $(\mathbf{1 0 3})\left(\mathrm{CB}_{1} \mathrm{~K}_{\mathrm{i}}=82.5 \mathrm{nM} ; \mathrm{CB}_{2} \mathrm{~K}_{\mathrm{i}}=11.2 \mathrm{nM}\right)$ one may note the nearly 40 -fold increase in $\mathrm{CB}_{1}$ as compared to the 10 -fold increase at $\mathrm{CB}_{2}{ }^{266}$ The preference of $\mathrm{C}$-1-methoxy for $\mathrm{CB}_{2}$ receptors holds true when comparing C-3-(1',1'-dimethylheptyl)- 


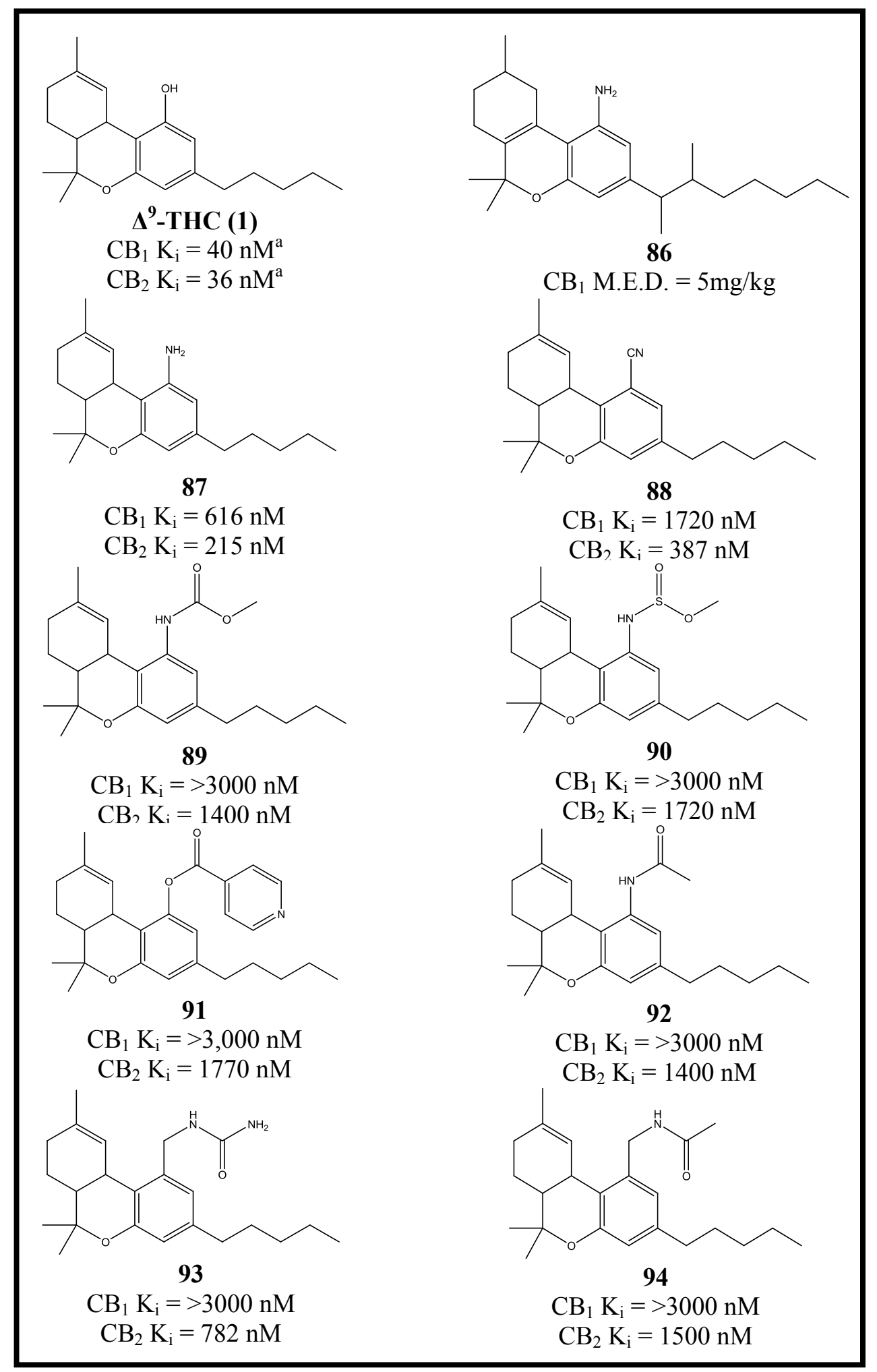

Figure 4.4. Amino and aminomethyl derivatives

Note: ${ }^{\text {aJ.W. Huffman }}{ }^{265}$ M.E.D. stands for Minimum Effective Dose 
Table 4.1. $\quad \mathrm{CB}_{1} / \mathrm{CB}_{2} \mathrm{~K}_{\mathrm{i}}$ ratios for selected compounds

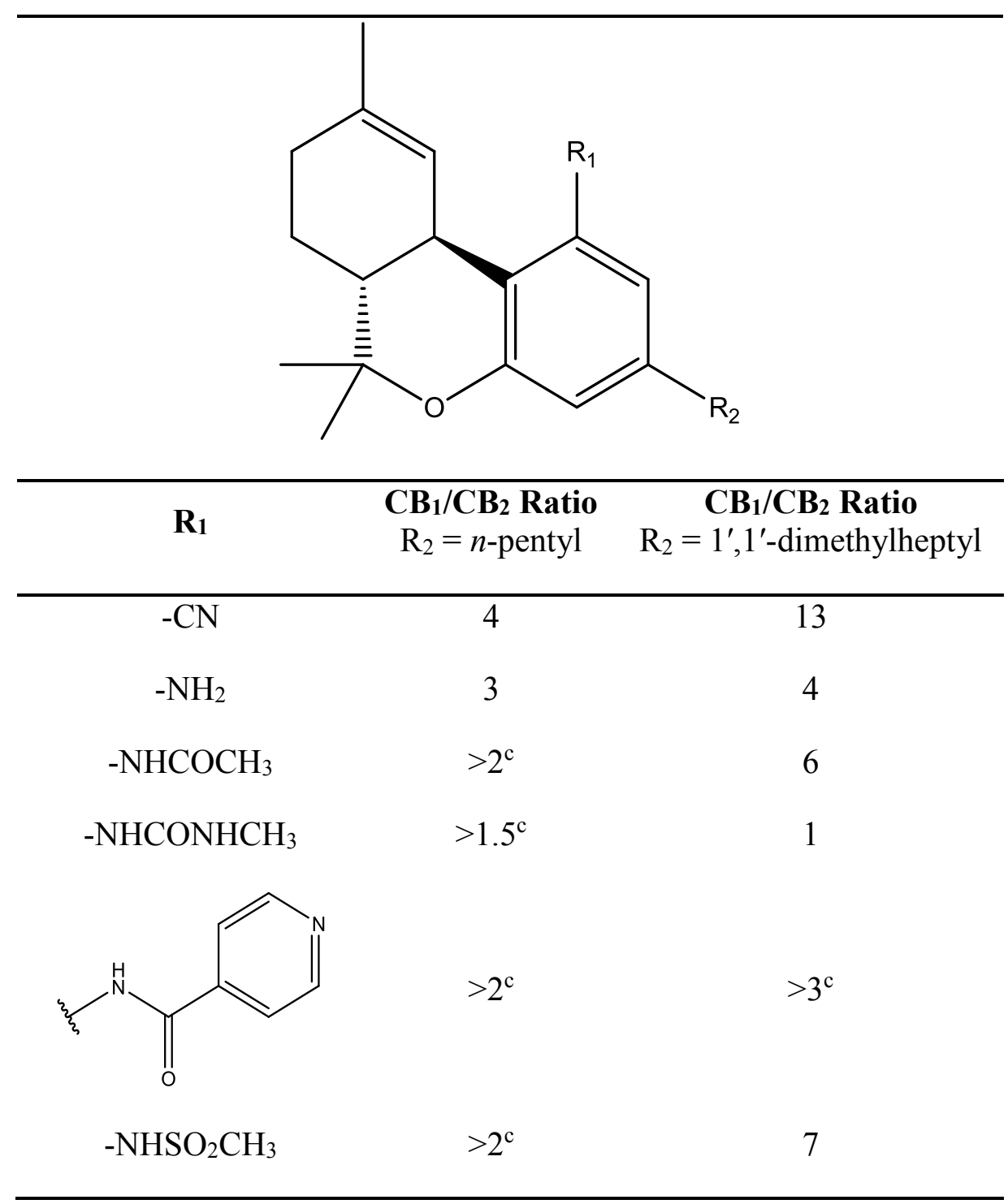

${ }^{c}$ Values cannot be calculated accurately due to a $\mathrm{CB}_{1}$ affinity in excess of $3 \mu \mathrm{M}$. 


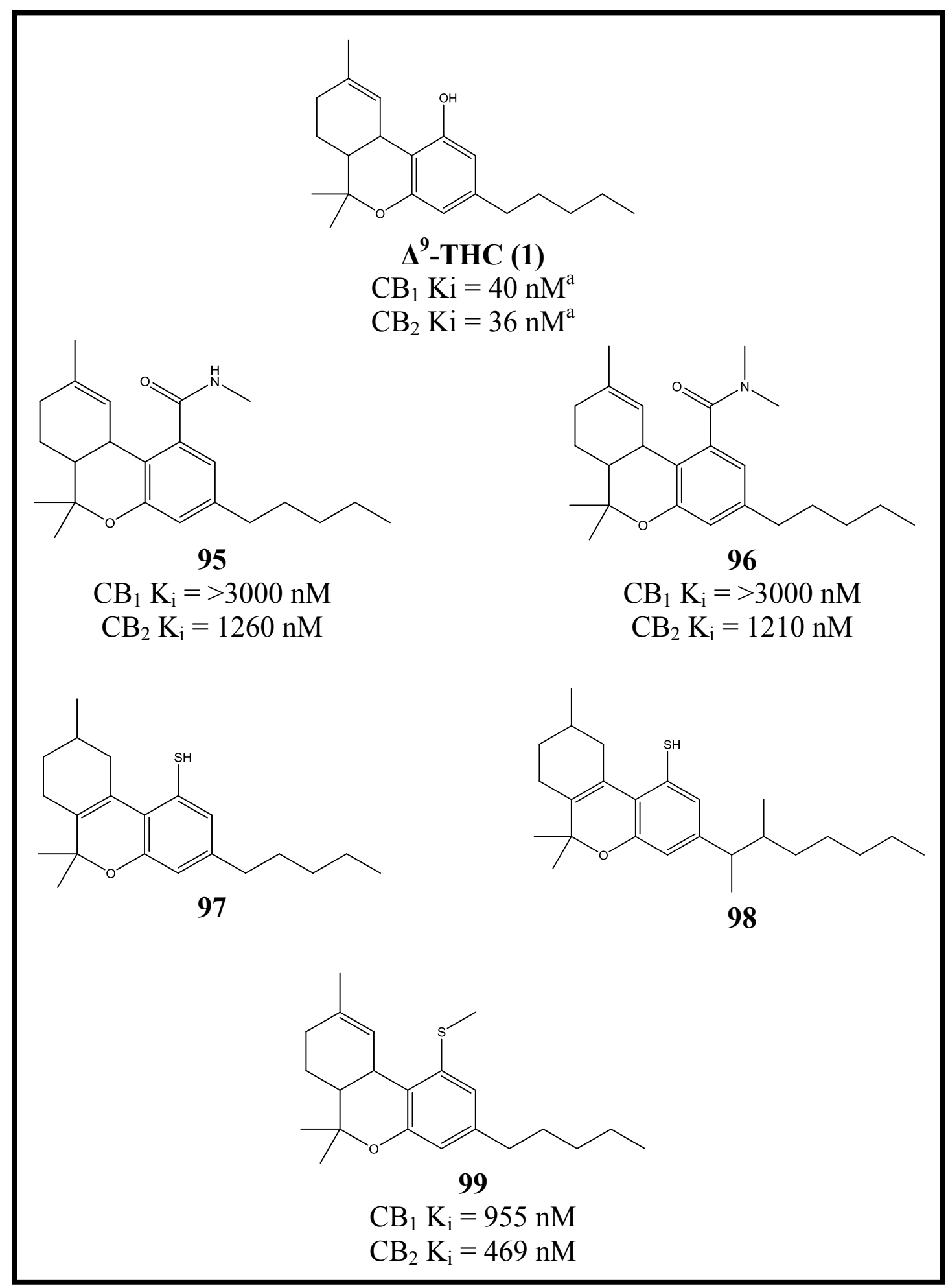

Figure 4.5. Amide and thiol derivatives

${ }^{\text {a J.W. Huffman }}{ }^{265}$ 


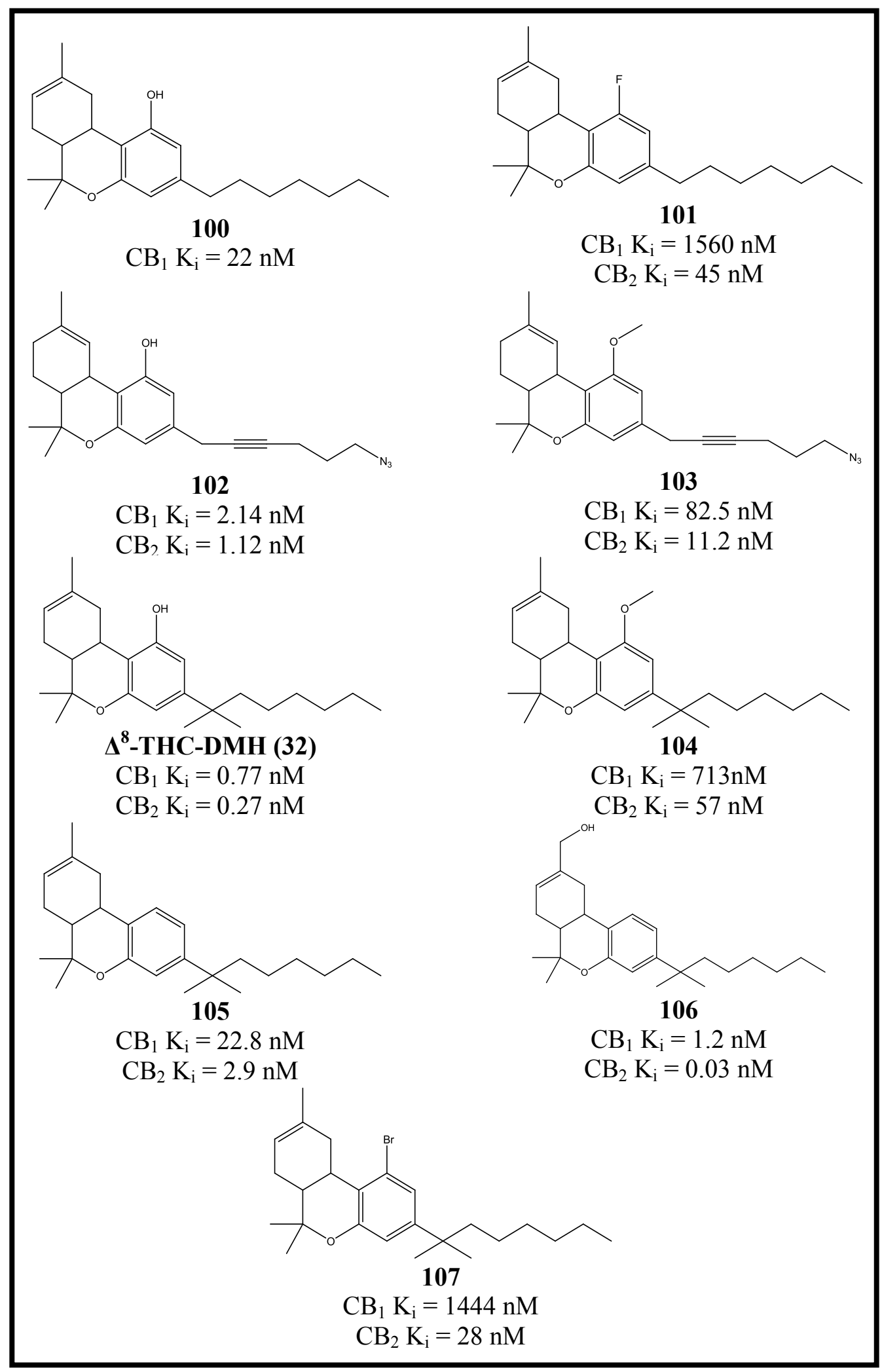

Figure 4.6. C-1 deoxy and methoxy derivatives 
$\Delta^{8}$-THC (104) $\left(\mathrm{CB}_{1} \mathrm{~K}_{\mathrm{i}}=713 \mathrm{nM} ; \mathrm{CB}_{2} \mathrm{~K}_{\mathrm{i}}=57 \mathrm{nM}\right)^{267}$ to C-3-(1',1'-dimethylheptyl)- $\Delta^{8}$ $\mathrm{THC}(32)\left(\mathrm{CB}_{1} \mathrm{~K}_{\mathrm{i}}=0.77 \mathrm{nM} ; \mathrm{CB}_{2} \mathrm{~K}_{\mathrm{i}}=0.27 \mathrm{nM}\right) .{ }^{242}$ Complete absence of a C-1hydroxyl allows for good selectivity of $\mathrm{CB}_{2}$ (Figure 4.6) ${ }^{250,267-270}$ This deoxy preference is exemplified by 1-deoxy-3-(1',1'-dimethylheptyl)- $\Delta^{8}$-THC (105) $\left(\mathrm{CB}_{1} \mathrm{~K}_{\mathrm{i}}=22.8 \mathrm{nM}\right.$; $\left.\mathrm{CB}_{2} \mathrm{~K}_{\mathrm{i}}=2.9 \mathrm{nM}\right)-\mathrm{a}$ trend augmented by addition of C-11-hydroxy, with 1-deoxy-11-hydroxy-3-(1',1'-dimethylheptyl)- $\Delta^{8}-\mathrm{THC}(\mathbf{1 0 6})\left(\mathrm{CB}_{1} \mathrm{~K}_{\mathrm{i}}=1.2 \mathrm{nM} ; \mathrm{CB}_{2} \mathrm{~K}_{\mathrm{i}}=\right.$ $0.03 \mathrm{nM}$ ) showing the highest $\mathrm{CB}_{1} / \mathrm{CB}_{2}$ ratio. ${ }^{250,267}$ The data show the $\mathrm{CB}_{2} \mathrm{LBP}$ has an inclination for C-1-deoxy or C-1-methoxy when compared to the $\mathrm{CB}_{1} \mathrm{LBP}^{2}$ - which may indicate that the hydrogen bond in the LBP may not be as necessary as it is in the $\mathrm{CB}_{1}$ LBP. Further derivation of these compounds utilizing $\mathrm{C}-1-\mathrm{Br}$ have been tested and showed a similar preference for $\mathrm{CB}_{2}$, again downplaying the need for LBP H-bonding (Figure 4.6). In this modification of the C-1 substituent, 3-(1', $1^{\prime}$-dimethylhexyl)-1-Br- $\Delta^{8}$ THC (107) $\left(\mathrm{CB}_{1} \mathrm{~K}_{\mathrm{i}}=1444 \mathrm{nM} ; \mathrm{CB}_{2} \mathrm{~K}_{\mathrm{i}}=28 \mathrm{nM}\right)$ exhibited the best $\mathrm{CB}_{1} / \mathrm{CB}_{2}$ ratio. ${ }^{270}$

\subsubsection{Modifying the B-ring}

In most synthetic and all naturally occurring cannabinoids, the B ring is a pyran; however, there are synthetic derivatives wherein this ring exists as a piperidine (108) (Figure 4.7) $\left(\mathrm{CB}_{1} \mathrm{~K}_{\mathrm{i}}=0.6 \mathrm{nM}\right)$ without any loss of activity compared to the pyran parent compound. ${ }^{271,272}$ Further derivation of the $\mathrm{B}$ ring into $\Delta^{6 \mathrm{a}, 10 \mathrm{a}}$ derivatives or oxepane derivatives gives some insight into the binding of these compounds into the LBP. For example, $\Delta^{6 a}$,10a -lactones (109) (Figure 4.7) show inactivity at testing doses in a muricidal rat model $(>10 \mathrm{mg} / \mathrm{kg})^{273}$; however, the $\Delta^{6 \mathrm{a}, 10 \mathrm{a}}-\mathrm{C}-6$-gem-dimethyl (110) (Figure 4.7) showed substantially better activity $(0.62 \mathrm{mg} / \mathrm{kg})^{273}$ over $\Delta^{9}$-THC (1) (Figure 4.7) $(5.0 \mathrm{mg} / \mathrm{kg})^{273}$ in the same model. This demonstrates that $\Delta^{6 \mathrm{a}, 10 \mathrm{a}}$ increases efficacy; however, there is a need for hydrophobic substituents at C-6 to maximize this benefit.

Expansion of the B ring to the oxepane and substituting C-6 and C-7 with concomitant unsaturation $\left(\Delta^{7 \mathrm{a}, 11 \mathrm{a}}\right)$ shows the limitations of the binding pocket about the tricyclic core. In the case of $\Delta^{7 \mathrm{a}, 11 \mathrm{a}}$ compounds where both C-6 and C-7 are unsubstituted (111) (Figure 4.7) $(1.25 \mathrm{mg} / \mathrm{kg})^{273}$, there is a gain relative to $\Delta^{9}-\mathrm{THC}(5.0 \mathrm{mg} / \mathrm{kg})(\mathbf{1})$, but

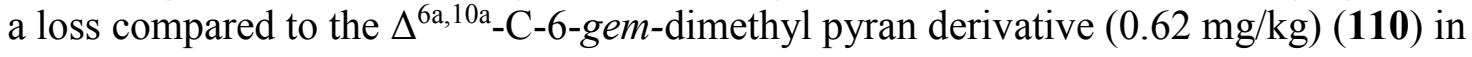
the muricidal rat model. Further exploration of the oxepan derivatives shows that C-6gem-dimethyl (112) (Figure 4.7) $(2.5 \mathrm{mg} / \mathrm{kg})^{273}$ is better than a C-6-lactone (113) (Figure 4.7) $(5 \mathrm{mg} / \mathrm{kg}),{ }^{273}$ which further shows the need for hydrophobic substitution at this position. Interestingly, a C-7-gem-dimethyl substitution, with (114) (Figure 4.7) or without (115) (Figure 4.7) C-6-ketone, results in completely inactive compounds at tested doses $(>10 \mathrm{mg} / \mathrm{kg})^{273}$ - showing C-7 substitution may be detrimental.

\subsubsection{Modifying the C-ring}

As is the case for the two novel classes examined in this chapter, unsaturation of the C-ring resulting in either $\Delta^{8}$ or $\Delta^{9}$ compounds is not necessary for activity. ${ }^{274-276}$ For 


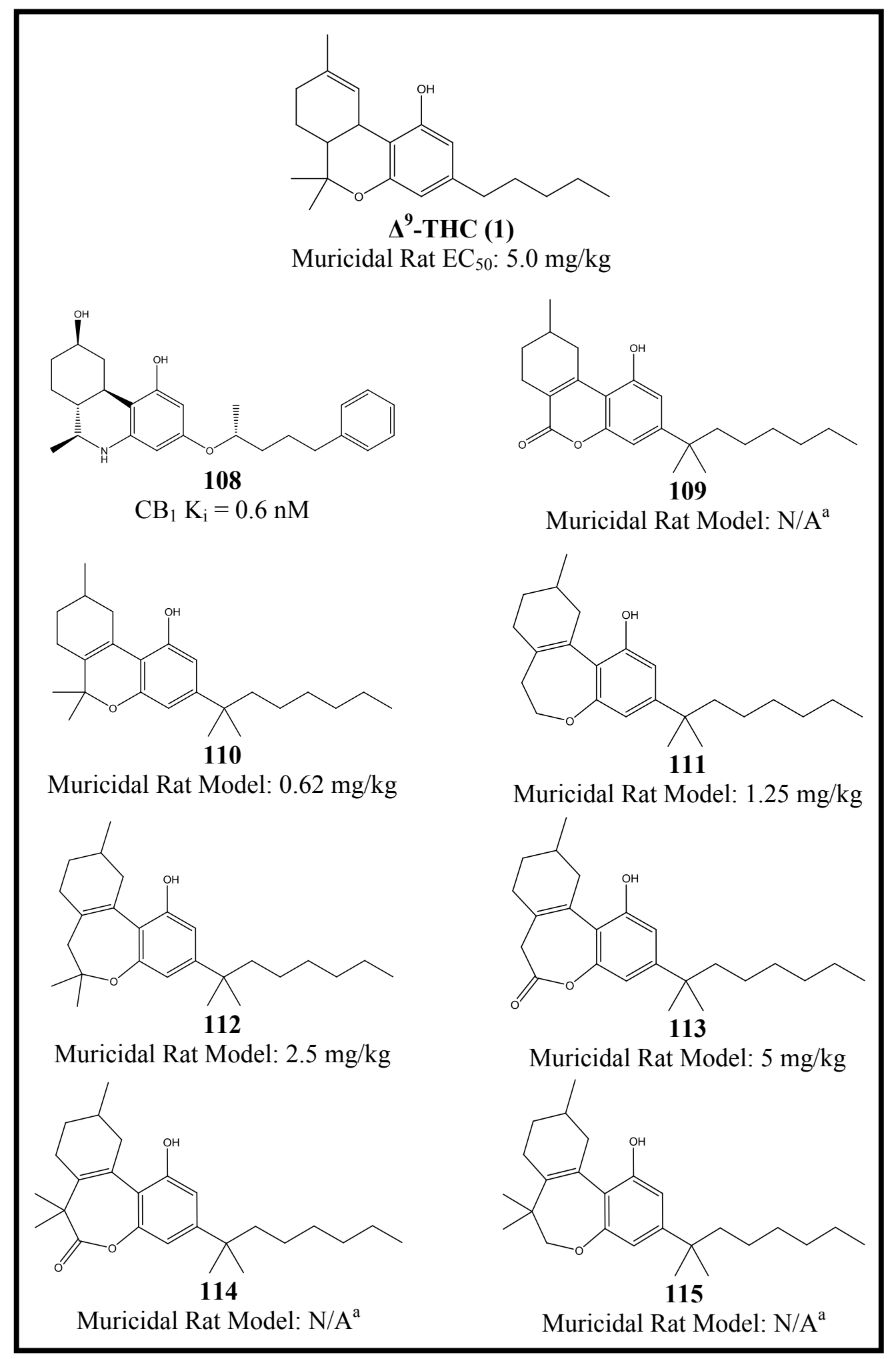

Figure 4.7. B-ring modifications

${ }^{a}$ Not Active 
both the hot plate and anti-writhing test models, conversion of $\Delta^{9}$-THC (1) (Figure 4.8) $\left(>100 \mathrm{mg} / \mathrm{kg}\right.$ and $43 \mathrm{mg} / \mathrm{kg}$, respectively) to a $\Delta^{6 \mathrm{a}, 10 \mathrm{a}}$ derivative (116) (Figure 4.8) (9.3 $\mathrm{mg} / \mathrm{kg}$ and $3.0 \mathrm{mg} / \mathrm{kg}$, respectively) showed a dramatic decrease in $\mathrm{EC}_{50} .^{276}$ Completeunsaturation resulting in a benzene ring, in conjunction with a $\mathrm{C}-6$ ketone moiety $(117,119,121)$ (Figure 4.8), yields compounds near or better at $\mathrm{CB}_{2}$ receptors than those containing C-6-gem-dimethyl $(118,120,122)$ (Figure 4.8) - it is noteworthy, that the $\mathrm{C}-6$ ketone compounds did bear a great deal more selectivity for $\mathrm{CB}_{2}$ over $\mathrm{CB}_{1}{ }^{277}$ Along with this, conversion of the C-ring to a heterocyclic ring does not impede or improves activity. ${ }^{274-276}$ In fact, converting the C-ring to pyridine $(\mathbf{1 2 3 , 1 2 4})$ (Figure 4.9) increased the efficacy of compounds over THC (1) (Figure 4.9) in both the rat tail flick and acetic acid writhing tests. ${ }^{274}$ Conversion of these pyridine C-ring derivatives to pyridine oxide rings $(\mathbf{1 2 5}, \mathbf{1 2 6})$ (Figure 4.9) causes a substantial drop in efficacy, ${ }^{274}$ suggesting that destabilization of the pi-ring system and/or size constraints about the Cring cause an issue. In cases where the $\mathrm{C}$-ring was a $\Delta^{6 \mathrm{a}, 10 \mathrm{a}}$-tetrahydropyridine $(\mathbf{1 2 7}, \mathbf{1 2 8})$ (Figure 4.9), compounds exhibited an activity equivalent to or worse than $\Delta^{9}$-THC (1) (Figure 4.9) except in cases where the substituent on the tetrahydropyridine nitrogen was a small aliphatic group. ${ }^{275}$ There are references ${ }^{278,279}$ and compounds synthesized that attempt to modify C-11 into esters and ethers as an attempt to garner a prodrug and/or peripherally restricted compound. I discounted these modifications in this analysis because they are inactive until converted, at which time the active drug's properties predominate.

\subsection{Non-Classical Cannabinoids - Heterocyclic Ring-Containing Compounds}

\subsubsection{Aminoalkylindoles (AAIs)}

The aminoalkylindole (AAI) compounds, originally developed in the early to mid-1990s as non-steroidal anti-inflammatory drugs (NSAIDs), sought to inhibit the pain and inflammation pathways while diverting from the ulcerogenic properties common among non-COX-2 specific NSAIDs. ${ }^{280}$ These compounds have found favor and infamy in the synthetic cannabis designer drug realm. Some of the compounds herein are found in trace amounts in over-the-counter "herbal incense." Recreational use of these herbal incense products has led to serious injury and death because of the cannabinoids hallucinogenic properties. Due to these serious issues, addictive potential and nonregulated production and use, the DEA has classified many of these compounds as Schedule I controlled substances, despite their therapeutic potential. Structurally, they have no resemblance to classical cannabinoids; however, the eventual assignment of antinociception via cannabinoid receptors ${ }^{281,282}$ has cemented these compounds as part of the cannabinoid family. By far the most widely studied of these compounds is WIN-55,212-2 (Figure 4.10). WIN-55,212-2 is thus know to be a potent agonist at both $\mathrm{CB}_{1}$ and $\mathrm{CB}_{2}$. The close examination of this molecule has allowed the study of cannabinoids to draw some potential conclusions with regard to requirements and/or potential switches for functional activity. 


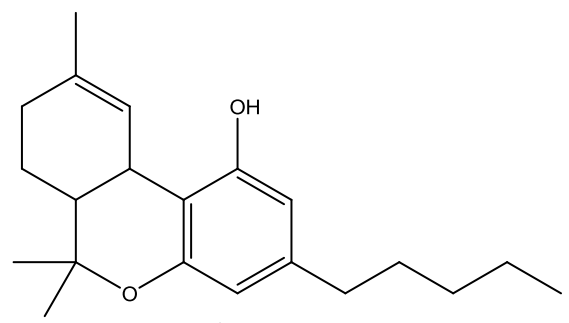

$\Delta^{9}$-ТHC (1)

Hot plate: $>100 \mathrm{mg} / \mathrm{kg}$ Anti-writhing: $43 \mathrm{mg} / \mathrm{kg}$

CB1 Ki $=40 \mathrm{nM}^{\mathrm{a}}$ $\mathrm{CB} 2 \mathrm{Ki}=36 \mathrm{nM}^{\mathrm{a}}$

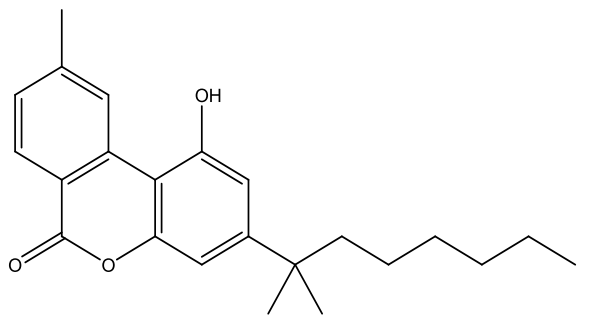

117

$\mathrm{CB}_{1} \mathrm{~K}_{\mathrm{i}}=39 \mathrm{nM}$

$\mathrm{CB}_{2} \mathrm{~K}_{\mathrm{i}}=3.1 \mathrm{nM}$

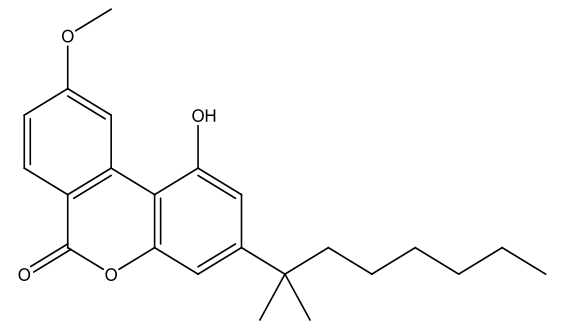

119

$\mathrm{CB}_{1} \mathrm{~K}_{\mathrm{i}}=360 \mathrm{nM}$

$\mathrm{CB}_{2} \mathrm{~K}_{\mathrm{i}}=6.7 \mathrm{nM}$

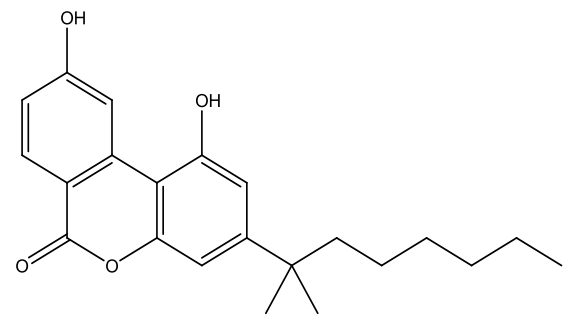

121

$\mathrm{CB}_{1} \mathrm{~K}_{\mathrm{i}}=400 \mathrm{nM}$

$\mathrm{CB}_{2} \mathrm{~K}_{\mathrm{i}}=0.82 \mathrm{nM}$

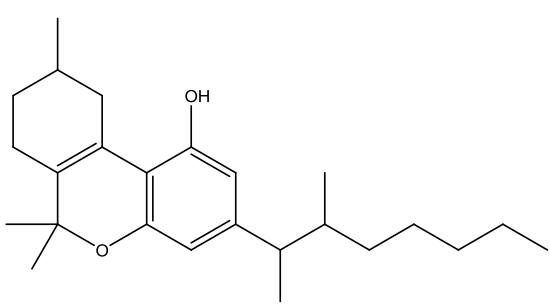

116

Hot plate: $9.3 \mathrm{mg} / \mathrm{kg}$ Anit-writhing: $3.0 \mathrm{mg} / \mathrm{kg}$

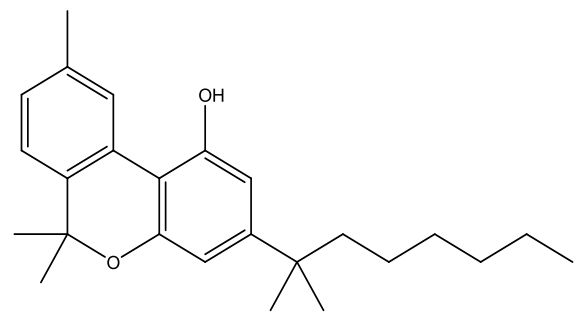

118

$\mathrm{CB}_{1} \mathrm{~K}_{\mathrm{i}}=0.95 \mathrm{nM}$

$\mathrm{CB}_{2} \mathrm{~K}_{\mathrm{i}}=1.1 \mathrm{nM}$

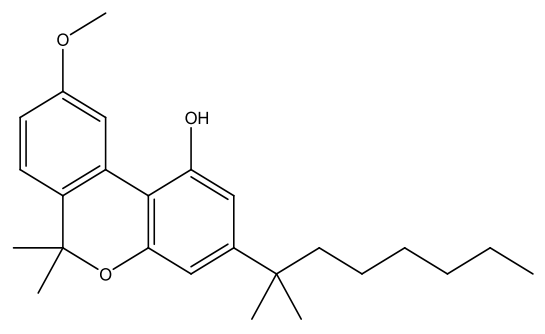

120

$\mathrm{CB}_{1} \mathrm{~K}_{\mathrm{i}}=5.4 \mathrm{nM}$

$\mathrm{CB}_{2} \mathrm{~K}_{\mathrm{i}}=5.9 \mathrm{nM}$

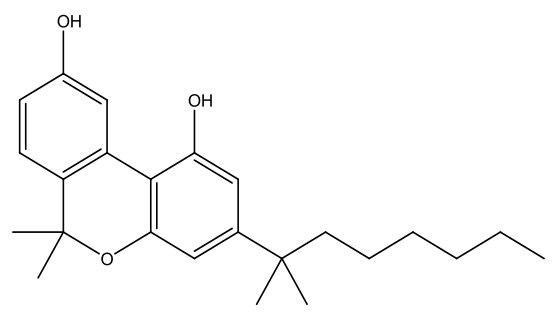

122

$\mathrm{CB}_{1} \mathrm{~K}_{\mathrm{i}}=2.6 \mathrm{nM}$

$\mathrm{CB}_{2} \mathrm{~K}_{\mathrm{i}}=4.8 \mathrm{nM}$

Figure 4.8. C-ring unsaturation

${ }^{\mathrm{a}}$ J.W. Huffman ${ }^{265}$ 


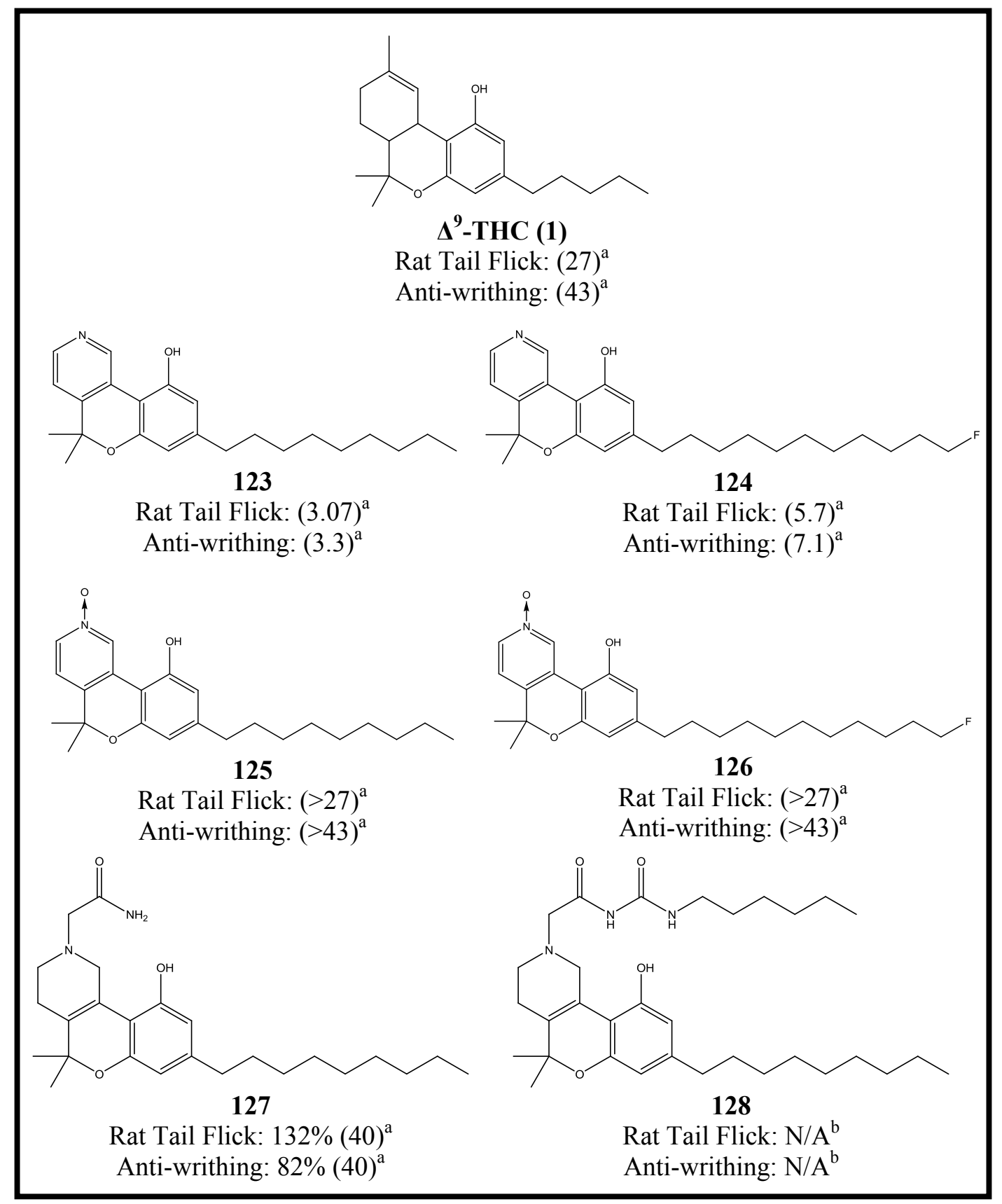

Figure 4.9. Heterocyclic derivatives of the C-ring

Notes: ${ }^{a}$ Numbers in parentheses are oral doses in $\mathrm{mg} / \mathrm{kg}$, percentages are percent reduction vs. control (THC). ${ }^{\text {b }}$ /A: Not Active 


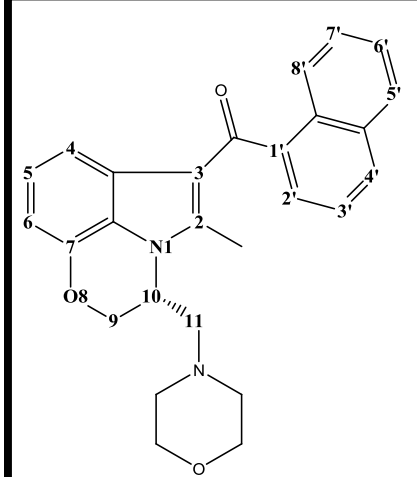

WIN-55,212-2

$\mathrm{CB}_{1} \mathrm{~K}_{\mathrm{i}}=1.9 \mathrm{nM}$

$\mathrm{CB}_{2} \mathrm{~K}_{\mathrm{i}}=0.3 \mathrm{nM}$

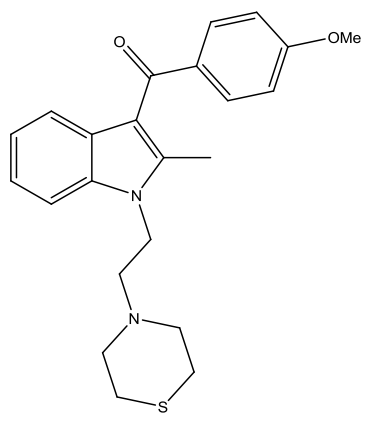

130

$\mathrm{IC}_{50}{ }^{\mathrm{b}}=622 \mathrm{nM}^{\mathrm{c}}$

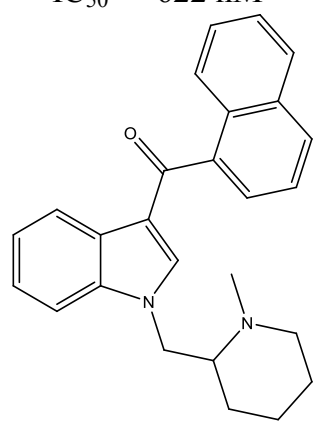

133

$\mathrm{IC}_{50}{ }^{\mathrm{b}}=1.22 \mathrm{nM}^{\mathrm{d}}$

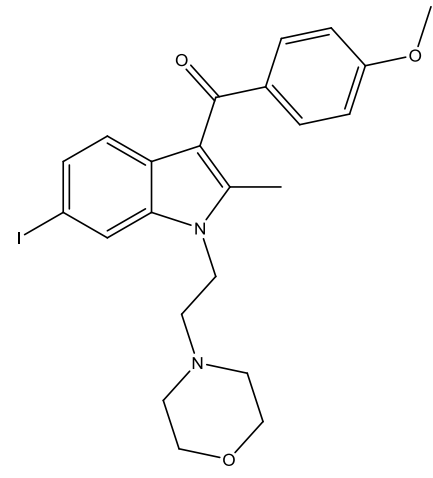

AM-630

$\mathrm{CB}_{1} \mathrm{~K}_{\mathrm{i}}=>5000 \mathrm{nM}$

$\mathrm{CB}_{2} \mathrm{~K}_{\mathrm{i}}=31.2 \mathrm{nM}$

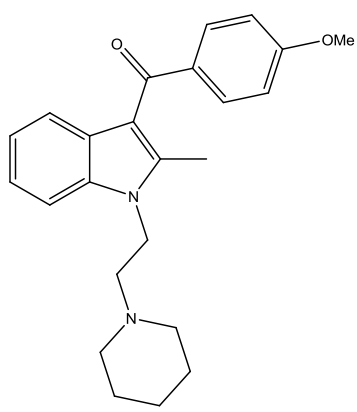

131

$\mathrm{IC}_{50}{ }^{\mathrm{b}}=34 \% @ 3000 \mathrm{nM}^{\mathrm{c}}$

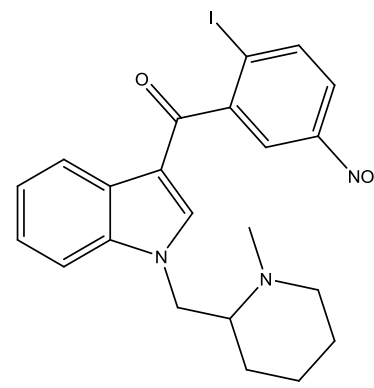

134

$\mathrm{CB}_{1} \mathrm{~K}_{\mathrm{i}}=280 \mathrm{nM}^{\mathrm{e}}$

$\mathrm{CB}_{2} \mathrm{~K}_{\mathrm{i}}=3.4 \mathrm{nM}^{\mathrm{e}}$

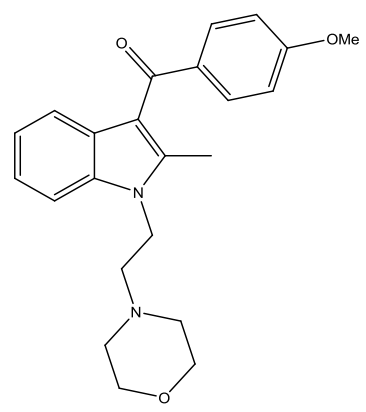

Pravadoline (129)

$\mathrm{CB}_{1} \mathrm{~K}_{\mathrm{i}}=2510 \mathrm{nM}^{\mathrm{a}}$

$\mathrm{CB}_{2} \mathrm{~K}_{\mathrm{i}}=320 \mathrm{nM}^{\mathrm{a}}$

$\mathrm{IC}_{50}{ }^{\mathrm{b}}=3155 \mathrm{nM}^{\mathrm{c}}$

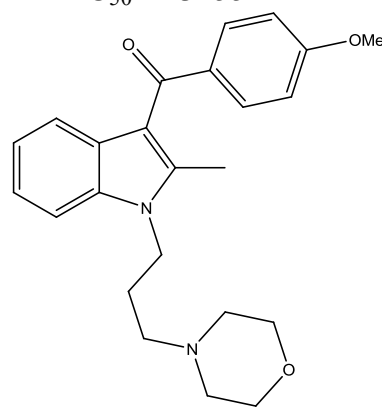

132

$\mathrm{IC}_{50}^{\mathrm{b}}=-6 \% @ 1000 \mathrm{nM}^{\mathrm{c}}$

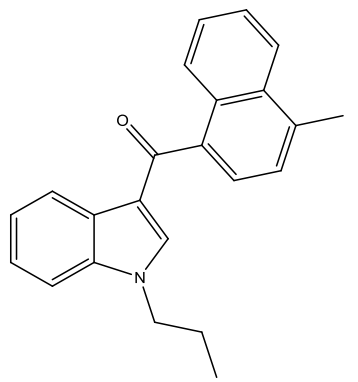

135

$\mathrm{CB}_{1} \mathrm{~K}_{\mathrm{i}}=1054 \mathrm{nM}^{\mathrm{f}}$

$\mathrm{CB}_{2} \mathrm{~K}_{\mathrm{i}}=6.1 \mathrm{nM}^{\mathrm{f}}$

Figure 4.10. Derivations of N-1 of aminoalkylindoles

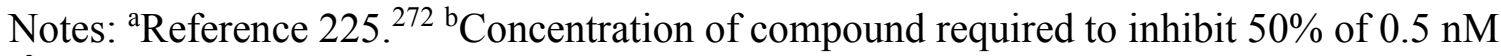
$\left[{ }^{3} \mathrm{H}\right] \mathrm{WIN}-55,212-2$ binding in rate cerebellum membranes as described in reference $\mathrm{XX}$. Values are the $\mathrm{IC}_{50}$ or percent inhibition at the highest tested dose $(\mathrm{nM})$. Negative values indicate stimulation rather than inhibition. ${ }^{\mathrm{c}}$ Reference $247 .{ }^{282} \mathrm{~d}$ Reference $248 .{ }^{283}$ ${ }^{\mathrm{e}}$ Reference $249 .{ }^{284}$ Reference $250 .{ }^{285}$

Within the aminoalkylindoles, four pharmacophoric elements exist: 
Study of WIN-55,212-2 led to a more refined understanding of the $\mathrm{CB}_{1}$ and $\mathrm{CB}_{2} \mathrm{LBP}$.

1. N-1 substituents

2. C-2 substituents

3. C-3 substituents

4. Indole ring substituents and derivatives

4.3.1.1. $\mathrm{N}-1$ substitutions of aminoalkylindoles. Other groups have synthesized and tested a number of indole analogs bearing different aminoalkyl substituents at $\mathrm{N}-1 .{ }^{282}$ One such study found the aminoethyl substitution as an optimal requirement with morpholino (129), thiomorpholino (130) and piperidino (131) analogs - aminopropyl morpholino (132) demonstrated an induced rather than inhibitory binding for $\left[{ }^{3} \mathrm{H}\right]-W I N-55,212-2$ (Figure 4.10). The respective acyclic amine, deemed to be possible metabolites, and piperazine analogs demonstrated activity similar to that of $\mathbf{1 3 2} .^{282}$

The Sterling Winthrop and Makriyannis laboratories further explored structural requirements at the $\mathrm{N}-1$ position by synthesizing novel analogs in which the aminoalkyl chain of the indole ring is attached to a heterocyclic amine through a $\mathrm{C}-\mathrm{C}$ bond. These analogs are generally more potent compared to the $\mathrm{C}-\mathrm{N}$ analogs and exhibit more favorable physicochemical properties. Potency was optimum for $\mathrm{N}$-methylpiperidinyl-2-methyl substitution at the N-1 position (133, Figure 4.10), with activity residing predominately in the $\mathrm{R}$-enantiomer. ${ }^{283}$

Mackriyannis, et al. developed AM-1241 (134, Figure 4.10), a highly $\mathrm{CB}_{2}$-selective and potent agonist. ${ }^{284,286}$ Design of this molecule incorporated the $\mathrm{N}$-methylpiperidinyl-2-methyl substituent at the $\mathrm{N}-1$ position and a novel 2-iodo-5-nitrobenzoyl group at C-3. AM-1241 exhibits remarkably high peripheral analgesia in vivo and does not produce catalepsy, hypothermia, inhibition of spontaneous locomotor activity or impairment of performance on the rotarod apparatus. This agent could thus see higher use in experimental neuropathic pain model experiments. ${ }^{287,288}$

Replacement of the aminoalkyl substituent by an alkyl chain results in N-alkyl indoles (non-aminoalkylindoles) (e.g., 135, Figure 4.10). The SAR of cannabimimetic 2methylindoles indicates that compounds with $\mathrm{N}$-alkyl substituents from n-propyl to nhexyl have good affinities for both $\mathrm{CB}_{1}$ and $\mathrm{CB}_{2}$ receptors and a preference for $\mathrm{CB}_{2}$. Previous reports of the in vivo potencies for these compounds are consistent with their receptor affinities. ${ }^{285,289,290}$

4.3.1.2. C-2 substituents of aminoalkylindoles. Analysis of the effect of C-2 substitution on cannabinoid receptor affinity in aminoalkylindoles reveals a strong preference for a small substituent at $\mathrm{C}$-2. Thus, hydrogen or methyl groups are well tolerated with the $\mathrm{C}-2 \mathrm{H}$ analogs (e.g., 136, Figure 4.11) $\left(\mathrm{CB}_{1} \mathrm{~K}_{\mathrm{i}}=245 \mathrm{nM} ; \mathrm{CB}_{2} \mathrm{~K}_{\mathrm{i}}=11\right.$ $\mathrm{nM}$ ) exhibiting slightly higher affinities for the $\mathrm{CB}_{2}$ than $\mathrm{C}-2$ methyl analogs (137, Figure 4.11) $\left(\mathrm{CB}_{1} \mathrm{~K}_{\mathrm{i}}=8 \mathrm{nM} ; \mathrm{CB}_{2} \mathrm{~K}_{\mathrm{i}}=29 \mathrm{nM}\right) .{ }^{282,291,292}$ Recently, researchers at BristolMyers-Squibb reported their discovery of indazole carboxamides (e.g., 138, Figure 4.11), replacing the $\mathrm{C}-2$ carbon of 3-amido aminoalkylindoles (136) with nitrogen. The indazole analog 138 exhibits high affinity for the $\mathrm{CB}_{2}$ receptor $(\mathrm{Ki}=2.0 \mathrm{nM})$ compared to the 


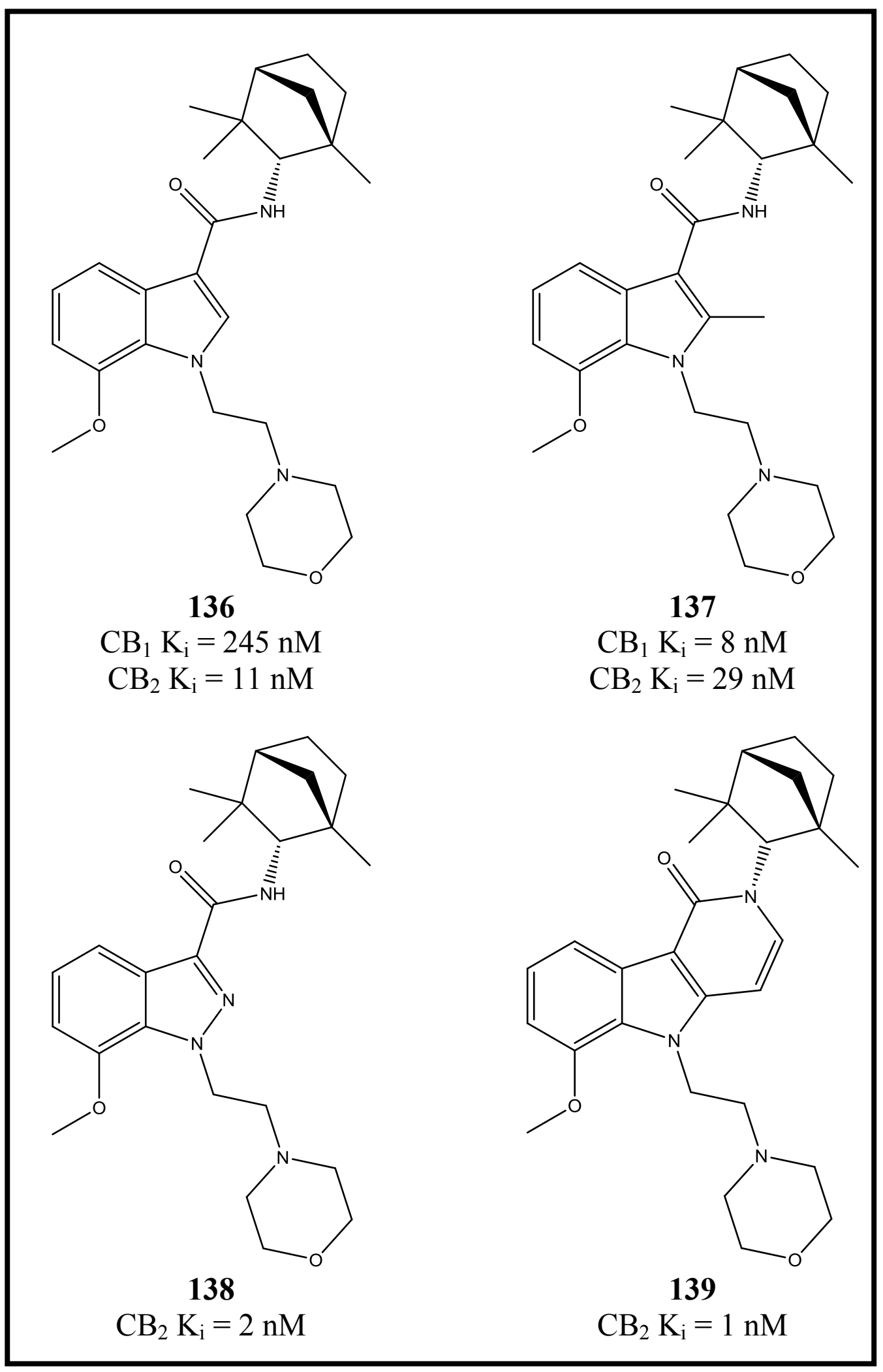

Figure 4.11. Modifications to C-2 of aminoalkylindoles 
corresponding AAI analogs. ${ }^{292}$ Indolopyridones (e.g., 139, Figure 4.11), which areconformationally restricted $\mathrm{C}-3$ amido aminoalkylindoles, exhibit increased affinities for the $\mathrm{CB}_{2}$ receptor $(\mathrm{Ki}=1.0 \mathrm{nM})$ and possess anti-inflammatory properties when administered orally in an in vivo murine inflammation model. ${ }^{292}$

4.3.1.3. C-3 substituents of aminoalkylindoles. Pravadoline (129, Figure 4.10), was originally developed as an NSAID to circumvent the gastric issues of currently available NSAIDs; however, it was found to be anti-nociceptive via $\mathrm{CB}_{2}$, and it serves as the prototype for elucidating C-3 substituent variations. ${ }^{280,282}$ Additionally, this compound preceded and led to the discovery of WIN-55,212-2. ${ }^{281,293}$ The $p$-methoxy of 129 gives good activity; however, ortho-substitution of the benzene ring with other groups such as $\mathrm{CH}_{3}, \mathrm{OH}, \mathrm{Cl}, \mathrm{CN}$, or $\mathrm{F}$ (e.g. 140, Figure 4.12) diminishes activity. The presence of an ethyl group at the para position (141, Figure 4.12) improves potency, but further increase in chain length results in diminished potency. The 1-naphthoyl (142, Figure 4.12) $\left(\mathrm{IC}_{50}=19 \mathrm{nM}\right)$ substitution at $\mathrm{C}-3$ is more potent than the 2-napthoyl (143, Figure 4.12) $\left(\mathrm{IC}_{50}=128 \mathrm{nM}\right)$ analog. Replacement of the naphthyl ring with an alkyl (e.g., $\mathrm{CH}_{3}$ ) or alkenyl $\left[\left(\mathrm{CH}_{3}\right)_{2} \mathrm{C}=\mathrm{CH}\right]$ groups results in complete loss of $\mathrm{CB}_{1}$ receptor affinity $\left(\mathrm{K}_{\mathrm{i}}>10,000 \mathrm{nM}\right){ }^{289}$

NMR and X-ray crystallography studies of $\mathbf{1 2 9}$ and its C-2 H analog have revealed that aminoalkylindoles can exist in two distinct conformations based on the orientation of the C-3 aryl system. ${ }^{260,280}$ In the s-trans (Figure 4.13A) conformation, which predominates when the $\mathrm{C}-2$ substitution is hydrogen, the aryl group is nearest $\mathrm{C}-2$, while the carbonyl oxygen locates near $\mathrm{C}-4$. In the s-cis (Figure 4.13B) conformation, which predominates when the $\mathrm{C}-2$ substituent is a methyl group, the conformational preference shows the aryl ring to be located near C-4, and the carbonyl oxygen near C-2.

Naphthylidene-substituted alkylindenes (e.g., 144, Figure 4.12) were originally designed to circumvent the CNS side effects of $\mathbf{1 2 9} .{ }^{294}$ These analogs were tested as a mixture of $\mathrm{E}$ - and $\mathrm{Z}$-isomers and exhibited higher $\mathrm{CB}_{1}$ affinity compared to 129. Later, it was shown that the $\mathrm{CB}_{1}$ and $\mathrm{CB}_{2}$ affinities and pharmacological potencies were higher for the E-isomer (144) compared to the Z-isomer. ${ }^{260} \mathrm{By}$ removing the carbonyl oxygen of the $\mathrm{C}-3$ aryl group in AAIs, having an unsubstituted $\mathrm{C}-2$, one gets a moderate reduction in affinity for $\mathrm{CB}_{1}$ relative to their carbonyl precursors. ${ }^{295}$ This loss of affinity is larger in the 2-methyl substituted analogs (145, Figure 4.12). Both observations support the hypothesis that the s-trans conformation of AAIs, such as WIN-55,212-2, is the preferred conformation for interaction at both $\mathrm{CB}_{1}$ and $\mathrm{CB}_{2}$ receptors, and that aromatic stacking of the ligands with aromatic residues in helices 3,4 , and 5 of both receptors may be an important interaction for AAIs at these receptors. ${ }^{260,295,296}$

The spatial and electronic requirements of the $\mathrm{C}-3$ substituent were further explored by Bristol-Myers-Squibb, introducing a C-3 amide group. The AAI C-3 amide ligand 146 (Figure 4.12) with a methoxy group at $C-7$, exhibited high $\mathrm{CB}_{2}$ affinity $\left(\mathrm{K}_{\mathrm{i}}=\right.$ $8 \mathrm{nM})$ and selectivity $\left(\mathrm{CB}_{1} / \mathrm{CB}_{2}=500\right) \cdot{ }^{291}$ Replacement of the amino acid moiety in 146 with the $S$-fenchylamine component resulted in slightly reduced affinity for the $\mathrm{CB}_{2}$ receptor $\left(\mathrm{K}_{\mathrm{i}}=30 \mathrm{nM}\right)$; however, when hydrogen replaced the 2-methyl group in indole, the resulting ligand $\left(\mathbf{1 4 7}\right.$, Figure 4.12) showed improved $\mathrm{CB}_{2}$ affinity $\left(\mathrm{K}_{\mathrm{i}}=11 \mathrm{nM}\right) \cdot{ }^{291}$ 


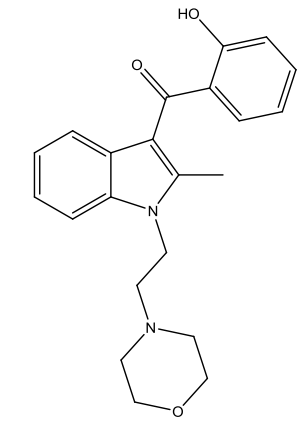

140

$\mathrm{IC}_{50}{ }^{\mathrm{a}}=-2 \% @ 1000 \mathrm{nM}$

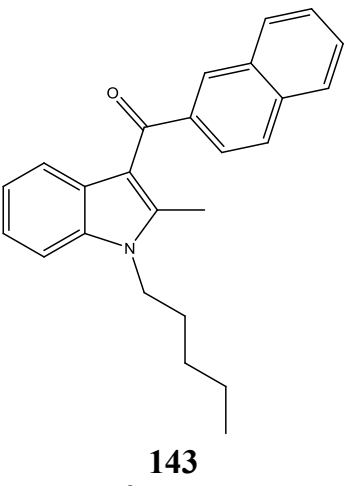

$\mathrm{IC}_{50}{ }^{\mathrm{a}}=128 \mathrm{nM}$

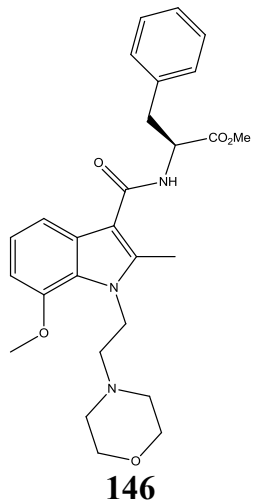

$\mathrm{CB}_{1} \mathrm{~K}_{\mathrm{i}}=4000 \mathrm{nM}$

$\mathrm{CB}_{2} \mathrm{~K}_{\mathrm{i}}=8 \mathrm{nM}$

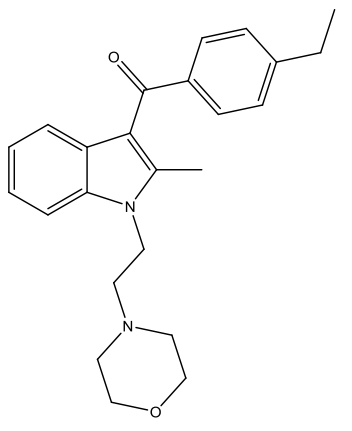

141

$\mathrm{IC}_{50}{ }^{\mathrm{a}}=306 \mathrm{nM}$

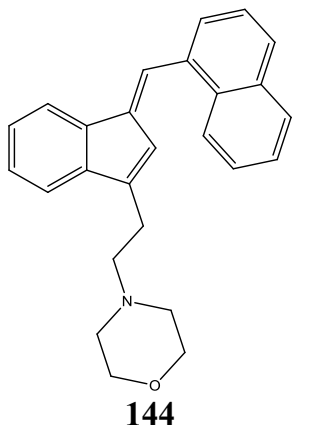

$\mathrm{IC}_{50}{ }^{\mathrm{a}}=1 \mathrm{nM}$

A $\quad \mathrm{R}=\mathrm{H} ; \mathrm{CB}_{1} \mathrm{~K}_{\mathrm{i}}=113 \mathrm{nM}$

B $\mathrm{R}=\mathrm{CH}_{3} ; \mathrm{CB}_{1} \mathrm{~K}_{\mathrm{i}}=41 \mathrm{nM}$

C $\mathrm{R}=\mathrm{OMe} ; \mathrm{CB}_{1} \mathrm{~K}_{\mathrm{i}}=20 \mathrm{nM}$

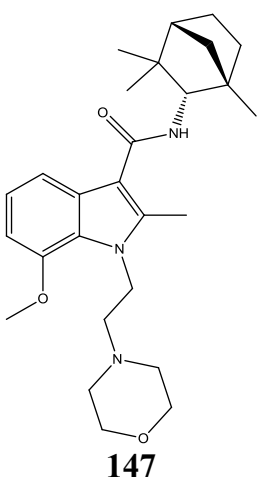

$\mathrm{CB}_{1} \mathrm{~K}_{\mathrm{i}}=245 \mathrm{nM}$

$\mathrm{CB}_{2} \mathrm{~K}_{\mathrm{i}}=11 \mathrm{nM}$

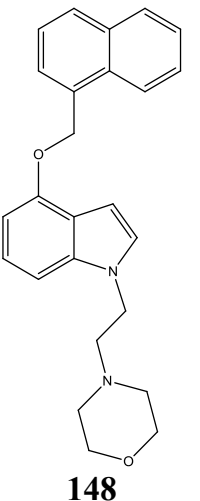

$\mathrm{CB}_{1} \mathrm{~K}_{\mathrm{i}}=221 \mathrm{nM}$

Figure 4.12. Modifications to C-3 of aminoalkylindoles

${ }^{a}$ Concentration of compound required to inhibit $50 \%$ of $0.5 \mathrm{nM}\left[{ }^{3} \mathrm{H}\right]$ WIN-55,212-2 binding in rate cerebellum membranes as described in reference 238 . Values are the $\mathrm{IC}_{50}$ or percent inhibition at the highest tested dose (nM). Negative values (140) indicate stimulation rather than inhibition. 


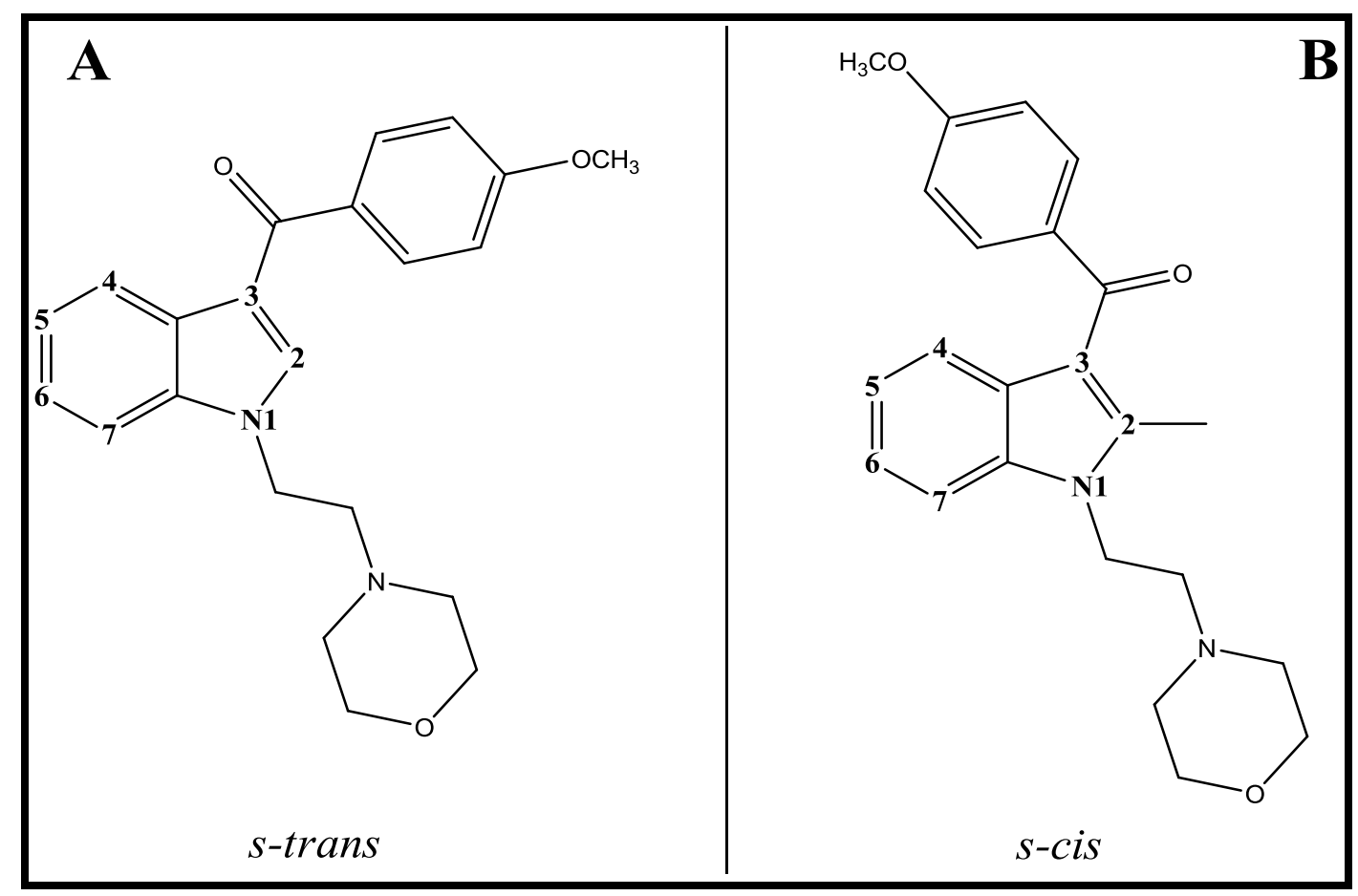

Figure 4.13. Confirmations of Pravadoline (129) 
Derivation of the 4-alkyloxy indole analogs (e.g., 148, Figure 4.12) resulted from the translocation of the C-3 substituent of AAIs to C-4 via an ether linkage. Some of these exhibited in vivo cannabimimetic activity, but most of them lacked cannabinoid receptor affinity. ${ }^{297}$

4.3.1.4. Indole Ring Substituents and Modifications. Introduction of a methyl group at C-4 or various substituents such as $\mathrm{CH}_{3}, \mathrm{OCH}_{3}, \mathrm{~F}, \mathrm{Br}$ or $\mathrm{OH}$ at $\mathrm{C}-5$ of 129 diminishes affinity. Conversely, $\mathrm{C}-6$ substitution with $\mathrm{CH}_{3}, \mathrm{OCH}_{3}$ or $\mathrm{Br}$ groups improves receptor affinity, but the ligands exhibit diminished agonist properties. ${ }^{282}$ Incorporation of an iodo group at C-6 led to AM-630 (Figure 4.10), a ligand that exhibits improved affinity as well as selectivity for $\mathrm{CB}_{2}{ }^{298,299}$ This compound is selective inverse agonist for $\mathrm{CB}_{2},{ }^{300}$ and I have since confirmed confirmed through use of the ACTOne assay.

Substitution at C-7 (Figure 4.11; Figure 4.12) gives modest improvement in binding affinity. Potent analogs came to be by conformationally restricting the $\mathrm{N}-1$ side chain through the formation of a six membered ring between the N-1 and C-7 substituents (not shown). ${ }^{281}$ For $\mathrm{N}$-alkyl indoles, replacement of the indole phenyl ring with a cyclohexyl ring led to an analog with reduced affinities for both $\mathrm{CB}_{1}$ and $\mathrm{CB}_{2}{ }^{301}$ Removal of the phenyl ring in AAIs or non-AAIs led to a pyrrole class. ${ }^{290,301}$ Most of the pyrrole-derived analogs are less potent than the corresponding indole derivatives; however, the 4-bromopyrrole analog shows high affinity for $\mathrm{CB}_{1}$ and $\mathrm{CB}_{2}\left(\mathrm{EC}_{50}=13.3\right.$ $\mathrm{nM}$ for $\mathrm{rCB}_{1}$ and $6.8 \mathrm{nM}$ for $\mathrm{hCB}_{2}$ ), comparable to $\mathrm{WIN}-55,212-2 .{ }^{301}$

\subsubsection{Diarylpyrazoles}

The most widely studied compound of the diarylpyrazole class is SR-141716A (Rimonabant) (Figure 4.14) developed by Rinaldi-Carmona and co-workers at Sanofi. ${ }^{302}$ Originally touted as a "wonder drug" for use in anti-obesity treatment, it was approved for use in Europe in 2006; however, it was withdrawn from the market in 2009 for the same reason approval was never granted in the United States of America - psychiatric side effects, some quite serious. ${ }^{211}$ This highly potent and selective $\mathrm{CB}_{1}$ receptor ligand has served as a unique pharmacological and biochemical tool for further characterization of the $\mathrm{CB}_{1}$ cannabinoid receptor ${ }^{303,304}$ - serving as the prototype $\mathrm{CB}_{1}$ inverse agonist for evaluation of the compounds presented herein (Chapter 2). In vitro, SR-141716A antagonizes the inhibitory effects of cannabinoid agonists on both mouse vas deferens contractions $^{305}$ and adenylyl cyclase activity. ${ }^{306}$ SR-141716A also antagonizes the pharmacological and behavioral effects produced by $\mathrm{CB}_{1}$ agonists after intraperitoneal (IP) or oral (PO) administration. ${ }^{302}$

Other diarylpyrazole ligands that have contributed to our understanding of $\mathrm{CB}_{1}$ pharmacology are AM-251 and AM-281, both of which are specialized $\mathrm{CB}_{1}$ antagonists known as inverse agonists (Figure 4.14), and capable of displacing $\left[{ }^{3} \mathrm{H}\right] \mathrm{SR}-141716 \mathrm{~A}$ and $\left[{ }^{3} \mathrm{H}\right] \mathrm{CP}-55,940$ in CB1 receptor membrane preparations. ${ }^{303}$ Both AM-251 and AM-281 share the ability of SR-141716A to attenuate the responses to established cannabinoid receptor agonists like WIN-55,212-2 or CP-55,940; however, data show that AM-251 may have a more "CB 1 -selective" role than SR-141716A. ${ }^{307}$ In addition to AM-630 (93), the most notable $\mathrm{CB}_{2}$ receptor inverse agonist is SR-144528 (Figure 4.14), a 


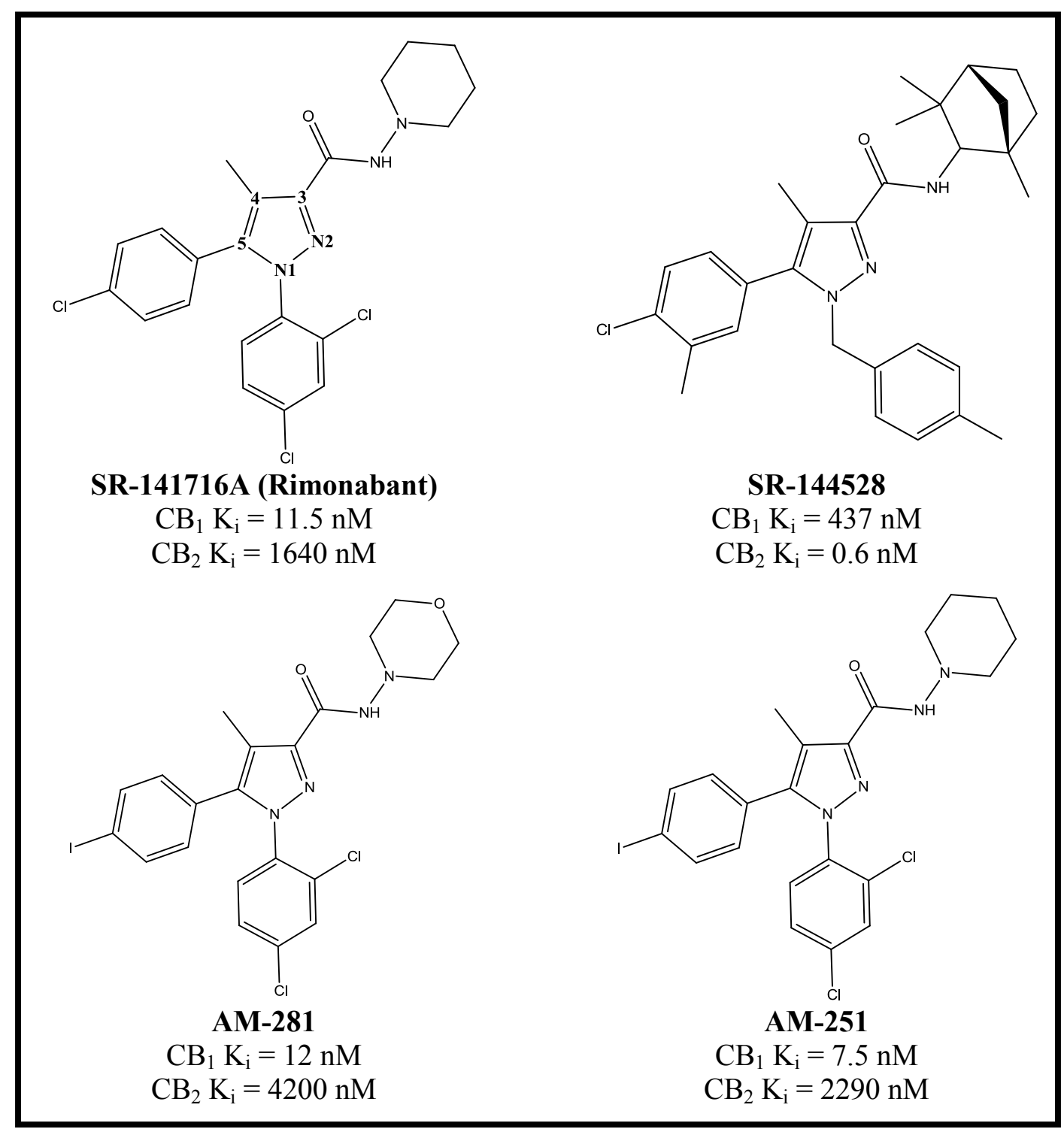

Figure 4.14. Known standards of diarylpyrazole class of cannabinoid ligands 
diarylpyrazole developed by Sanofi, exhibiting 700-fold selectivity for the $\mathrm{CB}_{2}$ receptor over $\mathrm{CB}_{1}{ }^{308}$ Structural requirements for SR-141716A-like compounds are similar to those found in the aminoalkylindoles:

1. N-1 substituents

2. C-3 substituents

3. C-4 substituents

4. C-5 substituents

4.3.2.1. N-1 substituents of diarylpyrazoles. 2,4-Dichlorophenyl (SR-141716A) is the optimal substituent for both high $\mathrm{CB}_{1}$ affinity and subtype selectivity. ${ }^{303,309}$ Its replacement with (5-isothiocyanate)-pentyl (149, Figure 4.15) group decreased $\mathrm{CB}_{1}$ affinity by a factor of four. ${ }^{310}$ The inclusion of strictly a phenyl (150, Figure 4.15), 4-butylphenyl (151, Figure 4.15) or a 4-pentylphenyl (152, Figure 4.15) group at N-1 significantly reduces affinity ${ }^{311,312}$ while n-pentyl (153, Figure 4.15), n-hexyl (154, Figure 4.15), n-heptyl (155, Figure 4.15) substitution retains good affinity, relative to SR-141716A. ${ }^{311}$ A 4-methylbenzyl group as represented in SR-144528 contributes optimal selectivity for CB2. ${ }^{308}$

In the 2,4-dichlorophenyl moiety of SR141716A, elimination of $p$-chloro (156, Figure 4.16) substitution of o-chloro with o-fluoro (157, Figure 4.16) or o-methoxy (158, Figure 4.16) groups led to low-affinity analogs. ${ }^{313}$ Replacement of the 2,4dichlorophenyl moiety of SR141716A with cycloalkyl (159, Figure 4.16) groups decreased both $\mathrm{CB}_{1}$ and $\mathrm{CB}_{2}$ affinities, while the 3-methyl and 4-methylcyclohexyl analogs exhibited moderate improvement in $\mathrm{CB}_{2}$ affinity without any enhancement in selectivity compared to SR-141716A. ${ }^{314}$

4.3.2.2. C-3 substituents of diarylpyrazoles. Alkylation of the amide group (e.g. 160) as well as replacement of the amide group by a ketone, alcohol or ether (e.g. 161) greatly decreases $\mathrm{CB}_{1}$ affinity (Figure 4.17) ${ }^{312,315}$ Replacement of the piperidinyl group with the respective five- or seven-membered heterocyclic rings or by a cyclohexyl group (e.g. 162, Figure 4.17) does not alter $\mathrm{CB}_{1}$ binding affinity. While replacement of the piperidinyl group with a morpholine group (163, Figure 4.17) leads to reduction in $\mathrm{CB}_{1}$ affinity. However, this seems to be true only in cases where the C-5-p-phenyl substituent is not iodine, as AM-281 retains good activity and excellent specificity. ${ }^{303}$ To probe the structural and steric requirements of this pharmacophore, alkyl hydrazines, amines and hydroxyalkylamines replace the aminopiperidinyl moiety. ${ }^{316}$ For alkylamides, hydroxyalkyl amides and alkyl hydrazides, affinity for $\mathrm{CB}_{1}$ increased directly with increasing chain length (across this group of compounds) from ethyl to butyl up to and including pentyl. Increasing the carbon chain length beyond pentyl reduces affinity for both receptors. Alkylamide analogs (e.g. 164, Figure 4.17) exhibited enhanced $\mathrm{CB}_{1}$ selectivity when compared to SR-141716A, conversely hydroxyalkyl amide and alkylhydrazide analogs (e.g. 165, Figure 4.17) had both decreased affinities and equivalent to slightly worse selectivity for $\mathrm{CB}_{1}$ or $\mathrm{CB}_{2} .{ }^{316}$

4.3.2.3. C-4 substituents of diarylpyrazoles. Compounds with methyl, ethyl, bromo, or iodo substituents in the 4-position of the pyrazole ring are approximately 


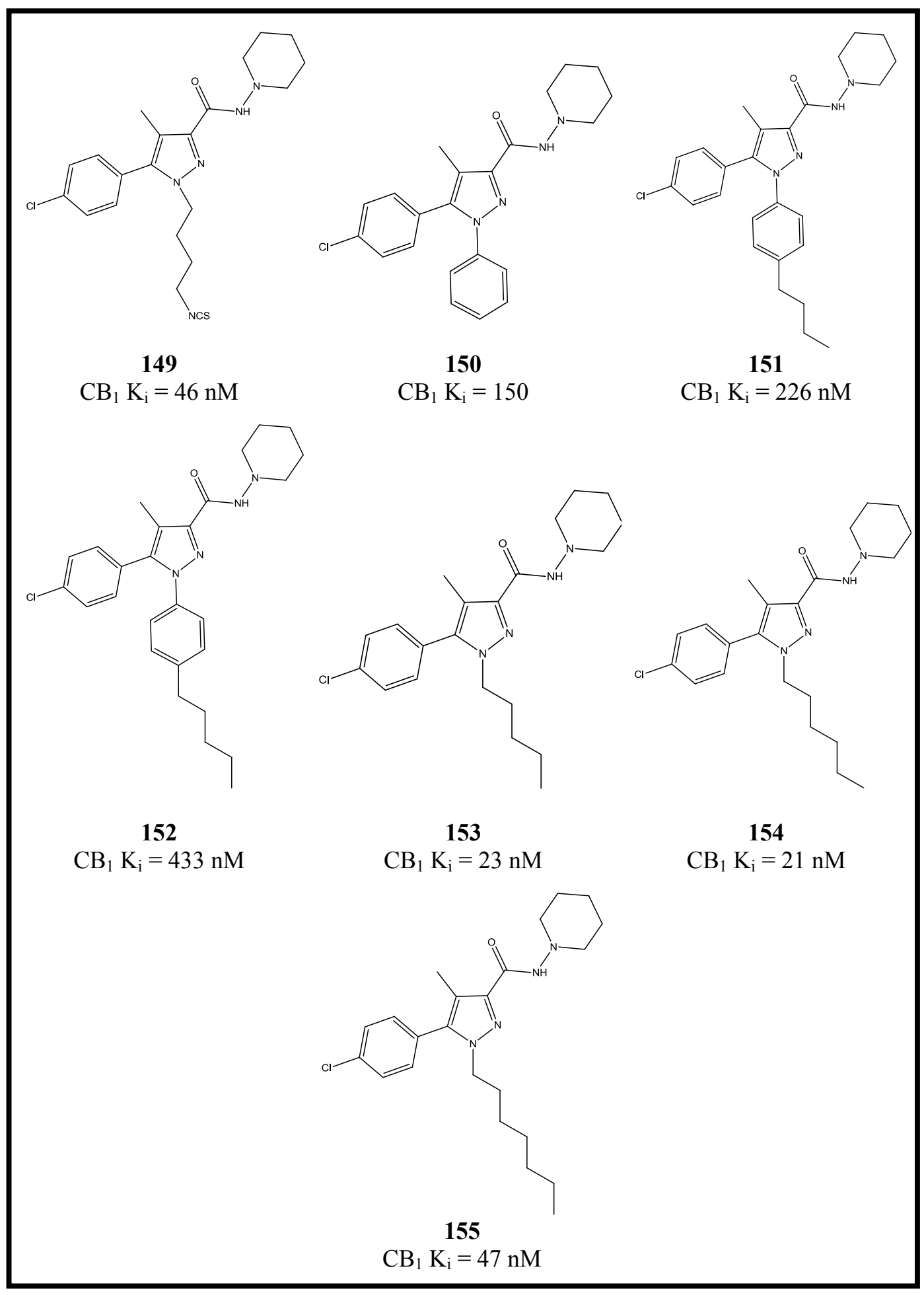

Figure 4.15. Alkyl N-1 modifications of diarylpyrazoles 


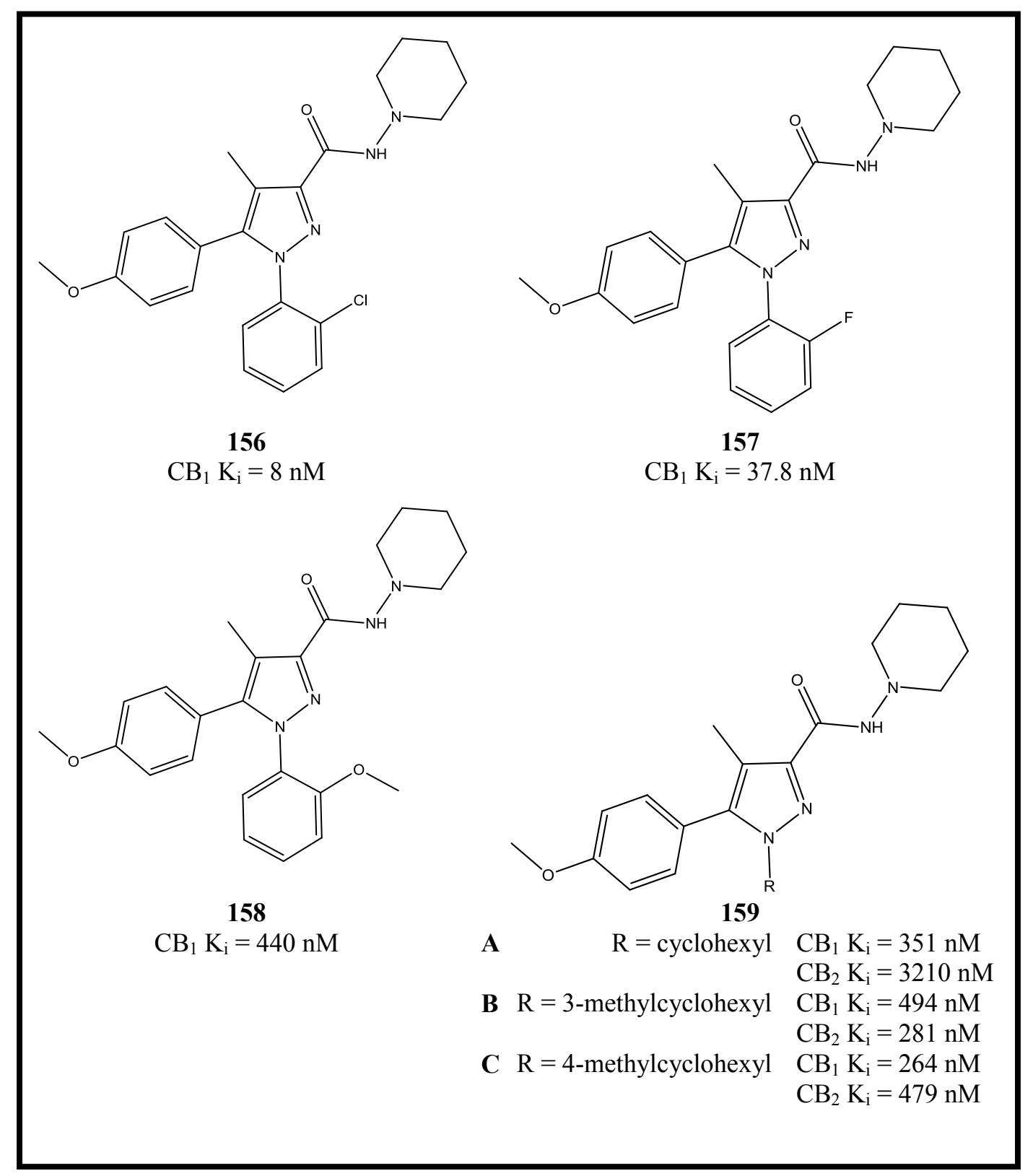

Figure 4.16. Aromatic and cycloalkyl modifications to N-1 of diarylpyrazoles 


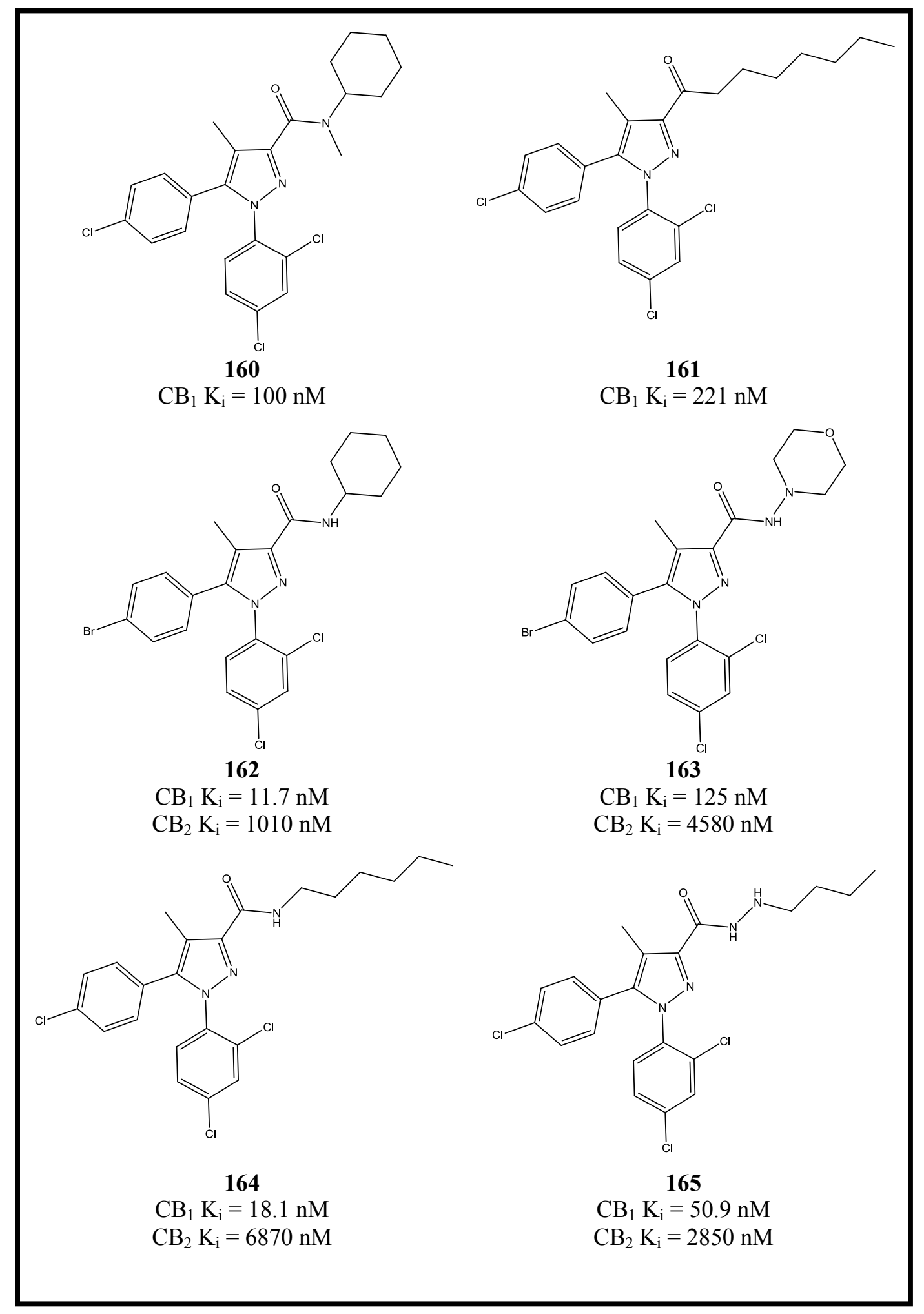

Figure 4.17. Modifications of C-3 of diarylpyrazoles 
equipotent, whereas replacement of methyl with hydrogen results in a 12-fold decrease in $\mathrm{CB}_{1}$ affinity. ${ }^{312}$

4.3.2.4. C-5 substituents of diarylpyrazoles. The 4-chloro group of the phenyl ring can be replaced by bromo (e.g. 162) or alkyl groups (e.g. 156) but not by nitro or amino groups. ${ }^{22,303,312}$ Replacement of 4-chloro with a 4-iodo substituent (e.g. AM-251) leads to significant $\mathrm{CB}_{1}$ affinity and selectivity. Conversely, replacement of the aromatic ring with alkyl groups abolishes $\mathrm{CB}_{1}$ affinity. ${ }^{303}$

Solvay first reported some rigid SR-141716A, tricyclic $\mathrm{CB}_{1}$-selective ligands in which the 4- and 5-substituents are conformationally restricted through the formation of a relatively rigid tricyclic system. ${ }^{272,317}$ In these compounds the 4-methyl group is connected with the ortho position of the aromatic 5-aryl substituent to form benzocycloheptapyrazole analogs, i.e. 166 (Figure 4.18) that exhibited higher $\mathrm{CB}_{1}$ affinity than the parent, SR-141716A..$^{272,317}$ Later Pinna and co-workers reported similar tricyclic pyrazole analogs in which the additional 7-membered ring of 166 was replaced by a five-membered ring (e.g. 167, Figure 4.18). ${ }^{318}$ Of note, most ligands in this class had high affinity and selectivity for $\mathrm{CB}_{2}$ compared to 166 and SR-141716A.

Solvay also reported a novel class of 3,4-disubstituted pyrazoline analogs exhibiting high $\mathrm{CB}_{1}$ selectivity (e.g., 168, Figure 4.18). ${ }^{319}$ Another novel class of $\mathrm{CB}_{1}$ antagonists that has received only limited attention includes the 3-alkyl-5-arylhydantoins (e.g. 169, Figure 4.18). ${ }^{320}$

\subsection{Novel Series of C-1'-Dimethyl-Hexahydro-Pyridine/Pyrimidine-THC Analogs (Hexahydro Series)}

\subsubsection{Rationale of design}

Previous work from our group saw the synthesis of cycloalkyl ${ }^{234}$ and aromatic ${ }^{235}$ substituents introduced at C-1'. Both groups of compounds saw marked improvement in affinity for $\mathrm{CB}_{1}$ and $\mathrm{CB}_{2}$ receptors (Chapter 3). To further expand upon this discovery, the novel pyridine and pyrimidine hexahydro compounds (Table 4.2) attempt to incorporate the advantages of the hexahydro compound's increased potency ${ }^{271,272,276}$ as well as the increase in selectivity for $\mathrm{CB}_{2}$ added by addition of nitrogen into the $\mathrm{ABC}$ ring system. ${ }^{264,271,272}$ The binding data (Table 4.3) do show that the addition of the nitrogen(s) to the A-ring does not negatively impact the binding affinity relative to the carbocyclic hexahydros. In fact, this small change lends some increase and a measure of selectivity toward CB2. With an increase in binding affinity, coupled with the presence of favorable potential $\mathrm{H}$-bonding interactions within the $\mathrm{LBP}$ of both $\mathrm{CB}_{1}$ and $\mathrm{CB}_{2}$ and the potential for a more therapeutically favorable preference of $\mathrm{CB}_{2}$ over $\mathrm{CB}_{1}$, a need existed to establish a functional mechanism for these compounds.

Analysis of compound 172 via ${ }^{1} \mathrm{H}_{-}{ }^{15} \mathrm{~N}$ HSQC, ${ }^{1} \mathrm{H}-{ }^{13} \mathrm{C}$ HSQC and ${ }^{1} \mathrm{H}-{ }^{1} \mathrm{H}$ NOSY shows that the compound exists as the acetamide form (Figure 4.19). A diagnostic amide peak in the IR sepctra $(1640$ - 1690) was used to confirm that amide structure of the 


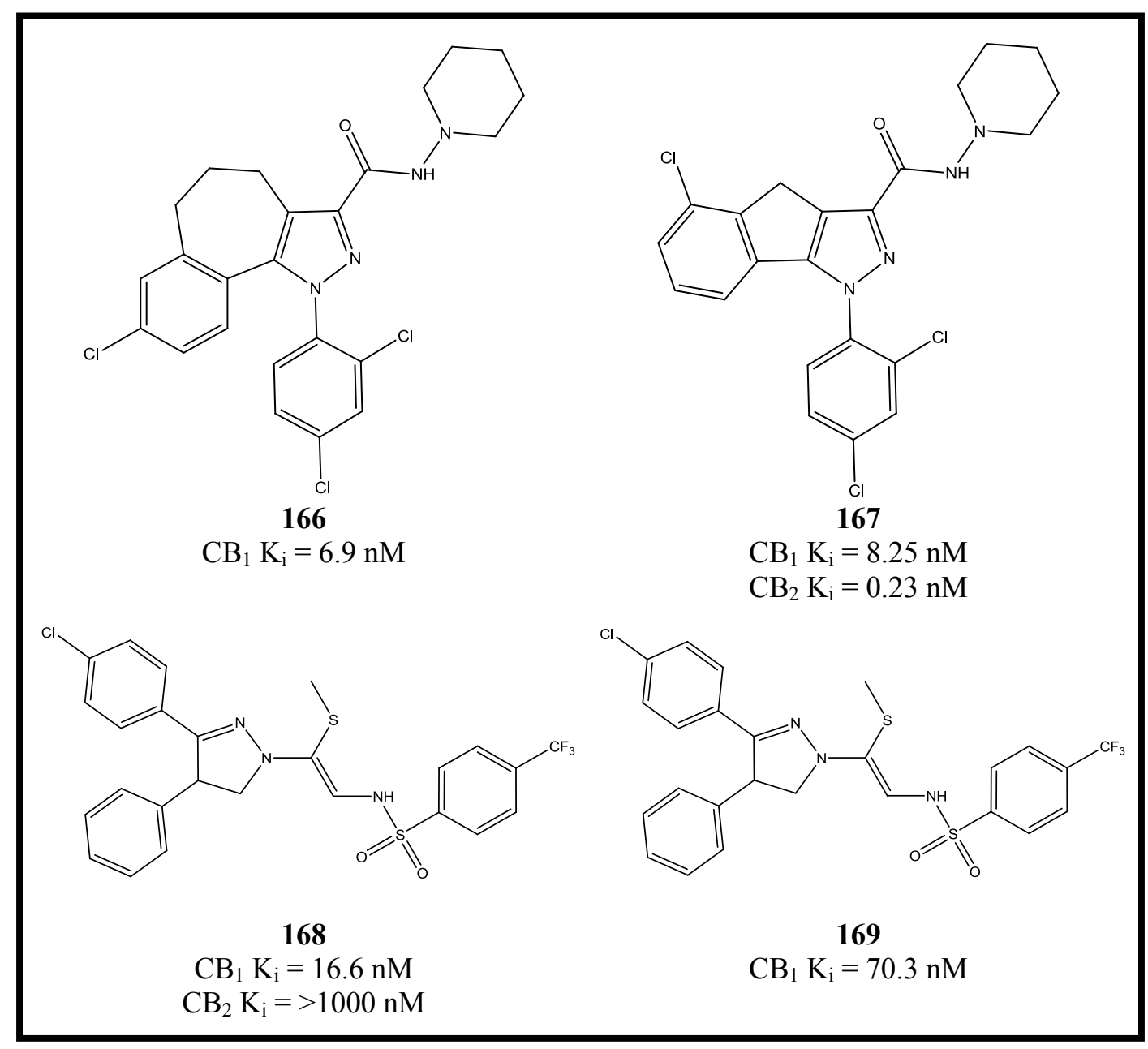

Figure 4.18. Conformationally constrained and non-traditional diarylpyrazoles 
Table 4.2. Hexahydro-THC derivatives

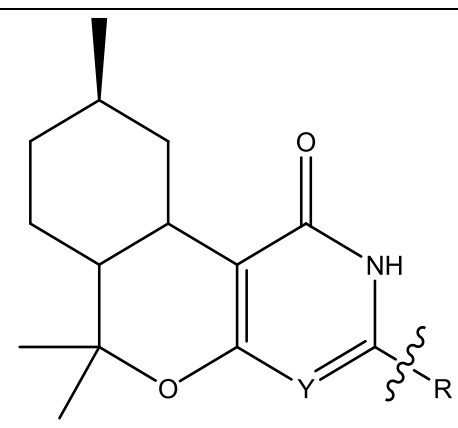

Pyridine Hexahydro-THC

Pyrimidine Hexahydro-THC

Compound $\mathrm{Y}$


Table 4.3. Binding affinities for hexahydro series

\begin{tabular}{ccc}
\hline Compound & CB $_{1} \mathbf{K}_{\mathbf{i}}(\mathbf{n M})$ & $\mathbf{C B}_{\mathbf{2}} \mathbf{K}_{\mathbf{i}}(\mathbf{n M})$ \\
\hline $\mathbf{1 7 0}$ & $1481(\mathrm{~N}=1)$ & $678( \pm 347.1)$ \\
$\mathbf{1 7 1}$ & - & - \\
$\mathbf{1 7 2}$ & - & $83( \pm 42.51)$ \\
$\mathbf{1 7 3}$ & - & - \\
$\mathbf{1 7 4}$ & $55(\mathrm{~N}=1)$ & $8.33( \pm 1.53)$ \\
$\mathbf{1 7 5}$ & $18.5( \pm 16.26)$ & $1.67( \pm 1.15)$ \\
$\mathbf{1 7 6}$ & $>10,000$ & $137( \pm 13.23)$ \\
$\mathbf{1 7 7}$ & - & $52.33( \pm 28.18)$ \\
\hline
\end{tabular}




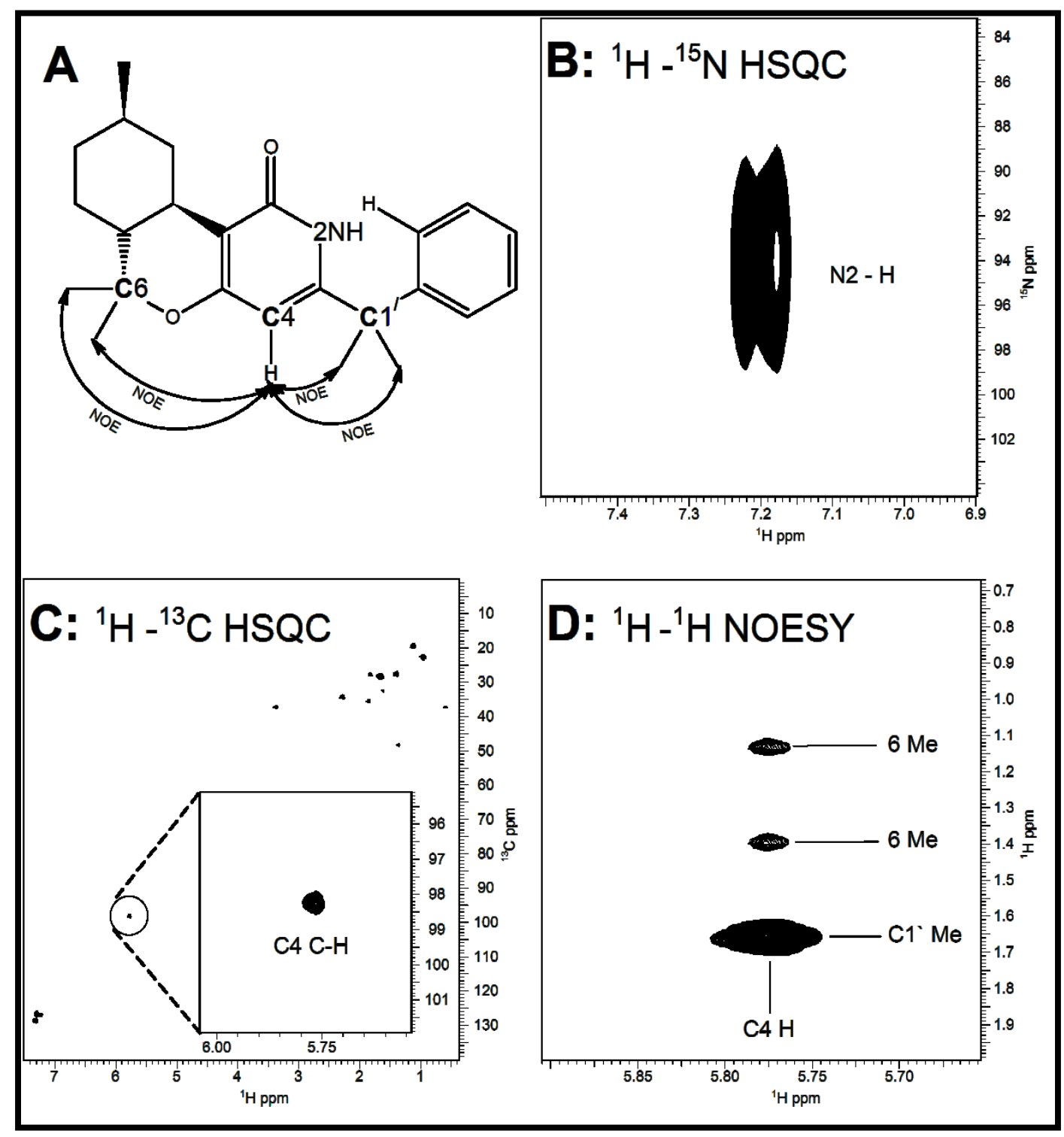

Figure 4.19. NMR structure confirmation of the pyridine hexahydro compounds

Notes: Panel A shows the actual structure of compound $\mathbf{1 7 2}$ in solution, and a possible way (in an aqueous environment) it would exist when interacting with the LBP. Panel B shows the existence of the $\mathrm{N}-\mathrm{H}$ and thus amide composition of the A-ring. Panel $\mathrm{C}$ shows that position 4 is $\mathrm{C}$, and thus the $\mathrm{N}-\mathrm{H}$ must be at position 2 of the A-ring. Panel D shows the NOE correlation between the C-4 hydrogen and the C-6 methyl groups (weak) and C1 ' methyl groups (strong). 
remaining pyridine-related structures. Initial findings via the ${ }^{1} \mathrm{H}-{ }^{15} \mathrm{~N}$ HSQC show that the nitrogen of the pyridine ring exists, at least in solvent, with a hydrogen atom attached to it; however, the question was if it remained at the 2 or 4 position of the A-ring. ${ }^{1} \mathrm{H}-{ }^{13} \mathrm{C}$ HSQC demonstrated that the 4 position was, indeed, a carbon with a hydrogen attached ${ }^{1} \mathrm{H}-{ }^{1} \mathrm{H}$ NOSY confirmed this finding by demonstrating weak coupling into both $\mathrm{C}-6$ methyl moieties and very strongly into both $\mathrm{C}-1$ ' methyl moieties.

\subsubsection{Objectives of the current study}

Utilizing this novel series of compounds, I explored the functional pharmacology of each in relation to $\mathrm{CB}_{1}, \mathrm{CB}_{2}$ and the parental HEK-293-CNG cell lines (covered in greater detail in Chapter 2) to better understand the mechanism of action (MoA) in a cell-based functional assay. Furthermore, to aid in explanation of the observed functional data, I will reconcile known literature with regard to binding and homology models.

\subsection{Functional Pharmacology}

\subsubsection{ACTOne assay}

Assay development and conductance, discussed in detail in Chapter 2. Briefly, stably transfected HEK-293 cells co-expressed with modified CNG channels along with $\mathrm{CB}_{1}$ or $\mathrm{CB}_{2}$ or their parental cells (without $\mathrm{CB}_{1}$ or $\mathrm{CB}_{2}$; HEK-CNG) were plated at an appropriate density in a clear, 96-well, Poly-D-Lysine plate and allowed to incubate at $37^{\circ} \mathrm{C}$ and $5 \%$ overnight. After the overnight incubation period, I added $100 \mu \mathrm{L}$ of ACTOne membrane potential dye to each well of the culture plate and allowed to sit in a dark area at room temperature for 60 additional minutes. After the 60 minute incubation period a baseline read in a BioTek Synergy 2 plate reader with an excitation of 540/25 $\mathrm{nm}$ and an emission of 590/20 nm. Following the baseline read, $50 \mu \mathrm{L}$ of drug stock solution containing (Figure 4.20): appropriate concentrations of drug, Forskolin and Ro 20-1724 in DPBS with 2.5\% DMSO was added to the cell culture plate. The plate was then placed on the plate reader with 1 read per well per minute with an excitation of $540 / 25 \mathrm{~nm}$ and an emission of 590/20 nm for 60 minutes.

Screening of compounds against $\mathrm{CB}_{1}$ and $\mathrm{CB}_{2}$ were completed as technical replicates of $n=6$ each. Screenings against HEK-CNG Parental and $\mathrm{CB}_{1} / \mathrm{CB}_{2}$ PTxsensitive $\mathrm{G}_{\mathrm{i} / \mathrm{o}}$-inactivation were completed as technical replicates of $\mathrm{n}=3$ each. Utilizing the lower number of replicates for HEK-CNG Parental and $\mathrm{CB}_{1} / \mathrm{CB}_{2} \mathrm{PTx}$-sensitive $\mathrm{G}_{\mathrm{i} / \mathrm{o}}$-inactivation was employed due to the expected lack of response from these compounds to those three (3) groups of assays, in an effort to maximize time and resource management without compromising data integrity or statistical significance.

Primary data were analyzed with customized VBA macros (Chapter 2), developed internally, within Microsoft Excel (Redmond, WA). The experimental maximum response (100\% activity) was determined by Column 11 (lowest [drug]) at each individual time point, and the experimental basal response was determined by the 


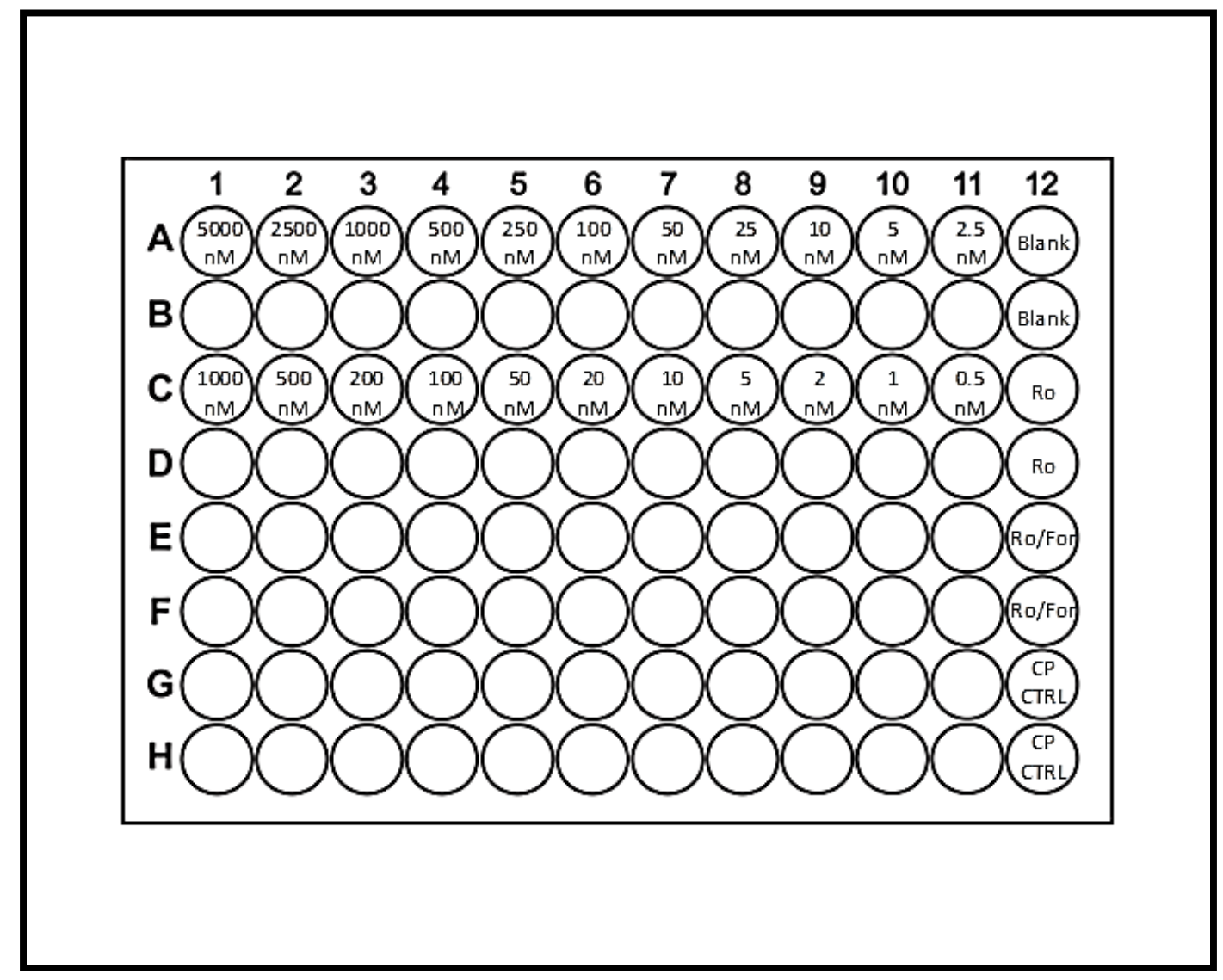

Figure 4.20. Drug plate layout and concentrations

Notes: Columns 1 through 11 contain test compound, and Column 12 contains control wells. All listed concentrations are the final concentrations once added to the testing plate, so in the drug plate all compounds are at $5 \mathrm{x}$ of the listed concentrations in Columns 1 through 11. Control wells (Column 12) are: "Blank" (cells, media and dye), "Ro" ("Blank" + $125 \mu \mathrm{M}$ Ro 20-1724 [25 $\mu \mathrm{M}$, Final]), "Ro/For" ("Ro" $+4 \mu \mathrm{M}$ Forskolin [800 nM, Final]) and "CP CTRL" ("Ro/For" $+25 \mu \mathrm{M}$ CP-55,940 [5 $\mu \mathrm{M}$, Final]). 
average response given by the 2 "Ro" control wells. All data were normalized with these minimum and maximum values via Equation 2.1. The $\mathrm{EC}_{50}$ values were calculated from concentration-response curves by non-linear regression and automatic outlier elimination analysis utilizing GraphPad Prism Software (San Diego, CA).

\subsection{2. $\mathrm{CB}_{1}$ functional activity for the Hexahydro series}

Plates were run as previously described (Section 4.5.1) in six (6) replicates with CP-55,940 as the internal control for all six (6) replicates (Figure 4.21; Table 4.4). All compounds, with the exception of compound 176, bind with good affinity to the $\mathrm{CB}_{1}$ receptor. ${ }^{259}$ Compounds 170, 171, 174 and 175 (Figure 4.22) demonstrated good agonist activity at $\mathrm{CB}_{1}$ with an $\mathrm{EC}_{50}$ of $78.9 \mathrm{nM}, 209 \mathrm{nM}, 276 \mathrm{nM}$ and $56.3 \mathrm{nM}$, respectively, with percent $\mathrm{CAMP}$ inhibition ranging from 72 to $77 \%, 50$ minutes after compound addition into the assay plate. These three (3) compounds exhibit a decrease in cAMP production equivalent to our standard of CP-55,940, albeit at a higher $\mathrm{EC}_{50}$ than for $\mathrm{CP}$ 55,940 . Special notation should be given to compound $\mathbf{1 7 3}$, because it shows itself to be a special type of antagonist, known as an inverse agonist - with an $\mathrm{EC}_{50}$ of $48.9 \mathrm{nM}$.

\subsection{3. $\mathrm{CB}_{2}$ functional activity for the Hexahydro series}

Plates were run as previously described (Section 4.5.1) in six (6) replicates with CP-55,940 as the internal control for all six (6) replicates (Figure 4.23; Table 4.4). All compounds in the series bind with good affinity to the $\mathrm{CB}_{2}$ receptor. ${ }^{259}$ As was the case with the $\mathrm{CB}_{1}$ functional activity assays, compounds 170, 171, 174 and 175 (Figure 4.24) demonstrated agonist activity; however, they were not equipotent in terms of $\mathrm{EC}_{50}$ or percent cAMP inhibited at $\mathrm{CB}_{2}$ compared to $\mathrm{CB}_{1}$. $\mathrm{EC}_{50}$ for compounds 170, 171, 174 and 175 were $333 \mathrm{nM}, 835 \mathrm{nM}, 682.1 \mathrm{nM}$ and $66.8 \mathrm{nM}$, respectively, with percent cAMP inhibition ranging from 27 to $51 \%$. Interestingly, compound 173 did not exhibit its inverse agonist activity against $\mathrm{CB}_{2}$, as it had for $\mathrm{CB}_{1}$.

\subsubsection{CNG parental functional activity for the Hexahydro series}

Plates were run as previously described (Section 4.5.1) in three (3) replicates with CP-55,940 as the internal control for all three (3) replicates (Figure 4.25; Table 4.4). As was expected, all compounds in the series failed to elucidate any change in the cAMP production of the cells, including the reference standard of CP-55,940.

\subsubsection{CB1 PTx-sensitive $G_{i} / 0$-inactivation for the Hexahydro series}

Plates were run as previously described (Section 4.5.1) in three (3) replicates with CP-55,940 as the internal control for all three (3) replicates (Figure 4.26; Table 4.4). All compounds failed to alter cAMP production, including the reference standard of CP-55,940. Only compounds 170, 171, 174 and 175 were screened in the PTx-sensitive $\mathrm{G}_{\mathrm{i} / \mathrm{o}}$-inactivation assays due to their ability to elucidate a response in the standard $\mathrm{CB}_{1}$ assay. 


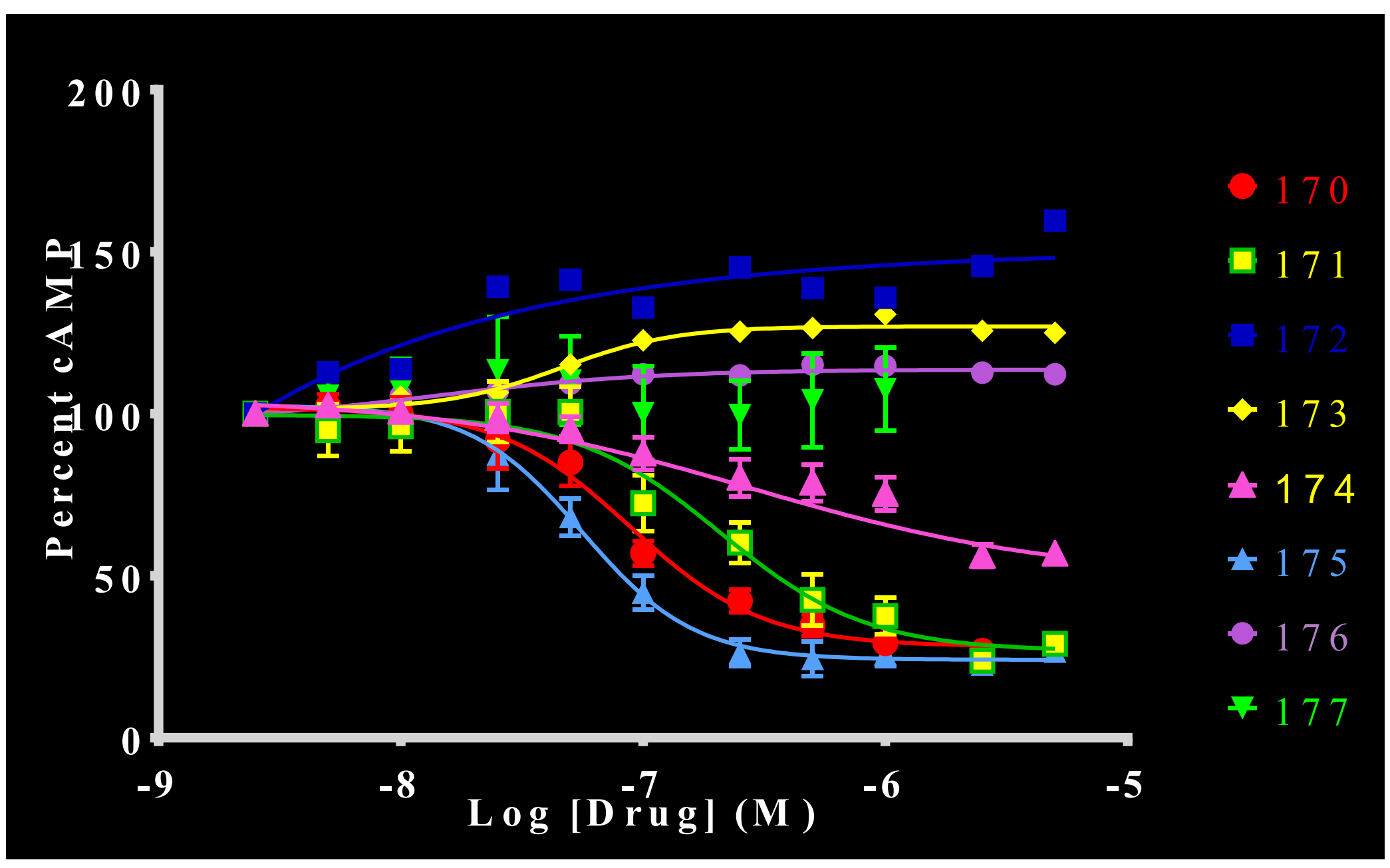

Figure 4.21. $\mathrm{CB}_{1}$ functional activity of hexahydro series compounds, 50 minutes after compound addition. 
Table 4.4. EC50 values for hexahydro series functional assays

\begin{tabular}{|c|c|c|c|c|c|}
\hline Compound & $\begin{array}{c}\mathrm{CB}_{1} \\
\text { EC50 } \\
\text { or } \\
\text { Concentration } \\
\text { (\% baseline) }\end{array}$ & $\begin{array}{c}\mathrm{CB}_{2} \\
\text { EC50 } \\
\text { or } \\
\text { Concentration } \\
\text { (\% baseline) }\end{array}$ & $\begin{array}{c}\text { CNG } \\
\text { EC50 (nM) }\end{array}$ & $\begin{array}{c}\text { CB }_{1} \text { PTx } \\
\text { EC }_{50}(\mathbf{n M})\end{array}$ & $\begin{array}{c}\mathrm{CB}_{2} \text { PTx } \\
\mathrm{EC}_{50}(\mathrm{nM})\end{array}$ \\
\hline 170 & $78.9^{\mathrm{a}}$ & $333^{a}$ & $\mathrm{n} / \mathrm{a}$ & $\mathrm{n} / \mathrm{a}$ & $\mathrm{n} / \mathrm{a}$ \\
\hline 171 & $209^{a}$ & $835^{\mathrm{a}}$ & $\mathrm{n} / \mathrm{a}$ & $\mathrm{n} / \mathrm{a}$ & $\mathrm{n} / \mathrm{a}$ \\
\hline 172 & $25(40 \%)^{b}$ & $\mathrm{n} / \mathrm{a}$ & $\mathrm{n} / \mathrm{a}$ & - & - \\
\hline 173 & $250(25 \%)^{b}$ & $\mathrm{n} / \mathrm{a}$ & $\mathrm{n} / \mathrm{a}$ & - & - \\
\hline 174 & $276^{\mathrm{a}}$ & $682.1^{\mathrm{a}}$ & $\mathrm{n} / \mathrm{a}$ & - & $\mathrm{n} / \mathrm{a}$ \\
\hline 175 & $56.3^{\mathrm{a}}$ & $66.8^{\mathrm{a}}$ & $\mathrm{n} / \mathrm{a}$ & $\mathrm{n} / \mathrm{a}$ & $\mathrm{n} / \mathrm{a}$ \\
\hline 176 & $100(12 \%)^{b}$ & $500(36 \%)^{b}$ & $\mathrm{n} / \mathrm{a}$ & - & - \\
\hline 177 & $\mathrm{n} / \mathrm{a}$ & $\mathrm{n} / \mathrm{a}$ & $\mathrm{n} / \mathrm{a}$ & - & - \\
\hline
\end{tabular}

Note: All concentrations are listed as $\mathrm{nM}$, percentages indicate the change from baseline. $\mathrm{n} / \mathrm{a}$ : Not active, based on no statistically significant change in cAMP from baseline. “_." Indicates test not performed. a Indicates a decrease in cAMP as compared to baseline. ${ }^{\mathrm{b}}$ Indicates an increase in cAMP as compared to baseline. 


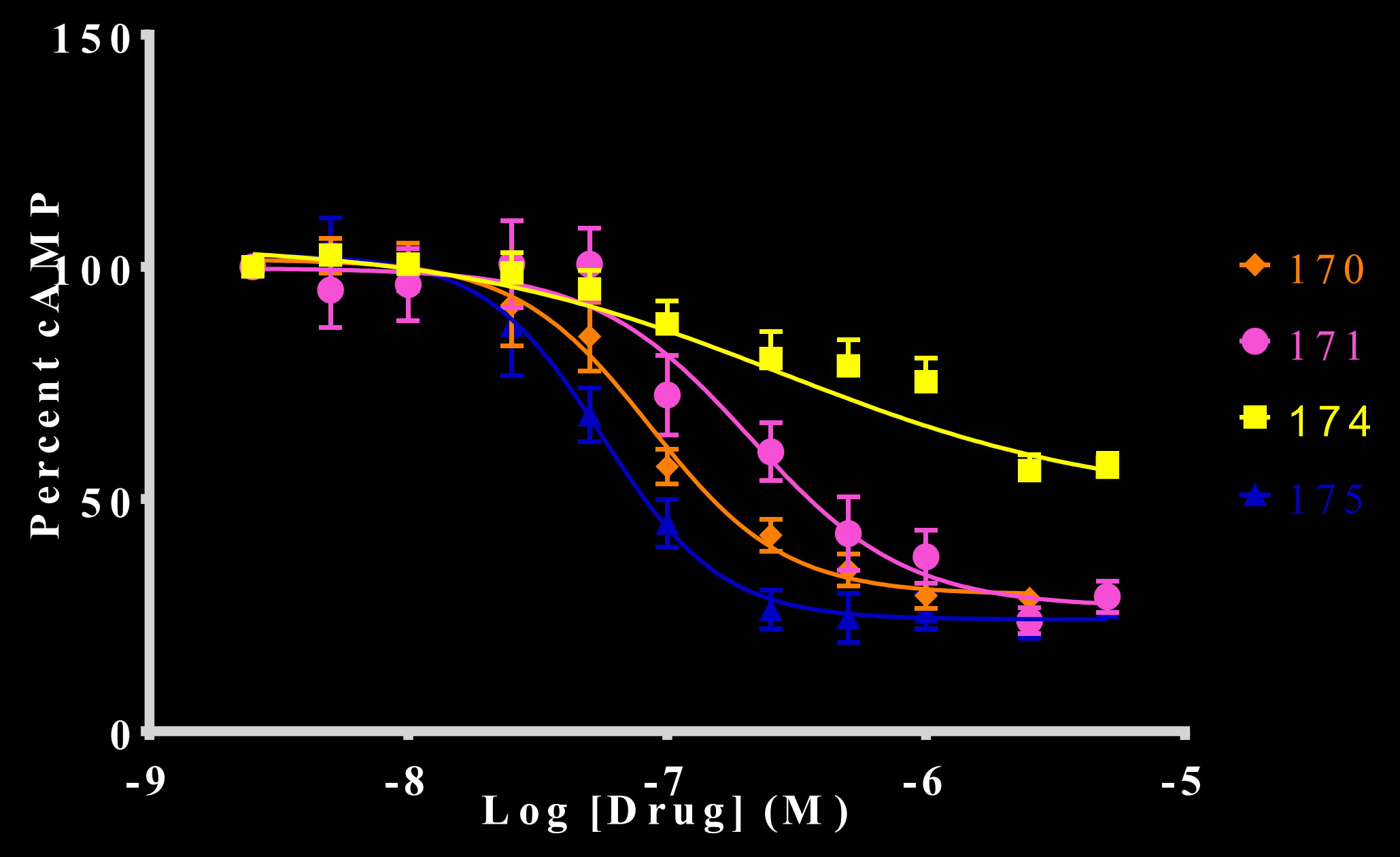

Figure 4.22. $\quad \mathrm{CB}_{1}$ functional activity for select hexahydro series compounds, 50 minutes after compound addition 


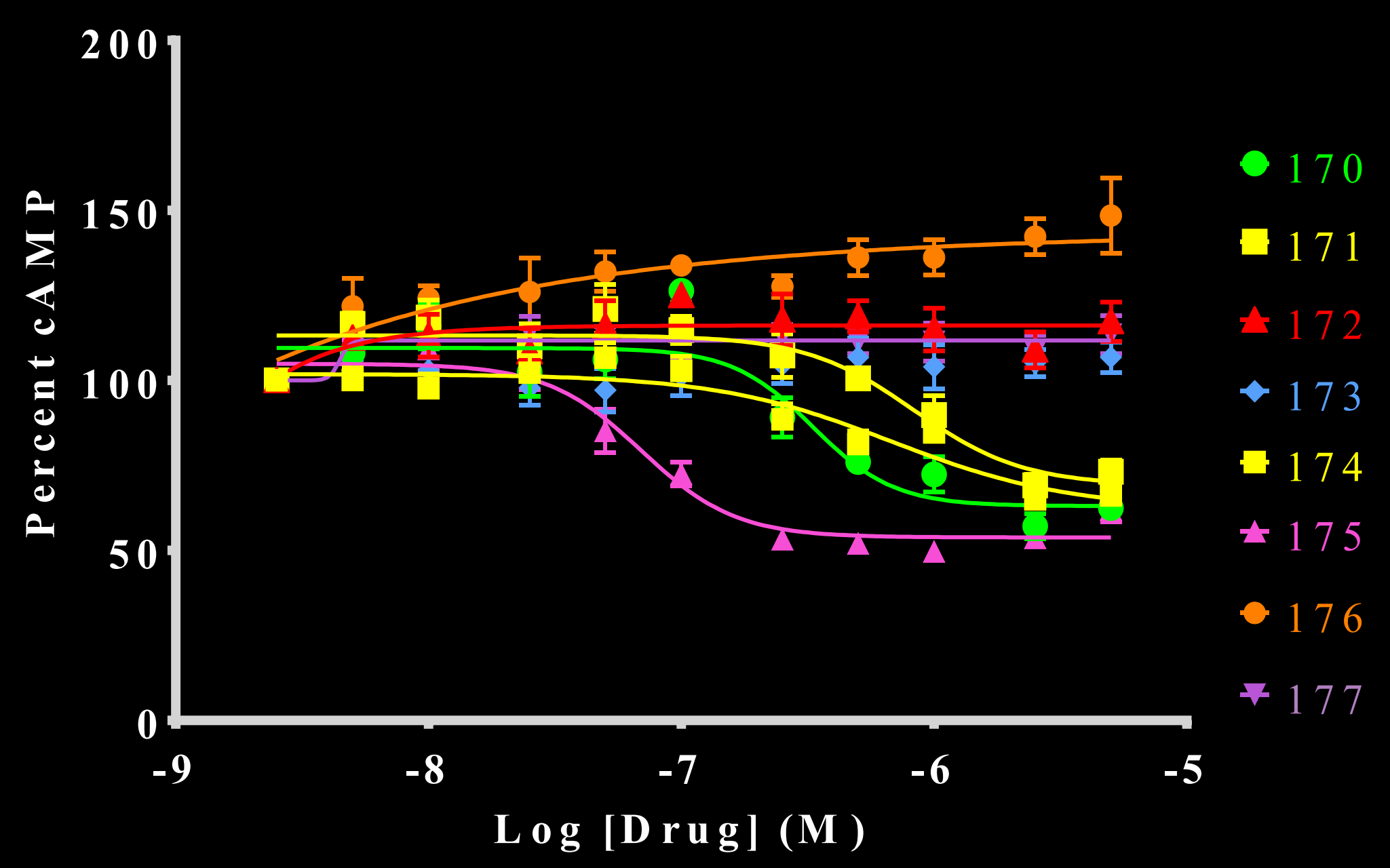

Figure 4.23. $\quad \mathrm{CB}_{2}$ functional activity for hexahydro series, 50 minutes after compound addition 


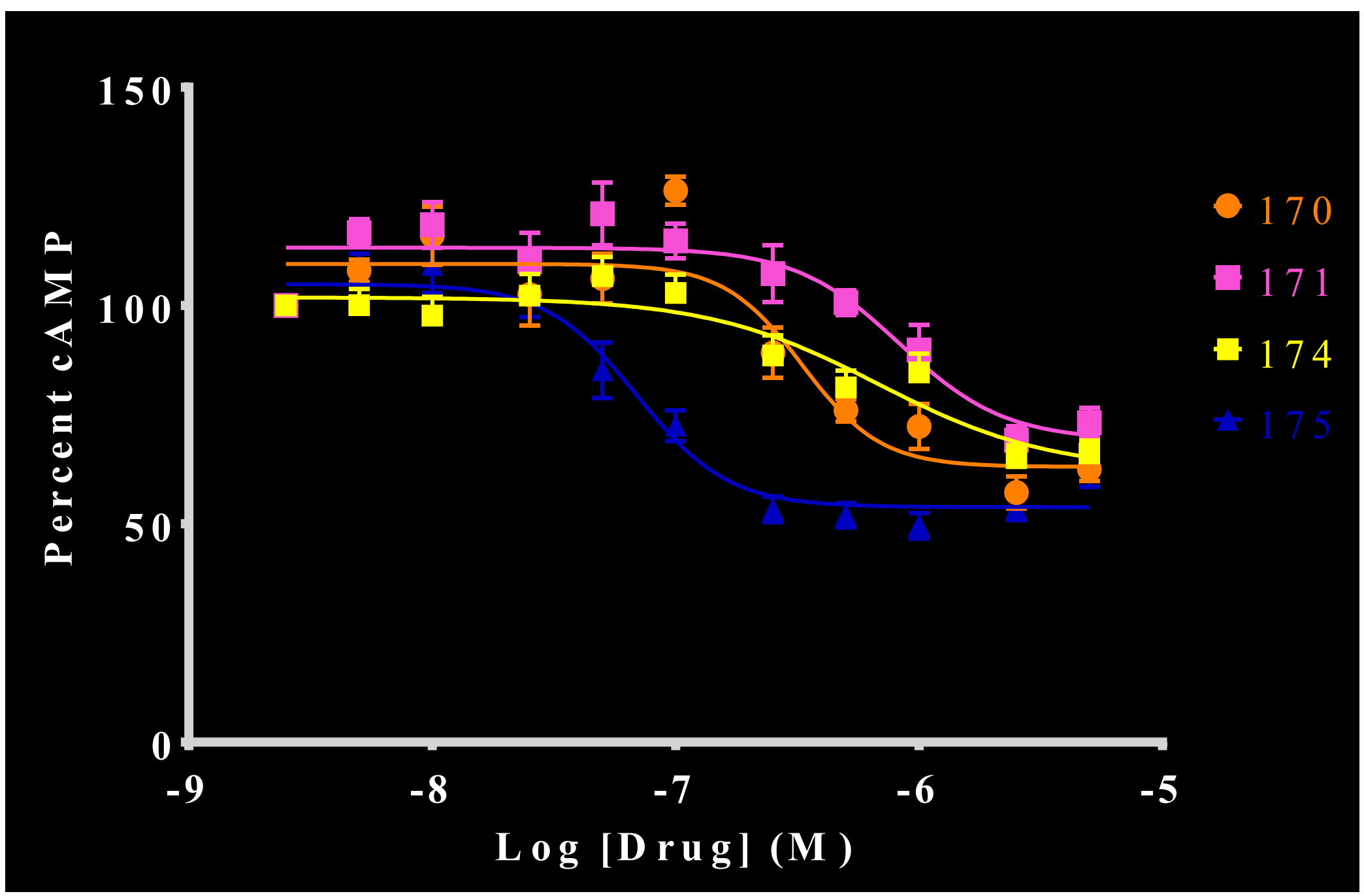

Figure 4.24. $\quad \mathrm{CB}_{2}$ functional activity for select hexahydro series compounds, 50 minutes after compound addition 


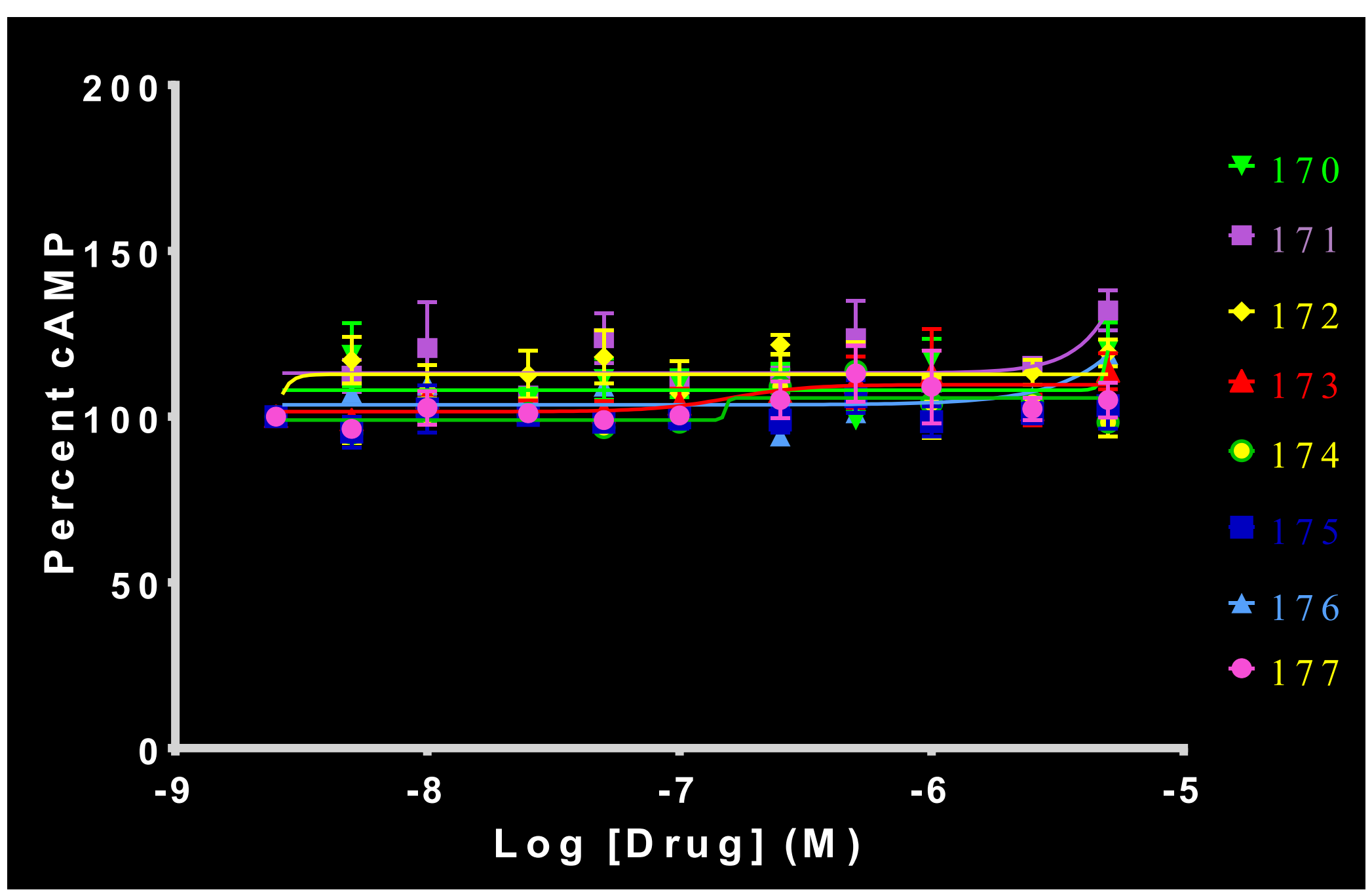

Figure 4.25. HEK-CNG parental cell functional activity for hexahydro series, $\mathbf{5 0}$ minutes after compound addition 


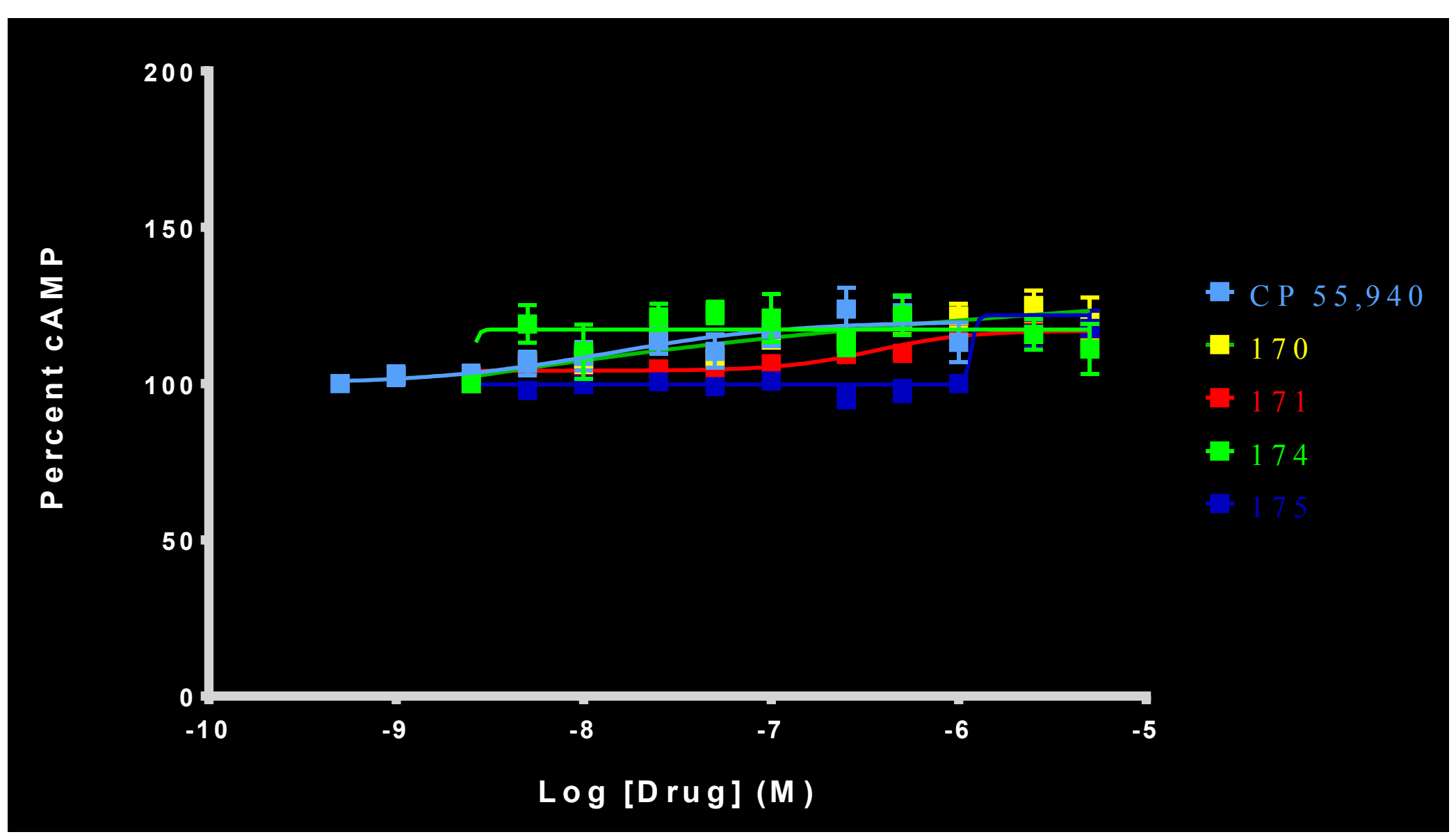

Figure 4.26. $\mathrm{CB}_{1}$ PTx-sensitive $\mathrm{G}_{\mathrm{i} / \mathrm{o}}$-inactivation of functional activity for hexahydro series, 50 minutes after compound addition 


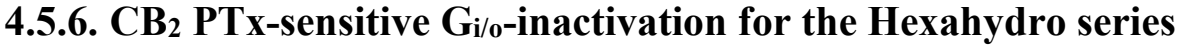

Plates were run as previously described (Section 4.5.1) in three (3) replicates with CP-55,940 as the internal control for all three (3) replicates of compounds 170, 171, 174 and 175 As was expected, all compounds failed to alter cAMP production, including the reference standard of CP-55,940 (Figure 4.27).

\subsection{Discussion}

\subsubsection{Interactions with the $\mathrm{CB}_{1} \mathrm{LBP}$}

As previously discussed (Section 1.2.2), $\mathrm{CB}_{1}$ receptors are 7 trans-membrane G-protein coupled receptors (GPCRs). ${ }^{14}$ Interaction of classical cannabinoids within the LBP of $\mathrm{CB}_{1}$ occurs primarily in domains 3, 6 and 7. Several homology models help to elucidate the residues important for binding and functional activity.

To generate hypotheses, gain some insight into the possible explanations and interplay between ligand and receptor, I performed docking of the compound. Dr. Chang of The University of California, Riverdale, kindly supplied the models for the CB1 receptor. Dr. Chang provided 4 models: "ACEA" - to mimic the endocannabinoid binding, "HU-210" - to mimic the classical core binding, "SR-141716A" - to mimic the inverse agonist binding and "WIN-55,212-2" - to mimic the non-classical agonist binding. ${ }^{249}$ The hexahydro series of compounds, being similar in form to that of HU-210, I believe adopt a similar binding mode within the LBP, thus this was the model used for docking studies.

4.6.1.1. C-1 hydroxyl. K192(3.28) plays a very important role in both receptor binding and function. Since it is a very flexible residue in the LBP, it may hydrogen bond with the C-1 hydroxyl, C-11 ("Northern") hydryoxyl, C-6 ("Southern") hydroxyl or even the benzochromene oxygen. ${ }^{250}$ However, newer data suggest that K192(3.28) plays a primary role as H-bond acceptor of the C-1 hydroxyl, ${ }^{251}$ presumably this occurs after being activated by donating a H-bond to F189(3.25) (Figure 4.28). ${ }^{249} \mathrm{~S} 383(7.35)$ interacts by donating a H-bond to either the benzchromene oxygen or the C-6 hydroxyl, if present (Figure 4.28). ${ }^{251}$ Additionally, data suggest that the resting state of the $\mathrm{CB}_{1}$ receptor exists when the salt bridge between $\mathrm{K} 192(3.28)$ and D366(6.58) is intact. ${ }^{252}$

The hexahydro series of compounds sought to further the functional activity and binding affinity of the KM Series (Chapter 3), and therein brought about novel activity. Within this series, aromatic substitutions yielded inverse agonists at $\mathrm{CB}_{1}$ and neutral antagoinsts $\mathrm{CB}_{2}$, while aliphatic substitutions at $\mathrm{C}-1^{\prime}$ yielded agonists at both $\mathrm{CB}_{1}$ and $\mathrm{CB}_{2}$. This is unique when considering the change between KM-233 (63), compounds 172 and $\mathbf{1 7 6}$ are the inclusion of a pyridine and pyrimidine A-ring, respectively. NMR data shows the hexahydro compounds exist in solution as an amide at the A-ring, which may contribute to the binding modes in the LBP and change in activity compared to the KM series. 


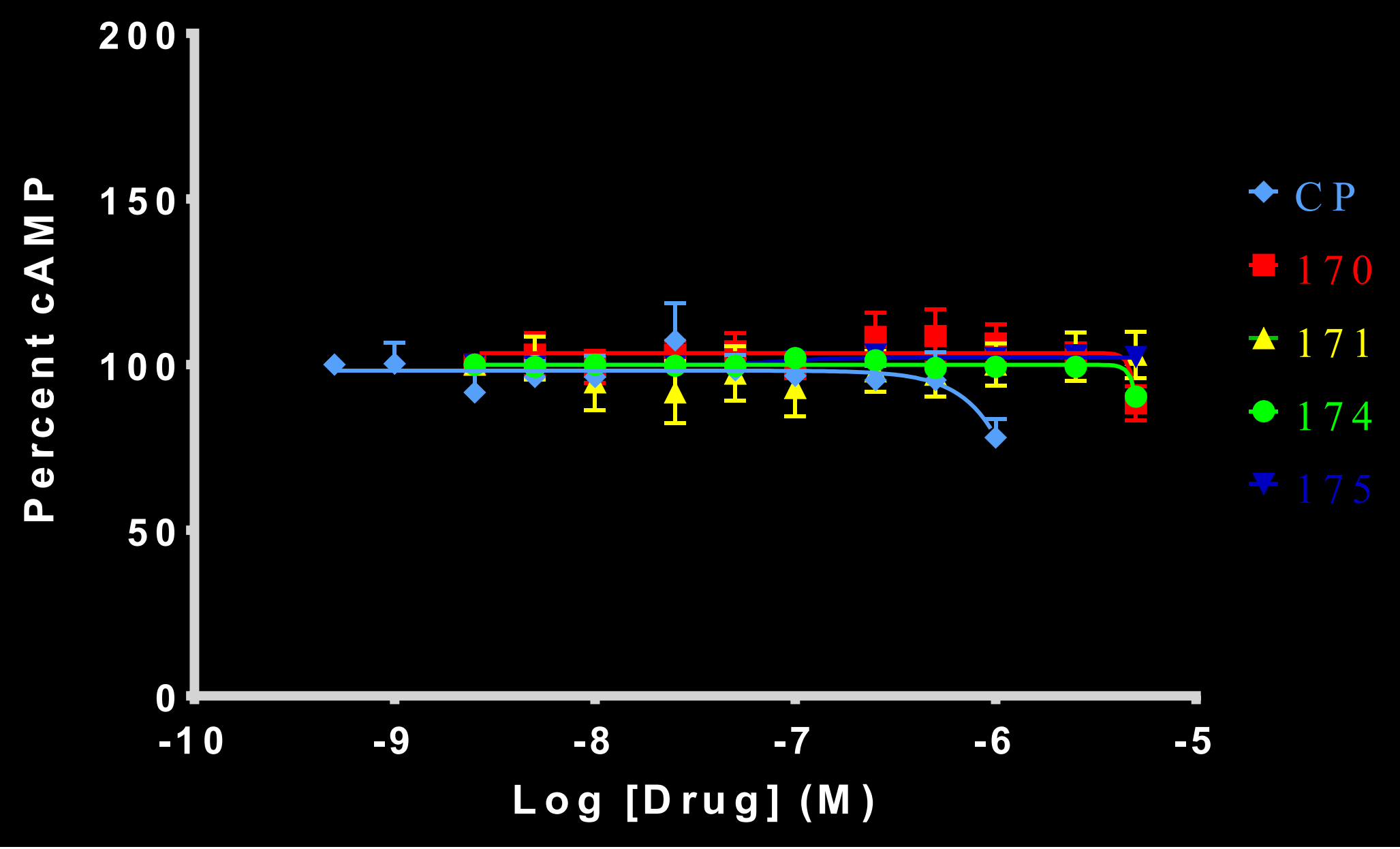

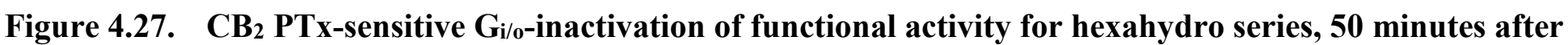
compound 


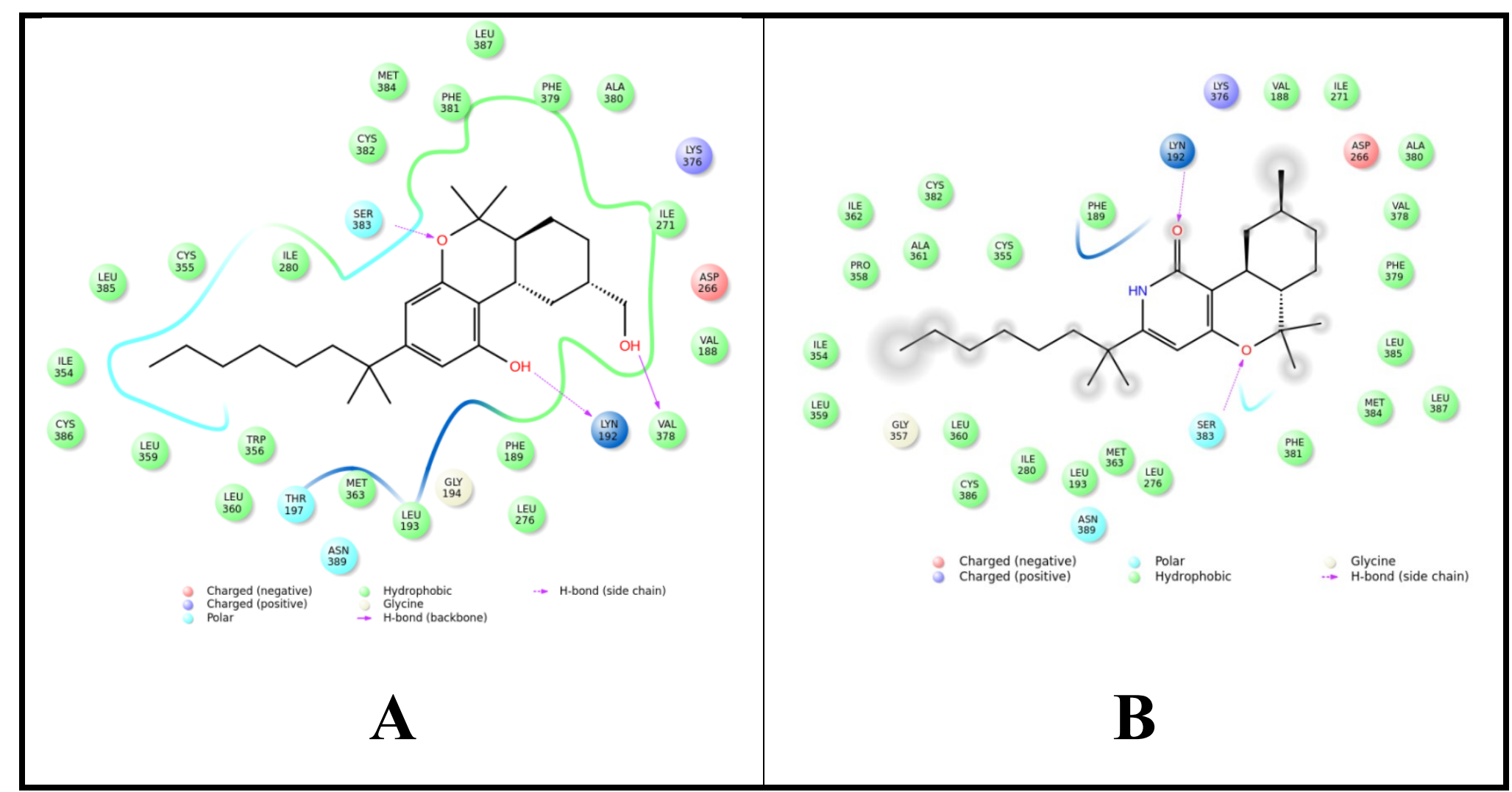

Figure 4.28. $\quad \mathrm{CB}_{1} \mathrm{LBP}$ - amide and benzchromene oxygen interactions of 170, as compared to HU-210 
Looking at compounds 170, 171, 174 and 175 and considering their agonist activity, two literature-reported interactions should be present for agonist activity:

1. The K192(3.38) and D366(6.58) salt bridge must be severed. ${ }^{252}$

2. Each of these ligands has to interact with both residues F200(3.36) and W279(5.43) to exert agonist effect. ${ }^{257}$

The first is the severance of the salt bridge by interacting with K192(3.38). I hypothesize that this novel series of compounds binds similarly to classical cannabinoids; however, the key difference lies in the presence of the amide moiety in the A-ring. This moiety may cause the $\mathrm{ABC}$ ring to bind more deeply into the tricyclic ( $\mathrm{ABC}$ ring) pocket to facilitate H-bonding between the amide N-H and K192(3.28) (Figure 4.28B).

4.6.1.2. C-3 lipophilic side chain. A predominantly lipophilic pocket exists, seemingly as two lobes - as evidenced in the fact that 3-(1',1'-dimethylheptyl)- $\Delta^{8}-\mathrm{THC}$ binds with higher affinity than does 3-heptyl- $\Delta^{8}-$ THC along with 3D-QSAR models which demonstrate this trait. ${ }^{253}$ In one homology model, residues V196(3.32), T197(3.33), F200(3.36), Y275(5.39), W279(5.43), L359(6.51) and M363(6.55) form a hydrophobic pocket which encompasses the A-ring and C-3 side chain of HU-210. ${ }^{254}$ More recent homology models form this pocket from I354(6.46), C355(6.47), W356(6.48), L359(6.51), L360(3.52) and M363(6.55) (Figure 4.29A). ${ }^{249}$ Interestingly, mutations studies conducted on this hydrophobic pocket show that M363A(6.55) mutation greatly impacts binding affinity for HU-210 but has virtually no impact on THC which would suggest that this is an important residue for C-1' substituted derivatives. ${ }^{255}$ The hexahydro series, given that it shares a C-1'-gem-dimethyl substituent, likely share a similar interaction with M363(6.55) (Figure 4.29B).

The second element suggested for an agonist is the simultaneous interaction with residues F200(3.36) and W279(5.43). Compounds 170, 171, 174 and 175, with their long, flexible, aliphatic C-3 substituents may still provide necessary interactions with both F200(3.36) and W279(5.43). The more compact and rigid aromatic substituents of compounds 176 and 177 may not provide these interactions as a result of the deeper binding into the tricyclic (ABC ring) pocket or stronger intra-molecular $\pi$ - $\pi$ interactions (Figure 4.30) and lack of activity.

Looking at the special case of compounds $172,173,176$ and 177 within the $\mathrm{CB}_{1} \mathrm{LBP}$, there is a unique situation. Given the current functional data generated from my assay conductance suggests that these compounds are members of a special class of antagonists, known as inverse agonists. Two key interactions are reported in literature to help stabilize the $\mathrm{CB}_{1}$ receptor in its inactive state:

1. A salt bridge between residues K192(3.28) and D366(6.58). ${ }^{252}$

2. A $\pi-\pi$ stacking, rotameric "toggle" switch between residues F200(3.36) and $\mathrm{W} 356(6.48){ }^{256,321,322}$

I hypothesize that in order for compounds to bind into the LBP, they must break the salt bridge between K192(3.28) and D366(6.58). Thus, the salt bridge represents an inactive 


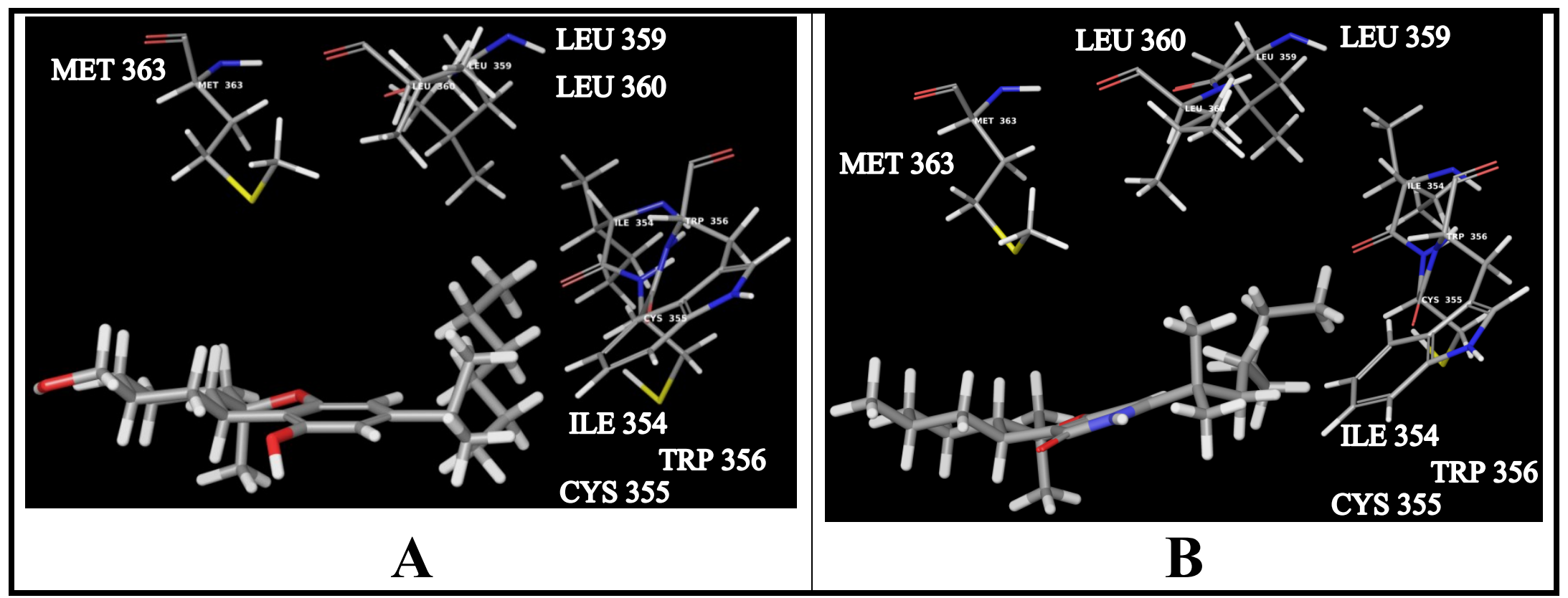

Figure 4.29. $\quad \mathrm{CB}_{1}$ hydrophobic $\mathrm{C}-3$ substituent binding pocket

Note: Panel A depicts HU-210 in the $\mathrm{CB}_{1}$ LBP, with focus given to the hydrophobic pocket. Panel B depicts Compound 170 in the $\mathrm{CB}_{1} \mathrm{LBP}$, with focus given to the hydrophobic pocket. 


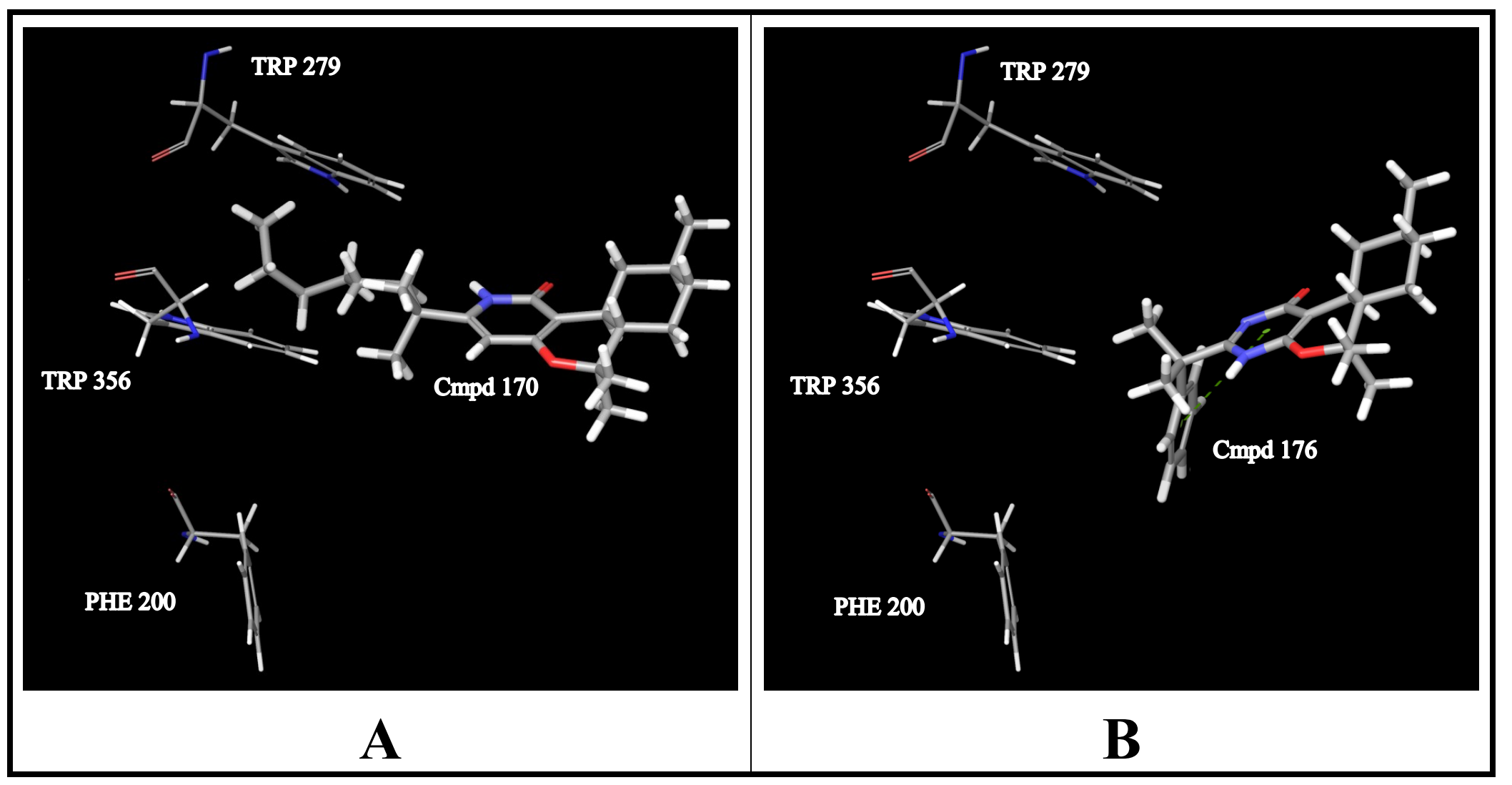

Figure 4.30. $\quad \mathrm{CB}_{1} \mathrm{LBP}$ - comparison of the aliphatic and aromatic hexahydro interactions

Note: Panel A depicts Compound 170 interacting with W279(5.43). Panel B depicts Compound 176 with a lack of interaction. 
state for the $\mathrm{CB}_{1}$ receptor; however, it does not represent a ligand-bound state of the receptor, because the act of binding ligand to receptor results in a severance of this salt bridge. The aromatic C-3 substituent of 172, 173, 176 and 177 would then potentially stabilize the "toggle" switch of F200(3.36) and W356(6.48) (Figure 4.31). The F200(3.36)/W356(6.48) stabilization is reported for inverse agonists. Given that the compounds in this series bind, but the aliphatic-substituted compounds lack of ability to stabilize the "toggle" switch points to a unique mechanism of aromatic substituents in $\mathrm{CB}_{1}$ LBP interactions. This hypothesis would be more consistent with the work of homology modeling of SR141716A ${ }^{322}$ and of McAllister, et al. ${ }^{256}$ - the group who thus behave as an inverse agonist. Dashed green lines indicate $\pi-\pi$ interactions. Helix 3 shown in yellow, helix 6 shown in blue. originally proposed the salt bridge ${ }^{252}$ concept. In addition, it is important to note, that in the KM series there exists a thiophene derivative 78, which was an agonist at $\mathrm{CB}_{1}$. Thus, stabilization of the "toggle" switch in conjunction with a lack of interaction with both F200(3.36) and W279(5.43) - which is suggested to arise from the inclusion of a heteroatomcontaining A-ring, though the exact root cause is currently unknown - give the unique $\mathrm{CB}_{1}$ mechanism.

4.6.1.3. ABC ring system. Residues $F 174(2.61)$ and $F 177(2.64)$ are not suggested to be involved in binding to HU-210, but rather they help stabilize the tricyclic (ABC ring) pocket where the ABC benzochromene ring binds the LBP. ${ }^{255}$ Residues F200(3.36) and W279(5.43) appear to play an important a role in both in terms of binding (in the case of AEA and CP-55,940) and receptor activation (Figure 4.30) ${ }^{257}$ That is to say, a ligand's hydrophobic substituents at the $C 3$ position may bind well without interacting with these two residues, but agonists MUST have interaction with these residues to exert their effect. ${ }^{254} \mathrm{~F} 189(3.25)$ is key at providing a $\pi-\pi$ stacking interaction with HU-210 (Figure 4.32A), and is instrumental in binding of AEA to the pocket as its mutation to alanine causes a 7-fold drop in affinity for the receptor. ${ }^{258} \mathrm{~F} 189(3.25)$ also seems to form the "ceiling of the anandamide binding pocket in $\mathrm{CB}_{1} .{ }^{257}$ From a $\pi-\pi$ stacking standpoint, the hexahydro series shares these interactions (Figure 4.32B) - though not depicted on the 2D output. However, the major difference lies in the compounds' ability to interact with the key residues, which were addressed previously.

4.6.1.4. (Optional) C-11 ("Northern") hydroxyl. While there is not data currently to confirm, logical extension of the known fact that $\mathrm{C}-11$ hydroxylation is the primary metabolite of the KM series would suggest that this would be a. metabolite of the hexahydro series. As a result of this metabolite potentially contributing to the overall activity of the compound in vivo, this potential interaction is discussed and explored. S383(7.35) shows itself to be equally important in binding of molecules along with stabilizing the LBP. S383(7.35) can hydrogen bond with the C-11 hydroxyl ${ }^{251}$ (if present) or the benzochromene oxygen ${ }^{249,254}$, but more importantly it helps to stabilize the LBP by maintaining the shape of the trans-membrane helix 1-2-7 binding pocket. ${ }^{251}$ The "Northern" hydroxyl group may also H-bond with K192(3.28); however, this is not a preferred interaction in several recent homology models. 


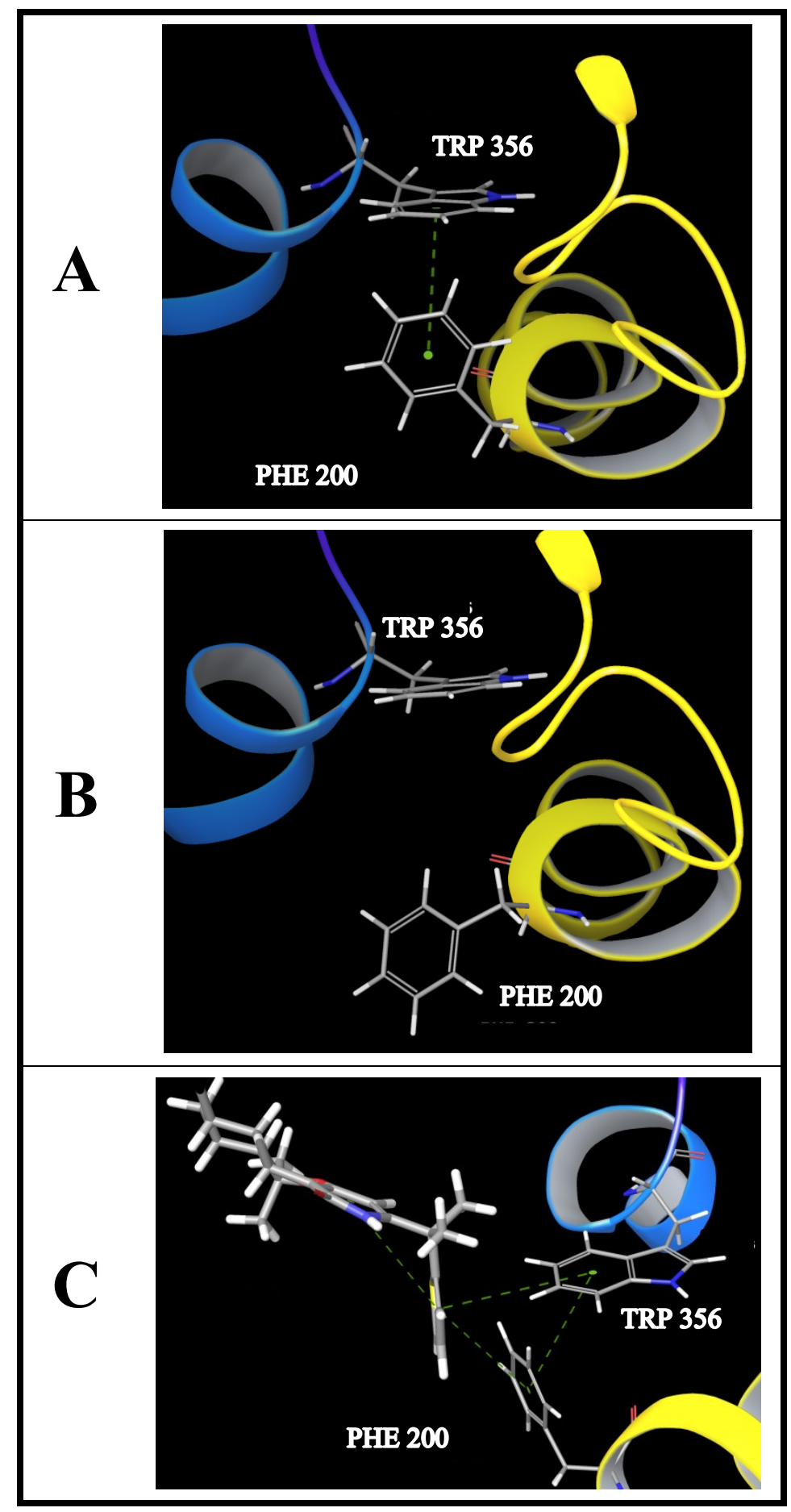

Figure 4.31. Stabilization of the CB1 LBP in the inactive state

Note: Panel A depicts the two (2) key residues involved in stabilizing the inactive state of $\mathrm{CB}_{1}$. Panel B depicts these two (2) residues in the active state of the receptor. Panel $\mathrm{C}$ is the hypothesized interaction of $\mathbf{1 7 3}$ to stabilize the $\mathrm{CB}_{1}$ receptor in the inactive state. 


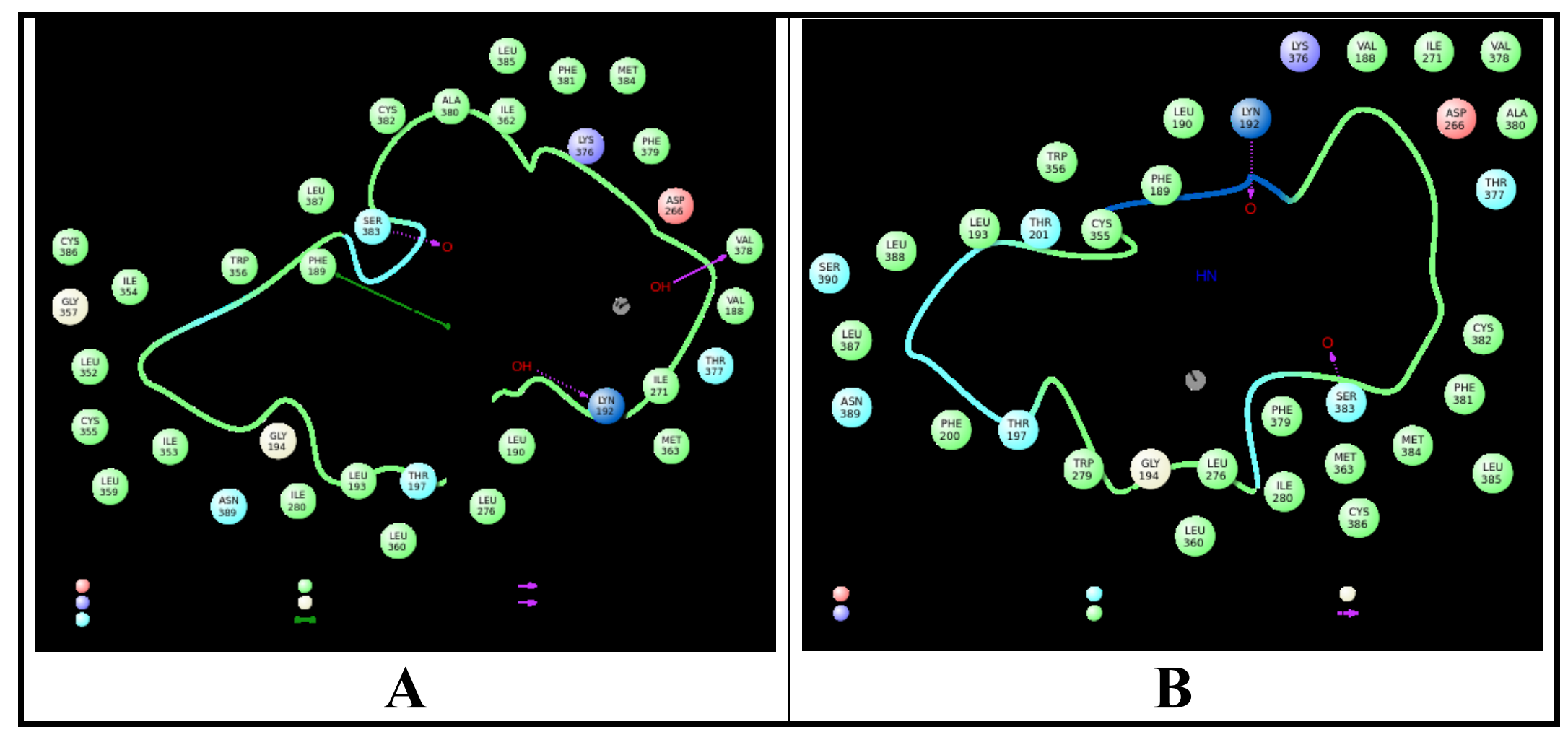

Figure 4.32. CB1 LBP proposed $\pi-\pi$ stacking and key residue interactions 


\subsubsection{Interactions with the $\mathrm{CB}_{2} \mathrm{LBP}$}

As previously discussed (Section 1.2.3), $\mathrm{CB}_{2}$ receptors are also 7 trans-membrane GPCRs; however, $\mathrm{CB}_{2}$ only shares $48 \%$ homology with $\mathrm{CB}_{1}-$ a trait unique to the $\mathrm{CB}_{1}$ and $\mathrm{CB}_{2}$ receptors among GPCRs, which generally share a much higher level of homology among receptors of the same family. ${ }^{14}$ The SAR of the $\mathrm{CB}_{2}$ LBP has yet to be fully developed - even less so with the classical cores. $\mathrm{B} / \mathrm{C}$ ring trans junction. With data relatively limited for $\mathrm{CB}_{2}$ 's $\mathrm{LBP}$, especially in the arena of classical core compounds, one must make some inferences based on available aminoalkylindole derivative homology model data. One such extrapolation lies in WIN-55,212-2's biologically assumed conformation to be s-trans-conformer. ${ }^{260}$ Docking the s-cis-conformer, there are steric interactions with V113(3.32) (Figure 4.33). ${ }^{261}$ Existence of this clash may suggest a trans junction of the $\mathrm{B} / \mathrm{C}$ ring allows for proper orientation in the LBP to circumvent this steric interaction with V113(3.32) within the classical core molecules. By extension, WIN55,212-3, the S-(-) isomer of WIN-55,212-3, may also orient the morpholino side chain such to prevent critical amino acids interactions in the $\mathrm{CB}_{2} \mathrm{LBP}$ as discussed below.Taking WIN-55,212-2 and ascribing its moieties to those of the representative example of 172 (Figure 4.34), one can see that there are many similarities. While the similarities are numerous, the differences are profound as well, because the steric interactions of WIN-55,212-2 with V113(3.32) are apparently non-existent with the hexahydro series. The steric issue comes in with what I hypothesize to be the "northern" aliphatic hydroxyl region, or C-11 substituted derivatives of $\Delta^{8}$-THC, thus the hexahydro series has little to contend with in this arena.

\subsubsection{C-1 Hydroxyl, benzchromene oxygen and (optional) C-6 hydroxyl.} Mutation studies with HU-210, CP-55,940 and WIN-55,212-2 have shown that binding of both HU-210 and CP-55,940 experience negative consequence with a S292A mutation. This mutation-induced change of binding strongly suggests S292(7.46) is involved in H-bonding with the benzchromene oxygen - assuming a similar LBP arrangements relative to the $\mathrm{CB}_{1}$ LBP. ${ }^{323}$ Though WIN 55-212-2's ketone is in suggest to be in proximity to residue $\mathrm{S} 193(5.42)$ it does not appear to hydrogen bond. ${ }^{261}$ In the case where amide linkages exist between the aryl groups, it is suggested that S193(5.42) does act as a H-bond donor. ${ }^{324,325}$

4.6.2.2. Hydrophobic and $\pi$ - $\pi$ stacking. As has been the case previously, it remains so that many of the interactions derive from the aminoalkylindole derivative WIN-55,212-2. Beginning with the indole ring of WIN-55,212-2, there are reported $\pi-\pi$ stacking interactions between it and residues F197(5.46), W258(6.48) and F117(3.36). ${ }^{261}$ There also exists a shared $\pi-\pi$ stacking between the indole ring and napthyl ring with residue W194(5.43). ${ }^{261}$ The napthyl ring has an added $\pi$ - $\pi$ stack with residue F106(3.25). ${ }^{261}$ More recent 2-pyridone derivatives add additional residues to the binding pocket for $\pi$ - $\pi$ stacking, namely Y 190(5.39) and F281(7.35) along with a C288(7.42) residue. ${ }^{324,325}$ For the 2-pyridone analog containing N-butyl, there involves a hydrophobic interaction with I198(5.47). ${ }^{325}$

4.6.2.3. Proposed interactions of the hexahydro series with the $C_{2}$ LBP. Due to their similarities, compounds 170, 171, 174 and 175 are the aliphatic constellation and 


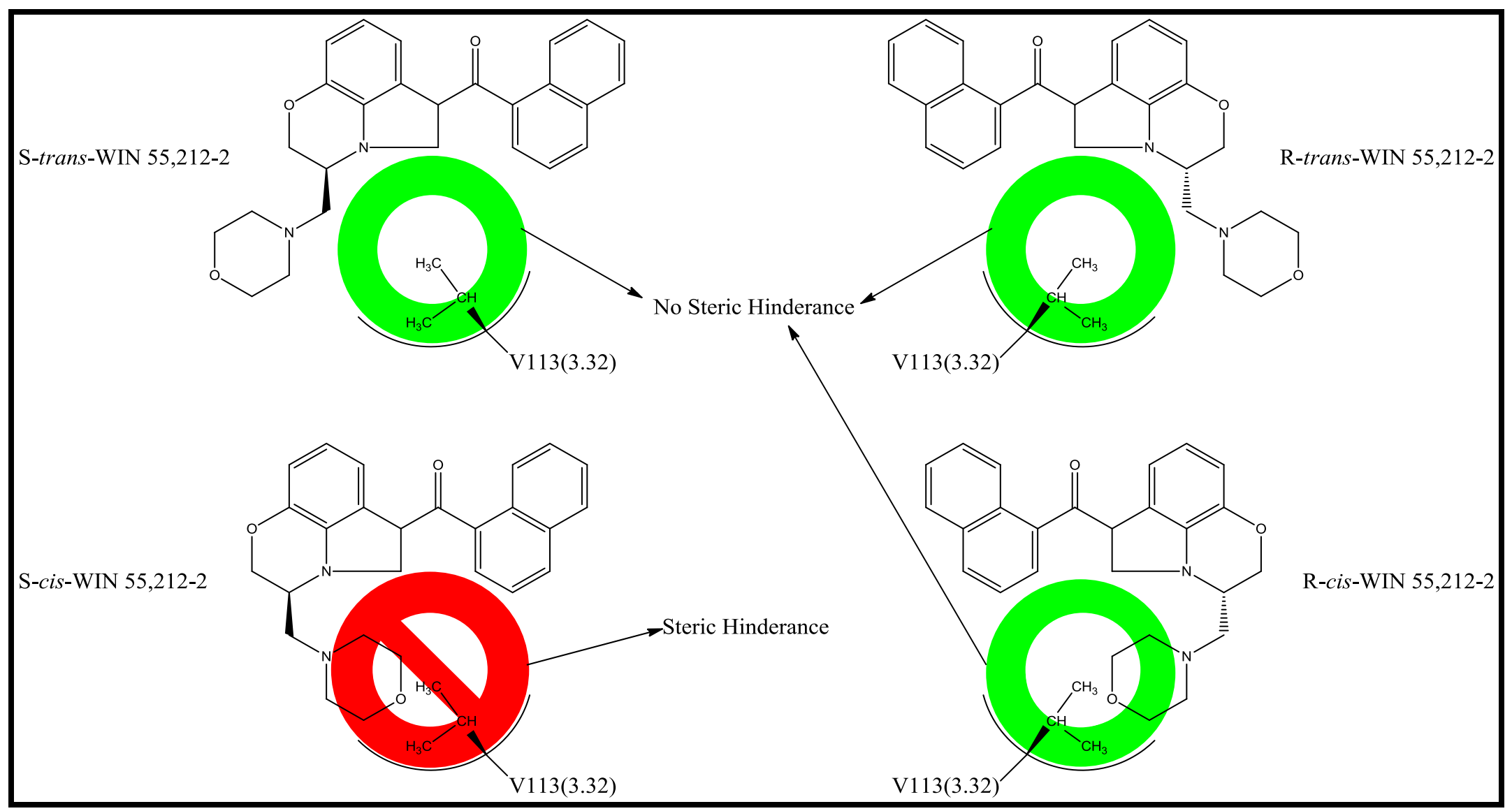

Figure 4.33. $\mathrm{CB}_{2}$ LBP steric hinderance of cis and trans isomers of WIN-55,212-2 


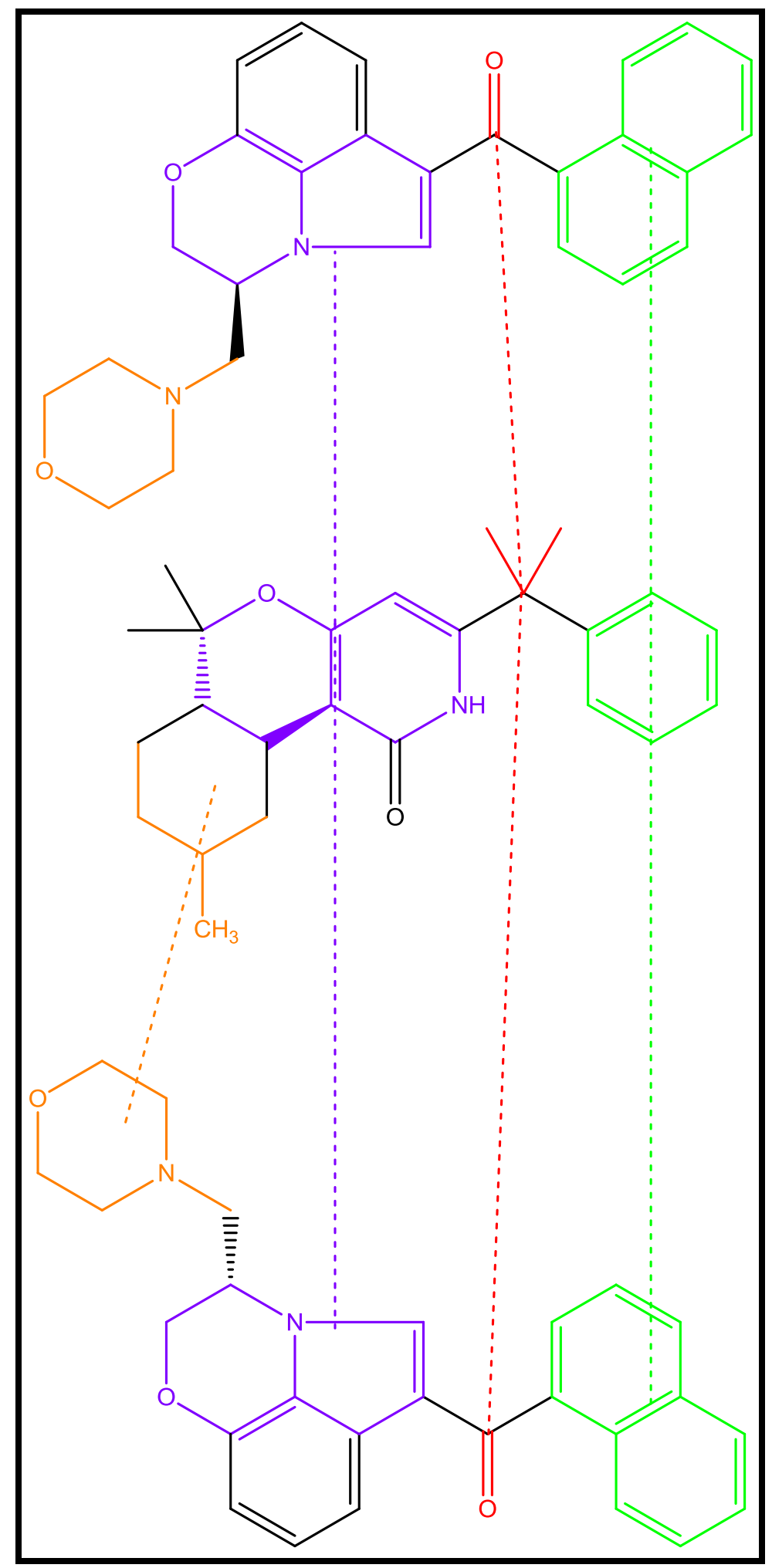

Figure 4.34. Comparison of analogous functional groups of WIN-55,212-2, 172 and WIN-55,212-3 
compounds 172, 173, 176 and 177 are the aromatic constellation. Similar to the KM Series, I hypothesize that the binding mode for the hexahydro series is more similar to $\mathrm{THC}$, in that the $\mathrm{ABC}$ ring occupies the tricyclic ( $\mathrm{ABC}$ ring) pocket of the LBP and the C-3 substituents project into the major pocket. Therein the major pocket lies the key residue of W258(6.48), the rotameric "toggle" switch for $\mathrm{CB}_{2}$ - proposed to H-bond with L255(6.45) to open place C2.57(6.47) in optimal range for agonist interaction. ${ }^{326}$ As was the case in $\mathrm{CB}_{1}$, compounds 170, 171, 174 and 175, with their long, flexible, aliphatic $\mathrm{C}$ 3 substituents can then still provide necessary interactions with W258(6.48) while still allowing the rotameric change from gauche+ to trans of the residue (Figure 4.35).

\subsection{Conclusions}

The pyridine and pyrimidine hexahydro series of compounds represent a novel series of compounds, which may also give insight into the binding mode and residue interactions of these compounds in the $\mathrm{CB}_{1} \mathrm{LBP}$. The presence of the amide moiety in the A-ring causes these series of compounds to bind further into the tricyclic ( $\mathrm{ABC}$ ring) pocket of the $\mathrm{CB}_{1}$ LBP making interaction with the two (2) key residues, F200(3.36) and W279(5.43), possible only with a long, flexible C-3 substituent - such as that found on 170, 171, 174 and 175. Unique mechanisms with regard to heterocyclic stabilization of the $\mathrm{CB}_{1}$ receptor's inactive state by aiding in the $\pi-\pi$ stacking of residues F200(3.36) and W356(6.48) to yield an inverse agonist. Interactions within the $\mathrm{CB}_{2} \mathrm{LBP}$ can only be postulated in the absence of a refined model. Hoewever, aromatic C-3 substituents seem to stabilize the gauche + rotamer of $\mathrm{W} 258(6.48)$ in order to yield an antagonist at $\mathrm{CB}_{2}-$ aliphatic substituents allow for rotation of W258(6.48) and thus receptor activation.

Future studies to test these hypotheses would be to conduct homology model studies with these compounds - more specifically to reconcile the divergence of binding mode for these compounds and further solidify key residues. Additionally, synthesis of other derivatives would help to determine a more exact functional pharmacophore. Lastly, testing of the antagonist compounds against known $\mathrm{CB}_{1}$ and $\mathrm{CB}_{2}$ agonists will confirm this mechanism and establish $\mathrm{EC}_{50}$ doses for these compounds. 


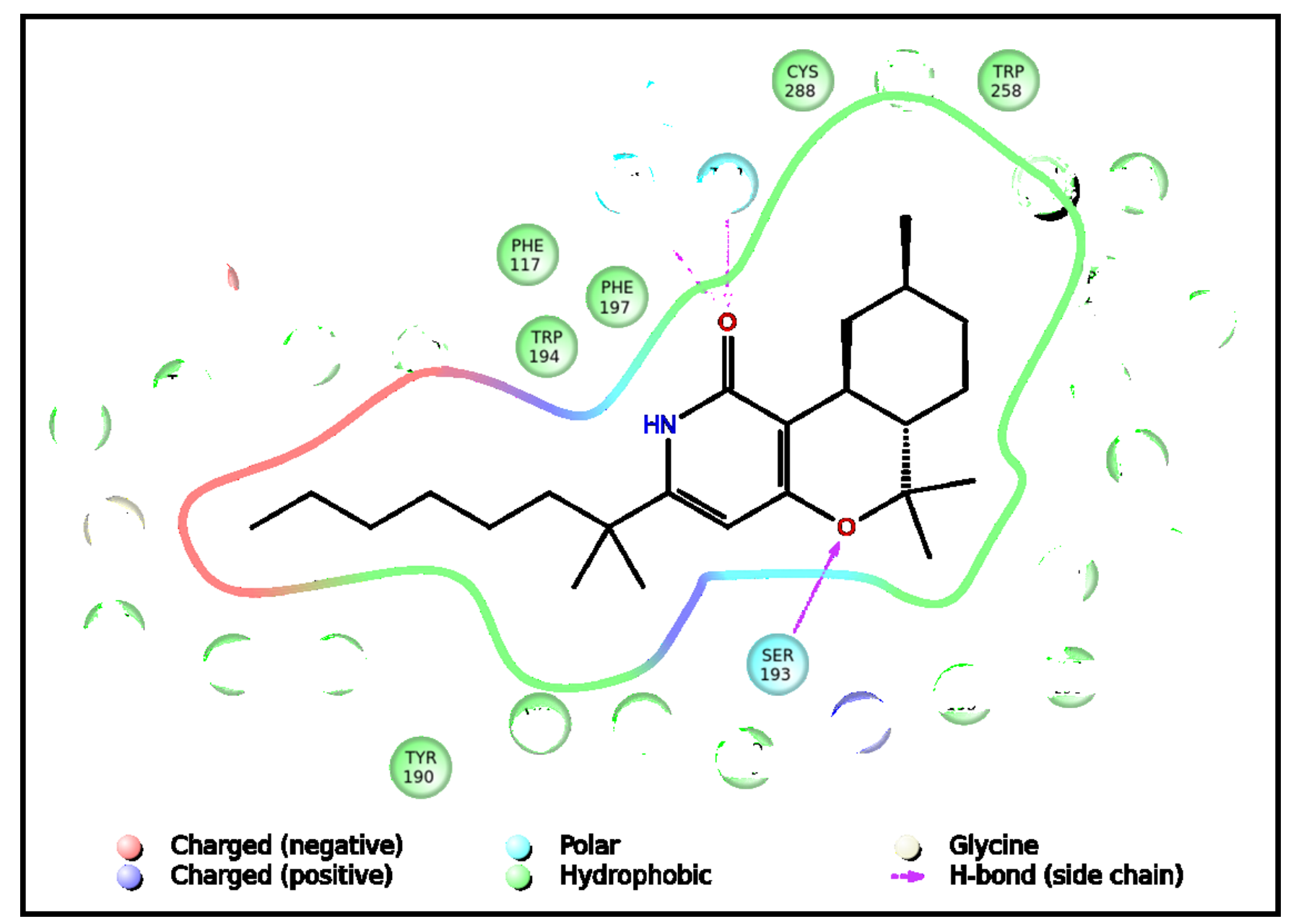

Figure 4.35. Proposed $C_{2}$ LBP residues for compound 170 


\section{CHAPTER 5. FUNCTIONAL EVALUATION OF A NOVEL CLASS OF TRIARYL CANNABINERGIC LIGANDS}

\subsection{Introduction}

As previously discussed (Section 1.1), classical cannabinoids are tricyclic compounds possessing a benzopyran core - these are of either natural origin or synthetic analogues thereof. The naturally occurring, prototypes for these compounds are $\Delta^{9}$-THC and $\Delta^{8}-\mathrm{THC}$, which both bind to $\mathrm{CB}_{1}$ and $\mathrm{CB}_{2}$ receptors and exert a partial agonist activity. ${ }^{14}$ Chapter 3 and Chapter 4 have been examples of the extensive study and mining of these classical core compounds for functional pharmacophoric elements. To further the field and probe $\mathrm{CB}_{1}$ and $\mathrm{CB}_{2}$ further, new chemical scaffolds are essential. To move away from the classical core and parse out even more functional pharmacophoric elements, the non-classical cannabinoids came into their own right. These non-classical cannabinoids sought to simplify the overall structure of cannabinoid ligands and to augment the understanding of $\mathrm{CB}_{1}$ and $\mathrm{CB}_{2}$ ligand requirements.

It has been established through study of these non-classical cannabinoids that simplifying the overall structure to that of an AC-bicyclic increases the affinity for both $\mathrm{CB}_{1}$ and $\mathrm{CB}_{2}$; however, transition from $\mathrm{AC}$-bicyclic to $\mathrm{ACD}$-tricyclic allows for selectivity between $\mathrm{CB}_{1}$ and $\mathrm{CB}_{2}$ (Section 5.2.1). Merging traits of the classical with these AC-bicyclic and ACD-tricyclic compounds gave rise to a hybrid crossover series, with increased affinity for both without any appreciable selectivity (Section 5.2). The biaryl class of cannabinoid compounds sought to take the best of the classical C-3 substituent and merge it with the bicyclic structure of the AC-bicyclic compounds, resulting in a series with very good affinity and specificity towards $\mathrm{CB}_{2}$ (Section 5.2.3). A final derivative in the non-classical cannabinoid derivatives was that of the triaryl bis-sulfones, which were the result of the diarylpyrazole derivatives and resulted in extremely potent and selective compounds at $\mathrm{CB}_{2}$ (Section 5.2.4).

Taking this non-classical knowledge and amalgamating it with the previous classical, KM series and hexahydro-heteroaromatic compounds, gave rise to 4 distinct classes of triaryl cannabinoid ligands: HB series, SMM series, pyridine triaryl series and pyrimidine triaryl series (Figure 5.1; Section 5.3). The aim of this study is to utilize the ACTOne Assay (Chapter 2) to explore the functional effects of the HB series (Section 5.4.2), SMM series (Section 0), pyridine triaryl series (Section 5.4.4) and pyrimidine triaryl series (Section 5.4.5). Following this, I will reconcile these data against current literature standards' binding and homology models to propose hypothetical interactions within the $\mathrm{CB}_{1}$ and $\mathrm{CB}_{2} \mathrm{LBP}$ to further develop the $\mathrm{SAR}$, functional pharmacophore and unique MoA of these compounds. 


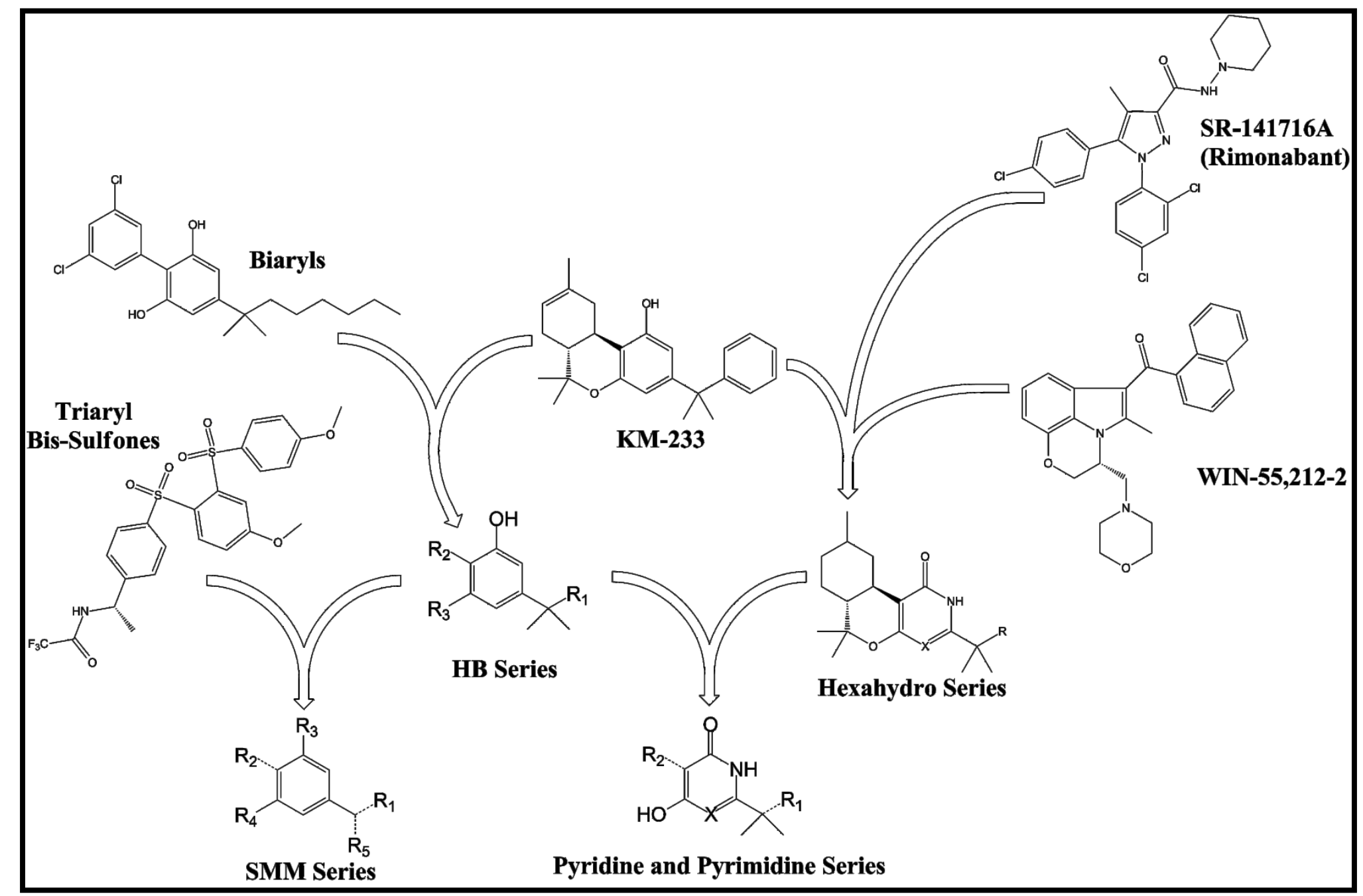

Figure 5.1. Triaryl series design flow 


\subsection{Non-Classical Cannabinoids}

\subsubsection{AC-bicyclic and ACD-tricyclic compounds}

These series of compounds were developed by Pfizer and omit the B-ring of the classical cannabinoid compounds, which is generally a pyran ring. The best known of these series is CP-55,940 (Figure 5.2), which works to incorporate many of the previously mentioned elements of the classical cannabinoids without, itself, being a classical cannabinoid. This AC-bicyclic structure has very good affinity and no specificity for $\mathrm{CB}_{1}$ and $\mathrm{CB}_{2}\left(\mathrm{CB}_{1} \mathrm{~K}_{\mathrm{i}}=0.6 \mathrm{nM} ; \mathrm{CB}_{2} \mathrm{~K}_{\mathrm{i}}=0.7 \mathrm{nM}\right)$. Closely related to CP-55,940 is CP-47,497 (Figure 5.2) which incorporates all of the same elements with the notable exception of a southern aliphatic hydroxyl (SAH). Lack of the SAH in $\mathrm{CP}-47,497$ causes almost a 4 -fold increase in affinity for the $\mathrm{CB}_{1}$ receptor $\left(\mathrm{CB}_{1} \mathrm{~K}_{\mathrm{i}}=2.1\right.$ $\mathrm{nM}$ ). While this is in no way a poor compound, it does show the added benefit of the $\mathrm{SAH}$ moiety in these compounds. Knowing that the SAH is a useful component of the cannabinoid compounds, a more conformationally restricted ACD-tricyclic derivative was synthesized with CP-55,244 (Figure 5.2) $\left(\mathrm{CB}_{1} \mathrm{~K}_{\mathrm{i}}=0.21 \mathrm{nM}\right)$. Restricting the $\mathrm{SAH}$ to project into the tricyclic ( $\mathrm{ABC}$ ring) pocket allows for a compound that is more potent than the prototype $\mathrm{CP}-55,940$. Still missing was the lack of specificity for targeting $\mathrm{CB}_{2}$ over $\mathrm{CB}_{1}$; however, the advent of the $\mathrm{AC}$ "D"-tricyclic ligand of HU-308 (Figure 5.2) $\left(\mathrm{CB}_{1} \mathrm{~K}_{\mathrm{i}}=>10,000 \mathrm{nM} ; \mathrm{CB}_{2} \mathrm{~K}_{\mathrm{i}}=22.7 \mathrm{nM}\right)$ helped to change that.

\subsubsection{Hybrid cross-over compounds}

After the advent of the non-classical cannabinoid compounds, and with good understanding of classical cannabinoids, compounds were synthesized to take advantage of useful aspects of both - more namely the C3-1',1'-dimethylheptyl of the classical and the $\mathrm{SAH}$ of the non-classical compounds. These compounds served a unique purpose in the understanding of cannabinoids by providing three-dimensional probes for the $\mathrm{CB}_{1}$ and $\mathrm{CB}_{2} \mathrm{LBP}$. Data from studies conducted in the Makriyannis lab group demonstrated that C-6 $\beta$-hydroxypropyl (178a, Figure 5.3) $\left(\mathrm{CB}_{1} \mathrm{~K}_{\mathrm{i}}=70.5 \mathrm{nM}\right)$ had a higher affinity for $\mathrm{CB}_{1}$ than did its $\alpha$-hydroxypropyl (178b, Figure 5.3) $\left(\mathrm{CB}_{1} \mathrm{~K}_{\mathrm{i}}=1353 \mathrm{nM}\right)$ epimer. $^{327-}$ ${ }^{329}$ Further derivation of these hybrid compounds led to unsaturation at the 1' position of the C-6 SAH substituent. The alkyne (179, Figure 5.3) demonstrates great affinity for both $\mathrm{CB}_{1}\left(\mathrm{~K}_{\mathrm{i}}=1.2 \mathrm{nM}\right)$ and $\mathrm{CB}_{2}\left(\mathrm{~K}_{\mathrm{i}}=0.3 \mathrm{nM}\right)$, with a slight affinity for $\mathrm{CB}_{2}$. The alkene (180, Figure 5.3) demonstrates good affinity for both $\mathrm{CB}_{1}\left(\mathrm{~K}_{\mathrm{i}}=0.7 \mathrm{nM}\right)$ and $\mathrm{CB}_{2}\left(\mathrm{~K}_{\mathrm{i}}=\right.$ $8.6 \mathrm{nM}$ ), with a $>10$-fold affinity for $\mathrm{CB}_{1}-$ the $\mathrm{C} 1$ " cis isomer (not pictured) of $\mathbf{1 8 0}$ demonstrated a difference in activity $\left(\mathrm{CB}_{1} \mathrm{~K}_{\mathrm{i}}=2.24 \mathrm{nM} ; \mathrm{CB}_{2} \mathrm{~K}_{\mathrm{i}}=0.33 \mathrm{nM}\right) .{ }^{330}$ It is noteworthy, that the values listed for compounds $\mathbf{1 7 9}$ and $\mathbf{1 8 0}$ are for the racemic mixture, and that work by Thakur and co-workers demonstrated that the active isomer of $\mathbf{1 8 0}$ is, as drawn, the $6 \mathrm{~S}, 6 \mathrm{aR}, 9 \mathrm{R}, 10 \mathrm{aR}$ configuration. ${ }^{331}$ 


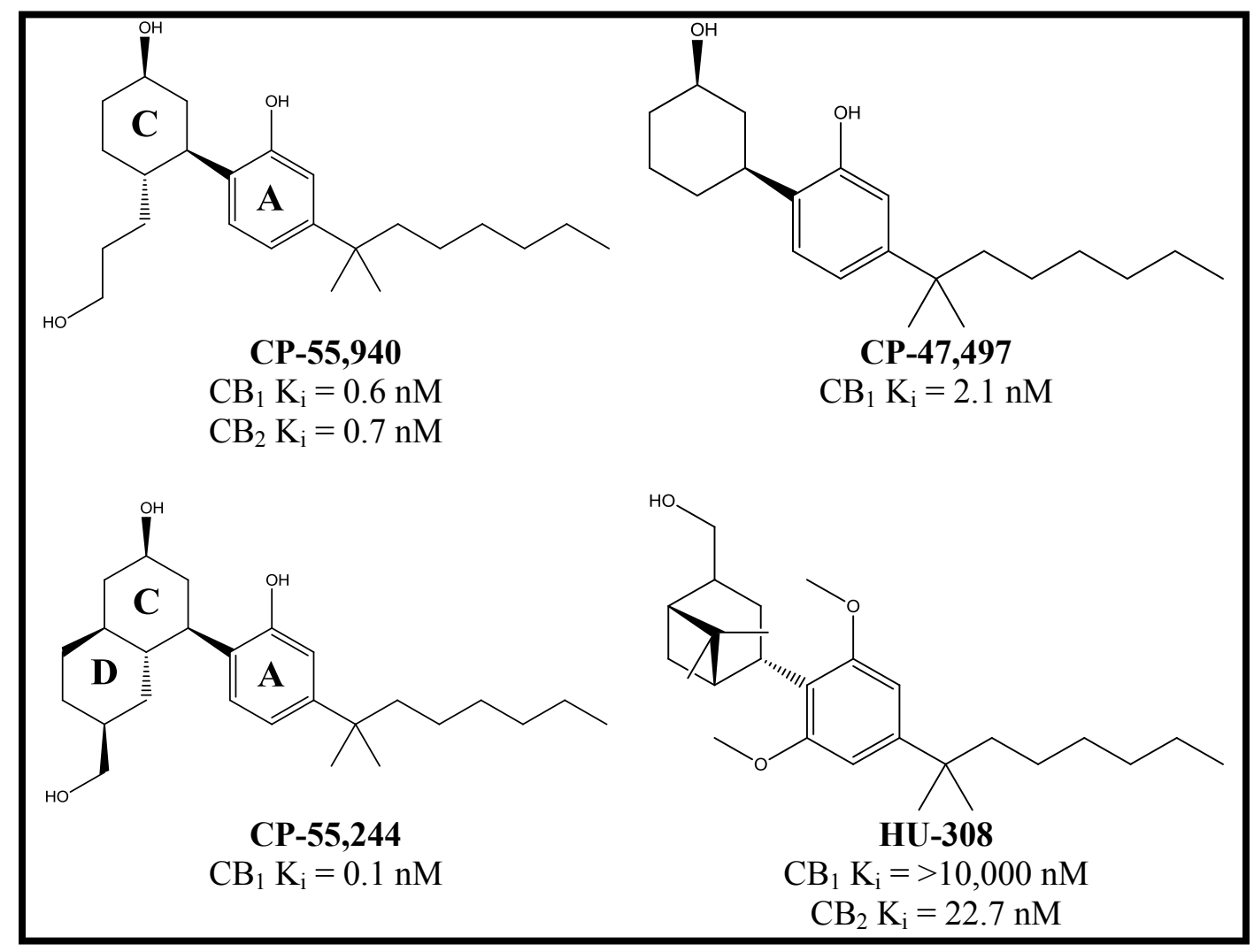

Figure 5.2. Non-classical cannabinoids 


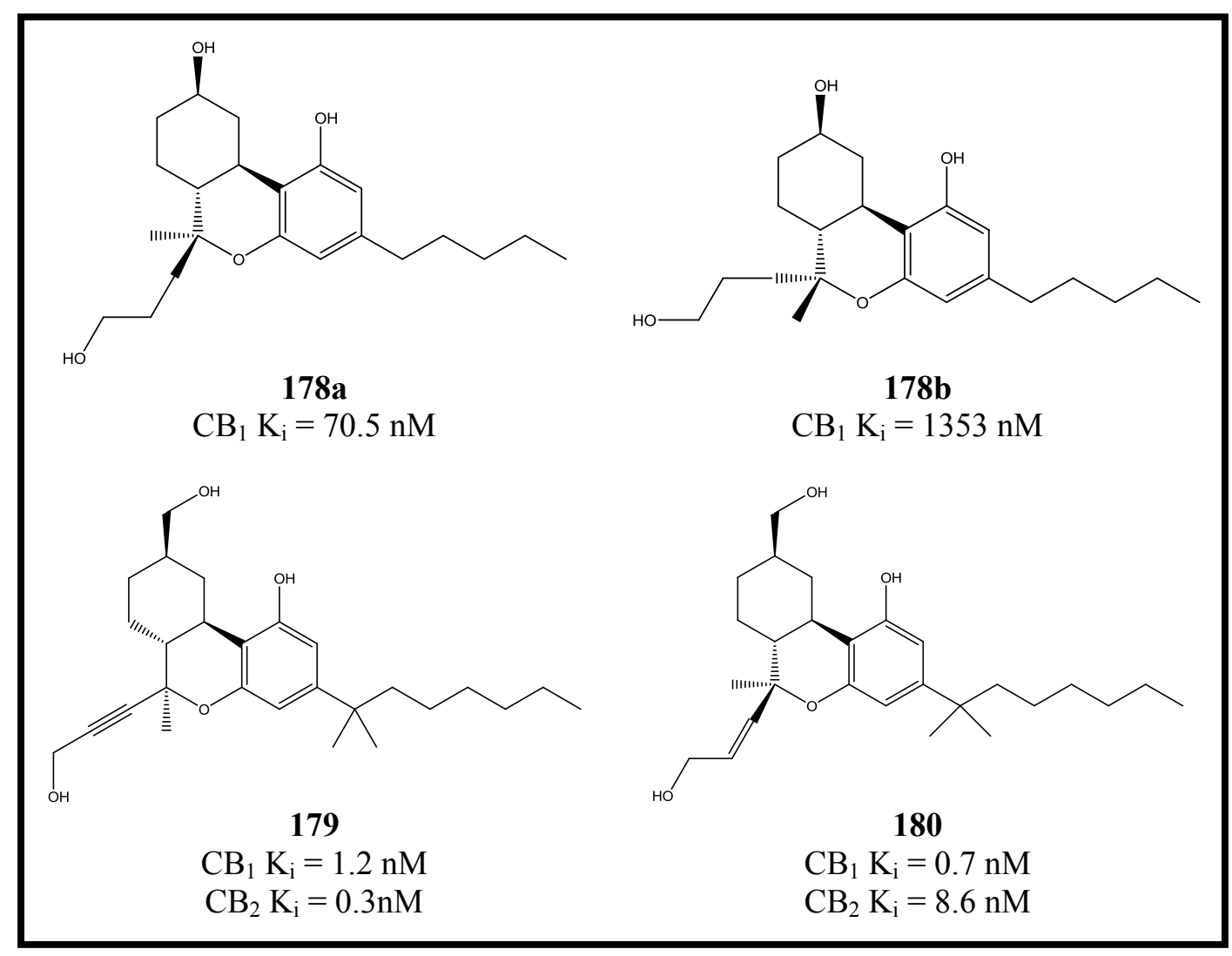

Figure 5.3. Hybrid cannabinoid ligands 


\subsubsection{Biaryl cannabinoids}

As an added attempt to bridge the gap among the non-classical cannabinoids and further hone in upon a $\mathrm{CB}_{2}$-selective compound, the biphenyl compounds were developed by Makriyannis' group. These compounds sought to build upon the work at Pfizer with the "CP" compounds, and add the selectivity of HU-308. The group, overall, has good affinity, with the prototype compound (181, Figure 5.4) having good affinity for both receptors, with a $\mathrm{CB}_{1} \mathrm{~K}_{\mathrm{i}}=2.6 \mathrm{nM}$ and $\mathrm{a} \mathrm{CB}_{2} \mathrm{~K}_{\mathrm{i}}=0.6 \mathrm{nM}$. Addition of more substituents to the "C"-ring (e.g. 182 and 183, Figure 5.4) led to a nearly 100-fold and 300-fold preference for $\mathrm{CB}_{2}$ over $\mathrm{CB}_{1}$, respectively. Incorporation of a heteroaromatic (furan) ring as the "C"-ring (184, Figure 5.4) confers a great deal of $\mathrm{CB}_{2}$ selectivity $\left(\mathrm{CB}_{1} / \mathrm{CB}_{2}\right.$ ratio = 90). ${ }^{332}$ This class of compounds has been disclosed in patent literature; however, little outside thereof has come to be known about these compounds.

\subsubsection{Triaryl bis-sulfones}

Initial discovery of these compounds was a direct result of work in the discovery and development of the diarylpyrazoles, such as SR-141716A. These were originally conceived as biaryl sulfone compounds (e.g. 185, Figure 5.5); however, analogs of this compound and an ultra-pure sample of this compound showed poor activity. Analysis of 185 via LCMS demonstrated a tainted sample - which in light of the pure sample's failure to elucidate effect, were likely contributing to the activity in vitro. Of these impurities, compound 186 (Figure 5.5) became the new lead molecule for development. ${ }^{333}$ Derivation of this lead compound centered initially on the substituents attached to the B- and C-ring, where investigation demonstrated a penchant for substitution at the 2 position of the C-ring and small alkyl/halogen substitution at the 4 position of the B-ring. ${ }^{334}$ Also examined were the linkages between A- and B-rings and B- and C-rings, with sulfone being the only link tolerated between B- and C-rings and ultimately the best choice for both junctions. ${ }^{334}$ Next in the elucidation and optimization for these compounds was the amide moiety, attached to the A-ring. Modifications about this position seemed to favor small substituents, with a preference for electron withdrawing groups. The primary example being the trifluoromethyl derivative (186) $\left(\mathrm{CB}_{1} \mathrm{~K}_{\mathrm{i}}=235 \mathrm{nM} ; \mathrm{CB}_{2} \mathrm{~K}_{\mathrm{i}}=0.3 \mathrm{nM}\right)$. This trait was further exemplified by the methylsulfonamide derivative (187, Figure 5.5) $\left(\mathrm{CB}_{1} \mathrm{~K}_{\mathrm{i}}=905 \mathrm{nM} ; \mathrm{CB}_{2} \mathrm{~K}_{\mathrm{i}}=0.4 \mathrm{nM}\right)-$ showing inverse agonist activity in the $\left[{ }^{35} \mathrm{~S}\right] \mathrm{GTP} \gamma \mathrm{S}$ assay, with an $\mathrm{EC}_{50}$ of $0.6 \mathrm{nM} .{ }^{333}$

Further development of $\mathbf{1 8 7}$ saw modifications to the C-ring, where o-F-phenyl (188, Figure 5.5) $\left(\mathrm{CB}_{2} \mathrm{~K}_{\mathrm{i}}=1.3 \mathrm{nM} ; \mathrm{CB}_{1} \mathrm{~K}_{\mathrm{i}} / \mathrm{CB}_{2} \mathrm{~K}_{\mathrm{i}}=4387\right)$ and 2-pyridine (189, Figure 5.5) $\left(\mathrm{CB}_{2} \mathrm{~K}_{\mathrm{i}}=2.9 \mathrm{nM} ; \mathrm{CB}_{1} \mathrm{~K}_{\mathrm{i}} / \mathrm{CB}_{2} \mathrm{~K}_{\mathrm{i}}=4403\right)$ exhibited similar selectivity ratios, but o-F-phenyl demonstrated a superior $\mathrm{CB}_{2} \mathrm{~K}_{\mathrm{i}} .{ }^{335}$ With a newly optimized C-ring, the amide moiety of the A-ring was re-examined, and data demonstrated that a trifluromethylsulfonamide (190, Figure 5.5) $\left(\mathrm{CB}_{2} \mathrm{~K}_{\mathrm{i}}=2 \mathrm{nM}\right)$ held top honors. ${ }^{335}$ The next step in optimization of the compound was to examine the halogen substitution on the B-ring; however, $\mathrm{Cl}$ held its place atop the possible moieties examined. ${ }^{335}$ To complete the cycle, the $\mathrm{C}$-ring was examined with the newly optimized trifluoromethylsulfonamide present on the A-ring, and as with the $\mathrm{Cl}$, o-F-phenyl retained its spot. ${ }^{335}$ 


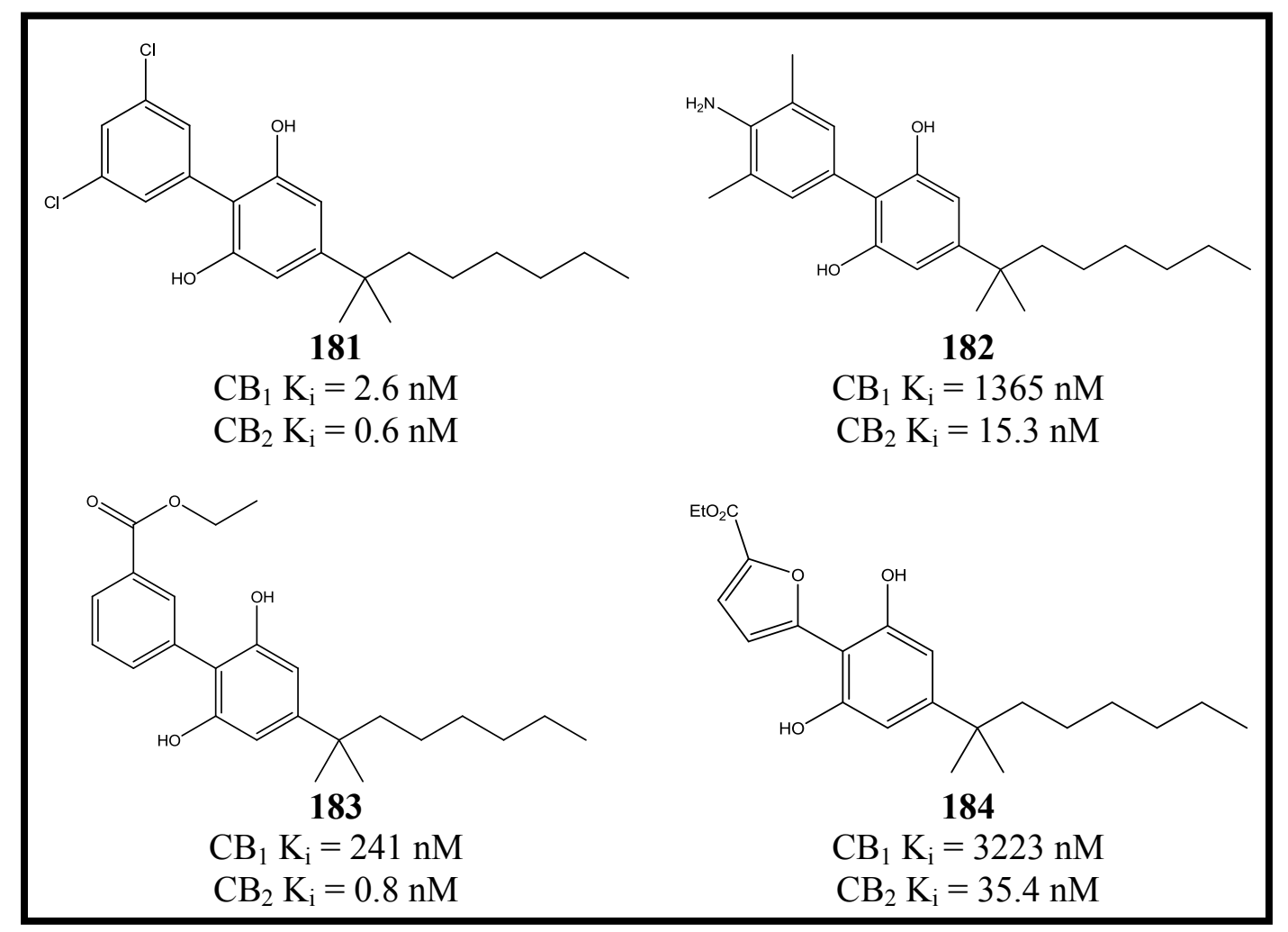

Figure 5.4. Biaryl cannabinoid ligands 


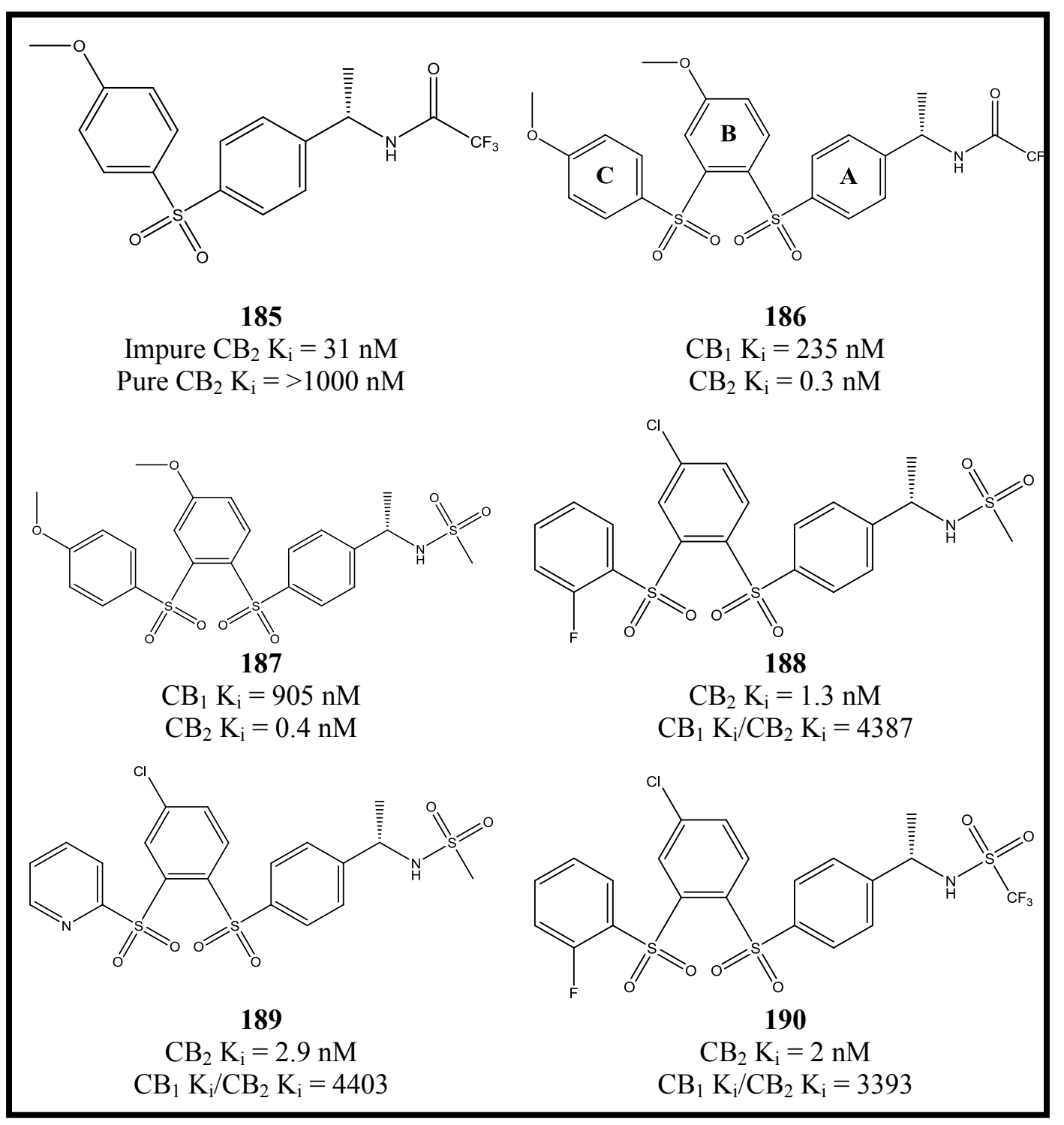

Figure 5.5. Triaryl bis-sulfone derivatives 


\subsection{Novel Series of Triaryl Cannabaergic Ligands}

\subsubsection{Rationale of design}

As previously examined in Chapter 3, the C-1'-dimethyl-aryl- $\Delta^{8}-\mathrm{THC}$ analogs demonstrated a unique mechanism in that they were agonists at $\mathrm{CB}_{1}$ and antagonists at $\mathrm{CB}_{2}$. Additionally, considering the increase in binding affinity gained with this novel series, they are good agents in their own right; however, as scientists are in the pursuit of as near perfection as one may attain, "good" is not good enough. Recall, too, the increase in binding affinity gained from the biaryl cannabinoids (Section 5.2.3) - though these particular cannabinoids did not lend themselves to specificity. With the combination of these two series, a third novel series was born - the HB series (Table 5.1).

Success of the HB series compounds in testing led one-step further in development - combine the qualities and attributes of the HB series with those of the triaryl bis-sulfones (Section 5.2.4). The hypothesis is that one may retain the wellestablished attributes of a $\mathrm{C}-1^{\prime}$ derivative while maximizing the hydrophilic interactions within the LBP - thus the SMM series (Table 5.2) came to be. To further hone activity, consideration was given to the classes of the aminoalkylindoles (Section 4.3.1) and diarylpyrazoles (Section 4.3.2), which have proven repeatedly that they are a potent classes of compounds. Additionally, the successes of the hexahydro heterocyclic derivatives discussed in Chapter $\mathbf{4}$ add another layer of consideration to the developing cannabinoid pharmacophore. Coupling all of these components with the SMM and HB series spawned two unique series to mimic the heterocyclic central core of the diarylpyrazoles and aminoalkylindoles. A heterocyclic A-ring afforded the pyridine (Table 5.3) and pyrimidine (Table 5.4) triaryl series of compounds. All were synthesized to separate affinity and/or activity to yield therapeutically viable compounds.

\subsubsection{Objectives of the study}

Utilizing these 4 novel series (Figure 5.6), I explored the functional pharmacology of each in relation to $\mathrm{CB}_{1}, \mathrm{CB}_{2}$ and the parental HEK-293-CNG cell lines (greater detail in Chapter 2) to better understand the functional activity. Further, reconciliation of known literature of binding and homology models helps to explain observed data and interaction of the ligands with the $\mathrm{LBP}$ of $\mathrm{CB}_{1}$ and/or $\mathrm{CB}_{2}$ receptors.

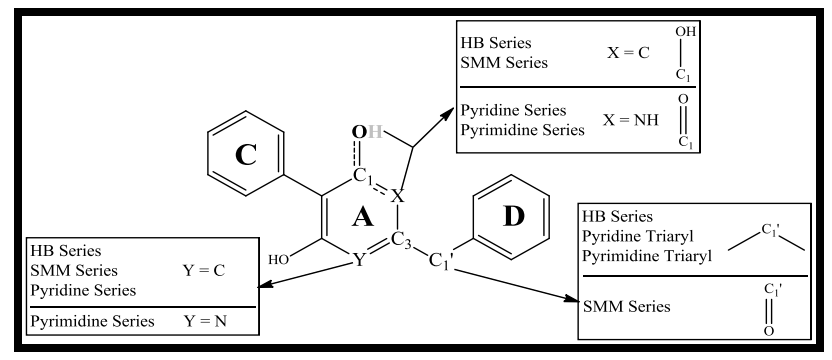

Figure 5.6. Generic triaryl structure nomenclature 
Table 5.1. HB triaryl series analogues

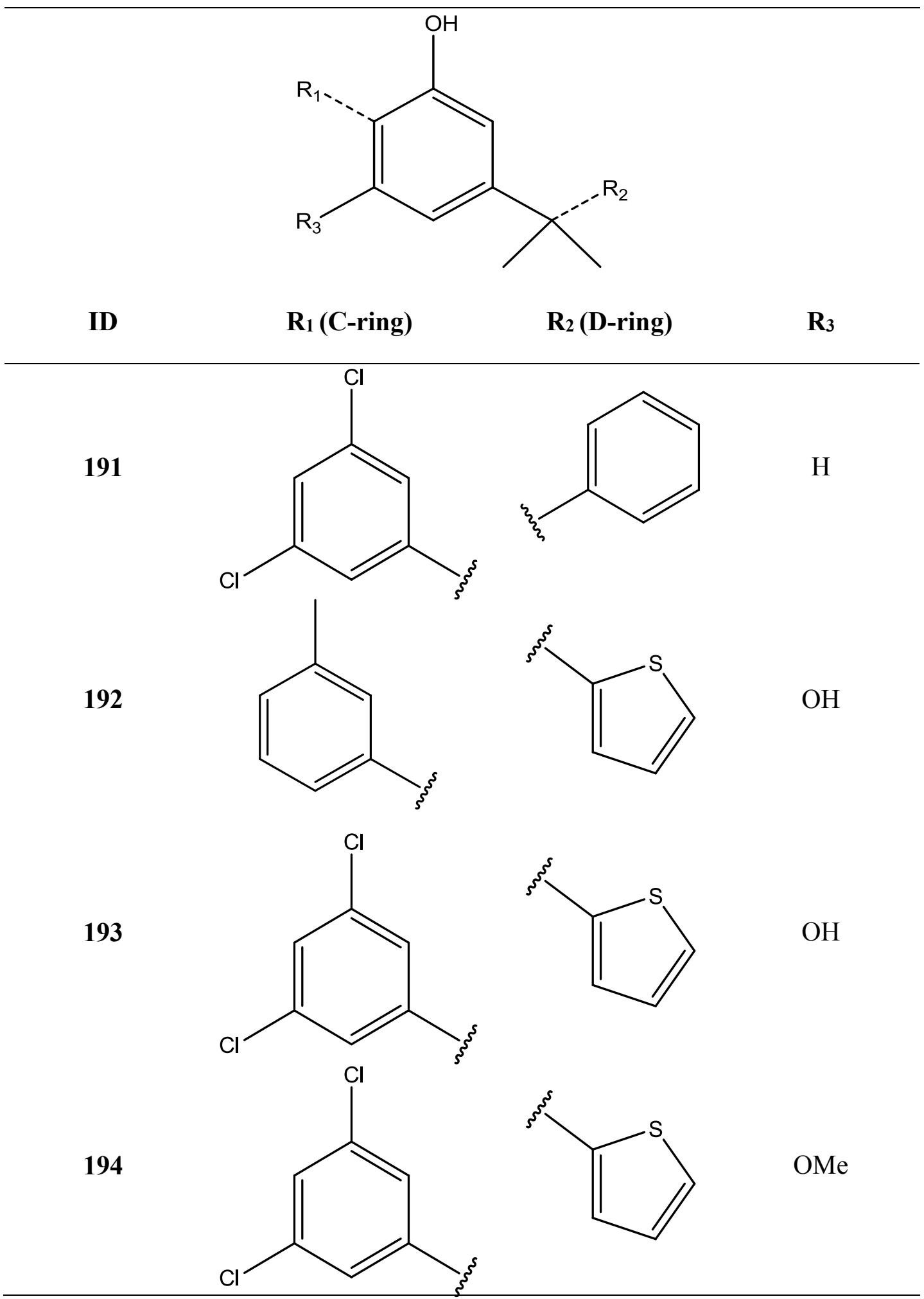


Table 5.2. SMM triaryl series analogues

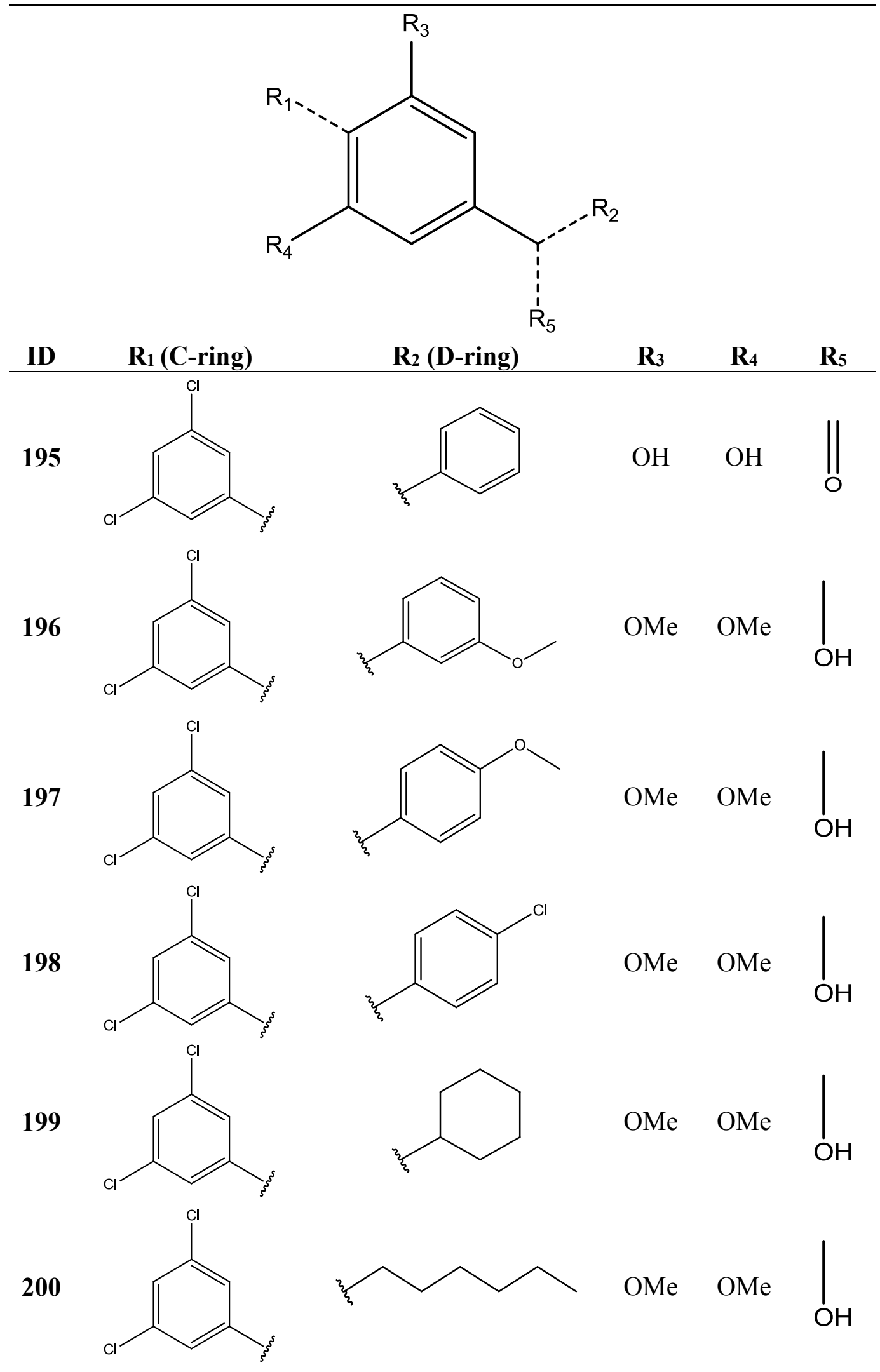


Table 5.2. (Continued)

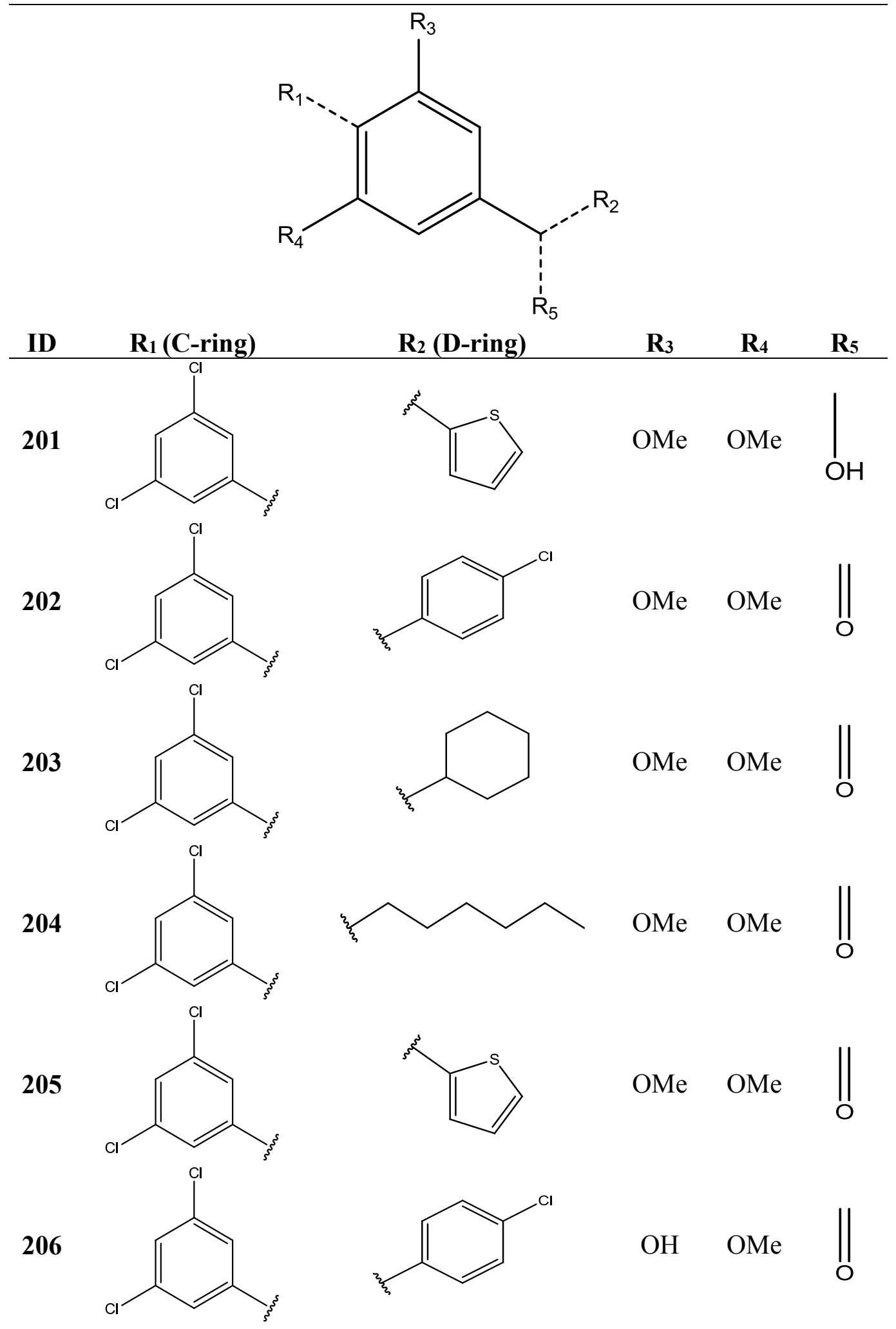


Table 5.2. (Continued)

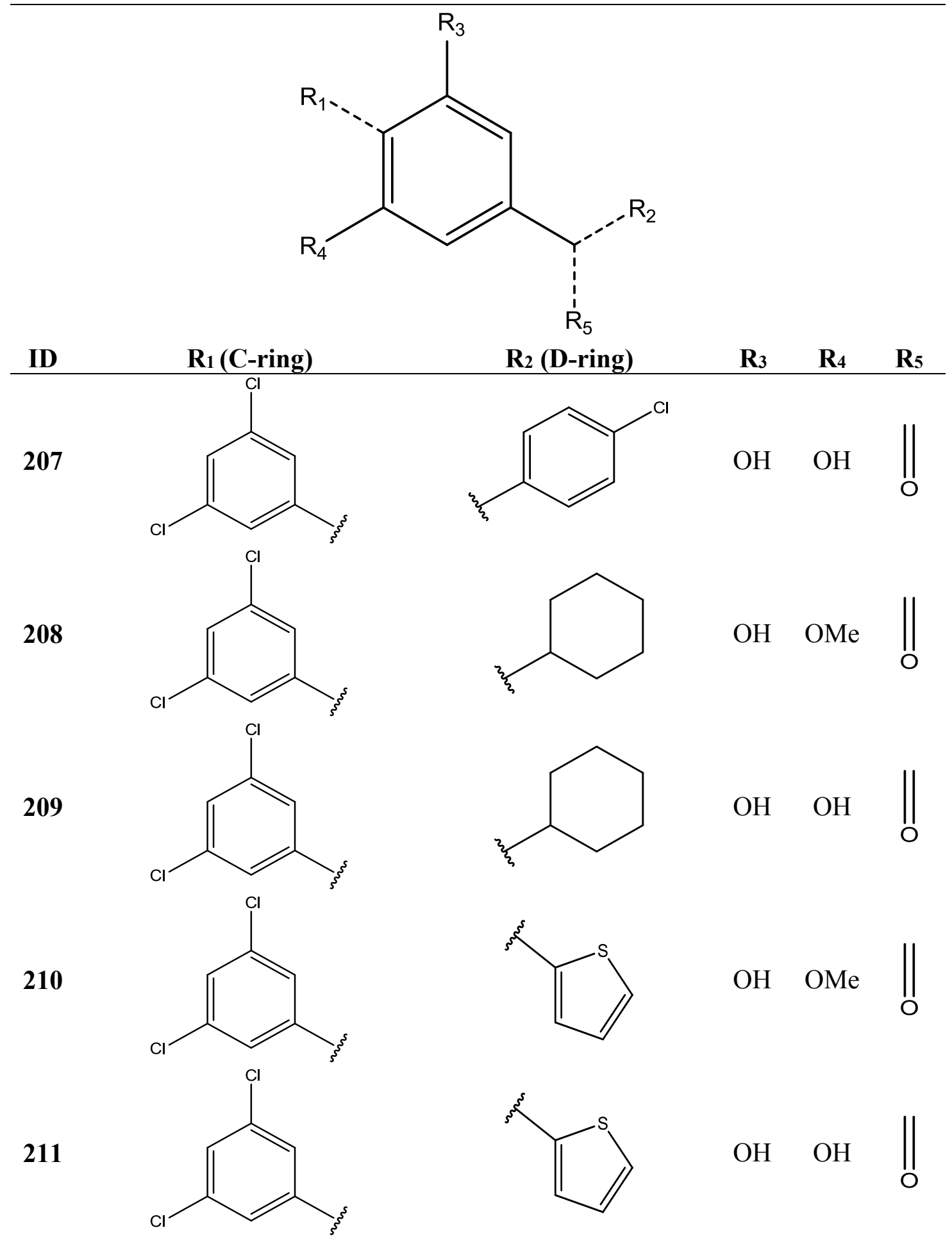


Table 5.2. (Continued)

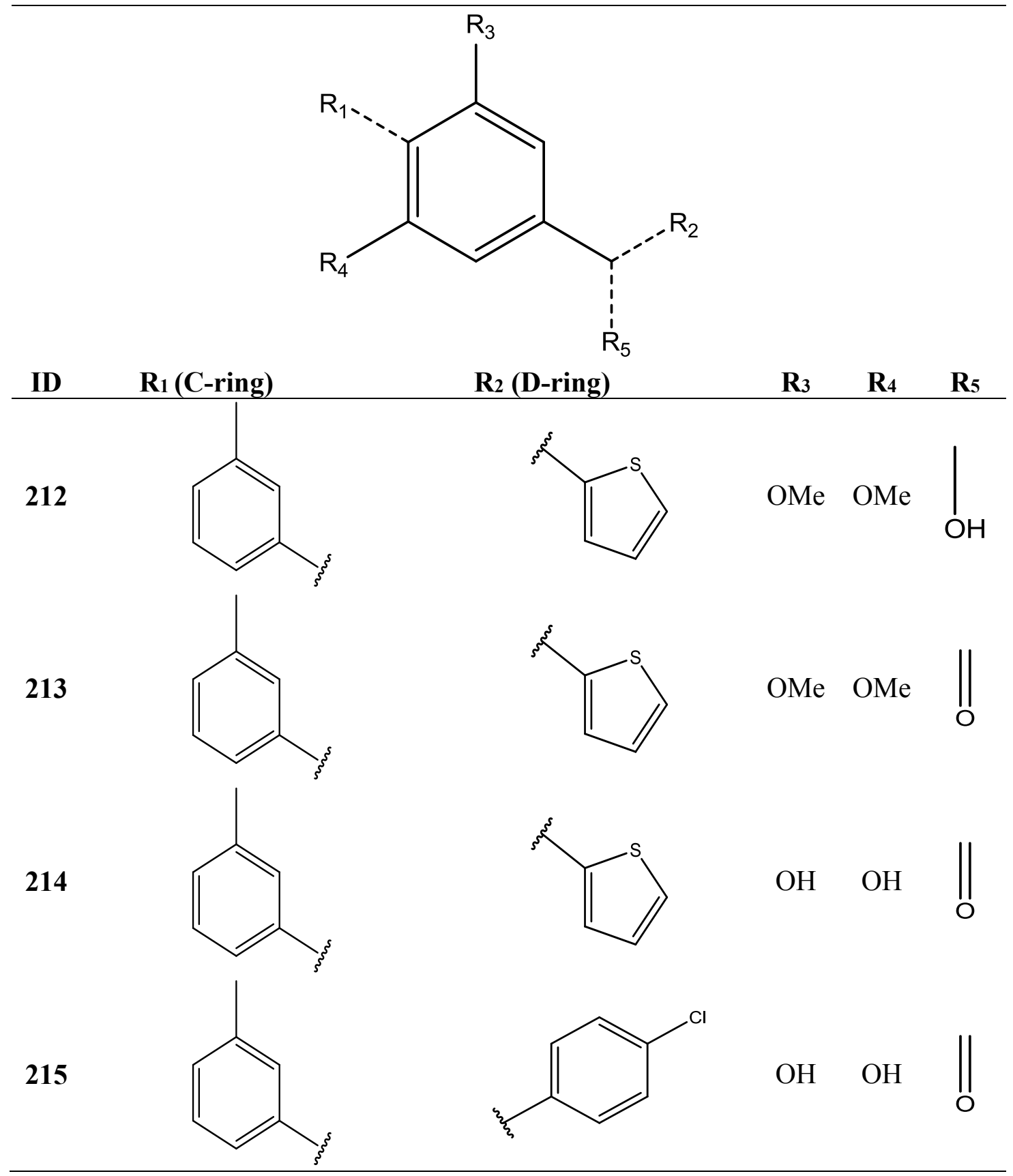


Table 5.3. Pyridine triaryl series compounds

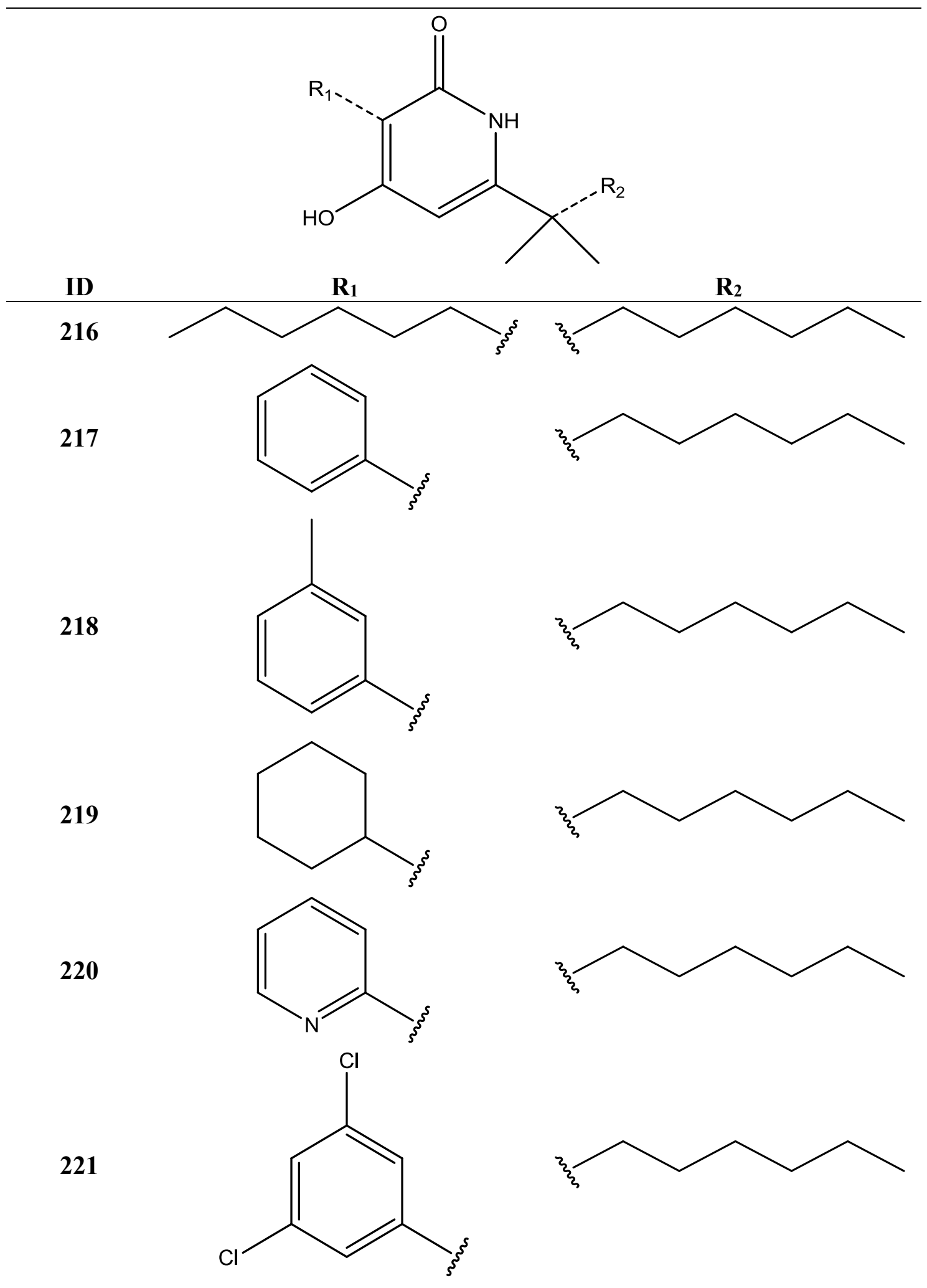


Table 5.3. (Continued)

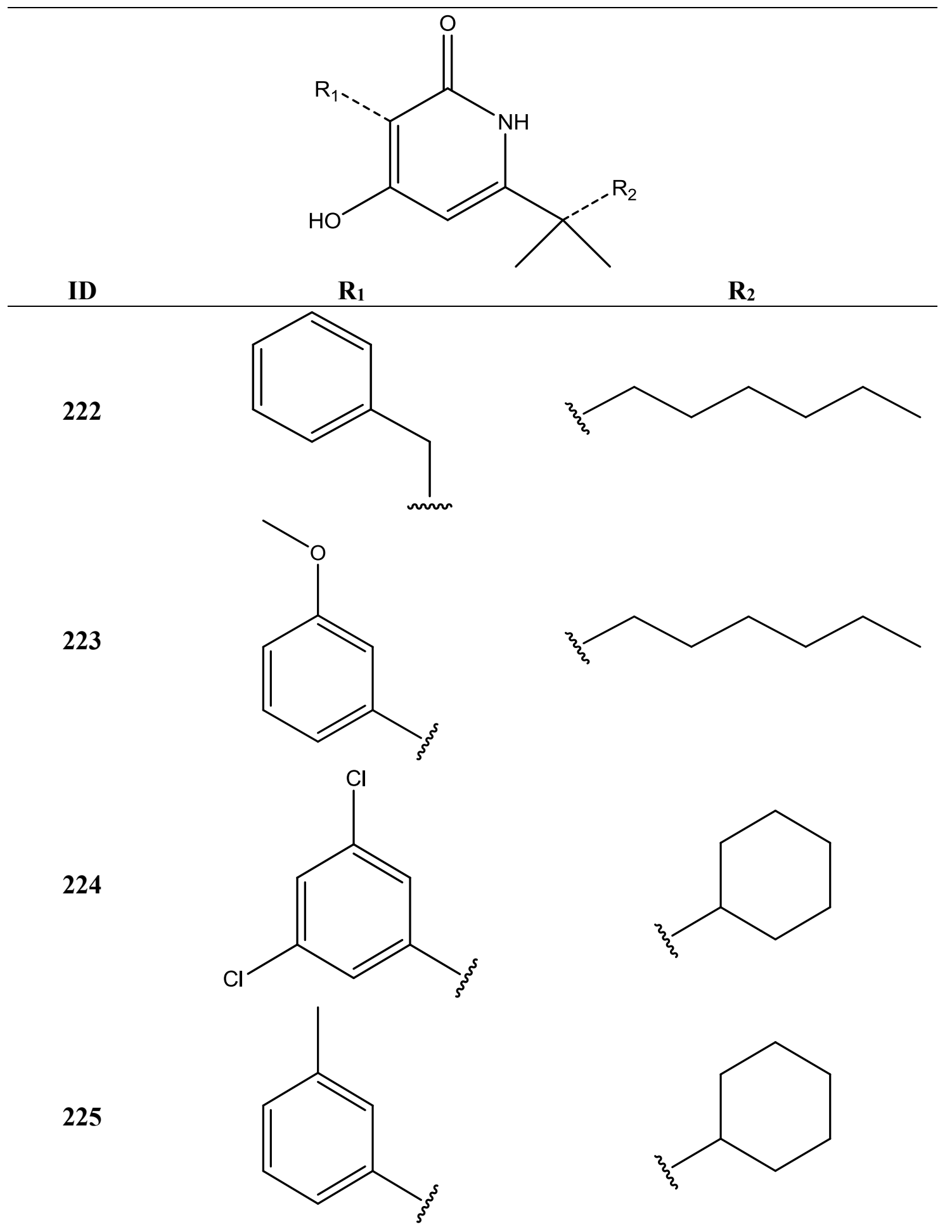


Table 5.3. (Continued)

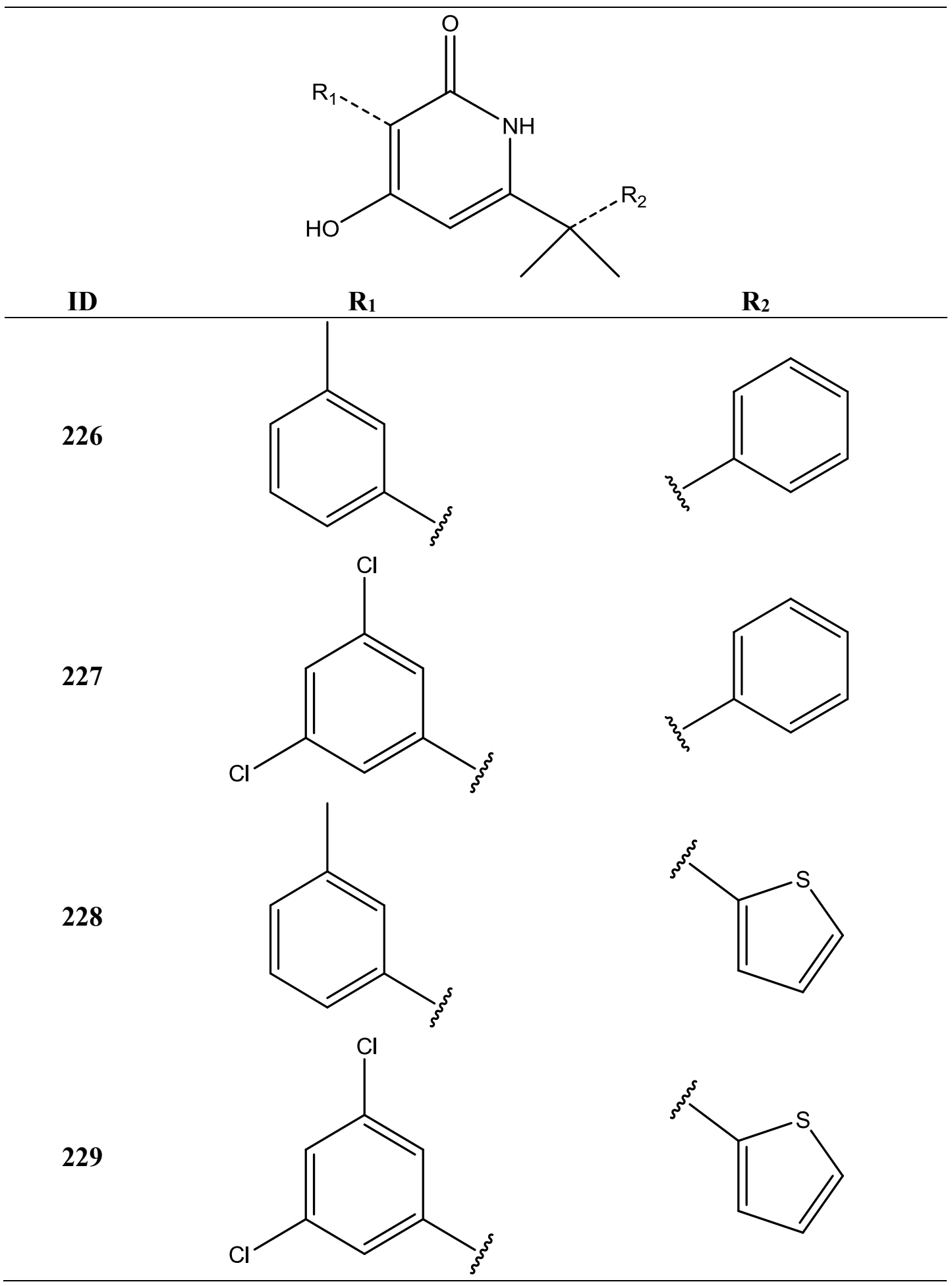


Table 5.4. Pyrimidine triaryl series compounds

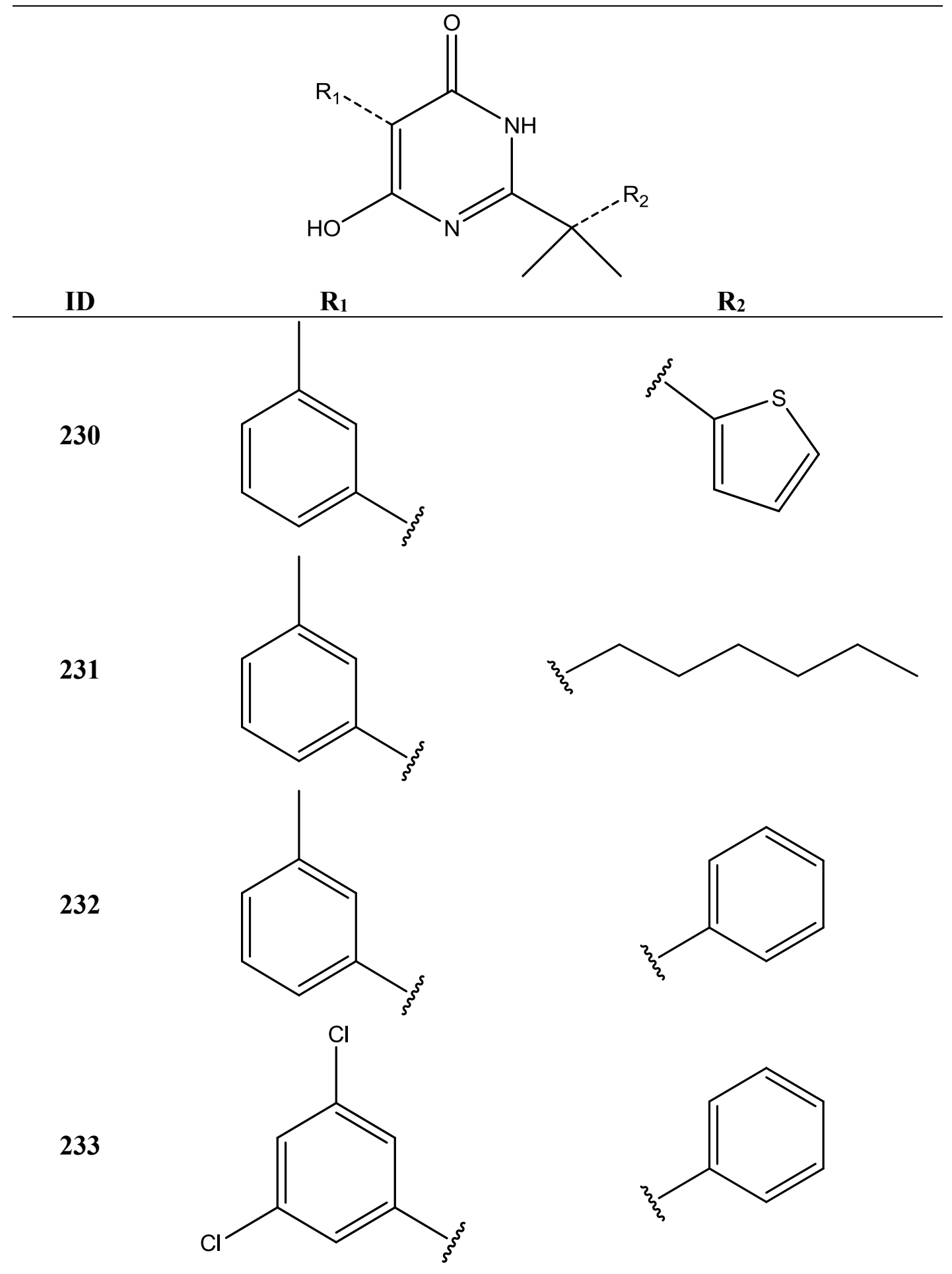


Table 5.4. (Continued)

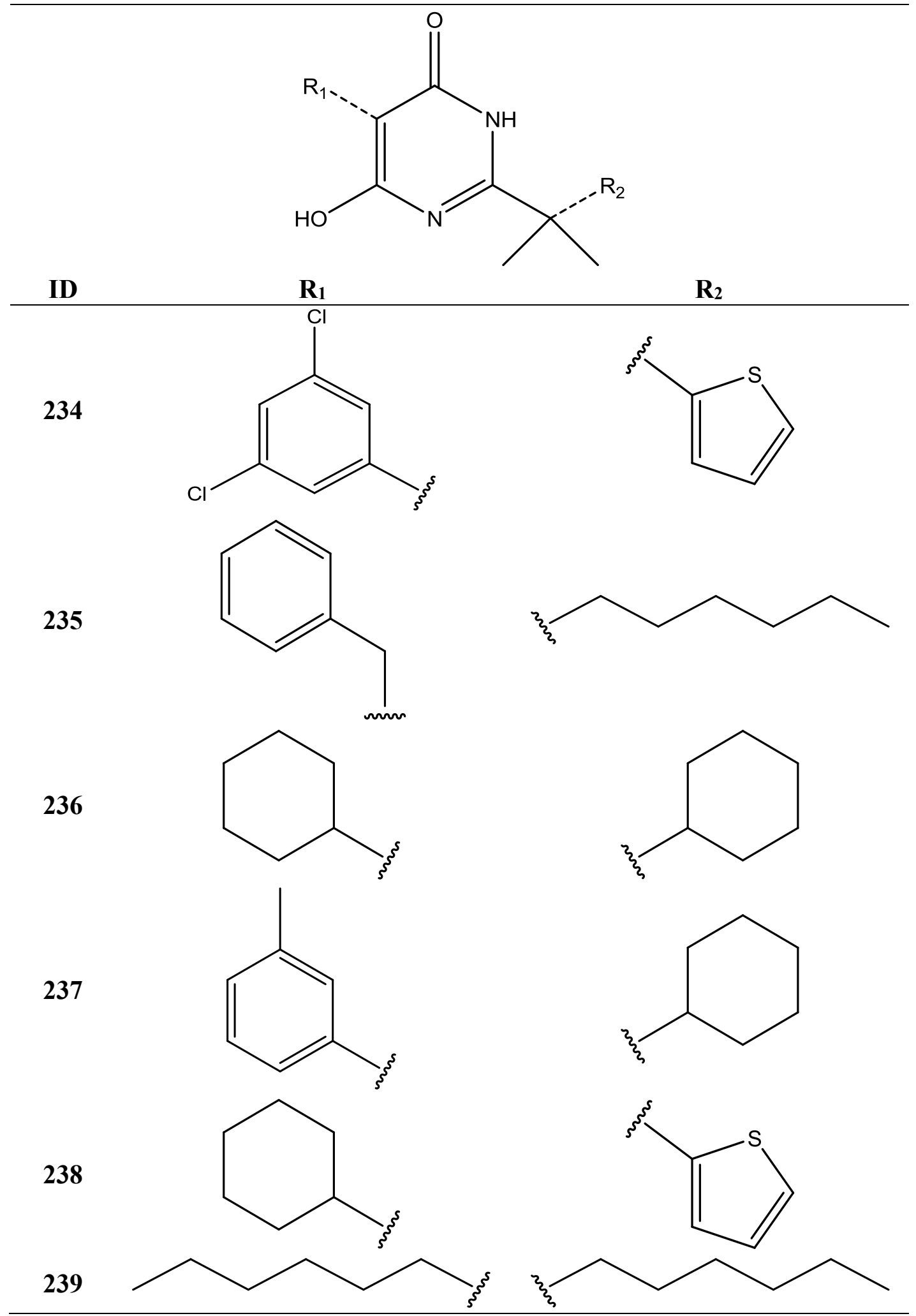




\subsection{Functional Pharmacology}

\subsubsection{ACTOne Assay}

Assay development and conductance was discussed in detail in Chapter 2. Briefly, stably transfected HEK-293 cells co-expressed with modified CNG channels along with $\mathrm{CB}_{1}$ or $\mathrm{CB}_{2}$ or their parental cells (without $\mathrm{CB}_{1}$ or $\mathrm{CB}_{2}$; HEK-CNG) were plated at an appropriate density in a clear, 96-well, Poly-D-Lysine plate and allowed to incubate at $37^{\circ} \mathrm{C}$ and $5 \%$ overnight. After the overnight incubation period, $100 \mu \mathrm{L}$ of ACTOne membrane potential dye was added to each well of the culture plate and allowed to sit in a dark area at room temperature for 60 additional minutes. After the 60 minute incubation period a baseline read in a BioTek Synergy 2 plate reader with an excitation of $540 / 25 \mathrm{~nm}$ and an emission of 590/20 nm. Following the baseline read, $50 \mu \mathrm{L}$ of drug stock solution containing appropriate concentrations of drug (Figure 5.7), Forskolin and Ro 20-2304 in DPBS with 2.5\% DMSO was added to the cell culture plate. The plate was then placed on the plate reader with 1 read per well per minute with an excitation of $540 / 25 \mathrm{~nm}$ and an emission of 590/20 nm for 60 minutes.

Screening of compounds against $\mathrm{CB}_{1}$ and $\mathrm{CB}_{2}$ were completed as technical replicates of $n=6$ each. A decrease in the number of replicates $(n=3)$ for HEK-CNG Parental and $\mathrm{CB}_{1} / \mathrm{CB}_{2}$ PTx-sensitive $\mathrm{G}_{\mathrm{i} / \mathrm{o}}$-inactivation was employed due to the expected lack of response from these compounds to those three (3) groups of assays, thus maximizing resource allocation and ensuring accurate data evaluation.

Primary data were analyzed with customized VBA macros (Chapter 2), developed internally, within Microsoft Excel (Redmond, WA). Column 11 (lowest [drug]) determined the experimental maximum response (100\% activity) at each individual time point, and the experimental basal response was determined by the average response given by the 2 blank control wells. All data were normalized with these minimum and maximum values via Equation 2.1. The $\mathrm{EC}_{50}$ values were calculated from concentration-response curves by non-linear regression and automatic outlier elimination analysis utilizing GraphPad Prism Software (San Diego, CA).

\subsubsection{Functional activity for HB triaryl series}

5.4.2.1. $\mathrm{CB}_{1}$ functional activity for the $\mathrm{HB}$ series. Plates were run as previously described (Section 4.5.1) in six (6) replicates with CP-55,940 as the internal control for all six (6) replicates (Figure 5.8). Compounds 192 and 193 demonstrated agonist activity at $\mathrm{CB}_{1}$ with an $\mathrm{EC}_{50}$ of $993 \mathrm{nM}, 1429 \mathrm{nM}$, respectively, with percent cAMP inhibition ranging from $46 \%$ to $57 \%, 50$ minutes after compound addition into the assay plate (Table 5.8). These two (2) compounds exhibit a decrease in cAMP production equivalent to our standard of CP-55,940, albeit at a higher $\mathrm{EC}_{50}$. Compound 191 was tested at a lower concentration gradient than the other three (3) compounds to examine the effects of its mono-hydroxy A-ring more directly with compound 195. However, data do suggest that shifting the concentrations to the higher range may result in curves more similar to 192 and 193, with a $10 \%$ decrease in cAMP at $1 \mu \mathrm{M}$ drug concentration. 


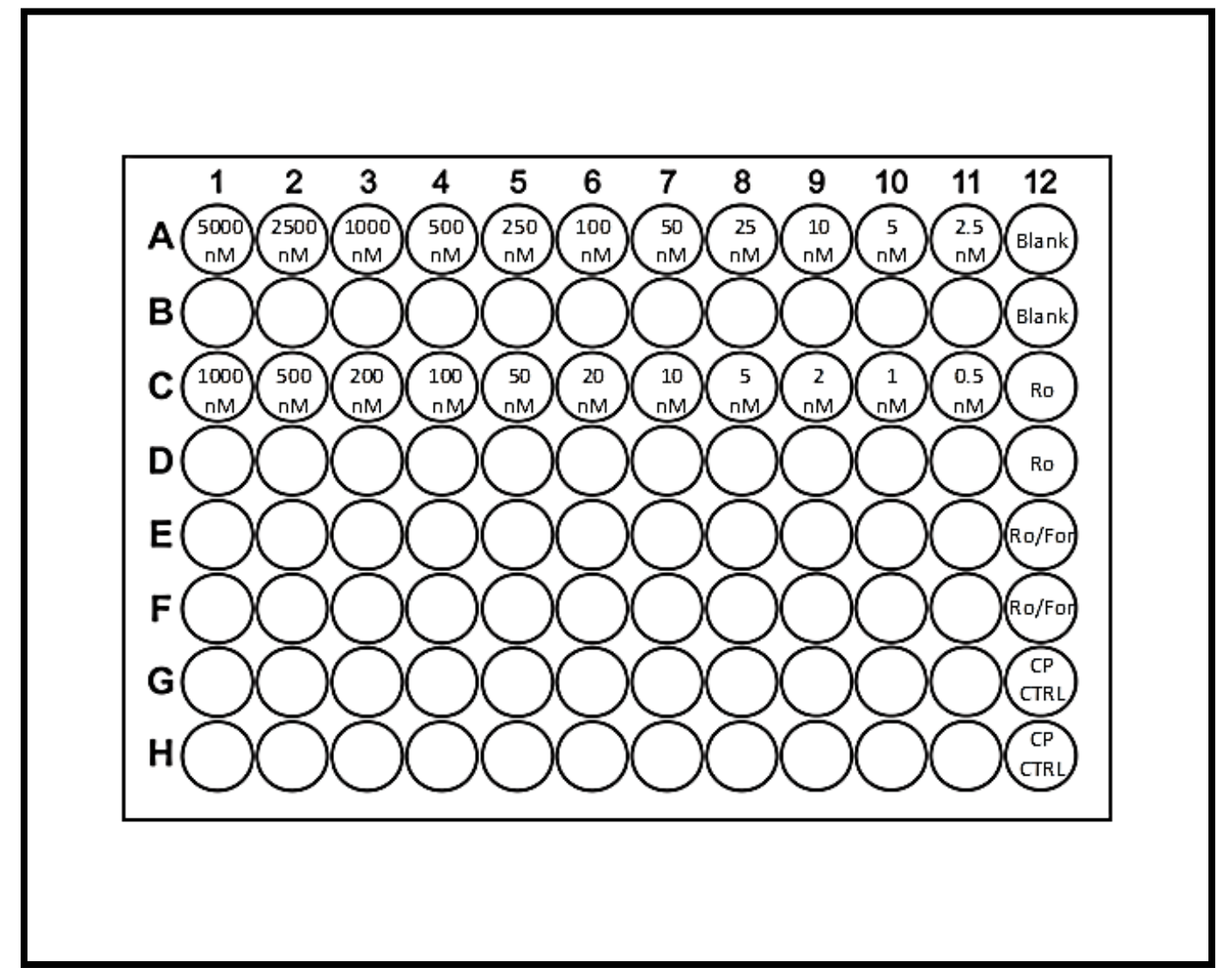

Figure 5.7. Drug plate layout and concentrations

Notes: Columns 1 through 11 contain test compound, and Column 12 contains control wells. All listed concentrations are the final concentrations once added to the testing plate, so in the drug plate all compounds are at $5 \mathrm{x}$ of the listed concentrations in Columns 1 through 11. Control wells (Column 12) are: "Blank" (cells, media and dye), "Ro" ("Blank" + $125 \mu$ M Ro 20-2304 [25 $\mu$ M, Final]), "Ro/For" ("Ro" $+4 \mu$ M Forskolin [800 nM, Final]) and "CP CTRL" ("Ro/For" + $25 \mu \mathrm{M}$ CP 55,940 [5 $\mu \mathrm{M}$, Final]). 


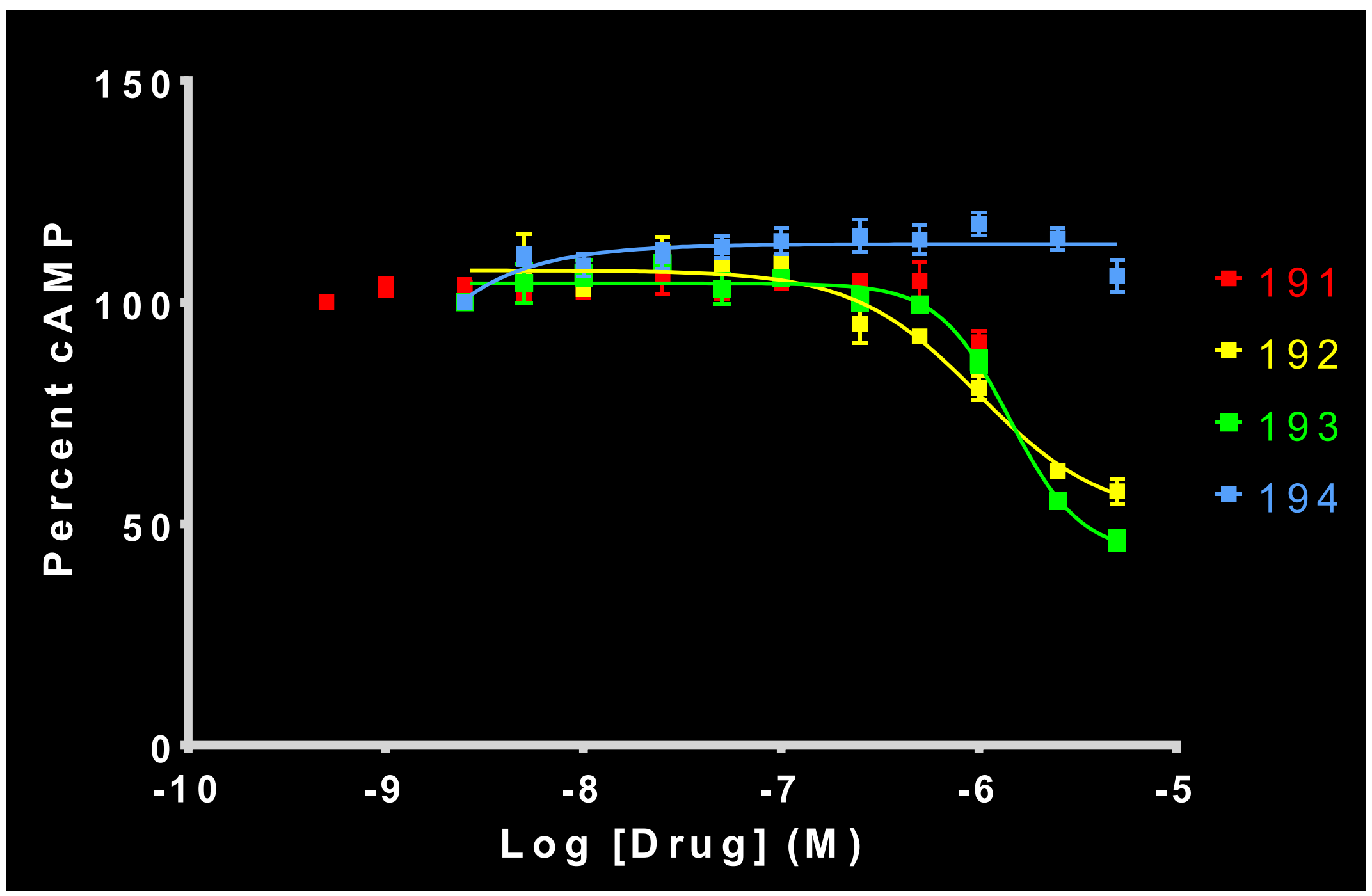

Figure 5.8. $\quad \mathrm{CB}_{1}$ functional activity of $\mathrm{HB}$ triaryl series, 50 minutes after compound addition 


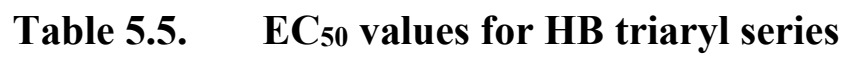

\begin{tabular}{cccccc}
\hline Compound & $\begin{array}{c}\text { CB1 } \\
\text { EC50 } \\
\text { or } \\
\text { Concentration } \\
\text { (\% baseline) }\end{array}$ & $\begin{array}{c}\text { CB2 } \\
\text { EC50 } \\
\text { or } \\
\text { Concentration } \\
\text { (\% baseline) }\end{array}$ & $\begin{array}{c}\text { HEK-CNG } \\
\text { EC50 (nM) }\end{array}$ & $\begin{array}{c}\text { CB1 PTx } \\
\text { EC50 (nM) }\end{array}$ & $\begin{array}{c}\text { CB2 PTx } \\
\text { EC50 (nM) }\end{array}$ \\
\hline 191 & $\mathrm{n} / \mathrm{a}$ & $25(10 \%) \mathrm{b}$ & $\mathrm{n} / \mathrm{a}$ & - & - \\
192 & $993 \mathrm{a}$ & $10.3 \mathrm{a}$ & $\mathrm{n} / \mathrm{a}$ & $\mathrm{n} / \mathrm{a}$ & $\mathrm{n} / \mathrm{a}$ \\
193 & $1429 \mathrm{a}$ & $7.4 \mathrm{a}$ & $\mathrm{n} / \mathrm{a}$ & $\mathrm{n} / \mathrm{a}$ & $\mathrm{n} / \mathrm{a}$ \\
194 & $1000(10 \%) \mathrm{a}$ & $1000(10 \%) \mathrm{a}$ & $\mathrm{n} / \mathrm{a}$ & - & $\mathrm{n} / \mathrm{a}$ \\
\hline
\end{tabular}

Notes: All concentrations are listed as nM, percentages indicate the change from baseline. n/a: Not active, based on no statistically significant change in cAMP from baseline. "-." Indicates test not performed. a Indicates a decrease in cAMP as compared to baseline. ${ }^{b}$ Indicates an increase in cAMP as compared to baseline 
5.4.2.2. $\mathrm{CB}_{2}$ functional activity for the HB Series. Plates were run as previously described (Section 5.4.1) in six (6) replicates with CP-55,940 as the internal control (Figure 5.9). All compounds in the series bind with good affinity to the $\mathrm{CB}_{2}$ receptor. ${ }^{259}$ Similar to $\mathrm{CB}_{1}$ functional activity assays 192 and 193 (Figure 5.9) demonstrated agonist activity; however, it was not as effective in terms the percent cAMP inhibited at $\mathrm{CB}_{2}$. Compounds 192 and 193 did demonstrate an $\mathrm{EC}_{50}$ that was significantly better than it had fared at $\mathrm{CB}_{1} . \mathrm{EC}_{50}$ for 192 and 193 was $10.3 \mathrm{nM}$ and $7.4 \mathrm{nM}$, with percent cAMP inhibition of approximately $17 \%$ and 14\%, respectively. Additionally, 191 and 194 showed a 10\% change in cAMP in an inverse agonist and agonist fashion, respectively.

5.4.2.3. CNG parental functional activity for the $\mathrm{HB}$ series. Plates were run as previously described (Section 5.4.1) in three (3) replicates with CP-55,940 as the internal control for all three (3) replicates (Figure 5.10). As was expected, all compounds in the series failed to elucidate any statistically significant change in the cAMP production of the cells, including the reference standard of CP-55,940.

5.4.2.4. $C_{1}$ PTx-sensitive $G_{i / 0}$-inactivation for the HB series. Plates were run as previously described (Section 5.4.1) in three (3) replicates with CP-55,940 as the internal control for all three (3) replicates (Figure 5.11). As was expected, all compounds failed to alter cAMP production, including the reference standard of CP-55,940. Only compounds 192 and 193 were screened in the PTx-sensitive $G_{i / o}$-inactivation assays due to their ability to elucidate a response in the standard $\mathrm{CB}_{1}$ functional assay.

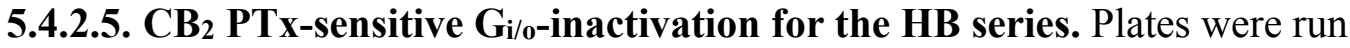
as previously described (Section 5.4.1) in three (3) replicates with CP-55,940 as the internal control for all three (3) replicates (Figure 5.12). As was expected, the tested compound failed to alter cAMP production, including the reference standard of CP-55,940. Only compound 192 was screened in the PTx-sensitive $\mathrm{G}_{\mathrm{i} / \mathrm{o}}$-inactivation assays due to its ability to elucidate a response in the standard $\mathrm{CB}_{2}$ functional assay.

\subsubsection{Functional activity for SMM Triaryl series}

5.4.3.1. $\mathrm{CB}_{1}$ functional activity for the SMM series. Plates were run as previously described (Section 5.4.1) in six (6) replicates - unless the compound was known to not bind, in which case it was run in three (3) replicates - with CP-55,940 as the internal control for all replicates (Figure 5.13). As evidenced in Figure 5.13, nearly half of the compounds had no appreciable effect on the cAMP levels. Of those with some alteration of cAMP activity, all demonstrated an increase; however, most did not result in curves with a good fit upon data analysis. Compounds 200, 206 and 210 showed a maximum effect at $5 \mu \mathrm{M}$ with an increase in cAMP of $48 \%, 32 \%$ and $24 \%$, respectively. Compounds 202 and 203 showed a maximum effect at $50 \mathrm{nM}$, with an increase in cAMP of $85 \%$ and 75\%, respectively. Three compounds 204, 211 and 213 (Figure 5.14), demonstrate inverse agonist activity at $\mathrm{CB}_{1}$, with an $\mathrm{EC}_{50}$ of $469 \mathrm{nM}, 1497 \mathrm{nM}$ and 153 $\mathrm{nM}$, respectively (Table 5.6), with percent cAMP induction ranging from $37 \%$ to $68 \%$ over basal. These compounds failed to attain the level of cAMP induction relative to our standard inverse agonist of SR-41716A (80\% increase); however, they are worth noting. 


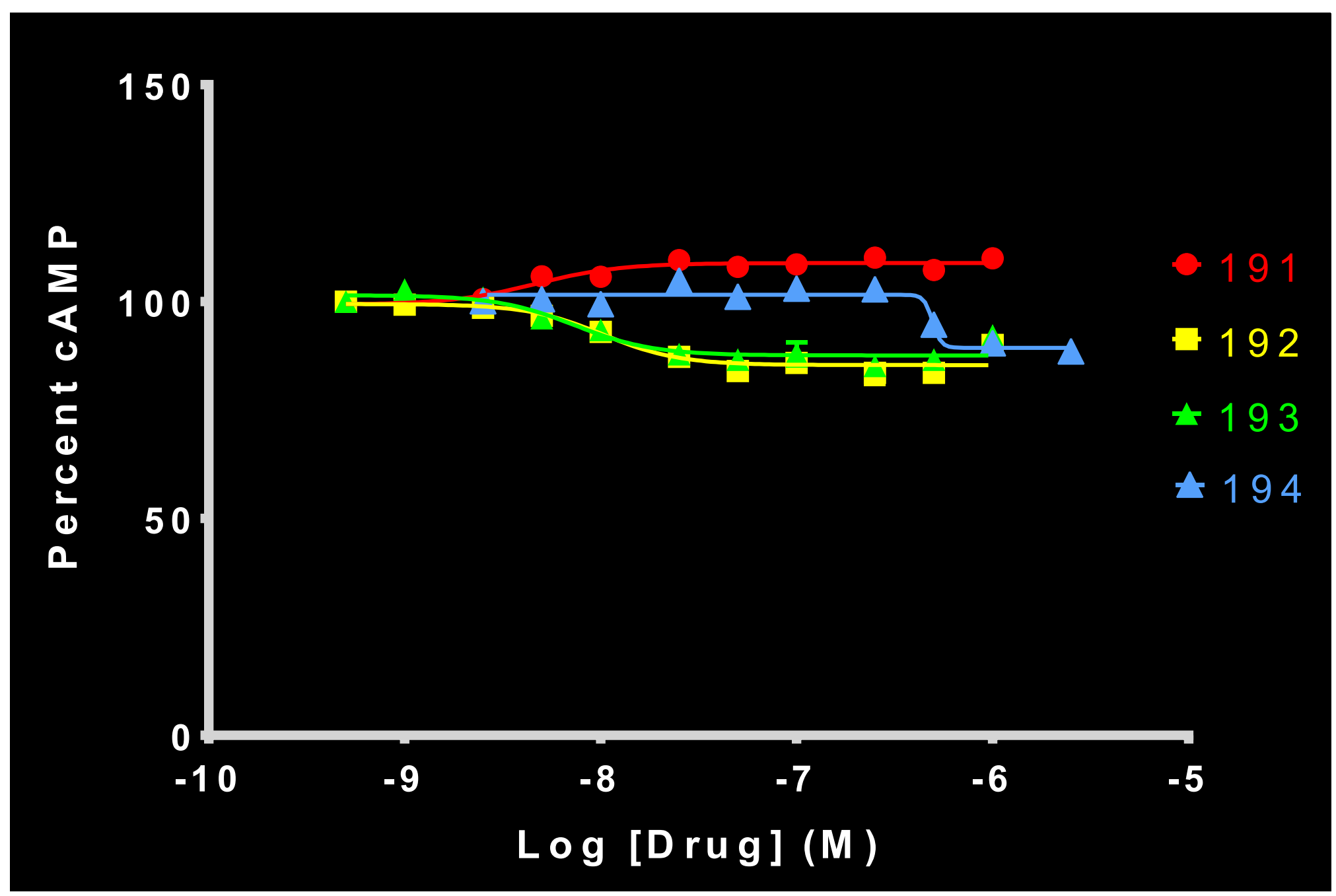

Figure 5.9. $\quad \mathrm{CB}_{2}$ functional activity of $\mathrm{HB}$ triaryl series, 50 minutes after compound addition 


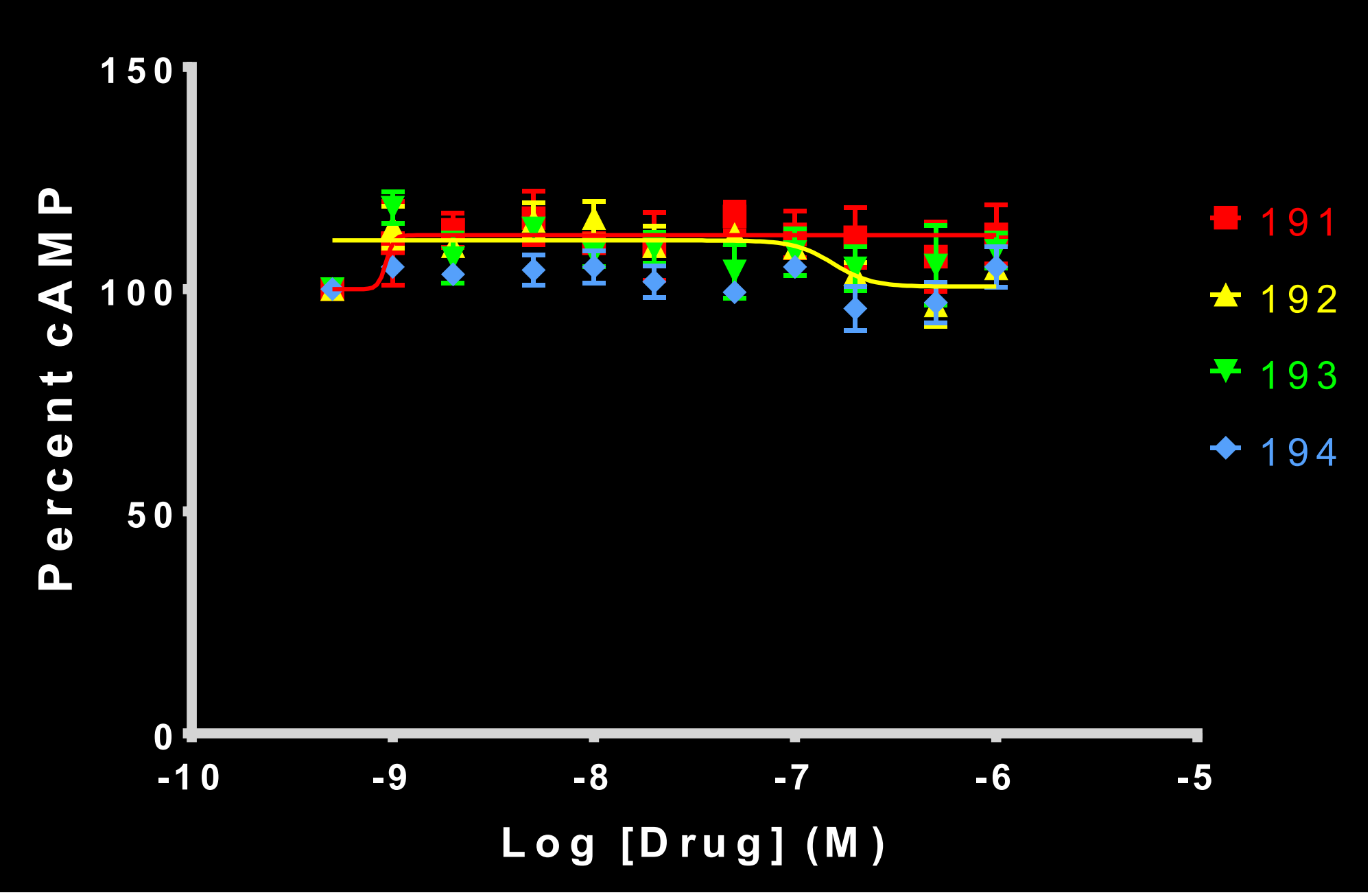

Figure 5.10. CNG functional activity of $\mathrm{HB}$ triaryl series, 50 minutes after compound addition 


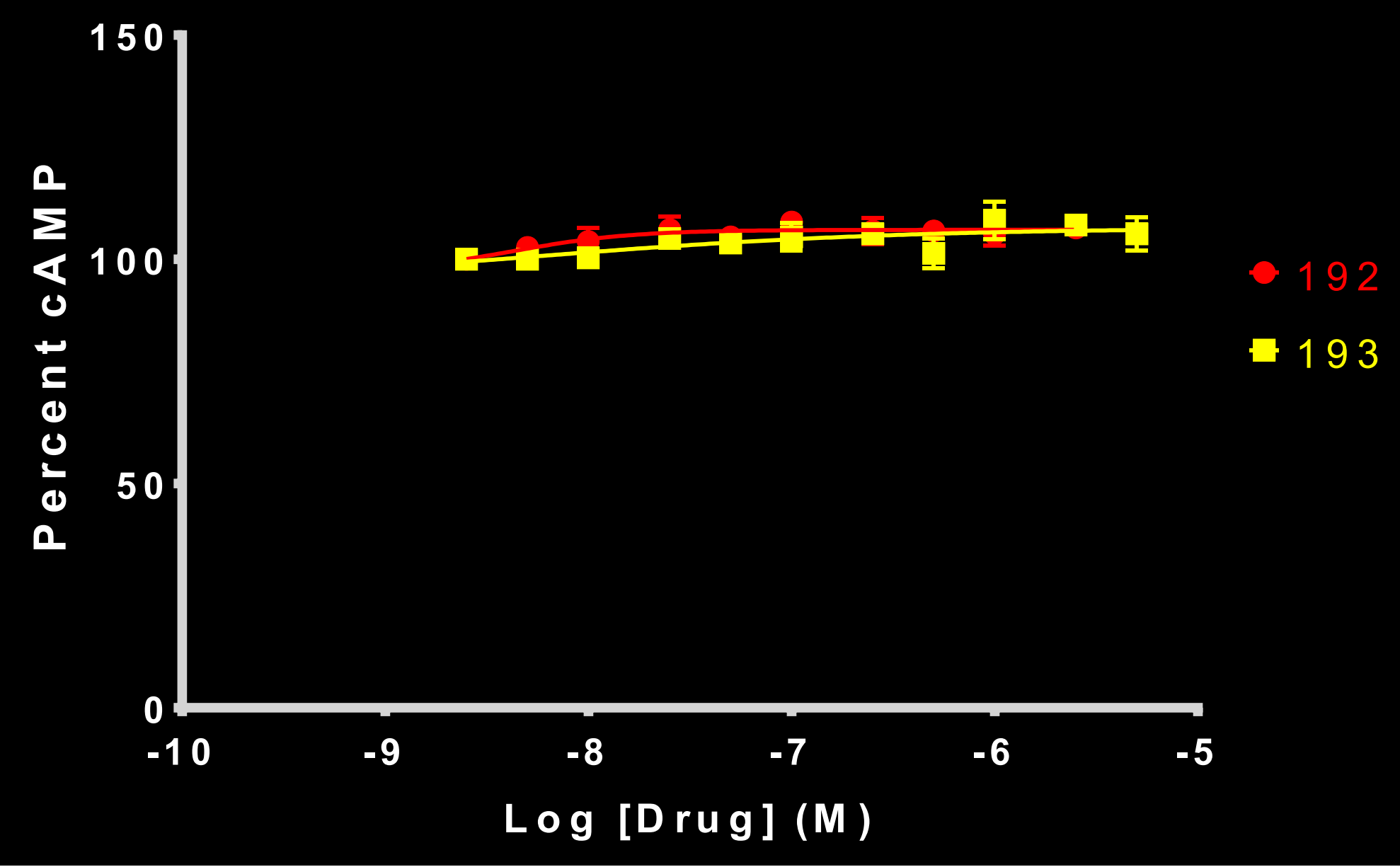

Figure 5.11. $\mathrm{CB}_{1}$ PTx-sensitive Gi/o-inactivation of $\mathrm{HB}$ triaryl series, 50 minutes after compound addition 


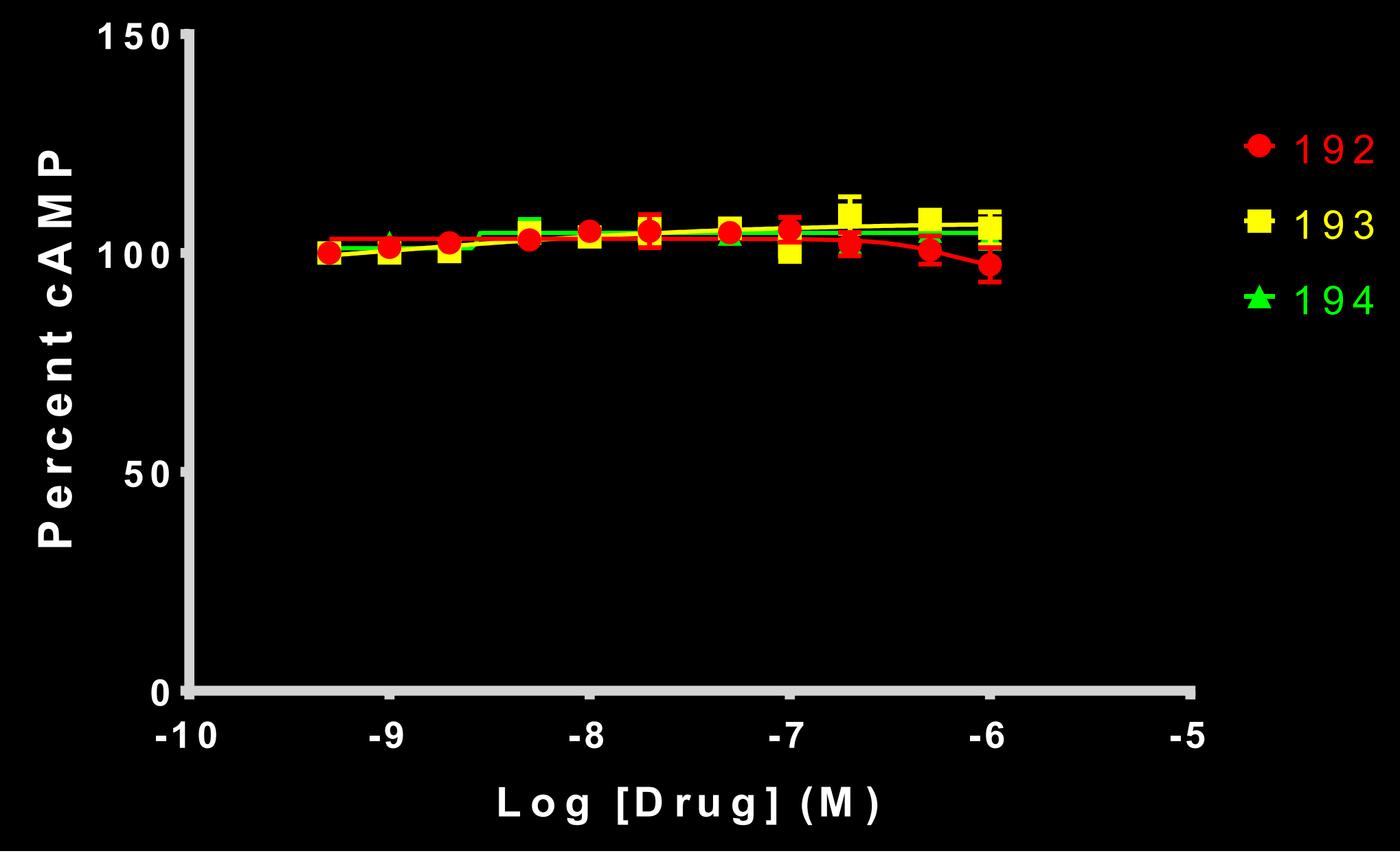

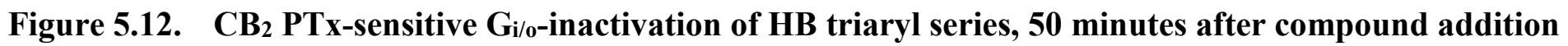




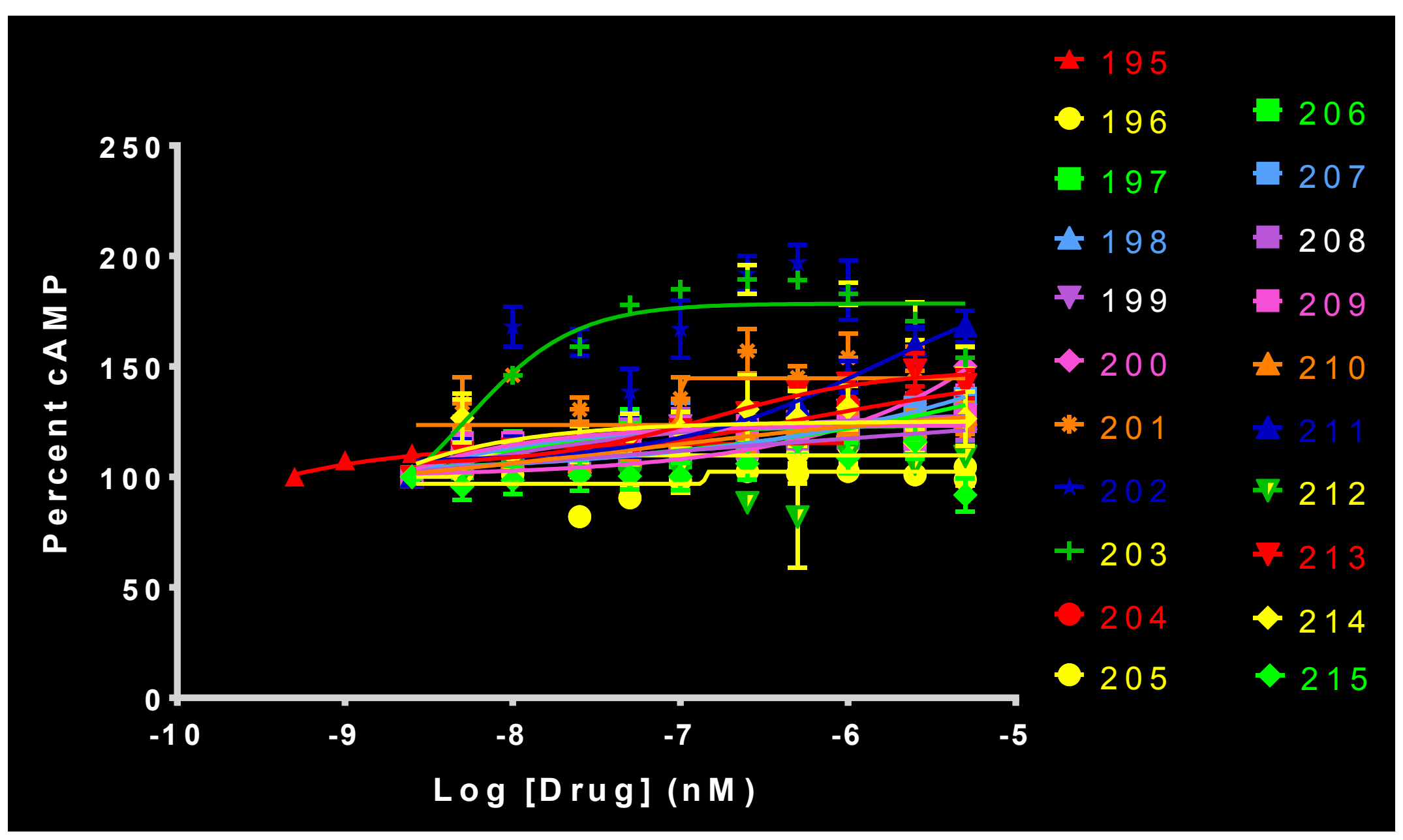

Figure 5.13. $\mathrm{CB}_{1}$ functional activity of SMM triaryl series, 50 minutes after compound addition 


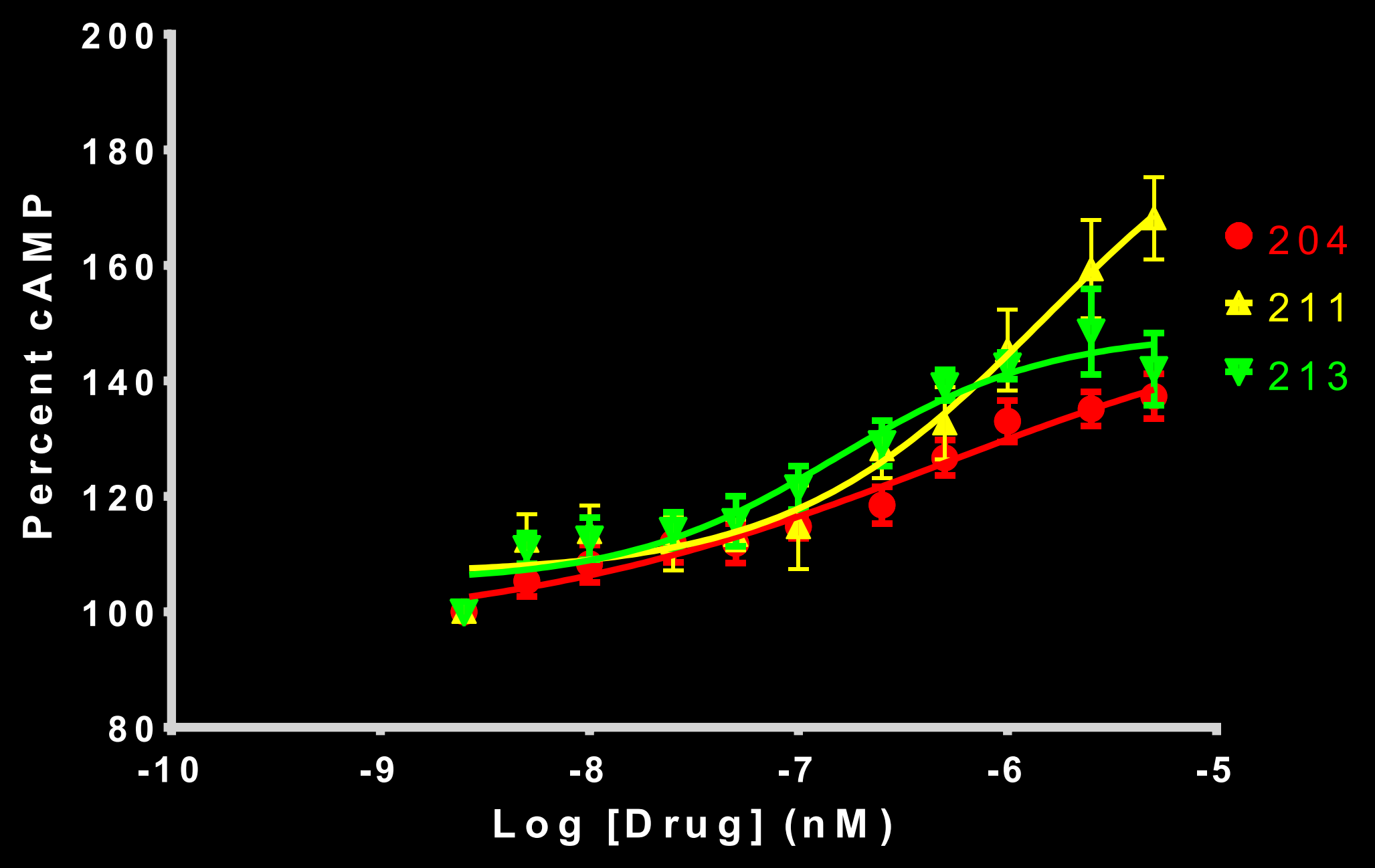

Figure 5.14. $\quad \mathrm{CB}_{1}$ functional activity of select $\mathrm{SMM}$ triaryl series compounds, 50 minutes after compound addition 
Table 5.6. $\quad \mathrm{EC}_{50}$ values for SMM triaryl series

\begin{tabular}{|c|c|c|c|c|c|}
\hline Compound & $\begin{array}{c}\mathrm{CB}_{1} \\
\text { EC50 } \\
\text { or } \\
\text { Concentration } \\
\text { (\% baseline) }\end{array}$ & $\begin{array}{c}\mathbf{C B}_{2} \\
\text { EC50 } \\
\text { or } \\
\text { Concentration } \\
\text { (\% baseline) }\end{array}$ & $\begin{array}{l}\text { HEK-CNG } \\
\mathrm{EC}_{50}(\mathrm{nM})\end{array}$ & $\begin{array}{c}\mathrm{CB}_{1} \text { PTx } \\
\mathrm{EC}_{50}(\mathrm{nM})\end{array}$ & $\begin{array}{c}\mathrm{CB}_{2} \text { PTx } \\
\mathrm{EC}_{50}(\mathrm{nM})\end{array}$ \\
\hline 195 & $\mathrm{n} / \mathrm{a}$ & 744 & $\mathrm{n} / \mathrm{a}$ & - & $\mathrm{n} / \mathrm{a}$ \\
\hline 196 & $\mathrm{n} / \mathrm{a}$ & - & $\mathrm{n} / \mathrm{a}$ & - & - \\
\hline 197 & $\mathrm{n} / \mathrm{a}$ & - & $\mathrm{n} / \mathrm{a}$ & - & - \\
\hline 198 & $\mathrm{n} / \mathrm{a}$ & - & $\mathrm{n} / \mathrm{a}$ & - & - \\
\hline 199 & $\mathrm{n} / \mathrm{a}$ & $5000(81 \%)^{b}$ & $\mathrm{n} / \mathrm{a}$ & - & - \\
\hline 200 & $5000(48 \%)^{b}$ & $5000(20 \%)^{b}$ & $\mathrm{n} / \mathrm{a}$ & - & - \\
\hline 201 & $\mathrm{n} / \mathrm{a}$ & - & $\mathrm{n} / \mathrm{a}$ & - & - \\
\hline 202 & $50(85 \%)^{b}$ & - & $\mathrm{n} / \mathrm{a}$ & - & - \\
\hline 203 & $50(75 \%)^{b}$ & $5000(50 \%)^{b}$ & $\mathrm{n} / \mathrm{a}$ & - & - \\
\hline 204 & $469^{\mathrm{b}}$ & $5000(43 \%)^{b}$ & $\mathrm{n} / \mathrm{a}$ & $\mathrm{n} / \mathrm{a}$ & - \\
\hline 205 & $\mathrm{n} / \mathrm{a}$ & - & $\mathrm{n} / \mathrm{a}$ & - & - \\
\hline 206 & $5000(32 \%)^{b}$ & $5000(97 \%)^{b}$ & $\mathrm{n} / \mathrm{a}$ & - & $\mathrm{n} / \mathrm{a}$ \\
\hline 207 & $\mathrm{n} / \mathrm{a}$ & $97.6^{\mathrm{b}}$ & $\mathrm{n} / \mathrm{a}$ & - & $\mathrm{n} / \mathrm{a}$ \\
\hline 208 & $\mathrm{n} / \mathrm{a}$ & $5000(73 \%)^{\mathrm{b}}$ & $\mathrm{n} / \mathrm{a}$ & - & - \\
\hline 209 & $\mathrm{n} / \mathrm{a}$ & $5000(110 \%)^{b}$ & $\mathrm{n} / \mathrm{a}$ & - & - \\
\hline 210 & $5000(24 \%)^{b}$ & $5000(85 \%)^{\mathrm{b}}$ & $\mathrm{n} / \mathrm{a}$ & - & - \\
\hline 211 & $1497^{\mathrm{b}}$ & $702^{\mathrm{b}}$ & $\mathrm{n} / \mathrm{a}$ & $\mathrm{n} / \mathrm{a}$ & $\mathrm{n} / \mathrm{a}$ \\
\hline 212 & $\mathrm{n} / \mathrm{a}$ & - & $\mathrm{n} / \mathrm{a}$ & - & - \\
\hline 213 & $153^{b}$ & $5000(125 \%)^{b}$ & $\mathrm{n} / \mathrm{a}$ & $\mathrm{n} / \mathrm{a}$ & - \\
\hline 214 & $\mathrm{n} / \mathrm{a}$ & $5000(200 \%)^{b}$ & $\mathrm{n} / \mathrm{a}$ & - & $\mathrm{n} / \mathrm{a}$ \\
\hline 215 & $\mathrm{n} / \mathrm{a}$ & $5000(110 \%)^{b}$ & $\mathrm{n} / \mathrm{a}$ & - & $\mathrm{n} / \mathrm{a}$ \\
\hline
\end{tabular}

Notes: All concentrations are listed as nM, percentages indicate the change from baseline. n/a: Not active, based on no statistically significant change in cAMP from baseline. "_." Indicates test not performed. ${ }^{a}$ Indicates a decrease in cAMP as compared to baseline. ${ }^{b}$ Indicates an increase in cAMP as compared to baseline. 
5.4.3.2. $\mathrm{CB}_{2}$ functional activity for the $\mathrm{SMM}$ series. Plates were run as previously described (Section 5.4.1) in six (6) replicates - unless the compound was known to not bind, in which case it was run in three (3) replicates - with CP-55,940 as the internal control for all replicates (Figure 5.15). All compounds in the series bind with good affinity to the $C_{2}$ receptor, except 200, 201, 202, 205 and 212. ${ }^{259}$ Unlike the $C_{1}$ functional assays, none of the compounds in demonstrated agonist activity at $\mathrm{CB}_{2}$. Compounds 195 (744 nM), 206 (>5000 nM), 207 (>5000 nM), 211 (702 nM), 214 $(>5000 \mathrm{nM})$ and $215(>5000 \mathrm{nM})$ (Figure 5.16) all demonstrated induction of cAMP in excess of our standard(s) SR-144528 (and AM-630 (Table 5.5), with a percent cAMP induction ranging from $148 \%$ to $266 \%$ over baseline. It is important to note that the $\mathrm{EC}_{50}$ values for these compounds are not possible to calculate due to lack of a saturation response with all of the compounds except 195 and 211.

5.4.3.3. CNG parental functional activity for the SMM series. Plates were run as previously described (Section 5.4.1) in three (3) replicates with CP-55,940 as the internal control for all three (3) replicates (Figure 5.17). As was expected, all compounds in the series failed to elucidate any statistically significant change in the cAMP production of the cells, including the reference standard of CP-55,940.

5.4.3.4. $C_{1} P T x$-sensitive $G_{i / 0}$-inactivation for the $S M M$ series. Plates were run as previously described (Section 5.4.1) in three (3) replicates with CP-55,940 as the internal control for all replicates (Figure 5.18). As was expected, all compounds failed to alter cAMP production, including the reference standard of CP-55,940. Only compounds 204, 211 and 213 were screened in the PTx-sensitive $\mathrm{G}_{\mathrm{i} / \mathrm{o}}$-inactivation assays due to their ability to elucidate a response in the $\mathrm{CB}_{1}$ functional assay.

5.4.3.5. $\mathrm{CB}_{2} \mathbf{P T x}$-sensitive $\mathrm{G}_{\mathrm{i} / \mathbf{0}}$-inactivation for the $\mathrm{SMM}$ series. Plates were run as previously described (Section 5.4.1) in three (3) replicates with CP-55,940 as the internal control for all replicates (Figure 5.19). As was expected, the tested compound failed to alter cAMP production, including the reference standard of CP-55,940. Only compounds compounds 195, 206, 207, 211, 214 and 215 were screened in the PTxsensitive $\mathrm{G}_{\mathrm{i} / \mathrm{o}}$-inactivation assays due to their ability to elucidate a response in the $\mathrm{CB}_{2}$ functional assay.

\subsubsection{Functional activity for pyridine triaryl series}

5.4.4.1. CB $_{1}$ functional activity for the Pyridine Triaryl series. Plates were run as previously described (Section 5.4.1) in six (6) replicates - unless the compound was known to not bind, in which case it was run in two (2) replicates - with CP-55,940 as the internal control for all replicates (Figure 5.20). Only compounds 217, 218, 219 and 222 were known to bind with any appreciable affinity to $\mathrm{CB}_{1}$, and compound $\mathbf{2 2 3}$ has no binding data available, so it was treated as a known binder. ${ }^{259} \mathrm{EC}_{50}$ values for these compounds ranged from $76.1 \mathrm{nM}$ to $3238 \mathrm{nM}$, with a percent decrease in cAMP ranging from $27 \%$ to $65 \%$ (Table 5.7). Despite binding to $\mathrm{CB}_{1}$, compounds 219 and 222 did not have any impact on cAMP and showed themselves to be antagonists, the steady rise of 222 in Figure 5.21 is attributable to the drift that is occasionally noticed within the assay, as discussed in Chapter 2. 


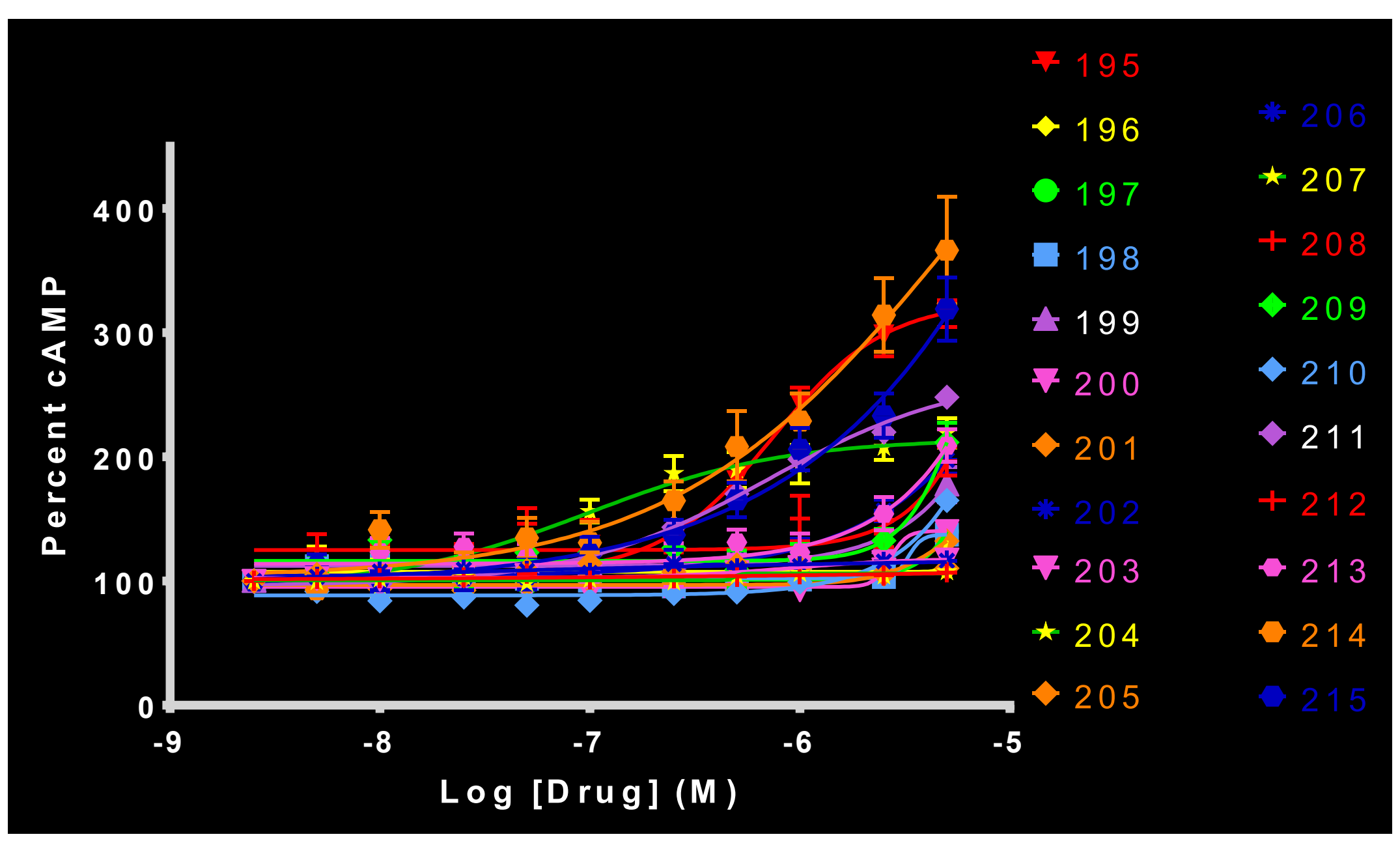

Figure 5.15. $\quad \mathrm{CB}_{2}$ functional activity of SMM triaryl series 


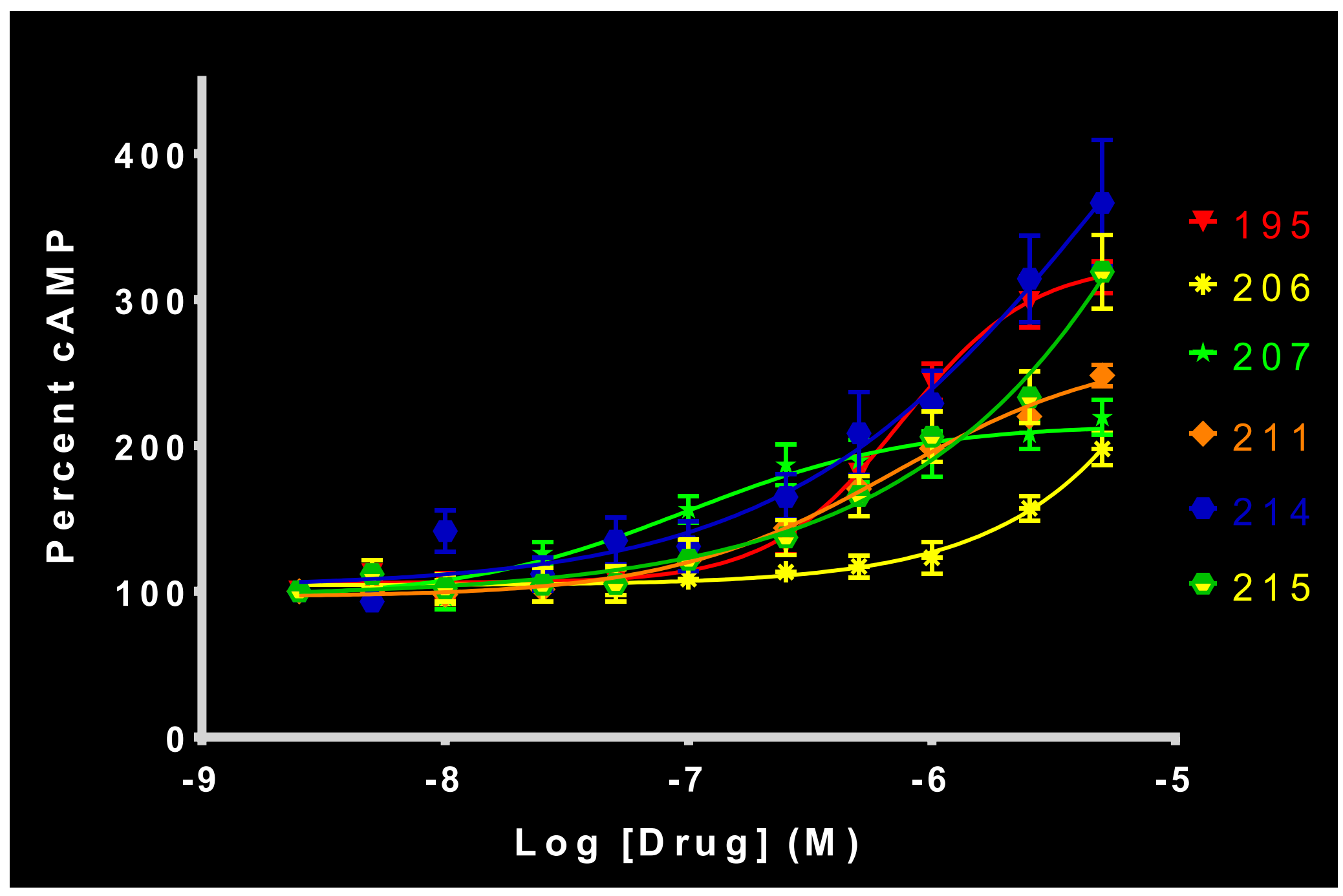

Figure 5.16. $\mathrm{CB}_{2}$ functional activity of select $\mathrm{SMM}$ triaryl series compounds 
Table 5.7. $\quad$ EC $_{50}$ values for pyridine triaryl series

\begin{tabular}{|c|c|c|c|c|c|}
\hline Compound & $\begin{array}{c}\mathrm{CB}_{1} \\
\mathrm{EC}_{50} \\
\text { or } \\
\text { Concentration } \\
\text { (\% baseline) } \\
\end{array}$ & $\begin{array}{c}\mathbf{C B}_{2} \\
\mathbf{E C}_{50} \\
\text { or } \\
\text { Concentration } \\
\text { (\% baseline) } \\
\end{array}$ & $\begin{array}{l}\text { HEK- } \\
\text { CNG } \\
\text { EC50 } \\
(\mathbf{n M})\end{array}$ & $\begin{array}{c}\mathrm{CB}_{1} \text { PTx } \\
\text { EC50 } \\
(\mathbf{n M})\end{array}$ & $\begin{array}{c}\mathbf{C B}_{2} \mathbf{P T} \mathbf{x} \\
\mathbf{E C}_{\mathbf{5 0}} \\
(\mathbf{n M})\end{array}$ \\
\hline 216 & $\mathrm{n} / \mathrm{a}$ & $\mathrm{n} / \mathrm{a}$ & $\mathrm{n} / \mathrm{a}$ & - & $\mathrm{n} / \mathrm{a}$ \\
\hline 217 & $5000(38 \%)^{\mathrm{a}}$ & $\mathrm{n} / \mathrm{a}$ & $\mathrm{n} / \mathrm{a}$ & $\mathrm{n} / \mathrm{a}$ & $\mathrm{n} / \mathrm{a}$ \\
\hline 218 & $5000(63 \%)^{\mathrm{a}}$ & $470.1^{\mathrm{a}}$ & $\mathrm{n} / \mathrm{a}$ & $\mathrm{n} / \mathrm{a}$ & $\mathrm{n} / \mathrm{a}$ \\
\hline 219 & $\mathrm{n} / \mathrm{a}$ & $108.6^{\mathrm{a}}$ & $\mathrm{n} / \mathrm{a}$ & $\mathrm{n} / \mathrm{a}$ & $\mathrm{n} / \mathrm{a}$ \\
\hline 220 & $\mathrm{n} / \mathrm{a}$ & - & - & - & - \\
\hline 221 & $5000(40 \%)^{* a}$ & $389.4^{\mathrm{a}}$ & $\mathrm{n} / \mathrm{a}$ & - & $\mathrm{n} / \mathrm{a}$ \\
\hline 222 & $\mathrm{n} / \mathrm{a}$ & $\mathrm{n} / \mathrm{a}$ & $\mathrm{n} / \mathrm{a}$ & $\mathrm{n} / \mathrm{a}$ & $\mathrm{n} / \mathrm{a}$ \\
\hline 223 & $5000(34 \%)^{\mathrm{a}}$ & $294.8^{\mathrm{a}}$ & $\mathrm{n} / \mathrm{a}$ & $\mathrm{n} / \mathrm{a}$ & $\mathrm{n} / \mathrm{a}$ \\
\hline 224 & $123.54 * b$ & - & $\mathrm{n} / \mathrm{a}$ & - & - \\
\hline 225 & $131.25 * \mathrm{~b}$ & - & $\mathrm{n} / \mathrm{a}$ & - & - \\
\hline 226 & $364.7 * \mathrm{~b}$ & $5000(43 \%)^{\mathrm{b}}$ & $\mathrm{n} / \mathrm{a}$ & - & $\mathrm{n} / \mathrm{a}$ \\
\hline 227 & $5000(16 \%)^{* b}$ & $5000(39 \%)^{b}$ & $\mathrm{n} / \mathrm{a}$ & - & $\mathrm{n} / \mathrm{a}$ \\
\hline 228 & $5000(21 \%)^{\mathrm{b}}$ & $5000(30 \%)^{b}$ & $\mathrm{n} / \mathrm{a}$ & - & $\mathrm{n} / \mathrm{a}$ \\
\hline 229 & $5000(21 \%)^{b}$ & $5000(30 \%)^{b}$ & $\mathrm{n} / \mathrm{a}$ & - & $\mathrm{n} / \mathrm{a}$ \\
\hline
\end{tabular}

Notes: All concentrations are listed as $\mathrm{nM}$, percentages indicate the change from baseline. n/a: Not active, based on no statistically significant change in cAMP from baseline. "_." Indicates test not performed. ${ }^{a}$ Indicates a decrease in cAMP as compared to baseline. ${ }^{\mathrm{b}}$ Indicates an increase in cAMP as compared to baseline. " $*$ " $=\mathrm{N}=3$. 


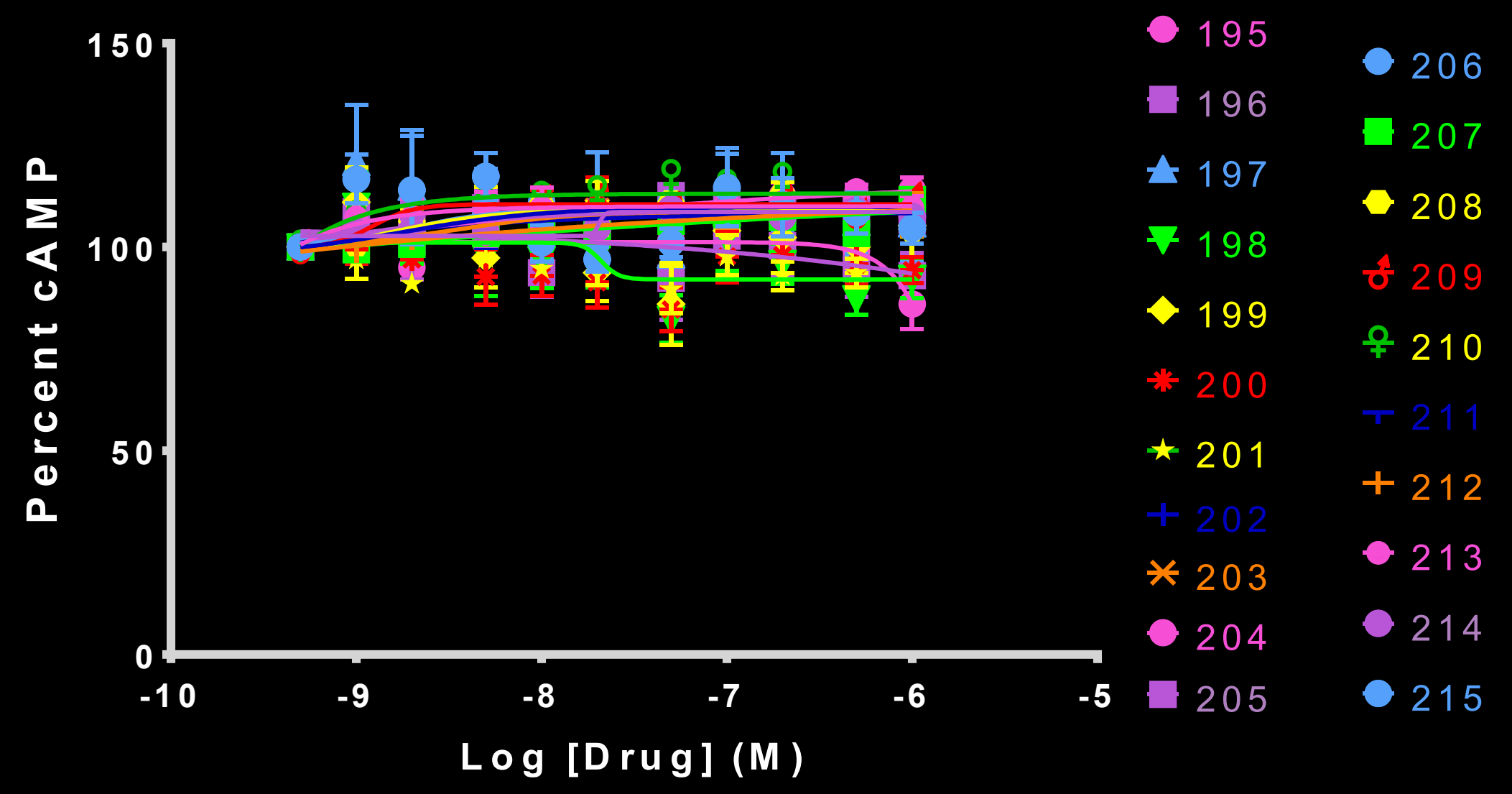

Figure 5.17. CNG functional activity for SMM triaryl series 


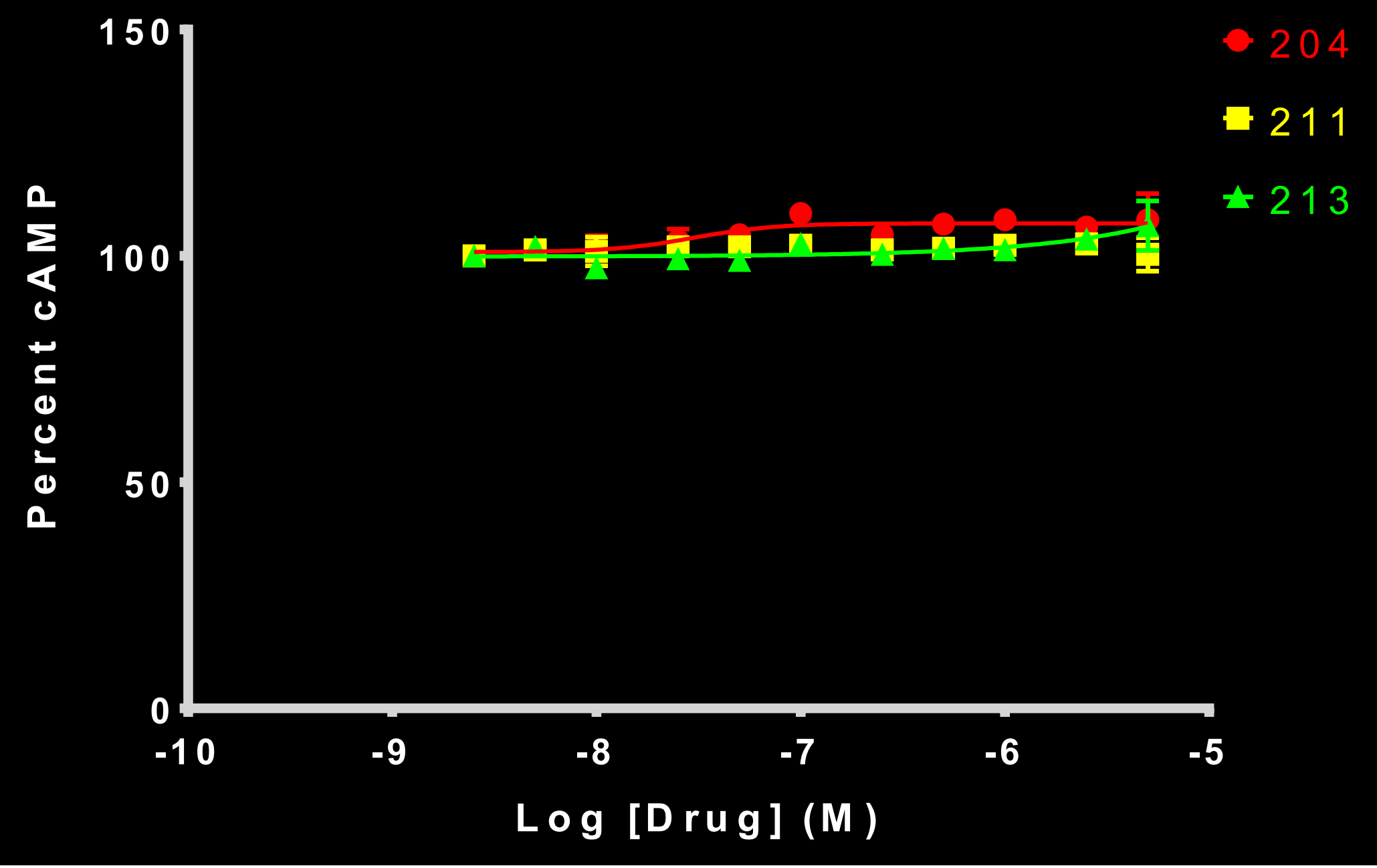

Figure 5.18. $\quad C_{1}$ PTx-sensitive $G_{i / 0}$-inactivation for selected $S M M$ triaryl series compounds 


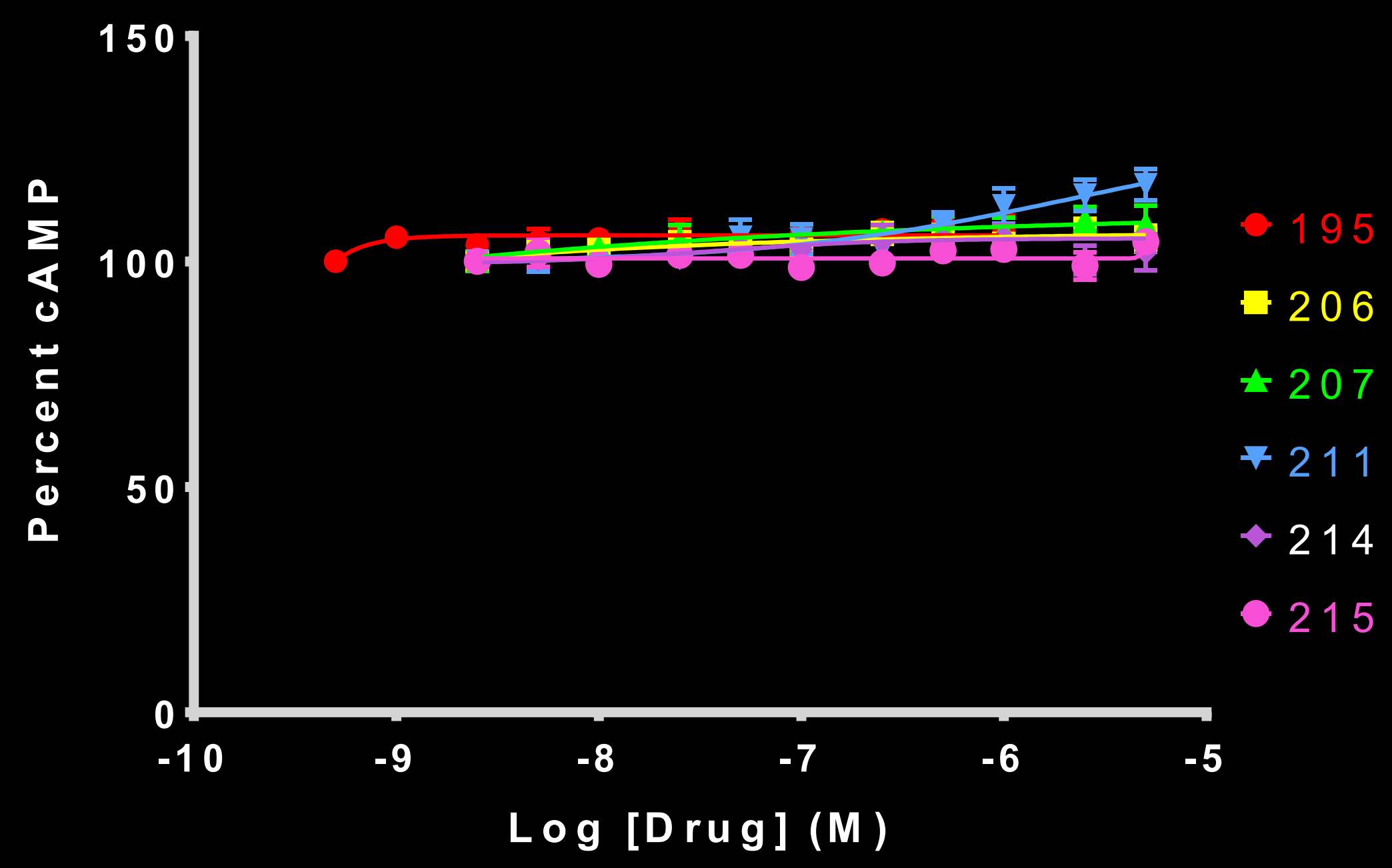

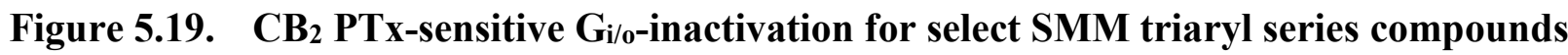




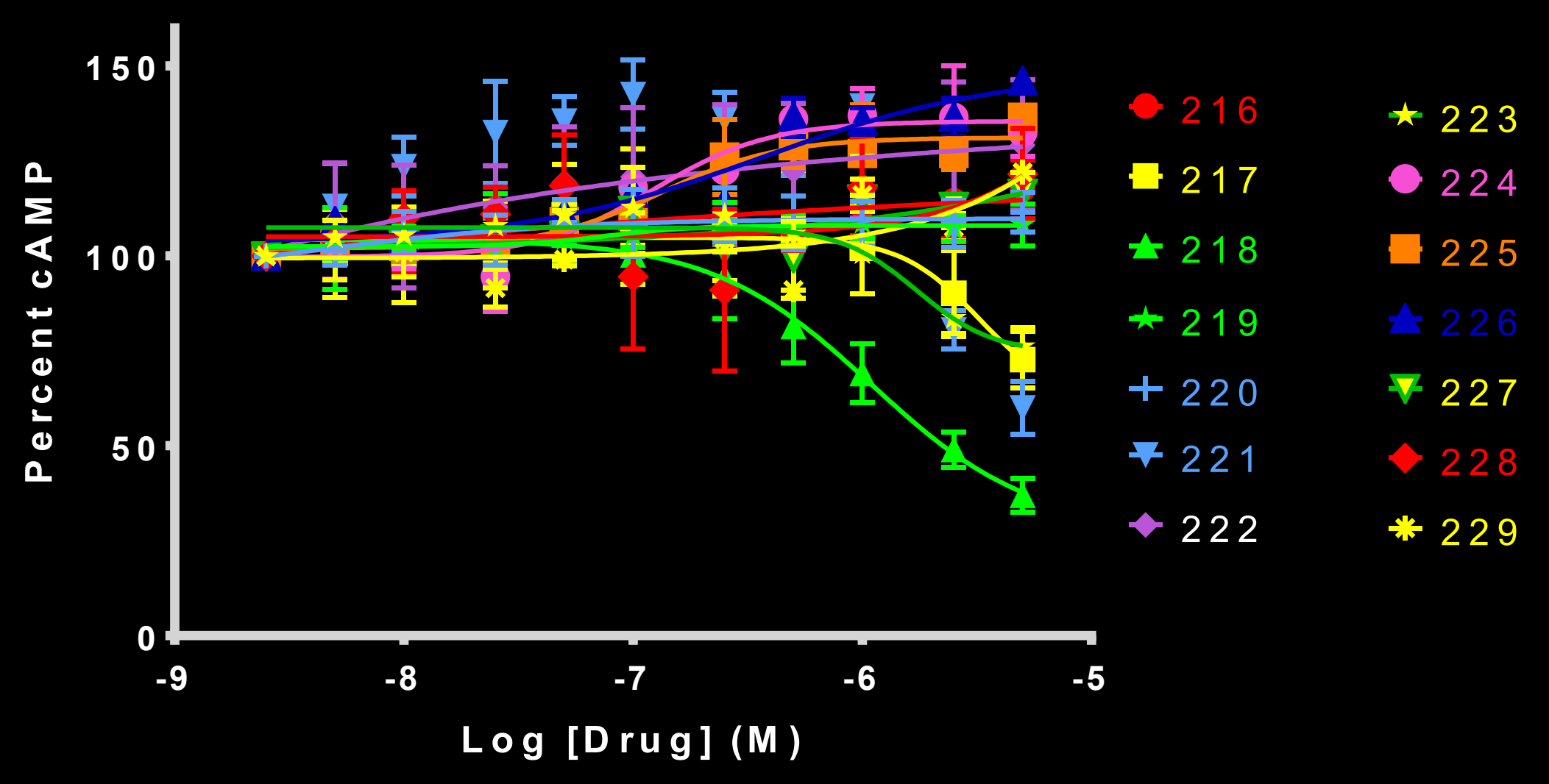

Figure 5.20. $\mathrm{CB}_{1}$ functional data for pyridine triaryl compounds 


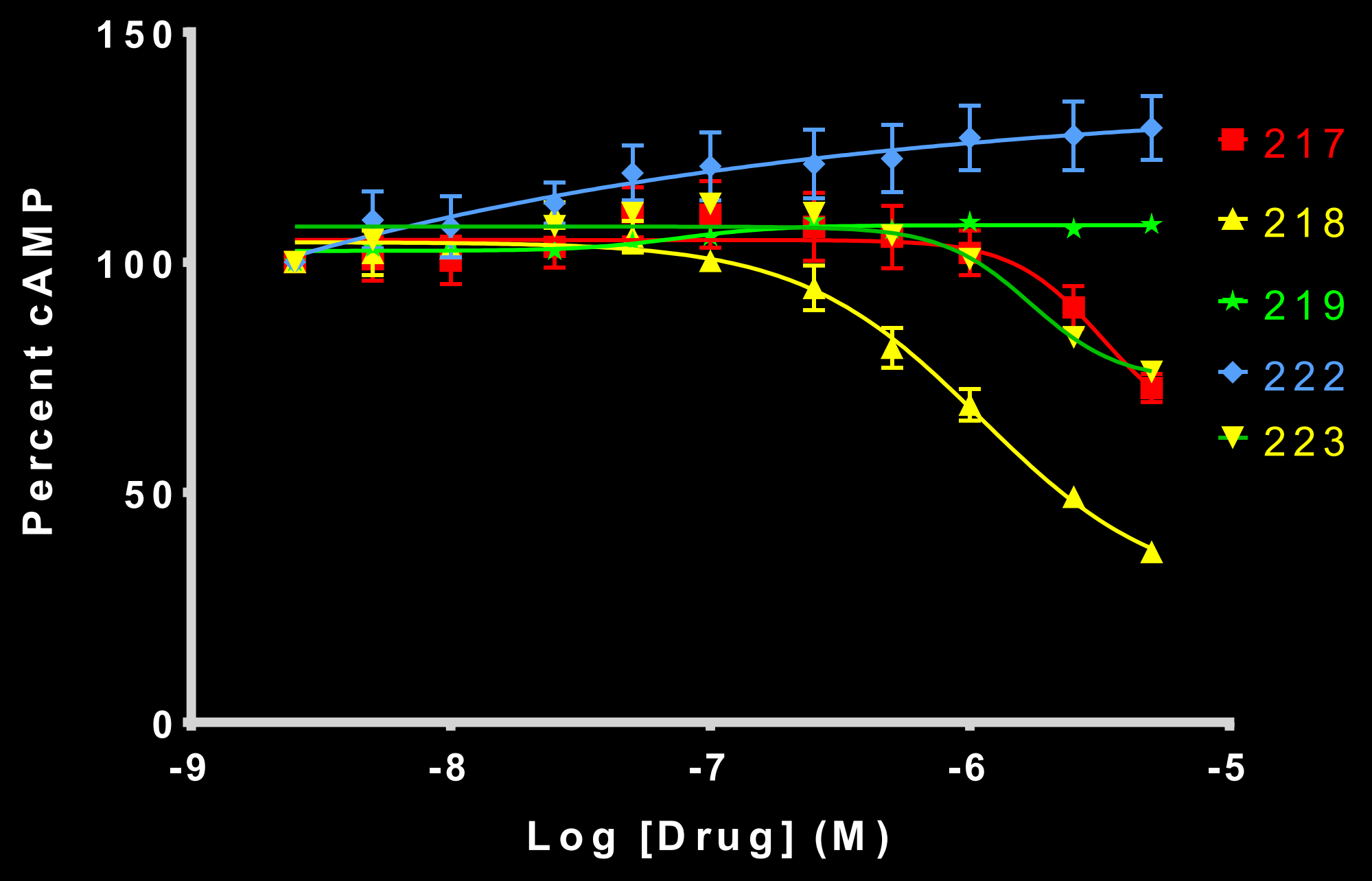

Figure 5.21. $\quad \mathrm{CB}_{1}$ functional activity of known binders within pyridine triaryl series 
As evidenced in Figure 5.22, some of the compounds (224, 225, 226 and 227) demonstrated a functional effect without having any known binding affinity for the $\mathrm{CB}_{1}$ LBP. Of great intrigue is the fact that the compounds containing either a $\mathrm{C}-1^{\prime}$ cyclohexyl or phenyl demonstrated an inverse agonist activity at $\mathrm{CB}_{1}$. Compound 227 and 221 did not elucidate a discernable curve upon analysis, and this could be because these non-binding compounds were not run in a large enough sample size to obtain a viable curve. These data taken in the context of the whole warrants further investigation of this compound.

5.4.4.2. $\mathrm{CB}_{2}$ functional activity for the pyridine triaryl series. Plates were run as previously described (Section 5.4.1) in six (6) replicates - unless the compound was known to not bind, in which case it was run in three (3) replicates - with CP-55,940 as the internal control for all replicates (Figure 5.23). All compounds in the series bind with good affinity to the $\mathrm{CB}_{2}$ receptor, except 224, 225 and 220. ${ }^{259}$ As with the $\mathrm{CB}_{1}$ assay, there is mixed activity within this class of compounds. Compounds 218, 219, 221 and 223 (Table 5.3) all demonstrated inhibition of cAMP production, with and $\mathrm{EC}_{50}$ of 470.1 $\mathrm{nM}, 108.6 \mathrm{nM}, 389.4 \mathrm{nM}$ and $294.8 \mathrm{nM}$, respectively (Figure 5.24; Table 5.7), with a percent cAMP reduction ranging from $11.5 \%$ to $45 \%$.

Additional compounds in this series known to bind are 216, 217, 222, 226, 227, 228 and 229 (Table 5.3). Within these compounds, neither 216, 217 nor 222 (all with linear aliphatic C-3 substituents) showed any change in cAMP production - in fact, 216 and 217 were virtually identical in their lack of activity; however, 226, 227, 228 and 229 all demonstrated a very weak inverse agonist activity (Figure 5.25). The $\mathrm{EC}_{50}$ for these compounds was not determined, as they are all in excess of my limit of $5 \mu \mathrm{M}$; however there was an approximately $40 \%$ increase in cAMP for these compounds at $5 \mu \mathrm{M}$.

5.4.4.3. CNG parental functional activity for the pyridine triaryl series. Plates were run as previously described (Section 5.4.1) in three (3) replicates with CP-55,940 as the internal control for all three (3) replicates (Figure 5.26). As was expected, all compounds in the series failed to elucidate any statistically significant change in the cAMP production of the cells, including the reference standard of CP-55,940.

5.4.4.4. $C_{1} P T x$-sensitive $G_{i / 0}$-inactivation for the pyridine triaryl series. Plates were run as previously described (Section 5.4.1) in three (3) replicates with CP-55,940 as the internal control for all replicates (Figure 5.27). As was expected, all compounds failed to alter cAMP production, including the reference standard of CP-55,940. Only compounds $\mathbf{2 1 7 , 2 1 8}$, and 223 were screened in the PTx-sensitive $\mathrm{G}_{\mathrm{i} / \mathrm{o}^{-}}$ inactivation assays due to their ability to elucidate a response in the $\mathrm{CB}_{1}$ functional assay.

\subsubsection{5. $\mathrm{CB}_{2} P T x$-sensitive $G_{i / 0}$-inactivation for the pyridine triaryl series.} Plates were run as previously described (Section 5.4.1) in three (3) replicates with CP-55,940 as the internal control for all replicates (Figure 5.28). As was expected, all compounds failed to alter cAMP production, including the reference standard of CP-55,940. Only compounds 217, 218, 219, 222 and 223 were screened in the PTxsensitive $\mathrm{G}_{\mathrm{i} / \mathrm{o}}$-inactivation assays due to their ability to elucidate a response in the $\mathrm{CB}_{1}$ functional assay. 


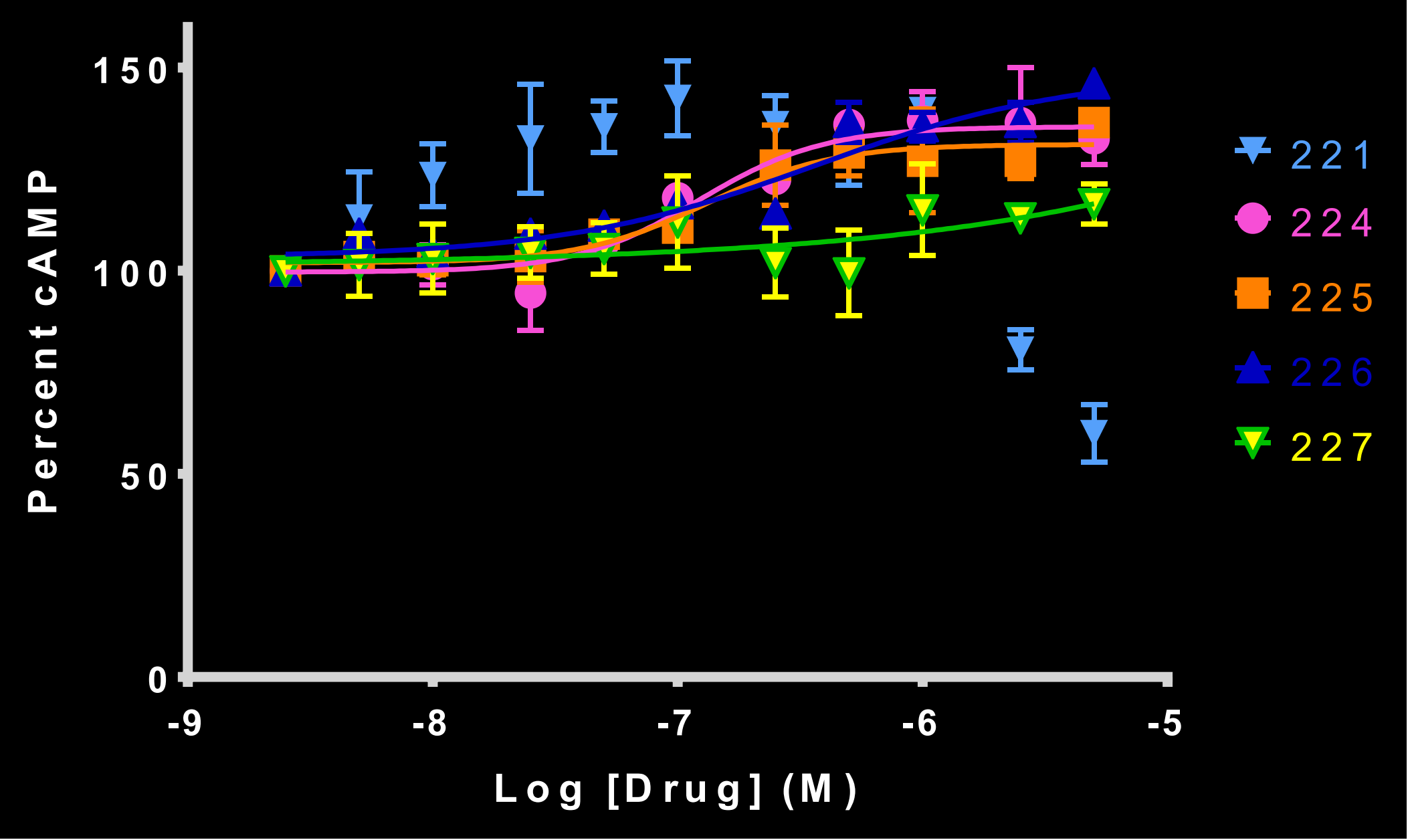

Figure 5.22. $\quad \mathrm{CB}_{1}$ active compounds with unknown $K_{i}$ values. 


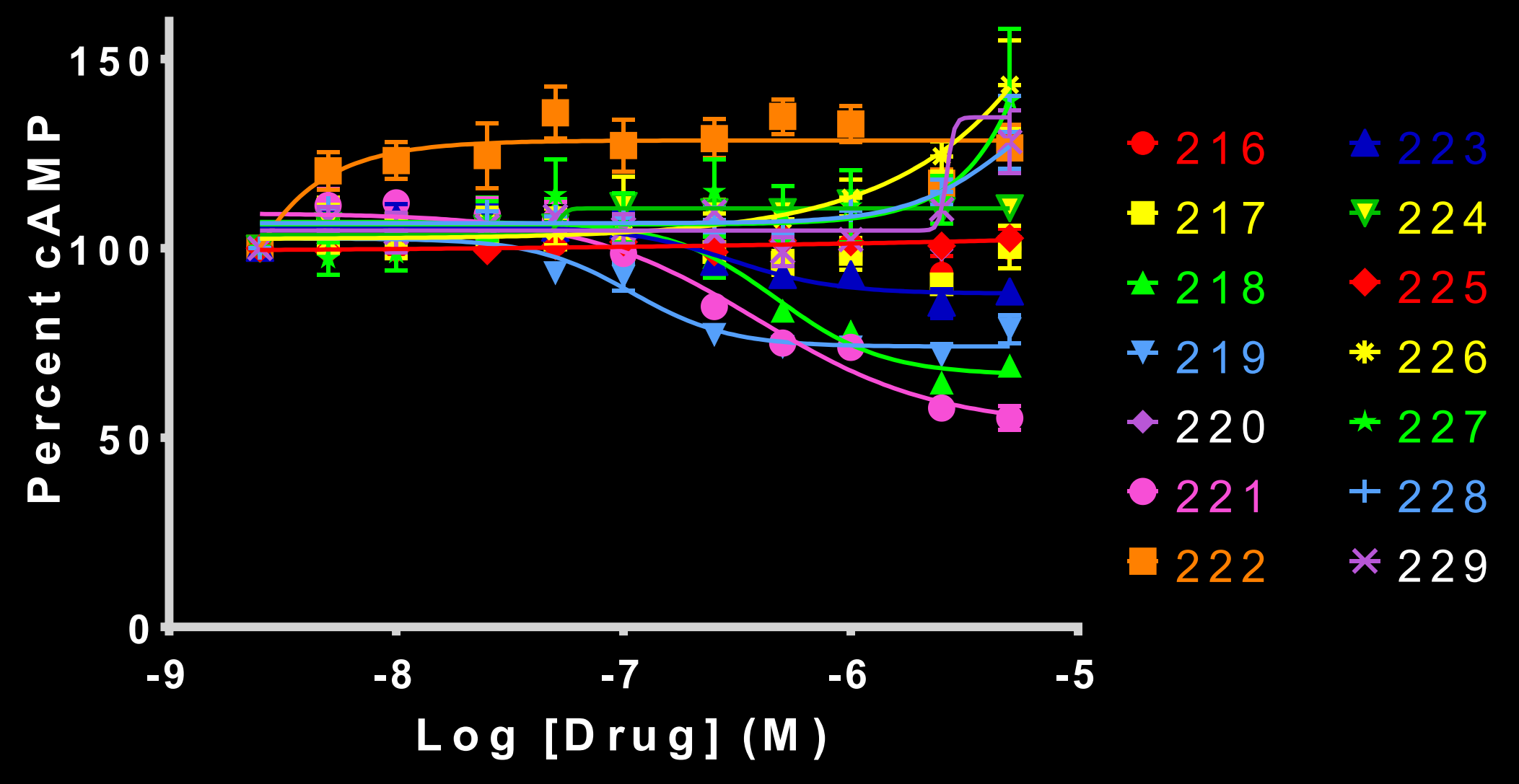

Figure 5.23. $\quad \mathrm{CB}_{2}$ functinal data for pyridine triaryls 


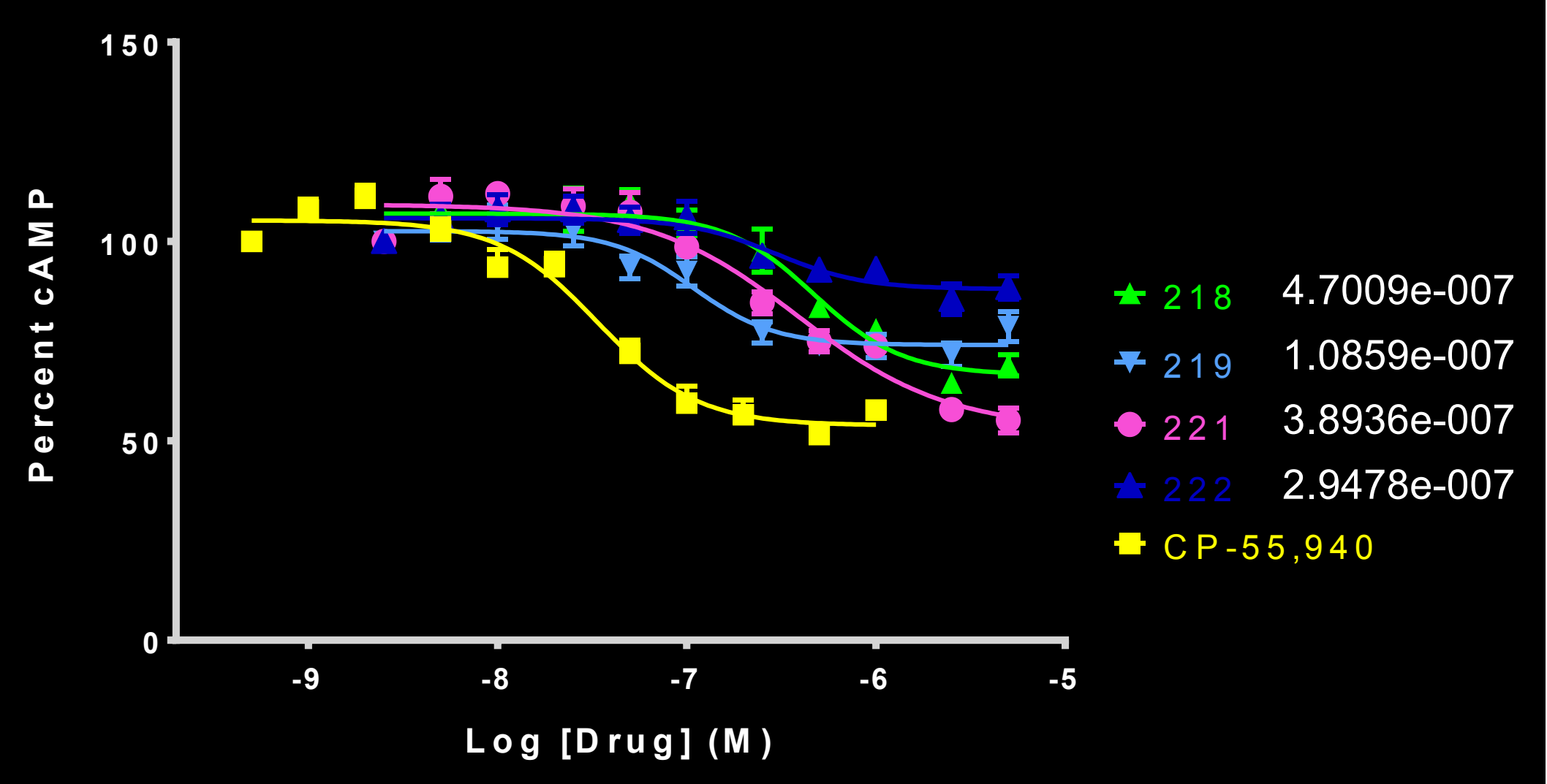

Figure 5.24. CB2 functional agonists of the pyridine triaryl series 


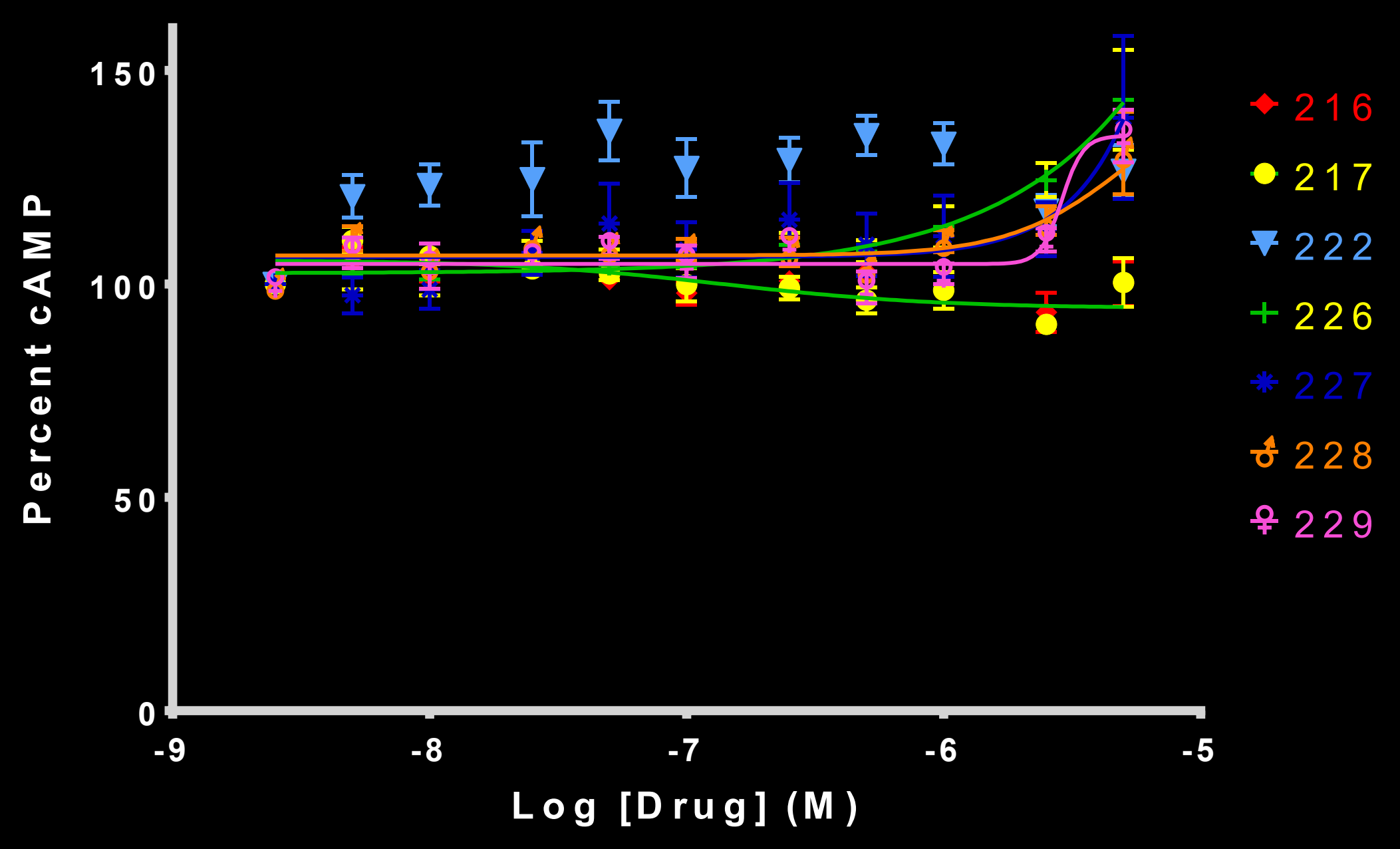

Figure 5.25. $\quad \mathrm{CB}_{2}$ functional activity of non-agonists with known binding within the pyridine triaryl series 


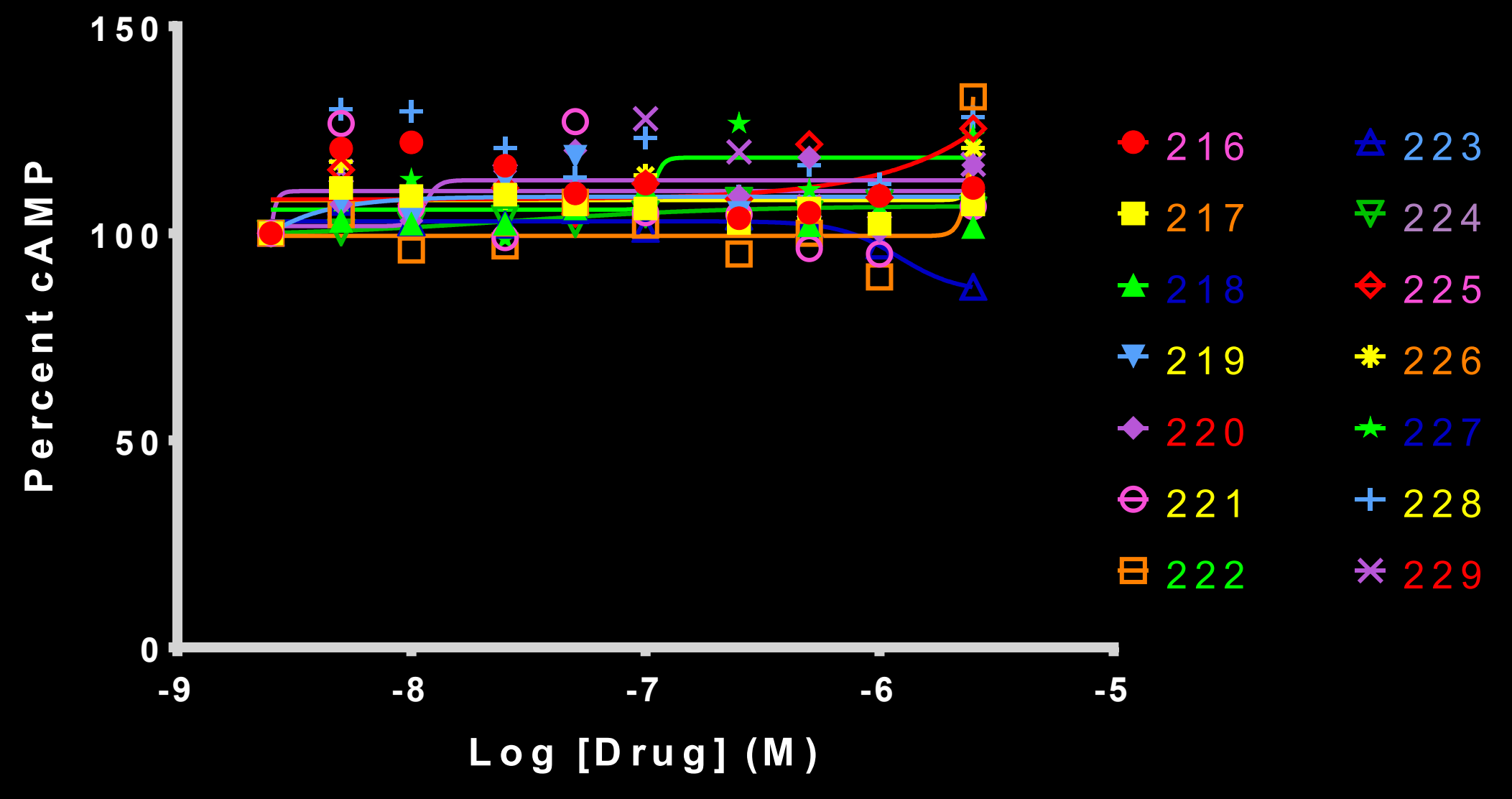

Figure 5.26. HEK-CNG functional assay with pyridine triaryl series 


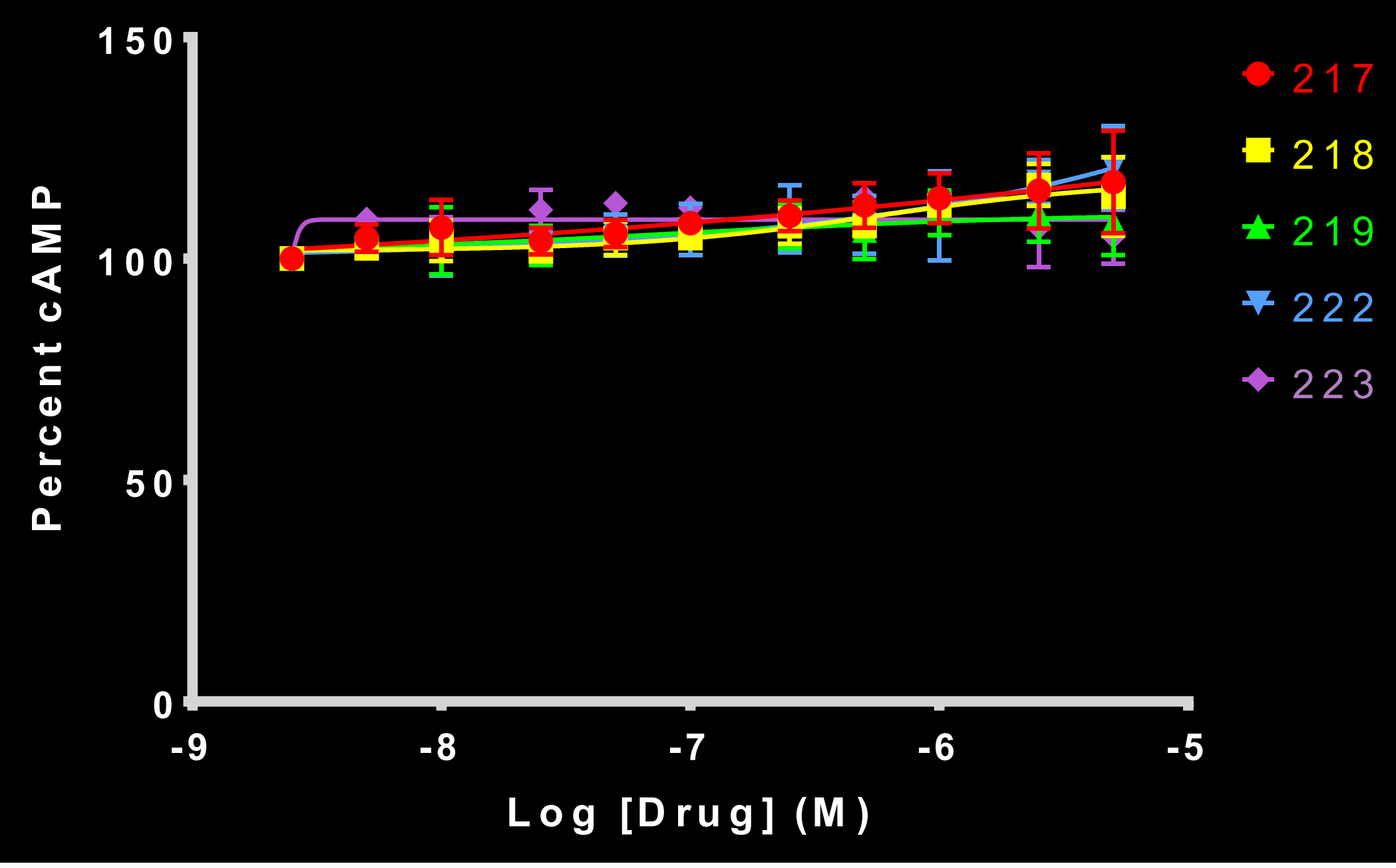

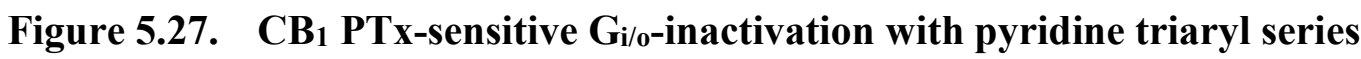




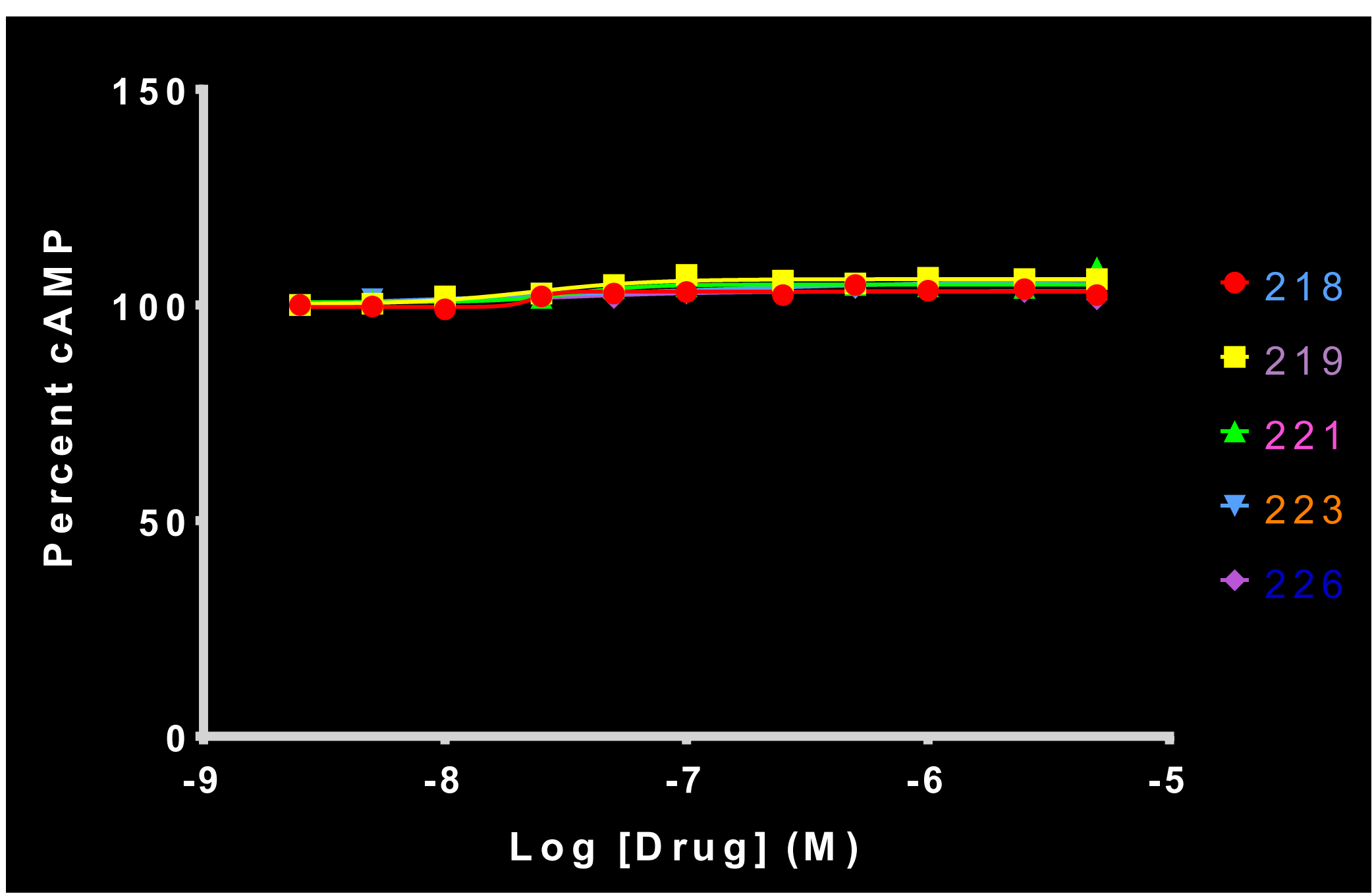

Figure 5.28. $\quad C_{2}$ PTx-sensitive $G_{i / 0}$-inactivation with pyridine triaryl series 


\subsubsection{Functional activity for pyrimidine triaryl series}

5.4.5.1. CB1 functional activity in the pyrimidine triaryl series. Plates were run as previously described (Section 5.4.1) in six (6) replicates - except the known nonbinding 231, which was run in two (2) replicates - with CP-55,940 as the internal control for all replicates (Figure 5.29). $\mathrm{EC}_{50}$ values for these compounds were inconclusive due to a lack of saturation response; however, the percent increase ranged from $8 \%$ to $27 \%$. This increase is over the non-specific increase noted in the HEK-CNG cells but the significance remains to be determined and was not treated as significant.

5.4.5.2. $\mathrm{CB}_{2}$ functional activity in the pyrimidine triaryl series. Plates were run as previously described (Section 5.4.1) in six (6) replicates - with CP-55,940 as the internal control (Figure 5.30). Only compounds 231 and 233 are known to bind with good affinity to $\mathrm{CB}_{2}$, all others are unknown and treated as if they bind $\mathrm{CB}_{2} \cdot{ }^{259}$ Contrary to the $\mathrm{CB}_{1}$ assay, 3 compounds set themselves apart and demonstrated agonist activity. Compounds 231, 236, 238 and 239 showed signs of partial agonist activity with $\mathrm{EC}_{50}$ values of $475.4 \mathrm{nM}, 167.9 \mathrm{nM}, 5 \mu \mathrm{M}(21 \%)$ and $5 \mu \mathrm{M}(29 \%)$, respectively, with a percent inhibition of cAMP for all compounds of approximately $30 \%$ (Figure 5.31; Table 5.8).

5.4.5.3. CNG parental functional activity in the pyrimidine triaryl series. Plates were run as previously described (Section 5.4.1) in three (3) replicates with CP-55,940 as the internal control for all three (3) replicates (Figure 5.32). As was expected, all compounds in the series failed to elucidate any change in the cAMP production of the cells, including the reference standard of CP-55,940.

5.4.5.4. $C_{1} P T x$-sensitive $G_{i / 0}$-inactivation in the pyrimidine triaryl series. Plates were run as previously described (Section 5.4.1) in three (3) replicates with CP-55,940 as the internal control for all replicates (Figure 5.33). As was expected, all compounds failed to alter cAMP production, including the reference standard of CP-55,940. All compounds were tested except 231, due to its known lack of binding affinity and all other compounds appearing to be inverse agonists.

5.4.5.5. $\mathrm{CB}_{2} P T x$-sensitive $\mathrm{G}_{\mathrm{i} / \mathrm{o}}$-inactivation in the pyrimidine triaryl series. Plates were run as previously described (Section 5.4.1) in three (3) replicates with CP-55,940 as the internal control for all replicates (Figure 5.34). As was expected, all compounds failed to alter cAMP production, including the reference standard of CP-55,940. Only compounds 231, 236 and 239 were screened in the PTx-sensitive $\mathrm{G}_{\mathrm{i} / \mathrm{o}^{-}}$ inactivation assays due to their ability to elucidate a response in the $\mathrm{CB}_{1}$ functional assay.

\subsection{Discussion}

\subsubsection{Interactions with the $\mathrm{CB}_{1} \mathrm{LBP}$}

5.5.1.1. CB 1 LBP interactions with non-classical ketone moiety. Interactions within the $\mathrm{CB}_{1} \mathrm{LBP}$ for the non-classical cannabinoids differs among the main branches. Most differences center around the presence or absence of interactions with K192(3.28). 


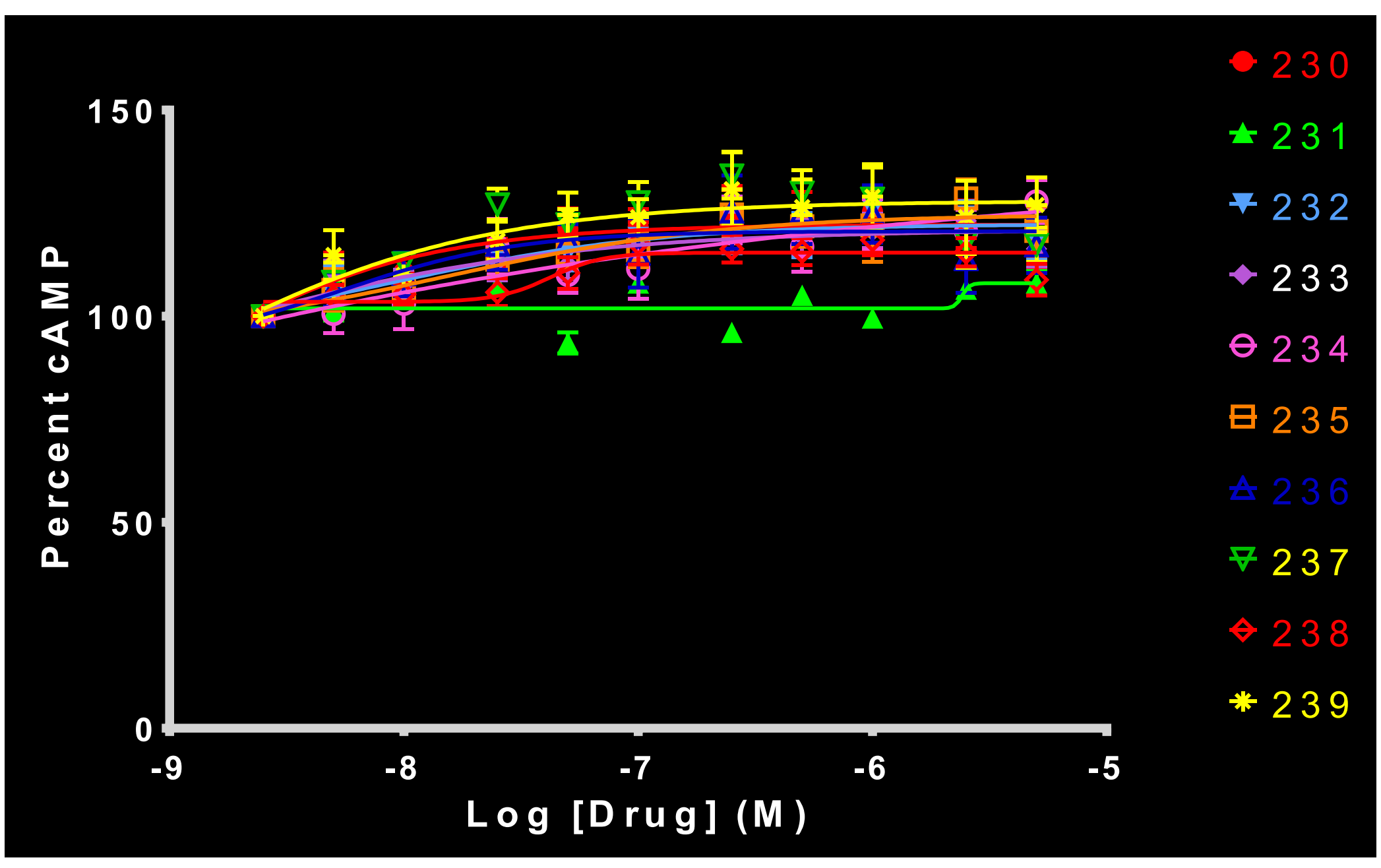

Figure 5.29. $\quad \mathrm{CB}_{1}$ functional activity for pyrimidine triaryl series 


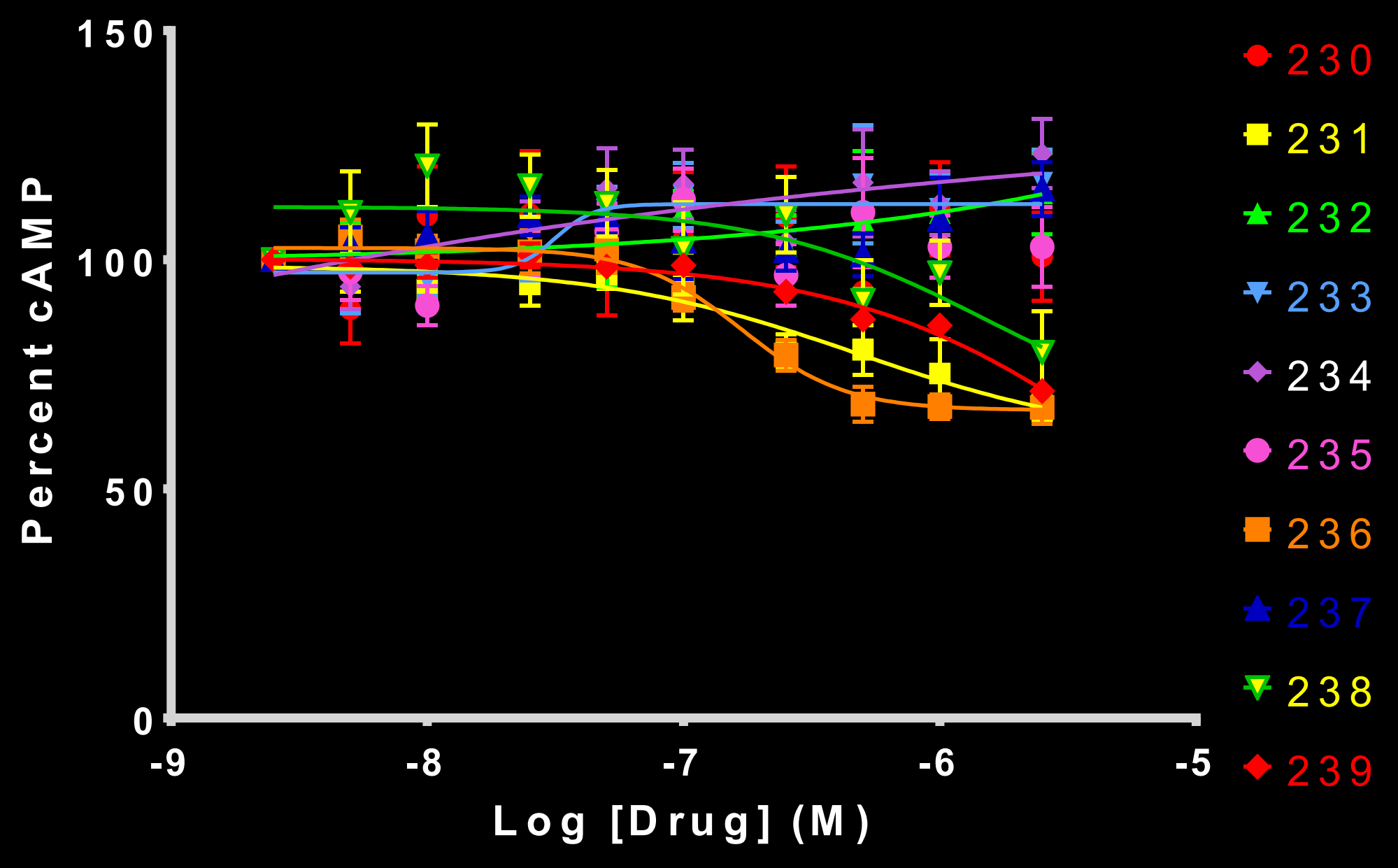

Figure 5.30. $\quad \mathbf{C B}_{2}$ functional activity of pyrimidine triaryl series 


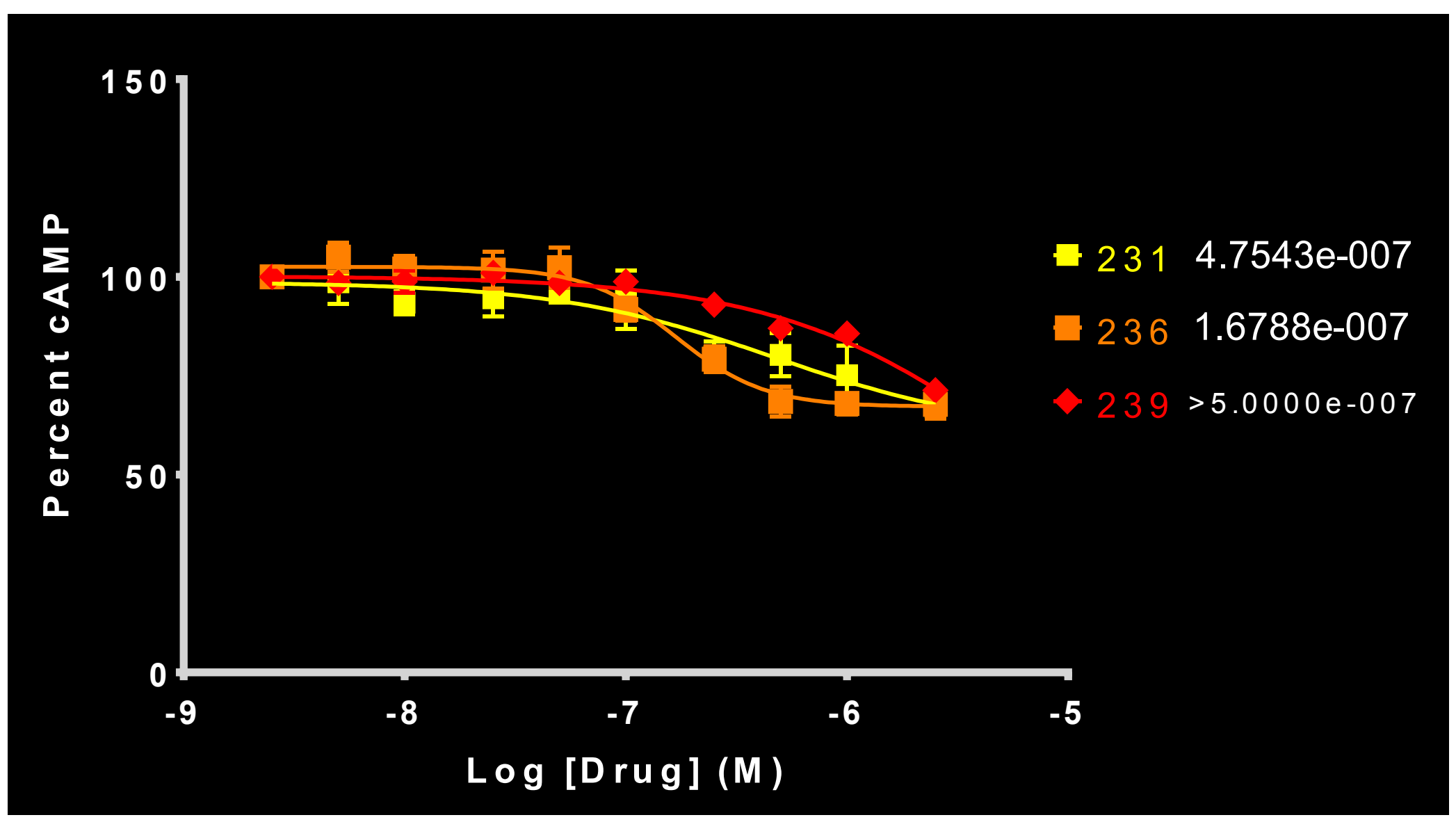

Figure 5.31. $\mathrm{CB}_{2}$ functional activity for select agonists in pyrimidine triaryl series 
Table 5.8. $\quad$ EC $_{50}$ values for pyrimidine triaryl series

\begin{tabular}{|c|c|c|c|c|c|}
\hline Compound & $\begin{array}{c}\mathrm{CB}_{1} \\
\text { EC50 }_{50} \\
\text { or } \\
\text { Concentration } \\
\text { (\% baseline) } \\
\end{array}$ & $\begin{array}{c}\mathrm{CB}_{2} \\
\mathrm{EC}_{50} \\
\text { or } \\
\text { Concentration } \\
\text { (\% baseline) } \\
\end{array}$ & $\begin{array}{l}\text { HEK- } \\
\text { CNG } \\
\text { EC } \\
(\mathbf{n M} M)\end{array}$ & $\begin{array}{c}\text { CB }_{1} \text { PTx } \\
\text { EC }_{50} \\
(\mathbf{n M})\end{array}$ & $\begin{array}{c}\mathbf{C B}_{2} \mathbf{P T} \mathbf{x} \\
\mathbf{E C}_{50} \\
(\mathbf{n M})\end{array}$ \\
\hline 230 & $\mathrm{n} / \mathrm{a}$ & $\mathrm{n} / \mathrm{a}$ & $\mathrm{n} / \mathrm{a}$ & $\mathrm{n} / \mathrm{a}$ & - \\
\hline 231 & - & $475.4^{\mathrm{a}}$ & $\mathrm{n} / \mathrm{a}$ & - & $\mathrm{n} / \mathrm{a}$ \\
\hline 232 & $\mathrm{n} / \mathrm{a}$ & $5000(14 \%)^{b}$ & $\mathrm{n} / \mathrm{a}$ & $\mathrm{n} / \mathrm{a}$ & - \\
\hline 233 & $\mathrm{n} / \mathrm{a}$ & $\mathrm{n} / \mathrm{a}$ & $\mathrm{n} / \mathrm{a}$ & $\mathrm{n} / \mathrm{a}$ & - \\
\hline 234 & $\mathrm{n} / \mathrm{a}$ & $5000(23 \%)^{b}$ & $\mathrm{n} / \mathrm{a}$ & $\mathrm{n} / \mathrm{a}$ & - \\
\hline 235 & $\mathrm{n} / \mathrm{a}$ & $\mathrm{n} / \mathrm{a}$ & $\mathrm{n} / \mathrm{a}$ & $\mathrm{n} / \mathrm{a}$ & - \\
\hline 236 & $\mathrm{n} / \mathrm{a}$ & $167.9^{\mathrm{a}}$ & $\mathrm{n} / \mathrm{a}$ & $\mathrm{n} / \mathrm{a}$ & - \\
\hline 237 & $\mathrm{n} / \mathrm{a}$ & $5000(14 \%)^{b}$ & $\mathrm{n} / \mathrm{a}$ & $\mathrm{n} / \mathrm{a}$ & $\mathrm{n} / \mathrm{a}$ \\
\hline 238 & $\mathrm{n} / \mathrm{a}$ & $5000(21 \%)^{\mathrm{a}}$ & $\mathrm{n} / \mathrm{a}$ & $\mathrm{n} / \mathrm{a}$ & - \\
\hline 239 & $\mathrm{n} / \mathrm{a}$ & $5000(29 \%)^{\mathrm{a}}$ & $\mathrm{n} / \mathrm{a}$ & $\mathrm{n} / \mathrm{a}$ & $\mathrm{n} / \mathrm{a}$ \\
\hline
\end{tabular}

Notes: All concentrations are listed as $\mathrm{nM}$, percentages indicate the change from baseline. n/a: Not active, based on no statistically significant change in cAMP from baseline. "_." Indicates test not performed. ${ }^{a}$ Indicates a decrease in cAMP as compared to baseline. ${ }^{b}$ Indicates an increase in cAMP as compared to baseline. 


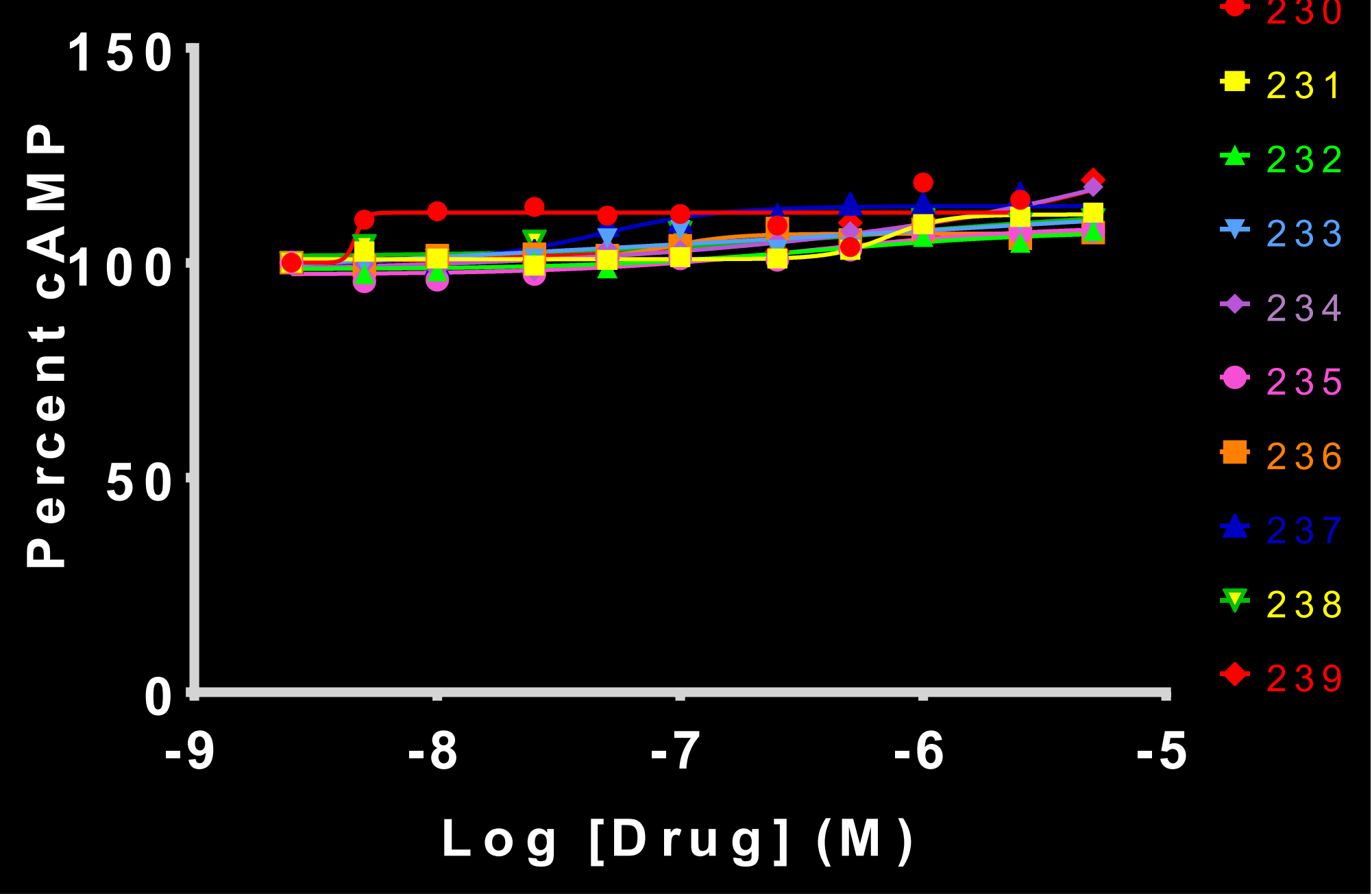

Figure 5.32. HEK-CNG functional activity of the pyrimidine triaryl series 


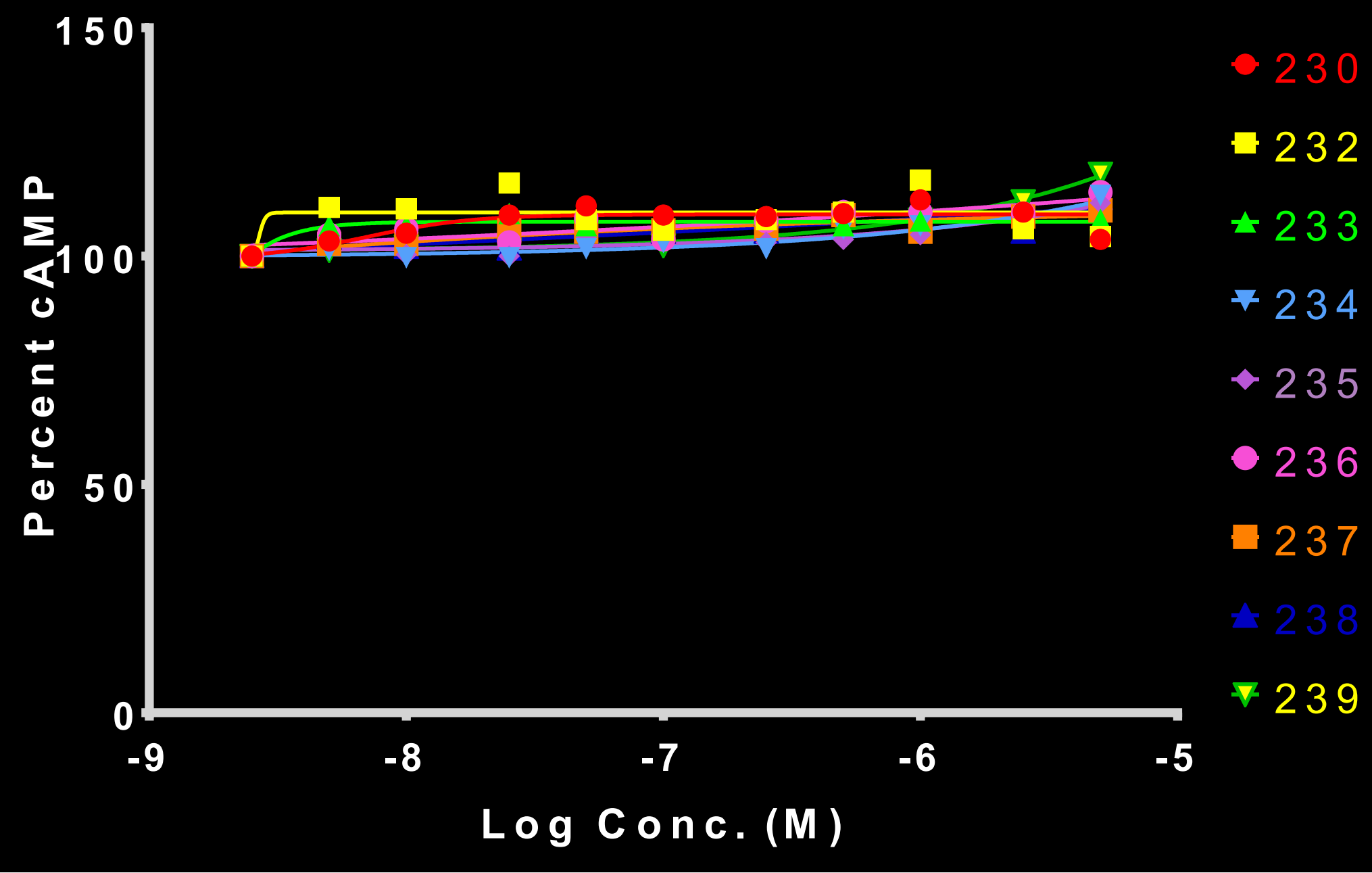

Figure 5.33. $\quad \mathrm{CB}_{1} \mathrm{PTx}$-sensitive $\mathrm{G}_{\mathrm{i} / \mathrm{o}}$-inactivation for select pyrimidine triaryl compounds 


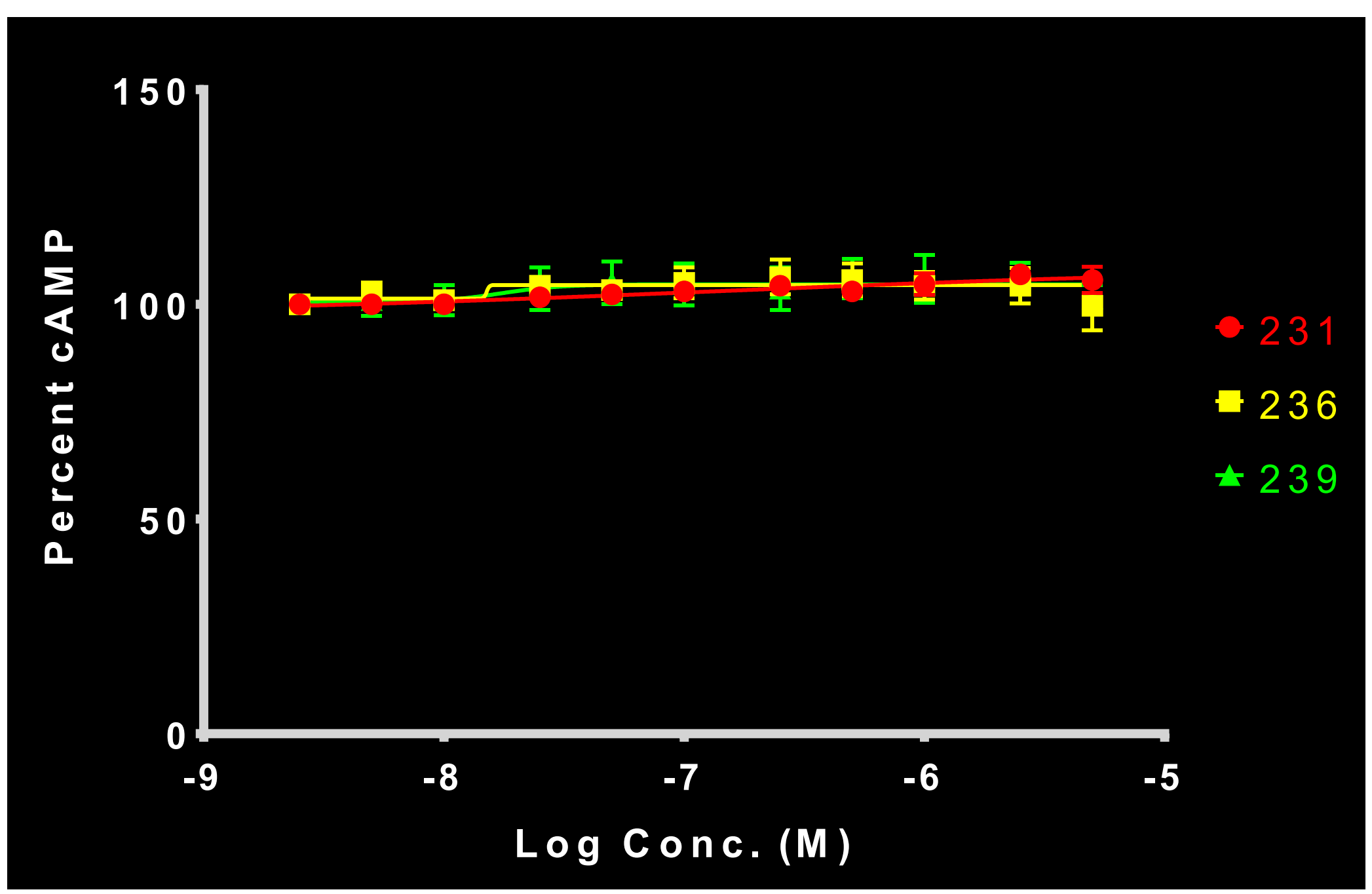

Figure 5.34. $\mathrm{CB}_{2} \mathrm{PT} x$-sensitive $\mathrm{G}_{\mathrm{i} / \mathrm{o}}$-inactivation for select pyrimidine triaryl compounds 
For the diarylpyrazoles (prototype, SR-141716A), K192(3.28) plays an important role in H-bonding with the carbamide oxygen (Figure 5.35A). ${ }^{249,252,257,336}$ In the case of WIN-55,212-2 there are conflicting data regarding K192(3.28) - it is held that the interaction between K192(3.28) and F189(3.25) help to hold each other in proper orientation to facilitate one, the other or both interacting with the ligand. ${ }^{249,254}$ Mutation of K192A(3.28) will negatively impact the binding of SR-141716A and the classical or endogenous ligands, but has a negligible effect on WIN-55,212-2 ${ }^{337}$ - further cause to keep this interaction in perspective and consider it only in certain instances with the nonclassical agents. Overall, this is not as integral an interaction with the non-classical agents as it was with the classical ligands. Additionally, SR-141716A literature shows interactions with $\mathrm{N} 187(3.23)$ and $\mathrm{S} 173(2.60)$ as H-bond interactions on the ketone. ${ }^{249}$ Mutagenesis S173A(2.60) has little effect on CP-55,940 binding, and thus is hypothesized to have little effect on SR-141716A, ${ }^{251}$ though it has not been tested to date.

Within the HB triaryl series of compounds, functional data show compounds 192 and 193 exert agonist activity at $\mathrm{CB}_{1}$, with $\mathrm{EC}_{50}$ values of $993.4 \mathrm{nM}$ and $2008 \mathrm{nM}$, respectively and total percent inhibition of cAMP production at around $50 \%$. While these are not as potent as our reference compound, CP-55,940, they do achieve a similar level of efficacy and are qualified as a full agonist. Also worth noting is the fact that a methoxy group (194) or a mono hydroxyl substitution (191) present on the A ring results in a compound that has antagonist activity at $\mathrm{CB}_{1}$. Thus, our first requirement would be for 1,5-dihydroxy on the A-ring of our triaryl series of compounds. The need for this 1,5-dihydroxy A-ring points strongly to the importance of K192(3.28) in H-bonding with these molecules (Figure 5.35B). The current homology model ${ }^{249}$ used for these cursory data show a possible involvement of S383(7.37) (Figure 5.35B). A similar trend shows within the SMM series, although these do not have the same impact.

The SMM triaryl series, gives us another valuable insight into the SAR of the triaryl compounds, as well as building upon previous data within the HB triaryl series. The first confirmation of SAR findings within this series is the utility of a 3,5-dihydroxy A-ring, as compounds 196, 197, 198, 199, 200, 201, 202, 203, 205, 206, 208, 210 and 212 all contain either a 1,5-dimethoxy or a mono-hydroxy A-ring and lack any effect at $\mathrm{CB}_{1}$. However, within this series, we see certain situations where a 1,5-dimethoxy is of benefit. One such case is the presence of a 3,5-dichloro C-ring with a heteroaromatic "D"-ring (205), as compared to a $m$-toluyl C-ring with a heteroaromatic " $D$ "-ring (213). $\mathbf{2 0 5}$ does not elucidate any response of cAMP production at $\mathrm{CB}_{1}$, whereas 213 demonstrates a weak inverse agonist activity. Di-deprotection of $\mathbf{2 0 5}$ results in $\mathbf{2 1 1}$, showing activity similar to $\mathbf{2 1 3}$ - the mono-deprotected product, $\mathbf{2 1 0}$, shows antagonist activity at $\mathrm{CB}_{1}$. Conversely, di-deprotection of 213 to yield 214 results in no impact of cAMP production at $\mathrm{CB}_{1}$. From this, I hypothesize that heteroaromatic " $\mathrm{D}$ "-rings coupled to electronegative $\mathrm{C}$-rings require deprotection for activity at $\mathrm{CB}_{1}$, whereas these same " $\mathrm{D}$ "-rings coupled to non-electronegative C-rings require di-protected A-ring hydroxyls (Figure 5.35D) to alter cAMP production.

5.5.1.2. CB 1 LBP $\pi$ - $\pi$ stacking interactions. Far and away, the $\pi$ - $\pi$ stacking interactions within the LBP are the most important to consider for the non-classical agents. Considering WIN-55,212-2 and SR-141716A, there exists a great deal of 


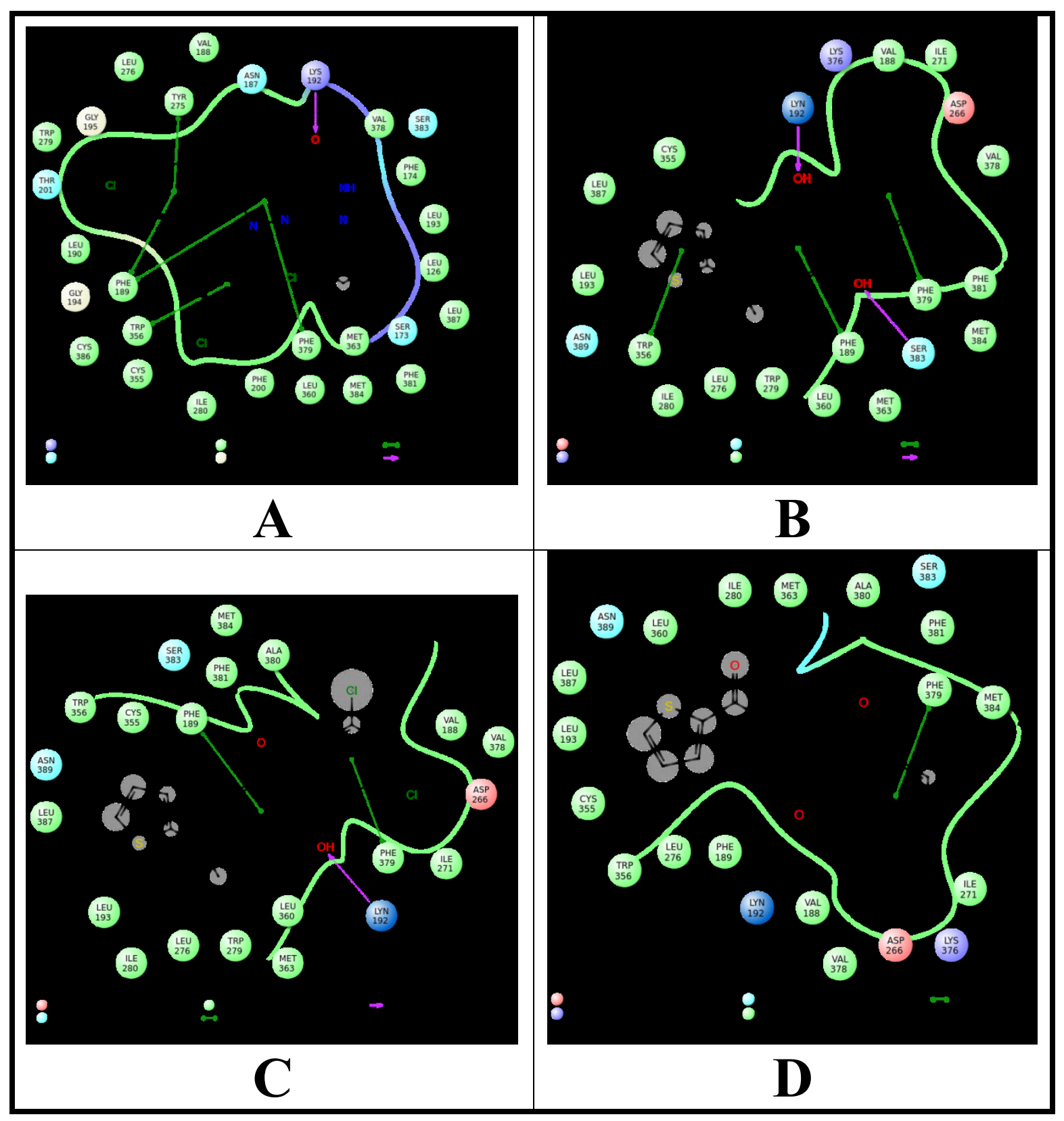

Figure 5.35. CB 1 LBP hydrophilic interactions

Note: Panel A depicts SR-141716A and the proposed LBP residue interactions. Panel B depicts 193 and the proposed LBP residue interactions. Panel C depicts 194 and the proposed LBP residue interactions - loss of one of the hydroxyl groups results in an antagonist. Panel D depicts 213 and the proposed LBP interactions. Green lines indicate $\pi-\pi$ interactions. Dashed pink arrows indicate H-bond interactions. 
common amino acids within the LBP of $\mathrm{CB}_{1}$. Commonalities are: F174(2.61), F189(3.25), F200(3.36), Y275(5.39), W279(5.43), W356(6.48), F379(7.35) and F381(7.35). Of these shared residues, F200(3.36) and W279(5.43) show themselves to be crucial in binding, as the mutation F200A(3.36) causes a precipitous drop in binding affinity for both WIN-55,212-2 and SR-141716A, and W356(6.48) adds itself as an additional imperative interaction for SR-141716A. ${ }^{249,252,257}$ One additional residue, F177(2.64), shows itself as important for binding of WIN-55,212-2 but not SR-141716A. F174(2.61) and F177(2.64) have been demonstrated in classical cannabinoid SAR to be important in maintaining patency of the minor groove of the LBP; however, they have not shown themselves to be involved in the binding of those ligands. ${ }^{255}$ In the case of WIN-55,212-2, they coordinate with the naphthyl moiety of the molecule as a $\pi-\pi$ stack. ${ }^{249}$ SR-141716A shows no additional unique $\pi-\pi$ interactions relative to WIN55,212-2.

\subsubsection{CB1 LBP additional known interactions of non-classical agents.} Remaining interactions for our prototype non-classical agents are only for SR-141716A. Remaining interactions are hydrophilic interactions with S383(7.39) and M384(7.40). These residues are suggested to stabilize the pyrazole ring of SR-141716A within the LBP, and may play an important role in augmenting the binding potential of ligands built off its scaffold. ${ }^{249}$ Mutagenesis S383A(7.39) has a profound effect on binding of CP-55,940 to $\mathrm{CB}_{1}$, and so it is hypothesized that this may play a more critical role in binding of SR-141716A but not WIN-55,212-2. ${ }^{251}$

5.5.1.4. CB 1 LBP interactions of the hydrophobic pocket. As a result of the physical nature of the non-classical agents is a more compact, aromatic nature, interactions within the hydrophobic pocket of the LBP are not discussed per se; however, some of the key interactions from the $\pi-\pi$ stacking and receptor activation/stabilization are located in this region. For purposes of discussion, I will consider the classical agent of HU-210 and its interaction within the LBP. A predominantly lipophilic pocket exists, seemingly as two lobes - as evidenced in the fact that $3-\left(1^{\prime}, 1^{\prime}\right.$-dimethylheptyl $)-\Delta^{8}-\mathrm{THC}$ binds with higher affinity than does 3-heptyl- $\Delta^{8}-$ THC along with 3D-QSAR models which demonstrate this trait. ${ }^{253}$ In one homology model, residues V196(3.32), T197(3.33), F200(3.36), Y275(5.39), W279(5.43), L359(6.51) and M363(6.55) form a hydrophobic pocket which encompasses the A-ring and C-3 side chain of HU-210. ${ }^{254}$ More recent homology models form this pocket from I354(6.46), C355(6.47), W356(6.48), L359(6.51), L360(3.52) and M363(6.55) (Figure 5.36). ${ }^{249}$ Interestingly, mutations studies conducted on this hydrophobic pocket show that M363A(6.55) mutation greatly impacts binding affinity for HU-210 but has virtually no impact on THC which would suggest that this is an important residue for $\mathrm{C}-1$ ' substituted derivatives. ${ }^{255}$

The novel SMM triaryl series offers insight within the C-3 binding domain. One such element found within the SMM triaryl series is the function of a ketone at C-1'. Recalling that 192 and 193 are both 1',1'-gem-dimethyl derivatives and that they exert full agonist activity, we compare them to 214 and 211, respectively. By exchanging the C-1' substituent, we either flip or erase the agonist activity. In the case of 192/214 we move from a full agonist (192) to an antagonist (214) at $\mathrm{CB}_{1}$ and with $\mathbf{1 9 3 / 2 1 1}$ the functional activity transitions from a full agonist (193) to an inverse agonist (211). 


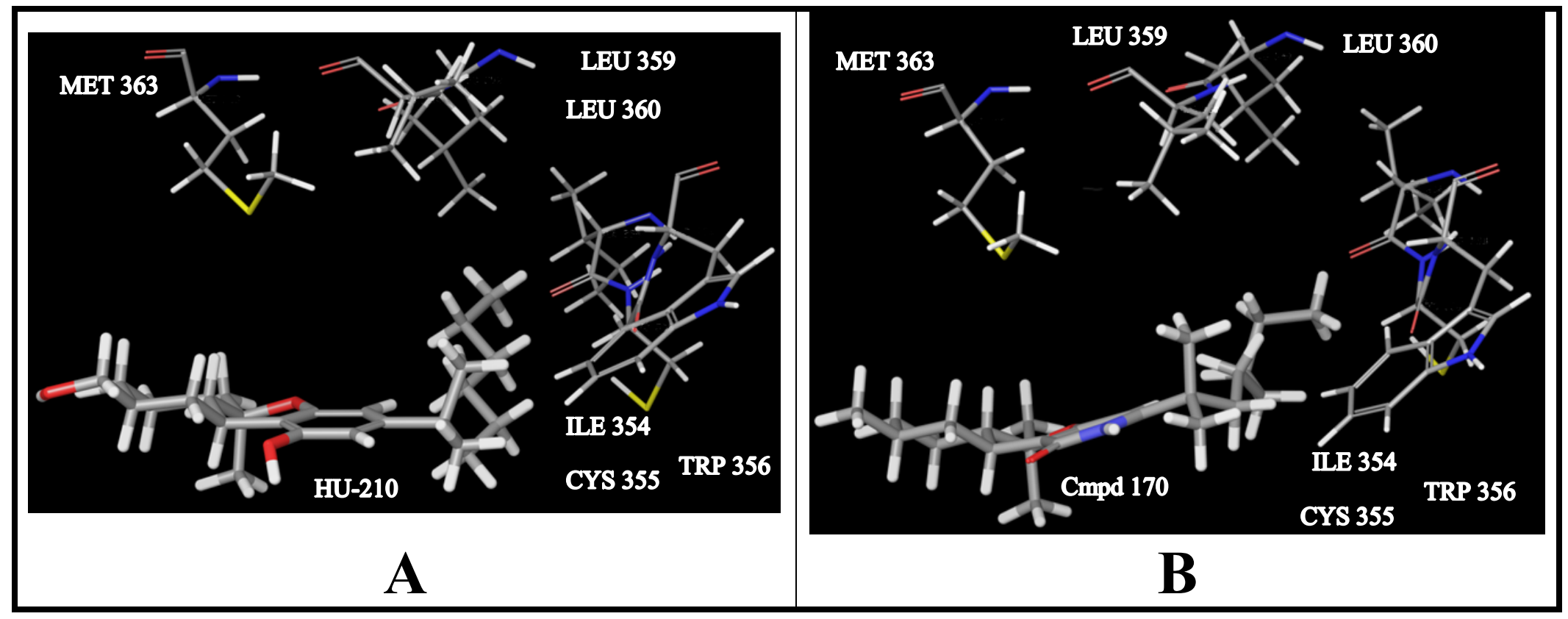

Figure 5.36. $\quad \mathrm{CB}_{1}$ hydrophobic $\mathrm{C}-3$ substituent binding pocket

Note: Panel A depicts HU-210 in the $\mathrm{CB}_{1}$ LBP, with focus given to the hydrophobic pocket. Panel B depicts Compound 170 in the $\mathrm{CB}_{1} \mathrm{LBP}$, with focus given to the hydrophobic pocket. 
Bearing in mind that agonists at $\mathrm{CB}_{1}$ cause the negative psychotropic effects of cannabinoids, this simple change - since 214 and 211 are the precursors to 192 and 193 , respectively - results in a very desirable therapeutic implication, as the psychotropic effects of these cannab inoid derivatives could prove to be rendered null in whole-animal studies. However, this is not the only gem gathered from the SMM series data in functional assay.

Related to the presence or absence of an electro-negative C-ring is the fact that a C-3 heptyl substituent with C-1' ketone (204) demonstrates inverse agonist activity, while a C-3 cyclohexyl with $\mathrm{C}-1$ ' ketone (203) shows antagonist activity. The implications here are that the linear chain fares better with di-protected hydroxyls, while the cycloalkyl has no cAMP impact regardless of its state of A-ring deprotection (di-protected (203), monodeprotected (208) or di-deprotected (209)). It is also of note that the C-3 heptyl (204) derivative is an inverse agonist only at $\mathrm{CB}_{1}$. These trends take a slight detour with the heterocyclic derivatives.

The pyridine and pyrimidine triaryl series are a constellation due to their similarities. Looking at the C-3 aliphatic chains, we see that compounds 217, 218, 219, 221 and 223 have agonist activity at $\mathrm{CB}_{1}$. Comparatively, 222, 224, 225, 226 and 227 have either an aromatic or cyclohexyl C-3 substituent and exhibit an inverse agonist activity. Though the current lack of further study and/or more robust modelling capabilities do not allow for a more precise diagnosis of mechanism, it is noteworthy and warrants further study. Inclusion of a heteroaromatic C-3 substituent seems to yield a non-binding compound with an obvious null effect on cAMP production. None of the pyrimidine compounds exerted any effect on $\mathrm{CB}_{1}$; however, to call them antagonist would not be prudent, as we do not currently have binding data on these compounds.

\subsubsection{Interactions with the $\mathrm{CB}_{2} \mathrm{LBP}$}

Given the varied functional activity of the triaryl compounds at $\mathrm{CB}_{1}$, and the fact that $\mathrm{CB}_{2}$ only shares a $48 \%$ homology with $\mathrm{CB}_{1}$, the potential for more targeted exploitation of this receptor exists. Though the SAR of the $\mathrm{CB}_{2} \mathrm{LBP}$ is not as wellcharacterized as $\mathrm{CB}_{1}$, there are still data which help to highlight some of the important residues and interactions within the LBP. Unfortunately, without a validated $\mathrm{CB}_{2}$ model, insights into the triaryl series are not feasible.

\subsubsection{C-1 Hydroxyl, Benzchromene Oxygen and (optional) C-6 Hydroxyl.} Mutation studies with HU-210, CP-55,940 and WIN-55,212-2 have shown that binding of both HU-210 and CP-55,940 experience negative consequence with a S292A mutation. This mutation-induced change of binding strongly suggests S292(7.46) is involved in H-bonding with the benzchromene oxygen - assuming a similar LBP arrangements relative to the $\mathrm{CB}_{1}$ LBP. ${ }^{323}$ Though WIN 55-212-2's ketone is in proximity to residue S193(5.42) some data suggest it does not hydrogen bond. ${ }^{261}$ In the case where amide linkages exist between the aryl groups, S193(5.42) does act as a H-bond donor. ${ }^{324,325}$ These interactions may help to provide nsight into why di-protected triaryls do not behave as their di-deprotected counterparts. 
As was the case in $\mathrm{CB}_{1}$, so it remains in $\mathrm{CB}_{2}$, the requirement for 1,5-dihydroxy A-ring, as 191 and 194 failed to cause a change in the cAMP production. Next, it is evident that an $m$-toluyl $\mathrm{C}$-ring (192) shows agonist activity at both $\mathrm{CB}_{1}$ and $\mathrm{CB}_{2}$, and has an increased potency at $\mathrm{CB}_{2}$, with an $\mathrm{EC}_{50}$ of $18.7 \mathrm{nM}$ and a $35 \%$ reduction in $\mathrm{cAMP}$ production. While 192 is only a partial agonist of $\mathrm{CB}_{2}$ (not as efficacious as CP-55,940), it is in line with CP-55,940's potency.

Considering now the SMM series, again the SAR requirements for $\mathrm{CB}_{1}$ begin to reemerge as similarly pertinent in $\mathrm{CB}_{2}$. First, comparing 193 to $\mathbf{2 1 1}$, we see how a $\mathrm{C}-1^{\prime}$ ketone again allows a change to an inverse agonist from a neutral antagonist. It becomes evident within the SMM triaryl series at $\mathrm{CB}_{2}$ that the $\mathrm{C}-1^{\prime}$ ' substituent must be a ketone for this inverse agonist, as 195 is a very effective $\mathrm{CB}_{2}$ inverse agonist, but when $\mathrm{C}-\mathrm{1}^{\prime}$ is a hydroxyl (the direct precursor of 195), all activity is lost.

5.5.2.2. Hydrophobic and $\pi-\pi$ stacking. As has been the case previously, it remains so that many of the interactions derive from the aminoalkylindole, WIN-55,2122 , and play a central role in non-classical binding. Beginning with the indole ring of WIN $55,212-2$, there are $\pi-\pi$ stacking interactions between it and residues F197(5.46), W258(6.48) and F117(3.36). ${ }^{261}$ There also exists a shared $\pi-\pi$ stacking between the indole ring and napthyl ring with residue W194(5.43). ${ }^{261}$ The napthyl ring has a proposed added $\pi-\pi$ stack with residue F106(3.25). ${ }^{261}$ More recent 2-pyridone derivatives add additional residues to the binding pocket for $\pi-\pi$ stacking, namely Y 190(5.39) and F281(7.35) along with C288(7.42) ${ }^{324,325}$ For the 2-pyridone analog containing N-butyl, there is a proposed hydrophobic interaction with I198(5.47). ${ }^{325}$ Though little data exists, it is suggested that $\mathrm{SR}-144528$ (a CB 2 -selective inverse agonist) has interaction with $\mathrm{W} 194(5.43)$ and F197(5.46), ${ }^{338}$ which may play a role in stabilizing the inactive state and impart inverse agonist activity. However, the 2-pyridone shows agonist activity and interaction with W194(5.43) and F197(5.46), so interaction with these residues does not impart inverse agonist activity. Considering the triaryl composition of this series of cannabinergic agents, it is logical to hypothesize that the $\pi-\pi$ interactions are important to receptor recognition and function. Future modelling is necessary and will be used to identify potential interactions within the $\mathrm{CB}_{2} \mathrm{LBP}$ for further ligand refinement.

From the SMM series, one may speculate about another $\mathrm{CB}_{2} \mathrm{SAR}$ - the need for an aromatic D-ring to obtain inverse agonist activity (Figure 5.37). Compounds 195, 206, 207, 211, 213 and 215 all contain aromatic D-rings, and when compared to 204 with its linear aliphatic and 203, 208 and 209 with their cycloalkyl, we see that non-aromatic compounds take on a neutral antagonist role. Interestingly, the effect of deprotection is not as staunch in the $\mathrm{CB}_{2}$ receptor studies; however, there is a requirement for at least a mono-deprotected A-ring, as evidenced in 202 (di-methoxy), 206 (mono-methoxy) and 207 (di-hydroxy). Compound 206, a mono-deprotected A-ring, and its successor 207, a di-hydroxy A-ring, demonstrate the same trend, with the efficacy being similar at high concentrations, but the potency of $207\left(\mathrm{EC}_{50} 89.7 \mathrm{nM}\right)$ greatly exceeds that of $206\left(\mathrm{EC}_{50}\right.$ $>5000 \mathrm{nM}$ ) (Figure 5.37). These data suggest the $\mathrm{CB}_{2}$ pocket has a varied hydrophilic requirement of the $\mathrm{CB}_{1}$ pocket and could allow for more selective targeting of compounds. However, the heteroaromatic compound data do not show the same bifurcation of $\mathrm{CB}_{1}$ and $\mathrm{CB}_{2}$, at least with the pyridine derivatives. 


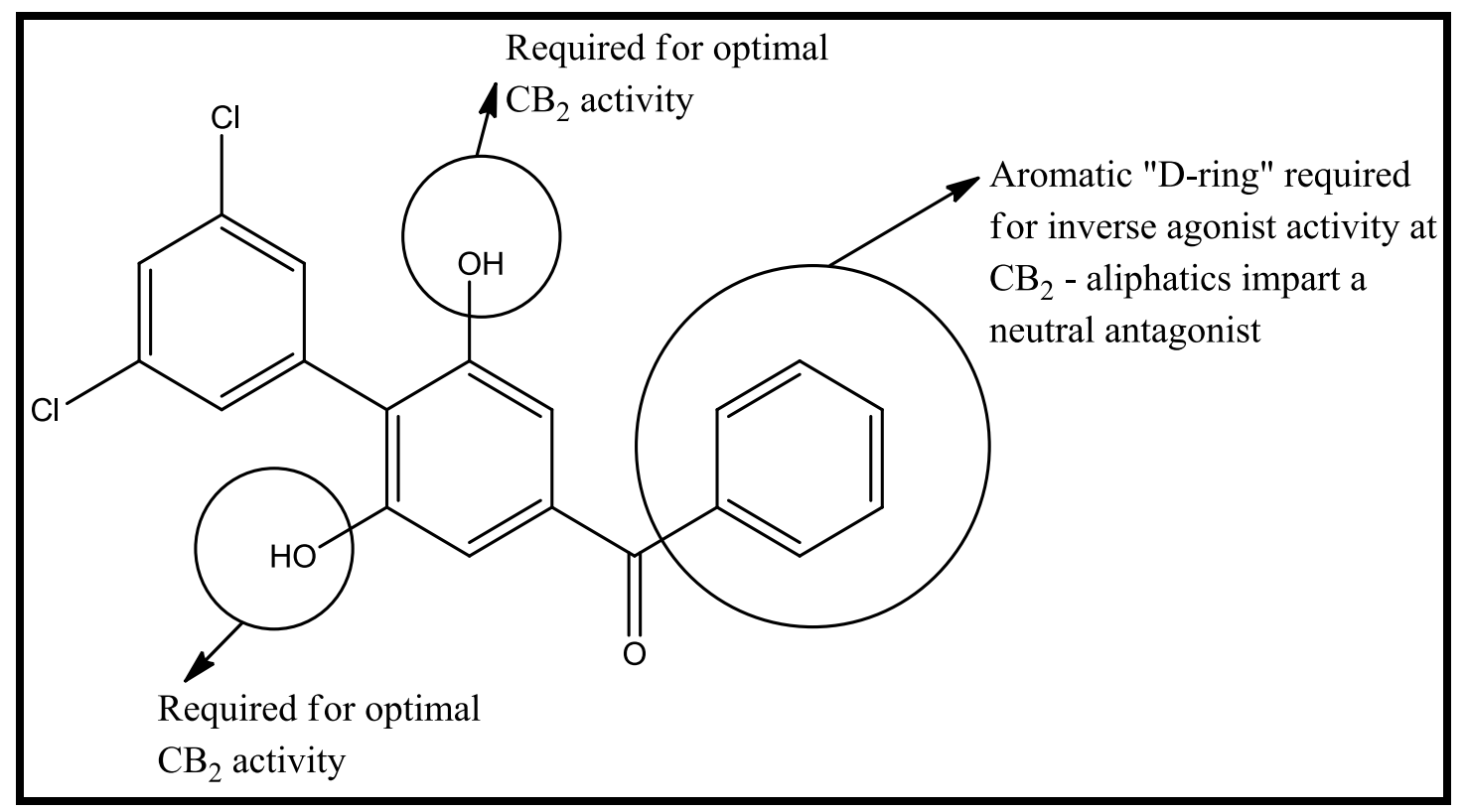

Figure 5.37. $\mathrm{CB}_{2}$ required D-ring and A-ring elements for compound 195 
At $\mathrm{CB}_{2}$, the pyridine trend holds, with compounds 217, 218, 219, 221 and 223 having agonist activity, and 222, 224, 225, 226 and 227 having inverse agonist activity (Figure 5.38). It is at $\mathrm{CB}_{2}$ where we begin to see the utility of the pyrimidine series, with 231, 236 and 239, all with aliphatic C-3 substituents demonstrating agonist activity. With the exception of 231, these compounds have saturated aliphatic substituents at both $\mathrm{C}-3$ and C-6 of the A-ring. Comparing 236 to 237 we see the effect of an aromatic substitution at C-6, with a loss of agonist activity. Looking at 231 and 235, we see a loss of activity. This holds when comparing $\mathbf{2 3 6}$ to $\mathbf{2 3 8}$ where one sees the incorporation of a heteroaromatic ring at $\mathrm{C}-1^{\prime}$ results in a non-active compound (Figure 5.39).

\subsection{Conclusions}

In this chapter, I have demonstrated by utilization of a functional assay how small synthetic changes help to yield more selective or opposing actions on $\mathrm{CB}_{1}$ and $\mathrm{CB}_{2}$. The first of which is the ability to convert from an agonist to an inverse agonist by the exchange of theC-1'-gem-dimethyl substituent for $\mathrm{C}-1^{\prime}$ ketone - a trend that holds true in both $\mathrm{CB}_{1}$ and $\mathrm{CB}_{2}$.

Additionally, I demonstrated how to target $\mathrm{CB}_{2}$ more effectively by monodeprotection of the 3 and 5 position of the A-ring, as this is permissible in $\mathrm{CB}_{2}$ assays but not in $\mathrm{CB}_{1}$. Lastly, I provided evidence of functional interchange within a given series incorporation of a heterocyclic A-ring allows for linear aliphatic C-3 substituents to exert agonist activity and cycloalkyl/aromatic substituents to provide an inverse agonist effect. In addition, this heterocyclic A-ring with cycloalkyl/aromatic C-3 substituent provides evidence of a non-competitive inverse agonist.

Future directions for this project are to synthesized matched derivatives within each of the four (4) triaryl series to ensure that we have a more accurate comparison of these SAR findings via functional assay validation. Additionally, further derivation of heterocyclic D-ring and C-ring, along with cycloalkyl C-ring derivatives - matched across the 4 series - will help to solidify this trait. Unsaturation along the C-3 heptyl chain, coupled with chain extension and redaction, would help to probe the depth and orientation of the pocket to optimize their activity. Finally, as we saw in the HB and SMM triaryl series, changing the pyridine and pyrimidine to $\mathrm{C}-1^{\prime}$ ketone could further solidify this as a key interchange for activity in the $\mathrm{CB}_{1}$ and $\mathrm{CB}_{2}$ receptor. 


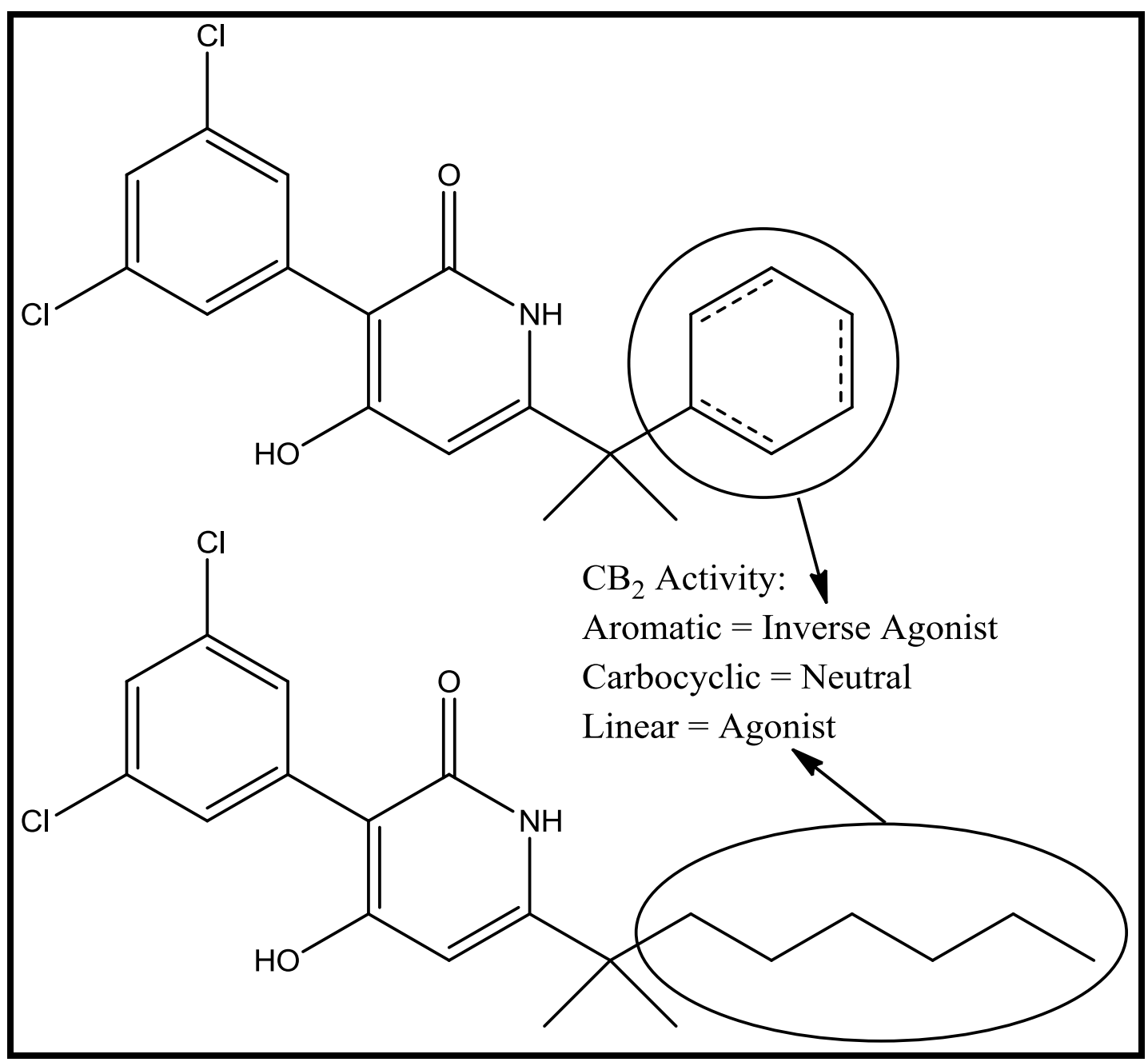

Figure 5.38. $\quad \mathrm{CB}_{2}$ required $\mathrm{C}-\mathrm{1}^{\prime}$ elements for pyridine triaryls 


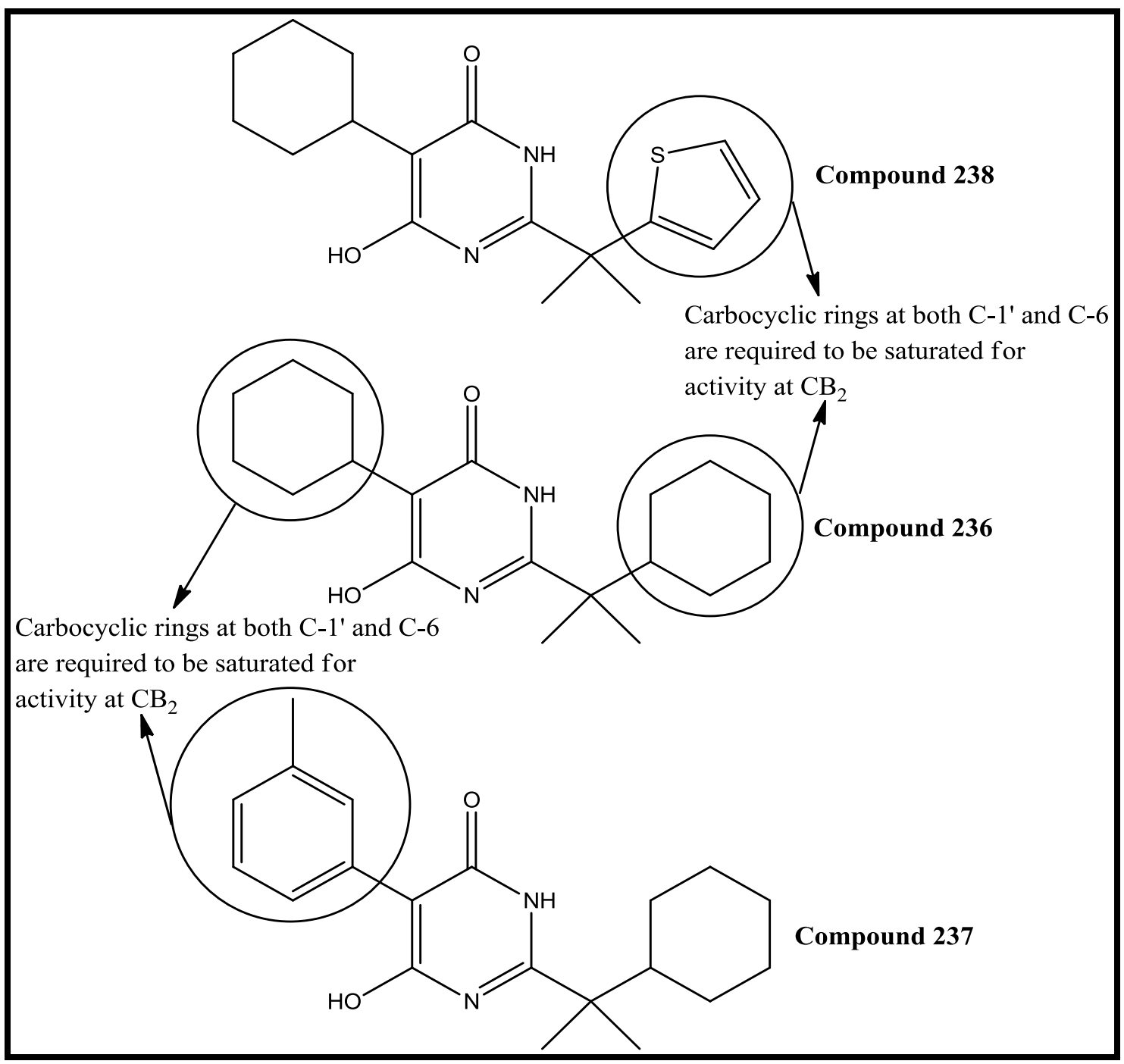

Figure 5.39. $\quad \mathrm{CB}_{2}$ required $\mathrm{C}-6$ and $\mathrm{C}-\mathbf{1}^{\prime}$ elements for pyrimidine triaryls 


\section{CHAPTER 6. FUNCTIONAL PHARMACOPHORE}

As has been demonstrated, minor changes in a compound's structure - be it a bioisosteric replacement, heteroatom substitution or a change in the oxidation state of one atom - have generated functionally distinct and potentially therapeutically significant molecules. One of the first examples presented was the interaction of the KM series (Figure 6.1) with the $\mathrm{CB}_{1}$ and $\mathrm{CB}_{2}$ receptors, wherein it was shown that these compounds are $\mathrm{CB}_{1}$ agonists and $\mathrm{CB}_{2}$ antagonists - a trait not displayed in other compounds with a classical core scaffold. Next, compounds built directly from this series sought to incorporate one (pyridine) or two (pyrimidine) nitrogen atoms in the A-ring of the classical benzchromene core (Figure 6.1). From the hexahydro series it was shown that aliphatic $\mathrm{C}-1^{\prime}$ substituents were able to retain the traditional agonist properties of classical core compounds; however, pyridine A-ring with $\mathrm{C}-1^{\prime}$-phenyl demonstrate $\mathrm{CB}_{1}$ inverse agonist activity and pyrimidine A-ring with $\mathrm{C}-1$ '-phenyl $\mathrm{CB}_{2}$ inverse agonists. Finally, the triaryl series (Figure 6.1) demonstrated another unique and potentially therapeutically useful switch. Within this series, a small change at the $\mathrm{C}-\mathrm{1}^{\prime}$, going from a C-1'-gem-dimethyl to a C-1' ketone, results in a switch from a full agonist to an inverse agonist. All of these minor chemical changes result in such staunch, sometimes opposing and potentially therapeutically exploitable switches and options. This leads to the question: What is happening within the respective receptors, and what residues may be involved that could be even further exploited for new therapeutic agents? Herein, I will summarize and highlight interactions, which may help to direct and drive future development. Further, with a validated $\mathrm{CB}_{1}$ homolgy model, I will propose LBP residues and interactions, which may further highlight the potentially therapeutically exploitable facets of the $\mathrm{CB}_{1}$ receptor. Unfortunately, I lack access to a validated $\mathrm{CB}_{2}$ homology model, and thus the same propositions are not possible.

To generate hypotheses and to gain some insight into the possible explanations and interplay between ligand and receptor, docking of the compounds was performed. Dr. Chang of The University of California, Riverdale, kindly supplied the models for the CB1 receptor. ${ }^{249}$ Compounds in the KM series and the hexahydro (pyridine and pyrimidine) series were docked and compared in the "HU-210" model. Classical and non-classical inverse agonists were docked and compared in the "SR-141716A" model. Finally, non-classical agonists were docked and compared in the "WIN-55,212-2" model. All tested/docked compounds were minimized and prepped with LigPrep, receptor grid generation and docking was completed utilizing these prepared ligands in GLIDE.

\subsection{CB1-Specific Proposed Functional Switches}

Within the $\mathrm{CB}_{1}$ "HU-210" model, one can see that HU-210 and $\Delta^{9}$-THC occupy nearly the same space (Figure 6.2). As previously mentioned (Section 3.5.1), the KM series may exhibit a binding mode in the $\mathrm{CB}_{1}$ pocket that very closely mimics that of $\Delta^{9}$ THC (Figure 6.3). One notable exception is that the pentyl side chain of $\Delta^{9}$-THC projects into the hydrophobic pocket more than the aromatic xylyl of 67. The aromatic xylyl, in this preliminary model, shows a preferential interaction with residue W356(6.48). The 


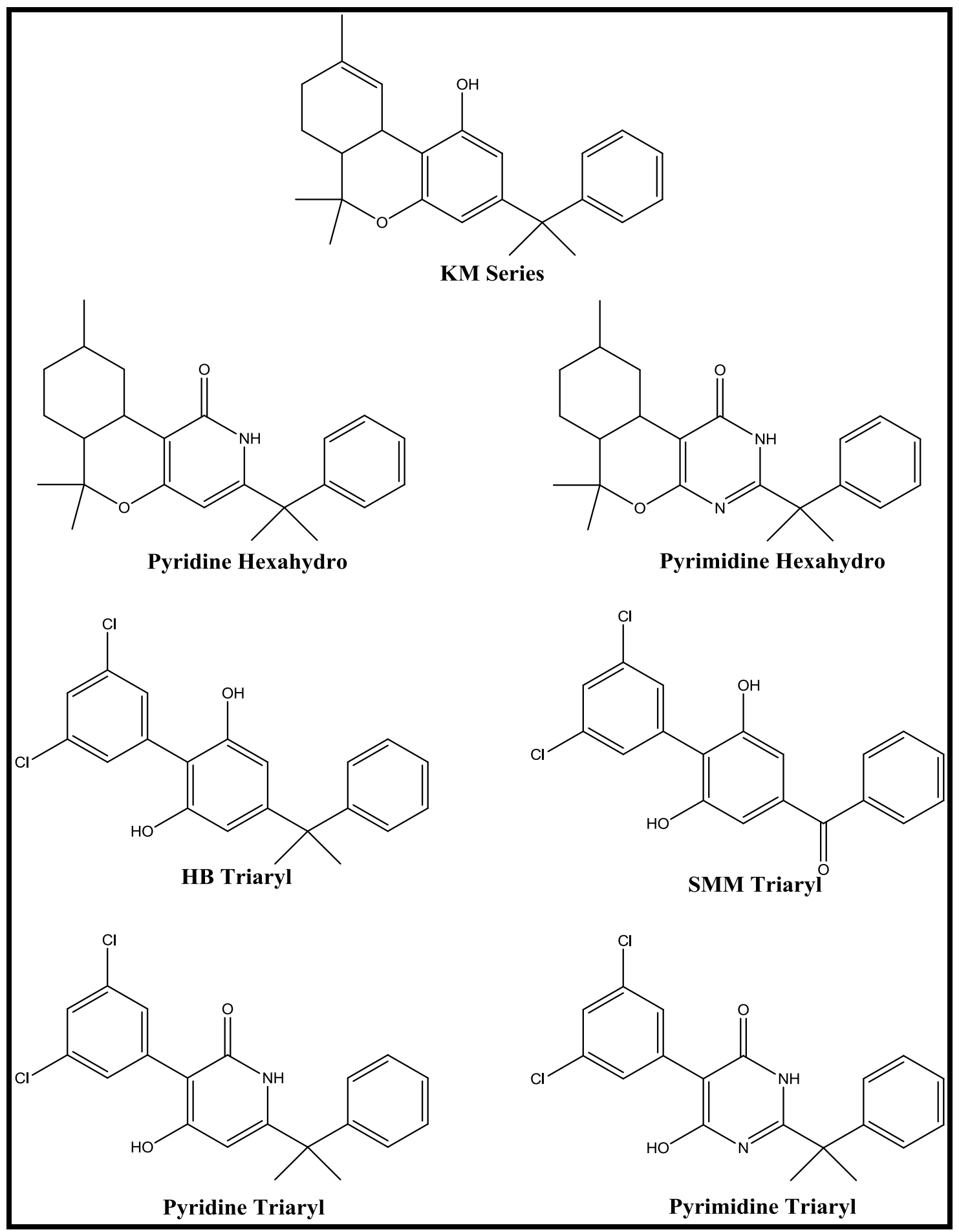

Figure 6.1. Representative examples from each of the novel cannabinergic agents 


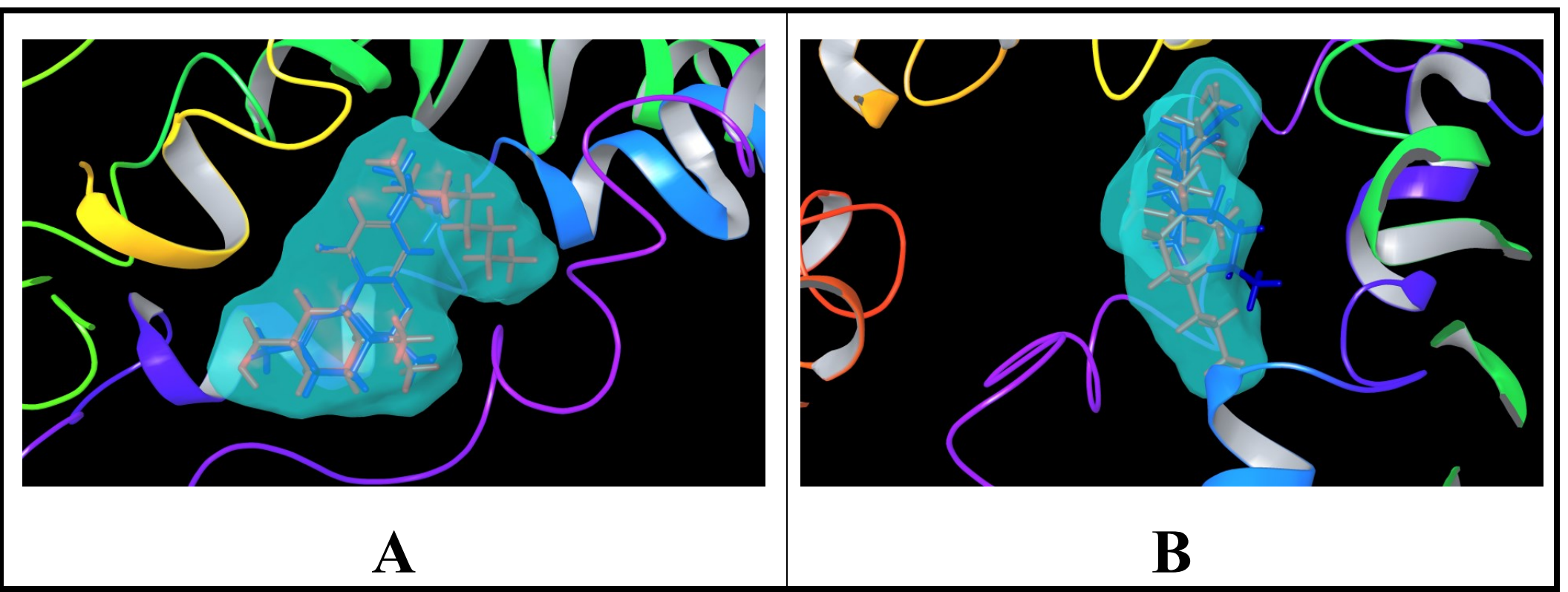

Figure 6.2. $\quad \mathrm{CB}_{1}$ spatial relationship between HU-210 and $\Delta^{9}$-THC

Note: Panel A shows the front view. Panel B shows the side view. For ease of visualization with the stacked molecules, HU210 is colored red and $\Delta^{9}$-THC is colored blue. 


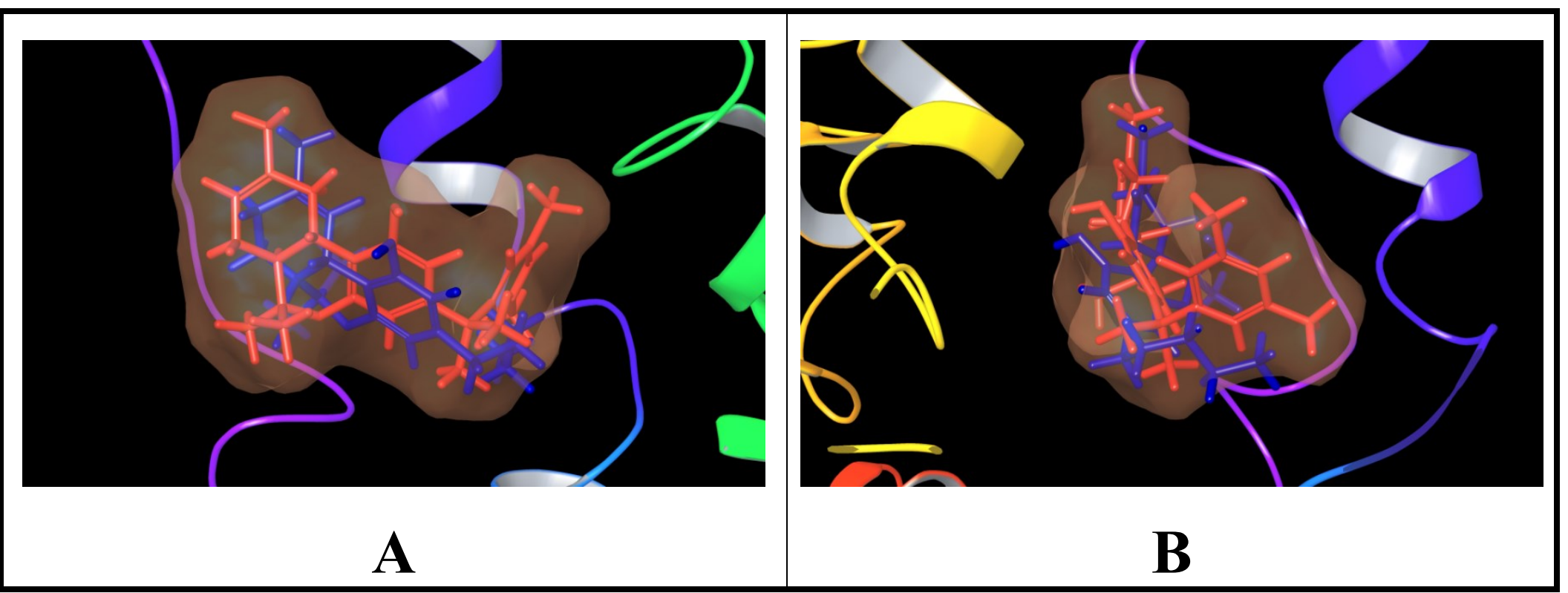

Figure 6.3. CB1 spatial relationship between Compound 67 and $\Delta^{9}$-THC

Note: Panel A shows the front view. Panel B shows the side view. For ease of visualization with the stacked molecules, $\mathbf{6 7}$ is colored red and $\Delta^{9}$-THC is colored blue. 
correlation of $\Delta^{9}$-THC to both HU-210 (the basis for this particular model) and $\mathbf{6 7}$ allow for my hypothesis about requirements and exploitations of the $\mathrm{CB}_{1} \mathrm{LBP}$.

\subsubsection{Common residue interactions}

Literature demonstrates time and again that F189(3.25) is important, if not crucial for binding of the endocannabinoids, such as AEA. In fact, the F189A CB 1 mutant shows a 7-fold drop in affinity for AEA compared to w.t. The current model used for this examination, while rudimentary at best, does show the importance of an aromatic A-ring for interaction with this residue in the LBP (Figure 6.4). This interaction, I believe, is what secures and properly orients the molecule in the LBP.

For most compounds, the classical core KM series and hexahydro series included, K192(3.28) plays an integral role in binding with the $\mathrm{C} 1$ hydroxy or amide oxygen (Figure 6.4). This holds true for most of the triaryl series, as well; however, other factors begin to outweigh the need for K192(3.28) in binding and exerting a functional effect. That said, having a moiety at $\mathrm{C} 1$ or an analogous position is one of the first and strongest considerations to be made in the design of targeted molecules.

As previously mentioned (Section 3.5.1.2), M363(6.55) has an extremely profound effect on the binding affinity of HU-210 when methionine is exchanged for alanine in the M363A mutant receptor. This precipitous drop in affinity does not hold true for $\Delta^{8}$-THC, so it is a logical conclusion that the $\mathrm{C1}^{\prime}$-gem-dimethyl group interacts with this residue. This bears itself out in the non-molecular-dynamic-docking into the "HU-210" homology model, as well, with M363(6.55) showing good contact with the C1'-gem-dimethyl of 67, but not so with $\Delta^{9}$-THC (Figure 6.5). This residue does seem to provides a viable target to impart increased $\mathrm{CB}_{1}$ affinity, but is not necessary for function.

For receptor activation to occur, interaction with W279(5.43) is essential, according to literature. The interactions, according to the current model, coupled with the functional data suggest that the interaction may be via $\pi-\pi$ stacking or aliphatic interactions. The constant is that a functionally active compound models with some sort of interaction with this residue (Figure 6.4).

\subsubsection{The W356(6.48) "toggle" switch}

As previously discussed (Section 3.4.2), the KM series of compounds has an aromatic C-1' substituent, and are agonists at $\mathrm{CB}_{1}$. Based on the current "HU-210" homology model, the aromatic substituent interact simultaneously with W279(5.43) and W356(6.48). Spatially, W356(6.48) is flanked by W279(5.43) and F200(3.36), and it is its interaction with one or the other which seemingly determines receptor activation (W279(5.43)) or stasis (F200(3.36)). Looking at the interaction of 67, an established $\mathrm{CB}_{1}$ agonist, the model suggests a $\pi-\pi$ stack which links W279(5.43) to W356(6.48) (Figure 6.6) - presumably leading to receptor activation. To the converse of this point, 172 is similar to 67 except for the fact that the A-ring contains a nitrogen at position 2 and the $\mathrm{C}-1$ hydroxyl is now an amide oxygen. This is a relatively small change to the molecule, 


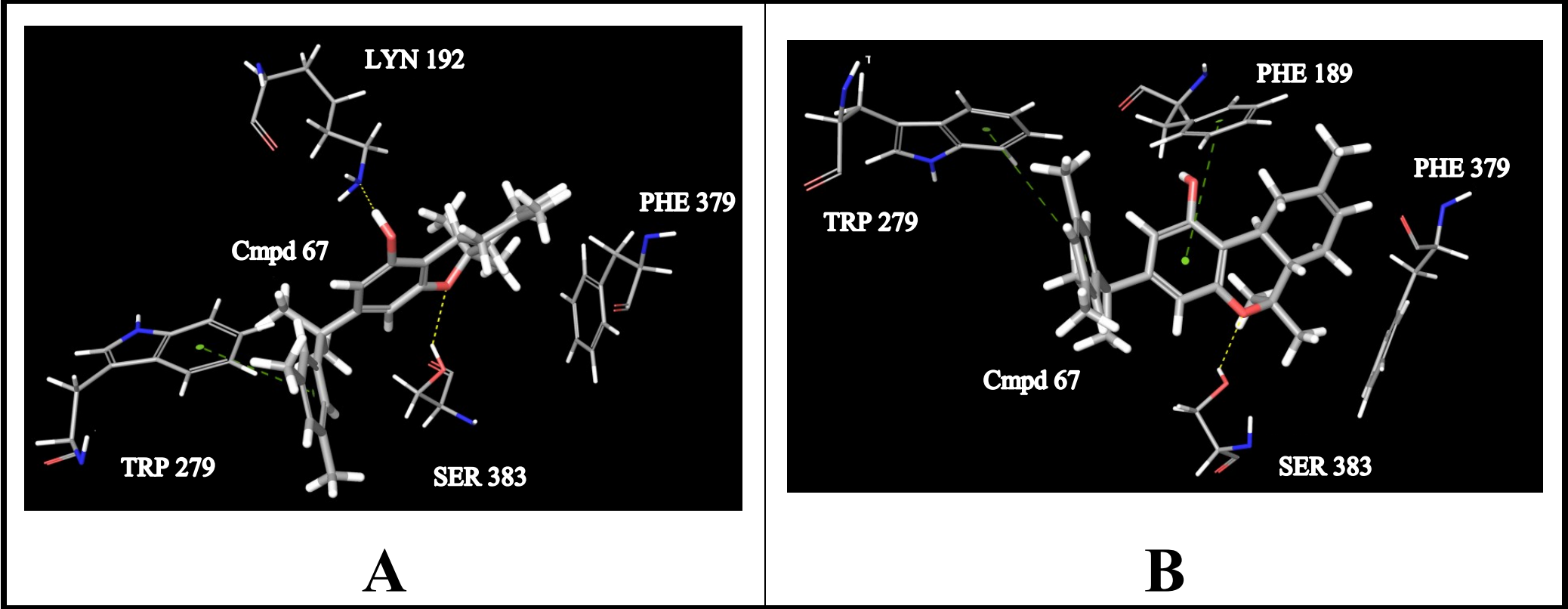

Figure 6.4. $\quad$ CB1 LBP common residues for classical core compound 67

Note: Depicts the common residues found in classical core interactions. Panel A is side view with F189(3.35) removed. Panel B is the top view with K192(3.28) removed. Dashed green lines indicate $\pi$ - $\pi$ interactions, dashed yellow lines indicate H-bond. 


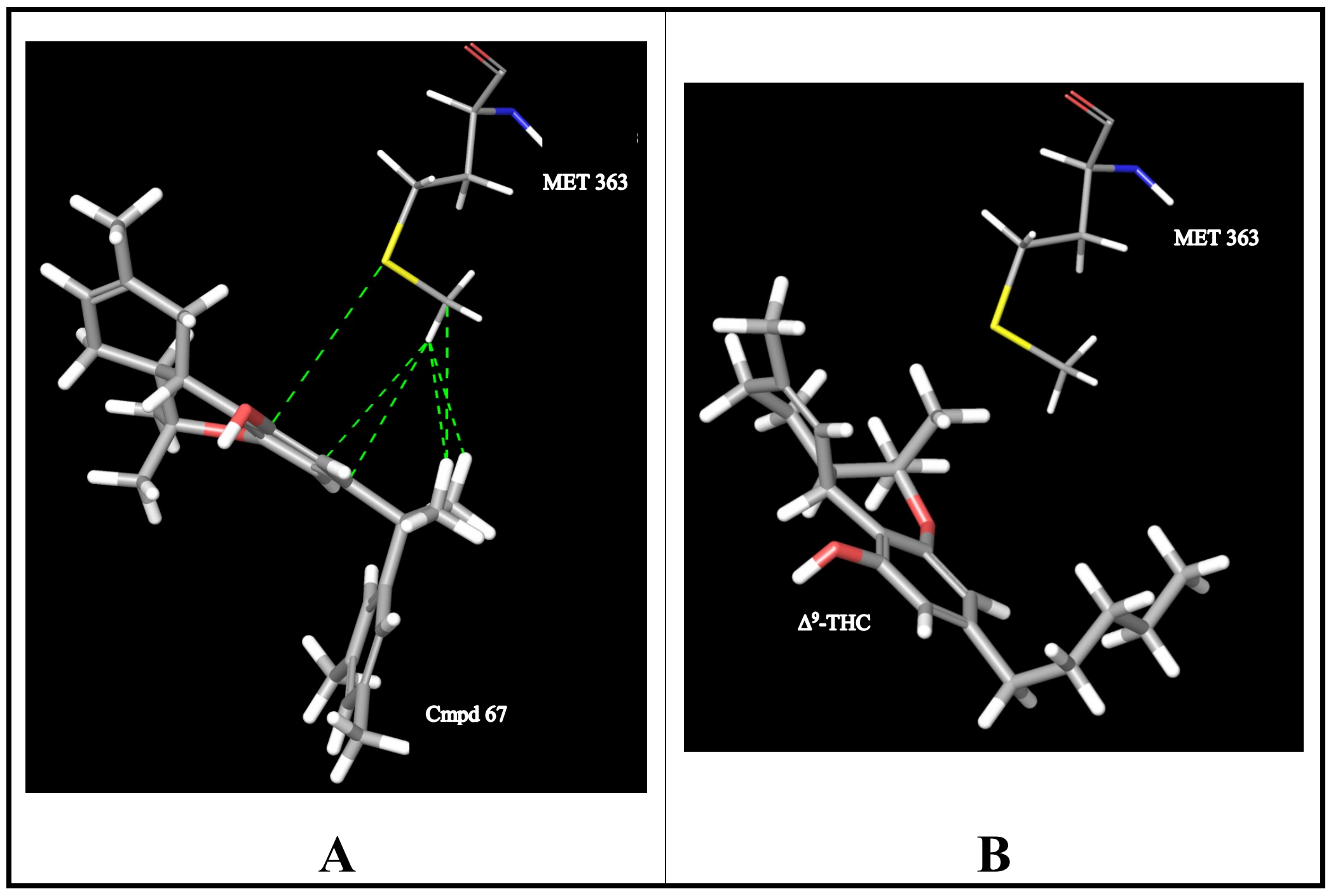

Figure 6.5. $\quad \mathrm{CB}_{1}$ M363(6.55) contact with compound 67 and $\Delta^{9}$-THC

Note: Dashed green lines indicate "good" contact between receptor and ligand. 


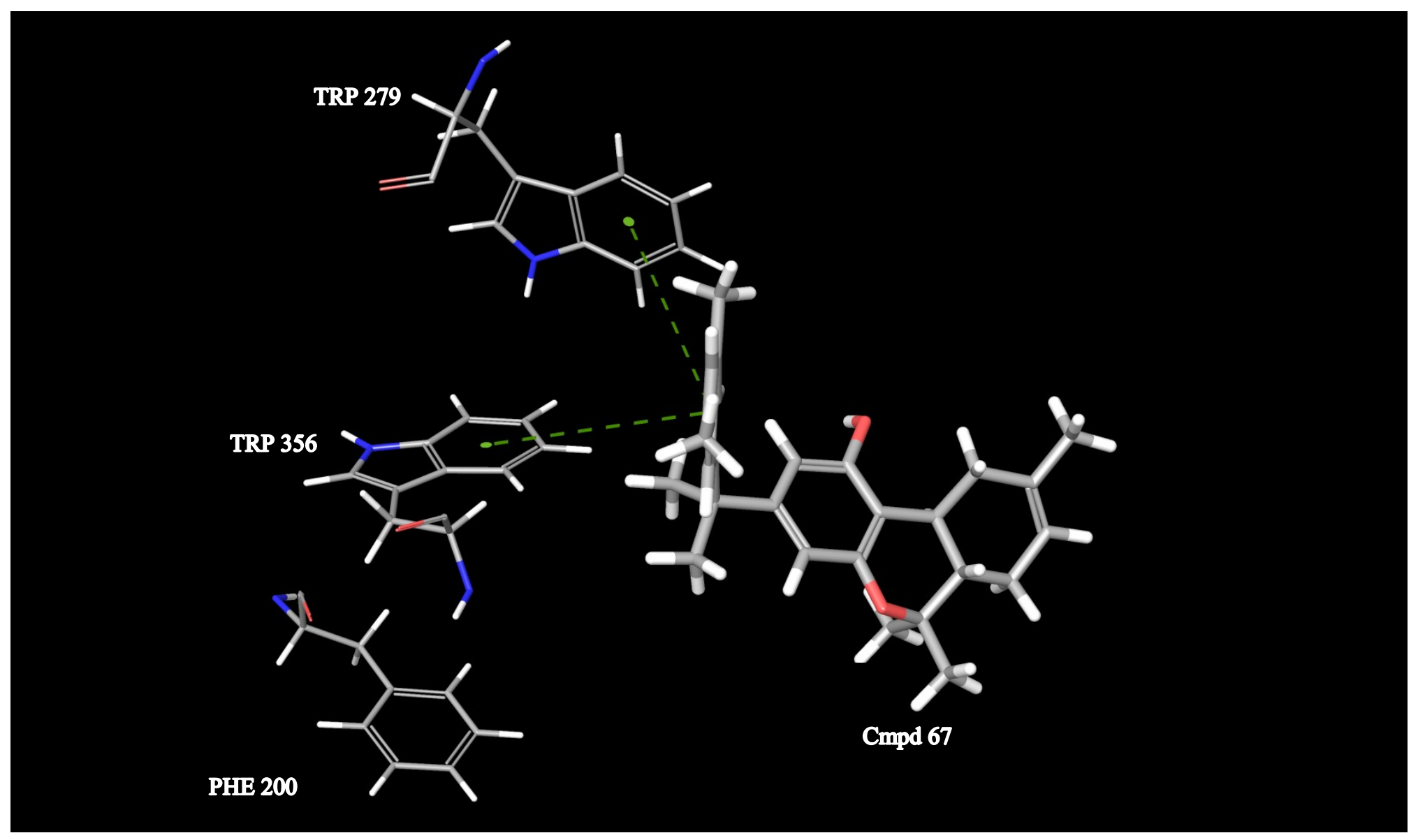

Figure 6.6. CB $\quad$ LBP W356(6.48) "toggle" switch presumed to be activated by compound 67

Note: Dashed green lines indicate $\pi-\pi$ stacking interactions. 
but it has a large impact on its function. Based on the model, this small change in the A-ring alters the binding and preference of the aromatic $\mathrm{C}-1$ ' substituent to now colocalize and $\pi-\pi$ stack with W356(6.48) and F200(3.36) (Figure 6.7)- resulting in an inverse agonist. I hypothesize that this shift is due to the amide in the A-ring altering how the hydrophilic interactions take place and the overall conformation in the LBP.

\subsubsection{The lack of hydrophilic interaction within the LBP}

When examining the triaryl series of compounds, another unique trait began to emerge, which was quite contrary to previously held belief about the rigors and requirement for binding. As previously mentioned, K192(3.28) and S173(2.60) play a key role in H-bond formation with the ligand and subsequent stabilization in the LBP and/or a conformational change induction. That said, the requirement for hydrogen bond donor groups on the A-ring is negated when $\mathrm{C}-1$ ' is a heteroaromatic group, as in the case of 214. Too, stabilization of the W356(6.48) "toggle" switch in the inactive state is required for inverse agonist activity. The "HU-210" model does suggest a stabilization of the "toggle" switch, it is conceivable that the interaction could be occurring with the aromatic thiol of $\mathbf{2 1 4}$ is near enough to have potential to interact in a dynamic system (Figure 6.8). To fully test this from a functional standpoint, one would need to synthesize a derivative with 1,5-dimethoxy and various $\mathrm{C}-1^{\prime}$ heteroaromatic groups and potentially anoxic A-ring derivatives. Synthesis of these additional derivatives coupled with more robust modelling and molecular dynamics runs would help to further elucidate the root cause of this interesting functional switch.

\subsubsection{C-1' importance beyond binding affinity}

Previously, it was brought to light that M363(6.55) played a key role in increasing the binding affinity of classical core compounds, substituted with $\mathrm{C}$-1'-gem-dimethyls. In the case of the triaryl compounds wherein the $\mathrm{C}-1$ ' substituent is a ketone, the compound turns from a full agonist to an inverse agonist and in many cases an extremely potent one, at that. Looking at the model, it is evident that there exists a small pocket in the LBP in the vicinity of $\mathrm{C}-1$ ' which contains a good number of cysteine residues - namely C355(5.47), C382(7.38) and C386(7.42). Further, looking at superimposed, docked ligands 192 and 214 (Figure 6.9), which are identical except for their C-1' substituent and functional activity, one may see that the thiophene ring of 192 projects into this pocket while the $\mathrm{C}-\mathbf{1}^{\prime}$ ketone of $\mathbf{2 1 4}$ occupies this space. It is my hypothesis that the ketone's interaction with this cysteine pocket contributes to the inverse agonist activity of the compound through induction of a conformational change of the receptor. However, to fully validate, confirm and test this, more detailed binding studies, in conjunction with mutation and/or more detailed modelling and dynamic simulations would be necessary and may represent a potential direction to expand upon this current project. 


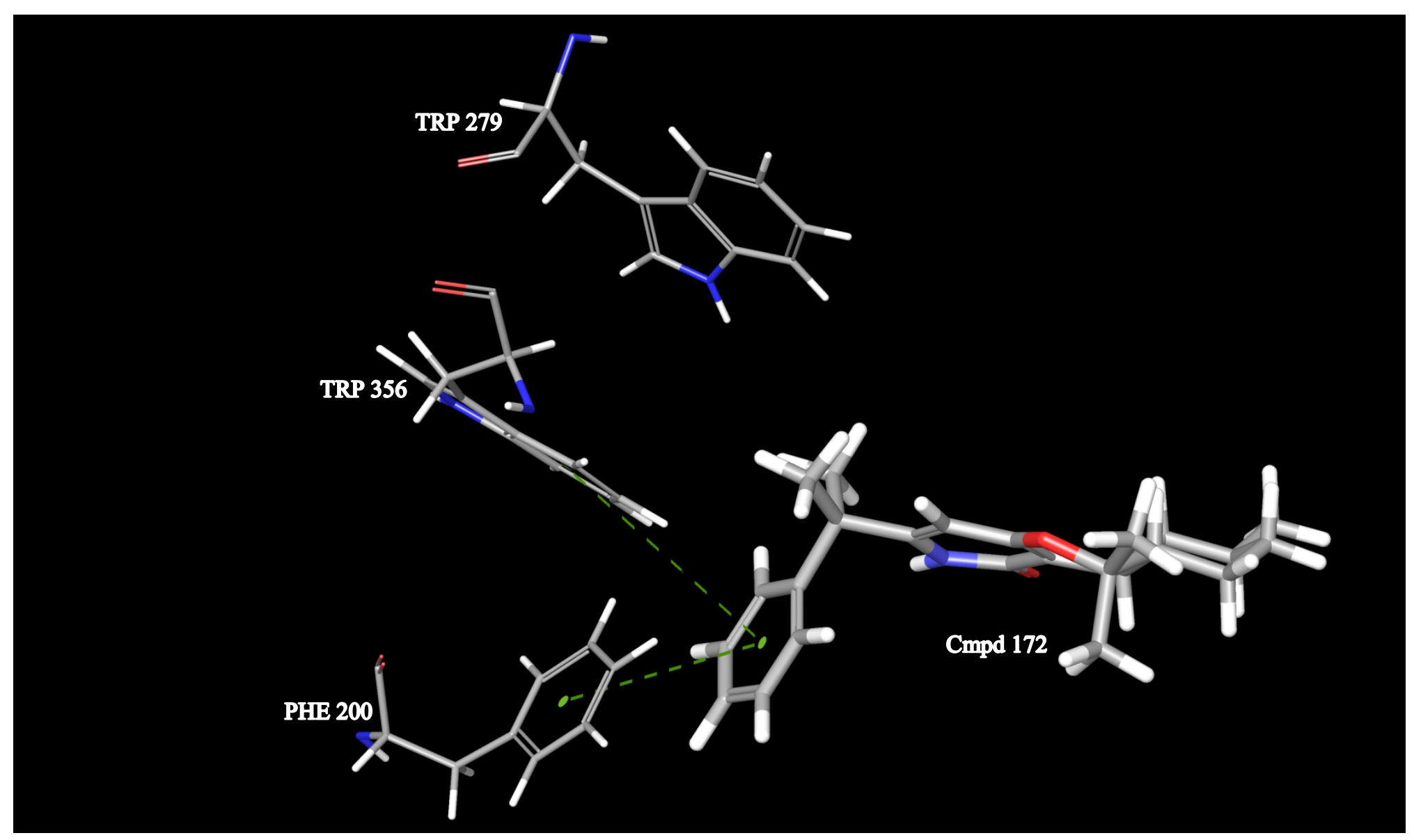

Figure 6.7. CB $\quad$ LBP W356(6.48) "toggle" switch presumed to be deactivated by compound 172

Note: Dashed green lines indicate $\pi-\pi$ stacking interactions. 


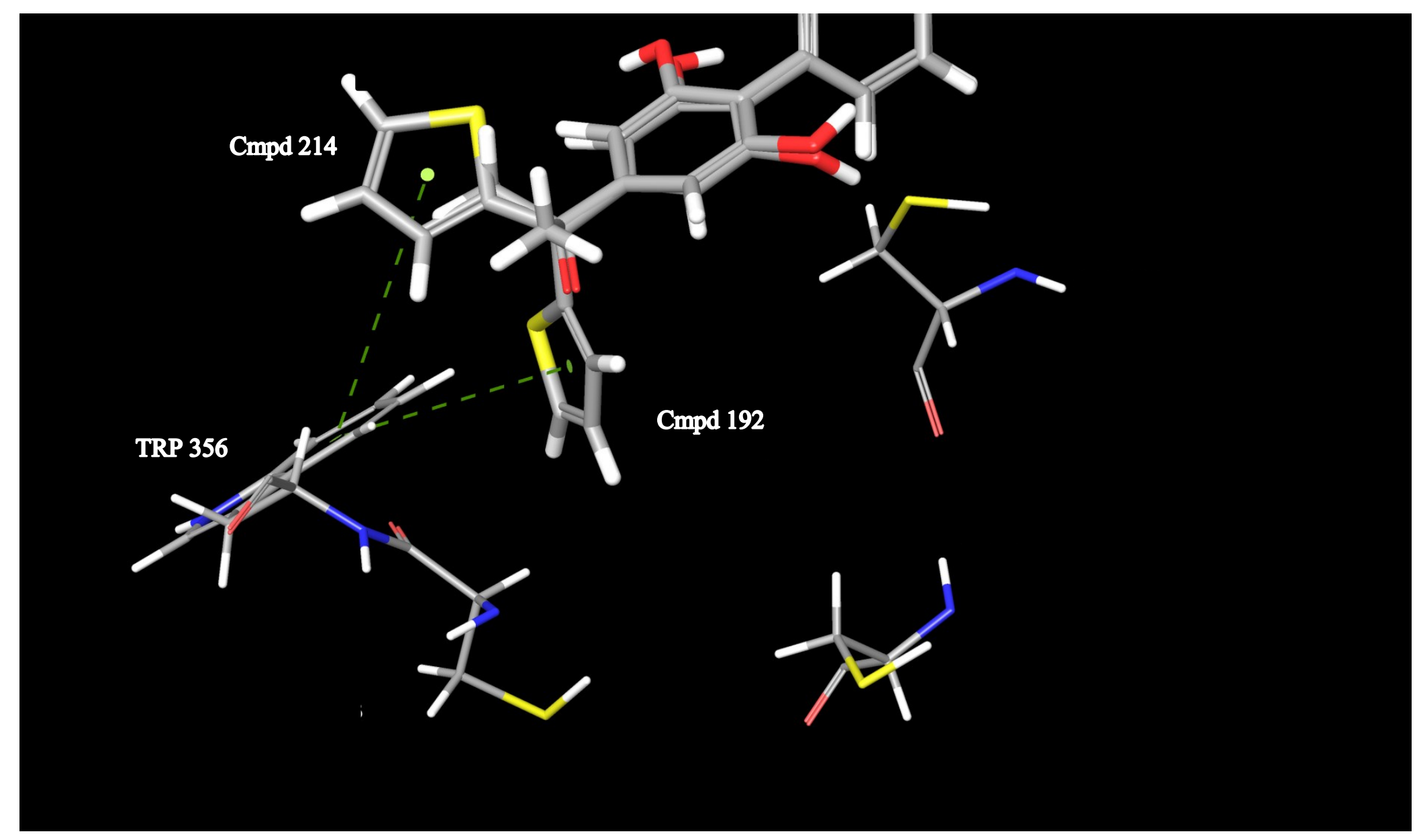

Figure 6.8. $\quad \mathrm{CB}_{1}$ interaction of 214 and 192 with the W356 "toggle" switch 

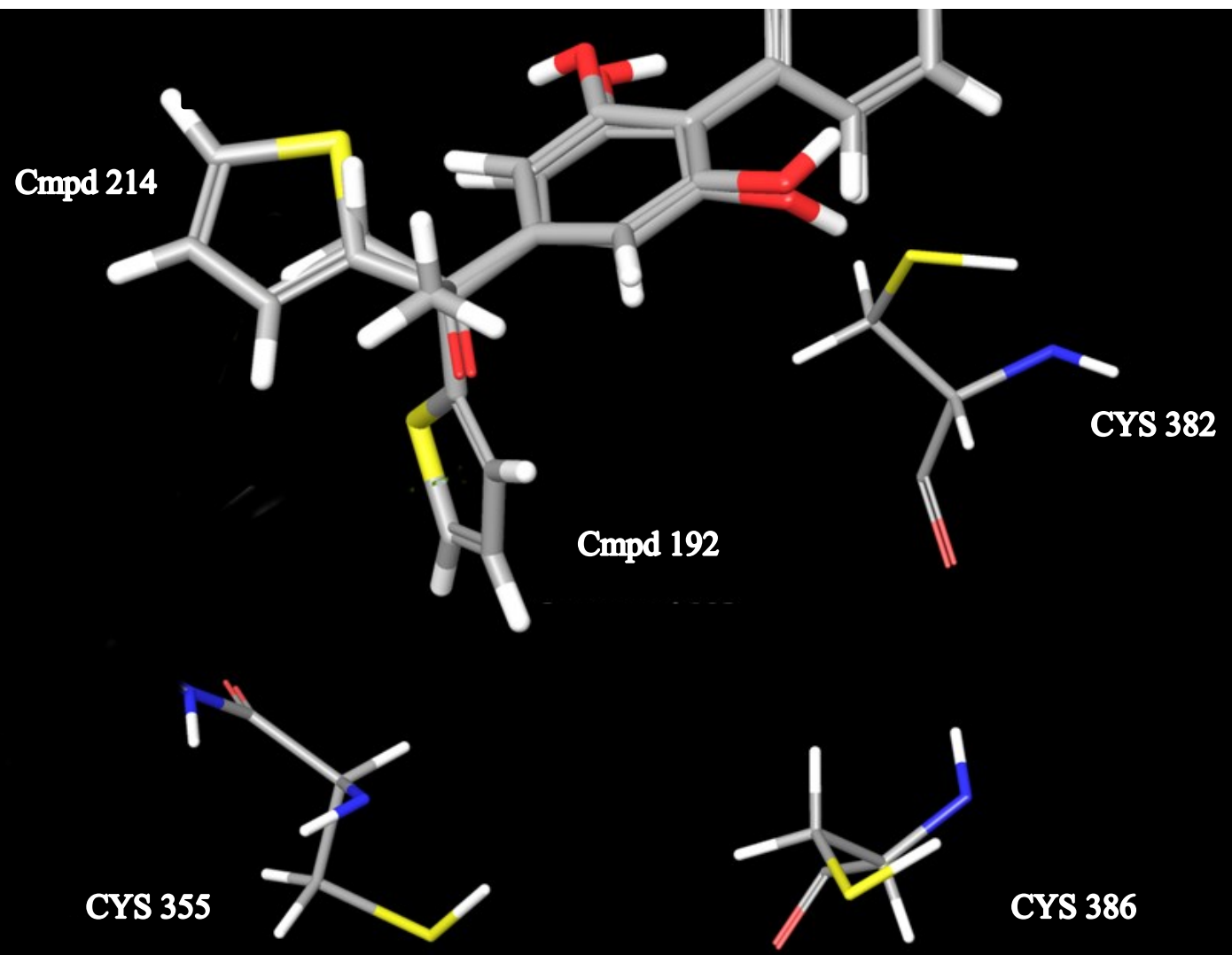

Figure 6.9. $\quad \mathrm{CB}_{1}$ LBP cysteine pocket 


\subsection{CB2-Specific Proposed Functional Switches}

\subsubsection{Common residue interactions}

Most of the common interactions currently well documented in $\mathrm{CB}_{2}$ modelling literature and precedent center around WIN-55,212-2. Residues F117(3.36), W194(5.43), F197(5.46) and W258(6.48) make up the bulk of the common aromatic interactions. Additionally, as was the case with $\mathrm{CB}_{1}$, a hydrophilic residue - usually S193(5.42), S292(7.46) or T114(3.33) - resides in the LBP and interacts with the ligand, if applicable. Currently, I am unable to utilize a validated homology model for $\mathrm{CB}_{2}$, unlike $\mathrm{CB}_{1}$. As a result, the key residues and/or binding sites - be they orthosteric or allosteric - cannot be given with sufficient certainty. However, there are certain moieties which seem to impart specific activity.

\subsubsection{The Di-hydroxy A-ring}

The first evidence of this requirement comes in the HB triaryl series. Compound 191, a mono-hydroxy A-ring, showed a very modest $10 \%$ increase in cAMP production relative to baseline. Compound 194, a mono-deprotected A-ring, showed an agonist activity, with an approximately $15 \%$ decrease in cAMP, but only at the upper end of the tested concentrations. Compound 193, the di-deprotected A-ring, analog of 194 demonstrated the same $15 \%$ decrease efficacy as 193; however, the potency of $194\left(\mathrm{EC}_{50}\right.$ $7.4 \mathrm{nM}$ ) greatly exceeded that of 194 (EC50 $494 \mathrm{nM}$ ) (Figure 6.10). Additionally, compound 192, another di-hydroxy A-ring analog, demonstrated an efficacy and potency nearly directly parallel to 194. Further foray into the necessity of this moiety being present appears in the SMM series. Compound 206, a mono-deprotected A-ring, and its successor 207, a di-hydroxy A-ring, demonstrate the same trend, with the efficacy being similar at high concentrations, but the potency of $207\left(\mathrm{EC}_{50} 89.7 \mathrm{nM}\right)$ greatly exceeds that of $206\left(\mathrm{EC}_{50}>5000 \mathrm{nM}\right)$. Lastly, the majority of the di-protected SMM triaryl series failed to elucidate any change in cAMP production in the $\mathrm{CB}_{2}$ assay - except for 199 and 213 at $5000 \mathrm{nM}$. Taken as a whole, the first requirement for optimal activity is the presence of a 1,3-dihydroxy A-ring.

Further, as alluded to during the introduction, one can see that making a modification to the A-ring composition has a relatively profound effect upon the functional activity of the compound. Take for example, 195, 227 and 233 with a carbocyclic, pyridine and pyrimidine A-ring, respectively - all have a 3,5-dichloro C-ring and a phenyl D-ring; however, 227 and 233 have a C-1'-gem-dimethyl and 195 has a C-1'-ketone (Figure 6.11). The difference in these compounds is quite profound, with 195 showing great $\mathrm{CB}_{2}$ inverse agonist activity, 227 showing appreciable activity at the highest tested concentration $(5000 \mathrm{nM})$ and $\mathbf{2 3 3}$ showing virtually no activity. This raises an interesting question: is this change in functional activity due to the heteroaromatic A-ring or the C-1'-gem-dimethyls? Further synthesis and expansion of this series is warranted to help gain some more definitive insight into the impact of a heteroaromatic A-ring and its help or hindrance of targeting agents within the cannabinoid system. 


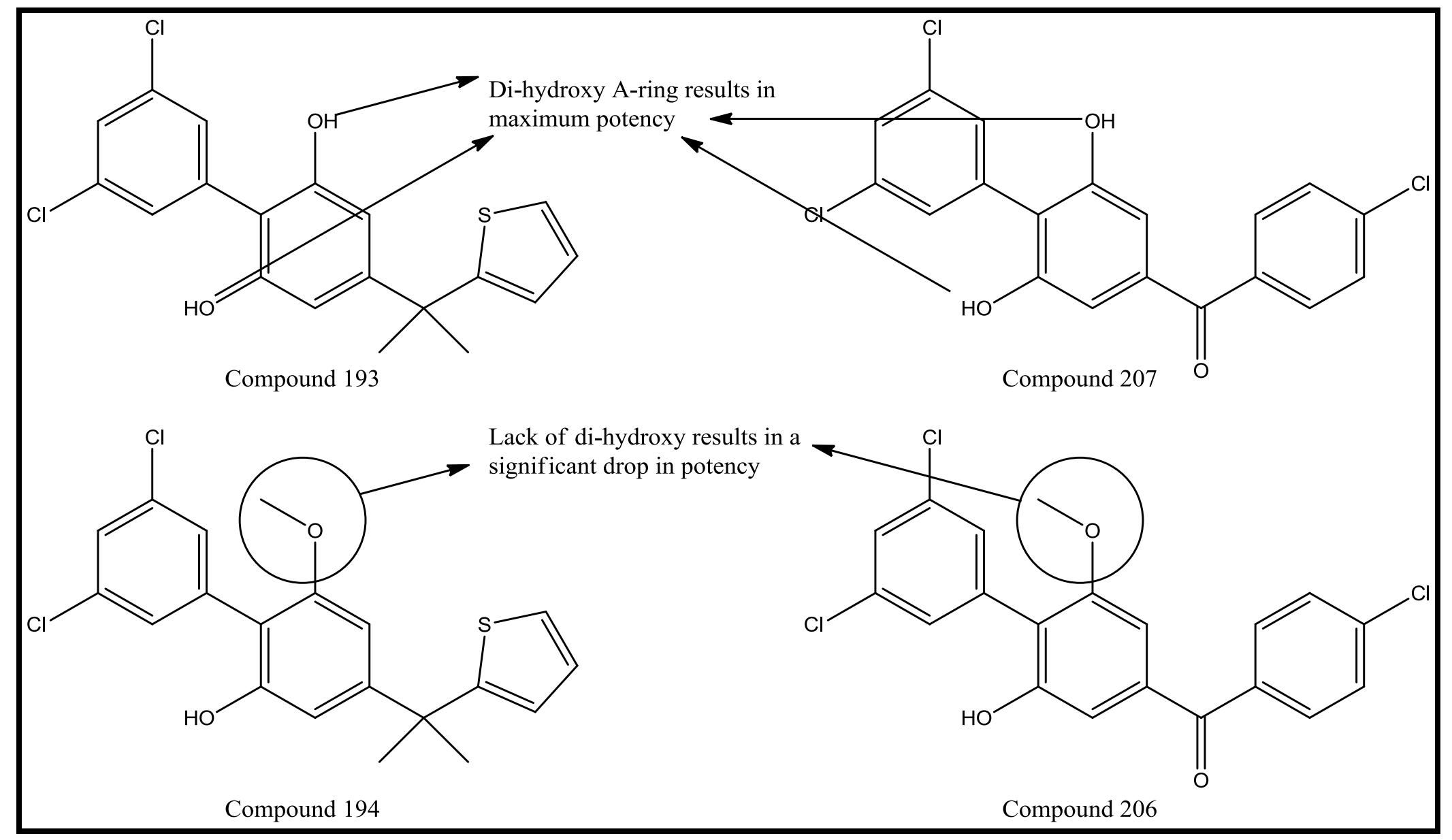

Figure 6.10. $\mathrm{CB}_{2}$ compounds demonstrating the requirement for di-hydroxy A-ring 


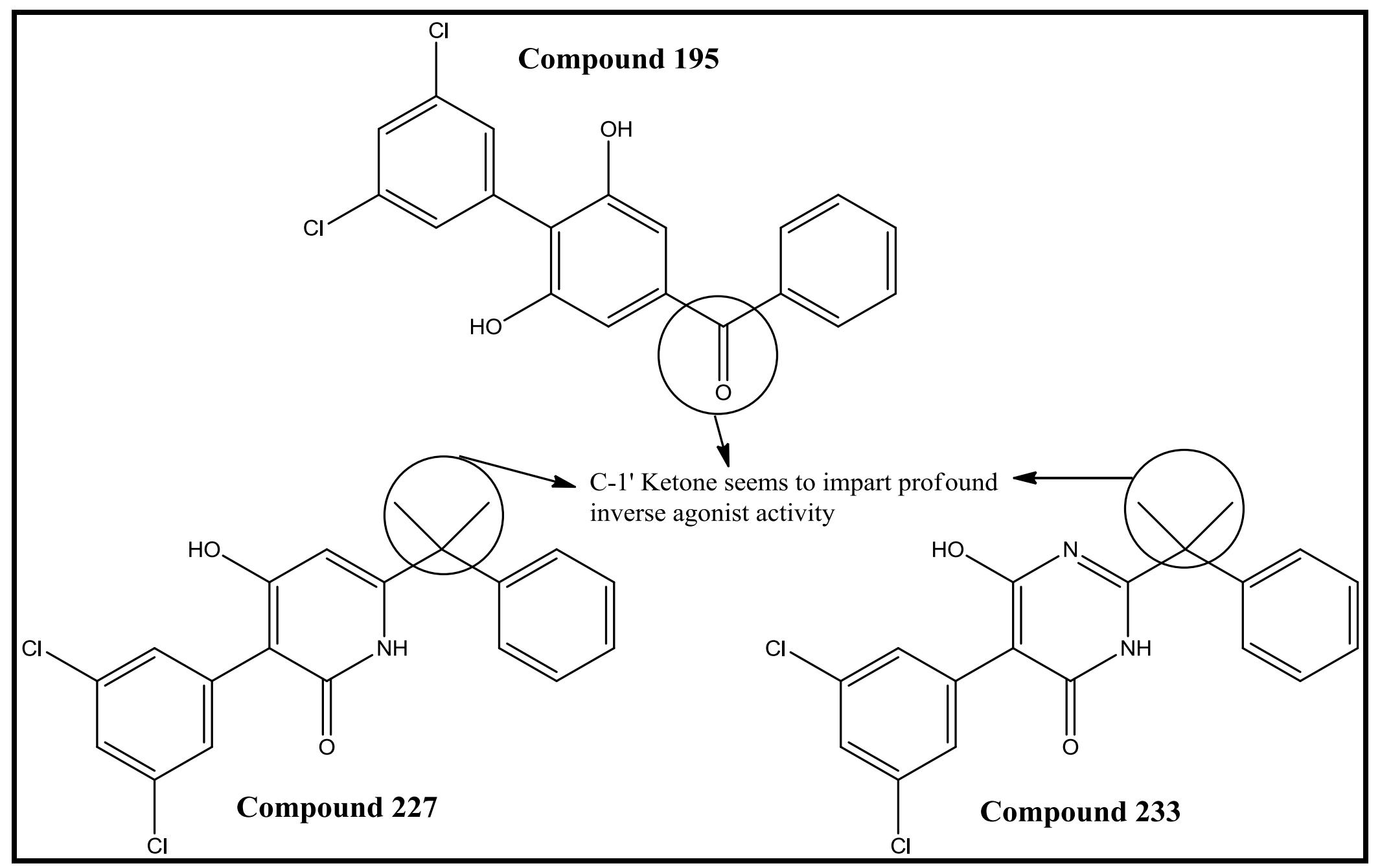

Figure 6.11. $\mathrm{CB}_{2} \mathrm{C}-\mathbf{1}^{\prime}$ substituents and functional changes 


\subsubsection{The C-1' substituent effect}

As was the case with $\mathrm{CB}_{1}, \mathrm{C}-1^{\prime}$ becomes extremely important when dealing with the functional switches and changes at $\mathrm{CB}_{2}$. Comparing 192 to 214, one can see that the only difference betwixt the two is a ketone vs. C-1'-gem-dimethyl substitution (Figure 6.12). The functional data shows that a change from C-1'-gem-dimethyl to ketone results in a compound switching from an agonist to a profoundly effective inverse agonist, respectively. Further, a ketone seems to be necessary, as a C-1'-hydroxy results in no effect when all else is equal and compared to a $\mathrm{C}-1^{\prime}-\mathrm{ketone}$. Though it is not clear if this effect is due to the aforementioned di-protected A-ring or the hydroxyl itself - as the current library of compounds only has concomitant expression of the C-1'-hydroxyl and di-protected A-ring. One question that does arise from this study and data is whether testing of the $\mathrm{C}-1$ '-ketone moiety in the known $\mathrm{CB}_{2}$-binding $\mathrm{KM}$ series compounds would result in a similar or more potent/effective inverse agonist compound at $\mathrm{CB}_{2}$.

\subsubsection{The D-ring substituent effect}

The last component of the functional SAR garnered from this study is the D-ring effect. From this study, it is evident that incorporation of the previous two elements - 1,5dihydroxy A-ring and C-1'-ketone - requires, too, the presence of an aromatic D-ring for maximum potency and efficacy. Example of this comes in the form of the cycloalkyl derivative of 209 as compared to its aromatic counterpart of 195 (Figure 6.13). Compound 209 exerts an inverse agonist effect (111\% increase over baseline) at the maximum concentration tested $(5000 \mathrm{nM})$, as compared to the increase in both potency (744 $\mathrm{nM})$ and efficacy (215\% increase over baseline) for compound 195.

\subsection{Summary}

Overall, as this data shows that very small changes in the structure of a compound can have significant impact upon its activity or affinity for $\mathrm{CB}_{1}$ and/or $\mathrm{CB}_{2}$. To understand this causal relationship with more certainty, a few key advances need to occur:

- A reliably predictable model (or crystal structure) for both the $\mathrm{CB}_{1}$ and $\mathrm{CB}_{2}$ receptor needs to be created and validated against a bevy of current literature standard cannabinoid compounds.

- For the compounds presented as products of this lab, matched sets, incorporating as similar as possible D-rings, $\mathrm{C}$-1' substituents, C-rings and A-ring oxidation, across all the series need to be synthesized and tested - in doing so, it will allow more definite trends to emerge.

- A refinement of the functional assay to test for and qualify antagonists - not only from a potency standpoint, but also from a binding site standpoint, as some of these switches may be due to an all-together unique binding site.

- A validation and assimilation of the data generated from this assay to an in vivo model of specific desease states. 


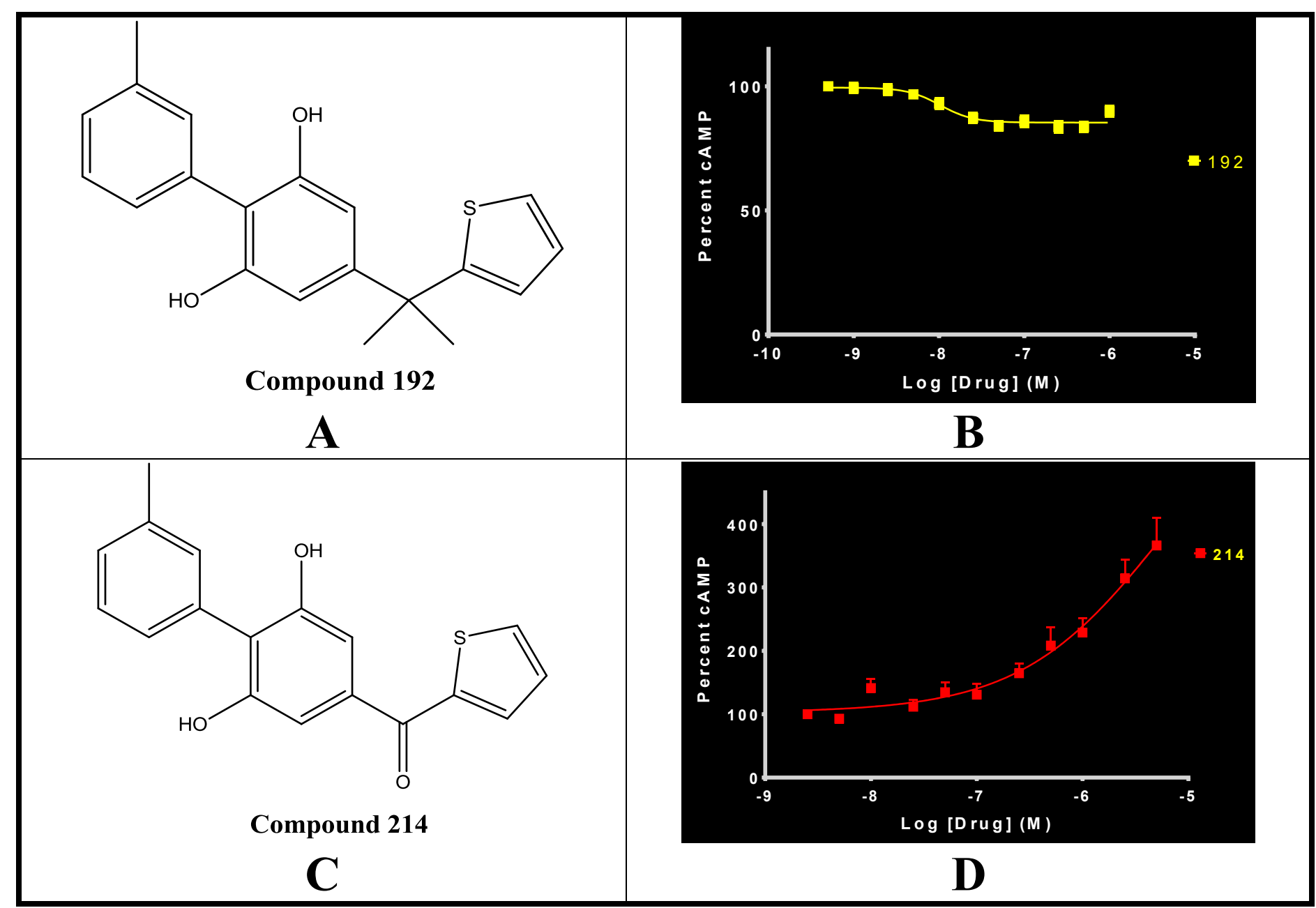

Figure 6.12. $\mathrm{CB}_{2} \mathrm{C}-\mathbf{1}^{\prime}$ substituent effects - switch from agonist to inverse agonist 


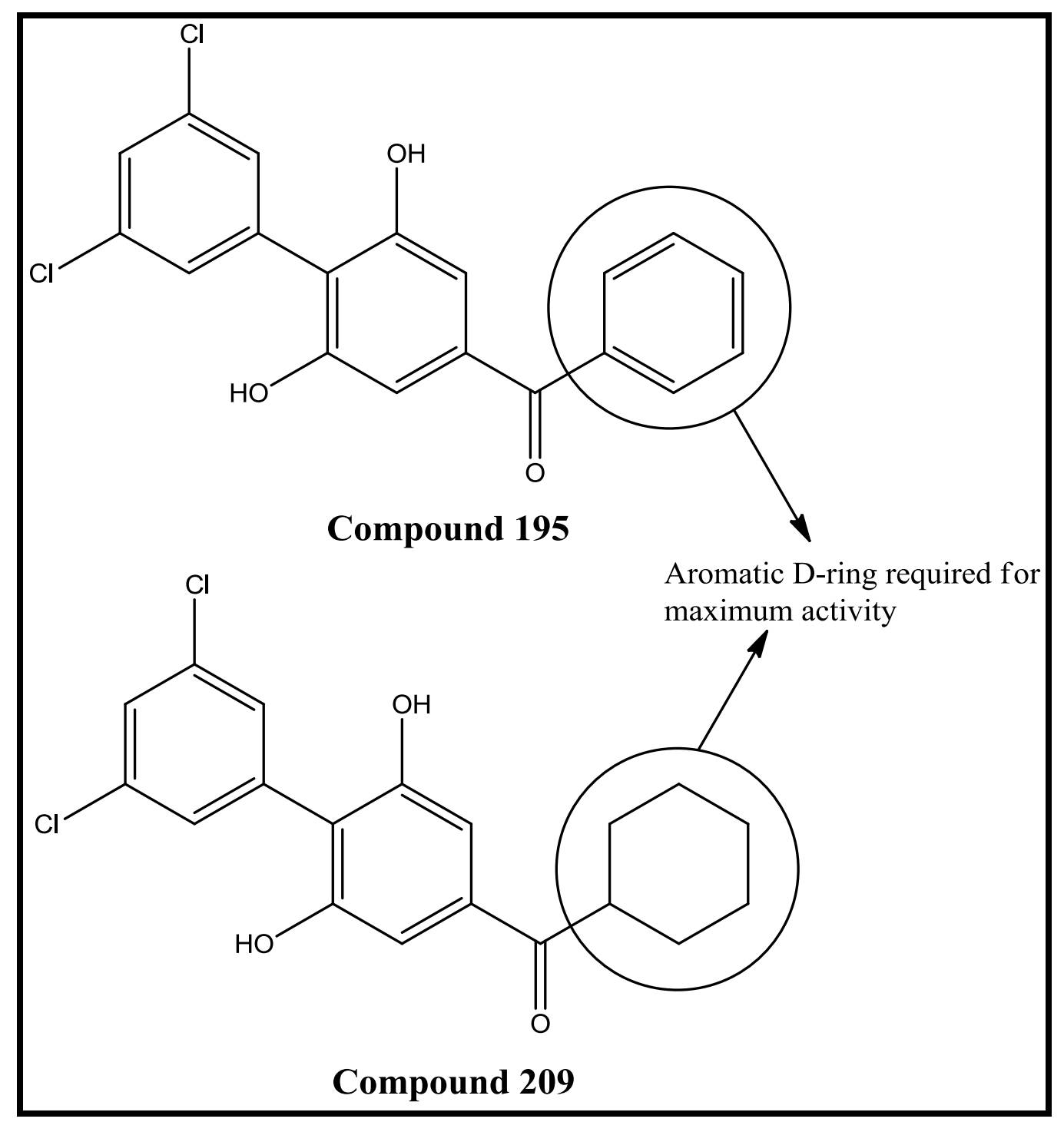

Figure 6.13. $\quad \mathrm{CB}_{2}$ aromatic D-ring requirement 
In summation, the field and this project has come leaps and bounds from where it originated not too long ago. It is an exciting time in the field of cannabinoid research, and emergence of better homology or crystal structure models coupled with expansion and refinement of this data will help to carry the field further. Combination of these approaches will help to ensure that cannabinoid research becomes more viable and targeted to usher it into its rightful place in therapy and treatment of various disease states. 


\section{LIST OF REFERENCES}

1. Mikuriya, T. H. Marijuana in medicine: past, present and future. California medicine 110, 34-40 (1969).

2. Mechoulam, R. in Milestones in Drug Therapy MDT (Birkhäuser Verlag,, Basel, 2005).

3. Mechoulam, R., Hanus, L. \& Fride, E. Towards cannabinoid drugs--revisited. Progress in medicinal chemistry 35, 199-243 (1998).

4. Walker, J. M. et al. The neurobiology of cannabinoid analgesia. Life Sci 65, 665673 (1999).

5. Kumar, R. N., Chambers, W. A. \& Pertwee, R. G. Pharmacological actions and therapeutic uses of cannabis and cannabinoids. Anaesthesia 56, 1059-1068 (2001).

6. Izzo, A. A. \& Coutts, A. A. Cannabinoids and the digestive tract. Handbook of experimental pharmacology, 573-598 (2005).

7. Croxford, J. L. \& Yamamura, T. Cannabinoids and the immune system: potential for the treatment of inflammatory diseases? Journal of neuroimmunology 166, 318, doi:10.1016/j.jneuroim.2005.04.023 (2005).

8. Wood, T. B., Spivey, W. T. N. \& Easterfield, T. H. XL.-Charas. The resin of Indian hemp. Journal of the Chemical Society, Transactions 69, 539-546, doi:10.1039/CT8966900539 (1896).

9. Cahn, R. S. 326. Cannabis indica resin. Part IV. The synthesis of some $2: 2-$ dimethyldibenzopyrans, and confirmation of the structure of cannabinol. Journal of the Chemical Society (Resumed), 1400-1405, doi:10.1039/JR9330001400 (1933).

10. Adams, R., Baker, B. R. \& Wearn, R. B. Structure of Cannabinol. III. Synthesis of Cannabinol, 1-Hydroxy-3-n-amyl-6,6,9-trimethyl-6-dibenzopyran1. JACS 62, 2204-2207, doi:10.1021/ja01865a083 (1940).

11. Adams, R., Harfenist, M. \& Loewe, S. New Analogs of Tetrahydrocannabinol. XIX. JACS 71, 1624-1628, doi:10.1021/ja01173a023 (1949).

12. Todd, A. R. Hashish. Experientia 2, 55-60, doi:10.1007/BF02163886 (1946).

13. Gaoni, Y. \& Mechoulam, R. Isolation, Structure, and Partial Synthesis of an Active Constituent of Hashish. JACS 86, 1646-1647, doi:10.1021/ja01062a046 (1964).

14. Howlett, A. C. et al. International Union of Pharmacology. XXVII. Classification of cannabinoid receptors. Pharmacological reviews 54, 161-202 (2002).

15. Mechoulam, R., Shani, A., Edery, H. \& Grunfeld, Y. Chemical Basis of Hashish Activity. Science 169, 611-612, doi:10.1126/science.169.3945.611 (1970).

16. Grunfeld, Y. \& Edery, H. Psychopharmacological activity of the active constituents of hashish and some related cannabinoids. Psychopharmacologia 14, 200-210, doi:10.1007/BF00404218 (1969).

17. Paton, W. D. Pharmacology of marijuana. Annual review of pharmacology 15, 191-220, doi:10.1146/annurev.pa.15.040175.001203 (1975).

18. Mechoulam, R. et al. Enantiomeric cannabinoids: stereospecificity of psychotropic activity. Experientia 44, $762-764$ (1988). 
19. Devane, W. A., Dysarz, F. A., 3rd, Johnson, M. R., Melvin, L. S. \& Howlett, A. C. Determination and characterization of a cannabinoid receptor in rat brain. Molecular pharmacology 34, 605-613 (1988).

20. Matsuda, L. A., Lolait, S. J., Brownstein, M. J., Young, A. C. \& Bonner, T. I. Structure of a cannabinoid receptor and functional expression of the cloned cDNA. Nature 346, 561-564, doi:10.1038/346561a0 (1990).

21. Munro, S., Thomas, K. L. \& Abu-Shaar, M. Molecular characterization of a peripheral receptor for cannabinoids. Nature 365, 61-65, doi:10.1038/365061a0 (1993).

22. Shire, D. et al. An amino-terminal variant of the central cannabinoid receptor resulting from alternative splicing. The Journal of biological chemistry 270, 37263731 (1995).

23. Griffin, G., Tao, Q. \& Abood, M. E. Cloning and pharmacological characterization of the rat $\mathrm{CB}(2)$ cannabinoid receptor. The Journal of pharmacology and experimental therapeutics 292, 886-894 (2000).

24. Welch, S. P. \& Eads, M. Synergistic interactions of endogenous opioids and cannabinoid systems. Brain research 848, 183-190 (1999).

25. Di Marzo, V. et al. Levels, metabolism, and pharmacological activity of anandamide in $\mathrm{CB}(1)$ cannabinoid receptor knockout mice: evidence for non$\mathrm{CB}(1)$, non- $\mathrm{CB}(2)$ receptor-mediated actions of anandamide in mouse brain. Journal of neurochemistry 75, 2434-2444 (2000).

26. Zimmer, A., Zimmer, A. M., Hohmann, A. G., Herkenham, M. \& Bonner, T. I. Increased mortality, hypoactivity, and hypoalgesia in cannabinoid CB1 receptor knockout mice. Proceedings of the National Academy of Sciences of the United States of America 96, 5780-5785 (1999).

27. Lauckner, J. E. et al. GPR55 is a cannabinoid receptor that increases intracellular calcium and inhibits M current. Proceedings of the National Academy of Sciences of the United States of America 105, 2699-2704, doi:10.1073/pnas.0711278105 (2008).

28. Pertwee, R. G. GPR55: a new member of the cannabinoid receptor clan? $\mathrm{Br} J$ Pharmacol 152, 984-986, doi:10.1038/sj.bjp.0707464 (2007).

29. Ryberg, E. et al. The orphan receptor GPR55 is a novel cannabinoid receptor. $\mathrm{Br}$ J Pharmacol 152, 1092-1101, doi:10.1038/sj.bjp.0707460 (2007).

30. Zygmunt, P. M. et al. Vanilloid receptors on sensory nerves mediate the vasodilator action of anandamide. Nature 400, 452-457, doi:10.1038/22761 (1999).

31. Smart, D. et al. The endogenous lipid anandamide is a full agonist at the human vanilloid receptor (hVR1). Br J Pharmacol 129, 227-230, doi:10.1038/sj.bjp.0703050 (2000).

32. Smart, D. \& Jerman, J. C. Anandamide: an endogenous activator of the vanilloid receptor. Trends in pharmacological sciences 21, 134 (2000).

33. Prather, P. L., Martin, N. A., Breivogel, C. S. \& Childers, S. R. Activation of cannabinoid receptors in rat brain by WIN 55212-2 produces coupling to multiple $\mathrm{G}$ protein alpha-subunits with different potencies. Molecular pharmacology $\mathbf{5 7}$, 1000-1010 (2000). 
34. Howlett, A. C., Song, C., Berglund, B. A., Wilken, G. H. \& Pigg, J. J.

Characterization of CB1 cannabinoid receptors using receptor peptide fragments and site-directed antibodies. Molecular pharmacology 53, 504-510 (1998).

35. Pertwee, R. G. The pharmacology of cannabinoid receptors and their ligands: an overview. International journal of obesity 30 Suppl 1, S13-18, doi:10.1038/sj.ijo.0803272 (2006).

36. Bramblett, R. D., Panu, A. M., Ballesteros, J. A. \& Reggio, P. H. Construction of a 3D model of the cannabinoid CB1 receptor: determination of helix ends and helix orientation. Life Sci 56, 1971-1982 (1995).

37. Reggio, P. H. \& SpringerLink (Online service). in The Receptors (Humana Press,, Totowa, NJ, 2009).

38. Pertwee, R. G. Pharmacology of cannabinoid CB1 and CB2 receptors. Pharmacology \& therapeutics 74, 129-180 (1997).

39. Mackie, K. \& Hille, B. Cannabinoids inhibit N-type calcium channels in neuroblastoma-glioma cells. Proceedings of the National Academy of Sciences of the United States of America 89, 3825-3829 (1992).

40. Bouaboula, M. et al. A selective inverse agonist for central cannabinoid receptor inhibits mitogen-activated protein kinase activation stimulated by insulin or insulin-like growth factor 1. Evidence for a new model of receptor/ligand interactions. The Journal of biological chemistry 272, 22330-22339 (1997).

41. Bouaboula, M. et al. Activation of mitogen-activated protein kinases by stimulation of the central cannabinoid receptor CB1. The Biochemical journal 312 ( Pt 2), 637-641 (1995).

42. Sanchez, C., Galve-Roperh, I., Rueda, D. \& Guzman, M. Involvement of sphingomyelin hydrolysis and the mitogen-activated protein kinase cascade in the Delta9-tetrahydrocannabinol-induced stimulation of glucose metabolism in primary astrocytes. Molecular pharmacology 54, 834-843 (1998).

43. Liu, J. et al. Functional CB1 cannabinoid receptors in human vascular endothelial cells. The Biochemical journal 346 Pt 3, 835-840 (2000).

44. Rueda, D., Galve-Roperh, I., Haro, A. \& Guzman, M. The CB(1) cannabinoid receptor is coupled to the activation of c-Jun N-terminal kinase. Molecular pharmacology 58, 814-820 (2000).

45. Galve-Roperh, I. et al. Anti-tumoral action of cannabinoids: involvement of sustained ceramide accumulation and extracellular signal-regulated kinase activation. Nature medicine 6, 313-319, doi:10.1038/73171 (2000).

46. Felder, C. C. et al. Comparison of the pharmacology and signal transduction of the human cannabinoid CB1 and CB2 receptors. Molecular pharmacology 48, 443-450 (1995).

47. Bouaboula, M., Bianchini, L., McKenzie, F. R., Pouyssegur, J. \& Casellas, P. Cannabinoid receptor $\mathrm{CB} 1$ activates the $\mathrm{Na}+\mathrm{H}+$ exchanger NHE-1 isoform via Gi-mediated mitogen activated protein kinase signaling transduction pathways. FEBS letters 449, 61-65 (1999).

48. Wartmann, M., Campbell, D., Subramanian, A., Burstein, S. H. \& Davis, R. J. The MAP kinase signal transduction pathway is activated by the endogenous cannabinoid anandamide. FEBS letters 359, 133-136 (1995). 
49. Sanchez, C. et al. The $\mathrm{CB}(1)$ cannabinoid receptor of astrocytes is coupled to sphingomyelin hydrolysis through the adaptor protein fan. Molecular pharmacology 59, 955-959 (2001).

50. Blazquez, C., Sanchez, C., Daza, A., Galve-Roperh, I. \& Guzman, M. The stimulation of ketogenesis by cannabinoids in cultured astrocytes defines carnitine palmitoyltransferase I as a new ceramide-activated enzyme. Journal of neurochemistry 72, 1759-1768 (1999).

51. Guzman, M., Galve-Roperh, I. \& Sanchez, C. Ceramide: a new second messenger of cannabinoid action. Trends in pharmacological sciences 22, 19-22 (2001).

52. Pettit, D. A., Anders, D. L., Harrison, M. P. \& Cabral, G. A. Cannabinoid receptor expression in immune cells. Advances in experimental medicine and biology 402, 119-129 (1996).

53. Schatz, A. R., Lee, M., Condie, R. B., Pulaski, J. T. \& Kaminski, N. E. Cannabinoid receptors $\mathrm{CB} 1$ and $\mathrm{CB} 2$ : a characterization of expression and adenylate cyclase modulation within the immune system. Toxicology and applied pharmacology 142, 278-287, doi:10.1006/taap.1996.8034 (1997).

54. Cichero, E. et al. Homology modeling in tandem with 3D-QSAR analyses: A computational approach to depict the agonist binding site of the human CB2 receptor. European Journal of Medicinal Chemistry 46, 4489-4505, doi:10.1016/j.ejmech.2011.07.023 (2011).

55. Griffin, G. et al. Evaluation of the cannabinoid CB2 receptor-selective antagonist, SR144528: further evidence for cannabinoid CB2 receptor absence in the rat central nervous system. Eur J Pharmacol 377, 117-125 (1999).

56. Ashton, J. C., Friberg, D., Darlington, C. L. \& Smith, P. F. Expression of the cannabinoid CB2 receptor in the rat cerebellum: an immunohistochemical study. Neurosci Lett 396, 113-116, doi:10.1016/j.neulet.2005.11.038 (2006).

57. Gong, J. P. et al. Cannabinoid CB2 receptors: immunohistochemical localization in rat brain. Brain research 1071, 10-23, doi:10.1016/j.brainres.2005.11.035 (2006).

58. Van Sickle, M. D. et al. Identification and functional characterization of brainstem cannabinoid CB2 receptors. Science 310, 329-332, doi:10.1126/science.1115740 (2005).

59. Galiegue, S. et al. Expression of central and peripheral cannabinoid receptors in human immune tissues and leukocyte subpopulations. European journal of biochemistry / FEBS 232, 54-61 (1995).

60. Hegde, V. L., Nagarkatti, M. \& Nagarkatti, P. S. Cannabinoid receptor activation leads to massive mobilization of myeloid-derived suppressor cells with potent immunosuppressive properties. European Journal of Immunology 40, 3358-3371, doi:10.1002/eji.201040667 (2010).

61. Marchese, A. et al. Discovery of three novel orphan G-protein-coupled receptors. Genomics 56, 12-21, doi:10.1006/geno.1998.5655 (1999).

62. Sawzdargo, M. et al. Identification and cloning of three novel human G proteincoupled receptor genes GPR52, PsiGPR53 and GPR55: GPR55 is extensively expressed in human brain. Brain research. Molecular brain research 64, 193-198 (1999). 
63. Henstridge, C. M. et al. The GPR55 ligand L-alpha-lysophosphatidylinositol promotes RhoA-dependent $\mathrm{Ca} 2+$ signaling and NFAT activation. FASEB journal : official publication of the Federation of American Societies for Experimental Biology 23, 183-193, doi:10.1096/fj.08-108670 (2009).

64. Oka, S., Nakajima, K., Yamashita, A., Kishimoto, S. \& Sugiura, T. Identification of GPR55 as a lysophosphatidylinositol receptor. Biochemical and biophysical research communications 362, 928-934, doi:10.1016/j.bbrc.2007.08.078 (2007).

65. Pertwee, R. G. et al. International Union of Basic and Clinical Pharmacology. LXXIX. Cannabinoid Receptors and Their Ligands: Beyond CB1 and CB2. Pharmacological reviews 62, 588-631, doi:10.1124/pr.110.003004 (2010).

66. Kress, M. \& Kuner, R. Mode of action of cannabinoids on nociceptive nerve endings. Experimental brain research. Experimentelle Hirnforschung. Experimentation cerebrale 196, 79-88, doi:10.1007/s00221-009-1762-0 (2009).

67. Pietr, M. et al. Differential changes in GPR55 during microglial cell activation. FEBS letters 583, 2071-2076, doi:10.1016/j.febslet.2009.05.028 (2009).

68. Ross, R. A. The enigmatic pharmacology of GPR55. Trends in pharmacological sciences 30, 156-163, doi:10.1016/j.tips.2008.12.004 (2009).

69. Staton, P. C. et al. The putative cannabinoid receptor GPR55 plays a role in mechanical hyperalgesia associated with inflammatory and neuropathic pain. Pain 139, 225-236, doi:10.1016/j.pain.2008.04.006 (2008).

70. Ridley, A. J. Rho GTPases and actin dynamics in membrane protrusions and vesicle trafficking. Trends in cell biology 16, 522-529, doi:10.1016/j.tcb.2006.08.006 (2006).

71. Johns, D. G. et al. The novel endocannabinoid receptor GPR55 is activated by atypical cannabinoids but does not mediate their vasodilator effects. $\mathrm{Br} J$ Pharmacol 152, 825-831, doi:10.1038/sj.bjp.0707419 (2007).

72. Whyte, L. S. et al. The putative cannabinoid receptor GPR55 affects osteoclast function in vitro and bone mass in vivo. Proceedings of the National Academy of Sciences of the United States of America 106, 16511-16516, doi:10.1073/pnas.0902743106 (2009).

73. Hillard, C. J. Role of cannabinoids and endocannabinoids in cerebral ischemia. Current pharmaceutical design 14, 2347-2361 (2008).

74. Libby, P. Inflammation in atherosclerosis. Nature 420, 868-874, doi:10.1038/nature01323 (2002).

75. Libby, P., Ridker, P. M. \& Maseri, A. Inflammation and atherosclerosis. Circulation 105, 1135-1143 (2002).

76. Mendizabal, V. E. \& Adler-Graschinsky, E. Cannabinoids as therapeutic agents in cardiovascular disease: a tale of passions and illusions. Br J Pharmacol 151, 427440, doi:10.1038/sj.bjp.0707261 (2007).

77. Randall, M. D., Harris, D., Kendall, D. A. \& Ralevic, V. Cardiovascular effects of cannabinoids. Pharmacology \& therapeutics 95, 191-202 (2002).

78. Varga, K., Lake, K., Martin, B. R. \& Kunos, G. Novel antagonist implicates the CB1 cannabinoid receptor in the hypotensive action of anandamide. Eur $J$ Pharmacol 278, 279-283 (1995).

79. Gulati, A. \& Srimal, R. C. Endothelin antagonizes the hypotension and potentiates the hypertension induced by clonidine. Eur J Pharmacol 230, 293-300 (1993). 
80. Lake, K. D., Compton, D. R., Varga, K., Martin, B. R. \& Kunos, G. Cannabinoidinduced hypotension and bradycardia in rats mediated by CB1-like cannabinoid receptors. The Journal of pharmacology and experimental therapeutics $\mathbf{2 8 1}$, 1030-1037 (1997).

81. Vidrio, H., Sanchez-Salvatori, M. A. \& Medina, M. Cardiovascular effects of (-)11-OH-delta 8-tetrahydrocannabinol-dimethylheptyl in rats. Journal of cardiovascular pharmacology 28, 332-336 (1996).

82. Pacher, P., Batkai, S. \& Kunos, G. Haemodynamic profile and responsiveness to anandamide of TRPV1 receptor knock-out mice. The Journal of physiology 558, 647-657, doi:10.1113/jphysiol.2004.064824 (2004).

83. Batkai, S., Pacher, P., Jarai, Z., Wagner, J. A. \& Kunos, G. Cannabinoid antagonist SR-141716 inhibits endotoxic hypotension by a cardiac mechanism not involving CB1 or CB2 receptors. American journal of physiology. Heart and circulatory physiology 287, H595-600, doi:10.1152/ajpheart.00184.2004 (2004).

84. Benowitz, N. L. \& Jones, R. T. Cardiovascular effects of prolonged delta-9tetrahydrocannabinol ingestion. Clinical pharmacology and therapeutics 18, 287297 (1975).

85. Benowitz, N. L., Rosenberg, J., Rogers, W., Bachman, J. \& Jones, R. T. Cardiovascular effects of intravenous delta-9-tetrahydrocannabinol: autonomic nervous mechanisms. Clinical pharmacology and therapeutics 25, 440-446 (1979).

86. Jones, R. T. Cardiovascular system effects of marijuana. Journal of clinical pharmacology 42, 58S-63S (2002).

87. Fredericks, A. B., Benowitz, N. L. \& Savanapridi, C. Y. The cardiovascular and autonomic effects of repeated administration of delta-9-tetrahydrocannabinol to rhesus monkeys. The Journal of pharmacology and experimental therapeutics 216, 247-253 (1981).

88. Ledent, C. et al. Unresponsiveness to cannabinoids and reduced addictive effects of opiates in CB1 receptor knockout mice. Science 283, 401-404 (1999).

89. Pacher, P. et al. Hemodynamic profile, responsiveness to anandamide, and baroreflex sensitivity of mice lacking fatty acid amide hydrolase. American journal of physiology. Heart and circulatory physiology 289, H533-541, doi:10.1152/ajpheart.00107.2005 (2005).

90. Pacher, P., Batkai, S. \& Kunos, G. The endocannabinoid system as an emerging target of pharmacotherapy. Pharmacological reviews 58, 389-462, doi:10.1124/pr.58.3.2 (2006).

91. Ford, W. R., Honan, S. A., White, R. \& Hiley, C. R. Evidence of a novel site mediating anandamide-induced negative inotropic and coronary vasodilatator responses in rat isolated hearts. Br J Pharmacol 135, 1191-1198, doi:10.1038/sj.bjp.0704565 (2002).

92. Bonz, A. et al. Cannabinoids acting on CB1 receptors decrease contractile performance in human atrial muscle. Journal of cardiovascular pharmacology $\mathbf{4 1}$, 657-664 (2003).

93. Wagner, J. A., Jarai, Z., Batkai, S. \& Kunos, G. Hemodynamic effects of cannabinoids: coronary and cerebral vasodilation mediated by cannabinoid $\mathrm{CB}(1)$ receptors. Eur J Pharmacol 423, 203-210 (2001). 
94. Kunos, G. et al. Endocannabinoids as cardiovascular modulators. Chemistry and physics of lipids 108, 159-168 (2000).

95. Hogestatt, E. D. \& Zygmunt, P. M. Cardiovascular pharmacology of anandamide. Prostaglandins, leukotrienes, and essential fatty acids 66, 343-351, doi:10.1054/plef.2001.0346 (2002).

96. O'Sullivan, S. E., Kendall, D. A. \& Randall, M. D. Characterisation of the vasorelaxant properties of the novel endocannabinoid N-arachidonoyl-dopamine (NADA). Br J Pharmacol 141, 803-812, doi:10.1038/sj.bjp.0705643 (2004).

97. Mukhopadhyay, S., Chapnick, B. M. \& Howlett, A. C. Anandamide-induced vasorelaxation in rabbit aortic rings has two components: $\mathrm{G}$ protein dependent and independent. American journal of physiology. Heart and circulatory physiology 282, H2046-2054, doi:10.1152/ajpheart.00497.2001 (2002).

98. Randall, M. D. et al. An endogenous cannabinoid as an endothelium-derived vasorelaxant. Biochemical and biophysical research communications 229, 114120, doi:10.1006/bbrc.1996.1766 (1996).

99. Harris, D., McCulloch, A. I., Kendall, D. A. \& Randall, M. D. Characterization of vasorelaxant responses to anandamide in the rat mesenteric arterial bed. The Journal of physiology 539, 893-902 (2002).

100. Peroni, R. N. et al. Participation of CGRP and prostanoids in the sex-linked differences of vascular anandamide effects in mesenteric beds of Sprague-Dawley rats. Eur J Pharmacol 557, 49-57, doi:10.1016/j.ejphar.2006.11.012 (2007).

101. Underdown, N. J., Hiley, C. R. \& Ford, W. R. Anandamide reduces infarct size in rat isolated hearts subjected to ischaemia-reperfusion by a novel cannabinoid mechanism. Br J Pharmacol 146, 809-816, doi:10.1038/sj.bjp.0706391 (2005).

102. Lepicier, P., Bouchard, J. F., Lagneux, C. \& Lamontagne, D. Endocannabinoids protect the rat isolated heart against ischaemia. Br J Pharmacol 139, 805-815, doi:10.1038/sj.bjp.0705313 (2003).

103. Mackie, K. \& Stella, N. Cannabinoid receptors and endocannabinoids: evidence for new players. The AAPS journal 8, E298-306, doi:10.1208/aapsj080234 (2006).

104. Libby, P. \& Theroux, P. Pathophysiology of coronary artery disease. Circulation 111, 3481-3488, doi:10.1161/CIRCULATIONAHA.105.537878 (2005).

105. Steffens, S. et al. Low dose oral cannabinoid therapy reduces progression of atherosclerosis in mice. Nature 434, 782-786, doi:10.1038/nature03389 (2005).

106. Buckley, N. E. et al. Immunomodulation by cannabinoids is absent in mice deficient for the cannabinoid CB(2) receptor. Eur J Pharmacol 396, 141-149 (2000).

107. Siegel, R., Naishadham, D. \& Jemal, A. Cancer statistics, 2013. CA: a cancer journal for clinicians 63, 11-30, doi:10.3322/caac.21166 (2013).

108. Preet, A. et al. Cannabinoid receptors, CB1 and CB2, as novel targets for inhibition of non-small cell lung cancer growth and metastasis. Cancer prevention research 4, 65-75, doi:10.1158/1940-6207.CAPR-10-0181 (2011).

109. Bhola, N. E. \& Grandis, J. R. Crosstalk between G-protein-coupled receptors and epidermal growth factor receptor in cancer. Frontiers in bioscience : a journal and virtual library 13, 1857-1865 (2008). 
110. Hart, S., Fischer, O. M. \& Ullrich, A. Cannabinoids induce cancer cell proliferation via tumor necrosis factor alpha-converting enzyme (TACE/ADAM17)-mediated transactivation of the epidermal growth factor receptor. Cancer research 64, 1943-1950 (2004).

111. Barsky, S. H., Roth, M. D., Kleerup, E. C., Simmons, M. \& Tashkin, D. P. Histopathologic and molecular alterations in bronchial epithelium in habitual smokers of marijuana, cocaine, and/or tobacco. Journal of the National Cancer Institute 90, 1198-1205 (1998).

112. Sanchez, M. G., Sanchez, A. M., Ruiz-Llorente, L. \& Diaz-Laviada, I. Enhancement of androgen receptor expression induced by (R)-methanandamide in prostate LNCaP cells. FEBS letters 555, 561-566 (2003).

113. Sanchez, M. G., Ruiz-Llorente, L., Sanchez, A. M. \& Diaz-Laviada, I. Activation of phosphoinositide 3-kinase/PKB pathway by $\mathrm{CB}(1)$ and $\mathrm{CB}(2)$ cannabinoid receptors expressed in prostate PC-3 cells. Involvement in Raf-1 stimulation and NGF induction. Cellular signalling 15, 851-859 (2003).

114. Mimeault, M., Pommery, N., Wattez, N., Bailly, C. \& Henichart, J. P. Antiproliferative and apoptotic effects of anandamide in human prostatic cancer cell lines: implication of epidermal growth factor receptor down-regulation and ceramide production. The Prostate 56, 1-12, doi:10.1002/pros.10190 (2003).

115. Nithipatikom, K., Isbell, M. A., Endsley, M. P., Woodliff, J. E. \& Campbell, W. B. Anti-proliferative effect of a putative endocannabinoid, 2-arachidonylglyceryl ether in prostate carcinoma cells. Prostaglandins \& other lipid mediators 94, 3443, doi:10.1016/j.prostaglandins.2010.12.002 (2011).

116. McKallip, R. J., Nagarkatti, M. \& Nagarkatti, P. S. Delta-9-tetrahydrocannabinol enhances breast cancer growth and metastasis by suppression of the antitumor immune response. J Immunol 174, 3281-3289 (2005).

117. Takeda, S., Yamamoto, I. \& Watanabe, K. Modulation of Delta9tetrahydrocannabinol-induced MCF-7 breast cancer cell growth by cyclooxygenase and aromatase. Toxicology 259, 25-32, doi:10.1016/j.tox.2009.01.024 (2009).

118. von Bueren, A. O., Schlumpf, M. \& Lichtensteiger, W. Delta(9)tetrahydrocannabinol inhibits 17 beta-estradiol-induced proliferation and fails to activate androgen and estrogen receptors in MCF7 human breast cancer cells. Anticancer research 28, 85-89 (2008).

119. McAllister, S. D., Christian, R. T., Horowitz, M. P., Garcia, A. \& Desprez, P. Y. Cannabidiol as a novel inhibitor of Id-1 gene expression in aggressive breast cancer cells. Molecular cancer therapeutics 6, 2921-2927, doi:10.1158/15357163.MCT-07-0371 (2007).

120. Caffarel, M. M. et al. Cannabinoids reduce ErbB2-driven breast cancer progression through Akt inhibition. Molecular cancer 9, 196, doi:10.1186/14764598-9-196 (2010).

121. Jones, T. H. et al. Thresholds of focal cerebral ischemia in awake monkeys. Journal of neurosurgery 54, 773-782, doi:10.3171/jns.1981.54.6.0773 (1981).

122. Garcia, J. H., Liu, K. F., Yoshida, Y., Chen, S. \& Lian, J. Brain microvessels: factors altering their patency after the occlusion of a middle cerebral artery (Wistar rat). The American journal of pathology 145, 728-740 (1994). 
123. Dirnagl, U., Iadecola, C. \& Moskowitz, M. A. Pathobiology of ischaemic stroke: an integrated view. Trends in neurosciences 22, 391-397 (1999).

124. Massi, P., Vaccani, A. \& Parolaro, D. Cannabinoids, immune system and cytokine network. Current pharmaceutical design 12, 3135-3146 (2006).

125. Rossi, B. et al. Inverse agonism of cannabinoid CB1 receptor blocks the adhesion of encephalitogenic $\mathrm{T}$ cells in inflamed brain venules by a protein kinase Adependent mechanism. Journal of neuroimmunology 233, 97-105, doi:10.1016/j.jneuroim.2010.12.005 (2011).

126. Kaplan, B. L., Springs, A. E. \& Kaminski, N. E. The profile of immune modulation by cannabidiol (CBD) involves deregulation of nuclear factor of activated T cells (NFAT). Biochemical pharmacology 76, 726-737, doi:10.1016/j.bcp.2008.06.022 (2008).

127. Mnich, S. J., Hiebsch, R. R., Huff, R. M. \& Muthian, S. Anti-inflammatory properties of CB1-receptor antagonist involves beta2 adrenoceptors. The Journal of pharmacology and experimental therapeutics 333, 445-453, doi:10.1124/jpet.109.163998 (2010).

128. Newton, C. A., Chou, P. J., Perkins, I. \& Klein, T. W. CB(1) and CB(2) cannabinoid receptors mediate different aspects of delta-9-tetrahydrocannabinol (THC)-induced T helper cell shift following immune activation by Legionella pneumophila infection. Journal of neuroimmune pharmacology : the official journal of the Society on NeuroImmune Pharmacology 4, 92-102, doi:10.1007/s11481-008-9126-2 (2009).

129. Borner, C., Hollt, V. \& Kraus, J. Activation of human T cells induces upregulation of cannabinoid receptor type 1 transcription. Neuroimmunomodulation 14, 281-286, doi:10.1159/000117809 (2007).

130. Schwarz, H., Blanco, F. J. \& Lotz, M. Anadamide, an endogenous cannabinoid receptor agonist inhibits lymphocyte proliferation and induces apoptosis. Journal of neuroimmunology 55, 107-115 (1994).

131. Carayon, P. et al. Modulation and functional involvement of CB2 peripheral cannabinoid receptors during B-cell differentiation. Blood 92, 3605-3615 (1998).

132. Mestre, L. et al. A cannabinoid agonist interferes with the progression of a chronic model of multiple sclerosis by downregulating adhesion molecules. Molecular and cellular neurosciences 40, 258-266, doi:10.1016/j.mcn.2008.10.015 (2009).

133. Stella, N. Cannabinoid and cannabinoid-like receptors in microglia, astrocytes, and astrocytomas. Glia 58, 1017-1030, doi:10.1002/glia.20983 (2010).

134. Nunez, E. et al. Glial expression of cannabinoid $\mathrm{CB}(2)$ receptors and fatty acid amide hydrolase are beta amyloid-linked events in Down's syndrome. Neuroscience 151, 104-110, doi:10.1016/j.neuroscience.2007.10.029 (2008).

135. Onaivi, E. S. Neuropsychobiological evidence for the functional presence and expression of cannabinoid CB2 receptors in the brain. Neuropsychobiology 54, 231-246, doi:10.1159/000100778 (2006).

136. Ashton, J. C. \& Glass, M. The cannabinoid CB2 receptor as a target for inflammation-dependent neurodegeneration. Current neuropharmacology 5, 7380 (2007). 
137. Carlisle, S. J., Marciano-Cabral, F., Staab, A., Ludwick, C. \& Cabral, G. A. Differential expression of the $\mathrm{CB} 2$ cannabinoid receptor by rodent macrophages and macrophage-like cells in relation to cell activation. International immunopharmacology 2, 69-82 (2002).

138. Maresz, K. et al. Direct suppression of CNS autoimmune inflammation via the cannabinoid receptor $\mathrm{CB} 1$ on neurons and CB2 on autoreactive T cells. Nature medicine 13, 492-497, doi:10.1038/nm1561 (2007).

139. Daaka, Y., Friedman, H. \& Klein, T. W. Cannabinoid receptor proteins are increased in Jurkat, human T-cell line after mitogen activation. The Journal of pharmacology and experimental therapeutics 276, 776-783 (1996).

140. Klein, T. W., Newton, C., Zhu, W., Daaka, Y. \& Friedman, H. delta 9Tetrahydrocannabinol, cytokines, and immunity to Legionella pneumophila. Proceedings of the Society for Experimental Biology and Medicine. Society for Experimental Biology and Medicine 209, 205-212 (1995).

141. Zarruk, J. G. et al. Cannabinoid type 2 receptor activation downregulates strokeinduced classic and alternative brain macrophage/microglial activation concomitant to neuroprotection. Stroke; a journal of cerebral circulation 43, 211219, doi:10.1161/STROKEAHA.111.631044 (2012).

142. Zhang, M. et al. CB2 receptor activation attenuates microcirculatory dysfunction during cerebral ischemic/reperfusion injury. Microvascular research 78, 86-94, doi:10.1016/j.mvr.2009.03.005 (2009).

143. Ren, X., Akiyoshi, K., Vandenbark, A. A., Hurn, P. D. \& Offner, H. $\mathrm{CD} 4+\mathrm{FoxP} 3+$ regulatory T-cells in cerebral ischemic stroke. Metabolic brain disease 26, 87-90, doi:10.1007/s11011-010-9226-6 (2011).

144. Hug, A. et al. Reduced efficacy of circulating costimulatory cells after focal cerebral ischemia. Stroke; a journal of cerebral circulation 42, 3580-3586, doi:10.1161/STROKEAHA.111.620948 (2011).

145. Hegde, V. L. et al. Attenuation of experimental autoimmune hepatitis by exogenous and endogenous cannabinoids: involvement of regulatory $\mathrm{T}$ cells. Molecular pharmacology 74, 20-33, doi:10.1124/mol.108.047035 (2008).

146. Wacnik, P. W. et al. Cannabinoids affect dendritic cell (DC) potassium channel function and modulate DC T cell stimulatory capacity. J Immunol 181, 3057-3066 (2008).

147. Tanikawa, T., Kurohane, K. \& Imai, Y. Regulatory effect of cannabinoid receptor agonist on chemokine-induced lymphocyte chemotaxis. Biological \& pharmaceutical bulletin 34, 1090-1093 (2011).

148. Lee, M., Yang, K. H. \& Kaminski, N. E. Effects of putative cannabinoid receptor ligands, anandamide and 2-arachidonyl-glycerol, on immune function in $\mathrm{B} 6 \mathrm{C} 3 \mathrm{~F} 1$ mouse splenocytes. The Journal of pharmacology and experimental therapeutics 275, 529-536 (1995).

149. Wiegand, F. et al. Respiratory chain inhibition induces tolerance to focal cerebral ischemia. Journal of cerebral blood flow and metabolism : official journal of the International Society of Cerebral Blood Flow and Metabolism 19, 1229-1237, doi:10.1097/00004647-199911000-00007 (1999). 
150. Zhang, Y. \& Lipton, P. Cytosolic Ca2+ changes during in vitro ischemia in rat hippocampal slices: major roles for glutamate and $\mathrm{Na}+$-dependent $\mathrm{Ca} 2+$ release from mitochondria. The Journal of neuroscience : the official journal of the Society for Neuroscience 19, 3307-3315 (1999).

151. Chen, Y., Tsai, Y. H. \& Tseng, S. H. The potential of tetrandrine as a protective agent for ischemic stroke. Molecules 16, 8020-8032, doi:10.3390/molecules16098020 (2011).

152. Mach, F., Montecucco, F. \& Steffens, S. Cannabinoid receptors in acute and chronic complications of atherosclerosis. Br J Pharmacol 153, 290-298, doi:10.1038/sj.bjp.0707517 (2008).

153. Pegorini, S., Zani, A., Braida, D., Guerini-Rocco, C. \& Sala, M. Vanilloid VR1 receptor is involved in rimonabant-induced neuroprotection. Br J Pharmacol 147, 552-559, doi:10.1038/sj.bjp.0706656 (2006).

154. Panikashvili, D., Mechoulam, R., Beni, S. M., Alexandrovich, A. \& Shohami, E. CB1 cannabinoid receptors are involved in neuroprotection via NF-kappa B inhibition. Journal of cerebral blood flow and metabolism : official journal of the International Society of Cerebral Blood Flow and Metabolism 25, 477-484, doi:10.1038/sj.jcbfm.9600047 (2005).

155. Du, J. et al. Involvement of ERK $1 / 2$ activation in electroacupuncture pretreatment via cannabinoid CB1 receptor in rats. Brain research 1360, 1-7, doi:10.1016/j.brainres.2010.07.034 (2010).

156. Mathew, R. J. et al. Time course of tetrahydrocannabinol-induced changes in regional cerebral blood flow measured with positron emission tomography. Psychiatry research 116, 173-185 (2002).

157. Mateo, I., Pinedo, A., Gomez-Beldarrain, M., Basterretxea, J. M. \& GarciaMonco, J. C. Recurrent stroke associated with cannabis use. Journal of neurology, neurosurgery, and psychiatry 76, 435-437, doi:10.1136/jnnp.2004.042382 (2005).

158. Wolff, V. et al. Cannabis use, ischemic stroke, and multifocal intracranial vasoconstriction: a prospective study in 48 consecutive young patients. Stroke; a journal of cerebral circulation 42, 1778-1780, doi:10.1161/STROKEAHA.110.610915 (2011).

159. Minati, L., Edginton, T., Bruzzone, M. G. \& Giaccone, G. Current concepts in Alzheimer's disease: a multidisciplinary review. American journal of Alzheimer's disease and other dementias 24, 95-121, doi:10.1177/1533317508328602 (2009).

160. Bayer, T. A., Buslei, R., Havas, L. \& Falkai, P. Evidence for activation of microglia in patients with psychiatric illnesses. Neurosci Lett 271, 126-128 (1999).

161. Heneka, M. T. \& O'Banion, M. K. Inflammatory processes in Alzheimer's disease. Journal of neuroimmunology 184, 69-91, doi:10.1016/j.jneuroim.2006.11.017 (2007).

162. Pratico, D. Evidence of oxidative stress in Alzheimer's disease brain and antioxidant therapy: lights and shadows. Annals of the New York Academy of Sciences 1147, 70-78, doi:10.1196/annals.1427.010 (2008).

163. Jo, D. G. et al. Evidence that gamma-secretase mediates oxidative stress-induced beta-secretase expression in Alzheimer's disease. Neurobiology of aging 31, 917925, doi:10.1016/j.neurobiolaging.2008.07.003 (2010). 
164. Riedel, G. \& Davies, S. N. Cannabinoid function in learning, memory and plasticity. Handbook of experimental pharmacology, 445-477 (2005).

165. Ramirez, B. G., Blazquez, C., Gomez del Pulgar, T., Guzman, M. \& de Ceballos, M. L. Prevention of Alzheimer's disease pathology by cannabinoids: neuroprotection mediated by blockade of microglial activation. The Journal of neuroscience : the official journal of the Society for Neuroscience 25, 1904-1913, doi:10.1523/JNEUROSCI.4540-04.2005 (2005).

166. Westlake, T. M., Howlett, A. C., Bonner, T. I., Matsuda, L. A. \& Herkenham, M. Cannabinoid receptor binding and messenger RNA expression in human brain: an in vitro receptor autoradiography and in situ hybridization histochemistry study of normal aged and Alzheimer's brains. Neuroscience 63, 637-652 (1994).

167. Benito, C. et al. Cannabinoid CB2 receptors and fatty acid amide hydrolase are selectively overexpressed in neuritic plaque-associated glia in Alzheimer's disease brains. The Journal of neuroscience : the official journal of the Society for Neuroscience 23, 11136-11141 (2003).

168. van der Stelt, M. et al. Endocannabinoids and beta-amyloid-induced neurotoxicity in vivo: effect of pharmacological elevation of endocannabinoid levels. Cellular and molecular life sciences : CMLS 63, 1410-1424, doi:10.1007/s00018-0066037-3 (2006).

169. Nadler, V., Mechoulam, R. \& Sokolovsky, M. Blockade of 45Ca2+ influx through the N-methyl-D-aspartate receptor ion channel by the non-psychoactive cannabinoid HU-211. Brain research 622, 79-85 (1993).

170. Eshhar, N., Striem, S. \& Biegon, A. HU-211, a non-psychotropic cannabinoid, rescues cortical neurones from excitatory amino acid toxicity in culture. Neuroreport 5, 237-240 (1993).

171. Ehrhart, J. et al. Stimulation of cannabinoid receptor 2 (CB2) suppresses microglial activation. Journal of neuroinflammation 2, 29, doi:10.1186/17422094-2-29 (2005).

172. Hampson, A. J., Grimaldi, M., Axelrod, J. \& Wink, D. Cannabidiol and (-)Delta9tetrahydrocannabinol are neuroprotective antioxidants. Proceedings of the National Academy of Sciences of the United States of America 95, 8268-8273 (1998).

173. Esposito, G. et al. Cannabidiol in vivo blunts beta-amyloid induced neuroinflammation by suppressing IL-1beta and iNOS expression. $\mathrm{Br} J$ Pharmacol 151, 1272-1279, doi:10.1038/sj.bjp.0707337 (2007).

174. Iuvone, T. et al. Neuroprotective effect of cannabidiol, a non-psychoactive component from Cannabis sativa, on beta-amyloid-induced toxicity in PC12 cells. Journal of neurochemistry 89, 134-141, doi:10.1111/j.1471-4159.2003.02327.x (2004).

175. Esposito, G., De Filippis, D., Carnuccio, R., Izzo, A. A. \& Iuvone, T. The marijuana component cannabidiol inhibits beta-amyloid-induced tau protein hyperphosphorylation through Wnt/beta-catenin pathway rescue in PC12 cells. Journal of molecular medicine 84, 253-258, doi:10.1007/s00109-005-0025-1 (2006).

176. Walther, S., Mahlberg, R., Eichmann, U. \& Kunz, D. Delta-9tetrahydrocannabinol for nighttime agitation in severe dementia. Psychopharmacology 185, 524-528, doi:10.1007/s00213-006-0343-1 (2006). 
177. Kirkham, T. C., Williams, C. M., Fezza, F. \& Di Marzo, V. Endocannabinoid levels in rat limbic forebrain and hypothalamus in relation to fasting, feeding and satiation: stimulation of eating by 2-arachidonoyl glycerol. Br J Pharmacol 136, 550-557, doi:10.1038/sj.bjp.0704767 (2002).

178. Hanus, L. et al. Short-term fasting and prolonged semistarvation have opposite effects on 2-AG levels in mouse brain. Brain research 983, 144-151 (2003).

179. Di Marzo, V. et al. Leptin-regulated endocannabinoids are involved in maintaining food intake. Nature 410, 822-825, doi:10.1038/35071088 (2001).

180. Izzo, A. A. et al. Peripheral endocannabinoid dysregulation in obesity: relation to intestinal motility and energy processing induced by food deprivation and refeeding. Br J Pharmacol 158, 451-461, doi:10.1111/j.1476-5381.2009.00183.x (2009).

181. Gomez, R. et al. A peripheral mechanism for CB1 cannabinoid receptordependent modulation of feeding. The Journal of neuroscience : the official journal of the Society for Neuroscience 22, $9612-9617$ (2002).

182. Matias, I., Bisogno, T. \& Di Marzo, V. Endogenous cannabinoids in the brain and peripheral tissues: regulation of their levels and control of food intake.

International journal of obesity 30 Suppl 1, S7-S12, doi:10.1038/sj.ijo.0803271 (2006).

183. Pagano, C. et al. The endogenous cannabinoid system stimulates glucose uptake in human fat cells via phosphatidylinositol 3-kinase and calcium-dependent mechanisms. The Journal of clinical endocrinology and metabolism 92, 48104819, doi:10.1210/jc.2007-0768 (2007).

184. Yan, Z. C. et al. Exercise reduces adipose tissue via cannabinoid receptor type 1 which is regulated by peroxisome proliferator-activated receptor-delta.

Biochemical and biophysical research communications 354, 427-433, doi:10.1016/j.bbrc.2006.12.213 (2007).

185. D'Eon, T. M. et al. The role of adipocyte insulin resistance in the pathogenesis of obesity-related elevations in endocannabinoids. Diabetes 57, 1262-1268, doi:10.2337/db07-1186 (2008).

186. Murdolo, G. et al. Insulin differentially modulates the peripheral endocannabinoid system in human subcutaneous abdominal adipose tissue from lean and obese individuals. Journal of endocrinological investigation 30, RC17-21 (2007).

187. Starowicz, K. M. et al. Endocannabinoid dysregulation in the pancreas and adipose tissue of mice fed with a high-fat diet. Obesity 16, 553-565, doi:10.1038/oby.2007.106 (2008).

188. Bellocchio, L., Cervino, C., Vicennati, V., Pasquali, R. \& Pagotto, U. Cannabinoid type 1 receptor: another arrow in the adipocytes' bow. Journal of neuroendocrinology 20 Suppl 1, 130-138, doi:10.1111/j.1365-2826.2008.01682.x (2008).

189. Bensaid, M. et al. The cannabinoid CB1 receptor antagonist SR141716 increases Acrp30 mRNA expression in adipose tissue of obese fa/fa rats and in cultured adipocyte cells. Molecular pharmacology 63, 908-914 (2003).

190. Jbilo, O. et al. The CB1 receptor antagonist rimonabant reverses the diet-induced obesity phenotype through the regulation of lipolysis and energy balance. FASEB journal : official publication of the Federation of American Societies for Experimental Biology 19, 1567-1569, doi:10.1096/fj.04-3177fje (2005). 
191. Wagner, I. V., Perwitz, N., Drenckhan, M., Lehnert, H. \& Klein, J. Cannabinoid type 1 receptor mediates depot-specific effects on differentiation, inflammation and oxidative metabolism in inguinal and epididymal white adipocytes. Nutrition and Diabetes 1, e16 (2011).

192. Bermudez-Siva, F. J. et al. Activation of cannabinoid CB1 receptors induces glucose intolerance in rats. Eur J Pharmacol 531, 282-284, doi:10.1016/j.ejphar.2005.12.016 (2006).

193. Getty-Kaushik, L. et al. The CB1 antagonist rimonabant decreases insulin hypersecretion in rat pancreatic islets. Obesity 17, 1856-1860, doi:10.1038/oby.2009.234 (2009).

194. Osei-Hyiaman, D. et al. Hepatic CB1 receptor is required for development of diet-induced steatosis, dyslipidemia, and insulin and leptin resistance in mice. The Journal of clinical investigation 118, 3160-3169, doi:10.1172/JCI34827 (2008).

195. Cavuoto, P., McAinch, A. J., Hatzinikolas, G., Cameron-Smith, D. \& Wittert, G. A. Effects of cannabinoid receptors on skeletal muscle oxidative pathways. Molecular and cellular endocrinology 267, 63-69, doi:10.1016/j.mce.2006.12.038 (2007).

196. Esposito, I. et al. The cannabinoid CB1 receptor antagonist rimonabant stimulates 2-deoxyglucose uptake in skeletal muscle cells by regulating the expression of phosphatidylinositol-3-kinase. Molecular pharmacology 74, 1678-1686, doi:10.1124/mol.108.049205 (2008).

197. Gary-Bobo, M. et al. Rimonabant reduces obesity-associated hepatic steatosis and features of metabolic syndrome in obese Zucker fa/fa rats. Hepatology 46, 122 129, doi:10.1002/hep.21641 (2007).

198. Janiak, P. et al. Blockade of cannabinoid CB1 receptors improves renal function, metabolic profile, and increased survival of obese Zucker rats. Kidney international 72, 1345-1357, doi:10.1038/sj.ki.5002540 (2007).

199. Despres, J. P. \& Lemieux, I. Abdominal obesity and metabolic syndrome. Nature 444, 881-887, doi:10.1038/nature05488 (2006).

200. Annuzzi, G. et al. Differential alterations of the concentrations of endocannabinoids and related lipids in the subcutaneous adipose tissue of obese diabetic patients. Lipids Health Dis 9, doi: 10.1186/1476-511x-9-43 (2010).

201. Bluher, M. et al. Dysregulation of the peripheral and adipose tissue endocannabinoid system in human abdominal obesity. Diabetes 55, 3053-3060, doi:10.2337/db06-0812 (2006).

202. Cote, M. et al. Circulating endocannabinoid levels, abdominal adiposity and related cardiometabolic risk factors in obese men. International journal of obesity 31, 692-699, doi:10.1038/sj.ijo.0803539 (2007).

203. Di Marzo, V. et al. Changes in plasma endocannabinoid levels in viscerally obese men following a 1 year lifestyle modification programme and waist circumference reduction: associations with changes in metabolic risk factors. Diabetologia 52, 213-217, doi:10.1007/s00125-008-1178-6 (2009).

204. Despres, J. P., Golay, A., Sjostrom, L. \& Rimonabant in Obesity-Lipids Study, G. Effects of rimonabant on metabolic risk factors in overweight patients with dyslipidemia. The New England journal of medicine 353, 2121-2134, doi:10.1056/NEJMoa044537 (2005). 
205. Scheen, A. J. et al. Efficacy and tolerability of rimonabant in overweight or obese patients with type 2 diabetes: a randomised controlled study. Lancet 368, 16601672, doi:10.1016/S0140-6736(06)69571-8 (2006).

206. Pi-Sunyer, F. X. et al. Effect of rimonabant, a cannabinoid-1 receptor blocker, on weight and cardiometabolic risk factors in overweight or obese patients: RIONorth America: a randomized controlled trial. Jama 295, 761-775, doi:10.1001/jama.295.7.761 (2006).

207. Van Gaal, L. F., Rissanen, A. M., Scheen, A. J., Ziegler, O. \& Rössner, S. Effects of the cannabinoid-1 receptor blocker rimonabant on weight reduction and cardiovascular risk factors in overweight patients: 1-year experience from the RIO-Europe study. The Lancet 365, 1389-1397, doi:10.1016/S01406736(05)66374-X (2005).

208. Aronne, L. J. et al. A clinical trial assessing the safety and efficacy of taranabant, a CB1R inverse agonist, in obese and overweight patients: a high-dose study. International journal of obesity 34, 919-935, doi:10.1038/ijo.2010.21 (2010).

209. Kipnes, M. S. et al. A one-year study to assess the safety and efficacy of the CB1R inverse agonist taranabant in overweight and obese patients with type 2 diabetes. Diabetes, Obesity and Metabolism 12, 517-531, doi:10.1111/j.14631326.2009.01188.x (2010).

210. Proietto, J. et al. A clinical trial assessing the safety and efficacy of the CB1R inverse agonist taranabant in obese and overweight patients: low-dose study. Int $J$ Obes 34, 1243-1254, doi:http://www.nature.com/ijo/journal/v34/n8/suppinfo/ijo201038s1.html (2010).

211. Kang, J. G. \& Park, C. Y. Anti-Obesity Drugs: A Review about Their Effects and Safety. Diabetes \& metabolism journal 36, 13-25, doi:10.4093/dmj.2012.36.1.13 (2012).

212. Pettypiece, S. Pfizer Halts Obesity Drug on Risk of U.S. Rejection (Update1), $<$ http://www.bloomberg.com/apps/news?pid=newsarchive\&sid=aevA_B.eWlOA \&refer $=$ healthcare\&tsid $=1>(2008)$.

213. Van Gaal, L., Pi-Sunyer, X., Després, J.-P., McCarthy, C. \& Scheen, A. Efficacy and Safety of Rimonabant for Improvement of Multiple Cardiometabolic Risk Factors in Overweight/Obese Patients: Pooled 1-year data from the Rimonabant in Obesity (RIO) program. Diabetes Care 31, S229-S240, doi:10.2337/dc08-s258 (2008).

214. Tam, J. et al. Peripheral CB1 cannabinoid receptor blockade improves cardiometabolic risk in mouse models of obesity. The Journal of clinical investigation 120, 2953-2966, doi:10.1172/JCI42551 (2010).

215. Harrison, C. \& Traynor, J. R. The [35S]GTPgammaS binding assay: approaches and applications in pharmacology. Life Sci 74, 489-508 (2003).

216. Bokoch, G. M., Katada, T., Northup, J. K., Ui, M. \& Gilman, A. G. Purification and properties of the inhibitory guanine nucleotide-binding regulatory component of adenylate cyclase. The Journal of biological chemistry 259, 3560-3567 (1984).

217. Sternweis, P. C. \& Robishaw, J. D. Isolation of two proteins with high affinity for guanine nucleotides from membranes of bovine brain. The Journal of biological chemistry 259, 13806-13813 (1984). 
218. Breivogel, C. S., Selley, D. E. \& Childers, S. R. Cannabinoid receptor agonist efficacy for stimulating [35S]GTPgammaS binding to rat cerebellar membranes correlates with agonist-induced decreases in GDP affinity. The Journal of biological chemistry 273, 16865-16873 (1998).

219. Mcguinness, D. et al. Characterizing Cannabinoid CB 2 Receptor Ligands Using DiscoveRx PathHunter $r^{\mathrm{TM}} \beta$-Arrestin Assay. Journal of Biomolecular Screening 14, 49-58, doi:10.1177/1087057108327329 (2009).

220. Atwood, B. K., Wager-Miller, J., Haskins, C., Straiker, A. \& Mackie, K. Functional selectivity in $\mathrm{CB}(2)$ cannabinoid receptor signaling and regulation: implications for the therapeutic potential of $\mathrm{CB}(2)$ ligands. Molecular pharmacology 81, 250-263, doi:10.1124/mol.111.074013 (2012).

221. van der Lee, M. M. C. et al. Pharmacological Characterization of Receptor Redistribution and $\beta$-Arrestin Recruitment Assays for the Cannabinoid Receptor 1. Journal of Biomolecular Screening 14, 811-823, doi:10.1177/1087057109337937 (2009).

222. Thomas, B. F., Gilliam, A. F., Burch, D. F., Roche, M. J. \& Seltzman, H. H. Comparative receptor binding analyses of cannabinoid agonists and antagonists. The Journal of pharmacology and experimental therapeutics 285, 285-292 (1998).

223. Hesley, J., Daijo, J. \& Ferguson, A. T. Stable, sensitive, fluorescence-based method for detecting cAMP. BioTechniques 33, 691-694 (2002).

224. Bevan, N. et al. Functional analysis of a human A1 adenosine receptor/green fluorescent protein/Gil $\alpha$ fusion protein following stable expression in $\mathrm{CHO}$ cells. FEBS letters 462, 61-65, doi:10.1016/S0014-5793(99)01467-2 (1999).

225. Kull, B. et al. Differences in the order of potency for agonists but not antagonists at human and rat adenosine A2A receptors. Biochemical pharmacology 57, 65-75, doi:10.1016/S0006-2952(98)00298-6 (1999).

226. Schulte, G. \& Fredholm, B. B. Human adenosine A(1), A(2A), A(2B), and A(3) receptors expressed in Chinese hamster ovary cells all mediate the phosphorylation of extracellular-regulated kinase 1/2. Molecular pharmacology 58, 477-482 (2000).

227. Salvatore, C. A., Jacobson, M. A., Taylor, H. E., Linden, J. \& Johnson, R. G. Molecular cloning and characterization of the human A3 adenosine receptor. Proceedings of the National Academy of Sciences of the United States of America 90, 10365-10369 (1993).

228. Seibold, A. et al. Localization of the Sites Mediating Desensitization of the $\beta 2$ Adrenergic Receptor by the GRK Pathway. Molecular pharmacology 58, 1162 1173, doi:10.1124/mol.58.5.1162 (2000).

229. Prystay, L., Gagne, A., Kasila, P., Yeh, L.-A. \& Banks, P. Homogeneous CellBased Fluorescence Polarization Assay for the Direct Detection of cAMP. Journal of Biomolecular Screening 6, 75-82, doi:10.1177/108705710100600203 (2001).

230. Kariv, I. I., Stevens, M. E., Behrens, D. L. \& Oldenburg, K. R. High Throughput Quantitation of cAMP Production Mediated by Activation of Seven Transmembrane Domain Receptors. J Biomol Screen 4, 27-32 (1999).

231. Wills, S. Drugs of abuse. 2nd edn, (Pharmaceutical Press, 2005). 
232. Izzo, A. A., Mascolo, N., Pinto, L., Capasso, R. \& Capasso, F. The role of cannabinoid receptors in intestinal motility, defaecation and diarrhoea in rats. Eur $J$ Pharmacol 384, 37-42 (1999).

233. Shook, J. E. \& Burks, T. F. Psychoactive cannabinoids reduce gastrointestinal propulsion and motility in rodents. The Journal of pharmacology and experimental therapeutics 249, 444-449 (1989).

234. Nadipuram, A. K., Krishnamurthy, M., Ferreira, A. M., Li, W. \& Moore Ii, B. M. Synthesis and testing of novel classical cannabinoids: exploring the side chain ligand binding pocket of the $\mathrm{CB} 1$ and CB2 receptors. Bioorg Med Chem 11, 3121-3132, doi:10.1016/S0968-0896(03)00238-4 (2003).

235. Krishnamurthy, M., Gurley, S. \& Moore Ii, B. M. Exploring the substituent effects on a novel series of $\mathrm{Cl}^{\prime}$-dimethyl-aryl $\Delta 8$-tetrahydrocannabinol analogs. Bioorg Med Chem 16, 6489-6500, doi:10.1016/j.bmc.2008.05.034 (2008).

236. Martin, B. R. et al. Manipulation of the Tetrahydrocannabinol Side Chain Delineates Agonists, Partial Agonists, and Antagonists. Journal of Pharmacology and Experimental Therapeutics 290, 1065-1079 (1999).

237. Papahatjis, D. P., Kourouli, T., Abadji, V., Goutopoulos, A. \& Makriyannis, A. Pharmacophoric Requirements for Cannabinoid Side Chains: Multiple Bond and C1 '-Substituted $\Delta 8$-Tetrahydrocannabinols. Journal of Medicinal Chemistry 41, 1195-1200, doi:10.1021/jm970277i (1998).

238. Busch-Petersen, J. et al. Unsaturated side chain beta-11hydroxyhexahydrocannabinol analogs. Journal of Medicinal Chemistry 39, 37903796, doi:10.1021/jm950934b (1996).

239. Griffin, G. et al. Separation of cannabinoid receptor affinity and efficacy in delta8-tetrahydrocannabinol side-chain analogues. Br J Pharmacol 132, 525-535, doi:10.1038/sj.bjp.0703827 (2001).

240. Martin, B. R., Razdan, R. K. \& Pertwee, R. G. Sulfonamide Cannabinoid Agonists and Antagoinists. US Patent (2005).

241. Crocker, P. J. et al. Development of agonists, partial agonists and antagonists in the $\Delta 8$-Tetrahydrocannabinol series. Tetrahedron 55, 13907-13926, doi:10.1016/S0040-4020(99)00849-2 (1999).

242. Huffman, J. W. et al. Structure-activity relationships for $1^{\prime}, 1^{\prime}$-dimethylalkyl- $\Delta 8$ tetrahydrocannabinols. Bioorg Med Chem 11, 1397-1410, doi:10.1016/S09680896(02)00649-1 (2003).

243. Liddle, J., Huffman, J. W., Wiley, J. L. \& Martin, B. R. Enantioselective synthesis and pharmacology of 11-hydroxy-(1'S,2'R)-dimethylheptyl- $\triangle 8$-THC. Bioorganic \& Medicinal Chemistry Letters 8, 2223-2226, doi:10.1016/S0960894X(98)00385-0 (1998).

244. Singer, M., Ryan, W. J., Saha, B., Martin, B. R. \& Razdan, R. K. Potent cyano and carboxamido side-chain analogues of 1', 1'-dimethyl-delta8tetrahydrocannabinol. Journal of Medicinal Chemistry 41, 4400-4407, doi:10.1021/jm9803875 (1998).

245. Papahatjis, D. P. et al. Pharmacophoric Requirements for the Cannabinoid Side Chain. Probing the Cannabinoid Receptor Subsite at $\mathrm{C1}^{\prime}$. Journal of Medicinal Chemistry 46, 3221-3229, doi:10.1021/jm020558c (2003). 
246. Papahatjis, D. P., Nahmias, V. R., Andreou, T., Fan, P. \& Makriyannis, A.

Structural modifications of the cannabinoid side chain towards C3-aryl and 1', 1'cycloalkyl-1'-cyano cannabinoids. Bioorganic \& Medicinal Chemistry Letters 16, 1616-1620, doi:10.1016/j.bmcl.2005.12.026 (2006).

247. Lu, D., Guo, J., Duclos, R. I., Bowman, A. L. \& Makriyannis, A. Bornyl- and Isobornyl- $\Delta 8$-tetrahydrocannabinols: A Novel Class of Cannabinergic Ligands. Journal of Medicinal Chemistry 51, 6393-6399, doi:10.1021/jm8005299 (2008).

248. Lu, D. et al. Adamantyl Cannabinoids: A Novel Class of Cannabinergic Ligands. Journal of Medicinal Chemistry 48, 4576-4585, doi:10.1021/jm058175c (2005).

249. Ai, R. \& Chang, C. E. Ligand-specific homology modeling of human cannabinoid (CB1) receptor. Journal of molecular graphics \& modelling 38, 155-164, doi:10.1016/j.jmgm.2012.05.002 (2012).

250. Huffman, J. W. et al. Synthesis and pharmacology of a very potent cannabinoid lacking a phenolic hydroxyl with high affinity for the CB2 receptor. Journal of Medicinal Chemistry 39, 3875-3877, doi:10.1021/jm960394y (1996).

251. Kapur, A. et al. Mutation studies of Ser7.39 and Ser2.60 in the human CB1 cannabinoid receptor: evidence for a serine-induced bend in CB1 transmembrane helix 7. Molecular pharmacology 71, 1512-1524, doi:10.1124/mol.107.034645 (2007).

252. McAllister, S. D. et al. An aromatic microdomain at the cannabinoid CB(1) receptor constitutes an agonist/inverse agonist binding region. Journal of Medicinal Chemistry 46, 5139-5152, doi:10.1021/jm0302647 (2003).

253. Durdagi, S., Papadopoulos, M. G., Papahatjis, D. P. \& Mavromoustakos, T. Combined 3D QSAR and molecular docking studies to reveal novel cannabinoid ligands with optimum binding activity. Bioorganic \& Medicinal Chemistry Letters 17, 6754-6763, doi:10.1016/j.bmcl.2007.10.044 (2007).

254. Salo, O. M. H., Lahtela-Kakkonen, M., Gynther, J., Järvinen, T. \& Poso, A. Development of a 3D Model for the Human Cannabinoid CB1 Receptor. Journal of Medicinal Chemistry 47, 3048-3057, doi:10.1021/jm031052c (2004).

255. Shim, J.-Y., Bertalovitz, A. C. \& Kendall, D. A. Identification of Essential Cannabinoid-binding Domains: STRUCTURAL INSIGHTS INTO EARLY DYNAMIC EVENTS IN RECEPTOR ACTIVATION. Journal of Biological Chemistry 286, 33422-33435, doi:10.1074/jbc.M111.261651 (2011).

256. McAllister, S. D. et al. Structural mimicry in class A G protein-coupled receptor rotamer toggle switches: the importance of the F3.36(201)/W6.48(357) interaction in cannabinoid CB1 receptor activation. The Journal of biological chemistry $\mathbf{2 7 9}$, 48024-48037, doi:10.1074/jbc.M406648200 (2004).

257. McAllister, S. D. et al. in 2002 Symposium on the Cannabinoids 76 (Burlington Vermont International Cannabinoid Research Society, Pacific Grove, CA, 2002).

258. Barnett-Norris, J. et al. Conformational memories and the endocannabinoid binding site at the cannabinoid CB1 receptor. Journal of Medicinal Chemistry $\mathbf{4 5}$, 3649-3659 (2002).

259. Moore II, B. M. et al. Unpublished Data.

260. Reggio, P. H. et al. The bioactive conformation of aminoalkylindoles at the cannabinoid CB1 and CB2 receptors: insights gained from (E)- and (Z)naphthylidene indenes. Journal of Medicinal Chemistry 41, 5177-5187, doi:10.1021/jm9801197 (1998). 
261. Song, Z. H., Slowey, C. A., Hurst, D. P. \& Reggio, P. H. The difference between the $\mathrm{CB}(1)$ and $\mathrm{CB}(2)$ cannabinoid receptors at position 5.46 is crucial for the selectivity of WIN55212-2 for CB(2). Molecular pharmacology 56, 834-840 (1999).

262. Martin, B. R. et al. Pharmacological evaluation of iodo and nitro analogs of $\Delta 8$ THC and $\triangle 9$-THC. Pharmacology Biochemistry and Behavior 46, 295-301, doi:10.1016/0091-3057(93)90356-X (1993).

263. Matsumoto, K., Stark, P. \& Meister, R. G. Cannabinoids. 1. 1-Amino- and 1mercapto-7,8,9,10-tetrahydro-6H-dibenzo [b,d]pyrans. Journal of Medicinal Chemistry 20, 17-24 (1977).

264. Burdick, D. et al. Synthesis and structure-activity relationship of substitutions at the $\mathrm{C}$-1 position of $\Delta 9$-tetrahydrocannabinol. Bioorganic \& Medicinal Chemistry Letters 20, 1424-1426, doi:10.1016/j.bmcl.2009.12.092 (2010).

265. Huffman, J. W. The search for selective ligands for the CB2 receptor. Current pharmaceutical design 6, 1323-1337 (2000).

266. Martin, B. R. et al. Assessment of structural commonality between tetrahydrocannabinol and anandamide. European Journal of Pharmacology 435, 35-42, doi:10.1016/S0014-2999(01)01527-8 (2002).

267. Huffman, J. W., Bushell, S. M., Miller, J. R. A., Wiley, J. L. \& Martin, B. R. 1Methoxy-, 1-deoxy-11-hydroxy- and 11-Hydroxy-1-methoxy- $\Delta 8$ tetrahydrocannabinols: new selective ligands for the CB2 receptor. Bioorg Med Chem 10, 4119-4129, doi:10.1016/S0968-0896(02)00331-0 (2002).

268. Gareau, Y. et al. Structure activity relationships of tetrahydrocannabinol analogues on human cannabinoid receptors. Bioorganic \& Medicinal Chemistry Letters 6, 189-194, doi:Doi 10.1016/0960-894x(95)00573-C (1996).

269. Huffman, J. W. et al. 3-(1',1'-Dimethylbutyl)-1-deoxy-delta8-THC and related compounds: synthesis of selective ligands for the CB2 receptor. Bioorg Med Chem 7, 2905-2914 (1999).

270. Huffman, J. W. et al. 1-Bromo-3-(1',1'-dimethylalkyl)-1-deoxy- $\Delta 8$ tetrahydrocannabinols: New selective ligands for the cannabinoid CB2 receptor. Bioorg Med Chem 18, 7809-7815, doi:10.1016/j.bmc.2010.09.061 (2010).

271. Melvin, L. S., Milne, G. M., Johnson, M. R., Wilken, G. H. \& Howlett, A. C. Structure-activity relationships defining the ACD-tricyclic cannabinoids: cannabinoid receptor binding and analgesic activity. Drug design and discovery 13, 155-166 (1995).

272. Pertwee, R. G. Cannabinoids. Handbook of Experimental Pharmacology, $<$ http://www.myilibrary.com?id=41243> (2005).

273. Matsumoto, K., Stark, P. \& Meister, R. G. Cannabinoids. 2. Synthesis and central nervous system activities of some B-ring homocannabinoid derivatives and related lactones. Journal of Medicinal Chemistry 20, 25-30 (1977).

274. Lee, C. M. et al. Cannabinoids. Synthesis and central nervous system activity of 8-substituted 10-hydroxy-5,5-dimethyl-5H-[1]benzopyrano[4,3-c]pyridine and derivatives. Journal of Medicinal Chemistry 20, 1508-1511 (1977).

275. Lee, C. M. et al. New azacannabinoids highly active in the central nervous system. Journal of Medicinal Chemistry 26, 278-280 (1983). 
276. Osgood, P. F., Howes, J. F., Razdan, R. K. \& Pars, H. G. Drugs derived from cannabinoids. 7. Tachycardia and analgesia structure-activity relationships in delta9-tetrahydrocannabinol and some synthetic analogues. Journal of Medicinal Chemistry 21, 809-811 (1978).

277. Khanolkar, A. D. et al. Cannabilactones: a novel class of CB2 selective agonists with peripheral analgesic activity. Journal of Medicinal Chemistry 50, 6493-6500, doi:10.1021/jm070441u (2007).

278. Blanc, J. A. et al. Adsorption losses from urine-based cannabinoid calibrators during routine use. Clinical Chemistry 39, 1705-1712 (1993).

279. Harvey, D. J. The mass spectra of the trimethylsilyl derivatives of cis- and transhexahydrocannabinol and their hydroxy and acid analogues. Biomedical mass spectrometry 8, 366-372 (1981).

280. Bell, M. R. et al. Antinociceptive (aminoalkyl)indoles. Journal of Medicinal Chemistry 34, 1099-1110 (1991).

281. D'Ambra, T. E. et al. Conformationally restrained analogues of pravadoline: nanomolar potent, enantioselective, (aminoalkyl)indole agonists of the cannabinoid receptor. Journal of Medicinal Chemistry 35, 124-135 (1992).

282. Eissenstat, M. A. et al. Aminoalkylindoles: structure-activity relationships of novel cannabinoid mimetics. Journal of Medicinal Chemistry 38, 3094-3105 (1995).

283. D'Ambra, T. E. et al. C-Attached aminoalkylindoles: potent cannabinoid mimetics. Bioorganic \& Medicinal Chemistry Letters 6, 17-22, doi:10.1016/0960894X(95)00560-G (1996).

284. Ibrahim, M. M. et al. Activation of CB2 cannabinoid receptors by AM1241 inhibits experimental neuropathic pain: pain inhibition by receptors not present in the CNS. Proceedings of the National Academy of Sciences of the United States of America 100, 10529-10533, doi:10.1073/pnas.1834309100 (2003).

285. Huffman, J. W. et al. Structure-activity relationships for 1-alkyl-3-(1naphthoyl)indoles at the cannabinoid $\mathrm{CB} 1$ and $\mathrm{CB} 2$ receptors: steric and electronic effects of naphthoyl substituents. New highly selective CB2 receptor agonists. Bioorg Med Chem 13, 89-112, doi:10.1016/j.bmc.2004.09.050 (2005).

286. Malan, T. P., Jr. et al. CB2 cannabinoid receptor-mediated peripheral antinociception. Pain 93, 239-245 (2001).

287. Ibrahim, M. M. et al. CB2 cannabinoid receptor activation produces antinociception by stimulating peripheral release of endogenous opioids. Proceedings of the National Academy of Sciences of the United States of America 102, 3093-3098, doi:10.1073/pnas.0409888102 (2005).

288. Herzberg, U., Eliav, E., Bennett, G. J. \& Kopin, I. J. The analgesic effects of $\mathrm{R}(+)$-WIN 55,212-2 mesylate, a high affinity cannabinoid agonist, in a rat model of neuropathic pain. Neurosci Lett 221, 157-160 (1997).

289. Huffman, J. W., Dai, D., Martin, B. R. \& Compton, D. R. Design, Synthesis and Pharmacology of Cannabimimetic Indoles. Bioorganic \& Medicinal Chemistry Letters 4, 563-566, doi:10.1016/S0960-894X(01)80155-4 (1994).

290. Wiley, J. L. et al. Structure-activity relationships of indole- and pyrrole-derived cannabinoids. The Journal of pharmacology and experimental therapeutics 285, 995-1004 (1998). 
291. Hynes, J., Jr. et al. C-3 Amido-indole cannabinoid receptor modulators. Bioorganic \& Medicinal Chemistry Letters 12, 2399-2402 (2002).

292. Wrobleski, S. T. et al. Rational design and synthesis of an orally active indolopyridone as a novel conformationally constrained cannabinoid ligand possessing antiinflammatory properties. Journal of Medicinal Chemistry 46, 2110-2116, doi:10.1021/jm020329q (2003).

293. Compton, D. R., Gold, L. H., Ward, S. J., Balster, R. L. \& Martin, B. R. Aminoalkylindole analogs: cannabimimetic activity of a class of compounds structurally distinct from delta 9-tetrahydrocannabinol. Journal of Pharmacology and Experimental Therapeutics 263, 1118-1126 (1992).

294. Kumar, V. et al. Morpholinoalkylindenes as antinociceptive agents: Novel cannabinoid receptor agonists. Bioorganic \& Medicinal Chemistry Letters 5, 381386, doi:10.1016/0960-894X(95)00040-Z (1995).

295. Huffman, J. W. et al. 3-Indolyl-1-naphthylmethanes: new cannabimimetic indoles provide evidence for aromatic stacking interactions with the $\mathrm{CB}(1)$ cannabinoid receptor. Bioorg Med Chem 11, 539-549 (2003).

296. Burley, S. K. \& Petsko, G. A. Aromatic-aromatic interaction: a mechanism of protein structure stabilization. Science 229, 23-28 (1985).

297. Dutta, A. K. et al. Synthesis, pharmacology, and molecular modeling of novel 4alkyloxy indole derivatives related to cannabimimetic aminoalkyl indoles (AAIs). Bioorg Med Chem 5, 1591-1600 (1997).

298. Hosohata, K. et al. AM630 is a competitive cannabinoid receptor antagonist in the guinea pig brain. Life Sci 61, PL115-118 (1997).

299. Pertwee, R. et al. AM630, a competitive cannabinoid receptor antagonist. Life Sci 56, 1949-1955 (1995).

300. Ross, R. A. et al. Agonist-inverse agonist characterization at $\mathrm{CB} 1$ and $\mathrm{CB} 2$ cannabinoid receptors of L759633, L759656, and AM630. Br J Pharmacol 126, 665-672, doi:10.1038/sj.bjp.0702351 (1999).

301. Tarzia, G. et al. Synthesis and structure-activity relationships of a series of pyrrole cannabinoid receptor agonists. Bioorg Med Chem 11, 3965-3973 (2003).

302. Rinaldi-Carmona, M. et al. SR141716A, a potent and selective antagonist of the brain cannabinoid receptor. FEBS letters 350, 240-244 (1994).

303. Lan, R. et al. Structure-activity relationships of pyrazole derivatives as cannabinoid receptor antagonists. Journal of Medicinal Chemistry 42, 769-776, doi:10.1021/jm980363y (1999).

304. Nakamura-Palacios, E. M., Moerschbaecher, J. M. \& Barker, L. A. The Pharmacology of SR 141716A: A Review. CNS Drug Reviews 5, 43-58, doi:10.1111/j.1527-3458.1999.tb00085.x (1999).

305. Pertwee, R. G., Fernando, S. R., Nash, J. E. \& Coutts, A. A. Further evidence for the presence of cannabinoid CB1 receptors in guinea-pig small intestine. $\mathrm{Br} J$ Pharmacol 118, 2199-2205 (1996).

306. Bonhaus, D. W., Chang, L. K., Kwan, J. \& Martin, G. R. Dual activation and inhibition of adenylyl cyclase by cannabinoid receptor agonists: evidence for agonist-specific trafficking of intracellular responses. The Journal of pharmacology and experimental therapeutics 287, 884-888 (1998). 
307. Hajos, N. \& Freund, T. F. Pharmacological separation of cannabinoid sensitive receptors on hippocampal excitatory and inhibitory fibers. Neuropharmacology 43, 503-510 (2002).

308. Rinaldi-Carmona, M. et al. SR 144528, the first potent and selective antagonist of the CB2 cannabinoid receptor. The Journal of pharmacology and experimental therapeutics 284, 644-650 (1998).

309. Barth, F. \& Rinaldi-Carmona, M. The development of cannabinoid antagonists. Current medicinal chemistry 6, 745-755 (1999).

310. Howlett, A. C. et al. Azido- and isothiocyanato-substituted aryl pyrazoles bind covalently to the CB1 cannabinoid receptor and impair signal transduction. Journal of neurochemistry 74, 2174-2181 (2000).

311. Shim, J. Y., Welsh, W. J., Cartier, E., Edwards, J. L. \& Howlett, A. C. Molecular interaction of the antagonist $\mathrm{N}$-(piperidin-1-yl)-5-(4-chlorophenyl)-1- (2,4dichlorophenyl)-4-methyl-1H-pyrazole-3-carboxamide with the CB1 cannabinoid receptor. Journal of Medicinal Chemistry 45, 1447-1459 (2002).

312. Wiley, J. L. et al. Novel pyrazole cannabinoids: insights into CB(1) receptor recognition and activation. The Journal of pharmacology and experimental therapeutics 296, 1013-1022 (2001).

313. Katoch-Rouse, R. et al. Synthesis, structure-activity relationship, and evaluation of SR141716 analogues: development of central cannabinoid receptor ligands with lower lipophilicity. Journal of Medicinal Chemistry 46, 642-645, doi:10.1021/jm020157x (2003).

314. Krishnamurthy, M., Li, W. \& Moore, B. M., 2nd. Synthesis, biological evaluation, and structural studies on N1 and C5 substituted cycloalkyl analogues of the pyrazole class of CB1 and CB2 ligands. Bioorg Med Chem 12, 393-404 (2004).

315. Dyck, B. et al. Potent imidazole and triazole CB1 receptor antagonists related to SR141716. Bioorganic \& Medicinal Chemistry Letters 14, 1151-1154, doi:10.1016/j.bmcl.2003.12.068 (2004).

316. Francisco, M. E. et al. Synthesis and structure-activity relationships of amide and hydrazide analogues of the cannabinoid $\mathrm{CB}(1)$ receptor antagonist $\mathrm{N}$ (piperidinyl)- 5-(4-chlorophenyl)-1-(2,4-dichlorophenyl)-4-methyl-1H-pyrazole3-carboxamide (SR141716). Journal of Medicinal Chemistry 45, 2708-2719 (2002).

317. Stoit, A. R. et al. Design, synthesis and biological activity of rigid cannabinoid CB1 receptor antagonists. Chem Pharm Bull (Tokyo) 50, 1109-1113 (2002).

318. Mussinu, J. M. et al. Tricyclic pyrazoles. Part 1: synthesis and biological evaluation of novel 1,4-dihydroindeno[1,2-c]pyrazol-based ligands for CB1and CB2 cannabinoid receptors. Bioorg Med Chem 11, 251-263 (2003).

319. Lange, J. H. et al. Synthesis, biological properties, and molecular modeling investigations of novel 3,4-diarylpyrazolines as potent and selective $\mathrm{CB}(1)$ cannabinoid receptor antagonists. Journal of Medicinal Chemistry 47, 627-643, doi:10.1021/jm031019q (2004).

320. Ooms, F. et al. Exploration of the pharmacophore of 3-alkyl-5arylimidazolidinediones as new $\mathrm{CB}(1)$ cannabinoid receptor ligands and potential antagonists: synthesis, lipophilicity, affinity, and molecular modeling. Journal of Medicinal Chemistry 45, 1748-1756 (2002). 
321. Singh, R. et al. Activation of the cannabinoid CB1 receptor may involve a W6 48/F3 36 rotamer toggle switch. The journal of peptide research : official journal of the American Peptide Society 60, 357-370 (2002).

322. Ai, R. \& Chang, C.-e. A. Ligand-specific homology modeling of human cannabinoid (CB1) receptor. Journal of Molecular Graphics and Modelling 38, 155-164, doi:10.1016/j.jmgm.2012.05.002 (2012).

323. Rhee, M. H. Functional role of serine residues of transmembrane dopamin VII in signal transduction of CB2 cannabinoid receptor. Journal of veterinary science $\mathbf{3}$, 185-191 (2002).

324. Kusakabe, K. et al. Design, synthesis, and binding mode prediction of 2-pyridonebased selective CB2 receptor agonists. Bioorg Med Chem 21, 2045-2055, doi:10.1016/j.bmc.2013.01.006 (2013).

325. Kusakabe, K. et al. Selective CB2 agonists with anti-pruritic activity: Discovery of potent and orally available bicyclic 2-pyridones. Bioorg Med Chem 21, 31543163, doi:10.1016/j.bmc.2013.03.030 (2013).

326. Tiburu, E. K. et al. Structural biology of human cannabinoid receptor-2 helix 6 in membrane-mimetic environments. Biochemical and biophysical research communications 384, 243-248, doi:10.1016/j.bbrc.2009.04.099 (2009).

327. Tius, M. A. et al. Classical/non-classical cannabinoid hybrids; Stereochemical requirements for the southern hydroxyalkyl chain. Life Sci 56, 2007-2012, doi:10.1016/0024-3205(95)00182-6 (1995).

328. Tius, M. A., Makriyannis, A., Long Zoua, X. \& Abadji, V. Conformationally restricted hybrids of CP-55,940 and HHC: Stereoselective synthesis and activity. Tetrahedron 50, 2671-2680, doi:10.1016/S0040-4020(01)86983-0 (1994).

329. Drake, D. J. et al. Classical/nonclassical hybrid cannabinoids: southern aliphatic chain-functionalized C-6beta methyl, ethyl, and propyl analogues. Journal of Medicinal Chemistry 41, 3596-3608, doi:10.1021/jm960677q (1998).

330. Harrington, P. E., Stergiades, I. A., Erickson, J., Makriyannis, A. \& Tius, M. A. Synthesis of Functionalized Cannabinoids. The Journal of Organic Chemistry 65, 6576-6582, doi:10.1021/jo000716c (2000).

331. Thakur, G. A. et al. Enantiomeric resolution of a novel chiral cannabinoid receptor ligand. Journal of biochemical and biophysical methods 54, 415-422 (2002).

332. Makriyannis, A. T. S. M. C. T., Lai, X.-Z. N. R. A. S. C. T. \& Lu, D. N. R. A. S. C. T. NOVEL BIPHENYL AND BIPHENYL-LIKE CANNABINOIDS. WO patent WO 2004/017920 A2 (2004).

333. Lavey, B. J. et al. Triaryl bis-sulfones as a new class of cannabinoid CB2 receptor inhibitors: identification of a lead and initial SAR studies. Bioorganic \& Medicinal Chemistry Letters 15, 783-786, doi:10.1016/j.bmcl.2004.11.007 (2005).

334. Shankar, B. B. et al. Triaryl bis-sulfones as cannabinoid-2 receptor ligands: SAR studies. Bioorganic \& Medicinal Chemistry Letters 15, 4417-4420, doi:10.1016/j.bmcl.2005.07.023 (2005).

335. Lavey, B. J. et al. Optimization of triaryl bis-sulfones as cannabinoid-2 receptor ligands. Bioorganic \& Medicinal Chemistry Letters 17, 3760-3764, doi:10.1016/j.bmcl.2007.04.028 (2007). 
336. Sitkoff, D. F. et al. Cannabinoid CB(1) receptor ligand binding and function examined through mutagenesis studies of F200 and S383. Eur J Pharmacol 651, 9-17, doi:10.1016/j.ejphar.2010.10.056 (2011).

337. Song, Z. H. \& Bonner, T. I. A lysine residue of the cannabinoid receptor is critical for receptor recognition by several agonists but not WIN55212-2. Molecular pharmacology 49, 891-896 (1996).

338. Poso, A. \& Huffman, J. W. Targeting the cannabinoid CB2 receptor: modelling and structural determinants of CB2 selective ligands. Br J Pharmacol 153, 335346, doi:10.1038/sj.bjp.0707567 (2008). 


\section{VITA}

BRET ALAN KOERTGE WAS BORN IN THE RURAL TOWN OF VINCENNES, INDIANA IN AUGUST OF 1982. GRADUATING HIGH SCHOOL IN 2000, HE THEN COMPLETED AN A.S.

DEGREE AT VINCENNES UNIVERSITY IN 2002. FROM THERE, HIS ACADEMIC PURSUITS LED TO A B.S. In PhARMACEUTICAL SCIENCE FROM PURDUE UNIVERSITY IN 2004, THEN A

Pharm.D. From The University of TenNessee, HeAlth SCIENCE CENTER IN 2009 AND Ultimately a Ph.D. In Pharmaceutical SCIENCE FROM The UniVERSiTy OF TENNESSEE, HeAlth SCIENCE CENTER IN 2015. 